\title{
Finite basis problem for semigroups of order six
}

\author{
Edmond W. H. Lee and Wen Ting Zhang
}

Dedicated to Professor Norman R. Reilly on the occasion of his 75th birthday

\begin{abstract}
Two semigroups are distinct if they are neither isomorphic nor anti-isomorphic. Although there exist 15973 pairwise distinct semigroups of order six, only four are known to be non-finitely based. In the present article, the finite basis property of the other 15969 distinct semigroups of order six is verified. Since all semigroups of order five or less are finitely based, the four known non-finitely based semigroups of order six are the only examples of minimal order.
\end{abstract}

\section{Contents}

Part I. Introduction

1. Main result

2. Preliminaries

3. Proof of the main theorem

Part II. Sufficient conditions for the finite basis property

4. On Condition 3 . . . . . . . . . 12

5. On Condition 7 .

6. On Condition 8 . $\quad$.

7. On Condition 9 .

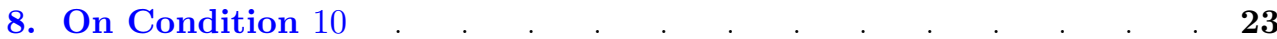

9. On Condition 14 .

10. On Condition 20 .

Part III. Finite basis property for the sporadic cases. . _ . . . $\quad$. 32

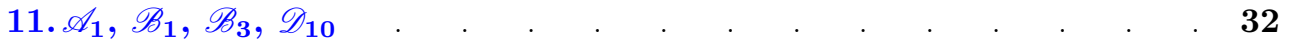

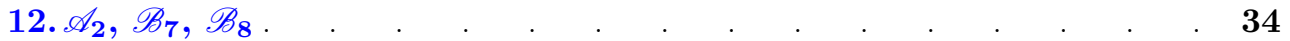

13. $\mathscr{A}_{3}, \mathscr{A}_{5}, \mathscr{A}_{6}, \mathscr{A}_{8}, \mathscr{A}_{9}, \mathscr{A}_{10}, \mathscr{G}_{4}, \mathscr{G}_{5} \quad$. $\quad . \quad . \quad . \quad . \quad . \quad . \quad . \quad . \quad . \quad 38$

14. $\mathscr{A}_{4}, \mathscr{A}_{7}, \mathscr{B}_{2}, \mathscr{G}_{3}$. . . . . . . . . . . . . . . . . . . . 40

15. $\mathscr{B}_{4}, \mathscr{B}_{5}, \mathscr{B}_{6}, \mathscr{B}_{9}, \mathscr{B}_{10}, \mathscr{E}_{1}$. . . . . . . . . . . . . . . 45

16. $\mathscr{C}_{1}, \mathscr{C}_{2}, \mathscr{C}_{3}, \mathscr{C}_{4}, \mathscr{D}_{3}, \mathscr{D}_{5}$. . . . . . . . . . . . . . . . . . . 49

$17 . \mathscr{C}_{5}, \mathscr{C}_{6}, \mathscr{C}_{10}, \mathscr{D}_{1}$. . . . . . . . . . . . . . . . . 52

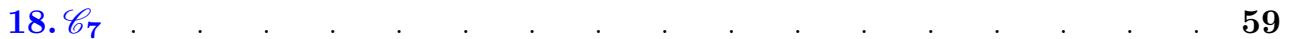

$19 . \mathscr{C}_{8}$.

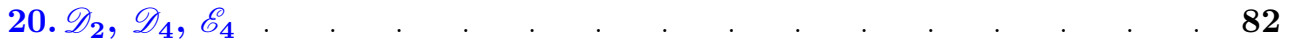

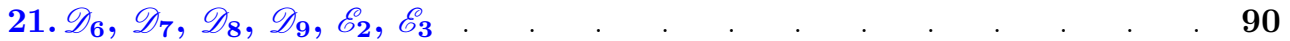

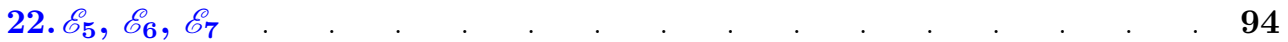

Received 5 May 2013; revised 21 June 2014.

2010 Mathematics Subject Classification 20M05 (primary), 20M07 (secondary).

W. T. Zhang, the corresponding author, was partially supported by the National Natural Science Foundation of China (nos. 10971086, 11371177, and 11401275) and the Natural Science Foundation of Gansu Province (no. 1107RJZA218). 
23. $\mathscr{E}_{8}, \mathscr{E}_{9}, \mathscr{E}_{10}, \mathscr{F}_{5}$

24. $\mathscr{F}_{1}, \mathscr{F}_{2}$.

25. $\mathscr{F}_{3}, \mathscr{F}_{4}$.

26. $\mathscr{F}_{6}, \mathscr{F}_{7}, \mathscr{F}_{8}$.

27. $\mathscr{F}_{9}, \mathscr{F}_{10}, \mathscr{G}_{1}, \mathscr{G}_{2}$.

References.

\section{PARt I. Introduction}

1. Main result

\subsection{Finite basis problem for semigroups of order five or less}

An algebra is finitely based if the identities it satisfies are finitely axiomatizable. In the 1950s, Lyndon initiated the investigation of identities satisfied by finite algebras; he first proved that all algebras of order two are finitely based [25], and shortly after exhibited a non-finitely based example with only seven elements [26]. Smaller examples of non-finitely based algebras have since been discovered $[\mathbf{3 1}, \mathbf{4 6}]$, the smallest of which are groupoids of order three, due to Ježek [11] and Murskiǔ [29]. In view of the pioneering result of Lyndon [25], non-finitely based algebras of order three are minimal with respect to being non-finitely based, or minimal non-finitely based.

The class of finite groups was the first significant class of finite algebras for which the finite basis property was established; this result, due to Oates and Powell [30], was published in 1964. In the years that followed, the finite basis property was also established for other classical finite algebras such as lattices [28], rings $[\mathbf{1 3}, \mathbf{2 4}]$, and Lie algebras [2]. The fundamental result of Oates and Powell [30] may have led to the conjecture that all finite semigroups are finitely based. But any hope for such a generalization was quickly extinguished when only a few years later, Perkins demonstrated that the monoid $B_{2}^{1}$, obtained by adjoining a unit element to the Brandt semigroup

$$
B_{2}=\left\langle a, b \mid a^{2}=b^{2}=0, a b a=a, b a b=b\right\rangle
$$

of order five, is non-finitely based [31]. The discovery of a non-finitely based semigroup with only six elements naturally focused attention upon the finite basis problem for semigroups of order less than six. This problem, first raised by Tarski [40] in 1966, attracted considerable interest $[4,8,12,41,42]$. A solution to the problem was eventually completed by Trahtman [43] in the early 1980s and published a few years later [45].

THEOREM 1.1. Every semigroup of order five or less is finitely based.

Refer to Shevrin and Volkov [39] for a more complete historical account of results leading to the proof of Theorem 1.1. This theorem is also a consequence of a recently established generalization [18].

\subsection{Minimal non-finitely based semigroups}

It follows from Theorem 1.1 that the semigroup $B_{2}^{1}$ and any other minimal non-finitely based semigroup are of order six. Since Perkins's publication of the semigroup $B_{2}^{1}$, only three other minimal non-finitely based semigroups have been discovered: the monoid $A_{2}^{1}$ obtained by adjoining a unit element to the 0 -simple semigroup

$$
A_{2}=\left\langle a, b \mid a^{2}=a b a=a, b a b=b, b^{2}=0\right\rangle,
$$


the semigroup $A_{2}^{g}$ obtained by adjoining a new element $g$ to $A_{2}$ where multiplication is given by $g^{2}=0$ and $g x=x g=g$ for all $x$ in $A_{2}$, and the $\mathscr{J}$-trivial semigroup

$$
L=\left\langle a, b \mid a^{2}=a, b^{2}=b, a b a=0\right\rangle .
$$

These four minimal non-finitely based semigroups can also be given by the following multiplication tables.

\begin{tabular}{c|ccccccc|ccccccc|c|ccccccc|ccccc}
$B_{2}^{1}$ & 1 & 2 & 3 & 4 & 5 & 6 & $A_{2}^{1}$ & 1 & 2 & 3 & 4 & 5 & 6 & $A_{2}^{g}$ & 1 & 2 & 3 & 4 & 5 & 6 & $L$ & 1 & 2 & 3 & 4 & 5 & 6 \\
\hline 1 & 1 & 1 & 1 & 1 & 1 & 1 & 1 & 1 & 1 & 1 & 1 & 1 & 1 & 1 & 1 & 1 & 1 & 1 & 1 & 6 & 1 & 1 & 1 & 1 & 1 & 1 & 1 \\
2 & 1 & 1 & 1 & 2 & 2 & 3 & 2 & 1 & 1 & 1 & 2 & 2 & 3 & 2 & 1 & 1 & 1 & 2 & 3 & 6 & 2 & 1 & 1 & 1 & 1 & 1 & 2 \\
3 & 1 & 2 & 3 & 1 & 3 & 1 & 3 & 1 & 2 & 3 & 2 & 3 & 3 & 3 & 1 & 2 & 3 & 2 & 3 & 6 & 3 & 1 & 1 & 1 & 1 & 1 & 3 \\
4 & 1 & 1 & 1 & 4 & 4 & 6 & 4 & 1 & 1 & 1 & 4 & 4 & 6 & 4 & 1 & 1 & 1 & 4 & 5 & 6 & 4 & 1 & 1 & 2 & 1 & 4 & 2 \\
5 & 1 & 2 & 3 & 4 & 5 & 6 & 5 & 1 & 2 & 3 & 4 & 5 & 6 & 5 & 1 & 4 & 5 & 4 & 5 & 6 & 5 & 1 & 1 & 3 & 1 & 5 & 3 \\
6 & 1 & 4 & 6 & 1 & 6 & 1 & 6 & 1 & 4 & 6 & 4 & 6 & 6 & 6 & 6 & 6 & 6 & 6 & 6 & 1 & 6 & 1 & 2 & 2 & 4 & 4 & 6
\end{tabular}

The non-finite basis property of the semigroup $A_{2}^{1}$ was independently established by Sapir [38] and Trahtman [44] in the 1980s. The semigroup $A_{2}^{g}$, discovered by Volkov (private communication, 3 April 2006), is non-finitely based by either Mashevitskiǔ [27] or Volkov [47]. The semigroup $L$ was recently shown to be non-finitely based by Zhang and Luo [50].

\subsection{Finite basis problem for semigroups of order six}

Two semigroups are distinct if they are neither isomorphic nor anti-isomorphic. There exist 15973 pairwise distinct semigroups of order six [33], among which 1373 are monoids [6] and 14600 are non-unital. In view of these large numbers, it is natural to question the existence of minimal non-finitely based semigroups that are distinct from the four known examples. The answer to this fundamental question clearly depends on a complete solution to the following problem.

Problem 1.2. Identify all finitely based semigroups of order six.

This problem was solved by Lee and Li for the special case of monoids. Specifically, they proved that among the 1373 pairwise distinct monoids of order six, only $B_{2}^{1}$ and $A_{2}^{1}$ are nonfinitely based semigroups [19]. The objective of the present article is to continue with the investigation by verifying that among the 14600 pairwise distinct non-unital semigroups of order six, only $A_{2}^{g}$ and $L$ are non-finitely based. A complete solution to Problem 1.2 is thus established.

Main Theorem. Among all distinct semigroups of order six, only $B_{2}^{1}, A_{2}^{1}, A_{2}^{g}$, and $L$ are non-finitely based.

Consequently, there exist precisely four distinct minimal non-finitely based semigroups. This result was first announced in Lee et al. [20].

\subsection{Organization}

The set of all sections in the present article, under the prerequisite relation, constitutes the directed tree in Figure 1; every section that follows $\S 2$ can be read independently. Notation and background information are given in $\S 2$. An outline of the proof of the main theorem is given in $\S 3$, while the finer details are deferred to $\S \S 4-27$. 


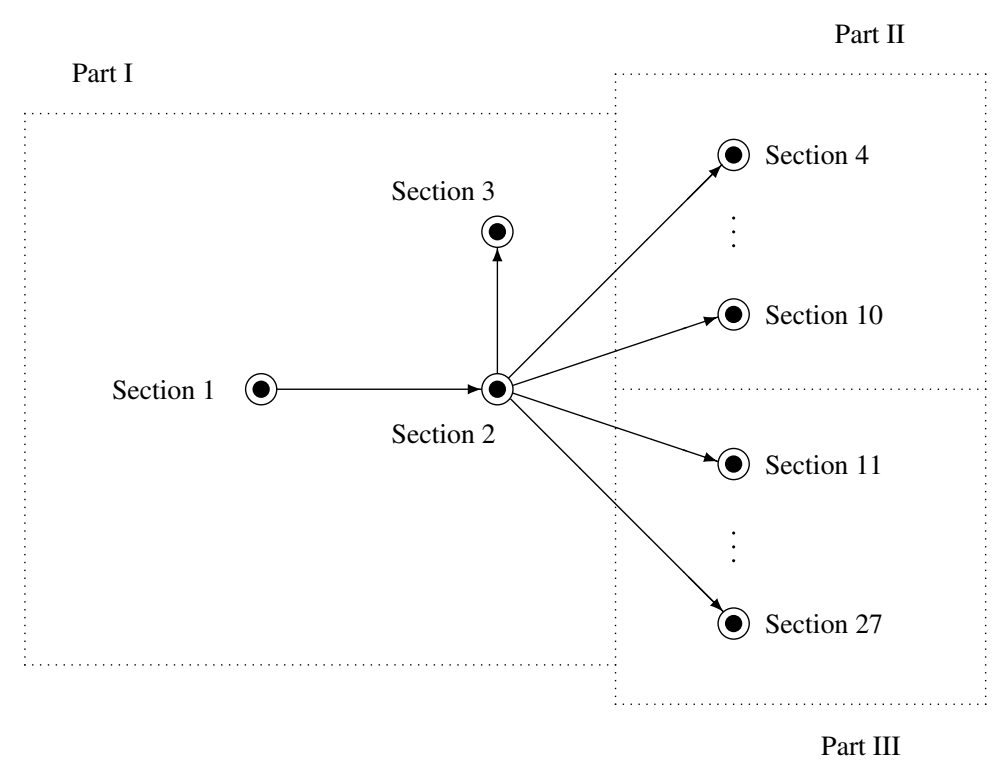

Figure 1. Prerequisites of sections.

Acknowledgements. The authors are indebted to the following colleagues: the anonymous referee for a detailed review of the article and for many helpful comments and suggestions, K. W. K. Lee for assistance in testing the 14600 non-unital semigroups of order six against Conditions 1-20 in $\S 3$ with Java, and J. D. Mitchell for independently performing the same test with GAP [7]. Special thanks are due to M. V. Volkov for invaluable advice over the years.

\section{Preliminaries}

\subsection{Words, identities, and varieties}

Let $\mathcal{X}$ be a countably infinite alphabet throughout. For any subset $\mathcal{Y}$ of $\mathcal{X}$, let $\mathcal{Y}^{+}$and $\mathcal{Y}^{*}$ denote the free semigroup and free monoid over $\mathcal{Y}$, respectively. Elements of $\mathcal{X}$ are called letters and elements of $\mathcal{X}^{*}$ are called words. For any word $\mathbf{w}$ :

- the head of $\mathbf{w}$, denoted by $\mathrm{h}(\mathbf{w})$, is the first letter occurring in $\mathbf{w}$;

- the tail of $\mathbf{w}$, denoted by $\mathrm{t}(\mathbf{w})$, is the last letter occurring in $\mathbf{w}$;

- the initial part of $\mathbf{w}$, denoted by $\operatorname{ini}(\mathbf{w})$, is the word obtained by retaining the first occurrence of each letter in w;

- the content of $\mathbf{w}$, denoted by con $(\mathbf{w})$, is the set of letters occurring in $\mathbf{w}$;

- the number of occurrences of a letter $x$ in $\mathbf{w}$ is denoted by $\operatorname{occ}(x, \mathbf{w})$;

- a letter $x$ is simple in $\mathbf{w}$ if $\operatorname{occ}(x, \mathbf{w})=1$;

- the set of simple letters of $\mathbf{w}$ is denoted by $\operatorname{sim}(\mathbf{w})$.

For any letters $x$ and $y$ of $\mathbf{w}$, let $\operatorname{occ}(x, y, \mathbf{w})$ denote the number of occurrences of $x$ before the first $y$ in $\mathbf{w}$. In other words, if $\operatorname{occ}(x, y, \mathbf{w})=m$, then retaining only the letters $x$ and $y$ in $\mathbf{w}$ results in a word in $x^{m} y\{x, y\}^{*}$. Two words $\mathbf{w}$ and $\mathbf{w}^{\prime}$ are disjoint if $\operatorname{con}(\mathbf{w}) \cap \operatorname{con}\left(\mathbf{w}^{\prime}\right)=\emptyset$.

An identity $\mathbf{w} \approx \mathbf{w}^{\prime}$ is nontrivial if $\mathbf{w} \neq \mathbf{w}^{\prime}$. A semigroup $S$ satisfies an identity $\mathbf{w} \approx \mathbf{w}^{\prime}$ if, for any substitution $\varphi: \mathcal{X} \rightarrow S$, the elements $\mathbf{w} \varphi$ and $\mathbf{w}^{\prime} \varphi$ of $S$ are equal. An identity $\mathbf{w} \approx \mathbf{w}^{\prime}$ containing one or more of the sans-serif letters from $\{\mathrm{H}, \mathrm{K}, \mathrm{T}\}$, with or without indices, represents the system that contains $\mathbf{w} \approx \mathbf{w}^{\prime}$ and any identity that can be obtained by eliminating all occurrences of some letters from $\{\mathrm{H}, \mathrm{K}, \mathrm{T}\}$. For instance, $x \mathrm{H} x \mathrm{~K} y \approx x \mathrm{H} y \mathrm{~K} x^{2}$ 
represents the system

$$
x h x k y \approx x h y k x^{2}, \quad x h x y \approx x h y x^{2}, \quad x^{2} k y \approx x y k x^{2}, \quad x^{2} y \approx x y x^{2} .
$$

Let $\Sigma$ be any set of identities. By Birkhoff's completeness theorem of equational logic [3], an identity $\mathbf{w} \approx \mathbf{w}^{\prime}$ is deducible from or implied by $\Sigma$ if some deduction sequence

$$
\mathbf{w}=\mathbf{z}_{0} \Rightarrow \mathbf{z}_{1} \Rightarrow \cdots \Rightarrow \mathbf{z}_{m}=\mathbf{w}^{\prime}
$$

exists, where each deduction $\mathbf{z}_{i} \Rightarrow \mathbf{z}_{i+1}$ involves an identity from $\Sigma$, that is, there exist words $\mathbf{e}_{i}, \mathbf{f}_{i} \in \mathcal{X}^{*}$, an identity $\mathbf{u}_{i} \approx \mathbf{v}_{i}$ in $\Sigma$, and an endomorphism $\varphi_{i}$ of $\mathcal{X}^{+}$such that $\mathbf{z}_{i}=\mathbf{e}_{i}\left(\mathbf{u}_{i} \varphi_{i}\right) \mathbf{f}_{i}$ and $\mathbf{z}_{i+1}=\mathbf{e}_{i}\left(\mathbf{v}_{i} \varphi_{i}\right) \mathbf{f}_{i}$. The deducibility of an identity $\mathbf{w} \approx \mathbf{w}^{\prime}$ from $\Sigma$ is indicated by $\mathbf{w} \underset{\sim}{\Sigma} \mathbf{w}^{\prime}$.

For any set $\Sigma$ of identities, the variety defined by $\Sigma$ is the class of all semigroups that satisfy all identities in $\Sigma$; in this case, $\Sigma$ is a basis for the variety. A variety is finitely based if it has a finite basis, and a semigroup is finitely based if it generates a finitely based variety. For any variety $\mathbf{V}$ and any set $\Sigma$ of identities, let $\mathbf{V} \Sigma$ denote the subvariety of $\mathbf{V}$ defined by $\Sigma$. For more information on universal algebra and semigroup varieties, refer to Burris and Sankappanavar [5], Shevrin and Volkov [39], and Volkov [49].

\subsection{Identities satisfied by some small semigroups}

For any nonempty subset $U$ of a semigroup $S$, the semigroup $S / U$ obtained from $S$ by identifying all elements in $U$ is called a quotient of $S$. A quotient of a subsemigroup of a semigroup $S$ is called a divisor of $S$. Any identity satisfied by a semigroup $S$ is also satisfied by all subsemigroups, quotients, and divisors of $S$.

The following small semigroups are required throughout the article:

$$
\begin{aligned}
J & =\left\langle a, b \mid a b=0, b a=a, b^{2}=b\right\rangle, \\
L_{2} & =\left\langle a, b \mid a^{2}=a b=a, b^{2}=b a=b\right\rangle, \\
R_{2} & =\left\langle a, b \mid a^{2}=b a=a, b^{2}=a b=b\right\rangle, \\
N_{2} & =\left\langle a \mid a^{2}=0\right\rangle, \\
N_{3} & =\left\langle a \mid a^{3}=0\right\rangle, \\
\mathbb{Z}_{2} & =\left\langle a \mid a^{2}=1\right\rangle .
\end{aligned}
$$

For any non-unital semigroup $S$, let $S^{1}$ denote the monoid obtained by adjoining a unit element to $S$. Let $\mathbf{J}, \mathbf{L}_{\mathbf{2}}^{\mathbf{1}}, \mathbf{N}_{\mathbf{2}}^{\mathbf{1}}, \mathbf{N}_{\mathbf{3}}, \mathbf{N}_{\mathbf{3}}^{\mathbf{1}}$ and $\mathbf{Z}_{2}$ denote the varieties generated by the semigroups $J, L_{2}^{1}$, $N_{2}^{1}, N_{3}, N_{3}^{1}$, and $\mathbb{Z}_{2}$, respectively.

Lemma 2.1. Let $\mathbf{w} \approx \mathbf{w}^{\prime}$ be any identity. Then:

(i) $L_{2}$ satisfies $\mathbf{w} \approx \mathbf{w}^{\prime}$ if and only if $\mathrm{h}(\mathbf{w})=\mathrm{h}\left(\mathbf{w}^{\prime}\right)$;

(ii) $R_{2}$ satisfies $\mathbf{w} \approx \mathbf{w}^{\prime}$ if and only if $\mathrm{t}(\mathbf{w})=\mathrm{t}\left(\mathbf{w}^{\prime}\right)$;

(iii) $L_{2}^{1}$ satisfies $\mathbf{w} \approx \mathbf{w}^{\prime}$ if and only if $\operatorname{ini}(\mathbf{w})=\operatorname{ini}\left(\mathbf{w}^{\prime}\right)$;

(iv) $N_{2}$ satisfies $\mathbf{w} \approx \mathbf{w}^{\prime}$ if and only if either $|\mathbf{w}|,\left|\mathbf{w}^{\prime}\right| \geqslant 2$ or $\mathbf{w}=\mathbf{w}^{\prime}$;

(v) $N_{2}^{1}$ satisfies $\mathbf{w} \approx \mathbf{w}^{\prime}$ if and only if $\operatorname{con}(\mathbf{w})=\operatorname{con}\left(\mathbf{w}^{\prime}\right)$ and $\operatorname{sim}(\mathbf{w})=\operatorname{sim}\left(\mathbf{w}^{\prime}\right)$;

(vi) $N_{3}$ satisfies $\mathbf{w} \approx \mathbf{w}^{\prime}$ if and only if either $|\mathbf{w}|,\left|\mathbf{w}^{\prime}\right| \geqslant 3 \operatorname{or} \operatorname{occ}(x, \mathbf{w})=\operatorname{occ}\left(x, \mathbf{w}^{\prime}\right)$ for all $x \in \mathcal{X}$

(vii) $N_{3}^{1}$ satisfies $\mathbf{w} \approx \mathbf{w}^{\prime}$ if and only if for each $x \in \mathcal{X}$, either $\operatorname{occ}(x, \mathbf{w})=\operatorname{occ}\left(x, \mathbf{w}^{\prime}\right) \leqslant 2$ or $\operatorname{occ}(x, \mathbf{w}), \operatorname{occ}\left(x, \mathbf{w}^{\prime}\right) \geqslant 3 ;$

(viii) $\mathbb{Z}_{2}$ satisfies $\mathbf{w} \approx \mathbf{w}^{\prime}$ if and only if $\operatorname{occ}(x, \mathbf{w}) \equiv \operatorname{occ}\left(x, \mathbf{w}^{\prime}\right)(\bmod 2)$ for all $x \in \mathcal{X}$.

Proof. These are easily verified. For instance, see Petrich and Reilly [32, Theorem V.1.9] for parts (i)-(iii) and Almeida [1, Lemma 6.1.4] for parts (v) and (vii). 
Lemma $2.2\left[\mathbf{1 0}\right.$, Lemma 7]. The semigroup $J$ satisfies an identity $\mathbf{w} \approx \mathbf{w}^{\prime}$ if and only if $\operatorname{con}(\mathbf{w})=\operatorname{con}\left(\mathbf{w}^{\prime}\right)$ and either one of the following conditions holds:

(i) $\mathrm{t}(\mathbf{w}) \in \operatorname{sim}(\mathbf{w}), \mathrm{t}\left(\mathbf{w}^{\prime}\right) \in \operatorname{sim}\left(\mathbf{w}^{\prime}\right)$, and $\mathrm{t}(\mathbf{w})=\mathrm{t}\left(\mathbf{w}^{\prime}\right)$;

(ii) $\mathrm{t}(\mathbf{w}) \notin \operatorname{sim}(\mathbf{w})$ and $\mathrm{t}\left(\mathbf{w}^{\prime}\right) \notin \operatorname{sim}\left(\mathbf{w}^{\prime}\right)$.

For any letters $x$ and $y$ of a word $\mathbf{w}$, write $x \prec_{\mathbf{w}} y$ to indicate that within $\mathbf{w}$, each occurrence of $x$ precedes the first $y$. In other words, if $x \prec_{\mathbf{w}} y$, then retaining only the letters $x$ and $y$ in $\mathbf{w}$ results in a word in $\{x\}^{+}\{y\}^{+}$. The simple part of a word $\mathbf{w}$, denoted by $\mathbf{w}_{\text {sim }}$, is the word obtained by retaining the simple letters of $\mathbf{w}$. For example, if $\mathbf{w}=x^{3} a b y c x z y^{2} e x t$, then $\mathbf{w}_{\text {sim }}=$ abczet.

Lemma 2.3. Suppose that $\mathbf{w} \approx \mathbf{w}^{\prime}$ is any identity satisfied by $J^{1}$. Then:

(i) $\operatorname{con}(\mathbf{w})=\operatorname{con}\left(\mathbf{w}^{\prime}\right)$ and $\operatorname{sim}(\mathbf{w})=\operatorname{sim}\left(\mathbf{w}^{\prime}\right)$;

(ii) for any letters $x \in \operatorname{con}(\mathbf{w})=\operatorname{con}\left(\mathbf{w}^{\prime}\right)$ and $y \in \operatorname{sim}(\mathbf{w})=\operatorname{sim}\left(\mathbf{w}^{\prime}\right)$, the conditions $x \prec_{\mathbf{w}} y$ and $x \prec_{\mathbf{w}^{\prime}} y$ are equivalent;

(iii) $\mathbf{w}_{\text {sim }}=\mathbf{w}_{\text {sim }}^{\prime}$.

Proof. (i) This follows from Lemma $2.1(\mathrm{v})$ because the subsemigroup $\{0, a, 1\}$ of $J^{1}$ is isomorphic to $N_{2}^{1}$.

(ii) Seeking a contradiction, suppose $x \prec_{\mathbf{w}} y$ and $x \nprec_{\mathbf{w}^{\prime}} y$ where $x \in \operatorname{con}(\mathbf{w})=\operatorname{con}\left(\mathbf{w}^{\prime}\right)$ and $y \in \operatorname{sim}(\mathbf{w})=\operatorname{sim}\left(\mathbf{w}^{\prime}\right)$. Then retaining the letters $x$ and $y$ in the identity $\mathbf{w} \approx \mathbf{w}^{\prime}$ results in an identity of the form $x^{p} y \approx x^{q} y x^{r}$ with $p, r \geqslant 1$ and $q \geqslant 0$. Since $J^{1}$ has a unit element, the semigroup $J$ satisfies $x^{p} y \approx x^{q} y x^{r}$, but this is impossible because $b^{p} a \neq b^{q} a b^{r}$ in $J$.

(iii) This is a consequence of part (ii).

\subsection{Connected words}

A word that is a single letter is called a singleton. A non-singleton word is connected if it cannot be written as a product of two nonempty disjoint words.

Let $\mathbf{A}_{\mathbf{0}}$ and $\mathbf{B}_{\mathbf{0}}$ denote the varieties generated by the following semigroups of order four:

$$
\begin{aligned}
& A_{0}=\left\langle a, b \mid a^{2}=a, b^{2}=b, b a=0\right\rangle, \\
& B_{0}=\left\langle a, b, c \mid a^{2}=a, b^{2}=b, a b=b a=0, a c=c b=c\right\rangle .
\end{aligned}
$$

Lemma 2.4. Let $\mathbf{w}$ and $\mathbf{w}^{\prime}$ be any words where $\mathbf{w}$ is connected. Then $A_{0}$ satisfies $\mathbf{w} \approx \mathbf{w}^{\prime}$ if and only if $\mathbf{w}^{\prime}$ is connected and $\operatorname{con}(\mathbf{w})=\operatorname{con}\left(\mathbf{w}^{\prime}\right)$.

Proof. This follows from Edmunds [8, proof of part 4 of the first proposition].

A semigroup $S$ is left idempotent-separable if, for any distinct $x, y \in S$, there exists an idempotent $e \in S$ such that $e x \neq e y$. A right idempotent-separable semigroup is dually defined. A semigroup is idempotent-separable if it is both left idempotent-separable and right idempotent-separable.

Lemma $2.5[\mathbf{2 1}, \S 3]$. The following semigroups are idempotent-separable: $A_{0}, B_{0}$, any monoid, and the direct product of any two idempotent-separable semigroups.

Lemma 2.6. Let $\mathbf{V}$ be the variety generated by any idempotent-separable semigroup $S$.

(i) If $A_{0} \in \mathbf{V}$, then $S$ has a basis that consists of identities formed by connected words.

(ii) If $B_{0} \in \mathbf{V}$ and $A_{0} \notin \mathbf{V}$, then $S$ has a basis that consists of identities formed by words that are products of pairwise disjoint connected words. 
Proof. This follows from Lee and Volkov [21, Proposition 3.2].

\subsection{Exclusion identities}

Let $S$ be any semigroup in a variety $\mathbf{V}$. An identity $\mathbf{w} \approx \mathbf{w}^{\prime}$ is called an exclusion identity for $S$ in $\mathbf{V}$ if the following are all satisfied:

(E1) $S$ does not satisfy $\mathbf{w} \approx \mathbf{w}^{\prime}$

(E2) any subvariety of $\mathbf{V}$ not containing $S$ satisfies $\mathbf{w} \approx \mathbf{w}^{\prime}$.

For any fixed integer $n \geqslant 2$, let $\mathbf{V}_{n}$ denote the variety defined by the identity

$$
\nu_{n}: x^{2 n} \approx x^{n}
$$

and let $\psi_{n}: \mathcal{X} \rightarrow \mathcal{X}^{+}$denote the substitution $x \mapsto h^{n} x h^{n}$ for all $x \in \mathcal{X}$.

Lemma 2.7 [15, Theorem 2]. Let $S$ be any non-unital semigroup such that $S, S^{1} \in \mathbf{V}_{n}$. Suppose that $\mathbf{w} \approx \mathbf{w}^{\prime}$ is an exclusion identity for $S$ in $\mathbf{V}_{n}$ with $h \notin \operatorname{con}\left(\mathbf{w w}^{\prime}\right)$. Then the identity $\mathbf{w} \psi_{n} \approx \mathbf{w}^{\prime} \psi_{n}$ is an exclusion identity for $S^{1}$ in $\mathbf{V}_{n}$.

Lemma 2.8. For each $n \geqslant 2$, the following is an exclusion identity for $A_{0}$ in $\mathbf{V}_{n}$ :

$$
\left(x^{n} y^{n}\right)^{n+1} \approx x^{n} y^{n} .
$$

Proof. By Lemma 2.4, the identity (2.1) is not satisfied by $A_{0}$. Suppose that a subvariety $\mathbf{V}$ of $\mathbf{V}_{n}$ does not satisfy the identity (2.1). Then there exists some semigroup $S \in \mathbf{V}$ with elements $a, b \in S$ such that

(a) $\left(a^{n} b^{n}\right)^{n+1} \neq a^{n} b^{n}$.

Let $T=\left\langle a^{n}, b^{n}\right\rangle$ be the subsemigroup of $S$ generated by the elements $a^{n}$ and $b^{n}$. Since $a^{2 n} \stackrel{\nu_{n}}{=} a^{n}$ and $b^{2 n} \stackrel{\nu_{n}}{=} b^{n}$, it is easily shown by (a) that

(b) $a^{n}, b^{n}, a^{n} b^{n}$, and $b^{n} a^{n}$ are distinct elements of $T$.

Suppose that there exists some $x \in\left\{a^{n}, b^{n}, a^{n} b^{n}\right\}$ such that $x \in T b^{n} a^{n} T$. Then

$$
a^{n} x b^{n} \in a^{n}\left\{a^{n}, b^{n}, a^{n} b^{n}\right\} b^{n} \stackrel{\nu_{n}}{=}\left\{a^{n} b^{n}\right\},
$$

so that $a^{n} x b^{n}=a^{n} b^{n}$. Therefore

$$
a^{n} b^{n}=a^{n} x b^{n} \in a^{n}\left(T b^{n} a^{n} T\right) b^{n} \stackrel{\nu_{n}}{\subseteq}\left\{\left(a^{n} b^{n}\right)^{2},\left(a^{n} b^{n}\right)^{3}, \ldots\right\},
$$

whence $a^{n} b^{n}=\left(a^{n} b^{n}\right)^{r+1}$ for some $r \geqslant 1$. But

$$
a^{n} b^{n}=\left(a^{n} b^{n}\right)^{r+1}=\left(a^{n} b^{n}\right)^{2 r+1}=\ldots=\left(a^{n} b^{n}\right)^{n r+1} \stackrel{\nu_{n}}{=}\left(a^{n} b^{n}\right)^{n+1},
$$

which is impossible by (a). Therefore $x$ does not exist, so that $\left\{a^{n}, b^{n}, a^{n} b^{n}\right\}$ and $T b^{n} a^{n} T$ are disjoint sets. It follows that

(c) $I=T b^{n} a^{n} T$ is an ideal of $T$ with $a^{n}, b^{n}, a^{n} b^{n} \notin I$.

By (b) and (c), the divisor $T /\left(T b^{n} a^{n} T\right)$ of $S$ is isomorphic to $A_{0}$. Hence $A_{0} \in \mathbf{V}$.

Lemma 2.9. For each $n \geqslant 2$, the following is an exclusion identity for $N_{2}^{1}$ in $\mathbf{V}_{n}$ :

$$
h^{n}\left(x h^{n}\right)^{n+1} \approx h^{n} x h^{n} .
$$

Proof. Suppose that $\mathbf{V}$ is any subvariety of $\mathbf{V}_{n}$ such that $N_{2} \notin \mathbf{V}$. Then by Lemma 2.1(iv), the variety $\mathbf{V}$ satisfies an identity $\alpha: x \approx x^{r+1}$ for some $r \geqslant 1$. The variety $\mathbf{V}$ also satisfies $x^{n+1} \approx x$ because

$$
x \stackrel{\alpha}{\approx} x^{r+1} \stackrel{\alpha}{\approx} x^{2 r+1} \stackrel{\alpha}{\approx} \ldots \stackrel{\alpha}{\approx} x^{n r+1} \stackrel{\nu_{n}}{\approx} x^{n+1}
$$


Hence the identity $x^{n+1} \approx x$ is an exclusion identity for $N_{2}$ in $\mathbf{V}_{n}$. By Lemma 2.7, the identity $\left(x^{n+1}\right) \psi_{n} \approx x \psi_{n}$ is an exclusion identity for $N_{2}^{1}$ in $\mathbf{V}_{n}$; this identity can be converted into the identity (2.2) by $\nu_{n}$.

2.5. Factors of length two formed by simple letters

For any word $\mathbf{w}$, let $\mathrm{F}_{\mathrm{SS}}(\mathbf{w})$ denote the set of factors of $\mathbf{w}$ of length two that are formed by simple letters:

$$
\mathrm{F}_{\mathrm{SS}}(\mathbf{w})=\left\{x y \in \mathcal{X}^{2} \mid \mathbf{w} \in \mathcal{X}^{*} x y \mathcal{X}^{*}, x, y \in \operatorname{sim}(\mathbf{w})\right\} .
$$

Lemma 2.10. Let $S$ be any semigroup that satisfies $\nu_{n}$ for some $n \geqslant 2$. Suppose that $S$ satisfies an identity $\mathbf{w} \approx \mathbf{w}^{\prime}$ with $\operatorname{con}(\mathbf{w})=\operatorname{con}\left(\mathbf{w}^{\prime}\right), \operatorname{sim}(\mathbf{w})=\operatorname{sim}\left(\mathbf{w}^{\prime}\right)$, and $\mathrm{F}_{\mathrm{SS}}(\mathbf{w}) \neq \mathrm{F}_{\mathrm{SS}}\left(\mathbf{w}^{\prime}\right)$. Then $S$ satisfies the identity

$$
x^{n} y x^{n} z x^{n} \approx x^{n} y z x^{n} .
$$

Proof. Generality is not lost by assuming $y z \in \mathrm{F}_{\mathrm{SS}}(\mathbf{w}) \backslash \mathrm{F}_{\mathrm{SS}}\left(\mathbf{w}^{\prime}\right)$. Let $\varphi: \mathcal{X} \rightarrow \mathcal{X}^{+}$denote the substitution

$$
t \mapsto \begin{cases}x^{n} y & \text { if } t=y, \\ z x^{n} & \text { if } t=z, \\ x^{n} & \text { otherwise. }\end{cases}
$$

Then the deduction $x^{n}(\mathbf{w} \varphi) x^{n} \stackrel{\nu_{n}}{\approx} x^{n} y z x^{n}$ holds because $y z$ is a factor of $\mathbf{w}$ and $y, z \in \operatorname{sim}(\mathbf{w})$. But since $y z$ is not a factor of $\mathbf{w}^{\prime}$ and $y, z \in \operatorname{sim}(\mathbf{w})=\operatorname{sim}\left(\mathbf{w}^{\prime}\right)$, either

$$
x^{n}\left(\mathbf{w}^{\prime} \varphi\right) x^{n} \stackrel{\nu_{n}}{\approx} x^{n} y x^{n} z x^{n} \quad \text { or } \quad x^{n}\left(\mathbf{w}^{\prime} \varphi\right) x^{n} \stackrel{\nu_{n}}{\approx} x^{n} z x^{n} y x^{n}
$$

holds. Hence $S$ satisfies either the identity (2.3) or the identity $\alpha: x^{n} y z x^{n} \approx x^{n} z x^{n} y x^{n}$. Since

$$
x^{n} y z x^{n} \stackrel{\alpha}{\approx} x^{n} z\left(x^{n} y\right) x^{n} \stackrel{\alpha}{\approx} x^{n} x^{n} y x^{n} z x^{n} \stackrel{\nu_{n}}{\approx} x^{n} y x^{n} z x^{n},
$$

the semigroup $S$ satisfies the identity (2.3) in either case.

\section{Proof of the main theorem}

The following twenty sufficient conditions for the finite basis property are required.

Condition 1 [37]. Any finite semigroup that satisfies the identities

$$
x^{13} \approx x, \quad x^{12} y^{12} x^{12} y^{12} \approx x^{12} y^{12}
$$

is finitely based.

Condition 2 [9, Theorem 6.1]. Any semigroup that satisfies the identities

$$
x y^{3} \approx x y, \quad x y x^{2} \approx x^{3} y, \quad x y x y \approx x y^{2} x
$$

is finitely based.

Condition 3. Any semigroup that satisfies the identities

$$
x^{3} y \approx x^{2} y, \quad x y^{3} \approx x y^{2}, \quad x y x \approx y x y, \quad x y x \approx x y x y
$$

is finitely based. 
Condition 4 [36, Proposition C]. Any semigroup that satisfies the identity

$$
x y z y \approx x y^{2} z
$$

is finitely based.

Condition 5 [16, Theorem 1.3]. Any semigroup that satisfies the identities

$$
x^{3} \approx x^{2}, \quad \text { xyxyx } \approx x y x, \quad x y x z x \approx x z x y x
$$

is finitely based.

Condition 6 [17, Theorem 3.3]. Any semigroup that satisfies the identities

$$
x^{3} \approx x^{2}, \quad x^{2} y x \approx x y x, \quad x y x^{2} \approx x y x, \quad x y x z x \approx x y z x
$$

is finitely based.

Condition 7. Any semigroup that satisfies the identities

$$
x^{4} \approx x^{2}, \quad x^{3} y x \approx x y x, \quad x y^{2} x \approx y x^{2} y, \quad x y z x \approx x z y x
$$

is finitely based.

Condition 8. Any semigroup that satisfies the identities

$$
x^{3} y z \approx x y z, \quad x^{3} y \approx x y^{3}, \quad x y x^{2} \approx x^{3} y, \quad x y x y \approx x y^{2} x
$$

is finitely based.

Condition 9. Any semigroup that satisfies the identities

$$
x^{4} \approx x^{2}, \quad x^{3} y x \approx x y x, \quad x y x y \approx x^{2} y^{2}, \quad x y^{2} z \approx x z y^{2}
$$

is finitely based.

Condition 10. Any semigroup that satisfies the identities

$$
x^{3} \approx x^{2}, \quad x^{2} y x \approx x y x, \quad x y x^{2} \approx x y x, \quad x^{2} y^{2} \approx y^{2} x^{2}, \quad x^{2} y z^{2} \approx x y z^{2} x
$$

is finitely based.

Condition 11 [18, Theorem 5.1]. Any semigroup that satisfies the identities

$$
x^{4} \approx x^{2}, \quad x^{3} y x \approx x y x, \quad x^{2} y x \approx x y x^{2}, \quad x^{2} y z^{2} \approx x y z^{2} x, \quad x y x z x \approx x z x y x
$$

is finitely based.

Condition 12 [22, Proposition 3.2]. Any semigroup that satisfies the identities

$$
x^{4} \approx x^{2}, \quad x(y x)^{3} \approx x y x, \quad x^{2} y^{3} x^{2} \approx x^{2} y x^{2}, \quad\left(x^{2} y^{2}\right)^{3} \approx\left(x^{2} y^{2}\right)^{2}, \quad x y x z x \approx x z x y x
$$

is finitely based.

Condition 13 [22, Proposition 3.2]. Any semigroup that satisfies the identities

$$
x^{5} \approx x^{2}, \quad x(y x)^{4} \approx x y x, \quad x^{2} y^{4} x^{2} \approx x^{2} y x^{2}, \quad\left(x^{3} y^{3}\right)^{3} \approx\left(x^{3} y^{3}\right)^{2}, \quad x y x z x \approx x z x y x
$$

is finitely based. 
Condition 14. Any semigroup that satisfies the identities

$x^{4} \approx x^{2}, \quad x y^{3} x \approx x y x, \quad x y x^{3} \approx x y x, \quad x^{2} y x \approx x y x^{2}, \quad x y x z x \approx x^{2} y z x, \quad x y x y \approx x y^{2} x$ is finitely based.

Condition 15 [23, Corollary 4.6]. Any semigroup that satisfies the identities

$x^{8} y \approx x^{2} y, \quad x y^{8} \approx x y^{2}, \quad x^{7} y x \approx x y x, \quad x^{2} y x \approx x y x^{2}, \quad x y x z x \approx x^{2} y z x, \quad x y x y \approx x^{2} y^{2}$

is finitely based.

Condition 16 [31, Theorem 22]. Any finite semigroup that satisfies the identity

$$
h x y k \approx h y x k
$$

is finitely based.

Condition 17 [35, Theorem A]. Any semigroup that satisfies the identity

$$
h x y x k \approx h x y^{2} k
$$

is finitely based.

Condition 18 [35, Theorem A]. Any semigroup that satisfies the identity

$$
h x k x y t \approx h x^{2} k y^{2} t
$$

is finitely based.

Condition 19 [19, Condition 9]. Any semigroup that satisfies the identities

$$
x^{4} \approx x^{2}, \quad x^{3} y x \approx x y x, \quad x y x^{2} \approx x^{3} y, \quad x y x y \approx x y^{2} x
$$

but violates both of the identities

$$
x y x y \approx x^{2} y^{2}, \quad x y^{3} x \approx x y x
$$

is finitely based.

Condition 20. Any semigroup that satisfies the identities

$$
\begin{gathered}
x^{3} \approx x^{2}, \quad x h x^{2} \approx x h x, \quad x^{2} h x k \approx x^{2} h k, \\
x h y k x y \approx x h y k y x, \quad x h y x y \approx x h y^{2} x, \quad x y k x y \approx x y k y x, \quad x y x y \approx x y^{2} x
\end{gathered}
$$

but violates the identity

$$
h^{2} x y x y \approx h^{2} x^{2} y^{2}
$$

is finitely based.

Conditions $3,7,8,9,10,14$, and 20 are established in $\S \S 4-10$.

It is routinely verified, with the aid of a computer, that 14534 of the 14600 pairwise distinct non-unital semigroups of order six are finitely based by Conditions 1-20 or their dual conditions. Multiplication tables of the 66 sporadic cases to which the twenty sufficient conditions do not apply are listed below in lexicographical order; the underlying set of each 
semigroup is $\{1,2,3,4,5,6\}$, and each multiplication table is represented by a $6 \times 6$ array where the $(i, j)$-entry denotes the product of the elements $i, j \in\{1,2,3,4,5,6\}$.

\begin{tabular}{|c|c|c|c|c|c|c|c|c|c|}
\hline 111111 & 111111 & 111111 & 111111 & 111111 & 111111 & 111111 & 111111 & 111111 & 111111 \\
\hline 111111 & 111111 & 111111 & 111111 & 111111 & 111111 & 111111 & 111111 & 111111 & 111111 \\
\hline 111113 & 111123 & 113156 & 113156 & 113333 & 113356 & 123156 & 123333 & 123456 & 123456 \\
\hline 111314 & 444444 & 121411 & 444444 & 114444 & 123456 & 444444 & 123456 & 124365 & 444444 \\
\hline 555555 & 111123 & 555555 & 113656 & 123456 & 555555 & 123656 & 125555 & 555555 & 444444 \\
\hline 123456 & 123456 & 556513 & 666666 & 124365 & 556613 & 666666 & 125634 & 666666 & 456123 \\
\hline$\S 11$ & $\S 12$ & $\S 13$ & $\S 14$ & $\S 13$ & $\S 13$ & $\S 14$ & $\S 13$ & $\S 13$ & $\S 13$ \\
\hline 111111 & 111111 & 111111 & 111111 & 111111 & 111111 & 111111 & 111111 & 111111 & 111111 \\
\hline & 111111 & 111112 & 111112 & 111112 & 111112 & 111112 & 111112 & 11 & 111112 \\
\hline 123456 & 333333 & 111112 & 111113 & 111113 & 111113 & 111113 & 111113 & 111113 & 111 \\
\hline 444444 & 333456 & 111214 & 111214 & 111214 & 111214 & 111214 & 111214 & 111224 & 111224 \\
\hline 445444 & 555555 & 555555 & 112111 & 112111 & 112112 & 113151 & 113153 & 112224 & 112224 \\
\hline 446445 & 553456 & 123456 & 121456 & 122456 & 122456 & 121416 & 121416 & 121456 & 122456 \\
\hline$\S 11$ & $\S 14$ & $\S 11$ & $\S 15$ & $\S 15$ & $\S 15$ & $\S 12$ & $\S 12$ & $\S 15$ & $\S 15$ \\
\hline 111111 & 111111 & 111111 & 111111 & 111111 & 111111 & 111111 & 111111 & 111111 & 111111 \\
\hline 111 & 111112 & 111112 & 111112 & 111112 & 111112 & 111112 & 111112 & 111112 & 12 \\
\hline 111113 & 111113 & 111113 & 111113 & 111113 & 111113 & 111113 & 111113 & 111 & 11 \\
\hline 112111 & 112111 & 112112 & 112112 & 112121 & 112122 & 112141 & 112141 & 112 & 113454 \\
\hline 555555 & 555555 & 555555 & 555555 & 112123 & 112123 & 113151 & 113151 & 113153 & 133454 \\
\hline 121456 & 122456 & 121456 & 122456 & 121446 & 122446 & 121416 & 122446 & 122446 & 113456 \\
\hline$\S 16$ & $\S 16$ & $\S 16$ & $\S 16$ & $\S 17$ & $\S 17$ & $\S 18$ & $\S 19$ & $I$ & $\S 17$ \\
\hline 111111 & 111111 & 111111 & 111111 & 111111 & 1111 & 111 & 111111 & 111 & 11 \\
\hline 12 & 111112 & 111112 & 111112 & 111112 & 111112 & 111112 & 111112 & 111 & 111112 \\
\hline 111113 & 111121 & 111121 & 111122 & 111122 & 111141 & 111141 & 111142 & 111142 & 112123 \\
\hline 113455 & 123444 & 444444 & 123444 & 444444 & 444444 & 444444 & 444444 & 444444 & 4444 \\
\hline 133455 & 123445 & 444445 & 123445 & 444445 & 555555 & 555555 & 555555 & 555555 & 112123 \\
\hline 133456 & 123446 & 123446 & 123446 & 123446 & 123416 & 123446 & 123416 & 123446 & 123456 \\
\hline$\S 17$ & $\S 20$ & $\S 16$ & $\S 20$ & $\S 16$ & $\S 21$ & $\S 21$ & $\S 21$ & $\S 21$ & $\$ 11$ \\
\hline 111111 & 111111 & 111111 & 111111 & 111111 & 111111 & 111 & 111 & 111 & 111111 \\
\hline 111 & 111112 & 111112 & 111113 & 111113 & 11 & & 11 & & 122 \\
\hline 112 & 333333 & 333333 & 111133 & 111133 & 11 & 333333 & 333333 & 333333 & 111133 \\
\hline 112224 & 333313 & 333313 & 111344 & 123444 & 444444 & 123451 & 123451 & 123453 & 444444 \\
\hline 112225 & 555555 & 555555 & 123455 & 123455 & 123455 & 123453 & 123541 & 123543 & 113156 \\
\hline 123556 & 123416 & 123436 & 123466 & 123466 & 123466 & 666666 & 666666 & 666666 & 133156 \\
\hline$\S 15$ & $\S 21$ & $\S 21$ & $\S 20$ & $\S 22$ & $\S 22$ & $\S 2$ & $\S 2$ & $\delta ?$ & $\delta 23$ \\
\hline 111111 & 111111 & 111111 & 111111 & 111111 & 1111 & 1111 & 111 & 111 & 111111 \\
\hline 111122 & 111122 & 111122 & 111122 & 111122 & 111122 & 111122 & 111122 & 111 & 111123 \\
\hline 111133 & 112133 & 123111 & 123122 & 333333 & 333333 & 333333 & 333333 & 333333 & 333333 \\
\hline 444444 & 444444 & 444444 & 444444 & 333344 & 444444 & 444444 & 444444 & 123 & 1234 \\
\hline 113456 & 123456 & 111456 & 111456 & 121156 & 111456 & 121456 & 333456 & 111156 & 111156 \\
\hline 133456 & 123456 & 444165 & 444165 & 121256 & 114456 & 124456 & 443456 & 666666 & 666666 \\
\hline$\S 24$ & $\S 24$ & $\S 25$ & $\S 25$ & $\S 23$ & $\S 26$ & $\S 26$ & $\S 26$ & $\S 27$ & $\S 27$ \\
\hline 11111 & 1111 & 1111 & 111111 & 111111 & 1111 & & & & \\
\hline 111 & 111223 & 121256 & 122256 & 122446 & & & & & \\
\hline 333333 & 333333 & 333333 & 122256 & 123456 & 123236 & & & & \\
\hline 123433 & 123456 & 121256 & 123456 & 644221 & 111456 & & & & \\
\hline 333356 & 123456 & 126256 & 555555 & 644221 & 145456 & & & & \\
\hline 666666 & 666666 & 666666 & 566612 & 666666 & 666661 & & & & \\
\hline$\S 27$ & $\S 27$ & $\S 14$ & $\S 13$ & $\S 13$ & $A_{2}^{g}$ & & & & \\
\hline
\end{tabular}

For each $\mathrm{X} \in\{\mathscr{A}, \mathscr{B}, \ldots, \mathscr{G}\}$, let $\mathrm{X}_{n}$ denote the $n$th semigroup in row (X) above. The semigroups $\mathscr{C}_{9}$ and $\mathscr{G}_{6}$ are isomorphic to the non-finitely based semigroups $L$ and $A_{2}^{g}$, respectively. The remaining 64 semigroups are shown to be finitely based in $\S \S 11-27$. Specifically, it is indicated below each multiplication table the section in which the semigroup is shown to be finitely based. Consequently, among the 14600 pairwise distinct non-unital semigroups of order six, only $A_{2}^{g}$ and $L$ are non-finitely based. 
As observed in $\S 1$, the monoids $B_{2}^{1}$ and $A_{2}^{1}$ are the only non-finitely based semigroups among the 1373 pairwise distinct monoids of order six [19]. The proof of the main theorem is thus complete.

REMARK 3.1. There is no redundancy with Conditions 1-20 since they are distinguished by semigroups with the following multiplication tables.

$\begin{array}{cccccccccc}111111 & 111111 & 111111 & 111111 & 111111 & 111111 & 111111 & 111111 & 111111 & 111111 \\ 121111 & 111111 & 111111 & 111111 & 111111 & 111111 & 111112 & 111111 & 111122 & 111122 \\ 113156 & 111133 & 111113 & 111113 & 111134 & 111133 & 111113 & 112111 & 111133 & 111211 \\ 44444 & 444444 & 111211 & 111114 & 113434 & 113434 & 113453 & 111456 & 111144 & 111144 \\ 113656 & 111456 & 113153 & 111335 & 111156 & 111155 & 113543 & 555555 & 122456 & 111155 \\ 666666 & 444165 & 111116 & 113456 & 115656 & 113456 & 121116 & 555614 & 144265 & 123156 \\ S_{1} & S_{2} & S_{3} & S_{4} & S_{5} & S_{6} & S_{7} & S_{8} & S_{9} & S_{10} \\ 11111 & 111116 & 111111 & 111111 & 111111 & 111111 & 111111 & 111111 & 111111 & 111111 \\ 11111 & 111236 & 111112 & 111112 & 111111 & 111111 & 111111 & 122456 & 111112 & 111111 \\ 111114 & 123116 & 123452 & 123152 & 111133 & 111113 & 111112 & 122456 & 113451 & 111134 \\ 111144 & 111456 & 124532 & 444444 & 444444 & 111114 & 123453 & 122456 & 444444 & 444444 \\ 113455 & 145116 & 125342 & 125132 & 123456 & 111135 & 555555 & 124456 & 445134 & 113456 \\ 113466 & 666661 & 111116 & 111416 & 123465 & 123156 & 111112 & 666666 & 121116 & 666666 \\ S_{11} & S_{12} & S_{13} & S_{14} & S_{15} & S_{16} & S_{17} & S_{18} & S_{19} & S_{20}\end{array}$

Specifically, the semigroup $S_{m}$ is finitely based by Condition $n$ if and only if $m=n$.

\section{PART II. SUFFICIENT CONDITIONS FOR THE FINITE BASIS PROPERTY}

\section{On Condition 3}

Let $\mathbf{X}_{(4.1)}$ denote the variety defined by the identities

$$
\begin{gathered}
x^{3} y \approx x^{2} y, \quad x y^{3} \approx x y^{2}, \\
x y x \approx y x y, \\
x y x \approx x y x y .
\end{gathered}
$$

Then Condition 3 is equivalent to the following result.

Proposition 4.1. All subvarieties of $\mathbf{X}_{(4.1)}$ are finitely based.

It is shown in $\S 4.1$ that $\mathbf{X}_{(4.1)}=\mathbf{A}_{\mathbf{0}} \vee \mathbf{N}_{\mathbf{3}}$. The proof of Proposition 4.1 is then given in $\S 4.2$.

\subsection{Identities satisfied by $\mathbf{X}_{(4.1)}$}

Lemma 4.2. For any $k \geqslant 2$, the identity (4.1c) implies the identity

$$
\left(x_{1} x_{2} \ldots x_{k}\right)^{3} \approx\left(x_{1} x_{2} \ldots x_{k}\right)^{2} .
$$

Proof. The identity (4.1c) clearly implies the identity $\left(x_{1} x_{2}\right)^{3} \approx\left(x_{1} x_{2}\right)^{2}$, which in turn implies the identity (4.2) for any $k \geqslant 2$.

Lemma 4.3 [21, Theorem 4.1]. The variety $\mathbf{A}_{\mathbf{0}}$ is defined by the identities (4.1) and

$$
x^{3} \approx x^{2} .
$$

Proposition 4.4. The equality $\mathbf{X}_{(4.1)}=\mathbf{A}_{\mathbf{0}} \vee \mathbf{N}_{\mathbf{3}}$ holds. 
Proof. It is routinely verified, either directly or by Lemmas 2.1(vi) and 4.3, that $A_{0}$ and $N_{3}$ satisfy the identities (4.1). Hence the inclusion $\mathbf{A}_{\mathbf{0}} \vee \mathbf{N}_{\mathbf{3}} \subseteq \mathbf{X}_{(4.1)}$ holds. To complete the proof, it remains to show that any nontrivial identity $\mathbf{w} \approx \mathbf{w}^{\prime}$ satisfied by $\mathbf{A}_{\mathbf{0}} \vee \mathbf{N}_{\mathbf{3}}$ is implied by the identities (4.1). Since $\mathbf{A}_{\mathbf{0}}$ satisfies the identity $\mathbf{w} \approx \mathbf{w}^{\prime}$, the identities $\{(4.1),(4.3)\}$ imply the identity $\mathbf{w} \approx \mathbf{w}^{\prime}$. Hence there exists a deduction sequence

$$
\mathbf{w}=\mathbf{z}_{0} \Rightarrow \mathbf{z}_{1} \Rightarrow \cdots \Rightarrow \mathbf{z}_{r}=\mathbf{w}^{\prime}
$$

where each deduction $\mathbf{z}_{j} \Rightarrow \mathbf{z}_{j+1}$ involves an identity from $\{(4.1),(4.3)\}$. For each $j$, since $A_{0}$ satisfies the identity $\mathbf{w} \approx \mathbf{z}_{j}$, it is easily shown that $\operatorname{con}(\mathbf{w})=\operatorname{con}\left(\mathbf{z}_{j}\right)$.

Let $\operatorname{con}(\mathbf{w})=\left\{x_{1}, \ldots, x_{k}\right\}$. If $k=1$, then the assumption $\mathbf{w} \neq \mathbf{w}^{\prime}$ and Lemma 2.1(vi) imply $|\mathbf{w}|,\left|\mathbf{w}^{\prime}\right| \geqslant 3$, whence the identity $\mathbf{w} \approx \mathbf{w}^{\prime}$ is implied by the identities (4.1a). Thus assume $k \geqslant 2$. Suppose that some deduction $\mathbf{z}_{j} \Rightarrow \mathbf{z}_{j+1}$ in (4.4) involves the identity (4.3). Then $\mathbf{z}_{j}=\mathbf{e u}^{p} \mathbf{f}$ and $\mathbf{z}_{j+1}=\mathbf{e u}^{q} \mathbf{f}$ for some $\mathbf{e}, \mathbf{f} \in \mathcal{X}^{*}, \mathbf{u} \in \mathcal{X}^{+}$, and $\{p, q\}=\{2,3\}$. If either $\mathbf{e} \neq \emptyset$ or $\mathbf{f} \neq \emptyset$, then

$$
\mathbf{z}_{j}=\mathbf{e u}^{p} \mathbf{f} \stackrel{(4.1 \mathrm{a})}{\approx} \mathbf{e u}^{q} \mathbf{f}=\mathbf{z}_{j+1}
$$

If $\mathbf{e}=\mathbf{f}=\emptyset$, then since $|\mathbf{u}| \geqslant|\operatorname{con}(\mathbf{w})| \geqslant 2$,

$$
\mathbf{z}_{j}=\mathbf{u}^{p} \stackrel{(4.2)}{\approx} \mathbf{u}^{q}=\mathbf{z}_{j+1}
$$

Therefore any deduction $\mathbf{z}_{j} \Rightarrow \mathbf{z}_{j+1}$ in (4.4) that involves the identity (4.3) can be replaced by a deduction that involves an identity from (4.1a) or (4.2). Hence the identity $\mathbf{w} \approx \mathbf{w}^{\prime}$ is implied by the identities $\{(4.1),(4.2)\}$. It follows from Lemma 4.2 that the identity $\mathbf{w} \approx \mathbf{w}^{\prime}$ is implied by the identities (4.1).

COROLlary 4.5. The variety $\mathbf{X}_{(4.1)}$ satisfies the identities

$$
\begin{gathered}
x^{2} y x \approx x y x^{2} \approx x y x, \\
x y x z x \approx x^{2} y z x .
\end{gathered}
$$

Proof. It is routinely shown, either directly or by Lemmas 2.1(vi) and 2.4, that $A_{0}$ and $N_{3}$ satisfy the identities (4.5). The result now follows from Proposition 4.4.

\subsection{Proof of Proposition 4.1}

Let $\mathbf{V}$ be any proper subvariety of $\mathbf{X}_{(4.1)}$. Then by Proposition 4.4 , either $A_{0} \notin \mathbf{V}$ or $N_{3} \notin \mathbf{V}$. Since

Case 1: $A_{0} \notin \mathbf{V}$. Then by Lemma 2.8, the variety $\mathbf{V}$ satisfies the identity $\alpha:\left(x^{2} y^{2}\right)^{3} \approx x^{2} y^{2}$.

$$
x^{2} y^{2} \stackrel{\alpha}{\approx} x^{2} y^{2} x^{2} y^{2} x^{2} y^{2} \stackrel{(4.5 \mathrm{~b})}{\approx} x^{4} y^{4} x^{2} y^{2} \stackrel{(4.5 \mathrm{a})}{\approx} x y x y
$$

the variety $\mathbf{V}$ satisfies the identity $x y x y \approx x^{2} y^{2}$. Since $\mathbf{V}$ satisfies the identities $\{(4.1),(4.5)\}$, it satisfies the identities in Condition 15 and so is finitely based.

Case 2: $N_{3} \notin \mathbf{V}$. Then it follows from Lemma 2.1(vi) that the variety $\mathbf{V}$ satisfies the identity $x^{3} \approx x^{2}$ and so, by Lemma 4.3 , is a subvariety of $\mathbf{A}_{\mathbf{0}}$. Since every subvariety of $\mathbf{A}_{\mathbf{0}}$ is finitely based [14, Corollary 4.3], the variety $\mathbf{V}$ is finitely based. 


\section{On Condition 7}

Let $\mathbf{X}_{(5.1)}$ denote the variety defined by the identities

$$
\begin{aligned}
x^{3} \mathrm{H} x & \approx x \mathrm{H} x, \\
x y^{2} x & \approx y x^{2} y, \\
x y z x & \approx x z y x .
\end{aligned}
$$

Then Condition 7 is equivalent to the following result.

Proposition 5.1. All subvarieties of $\mathbf{X}_{(5.1)}$ are finitely based.

It is shown in $\S 5.1$ that $\mathbf{X}_{(5.1)}=\mathbf{A}_{\mathbf{0}} \vee \mathbf{N}_{\mathbf{2}}^{1} \vee \mathbf{Z}_{2}$. The proof of Proposition 5.1 is then given in $\S 5.2$.

\subsection{Identities satisfied by $\mathbf{X}_{(5.1)}$}

Lemma 5.2. The identities (5.1) imply the identities

$$
\begin{gathered}
x \mathrm{H} x \mathrm{~K} x \approx x^{2} \mathrm{HK} x, \\
x \mathrm{H} y z \mathrm{~K} x \approx x \mathrm{H} z y \mathrm{~K} x, \\
x \mathrm{H} y \mathrm{~K} x y \approx x \mathrm{H} y \mathrm{~K} y x, \quad x y \mathrm{H} x \mathrm{~K} y \approx y x \mathrm{H} x \mathrm{~K} y .
\end{gathered}
$$

Proof. It is easily shown that the identity (5.1c) implies the identities (5.2a). Since

$$
\begin{gathered}
x \mathrm{H} y z \mathrm{~K} x \stackrel{(5.1 \mathrm{a})}{\approx} x^{3} \mathrm{H} y z \mathrm{~K} x \stackrel{(5.2 \mathrm{a})}{\approx} x \mathrm{H} x y z x \mathrm{~K} x \stackrel{(5.1 \mathrm{c})}{\approx} x \mathrm{H} x z y x \mathrm{~K} x \\
\stackrel{(5.2 \mathrm{a})}{\approx} x^{3} \mathrm{H} z y \mathrm{~K} x \stackrel{(5.1 \mathrm{a})}{\approx} x \mathrm{H} z y \mathrm{~K} x,
\end{gathered}
$$

the identities (5.1) imply the identities (5.2b). Since

$$
\begin{gathered}
x \mathrm{H} y \mathrm{~K} x y \stackrel{(5.1 \mathrm{a})}{\approx} x^{3} \mathrm{H} y^{3} \mathrm{~K} x y \stackrel{(5.2 \mathrm{a})}{\approx} x^{2} \mathrm{H} y^{2} \mathrm{~K} y x^{2} y \stackrel{(5.1 \mathrm{~b})}{\approx} x^{2} \mathrm{H} y^{2} \mathrm{~K} x y^{2} x \\
\stackrel{(5.2 \mathrm{a})}{\approx} x^{3} \mathrm{H} y^{3} \mathrm{~K} y x \stackrel{(5.1 \mathrm{a})}{\approx} x \mathrm{H} y \mathrm{~K} y x,
\end{gathered}
$$

the identities (5.1) imply $x \mathrm{H} y \mathrm{~K} x y \approx x \mathrm{H} y \mathrm{~K} y x$. By a symmetrical argument, the identities (5.1) also imply $x y \mathrm{H} x \mathrm{~K} y \approx y x \mathrm{H} x \mathrm{~K} y$. Hence the identities (5.1) imply the identities (5.2c).

In this section, a non-simple word $\mathbf{w}$ is said to be in canonical form if

$$
\mathbf{w}=x_{0}^{e_{0}} x_{1}^{e_{1}} \ldots x_{r}^{e_{r}} y_{1} \ldots y_{s} x_{0}
$$

where all of the following are satisfied:

(I) $x_{0}, x_{1}, \ldots, x_{r}$ are distinct non-simple letters of $\mathbf{w}$ in alphabetical order;

(II) $e_{0} \in\{1,2\}$ and $e_{1}, \ldots, e_{r} \in\{2,3\}$;

(III) $y_{1}, \ldots, y_{s}$ are distinct simple letters of $\mathbf{w}$ in alphabetical order.

Note that if the above word $\mathbf{w}$ in canonical form does not contain any simple letter, then $s=0$ and $y_{1} \ldots y_{s}=\emptyset$.

Lemma 5.3. Let $\mathbf{w}$ be any non-simple word with $\mathrm{h}(\mathbf{w})=\mathrm{t}(\mathbf{w})$. Then there exists some word $\overline{\mathbf{w}}$ in canonical form such that the identities (5.1) imply the identity $\mathbf{w} \approx \overline{\mathbf{w}}$. 
Proof. By Lemma 5.2, it suffices to convert w, using the identities $\{(5.1),(5.2)\}$, into a word in canonical form. By assumption, $\mathbf{w}=x \mathbf{u} x$ for some $\mathbf{u} \in \mathcal{X}^{*}$. The identities (5.2a) can be used to group every $x$ in $\mathbf{u}$ with the first $x$ in $\mathbf{w}$, resulting in a word $x^{e} \mathbf{u}^{\prime} x$, where $e=\operatorname{occ}(x, \mathbf{u})+1$ and $\mathbf{u}^{\prime}$ is obtained from $\mathbf{u}$ by removing all occurrences of $x$. The identities $(5.2 \mathrm{~b})$ can then be used to arrange the letters of $\mathbf{u}^{\prime}$ in any order. Specifically, the non-simple letters of $\mathbf{u}^{\prime}$ can be grouped to the left in alphabetical order to form a prefix of $\mathbf{u}^{\prime}$, while the simple letters of $\mathbf{u}^{\prime}$ can be grouped to the right in alphabetical order to form a suffix of $\mathbf{u}^{\prime}$. The resulting word is of the form $x^{e} x_{1}^{e_{1}} \ldots x_{r}^{e_{r}} y_{1} \ldots y_{s} x$ for some $e_{1}, \ldots, e_{r} \geqslant 2$. The identities (5.1a) can be used to reduce the exponents, so that $e \in\{1,2\}$ and $e_{1}, \ldots, e_{r} \in\{2,3\}$. The resulting word is in canonical form if $x$ alphabetically precedes $x_{1}$. If $x$ does not alphabetically precede $x_{1}$, then

$$
\begin{aligned}
x^{e} x_{1}^{e_{1}} x_{2}^{e_{2}} \ldots x_{r}^{e_{r}} y_{1} \ldots y_{s} x & \stackrel{(5.2 \mathrm{~b})}{\approx} x^{e} x_{1}^{e_{1}-1} x_{2}^{e_{2}} \ldots x_{r}^{e_{r}} y_{1} \ldots y_{s} x_{1} x \\
& \stackrel{(5.2 \mathrm{c})}{\approx} x_{1}^{e_{1}-1} x^{e} x_{2}^{e_{2}} \ldots x_{r}^{e_{r}} y_{1} \ldots y_{s} x x_{1} \\
& \stackrel{(5.2 \mathrm{~b})}{\approx} x_{1}^{e_{1}-1} x^{e+1} x_{2}^{e_{2}} \ldots x_{r}^{e_{r}} y_{1} \ldots y_{s} x_{1},
\end{aligned}
$$

and the factors $x^{e+1}, x_{2}^{e_{2}}, \ldots, x_{r}^{e_{r}}$ in the latter word can be alphabetically ordered by the identities $(5.2 \mathrm{~b})$, resulting in a word in canonical form.

Lemma 5.4. Let $\mathbf{w}$ be any connected word. Then there exists some word $\overline{\mathbf{w}}$ in canonical form such that the identities (5.1) imply the identity $\mathbf{w} \approx \overline{\mathbf{w}}$.

Proof. By Lemma 5.2, it suffices to convert w, using the identities $\{(5.1),(5.2)\}$, into a word in canonical form. Since $\mathbf{w}$ is connected, the letter $x=\mathrm{h}(\mathbf{w})$ occurs at least twice in $\mathbf{w}$. Hence $\mathbf{w}=x \mathbf{u} x \mathbf{v}$ for some $\mathbf{u}, \mathbf{v} \in \mathcal{X}^{*}$ such that $x \notin \operatorname{con}(\mathbf{v})$. If the factor $\mathbf{v}$ is empty, then the result follows from Lemma 5.3. Therefore assume that $\mathbf{v}$ is nonempty, whence $\mathbf{w}=x \mathbf{u} x y \mathbf{v}^{\prime}$ for some $y \in \mathcal{X}$ and $\mathbf{v}^{\prime} \in \mathcal{X}^{*}$. There are two cases to consider.

Case 1: $y \notin \operatorname{con}(\mathbf{u})$. Since $\mathbf{w}$ is connected, the factors $x \mathbf{u} x$ and $y \mathbf{v}^{\prime}$ cannot be disjoint. It follows that $\mathbf{u}$ and $\mathbf{v}^{\prime}$ share some common letter, whence $\mathbf{w}=x \mathbf{u} x y \mathbf{v}^{\prime} \stackrel{(5.2 \mathrm{~b})}{\approx} x \mathbf{u} y x \mathbf{v}^{\prime}$.

Case 2: $y \in \operatorname{con}(\mathbf{u})$. Then clearly $\mathbf{w}=x \mathbf{u} x y \mathbf{v}^{\prime} \stackrel{(5.2 \mathrm{c})}{\approx} x \mathbf{u} y x \mathbf{v}^{\prime}$.

In either case, the identities (5.2) can be used to interchange the last $x$ in $\mathbf{w}$ with the letter $y$ that immediately follows it. It is easily seen that the resulting word is connected. The same argument can be repeated to move the last $x$ in $\mathbf{w}$ to the right until it becomes the last letter of the word. Hence $\mathbf{w} \stackrel{(5.2)}{\approx} x \mathbf{u v} x$ and the result now holds by Lemma 5.3.

Proposition 5.5. The equality $\mathbf{X}_{(5.1)}=\mathbf{A}_{\mathbf{0}} \vee \mathbf{N}_{\mathbf{2}}^{1} \vee \mathbf{Z}_{2}$ holds.

Proof. By Lemma 2.5, the semigroup $A_{0} \times N_{2}^{1} \times \mathbb{Z}_{2}$ is idempotent-separable. Therefore by Lemma 2.6(i), there exists some basis $\Sigma$ for $A_{0} \times N_{2}^{1} \times \mathbb{Z}_{2}$ that consists of identities formed by connected words. It is routinely verified, either directly or by Lemmas 2.1 and 2.4, that $A_{0}$, $N_{2}^{1}$, and $\mathbb{Z}_{2}$ satisfy the identities (5.1). Hence the inclusion $\mathbf{A}_{\mathbf{0}} \vee \mathbf{N}_{\mathbf{2}}^{\mathbf{1}} \vee \mathbf{Z}_{2} \subseteq \mathbf{X}_{(5.1)}$ holds. To complete the proof, it remains to show that any identity $\mathbf{w} \approx \mathbf{w}^{\prime}$ in $\Sigma$ is implied by the identities (5.1).

By Lemma 5.4, the words $\mathbf{w}$ and $\mathbf{w}^{\prime}$ can be chosen to be in canonical form. Since $N_{2}^{1}$ satisfies $\mathbf{w} \approx \mathbf{w}^{\prime}$, it follows from Lemma 2.1(v) that $\operatorname{con}(\mathbf{w})=\operatorname{con}\left(\mathbf{w}^{\prime}\right)$ and $\operatorname{sim}(\mathbf{w})=\operatorname{sim}\left(\mathbf{w}^{\prime}\right)$, whence $\mathbf{w}=x_{0}^{e_{0}} x_{1}^{e_{1}} \ldots x_{r}^{e_{r}} y_{1} \ldots y_{s} x_{0}$ and $\mathbf{w}^{\prime}=x_{0}^{e_{0}^{\prime}} x_{1}^{e_{1}^{\prime}} \ldots x_{r}^{e_{r}^{\prime}} y_{1} \ldots y_{s} x_{0}$ for some $e_{0}, e_{0}^{\prime} \in\{1,2\}$ and $e_{1}, e_{1}^{\prime}, \ldots, e_{r}, e_{r}^{\prime} \in\{2,3\}$. Since $\mathbb{Z}_{2}$ satisfies $\mathbf{w} \approx \mathbf{w}^{\prime}$, it follows from Lemma 2.1(viii) that $e_{i}=e_{i}^{\prime}$ for all $i$. Hence the identity $\mathbf{w} \approx \mathbf{w}^{\prime}$ is trivial and is implied by the identities (5.1). 


\subsection{Proof of Proposition 5.1}

Let $\mathbf{V}$ be any proper subvariety of $\mathbf{X}_{(5.1)}$. Then by Proposition 5.5, the variety $\mathbf{V}$ does not contain one of the following semigroups: $A_{0}, N_{2}^{1}$, and $\mathbb{Z}_{2}$.

Case 1: $A_{0} \notin \mathbf{V}$. Then it follows from Lemma 2.8 that the variety $\mathbf{V}$ satisfies the identity $\alpha:\left(x^{2} y^{2}\right)^{3} \approx x^{2} y^{2}$. Since

$$
x^{2} y^{2} \stackrel{\alpha}{\approx} x^{2} y^{2} x^{2} y^{2} x^{2} y^{2} \stackrel{(5.1 \mathrm{c})}{\approx} x^{5} y^{5} x y \stackrel{(5.1 \mathrm{a})}{\approx} x y x y
$$

the variety $\mathbf{V}$ satisfies the identity $x y x y \approx x^{2} y^{2}$. It is then routinely shown that $\mathbf{V}$ satisfies the identities in Condition 15 and so is finitely based.

Case 2: $N_{2}^{1} \notin \mathbf{V}$. Then it follows from Lemma 2.9 that the variety $\mathbf{V}$ satisfies the identity $\beta: h^{2}\left(x h^{2}\right)^{3} \approx h^{2} x h^{2}$. Since

$$
x(y x)^{3} \stackrel{(5.1 \mathrm{a})}{\approx} x^{3} y x^{3} y x y x \stackrel{(5.2 \mathrm{a})}{\approx} x^{2} y x^{2} y x^{2} y x^{2} \stackrel{\beta}{\approx} x^{2} y x^{2} \stackrel{(5.2 \mathrm{a})}{\approx} x^{3} y x \stackrel{(5.1 \mathrm{a})}{\approx} x y x
$$

and

$$
x^{2} y x^{2} \stackrel{\beta}{\approx} x^{2} y x^{2} y x^{2} y x^{2} \stackrel{(5.2 \mathrm{a})}{\approx} x^{6} y^{3} x^{2} \stackrel{(5.1 \mathrm{a})}{\approx} x^{2} y^{3} x^{2},
$$

the variety $\mathbf{V}$ satisfies the identities $x(y x)^{3} \approx x y x$ and $x^{2} y^{3} x^{2} \approx x^{2} y x^{2}$. It is then routinely shown that $\mathbf{V}$ satisfies the identities in Condition 12 and so is finitely based.

Case 3: $\mathbb{Z}_{2} \notin \mathbf{V}$. Then it follows from Lemma 2.1(viii) that the variety $\mathbf{V}$ satisfies the identity $x^{3} \approx x^{2}$. It is easily shown that $\mathbf{V}$ satisfies the identities in Condition 6 and so is finitely based.

\section{On Condition 8}

Let $\mathbf{X}_{(6.1)}$ denote the variety defined by the identities

$$
x^{3} y z \approx x y z, \quad x^{3} y \approx x y^{3}, \quad x y x^{2} \approx x^{3} y, \quad x y x y \approx x y^{2} x .
$$

Then Condition 8 is equivalent to the following result.

Proposition 6.1. All subvarieties of $\mathbf{X}_{(6.1)}$ are finitely based.

Some preliminary results are established in $\S \S 6.1$ and 6.2. The proof of Proposition 6.1 is then given in $\S 6.3$.

6.1. Identities satisfied by $\mathbf{X}_{(6.1)}$

LEMma 6.2. The identities (6.1) imply the identities

$$
\begin{gathered}
x^{3} y z \approx x y^{3} z \approx x y z^{3} \approx x y z, \\
x y x^{2} z \approx x y z, \quad x y z y^{2} \approx x y z, \\
x \mathrm{H} y \mathrm{~K} x y \approx x \mathrm{H} y \mathrm{~K} y x .
\end{gathered}
$$

Proof. It is easily seen that the identities (6.1) imply the identities $\{(6.2 \mathrm{a}),(6.2 \mathrm{~b})\}$. Note that the fourth identity in (6.1) implies the identity $\alpha: y^{2} x^{2} y \approx y^{2} x y x$. Since

$$
\begin{gathered}
x \mathrm{H} y \mathrm{~K} x y \stackrel{(6.2 \mathrm{a})}{\approx} x \mathrm{H} y \mathrm{~K} x x^{2} y \stackrel{(6.2 \mathrm{~b})}{\approx} x \mathrm{H} y \mathrm{~K} x\left(y^{2} x^{2} y\right) \stackrel{\alpha}{\approx} x \mathrm{H} y \mathrm{~K} x y^{2} x y x \\
\stackrel{(6.2 \mathrm{~b})}{\approx} x \mathrm{H} y \mathrm{~K} x^{2} y x \stackrel{(6.2 \mathrm{~b})}{\approx} x \mathrm{H} y \mathrm{~K} y x,
\end{gathered}
$$

the identities (6.1) also imply the identities (6.2c). 


\subsection{A canonical form}

In this section, a word $\mathbf{w}$ with ini( $\mathbf{w})=x_{0} \ldots x_{m}$ is said to be in canonical form if the following are satisfied:

(I) if $m=0$, then $\mathbf{w}$ is any one of the words

$$
x_{0}, x_{0}^{2}, x_{0}^{3}, x_{0}^{4}
$$

(II) if $m=1$, then $\mathbf{w}$ is any one of the words

$$
x_{0} x_{1}, x_{0}^{2} x_{1}, x_{0}^{3} x_{1}, x_{0} x_{1}^{2}, x_{0}^{2} x_{1}^{2}, x_{0} x_{1} x_{0}, x_{0}^{2} x_{1} x_{0}, x_{0} x_{1}^{2} x_{0}, x_{0}^{2} x_{1}^{2} x_{0} ;
$$

(III) if $m \geqslant 2$, then

$$
\mathbf{w}=x_{0}^{e_{0}} \prod_{i=1}^{m}\left(x_{i}^{e_{i}} \mathbf{w}_{i}\right)
$$

where $e_{i} \in\{1,2\}$ and $\mathbf{w}_{i} \in\left\{x_{0}^{f_{0}} \ldots x_{i-1}^{f_{i-1}} \mid f_{0}, \ldots, f_{i-1} \in\{0,1\}\right\}$.

Lemma 6.3. Let $\mathbf{w}$ be any word. Then there exists some word $\overline{\mathbf{w}}$ in canonical form such that the identities (6.1) imply the identity $\mathbf{w} \approx \overline{\mathbf{w}}$.

Proof. Suppose ini $(\mathbf{w})=x_{0} \ldots x_{m}$. Then by Lemma 6.2, it suffices to convert $\mathbf{w}$, using the identities $\{(6.1),(6.2)\}$, into a word in canonical form. This is routine if $m \in\{0,1\}$. Hence assume $m \geqslant 2$. Then $\mathbf{w}$ can be written as $\mathbf{w}=\prod_{i=0}^{m}\left(x_{i} \mathbf{u}_{i}\right)$ for some $\mathbf{u}_{i} \in\left\{x_{0}, \ldots, x_{i}\right\}^{*}$. Since the letters in each $\mathbf{u}_{i}$ are non-first occurrences in $\mathbf{w}$, the identities (6.2c) can be used to arrange them, within $\mathbf{u}_{i}$, in any order. Specifically, any occurrence of $x_{i}$ in $\mathbf{u}_{i}$ can be moved to the left and grouped with the singleton factor $x_{i}$ that precedes $\mathbf{u}_{i}$, and any remaining letters in $\mathbf{u}_{i}$ can be ordered according to their indices. Thus $\mathbf{w}$ can be converted into the word in (6.4) with $e_{i} \geqslant 1$ and $\mathbf{w}_{i} \in\left\{x_{0}^{f_{0}} \ldots x_{i-1}^{f_{i-1}} \mid f_{0}, \ldots, f_{i-1} \geqslant 0\right\}$. The identities $\{(6.2 \mathrm{a}),(6.2 \mathrm{~b})\}$ can then be applied so that $e_{i} \in\{1,2\}$ and $\mathbf{w}_{i} \in\left\{x_{0}^{f_{0}} \ldots x_{i-1}^{f_{i-1}} \mid f_{0}, \ldots, f_{i-1} \in\{0,1\}\right\}$.

Lemma 6.4. Let $\mathbf{V}$ be any subvariety of $\mathbf{X}_{(6.1)}$ that satisfies an identity $\mathbf{w} \approx \mathbf{w}^{\prime}$.

(i) If $\operatorname{ini}(\mathbf{w}) \neq \operatorname{ini}\left(\mathbf{w}^{\prime}\right)$, then $\mathbf{V}$ is finitely based.

(ii) If $\operatorname{occ}(x, \mathbf{w}) \not \equiv \operatorname{occ}\left(x, \mathbf{w}^{\prime}\right)(\bmod 2)$ for some $x \in \mathcal{X}$, then $\mathbf{V}$ is finitely based.

(iii) If $\operatorname{occ}(x, y, \mathbf{w}) \not \equiv \operatorname{occ}\left(x, y, \mathbf{w}^{\prime}\right)(\bmod 2)$ for some $x, y \in \mathcal{X}$, then $\mathbf{V}$ is finitely based.

Proof. It is shown in each of the following parts that $\mathbf{V}$ satisfies the identity $x y x \approx x^{2} y$ and so is finitely based by Condition 4 .

(i) Suppose ini $(\mathbf{w}) \neq \operatorname{ini}\left(\mathbf{w}^{\prime}\right)$. Then $L_{2}^{1} \notin \mathbf{V}$ by Lemma 2.1(iii). If $x^{4}, x^{4} y^{4} z^{4}$, and $x^{4} z^{4} y^{4}$ are distinct elements in the $\mathbf{V}$-free semigroup $F_{\mathbf{V}}$ over $\mathcal{X}$, then they form a subsemigroup of $F_{\mathbf{V}}$ isomorphic to $L_{2}^{1}$, and this implies the contradiction $L_{2}^{1} \in \mathbf{V}$. Hence these three elements of $F_{\mathbf{V}}$ cannot be distinct, so that $\mathbf{V}$ satisfies either $x^{4} \approx x^{4} y^{4} z^{4}, x^{4} \approx x^{4} z^{4} y^{4}$, or $x^{4} y^{4} z^{4} \approx x^{4} z^{4} y^{4}$; it is easily seen that any one of the first two identities implies the third identity, whence $\mathbf{V}$ satisfies the third identity. It follows that $\mathbf{V}$ satisfies the identity $\alpha: x^{2} y^{2} z^{2} \approx x^{2} z^{2} y^{2}$. Since

$$
x y x \stackrel{(6.2 \mathrm{a})}{\approx} x^{3} y x \stackrel{(6.2 \mathrm{~b})}{\approx}\left(x^{2}(x y)^{2} y^{2}\right) y \stackrel{\alpha}{\approx} x^{2} y^{2}(x y)^{2} y \stackrel{(6.2)}{\approx} x^{2} y
$$

the variety $\mathbf{V}$ satisfies the identity $x y x \approx x^{2} y$.

(ii) Suppose $\operatorname{occ}(x, \mathbf{w}) \not \equiv \operatorname{occ}\left(x, \mathbf{w}^{\prime}\right)(\bmod 2)$ for some $x \in \mathcal{X}$. Then $\mathbf{V}$ satisfies the identity $\beta: x^{4} \approx x^{3}$. Since

$$
x y x \stackrel{(6.2 \mathrm{a})}{\approx} x^{3} y x^{3} \stackrel{\beta}{\approx} x^{4} y x^{4} \stackrel{(6.2 \mathrm{~b})}{\approx} x^{4} y \stackrel{(6.2 \mathrm{a})}{\approx} x^{2} y
$$

the variety $\mathbf{V}$ satisfies the identity $x y x \approx x^{2} y$. 
(iii) Suppose $\operatorname{occ}(x, y, \mathbf{w}) \not \equiv \operatorname{occ}\left(x, y, \mathbf{w}^{\prime}\right)(\bmod 2)$ for some $x, y \in \mathcal{X}$. Then by parts (i) and (ii), it suffices to further assume:

(a) $\operatorname{ini}(\mathbf{w})=\operatorname{ini}\left(\mathbf{w}^{\prime}\right)$;

(b) $\operatorname{occ}(x, \mathbf{w}) \equiv \operatorname{occ}\left(x, \mathbf{w}^{\prime}\right)(\bmod 2)$.

Write $\mathbf{w}=\mathbf{w}_{1} y \mathbf{w}_{2}$ and $\mathbf{w}^{\prime}=\mathbf{w}_{1}^{\prime} y \mathbf{w}_{2}^{\prime}$ where $\mathbf{w}_{1}, \mathbf{w}_{2}, \mathbf{w}_{1}^{\prime}, \mathbf{w}_{2}^{\prime} \in \mathcal{X}^{*}$ are such that $y \notin \operatorname{con}\left(\mathbf{w}_{1} \mathbf{w}_{1}^{\prime}\right)$. Let $e_{i}=\operatorname{occ}\left(x, \mathbf{w}_{i}\right)$ and $e_{i}^{\prime}=\operatorname{occ}\left(x, \mathbf{w}_{i}^{\prime}\right)$. Then $e_{1}=\operatorname{occ}(x, y, \mathbf{w}) \not \equiv \operatorname{occ}\left(x, y, \mathbf{w}^{\prime}\right)=e_{1}^{\prime}(\bmod 2)$ by assumption, whence $\left(e_{1}, e_{1}^{\prime}\right) \neq(0,0)$. If either $e_{1}$ or $e_{1}^{\prime}$ is zero, then (a) is violated. Thus $e_{1}, e_{1}^{\prime} \geqslant 1$. By symmetry, it suffices to assume:

(c) $e_{1} \in\{1,3,5, \ldots\}$;

(d) $e_{1}^{\prime} \in\{2,4,6, \ldots\}$.

Since $e_{1}+e_{2}=\operatorname{occ}(x, \mathbf{w}) \stackrel{(\mathrm{b})}{\equiv} \operatorname{occ}\left(x, \mathbf{w}^{\prime}\right)=e_{1}^{\prime}+e_{2}^{\prime}(\bmod 2)$, it follows that $e_{2} \not \equiv e_{2}^{\prime}(\bmod 2)$. Let $r \in\{0,1\}$ be such that $e_{2}+r \equiv 1(\bmod 2)$ and $e_{2}^{\prime}+r \equiv 0(\bmod 2)$. Let $\varphi$ denote the substitution

$$
t \mapsto \begin{cases}y^{2} & \text { if } t=y, \\ x^{2} & \text { if } t \in \mathcal{X} \backslash\{x, y\} .\end{cases}
$$

Then $\mathbf{w}_{1} \varphi=x^{e_{1}+2 k}$ for some $k \geqslant 0$, and $\mathbf{w}_{2} \varphi \in\left\{x, y^{2}\right\}^{*}$ with $\operatorname{occ}\left(x, \mathbf{w}_{2} \varphi\right)=e_{2}+2 \ell$ for some $\ell \geqslant 0$. In view of (c),

$$
\begin{aligned}
(\mathbf{w} \varphi) y x^{r} & =\mathbf{w}_{1} \varphi \cdot y \varphi \cdot \mathbf{w}_{2} \varphi \cdot y x^{r} \stackrel{(6.2 \mathrm{a})}{\approx} x y^{2}\left(\mathbf{w}_{2} \varphi\right) y x^{r} \stackrel{(6.2 \mathrm{~b})}{\approx} x y^{2} x^{e_{2}} y x^{r} \\
& \stackrel{(6.2 \mathrm{c})}{\approx} x y^{3} x^{e_{2}+r} \stackrel{(6.2 \mathrm{a})}{\approx} x y x .
\end{aligned}
$$

Similarly, $\mathbf{w}_{1}^{\prime} \varphi=x^{e_{1}^{\prime}+2 k^{\prime}}$ for some $k^{\prime} \geqslant 0$, and $\mathbf{w}_{2}^{\prime} \varphi \in\left\{x, y^{2}\right\}^{*}$ with $\operatorname{occ}\left(x, \mathbf{w}_{2}^{\prime} \varphi\right)=e_{2}^{\prime}+2 \ell^{\prime}$ for some $\ell^{\prime} \geqslant 0$. In view of $(d)$,

$$
\begin{aligned}
\left(\mathbf{w}^{\prime} \varphi\right) y x^{r} & =\mathbf{w}_{1}^{\prime} \varphi \cdot y \varphi \cdot \mathbf{w}_{2}^{\prime} \varphi \cdot y x^{r} \stackrel{(6.2 \mathrm{a})}{\approx} x^{2} y^{2}\left(\mathbf{w}_{2}^{\prime} \varphi\right) y x^{r} \stackrel{(6.2 \mathrm{~b})}{\approx} x^{2} y^{2} x^{e_{2}^{\prime}} y x^{r} \\
\stackrel{(6.2 \mathrm{c})}{\approx} & x^{2} y^{3} x^{e_{2}^{\prime}+r} \stackrel{(6.2 \mathrm{~b})}{\approx} x^{2} y^{3} \stackrel{(6.2 \mathrm{a})}{\approx} x^{2} y .
\end{aligned}
$$

Consequently, $\mathbf{V}$ satisfies the identity $x y x \approx x^{2} y$.

Lemma 6.5. Let $\mathbf{w}$ and $\mathbf{w}^{\prime}$ be any words in canonical form such that $|\operatorname{con}(\mathbf{w})| \geqslant 3$. Suppose:

(i) $\operatorname{ini}(\mathbf{w})=\operatorname{ini}\left(\mathbf{w}^{\prime}\right)$;

(ii) $\operatorname{occ}(x, \mathbf{w}) \equiv \operatorname{occ}\left(x, \mathbf{w}^{\prime}\right)(\bmod 2)$ for all $x \in \mathcal{X}$;

(iii) $\operatorname{occ}(x, y, \mathbf{w}) \equiv \operatorname{occ}\left(x, y, \mathbf{w}^{\prime}\right)(\bmod 2)$ for all $x, y \in \mathcal{X}$.

Then the words $\mathbf{w}$ and $\mathbf{w}^{\prime}$ are identical.

Proof. By assumption, ini $(\mathbf{w})=x_{0} \ldots x_{m}$ for some $m \geqslant 2$. It follows from (i) and (III) that

$$
\mathbf{w}=x_{0}^{e_{0}} \prod_{i=1}^{m}\left(x_{i}^{e_{i}} \mathbf{w}_{i}\right) \quad \text { and } \quad \mathbf{w}^{\prime}=x_{0}^{e_{0}^{\prime}} \prod_{i=1}^{m}\left(x_{i}^{e_{i}^{\prime}} \mathbf{w}_{i}^{\prime}\right)
$$

for some $e_{i}, e_{i}^{\prime} \in\{1,2\}$ and $\mathbf{w}_{i}, \mathbf{w}_{i}^{\prime} \in\left\{x_{0}^{f_{0}} \ldots x_{i-1}^{f_{i-1}} \mid f_{0}, \ldots, f_{i-1} \in\{0,1\}\right\}$. It is obvious that $\operatorname{occ}\left(x_{m}, \mathbf{w}\right)=e_{m}$ and $\operatorname{occ}\left(x_{m}, \mathbf{w}^{\prime}\right)=e_{m}^{\prime}$, so that $e_{m}=e_{m}^{\prime}$ by (ii). For each $i<m$, since $\operatorname{occ}\left(x_{i}, x_{i+1}, \mathbf{w}\right)=e_{i}$ and $\operatorname{occ}\left(x_{i}, x_{i+1}, \mathbf{w}^{\prime}\right)=e_{i}^{\prime}$, it follows from (iii) that $e_{i}=e_{i}^{\prime}$. Therefore $\left(e_{0}, \ldots, e_{m}\right)=\left(e_{0}^{\prime}, \ldots, e_{m}^{\prime}\right)$.

If $\left(\mathbf{w}_{1}, \ldots, \mathbf{w}_{m-1}\right)=\left(\mathbf{w}_{1}^{\prime}, \ldots, \mathbf{w}_{m-1}^{\prime}\right)$ and $\mathbf{w}_{m} \neq \mathbf{w}_{m}^{\prime}$, say $x_{j} \in \operatorname{con}\left(\mathbf{w}_{m}\right) \backslash \operatorname{con}\left(\mathbf{w}_{m}^{\prime}\right)$ for some $j<m$, then (ii) is violated because

$$
\begin{aligned}
\operatorname{occ}\left(x_{j}, \mathbf{w}\right) & =\operatorname{occ}\left(x_{j}, x_{0}^{e_{0}} x_{1}^{e_{1}} \mathbf{w}_{1} x_{2}^{e_{2}} \mathbf{w}_{2} \ldots x_{m-1}^{e_{m-1}} \mathbf{w}_{m-1}\right)+\operatorname{occ}\left(x_{j}, x_{m}^{e_{m}} \mathbf{w}_{m}\right) \\
& =\operatorname{occ}\left(x_{j}, x_{0}^{e_{0}^{\prime}} x_{1}^{e_{1}^{\prime}} \mathbf{w}_{1}^{\prime} x_{2}^{e_{2}^{\prime}} \mathbf{w}_{2}^{\prime} \ldots x_{m-1}^{e_{m-1}^{\prime}} \mathbf{w}_{m-1}^{\prime}\right)+1
\end{aligned}
$$


and

$$
\begin{aligned}
\operatorname{occ}\left(x_{j}, \mathbf{w}^{\prime}\right) & =\operatorname{occ}\left(x_{j}, x_{0}^{e_{0}^{\prime}} x_{1}^{e_{1}^{\prime}} \mathbf{w}_{1}^{\prime} x_{2}^{e_{2}^{\prime}} \mathbf{w}_{2}^{\prime} \ldots x_{m-1}^{e_{m-1}^{\prime}} \mathbf{w}_{m-1}^{\prime}\right)+\operatorname{occ}\left(x_{j}, x_{m}^{e_{m}^{\prime}} \mathbf{w}_{m}^{\prime}\right) \\
& =\operatorname{occ}\left(x_{j}, x_{0}^{e_{0}^{\prime}} x_{1}^{e_{1}^{\prime}} \mathbf{w}_{1}^{\prime} x_{2}^{e_{2}^{\prime}} \mathbf{w}_{2}^{\prime} \ldots x_{m-1}^{e_{m-1}^{\prime}} \mathbf{w}_{m-1}^{\prime}\right)+0 .
\end{aligned}
$$

Hence $\mathbf{w}_{m}=\mathbf{w}_{m}^{\prime}$. Suppose that $\ell<m$ is the least integer such that $\mathbf{w}_{\ell} \neq \mathbf{w}_{\ell}^{\prime}$, say $k<\ell$ is such that $x_{k} \in \operatorname{con}\left(\mathbf{w}_{\ell}\right) \backslash \operatorname{con}\left(\mathbf{w}_{\ell}^{\prime}\right)$. Then $\left(\mathbf{w}_{1}, \ldots, \mathbf{w}_{\ell-1}\right)=\left(\mathbf{w}_{1}^{\prime}, \ldots, \mathbf{w}_{\ell-1}^{\prime}\right)$, whence (iii) is violated because

$$
\begin{aligned}
\operatorname{occ}\left(x_{k}, x_{\ell+1}, \mathbf{w}\right) & =\operatorname{occ}\left(x_{k}, x_{0}^{e_{0}} x_{1}^{e_{1}} \mathbf{w}_{1} x_{2}^{e_{2}} \mathbf{w}_{2} \ldots x_{\ell-1}^{e_{\ell-1}} \mathbf{w}_{\ell-1}\right)+\operatorname{occ}\left(x_{k}, x_{\ell}^{e_{\ell}} \mathbf{w}_{\ell}\right) \\
& =\operatorname{occ}\left(x_{k}, x_{0}^{e_{0}^{\prime}} x_{1}^{e_{1}^{\prime}} \mathbf{w}_{1}^{\prime} x_{2}^{e_{2}^{\prime}} \mathbf{w}_{2}^{\prime} \ldots x_{\ell-1}^{e_{\ell-1}^{\prime}} \mathbf{w}_{\ell-1}^{\prime}\right)+1
\end{aligned}
$$

and

$$
\begin{aligned}
\operatorname{occ}\left(x_{k}, x_{\ell+1}, \mathbf{w}^{\prime}\right) & =\operatorname{occ}\left(x_{k}, x_{0}^{e_{0}^{\prime}} x_{1}^{e_{1}^{\prime}} \mathbf{w}_{1}^{\prime} x_{2}^{e_{2}^{\prime}} \mathbf{w}_{2}^{\prime} \ldots x_{\ell-1}^{e_{\ell-1}^{\prime}} \mathbf{w}_{\ell-1}^{\prime}\right)+\operatorname{occ}\left(x_{k}, x_{\ell}^{e_{\ell}^{\prime}} \mathbf{w}_{\ell}^{\prime}\right) \\
& =\operatorname{occ}\left(x_{k}, x_{0}^{e_{0}^{\prime}} x_{1}^{e_{1}^{\prime}} \mathbf{w}_{1}^{\prime} x_{2}^{e_{2}^{\prime}} \mathbf{w}_{2}^{\prime} \ldots x_{\ell-1}^{e_{\ell-1}^{\prime}} \mathbf{w}_{\ell-1}^{\prime}\right)+0 .
\end{aligned}
$$

Thus $\left(\mathbf{w}_{1}, \ldots, \mathbf{w}_{m}\right)=\left(\mathbf{w}_{1}^{\prime}, \ldots, \mathbf{w}_{m}^{\prime}\right)$, so that $\mathbf{w}=\mathbf{w}^{\prime}$.

\subsection{Proof of Proposition 6.1}

Let $\mathbf{V}$ be any proper subvariety of $\mathbf{X}_{(6.1)}$. Then $\mathbf{V}=\mathbf{X}_{(6.1)} \Sigma$ for some set $\Sigma$ of nontrivial identities. By Lemma 6.3, words that form the identities in $\Sigma$ can be chosen to be in canonical form. Let $\mathbf{w} \approx \mathbf{w}^{\prime}$ be any identity in $\Sigma$. By Lemma 6.4 , the variety $\mathbf{V}$ is finitely based if $\mathbf{w}$ and $\mathbf{w}^{\prime}$ violate any of the following conditions:

(a) $\operatorname{ini}(\mathbf{w})=\operatorname{ini}\left(\mathbf{w}^{\prime}\right)$;

(b) $\operatorname{occ}(x, \mathbf{w}) \equiv \operatorname{occ}\left(x, \mathbf{w}^{\prime}\right)(\bmod 2)$ for all $x \in \mathcal{X}$;

(c) $\operatorname{occ}(x, y, \mathbf{w}) \equiv \operatorname{occ}\left(x, y, \mathbf{w}^{\prime}\right)(\bmod 2)$ for all $x, y \in \mathcal{X}$.

Hence assume that $\mathbf{w}$ and $\mathbf{w}^{\prime}$ satisfy $(\mathrm{a})-(\mathrm{c})$. If the identity $\mathbf{w} \approx \mathbf{w}^{\prime}$ involves at least three distinct letters, then it is trivial by Lemma 6.5, contradicting the choice of identities for $\Sigma$. Therefore $\mathbf{w} \approx \mathbf{w}^{\prime}$ involves at most two distinct letters, whence $\mathbf{w}$ and $\mathbf{w}^{\prime}$ are from (6.3). Since $\mathbf{w} \approx \mathbf{w}^{\prime}$ is arbitrary in $\Sigma$, it follows that $\Sigma$ contains finitely many identities. (In fact, since the identities in $\Sigma$ satisfy (a)-(c), it is easily shown that $\Sigma \subseteq\left\{x^{3} \approx x, x^{4} \approx x^{2}, x^{3} y \approx x y\right\}$.)

REMARK 6.6. It is routinely shown that $N_{3}$ and the orthodox completely regular semigroup

$$
O=\left\langle a, b \mid a^{2}=a b=a, b^{2}=1\right\rangle
$$

of order four belong to the variety $\mathbf{X}_{(6.1)}$. From the proofs in this section, a proper subvariety of $\mathbf{X}_{(6.1)}$ satisfies one of the following identities:

$$
\begin{gathered}
x^{4} \approx x^{2}, \\
x y x \approx x^{2} y .
\end{gathered}
$$

By Lemma 2.1(vi) or direct verification, the semigroup $N_{3}$ does not satisfy the identity (6.5). The semigroup $O$ does not satisfy the identity (6.6) because $b a b \neq b^{2} a$ in $O$. Consequently, the variety $\mathbf{X}_{(6.1)}$ is generated by $N_{3}$ and $O$.

\section{On Condition 9}

Let $\mathbf{X}_{(7.1)}$ denote the variety defined by the identities

$$
x^{3} \mathrm{H} x \approx x \mathrm{H} x, \quad x y x y \approx x^{2} y^{2}, \quad x y^{2} z \approx x z y^{2} .
$$

Then Condition 9 is equivalent to the following result. 
Proposition 7.1. All subvarieties of $\mathbf{X}_{(7.1)}$ are finitely based.

It follows from Lemma 2.1(i) that the variety $\mathbf{X}_{(7.1)}$ contains the semigroup $L_{2}$. All subvarieties of $\mathbf{X}_{(7.1)}$ that contain $L_{2}$ are shown to be finitely based in $\S 7.2$, while those that do not contain $L_{2}$ are shown to be finitely based in $\S 7.3$. The proof of Proposition 7.1 is thus complete.

7.1. Identities satisfied by subvarieties of $\mathbf{X}_{(7.1)}$

Lemma 7.2. The identities (7.1) imply the identities

$$
\begin{aligned}
x^{4} \approx x^{2}, \quad x^{3} y x & \approx x y x, \quad x y x^{3} \approx x y x, \\
x \mathrm{H} x y x^{2} & \approx x \mathrm{H} x y, \\
h x^{2} y & \approx h y x^{2}, \\
h x y \mathrm{~K} x \mathrm{~T} y \approx h y x \mathrm{~K} x \mathrm{~T} y, \quad x \mathrm{H} x y \mathrm{~K} y & \approx x \mathrm{H} y x \mathrm{~K} y, \quad x \mathrm{H} y \mathrm{~K} x y \approx x \mathrm{H} y \mathrm{~K} y x .
\end{aligned}
$$

Proof. It is clear that the identities (7.1) imply the identities (7.2a)-(7.2c). Since

$$
\begin{aligned}
h y x \mathrm{~K} x \mathrm{~T} y & \stackrel{(7.2 \mathrm{a})}{\approx} h y y^{2} x^{2} x \mathrm{~K} x \mathrm{~T} y \stackrel{(7.2 \mathrm{c})}{\approx} h x^{2} y x y^{2} \mathrm{~K} x \mathrm{~T} y \stackrel{(7.1)}{\approx} h x^{3} y^{3} \mathrm{~K} x \mathrm{~T} y \\
& \stackrel{(7.2 \mathrm{a})}{\approx} h x y \mathrm{~K} x \mathrm{~T} y,
\end{aligned}
$$

the identities (7.1) imply $h x y \mathrm{~K} x \mathrm{~T} y \approx h y x \mathrm{~K} x \mathrm{~T} y$ in (7.2d). A similar argument shows that (7.1) also imply the other identities in $(7.2 \mathrm{~d})$.

Lemma 7.3. Let $\mathbf{V}$ be any subvariety of $\mathbf{X}_{(7.1)}$ that satisfies an identity $\mathbf{w} \approx \mathbf{w}^{\prime}$. Suppose that any of the following holds:

(i) either $\operatorname{con}(\mathbf{w}) \neq \operatorname{con}\left(\mathbf{w}^{\prime}\right)$ or $\operatorname{sim}(\mathbf{w}) \neq \operatorname{sim}\left(\mathbf{w}^{\prime}\right)$;

(ii) $\operatorname{occ}(x, \mathbf{w}) \not \equiv \operatorname{occ}\left(x, \mathbf{w}^{\prime}\right)(\bmod 2)$ for some $x \in \mathcal{X}$;

(iii) $\operatorname{occ}(x, y, \mathbf{w}) \not \equiv \operatorname{occ}\left(x, y, \mathbf{w}^{\prime}\right)(\bmod 2)$ for some $x \in \operatorname{con}(\mathbf{w})$ and $y \in \operatorname{sim}(\mathbf{w})$.

Then $\mathbf{V}$ is finitely based.

Proof. It is shown that each of (i)-(iii) implies that $\mathbf{V}$ satisfies the identity $x y x \approx x^{2} y$ and so is finitely based by Condition 4 .

Suppose that (i) holds. Then $N_{2}^{1} \notin \mathbf{V}$ by Lemma $2.1(\mathrm{v})$. Hence by Lemma 2.9 , the variety $\mathbf{V}$ satisfies the identity $h^{2}\left(x h^{2}\right)^{3} \approx h^{2} x h^{2}$; this identity can be converted into $\alpha: h^{2} x^{3} \approx h^{2} x$ by the identities (7.2b). Then $\mathbf{V}$ satisfies the identity $x y x \approx x^{2} y$ because

$$
x y x \stackrel{(7.2 \mathrm{a})}{\approx} x^{3} y x \stackrel{\alpha}{\approx} x^{3} y^{3} x \stackrel{(7.2 \mathrm{a})}{\approx} x y^{3} x \stackrel{(7.2 \mathrm{c})}{\approx} x y x y^{2} \stackrel{(7.2 \mathrm{~d})}{\approx} x^{2} y^{3} \underset{\approx}{\approx} x^{2} y .
$$

Suppose that (ii) holds. Then it is easily shown that $\mathbf{V}$ satisfies the identity $\beta: x^{3} \approx x^{2}$; it also satisfies the identity $x y x \approx x^{2} y$ because

$$
x y x \stackrel{(7.2 \mathrm{a})}{\approx} x^{3} y x^{3} \stackrel{\beta}{\approx} x^{2} y x^{2} \stackrel{(7.2 \mathrm{~b})}{\approx} x^{2} y .
$$

Suppose that (iii) holds, that is, $\operatorname{occ}(x, y, \mathbf{w}) \not \equiv \operatorname{occ}\left(x, y, \mathbf{w}^{\prime}\right)(\bmod 2)$ for some $x \in \operatorname{con}(\mathbf{w})$ and $y \in \operatorname{sim}(\mathbf{w})$. As established in the last two paragraphs, $\mathbf{V}$ is finitely based if (i) or (ii) holds. Hence it suffices to further assume that (i) and (ii) do not hold, that is, $\operatorname{con}(\mathbf{w})=\operatorname{con}\left(\mathbf{w}^{\prime}\right)$, $\operatorname{sim}(\mathbf{w})=\operatorname{sim}\left(\mathbf{w}^{\prime}\right)$, and $\operatorname{occ}(x, \mathbf{w}) \equiv \operatorname{occ}\left(x, \mathbf{w}^{\prime}\right)(\bmod 2)$. Since $y \in \operatorname{sim}(\mathbf{w})=\operatorname{sim}\left(\mathbf{w}^{\prime}\right)$, it follows that $\mathbf{w}=\mathbf{w}_{1} y \mathbf{w}_{2}$ and $\mathbf{w}^{\prime}=\mathbf{w}_{1}^{\prime} y \mathbf{w}_{2}^{\prime}$ for some $\mathbf{w}_{1}, \mathbf{w}_{2}, \mathbf{w}_{1}^{\prime}, \mathbf{w}_{2}^{\prime} \in(\mathcal{X} \backslash\{y\})^{*}$. Let $e_{i}=\operatorname{occ}\left(x, \mathbf{w}_{i}\right)$ and $e_{i}^{\prime}=\operatorname{occ}\left(x, \mathbf{w}_{i}^{\prime}\right)$. Then $e_{1}=\operatorname{occ}(x, y, \mathbf{w}), e_{1}^{\prime}=\operatorname{occ}\left(x, y, \mathbf{w}^{\prime}\right)$, and

$$
e_{1}+e_{2}=\operatorname{occ}(x, \mathbf{w}) \equiv \operatorname{occ}\left(x, \mathbf{w}^{\prime}\right)=e_{1}^{\prime}+e_{2}^{\prime} \quad(\bmod 2) .
$$


Since $e_{1} \not \equiv e_{1}^{\prime}(\bmod 2)$ by assumption, it follows that $e_{2} \not \equiv e_{2}^{\prime}(\bmod 2)$. By symmetry, it suffices to assume that $e_{1}$ is even and $e_{1}^{\prime}$ is odd. Then there exists some $r \in\{0,1\}$ such that $e_{2}+r$ is even and $e_{2}^{\prime}+r$ is odd. Let $\varphi$ denote the substitution $z \mapsto x^{2}$ for all $z \in \mathcal{X} \backslash\{x, y\}$. Then

$$
x^{2}(\mathbf{w} \varphi) x^{r+2}=x^{2}\left(\mathbf{w}_{1} \varphi\right) y\left(\mathbf{w}_{2} \varphi\right) x^{r+2} \stackrel{(7.2 \mathrm{a})}{\approx} x^{2} y x^{2} \stackrel{(7.2 \mathrm{~b})}{\approx} x^{2} y
$$

and

$$
x^{2}\left(\mathbf{w}^{\prime} \varphi\right) x^{r+2}=x^{2}\left(\mathbf{w}_{1}^{\prime} \varphi\right) y\left(\mathbf{w}_{2}^{\prime} \varphi\right) x^{r+2} \stackrel{(7.2 \mathrm{a})}{\approx} x y x,
$$

so that $\mathbf{V}$ satisfies the identity $x y x \approx x^{2} y$.

\subsection{Subvarieties of $\mathbf{X}_{(7.1)}$ containing $L_{2}$}

Proposition 7.4. Let $\mathbf{V}$ be any subvariety of $\mathbf{X}_{(7.1)}$ such that $L_{2} \in \mathbf{V}$. Then $\mathbf{V}$ is finitely based.

The proof of this result is given at the end of the subsection.

Lemma 7.5. Let $x \in \mathcal{X}$ and $\mathbf{w}, \mathbf{w}^{\prime} \in \mathcal{X}^{+}$be such that $\operatorname{con}(\mathbf{w})=\operatorname{con}\left(\mathbf{w}^{\prime}\right), \operatorname{sim}(\mathbf{w})=\operatorname{sim}\left(\mathbf{w}^{\prime}\right)$, and $x \notin \operatorname{con}\left(\mathbf{w} \mathbf{w}^{\prime}\right)$. Suppose that the words $\mathbf{w}$ and $\mathbf{w}^{\prime}$ begin with the same non-simple letter. Then $\mathbf{X}_{(7.1)}\left\{\mathbf{w} \approx \mathbf{w}^{\prime}\right\}=\mathbf{X}_{(7.1)}\left\{x \mathbf{w} \approx x \mathbf{w}^{\prime}\right\}$.

Proof. By assumption, $\mathbf{w}, \mathbf{w}^{\prime} \in z \mathcal{X}^{*} z \mathcal{X}^{*}$ with $z=\mathrm{h}(\mathbf{w})=\mathrm{h}\left(\mathbf{w}^{\prime}\right)$, so that the deductions $z^{2} \mathbf{w} \stackrel{(7.2 a)}{\approx} \mathbf{w}$ and $z^{2} \mathbf{w}^{\prime} \stackrel{(7.2 \mathrm{a} a)}{\approx} \mathbf{w}^{\prime}$ hold. Since the identity $x \mathbf{w} \approx x \mathbf{w}^{\prime}$ clearly implies the identity $z^{2} \mathbf{w} \approx z^{2} \mathbf{w}^{\prime}$, the inclusion $\mathbf{X}_{(7.1)}\left\{x \mathbf{w} \approx x \mathbf{w}^{\prime}\right\} \subseteq \mathbf{X}_{(7.1)}\left\{\mathbf{w} \approx \mathbf{w}^{\prime}\right\}$ holds. It is obvious that the inclusion $\mathbf{X}_{(7.1)}\left\{\mathbf{w} \approx \mathbf{w}^{\prime}\right\} \subseteq \mathbf{X}_{(7.1)}\left\{x \mathbf{w} \approx x \mathbf{w}^{\prime}\right\}$ also holds.

Let $\mathbf{w}$ be any non-simple word with $\mathrm{h}(\mathbf{w}) \in \operatorname{sim}(\mathbf{w})$ and let $x_{1}, \ldots, x_{r}$ be all its distinct non-simple letters listed in alphabetical order. In this section, such a word is said to be in canonical form if

$$
\mathbf{w}=\prod_{i=1}^{m}\left(y_{i} \mathbf{w}_{i}\right)
$$

for some $m \geqslant 1$ such that all of the following are satisfied:

(I) the letters $y_{1}, \ldots, y_{m}$ are all simple in $\mathbf{w}$;

(II) the letters of $\mathbf{w}_{1}, \ldots, \mathbf{w}_{m} \in \mathcal{X}^{*}$ are all non-simple in $\mathbf{w}$;

(III) $\mathbf{w}_{1}, \ldots, \mathbf{w}_{m-1} \in\left\{x_{1}^{e_{1}} \ldots x_{r}^{e_{r}} \mid e_{1}, \ldots, e_{r} \in\{0,1\}\right\}$;

(IV) $\mathbf{w}_{m}=x_{1}^{f_{1}} \ldots x_{r}^{f_{r}}$ for some $f_{1}, \ldots, f_{r} \in\{0,1,2,3\}$;

(V) if $\operatorname{occ}\left(x_{i}, \mathbf{w}_{1} \ldots \mathbf{w}_{m-1}\right)=0$, then $f_{i} \in\{2,3\}$;

(VI) if occ $\left(x_{i}, \mathbf{w}_{1} \ldots \mathbf{w}_{m-1}\right)=1$, then $f_{i} \in\{1,2\}$;

(VII) if occ $\left(x_{i}, \mathbf{w}_{1} \ldots \mathbf{w}_{m-1}\right) \geqslant 2$, then $f_{i} \in\{0,1\}$.

Lemma 7.6. Let $\mathbf{w}$ be any non-simple word with $\mathrm{h}(\mathbf{w}) \in \operatorname{sim}(\mathbf{w})$. Then there exists some word $\overline{\mathbf{w}}$ in canonical form such that the identities (7.1) imply the identity $\mathbf{w} \approx \overline{\mathbf{w}}$.

Proof. Generality is not lost by assuming that $x_{1}, \ldots, x_{r}$ are all the distinct non-simple letters of $\mathbf{w}$ listed in alphabetical order. By Lemma 7.2, it suffices to convert $\mathbf{w}$, using the identities (7.2), into a word in canonical form. It is clear that $\mathbf{w}$ can be written in the form (7.3) with (I) and (II) satisfied. The identities (7.2d) can be used to order the letters in each $\mathbf{w}_{i}$ alphabetically, resulting in $\mathbf{w}_{i} \in\left\{x_{1}^{e_{1}} \ldots x_{r}^{e_{r}} \mid e_{1}, \ldots, e_{r} \geqslant 0\right\}$. The identities (7.2a) can then be used to reduce the exponent of each $x_{j}$, so that $\mathbf{w}_{i} \in\left\{x_{1}^{e_{1}} \ldots x_{r}^{e_{r}} \mid e_{1}, \ldots, e_{r} \in\{0,1,2,3\}\right\}$. If $i<m$ is such that $\mathbf{w}_{i}$ contains the factor $x_{j}^{2}$, then this factor can be moved by the identity (7.2c) 
to the right and combined with the corresponding power of $x_{j}$ in $\mathbf{w}_{m}$. Hence (III) is satisfied, and $\mathbf{w}_{m}=x_{1}^{f_{1}} \ldots x_{r}^{f_{r}}$ for some $f_{1}, \ldots, f_{r} \geqslant 0$. The identities (7.2a) can be used to reduce the exponents $f_{1}, \ldots, f_{r}$ in $\mathbf{w}_{m}$ appropriately until (IV) is satisfied. Since $x_{i}$ is non-simple in $\mathbf{w}$, it follows that $\operatorname{occ}\left(x_{i}, \mathbf{w}_{1} \ldots \mathbf{w}_{m-1}\right)+f_{i}=\operatorname{occ}\left(x_{i}, \mathbf{w}\right) \geqslant 2$, whence (V) is satisfied. If $\operatorname{occ}\left(x_{i}, \mathbf{w}_{1} \ldots \mathbf{w}_{m-1}\right)=1$ and $f_{i}=3$, then the identities $(7.2 \mathrm{a})$ can be used to reduce the exponent $f_{i}$ to 1 . Thus (VI) is satisfied. Finally, if $\operatorname{occ}\left(x_{i}, \mathbf{w}_{1} \ldots \mathbf{w}_{m-1}\right) \geqslant 2$ and $f_{i} \in\{2,3\}$, then the identities $(7.2 \mathrm{~b})$ can be used to reduce the exponent $f_{i}$ to 0 or 1 , so that (VII) is satisfied.

Proof of Proposition 7.4. By assumption, $\mathbf{V}=\mathbf{X}_{(7.1)} \Sigma$ for some set $\Sigma$ of identities. The variety $\mathbf{V}$ is finitely based if $\Sigma$ contains some identity $\mathbf{w} \approx \mathbf{w}^{\prime}$ that satisfies any of (i)-(iii) in Lemma 7.3. Therefore it suffices to assume that every identity $\mathbf{w} \approx \mathbf{w}^{\prime}$ in $\Sigma$ satisfies all of the following:

(a) $\operatorname{con}(\mathbf{w})=\operatorname{con}\left(\mathbf{w}^{\prime}\right)$ and $\operatorname{sim}(\mathbf{w})=\operatorname{sim}\left(\mathbf{w}^{\prime}\right)$;

(b) $\operatorname{occ}(x, \mathbf{w}) \equiv \operatorname{occ}\left(x, \mathbf{w}^{\prime}\right)(\bmod 2)$ for all $x \in \mathcal{X}$;

(c) $\operatorname{occ}(x, y, \mathbf{w}) \equiv \operatorname{occ}\left(x, y, \mathbf{w}^{\prime}\right)(\bmod 2)$ for all $x \in \mathcal{X}$ and $y \in \operatorname{sim}(\mathbf{w})=\operatorname{sim}\left(\mathbf{w}^{\prime}\right)$.

Since $L_{2} \in \mathbf{V}$, it follows from Lemma 2.1(i) that any identity $\mathbf{w} \approx \mathbf{w}^{\prime}$ in $\Sigma$ satisfies the property that $\mathrm{h}(\mathbf{w})=\mathrm{h}\left(\mathbf{w}^{\prime}\right)$; by Lemma 7.5, the letter $\mathrm{h}(\mathbf{w})=\mathrm{h}\left(\mathbf{w}^{\prime}\right)$ can be assumed to be simple in $\mathbf{w}$ and $\mathbf{w}^{\prime}$. Hence by Lemma 7.6, the words $\mathbf{w}$ and $\mathbf{w}^{\prime}$ can be chosen to be in canonical form.

Let $\mathbf{w} \approx \mathbf{w}^{\prime}$ be any identity from $\Sigma$. By (a), the words $\mathbf{w}$ and $\mathbf{w}^{\prime}$ share the same simple letters. By (c), the order of appearance of these simple letters is the same in $\mathbf{w}$ and $\mathbf{w}^{\prime}$. Hence

$$
\mathbf{w}=\prod_{i=1}^{m}\left(y_{i} \mathbf{w}_{i}\right) \quad \text { and } \quad \mathbf{w}^{\prime}=\prod_{i=1}^{m}\left(y_{i} \mathbf{w}_{i}^{\prime}\right) .
$$

It follows from (a), (c), and (III) that

(d) $\mathbf{w}_{i}=\mathbf{w}_{i}^{\prime}$ for all $i<m$.

Now (IV) and (b) imply $\mathbf{w}_{m}=x_{1}^{f_{1}} \ldots x_{r}^{f_{r}}$ and $\mathbf{w}_{m}^{\prime}=x_{1}^{f_{1}^{\prime}} \ldots x_{r}^{f_{r}^{\prime}}$ for some $f_{i}, f_{i}^{\prime} \in\{0,1,2,3\}$ with $f_{i} \equiv f_{i}^{\prime}(\bmod 2)$. Suppose $f_{i}<f_{i}^{\prime}$ for some $i$. Then there are two cases: $\left(f_{i}, f_{i}^{\prime}\right)=(0,2)$ and $\left(f_{i}, f_{i}^{\prime}\right)=(1,3)$. If $\left(f_{i}, f_{i}^{\prime}\right)=(0,2)$, then

$$
\operatorname{occ}\left(x_{i}, \mathbf{w}_{1} \ldots \mathbf{w}_{m-1}\right) \geqslant 2 \text { by (VII) and } \operatorname{occ}\left(x_{i}, \mathbf{w}_{1}^{\prime} \ldots \mathbf{w}_{m-1}^{\prime}\right) \leqslant 1 \quad \text { by }(\mathrm{V}) \text { and }(\mathrm{VI}) \text {. }
$$

If $\left(f_{i}, f_{i}^{\prime}\right)=(1,3)$, then

$$
\operatorname{occ}\left(x_{i}, \mathbf{w}_{1} \ldots \mathbf{w}_{m-1}\right) \geqslant 1 \text { by (VI) and (VII) and } \operatorname{occ}\left(x_{i}, \mathbf{w}_{1}^{\prime} \ldots \mathbf{w}_{m-1}^{\prime}\right)=0 \text { by }(\mathrm{V}) \text {. }
$$

Since $\mathbf{w}_{1} \ldots \mathbf{w}_{m-1}=\mathbf{w}_{1}^{\prime} \ldots \mathbf{w}_{m-1}^{\prime}$ by $(\mathrm{d})$, both cases are impossible. Therefore $f_{i}=f_{i}^{\prime}$ for all $i$, whence $\mathbf{w}=\mathbf{w}^{\prime}$. Since the identity $\mathbf{w} \approx \mathbf{w}^{\prime}$ is arbitrary in $\Sigma$, every identity in $\Sigma$ is trivial. Consequently, the variety $\mathbf{V}$ coincides with $\mathbf{X}_{(7.1)}$ and is finitely based.

\subsection{Subvarieties of $\mathbf{X}_{(7.1)}$ not containing $L_{2}$}

Lemma 7.7. Let $\mathbf{V}$ be any subvariety of $\mathbf{X}_{(7.1)}$ that satisfies an identity $\mathbf{w} \approx \mathbf{w}^{\prime}$. Suppose $\mathrm{h}(\mathbf{w}) \neq \mathrm{h}\left(\mathbf{w}^{\prime}\right)$ with either $\mathrm{h}(\mathbf{w}) \in \operatorname{sim}(\mathbf{w})$ or $\mathrm{h}\left(\mathbf{w}^{\prime}\right) \in \operatorname{sim}\left(\mathbf{w}^{\prime}\right)$. Then $\mathbf{V}$ is finitely based.

Proof. If either $\operatorname{con}(\mathbf{w}) \neq \operatorname{con}\left(\mathbf{w}^{\prime}\right)$ or $\operatorname{sim}(\mathbf{w}) \neq \operatorname{sim}\left(\mathbf{w}^{\prime}\right)$, then the variety $\mathbf{V}$ is finitely based by Lemma 7.3. Therefore assume $\operatorname{con}(\mathbf{w})=\operatorname{con}\left(\mathbf{w}^{\prime}\right)$ and $\operatorname{sim}(\mathbf{w})=\operatorname{sim}\left(\mathbf{w}^{\prime}\right)$. Let $x=\mathrm{h}(\mathbf{w})$ and $y=\mathrm{h}\left(\mathbf{w}^{\prime}\right)$. By symmetry, it suffices to assume $x \in \operatorname{sim}(\mathbf{w})=\operatorname{sim}\left(\mathbf{w}^{\prime}\right)$. Let $\varphi$ denote the substitution $z \mapsto y^{2}$ for all $z \in \mathcal{X} \backslash\{x\}$. Then the deductions $\mathbf{w} \varphi \stackrel{(7.2)}{\approx} x y^{2}$ and $\mathbf{w}^{\prime} \varphi \stackrel{(7.2)}{\approx} y^{2} x$ hold, so that $\mathbf{V}$ satisfies the identity $x y^{2} \approx y^{2} x$; any subvariety of $\mathbf{X}_{(7.1)}$ that satisfies this identity is finitely based [9, Theorem 7.2]. 
Proposition 7.8. Let $\mathbf{V}$ be any subvariety of $\mathbf{X}_{(7.1)}$ such that $L_{2} \notin \mathbf{V}$. Then $\mathbf{V}$ is finitely based.

Proof. By assumption, $\mathbf{V}=\mathbf{X}_{(7.1)} \Sigma$ for some set $\Sigma$ of identities. Let

$$
\Sigma_{\oplus}=\left\{\mathbf{w} \approx \mathbf{w}^{\prime} \in \Sigma \mid \mathrm{h}(\mathbf{w})=\mathrm{h}\left(\mathbf{w}^{\prime}\right)\right\} \quad \text { and } \quad \Sigma_{\ominus}=\left\{\mathbf{w} \approx \mathbf{w}^{\prime} \in \Sigma \mid \mathrm{h}(\mathbf{w}) \neq \mathrm{h}\left(\mathbf{w}^{\prime}\right)\right\},
$$

so that $\Sigma=\Sigma_{\oplus} \cup \Sigma_{\ominus}$. By Lemma 2.1(i), the variety $\mathbf{X}_{(7.1)} \Sigma_{\oplus}$ contains $L_{2}$ and so is finitely based by Proposition 7.4. It remains to show that $\mathbf{X}_{(7.1)} \Sigma_{\ominus}$ is finitely based, so that the variety $\mathbf{V}=\mathbf{X}_{(7.1)} \Sigma_{\oplus} \cap \mathbf{X}_{(7.1)} \Sigma_{\ominus}$ is also finitely based.

By Lemmas 7.3 and 7.7 , the variety $\mathbf{V}$ is finitely based if some identity $\mathbf{w} \approx \mathbf{w}^{\prime}$ from $\Sigma_{\ominus}$ violates any of the following conditions:

(a) $\operatorname{con}(\mathbf{w})=\operatorname{con}\left(\mathbf{w}^{\prime}\right)$ and $\operatorname{sim}(\mathbf{w})=\operatorname{sim}\left(\mathbf{w}^{\prime}\right)$;

(b) $\mathrm{h}(\mathbf{w})$ is non-simple in $\mathbf{w}$ and $\mathrm{h}\left(\mathbf{w}^{\prime}\right)$ is non-simple in $\mathbf{w}^{\prime}$.

Hence it suffices to assume that any identity $\mathbf{w} \approx \mathbf{w}^{\prime}$ in $\Sigma_{\ominus}$ satisfies (a) and (b).

Let $\sigma: \mathbf{w} \approx \mathbf{w}^{\prime}$ be any identity from $\Sigma_{\ominus}$, so that $\mathrm{h}(\mathbf{w})=x \neq y=\mathrm{h}\left(\mathbf{w}^{\prime}\right)$. Let $\varphi: \mathcal{X} \rightarrow \mathcal{X}^{+}$ denote the substitution

$$
z \mapsto \begin{cases}x^{2} & \text { if } z=x, \\ y^{2} & \text { otherwise. }\end{cases}
$$

Then the deductions $\mathbf{w} \varphi \stackrel{(7.2)}{\approx} x^{2} y^{2}$ and $\mathbf{w}^{\prime} \varphi \stackrel{(7.2)}{\approx} y^{2} x^{2}$ hold, so that the identities $\{(7.2), \sigma\}$ imply the identity

$$
x^{2} y^{2} \approx y^{2} x^{2} .
$$

Thus $\mathbf{X}_{(7.1)}\{\sigma\}=\mathbf{X}_{(7.1)}\{(7.4), \sigma\}$. It follows from (a) and (b) that $\mathbf{w}=x \mathbf{h} y \mathbf{t}$ for some $\mathbf{h}, \mathbf{t} \in \mathcal{X}^{*}$ such that $x, y \in \operatorname{con}(\mathbf{h t})$. Since

$$
\mathbf{w} \stackrel{(7.2 \mathrm{a})}{\approx} x^{3} \mathbf{h} y^{3} \mathbf{t} \stackrel{(7.2 \mathrm{c})}{\approx} x^{2} y^{2} x \mathbf{h} y \mathbf{t} \stackrel{(7.4)}{\approx} y^{2} x^{2} x \mathbf{h} y \mathbf{t} \stackrel{(7.2 \mathrm{a})}{\approx} y^{2} \mathbf{w}
$$

the equality $\mathbf{X}_{(7.1)}\{\sigma\}=\mathbf{X}_{(7.1)}\{(7.4), \tilde{\sigma}\}$ holds, where $\widetilde{\sigma}$ is the identity $y^{2} \mathbf{w} \approx \mathbf{w}^{\prime}$ that satisfies the property $\mathrm{h}\left(y^{2} \mathbf{w}\right)=\mathrm{h}\left(\mathbf{w}^{\prime}\right)$.

Since the identity $\sigma$ is arbitrary in $\Sigma_{\ominus}$, the construction of $\widetilde{\sigma}$ from $\sigma$ in the preceding paragraph can be repeated on every identity in $\Sigma_{\ominus}$ to obtain $\widetilde{\Sigma}_{\ominus}=\left\{\widetilde{\sigma} \mid \sigma \in \Sigma_{\ominus}\right\}$. It follows that $\mathbf{X}_{(7.1)} \Sigma_{\ominus}=\mathbf{X}_{(7.1)}\{(7.4)\} \cap \mathbf{X}_{(7.1)} \widetilde{\Sigma}_{\ominus}$. By Lemma 2.1(i), the semigroup $L_{2}$ satisfies the identities in $\widetilde{\Sigma}_{\ominus}$ and so belongs to $\mathbf{X}_{(7.1)} \widetilde{\Sigma}_{\ominus}$. Therefore $\mathbf{X}_{(7.1)} \widetilde{\Sigma}_{\ominus}$ is finitely based by Proposition 7.4. Consequently, $\mathbf{X}_{(7.1)} \Sigma_{\ominus}$ is also finitely based.

\section{On Condition 10}

Let $\mathbf{X}_{(8.1)}$ denote the variety defined by the identities

$$
\begin{aligned}
x^{3} \approx x^{2}, \quad x^{2} y x & \approx x y x, \quad x y x^{2} \approx x y x \\
x^{2} y^{2} & \approx y^{2} x^{2} \\
x^{2} y z^{2} & \approx x y z^{2} x .
\end{aligned}
$$

Then Condition 10 is equivalent to the following result.

Proposition 8.1. All subvarieties of $\mathbf{X}_{(8.1)}$ are finitely based.

Some preliminary results are established in $\S \S 8.1$ and 8.2. The proof of Proposition 8.1 is then given in $\S 8.3$. 


\subsection{A canonical form}

Let $\mathbf{w}$ be any non-simple word and let $x_{1}, \ldots, x_{r}$ be all its distinct non-simple letters listed in alphabetical order. In this section, such a word is said to be in canonical form if

$$
\mathbf{w}=\mathbf{s}_{0} \prod_{i=1}^{m}\left(\mathbf{w}_{i} \mathbf{s}_{i}\right)
$$

for some $m \geqslant 1$ such that all of the following are satisfied:

(I) the letters of $\mathbf{s}_{0}, \mathbf{s}_{m} \in \mathcal{X}^{*}$ and $\mathbf{s}_{1}, \ldots, \mathbf{s}_{m-1} \in \mathcal{X}^{+}$are all simple in $\mathbf{w}$;

(II) $\mathbf{w}_{1}, \ldots, \mathbf{w}_{m} \in\left\{x_{1}^{e_{1}} \ldots x_{r}^{e_{r}} \mid e_{1}, \ldots, e_{r} \in\{0,2\}\right\}$ with $\mathbf{w}_{1}, \ldots, \mathbf{w}_{m} \neq \emptyset$

(III) $\operatorname{con}\left(\mathbf{w}_{1}\right) \subseteq \ldots \subseteq \operatorname{con}\left(\mathbf{w}_{m}\right)$.

Note that (II) and (III) imply:

(IV) $\mathbf{w}_{m}=x_{1}^{2} \ldots x_{r}^{2}$.

Lemma 8.2. Let $\mathbf{w}$ be any non-simple word. Then there exists some word $\overline{\mathbf{w}}$ in canonical form such that the identities (8.1) imply the identity $\mathbf{w} \approx \overline{\mathbf{w}}$.

Proof. It suffices to convert $\mathbf{w}$, using the identities (8.1), into a word in canonical form. Let $x_{1}, \ldots, x_{r}$ be all the distinct non-simple letters of $\mathbf{w}$ in alphabetical order. Clearly $\mathbf{w}$ can be written in the form (8.2) such that (I) is satisfied and $\mathbf{w}_{1}, \ldots, \mathbf{w}_{m} \in\left\{x_{1}, \ldots, x_{r}\right\}^{+}$. Suppose $x \in \operatorname{con}\left(\mathbf{w}_{i}\right) \backslash \operatorname{con}\left(\mathbf{w}_{i+1}\right)$, say $\mathbf{w}_{i}=\mathbf{a} x \mathbf{b}$ for some $\mathbf{a}, \mathbf{b} \in \mathcal{X}^{*}$. Then since the letters $x$ and $h=\mathrm{h}\left(\mathbf{w}_{i+1}\right)$ are non-simple in $\mathbf{w}$,

$$
\begin{aligned}
& \mathbf{w}=\ldots \mathbf{w}_{i} \mathbf{s}_{i} \cdot \mathbf{w}_{i+1} \mathbf{s}_{i+1} \ldots \stackrel{(8.1 \mathrm{a})}{\approx} \ldots \mathbf{a} x^{3} \mathbf{b s}_{i} \cdot h^{2} \mathbf{w}_{i+1} \mathbf{s}_{i+1} \ldots \\
& \stackrel{(8.1 \mathrm{c})}{\approx} \ldots \mathbf{a} x \mathbf{b s}_{i} \cdot h^{2} x^{2} \mathbf{w}_{i+1} \mathbf{s}_{i+1} \ldots \stackrel{(8.1 \mathrm{~b})}{\approx} \ldots \mathbf{w}_{i} \mathbf{s}_{i} \cdot x^{2} h^{2} \mathbf{w}_{i+1} \mathbf{s}_{i+1} \ldots \\
& \stackrel{(8.1 \mathrm{a})}{\approx} \ldots \mathbf{w}_{i} \mathbf{s}_{i} \cdot\left(x \mathbf{w}_{i+1}\right) \mathbf{s}_{i+1} \ldots,
\end{aligned}
$$

that is, the identities (8.1) can be used to convert $\mathbf{w}_{i+1}$ into $x \mathbf{w}_{i+1}$. It is easily seen how this procedure can be repeated until $\mathbf{w}$ is converted into a word of the form (8.2) with (I) and (III) satisfied. Since the letters of each $\mathbf{w}_{i}$ are non-simple in $\mathbf{w}$, apply the identities (8.1a) to replace each letter $x_{j}$ in $\mathbf{w}_{i}$ by its square $x_{j}^{2}$, apply the identity (8.1b) to alphabetically order these squares in $\mathbf{w}_{i}$, and apply the identities (8.1a) to eliminate any repeated squares. The resulting factor is of the form $x_{1}^{e_{1}} \ldots x_{r}^{e_{r}}$ with $e_{1}, \ldots, e_{r} \in\{0,2\}$. Therefore (II) is satisfied.

\subsection{Some finitely based subvarieties of $\mathbf{X}_{(8.1)}$}

Lemma 8.3. Suppose that $\mathbf{V}$ is any subvariety of $\mathbf{X}_{(8.1)}$ that satisfies an identity $\mathbf{w} \approx \mathbf{w}^{\prime}$ with either $\operatorname{con}(\mathbf{w}) \neq \operatorname{con}\left(\mathbf{w}^{\prime}\right)$ or $\operatorname{sim}(\mathbf{w}) \neq \operatorname{sim}\left(\mathbf{w}^{\prime}\right)$. Then $\mathbf{V}$ is finitely based.

Proof. It follows from the assumption and Lemma 2.1(v) that $N_{2}^{1} \notin \mathbf{V}$. Then by Lemma 2.9, the variety $\mathbf{V}$ satisfies the identity $\alpha: h^{2}\left(x h^{2}\right)^{3} \approx h^{2} x h^{2}$. Since

$$
x y x \stackrel{(8.1 \mathrm{a})}{\approx} x^{2} y x^{2} \underset{\approx}{\approx} x^{2}\left(y x^{2}\right)^{3} \stackrel{(8.1 \mathrm{a})}{\approx} x^{2}\left(y^{2} x^{2}\right)^{3} \stackrel{(8.1 \mathrm{~b})}{\approx} x^{8} y^{6} \stackrel{(8.1 \mathrm{a})}{\approx} x^{2} y^{2},
$$

the variety $\mathbf{V}$ satisfies the identity $x y x \approx x^{2} y^{2}$; any variety that satisfies this identity is finitely based [34].

Lemma 8.4. Suppose that $\mathbf{V}$ is any subvariety of $\mathbf{X}_{(8.1)}$ that satisfies an identity $\mathbf{w} \approx \mathbf{w}^{\prime}$ with $\mathrm{F}_{\mathrm{SS}}(\mathbf{w}) \neq \mathrm{F}_{\mathrm{SS}}\left(\mathbf{w}^{\prime}\right)$. Then $\mathbf{V}$ is finitely based. 
Proof. If either $\operatorname{con}(\mathbf{w}) \neq \operatorname{con}\left(\mathbf{w}^{\prime}\right)$ or $\operatorname{sim}(\mathbf{w}) \neq \operatorname{sim}\left(\mathbf{w}^{\prime}\right)$, then the variety $\mathbf{V}$ is finitely based by Lemma 8.3. Hence assume $\operatorname{con}(\mathbf{w})=\operatorname{con}\left(\mathbf{w}^{\prime}\right)$ and $\operatorname{sim}(\mathbf{w})=\operatorname{sim}\left(\mathbf{w}^{\prime}\right)$. By Lemma 2.10, the variety $\mathbf{V}$ satisfies the identity (2.3) with $n=2$. It follows that $\mathbf{V}$ satisfies the identities in Condition 6 and so is finitely based.

Lemma 8.5. Suppose that $\mathbf{V}$ is any subvariety of $\mathbf{X}_{(8.1)}$ that satisfies an identity $\mathbf{w} \approx \mathbf{w}^{\prime}$ with $\mathbf{w}_{\text {sim }} \neq \mathbf{w}_{\text {sim }}^{\prime}$. Then $\mathbf{V}$ is finitely based.

Proof. If either $\operatorname{con}(\mathbf{w}) \neq \operatorname{con}\left(\mathbf{w}^{\prime}\right)$ or $\operatorname{sim}(\mathbf{w}) \neq \operatorname{sim}\left(\mathbf{w}^{\prime}\right)$, then the variety $\mathbf{V}$ is finitely based by Lemma 8.3. Hence assume $\operatorname{con}(\mathbf{w})=\operatorname{con}\left(\mathbf{w}^{\prime}\right)$ and $\operatorname{sim}(\mathbf{w})=\operatorname{sim}\left(\mathbf{w}^{\prime}\right)$. Since $\mathbf{w}_{\text {sim }} \neq \mathbf{w}_{\text {sim }}^{\prime}$ by assumption, there exist some distinct letters $y, z \in \operatorname{sim}(\mathbf{w})=\operatorname{sim}\left(\mathbf{w}^{\prime}\right)$ such that $\mathbf{w} \in$ $\mathcal{X}^{*} y \mathcal{X}^{*} z \mathcal{X}^{*}$ and $\mathbf{w}^{\prime} \in \mathcal{X}^{*} z \mathcal{X}^{*} y \mathcal{X}^{*}$. Let $\varphi: \mathcal{X} \rightarrow \mathcal{X}^{+}$denote the substitution

$$
t \mapsto \begin{cases}x t x & \text { if } t \in\{y, z\} \\ x & \text { otherwise. }\end{cases}
$$

Then the deductions $\mathbf{w} \varphi \stackrel{(8.1 \mathrm{a})}{\approx} x y x z x$ and $\mathbf{w}^{\prime} \varphi \stackrel{(8.1 \mathrm{a})}{\approx} x z x y x$ hold so that $\mathbf{V}$ satisfies the identity $x y x z x \approx x z x y x$. Hence $\mathbf{V}$ satisfies the identities in Condition 11 and so is finitely based.

\subsection{Proof of Proposition 8.1}

Lemma 8.6. Let $\mathbf{w}$ and $\mathbf{w}^{\prime}$ be words and $x$ and $y$ be letters such that $x \in \operatorname{con}(\mathbf{w})=\operatorname{con}\left(\mathbf{w}^{\prime}\right)$ and $y \in \operatorname{sim}(\mathbf{w})=\operatorname{sim}\left(\mathbf{w}^{\prime}\right)$. Suppose that the first occurrence of $x$ precedes $y$ in $\mathbf{w}$, but $y$ precedes the first occurrence of $x$ in $\mathbf{w}^{\prime}$. Then the identities $\left\{(8.1), \mathbf{w} \approx \mathbf{w}^{\prime}\right\}$ imply the identity

$$
h^{2} y x^{2} h^{2} \approx x^{2} h^{2} y x^{2} h^{2} .
$$

Proof. By assumption, $\mathbf{w}=\mathbf{w}_{1} y \mathbf{w}_{2}$ and $\mathbf{w}^{\prime}=\mathbf{w}_{1}^{\prime} y \mathbf{w}_{2}^{\prime}$ for some $\mathbf{w}_{i}, \mathbf{w}_{i}^{\prime} \in(\mathcal{X} \backslash\{y\})^{*}$ such that $x \in \operatorname{con}\left(\mathbf{w}_{1}\right) \backslash \operatorname{con}\left(\mathbf{w}_{1}^{\prime}\right)$. Let $\varphi: \mathcal{X} \rightarrow \mathcal{X}^{+}$denote the substitution

$$
t \mapsto \begin{cases}x^{2} h^{2} & \text { if } t=x \\ y x^{2} h^{2} & \text { if } t=y \\ h^{2} & \text { otherwise. }\end{cases}
$$

Then since the deductions $h^{2}(\mathbf{w} \varphi) x^{2} h^{2} \stackrel{(8.1)}{\approx} x^{2} h^{2} y x^{2} h^{2}$ and $h^{2}\left(\mathbf{w}^{\prime} \varphi\right) x^{2} h^{2} \stackrel{(8.1)}{\approx} h^{2} y x^{2} h^{2}$ hold, the identities $\left\{(8.1), \mathbf{w} \approx \mathbf{w}^{\prime}\right\}$ imply the identity $(8.3)$.

Let $\mathbf{V}$ be any proper subvariety of $\mathbf{X}_{(8.1)}$. Then $\mathbf{V}=\mathbf{X}_{(8.1)} \Sigma$ for some nonempty set $\Sigma$ of nontrivial identities. By Lemmas $8.3-8.5$, the variety $\mathbf{V}$ is finitely based if some identity $\mathbf{w} \approx \mathbf{w}^{\prime}$ in $\Sigma$ does not satisfy any of the following conditions:

(a) $\operatorname{con}(\mathbf{w})=\operatorname{con}\left(\mathbf{w}^{\prime}\right)$ and $\operatorname{sim}(\mathbf{w})=\operatorname{sim}\left(\mathbf{w}^{\prime}\right)$;

(b) $\mathrm{F}_{\mathrm{SS}}(\mathbf{w})=\mathrm{F}_{\mathrm{SS}}\left(\mathbf{w}^{\prime}\right)$;

(c) $\mathbf{w}_{\text {sim }}=\mathbf{w}_{\text {sim }}^{\prime}$.

Hence assume that (a)-(c) hold for every identity $\mathbf{w} \approx \mathbf{w}^{\prime}$ in $\Sigma$.

Let $\mathbf{w} \approx \mathbf{w}^{\prime}$ be any identity in $\Sigma$. By $(\mathrm{a})$, the words $\mathbf{w}$ and $\mathbf{w}^{\prime}$ are either both simple or both non-simple. If the words $\mathbf{w}$ and $\mathbf{w}^{\prime}$ are both simple, then by $(\mathrm{c})$, the identity $\mathbf{w} \approx \mathbf{w}^{\prime}$ is contradictorily trivial. Therefore the words $\mathbf{w}$ and $\mathbf{w}^{\prime}$ are both non-simple, say with non-simple letters $x_{1}, \ldots, x_{r}$. By Lemma 8.2, the words $\mathbf{w}$ and $\mathbf{w}^{\prime}$ can be chosen to be in canonical form. It then follows from (a)-(c) that

$$
\mathbf{w}=\mathbf{s}_{0} \prod_{i=1}^{m}\left(\mathbf{w}_{i} \mathbf{s}_{i}\right) \quad \text { and } \quad \mathbf{w}^{\prime}=\mathbf{s}_{0} \prod_{i=1}^{m}\left(\mathbf{w}_{i}^{\prime} \mathbf{s}_{i}\right),
$$


where

(d) $\mathbf{w}_{m}=x_{1}^{2} \ldots x_{r}^{2}=\mathbf{w}_{m}^{\prime}$

by (IV). But since the identity $\mathbf{w} \approx \mathbf{w}^{\prime}$ is nontrivial, $\operatorname{con}\left(\mathbf{w}_{j}\right) \neq \operatorname{con}\left(\mathbf{w}_{j}^{\prime}\right)$ for some $j<m$, say $x_{i} \in \operatorname{con}\left(\mathbf{w}_{j}\right) \backslash \operatorname{con}\left(\mathbf{w}_{j}^{\prime}\right)$. Then $x_{i} \notin \operatorname{con}\left(\mathbf{w}_{1}^{\prime} \ldots \mathbf{w}_{j}^{\prime}\right)$ by (III), so that $x_{i} \in \operatorname{con}\left(\mathbf{w}_{j+1}^{\prime} \ldots \mathbf{w}_{m}^{\prime}\right)$. The letter $y=\mathrm{h}\left(\mathbf{s}_{j}\right) \in \operatorname{sim}(\mathbf{w})=\operatorname{sim}\left(\mathbf{w}^{\prime}\right)$ is preceded in $\mathbf{w}$ by the first occurrence of $x_{i}$ while $y$ precedes the first occurrence of $x_{i}$ in $\mathbf{w}^{\prime}$. Therefore by Lemma 8.6,

(e) $\mathbf{X}_{(8.1)}\left\{\mathbf{w} \approx \mathbf{w}^{\prime}\right\}=\mathbf{X}_{(8.1)}\left\{\mathbf{w} \approx \mathbf{w}^{\prime},(8.3)\right\}$.

Choose any letter $x_{\ell} \in \operatorname{con}\left(\mathbf{w}_{1}\right)$. Then $x_{\ell} \in \bigcap_{i=1}^{m} \operatorname{con}\left(\mathbf{w}_{i}\right)$ by (III). For any $i<m$, the factor $\mathbf{w}_{i}$ can be written in the form $\mathbf{w}_{i}=\mathbf{a} x_{\ell}^{2} \mathbf{b}$, where $\mathbf{a}=x_{1}^{e_{1}} \ldots x_{\ell-1}^{e_{\ell-1}}$ and $\mathbf{b}=x_{\ell+1}^{e_{\ell+1}} \ldots x_{r}^{e_{r}}$ for some $e_{1}, \ldots, e_{\ell-1}, e_{\ell+1}, \ldots, e_{r} \in\{0,2\}$. Then by (d),

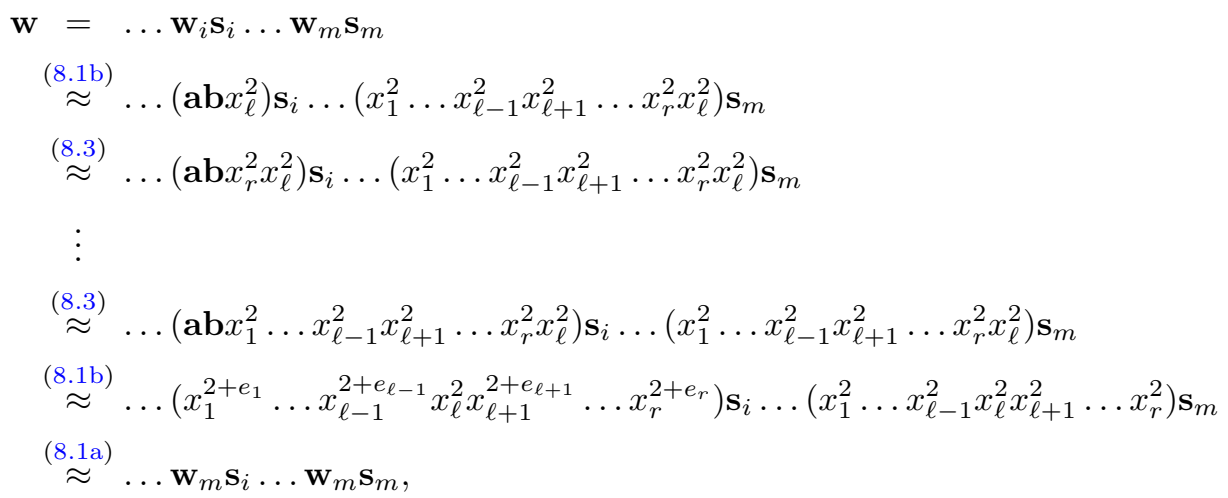

that is, $\mathbf{w}_{i}$ is converted into $\mathbf{w}_{m}$. This argument can be repeated on $\mathbf{w}_{j}$ for every $j<m$, so that the identities $\{(8.1),(8.3)\}$ imply the identity $\mathbf{w} \approx \mathbf{s}_{0} \prod_{i=1}^{m}\left(\mathbf{w}_{m} \mathbf{s}_{i}\right)$. Similarly, the identities $\{(8.1),(8.3)\}$ imply the identity $\mathbf{w}^{\prime} \approx \mathbf{s}_{0} \prod_{i=1}^{m}\left(\mathbf{w}_{m}^{\prime} \mathbf{s}_{i}\right)$. Therefore since

$$
\mathbf{w} \stackrel{(8.1),(8.3)}{\approx} \mathbf{s}_{0} \prod_{i=1}^{m}\left(\mathbf{w}_{m} \mathbf{s}_{i}\right) \stackrel{(\mathrm{d})}{=} \mathbf{s}_{0} \prod_{i=1}^{m}\left(\mathbf{w}_{m}^{\prime} \mathbf{s}_{i}\right) \stackrel{(8.1),(8.3)}{\approx} \mathbf{w}^{\prime}
$$

the identities $\{(8.1),(8.3)\}$ imply the identity $\mathbf{w} \approx \mathbf{w}^{\prime}$. It thus follows from (e) that the equality $\mathbf{X}_{(8.1)}\left\{\mathbf{w} \approx \mathbf{w}^{\prime}\right\}=\mathbf{X}_{(8.1)}\{(8.3)\}$ holds. But the identity $\mathbf{w} \approx \mathbf{w}^{\prime}$ is arbitrary in $\Sigma$. Therefore $\mathbf{X}_{(8.1)} \Sigma=\mathbf{X}_{(8.1)}\{(8.3)\}$. Consequently, $\mathbf{V}$ is finitely based.

\section{On Condition 14}

Let $\mathbf{X}_{(9.1)}$ denote the variety defined by the identities

$$
\begin{gathered}
x^{4} \approx x^{2}, \quad x y^{3} x \approx x y x, \quad x y x^{3} \approx x y x, \\
x^{2} y x \approx x y x^{2}, \quad x y x z x \approx x^{2} y z x, \\
x y x y \approx x y^{2} x .
\end{gathered}
$$

Then Condition 14 is equivalent to the following result.

Proposition 9.1. All subvarieties of $\mathbf{X}_{(9.1)}$ are finitely based.

It is shown in $\S 9.1$ that $\mathbf{X}_{(9.1)}=\mathbf{A}_{\mathbf{0}} \vee \mathbf{L}_{\mathbf{2}}^{\mathbf{1}} \vee \mathbf{Z}_{2}$. The proof of Proposition 9.1 is then given in $\S 9.2$. 


\subsection{Identities satisfied by $\mathbf{X}_{(9.1)}$}

Lemma 9.2. The identities (9.1) imply the identities

$$
\begin{aligned}
x \mathrm{H} x^{2} \mathrm{~K} x & \approx x \mathrm{HK} x, \\
x \mathrm{H} y^{3} \mathrm{~K} x & \approx x \mathrm{H} y \mathrm{~K} x, \\
x \mathrm{H} y \mathrm{~K} x y & \approx x \mathrm{H} y \mathrm{~K} y x, \\
x \mathrm{H} y z y \mathrm{~K} x & \approx x \mathrm{H} y^{2} z \mathrm{~K} x .
\end{aligned}
$$

Proof. It is easily seen that the identities (9.1) imply the identities (9.2a). Since

$$
x \mathrm{H} y^{3} \mathrm{~K} x \stackrel{(9.2 \mathrm{a})}{\approx} x \mathrm{H} x^{2} y^{3} x^{2} \mathrm{~K} x \stackrel{(9.1 \mathrm{a})}{\approx} x \mathrm{H} x^{2} y x^{2} \mathrm{~K} x \stackrel{(9.2 \mathrm{a})}{\approx} x \mathrm{H} y \mathrm{~K} x,
$$

the identities (9.1) imply the identities (9.2b). Since

$$
\begin{aligned}
x \mathrm{H} y \mathrm{~K} x y & \stackrel{(9.2 \mathrm{a})}{\approx} x \mathrm{H} y \mathrm{~K} y^{2} x y \stackrel{(9.2 \mathrm{a})}{\approx} x \mathrm{H} y \mathrm{~K} y x^{2} y x y \stackrel{(9.1 \mathrm{c})}{\approx} x \mathrm{H} y \mathrm{~K} y x^{2} y^{2} x \\
& \stackrel{(9.2 \mathrm{a})}{\approx} x \mathrm{H} y \mathrm{~K} y^{3} x \stackrel{(9.2 \mathrm{~b})}{\approx} x \mathrm{H} y \mathrm{~K} y x,
\end{aligned}
$$

the identities (9.1) imply the identities (9.2c). Since

$$
\begin{aligned}
x \mathrm{H} y^{2} z \mathrm{~K} x & \stackrel{(9.2 \mathrm{~b})}{\approx} x \mathrm{H} y(y z)^{3} \mathrm{~K} x \stackrel{(9.2 \mathrm{c})}{\approx} x \mathrm{H} y^{2} z^{3} y^{2} \mathrm{~K} x \stackrel{(9.1 \mathrm{~b})}{\approx} x \mathrm{H} y^{3} z^{3} y \mathrm{~K} x \\
\stackrel{(9.2 \mathrm{~b})}{\approx} x \mathrm{H} y z y \mathrm{~K} x, &
\end{aligned}
$$

the identities (9.1) imply the identities (9.2d).

In this section, a non-simple word $\mathbf{w}$ is said to be in canonical form if

$$
\mathbf{w}=x_{0}^{e_{0}} x_{1}^{e_{1}} \ldots x_{m}^{e_{m}} x_{0}
$$

for some distinct letters $x_{0}, \ldots, x_{m}$ with $e_{0}, \ldots, e_{m} \in\{1,2\}$. Note that if $m=0$, then the word $\mathbf{w}$ in $(9.3)$ is $x_{0}^{e_{0}+1}$.

Lemma 9.3. Let $\mathbf{w}$ be any connected word. Then there exists some word $\overline{\mathbf{w}}$ in canonical form such that the identities (9.1) imply the identity $\mathbf{w} \approx \overline{\mathbf{w}}$.

Proof. By Lemma 9.2, it suffices to convert $\mathbf{w}$, using the identities $\{(9.1),(9.2)\}$, into a word in canonical form. Since $\mathbf{w}$ is connected, the letter $x_{0}=\mathrm{h}(\mathbf{w})$ occurs at least twice in $\mathbf{w}$. There are two cases.

Case 1: $\mathrm{h}(\mathbf{w})=\mathrm{t}(\mathbf{w})$. Then $\mathbf{w}=x_{0} \mathbf{a} x_{0}$ for some $\mathbf{a} \in \mathcal{X}^{*}$. The identities (9.1b) can be used to group any $x_{0}$ in $\mathbf{a}$ with the first $x_{0}$ in $\mathbf{w}$, resulting in a word of the form $x_{0}^{e_{0}} \mathbf{b} x_{0}$, where $e_{0} \geqslant 1$ and $\mathbf{b} \in\left(\mathcal{X} \backslash\left\{x_{0}\right\}\right)^{*}$. The identities (9.2d) can be used to group any non-first occurrence of any letter $x$ in $\mathbf{b}$ with its first occurrence: $x_{0} \mathbf{c} x \mathbf{d} x \mathbf{e} x_{0} \stackrel{(9.2 \mathrm{~d})}{\approx} x_{0} \mathbf{c} x^{2} \mathbf{d e} x_{0}$. The resulting word is of the form (9.3) with $e_{i} \geqslant 1$. The identities $\{(9.2 \mathrm{a}),(9.2 \mathrm{~b})\}$ can be used to reduce each $e_{i}$ to a number in $\{1,2\}$, resulting in a word in canonical form.

Case $2: \mathrm{h}(\mathbf{w}) \neq \mathrm{t}(\mathbf{w})$. Then $\mathbf{w}=x_{0} \mathbf{a} x_{0} y \mathbf{b}$ for some $\mathbf{a}, \mathbf{b} \in \mathcal{X}^{*}$ and $y \in \mathcal{X}$ with $x_{0} \notin \operatorname{con}(y \mathbf{b})$. Since $\mathbf{w}$ is connected and its suffix $y \mathbf{b}$ does not contain the letter $x_{0}$, the factor $\mathbf{a}$ is nonempty. 
If $y \in \operatorname{con}(\mathbf{a})$, then $\mathbf{w}=x_{0} \mathbf{a} x_{0} y \mathbf{b} \stackrel{(9.2 \mathrm{c})}{\approx} x_{0} \mathbf{a} y x_{0} \mathbf{b}$. If $y \notin \operatorname{con}(\mathbf{a})$, then the connectedness of $\mathbf{w}$ implies that $\mathbf{a}$ and $\mathbf{b}$ share some common letter, whence

$$
\mathbf{w} \stackrel{(9.2 \mathrm{a})}{\approx} x_{0} \mathbf{a} x_{0}^{3} y \mathbf{b} \stackrel{(9.2 \mathrm{~d})}{\approx} x_{0} \mathbf{a} x_{0}^{2} y x_{0} \mathbf{b} \stackrel{(9.2 \mathrm{a})}{\approx} x_{0} \mathbf{a} y x_{0} \mathbf{b} .
$$

In any case, the identities (9.2) can be used to interchange the last $x_{0}$ in $\mathbf{w}$ with the letter $y$ that immediately follows it. This procedure can be repeated until the last $x_{0}$ in $\mathbf{w}$ is the last letter of the word. Hence $\mathbf{w} \stackrel{(9.2)}{\approx} x_{0} \mathbf{c} x_{0}$ for some $\mathbf{c} \in \mathcal{X}^{+}$. By Case 1 , the identities $\{(9.1),(9.2)\}$ can be used to convert $x_{0} \mathbf{c} x_{0}$ into a word in canonical form.

Proposition 9.4. The equality $\mathbf{X}_{(9.1)}=\mathbf{A}_{\mathbf{0}} \vee \mathbf{L}_{\mathbf{2}}^{\mathbf{1}} \vee \mathbf{Z}_{2}$ holds.

Proof. By Lemma 2.5, the semigroup $A_{0} \times L_{2}^{1} \times \mathbb{Z}_{2}$ is idempotent-separable. Therefore by Lemma 2.6(i), there exists some basis $\Sigma$ for $\mathbf{A}_{\mathbf{0}} \vee \mathbf{L}_{\mathbf{2}}^{\mathbf{1}} \vee \mathbf{Z}_{2}$ that consists of identities formed by connected words. It is routinely verified, either directly or by Lemmas 2.1 and 2.4, that $A_{0}$, $L_{2}^{1}$, and $\mathbb{Z}_{2}$ satisfy the identities (9.1). Hence the inclusion $\mathbf{A}_{\mathbf{0}} \vee \mathbf{L}_{\mathbf{2}}^{\mathbf{1}} \vee \mathbf{Z}_{2} \subseteq \mathbf{X}_{(9.1)}$ holds. To complete the proof, it remains to show that any identity $\mathbf{w} \approx \mathbf{w}^{\prime}$ in $\Sigma$ is implied by the identities (9.1).

By Lemma 9.3, there exist words $\overline{\mathbf{w}}$ and $\overline{\mathbf{w}}^{\prime}$ in canonical form such that the identities (9.1) imply $\mathbf{w} \approx \overline{\mathbf{w}}$ and $\mathbf{w}^{\prime} \approx \overline{\mathbf{w}}^{\prime}$. Since $\mathbf{A}_{\mathbf{0}} \vee \mathbf{L}_{\mathbf{2}}^{\mathbf{1}} \vee \mathbf{Z}_{2}$ satisfies the identity $\overline{\mathbf{w}} \approx \overline{\mathbf{w}}^{\prime}$, it follows from Lemma 2.1 parts (iii) and (viii) that $\operatorname{ini}(\overline{\mathbf{w}})=\operatorname{ini}\left(\overline{\mathbf{w}}^{\prime}\right)$ and $\operatorname{occ}(x, \overline{\mathbf{w}}) \equiv \operatorname{occ}\left(x, \overline{\mathbf{w}}^{\prime}\right)(\bmod 2)$ for all $x \in \mathcal{X}$. It is then easily shown that the words $\overline{\mathbf{w}}$ and $\overline{\mathbf{w}}^{\prime}$ are identical. Consequently, the identities (9.1) imply the identity $\mathbf{w} \approx \mathbf{w}^{\prime}$.

\subsection{Proof of Proposition 9.1}

Let $\mathbf{V}$ be any proper subvariety of $\mathbf{X}_{(9.1)}$. Then by Proposition 9.4 , the variety $\mathbf{V}$ does not contain one of the semigroups $A_{0}, L_{2}^{1}$, and $\mathbb{Z}_{2}$.

Case 1: $A_{0} \notin \mathbf{V}$. Then by Lemma 2.8, the variety $\mathbf{V}$ satisfies the identity $\alpha:\left(x^{2} y^{2}\right)^{3} \approx x^{2} y^{2}$. Since

$$
x^{2} y^{2} \stackrel{\alpha}{\approx} x^{2} y^{2} x^{2} y^{2} x^{2} y^{2} \stackrel{(9.1 \mathrm{~b})}{\approx} x y x^{5} y^{5} \stackrel{(9.1 \mathrm{a})}{\approx} x y x y
$$

the variety $\mathbf{V}$ satisfies the identity $x y x y \approx x^{2} y^{2}$. It is then routinely shown that $\mathbf{V}$ satisfies the identities in Condition 15 and so is finitely based.

Case 2: $L_{2}^{1} \notin \mathbf{V}$. If $x^{2}, x^{2} y^{2} z^{2} x^{2}$, and $x^{2} z^{2} y^{2} x^{2}$ are distinct elements in the $\mathbf{V}$-free semigroup $F_{\mathbf{V}}$ over $\mathcal{X}$, then they form a subsemigroup of $F_{\mathbf{V}}$ isomorphic to $L_{2}^{1}$, and this implies the contradiction $L_{2}^{1} \in \mathbf{V}$. Therefore these three elements of $F_{\mathbf{V}}$ cannot be distinct, whence $\mathbf{V}$ satisfies either $x^{2} \approx x^{2} y^{2} z^{2} x^{2}, x^{2} \approx x^{2} z^{2} y^{2} x^{2}$, or $x^{2} y^{2} z^{2} x^{2} \approx x^{2} z^{2} y^{2} x^{2}$; it is easily seen that any one of the first two identities implies the third identity, so that $\mathbf{V}$ satisfies the third identity. It follows that $\mathbf{V}$ satisfies the identity $\beta: x y^{2} z^{2} x \approx x z^{2} y^{2} x$. Since

$$
\begin{gathered}
x y x z x \stackrel{(9.2 \mathrm{~b})}{\approx} x y^{3} x z^{3} x \stackrel{(9.2 \mathrm{~d})}{\approx} x y^{2} x z^{2} y z x \stackrel{(9.1 \mathrm{~b})}{\approx} x y^{2} z^{2} x y z x \underset{\beta}{\approx} x z^{2} y^{2} x y z x \\
\stackrel{(9.2 \mathrm{~d})}{\approx} x z^{3} y^{3} x^{2} \stackrel{(9.2 \mathrm{~b})}{\approx} x z y x^{2} \stackrel{(9.1 \mathrm{~b})}{\approx} x z x y x,
\end{gathered}
$$

the variety $\mathbf{V}$ satisfies the identity $x y x z x \approx x z x y x$. It is routinely verified that $\mathbf{V}$ satisfies the identities in Condition 12 and so is finitely based.

Case 3: $\mathbb{Z}_{2} \notin \mathbf{V}$. Then by Lemma 2.1(viii), the variety $\mathbf{V}$ satisfies the identity $x^{3} \approx x^{2}$. It follows that $\mathbf{V}$ satisfies the identities in Condition 6 and so is finitely based. 


\section{On Condition 20}

Let $\mathbf{X}_{(10.1)}$ denote the variety defined by the identities

$$
\begin{aligned}
x \mathrm{H} x^{2} & \approx x \mathrm{H} x, \\
x^{2} y x z & \approx x^{2} y z, \\
x \mathrm{H} y \mathrm{~K} x y & \approx x \mathrm{H} y \mathrm{~K} y x .
\end{aligned}
$$

Then Condition 20 is equivalent to the following result.

Proposition 10.1. Any subvariety of $\mathbf{X}_{(10.1)}$ that does not satisfy the identity

$$
h^{2} x y x y \approx h^{2} x^{2} y^{2}
$$

is finitely based.

In this section, the following semigroup of order four is required:

$$
P_{2}=\left\langle a, b \mid a^{2}=a b=a, b^{2} a=b^{2}\right\rangle .
$$

Let $\mathbf{P}_{\mathbf{2}}^{1}$ denote the semigroup variety generated by the monoid $P_{2}^{1}$. It is shown in $\S 10.3$ that $\mathbf{X}_{(10.1)}=\mathbf{P}_{\mathbf{2}}^{\mathbf{1}} \vee \mathbf{J}$. The proof of Proposition 10.1 is then given in $\S 10.4$.

10.1. Some identities satisfied by subvarieties of $\mathbf{X}_{(10.1)}$

Lemma 10.2 [19, Corollary 6.6]. The variety $\mathbf{P}_{\mathbf{2}}^{\mathbf{1}}$ is defined by the identities (10.1) and

$$
x^{2} y x \approx x^{2} y .
$$

Lemma 10.3. The identity (10.2) is an exclusion identity for $P_{2}^{1}$ in $\mathbf{X}_{(10.1)}$.

Proof. Let $\mathbf{V}$ be any subvariety of $\mathbf{X}_{(10.1)}$ such that $P_{2} \notin \mathbf{V}$. Let $\overleftarrow{P}_{2}$ denote the dual semigroup of $P_{2}$. It is routinely shown that $A_{0}, B_{2}$, and $\overleftarrow{P}_{2}$ do not satisfy the identity (10.1b), so that $A_{0}, B_{2}, \overleftarrow{P}_{2} \notin \mathbf{V}$. It follows from Volkov [48, Theorem 2.1] that $\mathbf{V}$ satisfies the identity

$$
x y x y \approx x^{2} y^{2} .
$$

The semigroup $P_{2}$ does not satisfy the identity (10.4) because $b a b a \neq b^{2} a^{2}$. Therefore the identity (10.4) is an exclusion identity for $P_{2}$ in $\mathbf{X}_{(10.1)}$.

Recall that $\psi_{2}: \mathcal{X} \rightarrow \mathcal{X}^{+}$denotes the substitution $z \mapsto h^{2} z h^{2}$ for all $z \in \mathcal{X}$. By Lemma 2.7, the identity $(x y x y) \psi_{2} \approx\left(x^{2} y^{2}\right) \psi_{2}$ is an exclusion identity for $P_{2}^{1}$ in $\mathbf{X}_{(10.1)}$. Since

$$
(x y x y) \psi_{2}=h^{2} x h^{4} y h^{4} x h^{4} y h^{2} \stackrel{(10.1 \mathrm{c})}{\approx} h^{2} x h^{4} y h^{4} x h^{6} y \stackrel{(10.1 \mathrm{~b})}{\approx} h^{2} x y x y
$$

and

$$
\left(x^{2} y^{2}\right) \psi_{2}=h^{2} x h^{4} x h^{4} y h^{4} y h^{2} \stackrel{(10.1 \mathrm{c})}{\approx} h^{2} x h^{4} x h^{4} y h^{6} y \stackrel{(10.1 \mathrm{~b})}{\approx} h^{2} x^{2} y^{2},
$$

the identity (10.2) is an exclusion identity for $P_{2}^{1}$ in $\mathbf{X}_{(10.1)}$.

Lemma 10.4. Let $\mathbf{V}$ be any subvariety of $\mathbf{X}_{(10.1)}$ such that $N_{2}^{1} \in \mathbf{V}$ and $J \notin \mathbf{V}$. Then $\mathbf{V}$ satisfies the identity (10.3). 
Proof. Consider the elements $a=x^{2} y^{2}, b=x^{2} y, c=x^{2}$, and $d=b c=x^{2} y x^{2}$ in the $\mathbf{V}$-free semigroup $F_{\mathbf{V}}$. It follows from Lemma $2.1(\mathrm{v})$ that among these four elements, only $b$ and $d$ are possibly equal in $F_{\mathbf{V}}$ while any other pair of distinct elements are unequal in $F_{\mathbf{V}}$. Suppose $b \neq d$ in $F_{\mathbf{V}}$. Then $\{a, b, c, d\}$ constitutes the following subsemigroup $S$ of $F_{\mathbf{V}}$.

\begin{tabular}{c|cccc}
$S$ & $a$ & $b$ & $c$ & $d$ \\
\hline$a$ & $a$ & $a$ & $a$ & $a$ \\
$b$ & $a$ & $a$ & $d$ & $a$ \\
$c$ & $a$ & $b$ & $c$ & $d$ \\
$d$ & $a$ & $a$ & $d$ & $a$
\end{tabular}

The quotient $S /\{a, d\}$ is isomorphic to $J$, so that the contradiction $J \in \mathbf{V}$ is deduced. Thus the elements $b$ and $d$ must be equal in $F_{\mathbf{V}}$, whence $\mathbf{V}$ satisfies the identity $x^{2} y \approx x^{2} y x^{2}$. It follows that $\mathbf{V}$ satisfies the identity (10.3).

\subsection{A canonical form}

In this section, any word $\mathbf{w}$ with $t=\mathrm{t}(\mathbf{w}) \in \operatorname{sim}(\mathbf{w})$ is said to be in canonical form if

$$
\mathbf{w}=\left(\prod_{i=1}^{m}\left(x_{i} \mathbf{w}_{i}\right)\right) t
$$

where $x_{1}, \ldots, x_{m} \in \mathcal{X}$ and $\mathbf{w}_{1}, \ldots, \mathbf{w}_{m} \in \mathcal{X}^{*}$ are such that:

(I) $\operatorname{ini}(\mathbf{w})=x_{1} \ldots x_{m} t$;

(II) $\mathbf{w}_{i} \in\left\{x_{1}^{e_{1}} \ldots x_{i}^{e_{i}} \mid e_{1}, \ldots, e_{i} \in\{0,1\}\right\}$;

(III) $\operatorname{con}\left(\mathbf{w}_{i}\right) \cap \operatorname{con}\left(\mathbf{w}_{j}\right)=\emptyset$ whenever $i \neq j$.

It follows that

$(\mathrm{IV}) \operatorname{occ}(x, \mathbf{w}) \leqslant 2$ for any $x \in \mathcal{X}$.

Lemma 10.5. Let $\mathbf{w}$ be any word with $t=\mathrm{t}(\mathbf{w}) \in \operatorname{sim}(\mathbf{w})$. Then there exists some word $\overline{\mathbf{w}}$ in canonical form with $t=\mathrm{t}(\overline{\mathbf{w}}) \in \operatorname{sim}(\overline{\mathbf{w}})$ such that the identities (10.1) imply the identity $\mathbf{w} \approx \overline{\mathbf{w}}$.

Proof. It suffices to convert $\mathbf{w}$, using the identities (10.1), into a word in canonical form. Note that if $\mathbf{w}=\mathbf{a} x \mathbf{b} x \mathbf{c} x \mathbf{d} t$ for some $\mathbf{a}, \mathbf{b}, \mathbf{c}, \mathbf{d} \in \mathcal{X}^{*}$, then

$$
\mathbf{w} \stackrel{(10.1 \mathrm{a})}{\approx} \begin{cases}\mathbf{a} x \mathbf{b} x \mathbf{c d} t & \text { if } \mathbf{c}=\emptyset, \\ \mathbf{a} x \mathbf{b} x^{2} \mathbf{c} x \mathbf{d} t \stackrel{(10.1 \mathrm{~b})}{\approx} \mathbf{a} x \mathbf{b} x^{2} \mathbf{c d} t \stackrel{(10.1 \mathrm{a})}{\approx} \mathbf{a} x \mathbf{b} x \mathbf{c d} t & \text { if } \mathbf{c} \neq \emptyset .\end{cases}
$$

Therefore any third or later occurrence of a letter in $\mathbf{w}$ can be removed by the identities (10.1). Hence (IV) is satisfied.

Now the letter $t$ is simple in $\mathbf{w}$, so that ini $(\mathbf{w})=x_{1} \ldots x_{m} t$ for some $x_{1}, \ldots, x_{m} \in \mathcal{X}$. It is clear that $\mathbf{w}$ can be written in the form (10.5) with (I) satisfied and $\mathbf{w}_{i} \in\left\{x_{1}, \ldots, x_{i}\right\}^{*}$ for all $i$. It follows from (IV) that (III) is satisfied, and that within each $\mathbf{w}_{i}$, no letter occurs more than once. Since the letters in each $\mathbf{w}_{i}$ are non-first occurrences in $\mathbf{w}$, the identities (10.1c) can be used to order them according to their indices. Hence (II) is satisfied.

\subsection{A basis for $\mathbf{P}_{\mathbf{2}}^{\mathbf{1}} \vee \mathbf{J}$}

Lemma 10.6. Let $\mathbf{w} \approx \mathbf{w}^{\prime}$ be any identity satisfied by $N_{2}^{1}$ and let $t \in \mathcal{X} \backslash \operatorname{con}\left(\mathbf{w w}^{\prime}\right)$. Suppose $\mathrm{t}(\mathbf{w}) \notin \operatorname{sim}(\mathbf{w})$ and $\mathrm{t}\left(\mathbf{w}^{\prime}\right) \notin \operatorname{sim}\left(\mathbf{w}^{\prime}\right)$. Then $\mathbf{X}_{(10.1)}\left\{\mathbf{w} \approx \mathbf{w}^{\prime}\right\}=\mathbf{X}_{(10.1)}\left\{\mathbf{w} t \approx \mathbf{w}^{\prime} t\right\}$. 
Proof. Let $\sigma_{1}$ and $\sigma_{2}$ denote the identities $\mathbf{w} \approx \mathbf{w}^{\prime}$ and $\mathbf{w} t \approx \mathbf{w}^{\prime} t$, respectively. The inclusion $\mathbf{X}_{(10.1)}\left\{\sigma_{1}\right\} \subseteq \mathbf{X}_{(10.1)}\left\{\sigma_{2}\right\}$ is obvious. Let $x=\mathrm{t}(\mathbf{w})$ and $y=\mathrm{t}\left(\mathbf{w}^{\prime}\right)$. (Note that $x$ and $y$ need not be distinct.) The assumptions $x \notin \operatorname{sim}(\mathbf{w})$ and $y \notin \operatorname{sim}\left(\mathbf{w}^{\prime}\right)$ imply the deductions $\mathbf{w} \stackrel{(10.1 \mathrm{a})}{\approx} \mathbf{w} x$ and $\mathbf{w}^{\prime} \stackrel{(10.1 \mathrm{a})}{\approx} \mathbf{w}^{\prime} y$. It follows from Lemma $2.1(\mathrm{v})$ that $\operatorname{con}(\mathbf{w})=\operatorname{con}\left(\mathbf{w}^{\prime}\right)$ and $\operatorname{sim}(\mathbf{w})=\operatorname{sim}\left(\mathbf{w}^{\prime}\right)$, whence $y$ is non-simple in $\mathbf{w}$. Therefore $\mathbf{w}=\mathbf{a} y \mathbf{b} y \mathbf{c}$ for some $\mathbf{a}, \mathbf{b}, \mathbf{c} \in \mathcal{X}^{*}$. If $\mathbf{c}=\emptyset$, then

$$
\mathbf{w} x=\mathbf{a} y \mathbf{b} y x \stackrel{(10.1 \mathrm{a})}{\approx} \mathbf{a} y \mathbf{b} y y x=\mathbf{w} y x .
$$

If $\mathbf{c} \neq \emptyset$, then

$$
\mathbf{w} x \stackrel{(10.1 \mathrm{a})}{\approx} \mathbf{a} y \mathbf{b} y^{2} \mathbf{c} x \stackrel{(10.1 \mathrm{~b})}{\approx} \mathbf{a} y \mathbf{b} y^{2} \mathbf{c} y x \stackrel{(10.1 \mathrm{a})}{\approx} \mathbf{a} y \mathbf{b} y \mathbf{c} y x=\mathbf{w} y x .
$$

Therefore the deduction $\mathbf{w} x \stackrel{(10.1)}{\approx} \mathbf{w} y x$ holds in any case. Now since

$$
\mathbf{w} \stackrel{(10.1 \mathrm{a})}{\approx} \mathbf{w} x \stackrel{(10.1)}{\approx} \mathbf{w} y x \stackrel{(10.1 \mathrm{c})}{\approx} \mathbf{w} x y \stackrel{(10.1 \mathrm{a})}{\approx} \mathbf{w} y \stackrel{\sigma_{2}}{\approx} \mathbf{w}^{\prime} y \stackrel{(10.1 \mathrm{a})}{\approx} \mathbf{w}^{\prime},
$$

the identities $\left\{(10.1), \sigma_{2}\right\}$ imply $\sigma_{1}$. Hence the inclusion $\mathbf{X}_{(10.1)}\left\{\sigma_{2}\right\} \subseteq \mathbf{X}_{(10.1)}\left\{\sigma_{1}\right\}$ holds.

Proposition 10.7. The equality $\mathbf{X}_{(10.1)}=\mathbf{P}_{\mathbf{2}}^{\mathbf{1}} \vee \mathbf{J}$ holds.

Proof. It is routinely verified that $P_{2}^{1}$ and $J$ satisfy the identities (10.1), so that the inclusion $\mathbf{P}_{\mathbf{2}}^{\mathbf{1}} \vee \mathbf{J} \subseteq \mathbf{X}_{(10.1)}$ holds. Hence $\mathbf{P}_{\mathbf{2}}^{\mathbf{1}} \vee \mathbf{J}=\mathbf{X}_{(10.1)} \Sigma$ for some set $\Sigma$ of identities. Let $\mathbf{w} \approx \mathbf{w}^{\prime}$ be any identity from $\Sigma$. Since the subsemigroups $\left\{1, b a, b^{2}\right\}$ and $\left\{1, b, b^{2}\right\}$ of $P_{2}^{1}$ are isomorphic to $L_{2}^{1}$ and $N_{2}^{1}$, respectively, it follows from Lemma 2.1 parts (iii) and (v) that

(a) $\operatorname{ini}(\mathbf{w})=\operatorname{ini}\left(\mathbf{w}^{\prime}\right)$ and $\operatorname{sim}(\mathbf{w})=\operatorname{sim}\left(\mathbf{w}^{\prime}\right)$.

By Lemma 2.2 and since $J$ satisfies the identity $\mathbf{w} \approx \mathbf{w}^{\prime}$, one of the following holds:

(b) $\mathrm{t}(\mathbf{w})=\mathrm{t}\left(\mathbf{w}^{\prime}\right) \in \operatorname{sim}(\mathbf{w})=\operatorname{sim}\left(\mathbf{w}^{\prime}\right)$;

(c) $\mathrm{t}(\mathbf{w}) \notin \operatorname{sim}(\mathbf{w})$ and $\mathrm{t}\left(\mathbf{w}^{\prime}\right) \notin \operatorname{sim}\left(\mathbf{w}^{\prime}\right)$.

If (c) holds, then it follows from Lemma 10.6 that the identity $\mathbf{w} \approx \mathbf{w}^{\prime}$ in $\Sigma$ can be replaced by one that satisfies (b) without changing the definition of $\mathbf{X}_{(10.1)} \Sigma$. Therefore the identity $\mathbf{w} \approx \mathbf{w}^{\prime}$ can be assumed to satisfy both (a) and (b). By Lemma 10.5, the words $\mathbf{w}$ and $\mathbf{w}^{\prime}$ can be chosen to be in canonical form. It then follows from (a) and (b) that

$$
\mathbf{w}=\left(\prod_{i=1}^{m}\left(x_{i} \mathbf{w}_{i}\right)\right) t \quad \text { and } \quad \mathbf{w}^{\prime}=\left(\prod_{i=1}^{m}\left(x_{i} \mathbf{w}_{i}^{\prime}\right)\right) t,
$$

where $t=\mathrm{t}(\mathbf{w})=\mathrm{t}\left(\mathbf{w}^{\prime}\right)$. Let $\ell$ be the least integer such that $\operatorname{con}\left(\mathbf{w}_{\ell}\right) \neq \operatorname{con}\left(\mathbf{w}_{\ell}^{\prime}\right)$, say $k \leqslant \ell$ is such that $x_{k} \in \operatorname{con}\left(\mathbf{w}_{\ell}\right) \backslash \operatorname{con}\left(\mathbf{w}_{\ell}^{\prime}\right)$. Then $\operatorname{occ}\left(x_{k}, \mathbf{w}\right)=2$ by (IV), and $\mathbf{w}$ is of the form

$$
\mathbf{w}=\ldots x_{k} \mathbf{w}_{k} \ldots x_{\ell}(\underbrace{\ldots x_{k} \ldots}_{\mathbf{w}_{\ell}}) \ldots
$$

It follows from (a) and (IV) that $\operatorname{occ}\left(x_{k}, \mathbf{w}^{\prime}\right)=2$. But since $\operatorname{con}\left(\mathbf{w}_{i}\right)=\operatorname{con}\left(\mathbf{w}_{i}^{\prime}\right)$ for all $i<\ell$, the second $x_{k}$ in $\mathbf{w}^{\prime}$ occurs in $\mathbf{w}_{r}^{\prime}$ for some $r>\ell$, whence $\mathbf{w}^{\prime}$ is of the form

$$
\mathbf{w}^{\prime}=\ldots x_{k} \mathbf{w}_{k}^{\prime} \ldots x_{\ell} \mathbf{w}_{\ell}^{\prime} \ldots x_{r}(\underbrace{\ldots x_{k} \ldots}_{\mathbf{w}_{r}^{\prime}}) \ldots
$$

Let $\varphi: \mathcal{X} \rightarrow P_{2}^{1}$ denote the substitution

$$
z \mapsto \begin{cases}b & \text { if } z=x_{k} \\ a & \text { if } z=x_{r} \\ 1 & \text { otherwise }\end{cases}
$$


Then $\mathbf{w} \varphi=b^{2}$ and $\mathbf{w}^{\prime} \varphi=b a$, which is impossible. Therefore the integer $\ell$ does not exist, whence $\operatorname{con}\left(\mathbf{w}_{i}\right)=\operatorname{con}\left(\mathbf{w}_{i}^{\prime}\right)$ for all $i$. By (II), the identity $\mathbf{w} \approx \mathbf{w}^{\prime}$ is trivial and so is satisfied by $\mathbf{X}_{(10.1)}$. Since $\mathbf{w} \approx \mathbf{w}^{\prime}$ is arbitrary in $\Sigma$, it follows that $\mathbf{P}_{\mathbf{2}}^{\mathbf{1}} \vee \mathbf{J}=\mathbf{X}_{(10.1)} \Sigma=\mathbf{X}_{(10.1)}$.

\subsection{Proof of Proposition 10.1}

Let $S$ be any semigroup in $\mathbf{X}_{(10.1)}$ that does not satisfy the identity (10.2). By Proposition 10.7, the variety $\mathbf{V}$ generated by $S$ is a subvariety of $\mathbf{P}_{\mathbf{2}}^{\mathbf{1}} \vee \mathbf{J}$. Since $S$ is finitely based if $\mathbf{V}=\mathbf{P}_{\mathbf{2}}^{\mathbf{1}} \vee \mathbf{J}$, it suffices to assume $\mathbf{V} \neq \mathbf{P}_{\mathbf{2}}^{\mathbf{1}} \vee \mathbf{J}$, so that either $P_{2}^{1} \notin \mathbf{V}$ or $J \notin \mathbf{V}$. If $P_{2}^{1} \notin \mathbf{V}$, then it follows from Lemma 10.3 that $S$ satisfies the identity (10.2), contradicting the assumption. Hence $P_{2}^{1} \in \mathbf{V}$ and $J \notin \mathbf{V}$. By Lemma 10.4, the variety $\mathbf{V}$ satisfies the identity (10.3). It then follows from Lemma 10.2 that $\mathbf{V}=\mathbf{P}_{\mathbf{2}}^{\mathbf{1}}$, whence $\mathbf{V}$ is finitely based.

\section{PART III. FinITE BASIS PROPERTY FOR THE SPORADIC CASES}

$$
\text { 11. } \mathscr{A}_{1}, \mathscr{B}_{1}, \mathscr{B}_{3}, \mathscr{D}_{10}
$$

This section establishes the finite basis property of the following semigroups.

\begin{tabular}{c|ccccccc|cccccccc|ccccccc|cccccc}
$\mathscr{A}_{1}$ & 1 & 2 & 3 & 4 & 5 & 6 & & $\mathscr{B}_{1}$ & 1 & 2 & 3 & 4 & 5 & 6 & $\mathscr{B}_{3}$ & 1 & 2 & 3 & 4 & 5 & 6 & $\mathscr{D}_{10}$ & 1 & 2 & 3 & 4 & 5 & 6 \\
\hline 1 & 1 & 1 & 1 & 1 & 1 & 1 & 1 & 1 & 1 & 1 & 1 & 1 & 1 & 1 & 1 & 1 & 1 & 1 & 1 & 1 & 1 & 1 & 1 & 1 & 1 & 1 & 1 \\
2 & 1 & 1 & 1 & 1 & 1 & 1 & 2 & 1 & 1 & 1 & 1 & 1 & 1 & 2 & 1 & 1 & 1 & 1 & 1 & 2 & 2 & 1 & 1 & 1 & 1 & 1 & 2 \\
3 & 1 & 1 & 1 & 1 & 1 & 3 & 3 & 1 & 2 & 3 & 4 & 5 & 6 & 3 & 1 & 1 & 1 & 1 & 1 & 2 & 3 & 1 & 1 & 2 & 1 & 2 & 3 \\
4 & 1 & 1 & 1 & 3 & 1 & 4 & 4 & 4 & 4 & 4 & 4 & 4 & 4 & 4 & 1 & 1 & 1 & 2 & 1 & 4 & 4 & 4 & 4 & 4 & 4 & 4 & 4 \\
5 & 5 & 5 & 5 & 5 & 5 & 5 & 5 & 4 & 4 & 5 & 4 & 4 & 4 & 5 & 5 & 5 & 5 & 5 & 5 & 5 & 5 & 1 & 1 & 2 & 1 & 2 & 3 \\
6 & 1 & 2 & 3 & 4 & 5 & 6 & 6 & 4 & 4 & 6 & 4 & 4 & 5 & 6 & 1 & 2 & 3 & 4 & 5 & 6 & 6 & 1 & 2 & 3 & 4 & 5 & 6
\end{tabular}

Proposition 11.1. The variety $\mathbf{J} \vee \mathbf{L}_{2}^{1} \vee \mathbf{N}_{3}^{1}$ is defined by the identities

$$
\begin{aligned}
x^{3} \mathrm{H} x & \approx x^{2} \mathrm{H} x, \\
x y x z & \approx x^{2} y z, \\
x \mathrm{H} y \mathrm{~K} x y & \approx x \mathrm{H} y \mathrm{~K} y x .
\end{aligned}
$$

The proof of Proposition 11.1 is given in $\S 11.2$.

Let $S \in\left\{\mathscr{A}_{1}, \mathscr{B}_{1}, \mathscr{B}_{3}, \mathscr{D}_{10}\right\}$. By Proposition 11.1, it is routinely verified that $S \in \mathbf{J} \vee \mathbf{L}_{\mathbf{2}}^{\mathbf{1}} \vee \mathbf{N}_{\mathbf{3}}^{\mathbf{1}}$. Conversely, the following are isomorphic to $J, L_{2}^{1}$, and $N_{3}^{1}$ :

- the subsemigroups $\{1,2,6\},\{1,5,6\}$, and $\{1,3,4,6\}$ of $\mathscr{A}_{1}$;

- the subsemigroups $\{1,2,3\},\{1,3,4\}$, and $\{3,4,5,6\}$ of $\mathscr{B}_{1}$;

- the divisor $\{1,2,3,6\} /\{1,2\}$ and the subsemigroups $\{1,5,6\}$ and $\{1,2,4,6\}$ of $\mathscr{B}_{3}$;

- the divisor $\{1,2,3,5,6\} /\{1,2,3\}$ and the subsemigroups $\{1,4,6\}$ and $\{1,2,3,6\}$ of $\mathscr{D}_{10}$.

Hence the variety generated by $S$ contains $J, L_{2}^{1}$, and $N_{3}^{1}$, and so coincides with $\mathbf{J} \vee \mathbf{L}_{\mathbf{2}}^{\mathbf{1}} \vee \mathbf{N}_{\mathbf{3}}^{\mathbf{1}}$. Consequently, $S$ is finitely based by Proposition 11.1.

\subsection{Compact and pseudo-compact words}

A compact word is a word of the form $x_{1}^{e_{1}} \ldots x_{m}^{e_{m}}$, where $x_{1}, \ldots, x_{m} \in \mathcal{X}$ are distinct and $e_{1}, \ldots, e_{m} \in\{1,2,3\}$. A pseudo-compact word is a word of the form $\mathbf{c} x^{e} \mathbf{s} x$, where the following are satisfied:

(I) $\mathbf{c} \in \mathcal{X}^{*}$ is a compact word;

(II) $x \in \mathcal{X}$ with $e \in\{1,2\}$; 
(III) $\mathbf{s} \in \mathcal{X}^{+}$is a simple word;

(IV) $\mathbf{c}, x$, and $\mathbf{s}$ are pairwise disjoint.

Note that when the very last letter is removed from a pseudo-compact word $\mathbf{c} x^{e} \mathbf{s} x$, then the resulting word $\mathbf{c} x^{e} \mathbf{s}$ is compact.

Lemma 11.2. Let $\mathbf{w}$ be any word. Then there exists some word $\overline{\mathbf{w}}$ that is compact or pseudocompact such that the identities (11.1) imply the identity $\mathbf{w} \approx \overline{\mathbf{w}}$.

Proof. Generality is not lost by assuming ini $(\mathbf{w})=x_{1} \ldots x_{m}$. It suffices to convert $\mathbf{w}$, using the identities (11.1), into a word that is either compact or pseudo-compact. With the exception of the very last letter of $\mathbf{w}$, any non-first occurrence of a letter $x$ in $\mathbf{w}$ can be gathered, using the identity (11.1b), with the first occurrence of $x$ in $\mathbf{w}$. The resulting word $\mathbf{w}^{\prime}$ is of one of the following forms:

(a) $x_{1}^{e_{1}} \ldots x_{m}^{e_{m}}$ for some $e_{1}, \ldots, e_{m} \geqslant 1$;

(b) $x_{1}^{e_{1}} \ldots x_{m}^{e_{m}} x_{k}$ for some $k<m$ and $e_{1}, \ldots, e_{m} \geqslant 1$.

If $\mathbf{w}^{\prime}$ is of the form (a), then the identity $x^{4} \approx x^{3}$ from (11.1a) can be used to convert it into a compact word. Thus assume that $\mathbf{w}^{\prime}$ is of the form (b), that is,

$$
\mathbf{w}^{\prime}=\mathbf{c} \cdot x_{k}^{e_{k}} \cdot x_{k+1}^{e_{k+1}} \ldots x_{m}^{e_{m}} \cdot x_{k}
$$

where $\mathbf{c}=x_{1}^{e_{1}} \ldots x_{k-1}^{e_{k-1}}$. If $e_{k+1}=\ldots=e_{m}=1$, then it is clear that the identities (11.1a) can be used to convert $\mathbf{w}^{\prime}$ into a pseudo-compact word. Hence assume that $\ell \in\{k+1, \ldots, m\}$ is the greatest such that $e_{\ell} \geqslant 2$. Then

$$
\begin{aligned}
& \mathbf{w}^{\prime}=\mathbf{c} \cdot x_{k}^{e_{k}} \cdot x_{k+1}^{e_{k+1}} \ldots x_{\ell}^{e_{\ell}} x_{\ell+1} \ldots x_{m} \cdot x_{k} \\
& \stackrel{(11.1 \mathrm{~b})}{\approx} \mathbf{c} \cdot x_{k}^{e_{k}} \cdot x_{k+1}^{e_{k+1}} \ldots x_{\ell}^{e_{\ell}-1} x_{\ell+1} \ldots x_{m} \cdot x_{\ell} x_{k} \\
& \stackrel{(11.1 \mathrm{c})}{\approx} \mathbf{c} \cdot x_{k}^{e_{k}} \cdot x_{k+1}^{e_{k+1}} \ldots x_{\ell}^{e_{\ell}-1} x_{\ell+1} \ldots x_{m} \cdot x_{k} x_{\ell} \\
& \stackrel{(11.1 \mathrm{~b})}{\approx} \underbrace{\mathbf{c} \cdot x_{k}^{e_{k}+1} \cdot x_{k+1}^{e_{k+1}} \ldots x_{\ell}^{e_{\ell}-1} x_{\ell+1} \ldots x_{m} \cdot x_{\ell}}_{\mathbf{w}^{\prime \prime}},
\end{aligned}
$$

and the identities (11.1a) can be used to convert $\mathbf{w}^{\prime \prime}$ into a pseudo-compact word.

\subsection{Proof of Proposition 11.1}

It is routinely checked that $J, L_{2}^{1}$, and $N_{3}^{1}$ satisfy the identities (11.1). Thus it remains to show that any identity $\mathbf{w} \approx \mathbf{w}^{\prime}$ satisfied by $J, L_{2}^{1}$, and $N_{3}^{1}$ is deducible from the identities (11.1). By Lemma 11.2, the words $\mathbf{w}$ and $\mathbf{w}^{\prime}$ can be chosen to be compact or pseudo-compact. Hence the following holds by Lemma 2.1 parts (iii) and (vii):

(a) $\operatorname{ini}(\mathbf{w})=\operatorname{ini}\left(\mathbf{w}^{\prime}\right)$;

(b) for each $x \in \mathcal{X}$, either $\operatorname{occ}(x, \mathbf{w})=\operatorname{occ}\left(x, \mathbf{w}^{\prime}\right) \leqslant 2 \operatorname{or} \operatorname{occ}(x, \mathbf{w}), \operatorname{occ}\left(x, \mathbf{w}^{\prime}\right) \geqslant 3$.

It is then easily shown that if $\mathbf{w}$ and $\mathbf{w}^{\prime}$ are both compact, then they are identical. Therefore it suffices to assume that $\mathbf{w}$ is pseudo-compact, say

$$
\mathbf{w}=x_{1}^{e_{1}} \ldots x_{m}^{e_{m}} x^{e} y_{1} \ldots y_{n} x
$$

for some distinct letters $x_{1}, \ldots, x_{m}, x, y_{1}, \ldots, y_{n}$ with $m \geqslant 0$ and $n \geqslant 1$, and some exponents $e_{1}, \ldots, e_{m} \in\{1,2,3\}$ and $e \in\{1,2\}$.

Suppose that $\mathbf{w}^{\prime}$ is compact, so that by (a),

$$
\mathbf{w}^{\prime}=x_{1}^{e_{1}^{\prime}} \ldots x_{m}^{e_{m}^{\prime}} x^{e^{\prime}} y_{1}^{f_{1}} \ldots y_{n}^{f_{n}}
$$


for some $e_{1}^{\prime}, \ldots, e_{m}^{\prime}, e^{\prime}, f_{1}, \ldots, f_{n} \in\{1,2,3\}$. Then $f_{1}=\ldots=f_{n}=1$ by (b). But now $\mathbf{w}$ ends with the non-simple letter $x$ while $\mathbf{w}^{\prime}$ ends with the simple letter $y_{n}$, contradicting Lemma 2.2. Therefore $\mathbf{w}^{\prime}$ must be pseudo-compact, so that by (a),

$$
\mathbf{w}^{\prime}=x_{1}^{e_{1}^{\prime}} \ldots x_{m}^{e_{m}^{\prime}} x^{e^{\prime}} y_{1}^{f_{1}} \ldots y_{n}^{f_{n}} z
$$

for some $z \in\left\{x_{1}, \ldots, x_{m}, x, y_{1}, \ldots, y_{n}\right\}$. Since $\operatorname{occ}\left(y_{1}, \mathbf{w}\right)=\ldots=\operatorname{occ}\left(y_{n}, \mathbf{w}\right)=1$, it follows from (b) that $f_{1}=\ldots=f_{n}=1$ and $z \notin\left\{y_{1}, \ldots, y_{n}\right\}$. Now $x$ is non-simple in $\mathbf{w}$. Therefore, if $z=x_{i}$ for some $i$, then the definition of a pseudo-compact word forces the letter $x$ to be simple in $\mathbf{w}^{\prime}$, contradicting (b). Hence $z=x$ is the only possibility. It is then easily shown by (b) that the words $\mathbf{w}$ and $\mathbf{w}^{\prime}$ are identical.

$$
\text { 12. } \mathscr{A}_{2}, \mathscr{B}_{7}, \mathscr{B}_{8}
$$

This section establishes the finite basis property of the following semigroups.

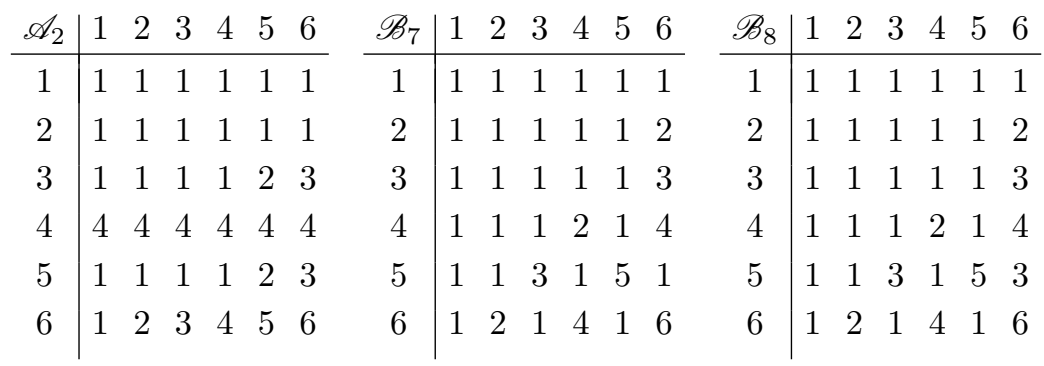

12.1. Finite basis property of $\mathscr{A}_{2}$

Proposition 12.1. The variety generated by $\mathscr{A}_{2}$ is defined by the identities

$$
\begin{aligned}
x^{3} y & \approx x^{2} y, \\
x y x z & \approx x^{2} y z, \\
x^{2} \mathrm{H} y^{2} \mathrm{~K} x & \approx x^{2} \mathrm{H} y^{2} \mathrm{~K} y .
\end{aligned}
$$

Lemma 12.2. Suppose that $\mathbf{w} \approx \mathbf{w}^{\prime}$ is any identity satisfied by $\mathscr{A}_{2}$. Then:

(i) $\operatorname{ini}(\mathbf{w})=\operatorname{ini}\left(\mathbf{w}^{\prime}\right)$;

(ii) $\operatorname{sim}(\mathbf{w})=\operatorname{sim}\left(\mathbf{w}^{\prime}\right)$;

(iii) either $|\mathbf{w}|,\left|\mathbf{w}^{\prime}\right| \geqslant 3$ or $\operatorname{occ}(x, \mathbf{w})=\operatorname{occ}\left(x, \mathbf{w}^{\prime}\right)$ for any $x \in \mathcal{X}$;

(iv) $\mathrm{t}(\mathbf{w}) \in \operatorname{sim}(\mathbf{w})$ if and only if $\mathrm{t}\left(\mathbf{w}^{\prime}\right) \in \operatorname{sim}\left(\mathbf{w}^{\prime}\right)$.

Proof. This follows from Lemmas 2.1 and 2.2 since the subsemigroups $\{1,4,6\},\{1,3,6\}$, $\{1,2,5\}$, and $\{1,2,6\}$ of $\mathscr{A}_{2}$ are isomorphic to $L_{2}^{1}, N_{2}^{1}, N_{3}$, and $J$, respectively.

In this subsection, a word $\mathbf{w}$ that contains at least two distinct letters is said to be in canonical form if

$$
\mathbf{w}=x_{1}^{e_{1}} \ldots x_{m}^{e_{m}} y
$$

where $x_{1}, \ldots, x_{m}, y \in \mathcal{X}$ and $e_{1}, \ldots, e_{m} \in\{1,2\}$ satisfy the following:

(I) the letters $x_{1}, \ldots, x_{m}$ are distinct while $y$ can be any letter;

(II) if $y=x_{i}$ and $e_{i}=2$ for some $i \geqslant 2$, then $e_{1}=\ldots=e_{i-1}=1$.

Lemma 12.3. Let $\mathbf{w}$ be any word that contains at least two distinct letters. Then there exists some word $\overline{\mathbf{w}}$ in canonical form such that the identities (12.1) imply the identity $\mathbf{w} \approx \overline{\mathbf{w}}$. 
Proof. It suffices to convert $\mathbf{w}$, using the identities (12.1), into a word in canonical form. Since $\mathbf{w}$ contains at least two distinct letters, $\mathbf{w}=\mathbf{w}^{\prime} y$ for some $\mathbf{w}^{\prime} \in \mathcal{X}^{+}$and $y \in \mathcal{X}$. Then the identities $\{(12.1 \mathrm{a}),(12.1 \mathrm{~b})\}$ can be used to convert $\mathbf{w}$ into a word of the form (12.2) with (I) satisfied. Suppose $y=x_{i}$ and $e_{i}=2$ for some $i \geqslant 2$. Then $\mathbf{w}=x_{1}^{e_{1}} \ldots x_{i-1}^{e_{i-1}} x_{i}^{2} x_{i+1}^{e_{i+1}} \ldots x_{m}^{e_{m}} x_{i}$. If $e_{1}=\ldots=e_{i-1}=1$, then $\mathbf{w}$ satisfies (II). Therefore suppose $e_{\ell}=2$ for some least integer $\ell \in\{1, \ldots, i-1\}$. Then

$$
\begin{aligned}
& \mathbf{w}=x_{1} \ldots x_{\ell-1} x_{\ell}^{2} x_{\ell+1}^{e_{\ell+1}} \ldots x_{i-1}^{e_{i-1}} x_{i}^{2} x_{i+1}^{e_{i+1}} \ldots x_{m}^{e_{m}} x_{i} \\
& \stackrel{(12.1 \mathrm{c})}{\approx} x_{1} \ldots x_{\ell-1} x_{\ell}^{2} x_{\ell+1}^{e_{\ell+1}} \ldots x_{i-1}^{e_{i-1}} x_{i}^{2} x_{i+1}^{e_{i+1}} \ldots x_{m}^{e_{m}} x_{\ell}
\end{aligned}
$$

so that (II) is satisfied.

Proof of Proposition 12.1. It is routinely checked that $\mathscr{A}_{2}$ satisfies the identities (12.1). Therefore it suffices to show that any identity $\mathbf{w} \approx \mathbf{w}^{\prime}$ satisfied by $\mathscr{A}_{2}$ is implied by the identities (12.1). If either $\mathbf{w}$ or $\mathbf{w}^{\prime}$ involves only one letter, say $\operatorname{con}(\mathbf{w})=\{x\}$, then it is easily shown by Lemma 12.2 parts (i) and (iii) that the identity $\mathbf{w} \approx \mathbf{w}^{\prime}$ is implied by the identity (12.1a). Hence assume that $\mathbf{w}$ and $\mathbf{w}^{\prime}$ each contains at least two distinct letters and so, by Lemma 12.3, can be chosen to be in canonical form. Since ini $(\mathbf{w})=\operatorname{ini}\left(\mathbf{w}^{\prime}\right)$ by Lemma 12.2(i),

$$
\mathbf{w}=x_{1}^{e_{1}} \ldots x_{m}^{e_{m}} y \quad \text { and } \quad \mathbf{w}^{\prime}=x_{1}^{e_{1}^{\prime}} \ldots x_{m}^{e_{m}^{\prime}} y^{\prime}
$$

Case 1: $y \in \operatorname{sim}(\mathbf{w})$. Then $y^{\prime} \in \operatorname{sim}\left(\mathbf{w}^{\prime}\right)$ by Lemma 12.2(iv), and $y=y^{\prime}$ by part (i) of the same lemma. Therefore $y, y^{\prime} \notin\left\{x_{1}, \ldots, x_{m}\right\}$, whence $e_{i}=\operatorname{occ}\left(x_{i}, \mathbf{w}\right)$ and $e_{i}^{\prime}=\operatorname{occ}\left(x_{i}, \mathbf{w}^{\prime}\right)$ for all $i$. Now Lemma 12.2(ii) implies $e_{i}=e_{i}^{\prime}$ for all $i$. Hence the identity $\mathbf{w} \approx \mathbf{w}^{\prime}$ is trivial and is implied by the identities (12.1).

Case 2: $y \notin \operatorname{sim}(\mathbf{w})$. Then $y^{\prime} \notin \operatorname{sim}\left(\mathbf{w}^{\prime}\right)$ by Lemma 12.2(iv). Seeking a contradiction, suppose $y \neq y^{\prime}$. By symmetry, it suffices to assume $y=x_{i}$ and $y^{\prime}=x_{j}$ with $i<j$. Then

$$
\mathbf{w}=x_{1}^{e_{1}} \ldots x_{i-1}^{e_{i-1}} \cdot x_{i}^{e_{i}} \cdot x_{i+1}^{e_{i+1}} \ldots x_{j-1}^{e_{j-1}} \cdot x_{j}^{e_{j}} \cdot x_{j+1}^{e_{j+1}} \ldots x_{m}^{e_{m}} \cdot x_{i}
$$

and

$$
\mathbf{w}^{\prime}=x_{1}^{e_{1}^{\prime}} \ldots x_{i-1}^{e_{i-1}^{\prime}} \cdot x_{i}^{e_{i}^{\prime}} \cdot x_{i+1}^{e_{i+1}^{\prime}} \ldots x_{j-1}^{e_{j-1}^{\prime}} \cdot x_{j}^{e_{j}^{\prime}} \cdot x_{j+1}^{e_{j+1}^{\prime}} \ldots x_{m}^{e_{m}^{\prime}} \cdot x_{j}
$$

Since $x_{i} \notin \operatorname{sim}(\mathbf{w})$, it follows from Lemma 12.2(ii) that $x_{i} \notin \operatorname{sim}\left(\mathbf{w}^{\prime}\right)$. Therefore $e_{i}^{\prime}=2$, whence $e_{j}^{\prime}=1$ by (II). Since $x_{j} \notin \operatorname{sim}\left(\mathbf{w}^{\prime}\right)$, it follows from Lemma 12.2(ii) that $x_{j} \notin \operatorname{sim}(\mathbf{w})$, whence $e_{j}=2$. Thus

$$
\mathbf{w}=x_{1}^{e_{1}} \ldots x_{i-1}^{e_{i-1}} \cdot x_{i}^{e_{i}} \cdot x_{i+1}^{e_{i+1}} \ldots x_{j-1}^{e_{j-1}} \cdot x_{j}^{2} \cdot x_{j+1}^{e_{j+1}} \ldots x_{m}^{e_{m}} \cdot x_{i}
$$

and

$$
\mathbf{w}^{\prime}=x_{1}^{e_{1}^{\prime}} \ldots x_{i-1}^{e_{i-1}^{\prime}} \cdot x_{i}^{2} \cdot x_{i+1}^{e_{i+1}^{\prime}} \ldots x_{j-1}^{e_{j-1}^{\prime}} \cdot x_{j} \cdot x_{j+1}^{e_{j+1}^{\prime}} \ldots x_{m}^{e_{m}^{\prime}} \cdot x_{j}
$$

Let $\varphi: \mathcal{X} \rightarrow \mathscr{A}_{2}$ denote the substitution

$$
z \mapsto \begin{cases}5 & \text { if } z=x_{j}, \\ 6 & \text { otherwise }\end{cases}
$$

Then $\mathbf{w} \varphi \in\{6\}^{*} \cdot 5^{2} \cdot\{6\}^{+}=\{1\}$ and $\mathbf{w}^{\prime} \varphi \in\{6\}^{*} \cdot 5 \cdot\{6\}^{*} \cdot 5=\{2\}$, which is the required contradiction.

Therefore $y=y^{\prime}$ is the only possibility, whence $y=y^{\prime}=x_{j}$ for some $j$, so that

$$
\mathbf{w}=x_{1}^{e_{1}} \ldots x_{j-1}^{e_{j-1}} \cdot x_{j}^{e_{j}} \cdot x_{j+1}^{e_{j+1}} \ldots x_{m}^{e_{m}} \cdot x_{j}
$$


and

$$
\mathbf{w}^{\prime}=x_{1}^{e_{1}^{\prime}} \ldots x_{j-1}^{e_{j-1}^{\prime}} \cdot x_{j}^{e_{j}^{\prime}} \cdot x_{j+1}^{e_{j+1}^{\prime}} \ldots x_{m}^{e_{m}^{\prime}} \cdot x_{j} .
$$

It follows from Lemma 12.2 (ii) that $\left(e_{1}, \ldots, e_{j-1}, e_{j+1}, \ldots, e_{m}\right)=\left(e_{1}^{\prime}, \ldots, e_{j-1}^{\prime}, e_{j+1}^{\prime}, \ldots, e_{m}^{\prime}\right)$. If $e_{j} \neq e_{j}^{\prime}$, say $\left(e_{j}, e_{j}^{\prime}\right)=(2,1)$, then

$$
\mathbf{w} \varphi \in\{6\}^{*} \cdot 5^{2} \cdot\{6\}^{*} \cdot 5=\{1\} \quad \text { and } \quad \mathbf{w}^{\prime} \varphi \in\{6\}^{*} \cdot 5 \cdot\{6\}^{*} \cdot 5=\{2\},
$$

which is impossible. Therefore $e_{j}=e_{j}^{\prime}$, whence the identity $\mathbf{w} \approx \mathbf{w}^{\prime}$ is trivial and is implied by the identities (12.1).

12.2. Finite basis property of $\mathscr{B}_{8}$

Proposition 12.4. The variety generated by $\mathscr{B}_{8}$ is defined by the identities

$$
\begin{gathered}
x^{3} \mathrm{H} x \approx x^{2} \mathrm{H} x, \\
x \mathrm{H} y z \mathrm{~K} x \approx x \mathrm{H} z y \mathrm{~K} x, \\
x \mathrm{H} y \mathrm{~K} x y \approx x \mathrm{H} y \mathrm{~K} y x, \quad x y \mathrm{H} x \mathrm{~K} y \approx y x \mathrm{H} x \mathrm{~K} y .
\end{gathered}
$$

Lemma 12.5. Let $S \in\left\{\mathscr{B}_{7}, \mathscr{B}_{8}\right\}$. Suppose that $\mathbf{w} \approx \mathbf{w}^{\prime}$ is any identity satisfied by $S$. Then for any $x \in \mathcal{X}$, either $\operatorname{occ}(x, \mathbf{w})=\operatorname{occ}\left(x, \mathbf{w}^{\prime}\right) \leqslant 2 \operatorname{or} \operatorname{occ}(x, \mathbf{w}), \operatorname{occ}\left(x, \mathbf{w}^{\prime}\right) \geqslant 3$.

Proof. This follows from Lemma 2.1(vii) since the subsemigroup $\{1,2,4,6\}$ of $S$ is isomorphic to $N_{3}^{1}$.

For the remainder of this section, a non-simple word $\mathbf{w}$ is said to be in canonical form if

$$
\mathbf{w}=x_{0}^{e_{0}} x_{1}^{e_{1}} \ldots x_{m}^{e_{m}} y_{1} \ldots y_{n} x_{0},
$$

where all of the following are satisfied:

(I) $x_{0}, x_{1}, \ldots, x_{m}$ are distinct non-simple letters of $\mathbf{w}$ in alphabetical order;

(II) $e_{0} \in\{1,2\}$ and $e_{1}, \ldots, e_{m} \in\{2,3\}$;

(III) $y_{1}, \ldots, y_{n}$ are distinct simple letters of $\mathbf{w}$ in alphabetical order.

Note that if all letters of the word $\mathbf{w}$ are non-simple, then $n=0$ and $y_{1} \ldots y_{n}=\emptyset$.

Lemma 12.6. Let $\mathbf{w}$ be any non-simple word with $\mathrm{h}(\mathbf{w})=\mathrm{t}(\mathbf{w})$. Then there exists some word $\overline{\mathbf{w}}$ in canonical form such that the identities (12.3) imply the identity $\mathbf{w} \approx \overline{\mathbf{w}}$.

Proof. It suffices to convert $\mathbf{w}$, using the identities (12.3), into a word in canonical form. By assumption, $\mathbf{w}=x \mathbf{w}^{\prime} x$ for some $\mathbf{w}^{\prime} \in \mathcal{X}^{*}$. Suppose that $x, x_{1}, \ldots, x_{m}$ are the non-simple letters of $\mathbf{w}$ and $y_{1}, \ldots, y_{n}$ are the simple letters of $\mathbf{w}$. Generality is not lost by assuming that $x_{1}, \ldots, x_{m}$ are in alphabetical order and $y_{1}, \ldots, y_{n}$ are in alphabetical order. Since the letters in the factor $\mathbf{w}^{\prime}$ of $\mathbf{w}$ are sandwiched between two occurrences of $x$, they can be rearranged by the identities (12.3b) to produce the word $x^{e} x_{1}^{e_{1}} \ldots x_{m}^{e_{m}} y_{1} \ldots y_{n} x$, where $e+1=\operatorname{occ}(x, \mathbf{w}) \geqslant 2$ and $e_{i}=\operatorname{occ}\left(x_{i}, \mathbf{w}\right) \geqslant 2$. The identities $(12.3 \mathrm{a})$ can then be used to reduce the exponents, so that $e \in\{1,2\}$ and $e_{1}, \ldots, e_{m} \in\{2,3\}$. The resulting word is in canonical form if $x$ alphabetically precedes $x_{1}$. If $x$ does not alphabetically precede $x_{1}$, then

$$
\begin{aligned}
& x^{e} x_{1}^{e_{1}} x_{2}^{e_{2}} \ldots x_{m}^{e_{m}} y_{1} \ldots y_{n} x \stackrel{(12.3 \mathrm{~b})}{\approx} x^{e} x_{1}^{e_{1}-1} x_{2}^{e_{2}} \ldots x_{m}^{e_{m}} y_{1} \ldots y_{n} x_{1} x \\
& \stackrel{(12.3 \mathrm{c})}{\approx} x_{1}^{e_{1}-1} x^{e} x_{2}^{e_{2}} \ldots x_{m}^{e_{m}} y_{1} \ldots y_{n} x x_{1} \\
& \stackrel{(12.3 \mathrm{~b})}{\approx} x_{1}^{e_{1}-1} x^{e+1} x_{2}^{e_{2}} \ldots x_{m}^{e_{m}} y_{1} \ldots y_{n} x_{1},
\end{aligned}
$$


and the factors $x^{e+1}, x_{2}^{e_{2}}, \ldots, x_{m}^{e_{m}}$ in the latter word can be alphabetically ordered by the identities (12.3b), resulting in a word in canonical form.

Lemma 12.7. Let $\mathbf{w}$ be any connected word. Then there exists some word $\overline{\mathbf{w}}$ in canonical form such that the identities (12.3) imply the identity $\mathbf{w} \approx \overline{\mathbf{w}}$.

Proof. It suffices to convert $\mathbf{w}$, using the identities (12.3), into a word in canonical form. Since $\mathbf{w}$ is connected, the letter $x=\mathrm{h}(\mathbf{w})$ occurs at least twice in $\mathbf{w}$. Hence $\mathbf{w}=x \mathbf{u} x \mathbf{v}$ for some $\mathbf{u}, \mathbf{v} \in \mathcal{X}^{*}$ such that $x \notin \operatorname{con}(\mathbf{v})$. If $\mathbf{v}=\emptyset$, then the result follows from Lemma 12.6. Therefore assume $\mathbf{v} \neq \emptyset$, whence $\mathbf{w}=x \mathbf{u} x y \mathbf{v}^{\prime}$ for some $y \in \mathcal{X}$ and $\mathbf{v}^{\prime} \in \mathcal{X}^{*}$. There are two cases.

Case 1: $y \notin \operatorname{con}(\mathbf{u})$. Since $\mathbf{w}$ is connected, the factors $x \mathbf{u} x$ and $y \mathbf{v}^{\prime}$ cannot be disjoint. It follows that $\mathbf{u}$ and $\mathbf{v}^{\prime}$ share some common letter, whence $\mathbf{w}=x \mathbf{u} x y \mathbf{v}^{\prime} \stackrel{(12.3 \mathrm{~b})}{\approx} x \mathbf{u} y x \mathbf{v}^{\prime}$.

Case 2: $y \in \operatorname{con}(\mathbf{u})$. Then clearly $\mathbf{w}=x \mathbf{u} x y \mathbf{v}^{\prime} \stackrel{(12.3 \mathrm{c})}{\approx} x \mathbf{u} y x \mathbf{v}^{\prime}$.

In any case, the identities (12.3) can be used to interchange the last $x$ in $\mathbf{w}$ with the letter $y$ that immediately follows it. It is easily seen that the resulting word $\mathbf{w}^{\prime}=x \mathbf{u} y x \mathbf{v}^{\prime}$ is connected. The same procedure can be repeated to move the last $x$ in $\mathbf{w}^{\prime}$ to the right until it becomes the last letter of the word. Hence $\mathbf{w} \stackrel{(12.3)}{\approx} x \mathbf{u v} x$ and the result now holds by Lemma 12.6.

Proof of Proposition 12.4. It is easily verified that $\mathscr{B}_{8}$ satisfies the identities (12.3) and is idempotent-separable. Since the subsemigroup $\{1,3,5,6\}$ of $\mathscr{B}_{8}$ is isomorphic to $A_{0}$, the variety generated by $\mathscr{B}_{8}$ contains $A_{0}$. It follows from Lemma 2.6(i) that $\mathscr{B}_{8}$ has a basis $\Sigma$ that consists of identities formed by connected words. Hence $\{(12.3)\} \cup \Sigma$ is also a basis for $\mathscr{B}_{8}$. By Lemma 12.7, the words that form the identities in $\Sigma$ can be chosen to be in canonical form. Let $\mathbf{w} \approx \mathbf{w}^{\prime}$ be any identity from $\Sigma$. Then

$$
\mathbf{w}=x_{0}^{e_{0}} x_{1}^{e_{1}} \ldots x_{m}^{e_{m}} y_{1} \ldots y_{n} x_{0} \quad \text { and } \quad \mathbf{w}^{\prime}=z_{0}^{f_{0}} z_{1}^{f_{1}} \ldots z_{p}^{f_{p}} t_{1} \ldots t_{q} z_{0}
$$

where $e_{0}, f_{0} \in\{1,2\}$ and $e_{1}, \ldots, e_{m}, f_{1}, \ldots, f_{p} \in\{2,3\}$. By Lemma 12.5:

(a) $\mathbf{w}$ and $\mathbf{w}^{\prime}$ share the same simple letters, so that $\left(y_{1}, \ldots, y_{n}\right)=\left(t_{1}, \ldots, t_{q}\right)$ by (III);

(b) $\mathbf{w}$ and $\mathbf{w}^{\prime}$ share the same non-simple letters, so that $\left(x_{0}, \ldots, x_{m}\right)=\left(z_{1}, \ldots, z_{p}\right)$ by (I).

It then follows from (II) and Lemma 12.5 that $e_{i}=f_{i}$ for all $i$, whence the identity $\mathbf{w} \approx \mathbf{w}^{\prime}$ is trivial. Since $\mathbf{w} \approx \mathbf{w}^{\prime}$ is arbitrary in $\Sigma$, every identity in $\Sigma$ is trivial. Consequently, the identities (12.3) form a basis for $\mathscr{B}_{8}$.

\subsection{Finite basis property of $\mathscr{B}_{7}$}

Proposition 12.8. The variety generated by $\mathscr{B}_{7}$ is defined by the identities (12.3) and

$$
x \mathrm{H} x y \mathrm{~K} y \approx x \mathrm{H} y x \mathrm{~K} y .
$$

Lemma 12.9. Let $\mathbf{w}$ be any product of pairwise disjoint connected words. Then there exists some word $\overline{\mathbf{w}}$ in canonical form such that the identities $\{(12.3),(12.4)\}$ imply the identity $\mathbf{w} \approx \overline{\mathbf{w}}$.

Proof. By assumption, $\mathbf{w}=\mathbf{w}_{1} \ldots \mathbf{w}_{k}$ for some connected words $\mathbf{w}_{1}, \ldots, \mathbf{w}_{k}$ that are pairwise disjoint. It suffices to convert $\mathbf{w}$, using the identities $\{(12.3),(12.4)\}$, into a word in canonical form. By Lemma 12.7, the identities (12.3) can be used to convert each $\mathbf{w}_{i}$ into 
a word in canonical form. Therefore $\mathbf{w}_{i} \stackrel{(12.3)}{\approx} x_{i} \mathbf{w}_{i}^{\prime} x_{i}$ for some $x_{i} \in \mathcal{X}$ and $\mathbf{w}_{i}^{\prime} \in \mathcal{X}^{*}$. Now

$$
\begin{gathered}
\mathbf{w} \stackrel{(12.3)}{\approx} x_{1} \mathbf{w}_{1}^{\prime} x_{1} \cdot x_{2} \mathbf{w}_{2}^{\prime} x_{2} \cdot x_{3} \mathbf{w}_{3}^{\prime} x_{3} \ldots x_{k-1} \mathbf{w}_{k-1}^{\prime} x_{k-1} \cdot x_{k} \mathbf{w}_{k}^{\prime} x_{k} \\
\stackrel{(12.4)}{\approx} \underbrace{x_{1} \mathbf{w}_{1}^{\prime} x_{2} \cdot x_{1} \mathbf{w}_{2}^{\prime} x_{3} \cdot x_{2} \mathbf{w}_{3}^{\prime} x_{4} \ldots x_{k-2} \mathbf{w}_{k-1}^{\prime} x_{k} \cdot x_{k-1} \mathbf{w}_{k}^{\prime} x_{k}}_{\mathbf{w}^{\prime}},
\end{gathered}
$$

where the word $\mathbf{w}^{\prime}$ is connected. Hence by Lemma 12.7, the identities (12.3) can be used to convert $\mathbf{w}^{\prime}$ into a word in canonical form.

Proof of Proposition 12.8. It is easily verified that the semigroup $\mathscr{B}_{7}$ satisfies the identities $\{(12.3),(12.4)\}$ and is idempotent-separable. Let $\mathbf{V}$ denote the variety generated by $\mathscr{B}_{7}$. Then $B_{0} \in \mathbf{V}$ because the subsemigroup $\{1,3,5,6\}$ of $\mathscr{B}_{7}$ is isomorphic to $B_{0}$. The semigroup $A_{0}$ does not satisfy the identities (12.4) because $a a b b \neq a b a b$ in $A_{0}$. Therefore $A_{0} \notin \mathbf{V}$, and it follows from Lemma 2.6(ii) that $\mathscr{B}_{7}$ has a basis $\Sigma$ that consists of identities formed by words that are products of pairwise disjoint connected words. Hence $\{(12.3),(12.4)\} \cup \Sigma$ is also a basis for $\mathscr{B}_{7}$. By Lemma 12.9, the words that form the identities in $\Sigma$ can be chosen to be in canonical form. Following the arguments in the proof of Proposition 12.4, every identity in $\Sigma$ is trivial. Consequently, the identities $\{(12.3),(12.4)\}$ form a basis for $\mathscr{B}_{7}$.

$$
\text { 13. } \mathscr{A}_{3}, \mathscr{A}_{5}, \mathscr{A}_{6}, \mathscr{A}_{8}, \mathscr{A}_{9}, \mathscr{A}_{10}, \mathscr{G}_{4}, \mathscr{G}_{5}
$$

\begin{tabular}{|c|c|c|c|c|c|c|c|c|c|c|c|c|c|c|c|c|c|c|c|c|c|c|c|c|c|c|}
\hline$z_{3}$ & 1 & 2 & & & & & $\mathscr{A}_{5}$ & & & & & & & $\mathscr{A}_{6}$ & & & & & & & Q/8 & & & & & \\
\hline 1 & 1 & 1 & 1 & 1 & 11 & 1 & 1 & 1 & 1 & 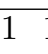 & 1 & & & 1 & \begin{tabular}{|l|}
1 \\
\end{tabular} & 1 & 1 & & 1 & & 1 & 1 & 1 & 1 & 1 & \\
\hline 2 & 1 & 1 & 1 & & 11 & & 2 & & 1 & 1 & 1 & 1 & & 2 & 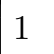 & 1 & 1 & 1 & 1 & & 2 & & 1 & & & \\
\hline 3 & 1 & 1 & 1 & & 56 & & 3 & & 1 & : & 3 & 3 & & & & 1 & 3 & 3 & 5 & & 3 & & 2 & & 33 & \\
\hline 4 & 1 & 2 & 2 & 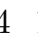 & 11 & & 4 & & 1 & 4 & 4 & 4 & & 4 & 1 & 2 & 3 & 4 & 5 & & 4 & & 2 & & 45 & \\
\hline 5 & 5 & 5 & 5 & . & 55 & & 5 & & 2 & 3 & 4 & & & $\partial$ & 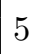 & 5 & 5 & 5 & 5 & & 5 & 1 & 2 & 5 & 5 & \\
\hline 6 & 5 & 5 & 5 & 5 & 13 & 3 & 6 & & 2 & 4 & 3 & 5 & & & 5 & 5 & 6 & 6 & 1 & & 6 & 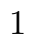 & 7 & & 3 & \\
\hline $\mathscr{A}_{9}$ & 1 & 2 & 3 & 4 & 5 & 6 & $\mathscr{L}_{10}$ & 1 & 2 & 3 & 4 & & 6 & $\mathscr{G}_{4}$ & 1 & 2 & 3 & 4 & 5 & & $\mathscr{G}_{5}$ & 1 & 2 & & 15 & \\
\hline 1 & 1 & 1 & & 1 & 11 & 1 & 4 & & 1 & 1 & 1 & & & 4 & \begin{tabular}{|l|}
1 \\
\end{tabular} & 1 & 1 & 1 & 1 & & 1 & 7 & 1 & & 1 & \\
\hline 2 & 1 & 1 & & & 1 & 1 & 2 & & 1 & 1 & 1 & & & 2 & 1 & 2 & 2 & 2 & 5 & & 2 & 1 & 2 & & 44 & \\
\hline 3 & 1 & 2 & & 4 & 5 & 6 & 3 & 1 & 2 & 3 & 4 & & & 3 & 1 & 2 & 2 & 2 & 5 & & 3 & 1 & 2 & 3 & & \\
\hline 4 & 1 & 2 & & & - & 5 & 4 & . & 4 & 4 & 4 & & & 4 & 1 & 2 & 3 & & 5 & & 4 & 6 & 4 & & 22 & \\
\hline J & 5 & & & & 5 & 5 & 5 & & 4 & 4 & 4 & & & 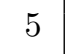 & 5 & 5 & 5 & & 5 & & 5 & 6 & 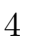 & & 2 & \\
\hline 6 & 0 & 6 & & & 6 & 6 & 6 & & & & 1 & & & 6 & 5 & 6 & 6 & 6 & 1 & & 6 & 6 & 6 & & $6 \quad 6$ & \\
\hline
\end{tabular}

This section establishes the finite basis property of the following semigroups.

Let $\mathbf{O}$ denote the variety generated by the semigroup $O$ in Remark 6.6.

Proposition 13.1. The variety $\mathbf{J} \vee \mathbf{O}$ is defined by the identities

$$
\begin{aligned}
x \mathrm{H} x^{3} & \approx x \mathrm{H} x, \\
x \mathrm{H} x^{2} y & \approx x \mathrm{H} y, \\
x \mathrm{H} y \mathrm{~K} x y & \approx x \mathrm{H} y \mathrm{~K} y x .
\end{aligned}
$$

The proof of Proposition 13.1 is given in $\S 13.3$.

Let $S \in\left\{\mathscr{A}_{3}, \mathscr{A}_{5}, \mathscr{A}_{6}, \mathscr{A}_{8}, \mathscr{A}_{9}, \mathscr{A}_{10}, \mathscr{G}_{4}, \mathscr{G}_{5}\right\}$. By Proposition 13.1, it is routinely verified that $S \in \mathbf{J} \vee \mathbf{O}$. Conversely, the following are isomorphic to $J$ and $O$ : 
- the subsemigroups $\{1,2,4\}$ and $\{1,3,5,6\}$ of $S^{\prime} \in\left\{\mathscr{A}_{3}, \mathscr{A}_{6}\right\}$;

- the subsemigroups $\{1,2,5\}$ and $\{3,4,5,6\}$ of $\mathscr{A}_{5}$;

- the subsemigroups $\{1,2,3\}$ and $\{3,4,5,6\}$ of $S^{\prime \prime} \in\left\{\mathscr{A}_{8}, \mathscr{A}_{9}\right\}$;

- the subsemigroups $\{1,2,3\}$ and $\{1,3,4,6\}$ of $\mathscr{A}_{10}$;

- the subsemigroups $\{2,3,4\}$ and $\{1,2,5,6\}$ of $\mathscr{G}_{4}$;

- the divisor $\{2,3,4,5\} /\{2,4\}$ and the subsemigroup $\{1,2,4,6\}$ of $\mathscr{G}_{5}$.

Hence the variety generated by $S$ contains $J$ and $O$, and so coincides with $\mathbf{J} \vee \mathbf{O}$. Consequently, $S$ is finitely based by Proposition 13.1 .

\subsection{Identities satisfied by $O$}

Lemma 13.2. Let $\mathbf{w} \approx \mathbf{w}^{\prime}$ be any identity satisfied by $O$. Then $\operatorname{occ}(x, y, \mathbf{w}) \equiv \operatorname{occ}\left(x, y, \mathbf{w}^{\prime}\right)$ $(\bmod 2)$ for any $x, y \in \mathcal{X}$.

Proof. Seeking a contradiction, $\operatorname{suppose} \operatorname{occ}(x, y, \mathbf{w}) \not \equiv \operatorname{occ}\left(x, y, \mathbf{w}^{\prime}\right)(\bmod 2)$ for some letters $x, y \in \mathcal{X}, \operatorname{say} \operatorname{occ}(x, y, \mathbf{w})=2 p$ and $\operatorname{occ}\left(x, y, \mathbf{w}^{\prime}\right)=2 q+1$ for some $p, q \geqslant 0$. Let $\varphi: \mathcal{X} \rightarrow O$ denote the substitution

$$
z \mapsto \begin{cases}b & \text { if } z=x, \\ a & \text { if } z=y, \\ 1 & \text { otherwise. }\end{cases}
$$

Then $\mathbf{w} \varphi=b^{2 p} \cdot a \ldots=a$ and $\mathbf{w}^{\prime} \varphi=b^{2 q+1} \cdot a \ldots=b a$ give the required contradiction.

Lemma 13.3. Let $\mathbf{w} \approx \mathbf{w}^{\prime}$ be any identity satisfied by $O$ and let $t \in \mathcal{X} \backslash \operatorname{con}\left(\mathbf{w w}^{\prime}\right)$. Suppose $\mathrm{t}(\mathbf{w}) \notin \operatorname{sim}(\mathbf{w})$ and $\mathrm{t}\left(\mathbf{w}^{\prime}\right) \notin \operatorname{sim}\left(\mathbf{w}^{\prime}\right)$. Then $(\mathbf{J} \vee \mathbf{O})\left\{\mathbf{w} \approx \mathbf{w}^{\prime}\right\}=(\mathbf{J} \vee \mathbf{O})\left\{\mathbf{w} t \approx \mathbf{w}^{\prime} t\right\}$.

Proof. Let $\sigma_{1}$ and $\sigma_{2}$ denote the identities $\mathbf{w} \approx \mathbf{w}^{\prime}$ and $\mathbf{w} t \approx \mathbf{w}^{\prime} t$, respectively. Then the inclusion $(\mathbf{J} \vee \mathbf{O})\left\{\sigma_{1}\right\} \subseteq(\mathbf{J} \vee \mathbf{O})\left\{\sigma_{2}\right\}$ is obvious. Let $x=\mathrm{t}(\mathbf{w})$ and $y=\mathrm{t}\left(\mathbf{w}^{\prime}\right)$. (Note that $x$ and $y$ need not be distinct.) Since $x \notin \operatorname{sim}(\mathbf{w})$ and $y \notin \operatorname{sim}\left(\mathbf{w}^{\prime}\right)$ by assumption, the deductions $\mathbf{w} \stackrel{(13.1 \mathrm{a})}{\approx} \mathbf{w} x^{2}$ and $\mathbf{w}^{\prime} \stackrel{(13.1 \mathrm{a})}{\approx} \mathbf{w}^{\prime} y^{2}$ hold. The subsemigroup $\{a, b a, 1\}$ of $O$ is isomorphic to $L_{2}^{1}$, so that $\operatorname{ini}(\mathbf{w})=\operatorname{ini}\left(\mathbf{w}^{\prime}\right)$ by Lemma $2.1\left(\right.$ iii). Therefore $y \in \operatorname{con}(\mathbf{w})$ and $\mathbf{w} x^{2} \stackrel{(13.1 \mathrm{~b})}{\approx} \mathbf{w} y^{2} x^{2}$. Since

$$
\mathbf{w} \stackrel{(13.1 \mathrm{a})}{\approx} \mathbf{w} x^{2} \stackrel{(13.1 \mathrm{~b})}{\approx} \mathbf{w} y^{2} x^{2} \stackrel{(13.1 \mathrm{c})}{\approx} \mathbf{w} x^{2} y^{2} \stackrel{(13.1 \mathrm{a})}{\approx} \mathbf{w} y^{2} \stackrel{\sigma_{2}}{\approx} \mathbf{w}^{\prime} y^{2} \stackrel{(13.1 \mathrm{a})}{\approx} \mathbf{w}^{\prime}
$$

the identities $\left\{(13.1), \sigma_{2}\right\}$ imply $\sigma_{1}$, so the inclusion $(\mathbf{J} \vee \mathbf{O})\left\{\sigma_{2}\right\} \subseteq(\mathbf{J} \vee \mathbf{O})\left\{\sigma_{1}\right\}$ follows.

\subsection{A canonical form}

In this section, any word $\mathbf{w}$ with $t=\mathrm{t}(\mathbf{w}) \in \operatorname{sim}(\mathbf{w})$ is said to be in canonical form if

$$
\mathbf{w}=\left(\prod_{i=1}^{m}\left(x_{i} \mathbf{w}_{i}\right)\right) t,
$$

where $x_{1}, \ldots, x_{m} \in \mathcal{X}$ and $\mathbf{w}_{1}, \ldots, \mathbf{w}_{m} \in \mathcal{X}^{*}$ are such that:

(I) $\operatorname{ini}(\mathbf{w})=x_{1} \ldots x_{m} t$;

(II) $\mathbf{w}_{i} \in\left\{x_{1}^{e_{1}} \ldots x_{i}^{e_{i}} \mid e_{1}, \ldots, e_{i} \in\{0,1\}\right\}$.

Lemma 13.4. Let $\mathbf{w}$ be any word with $t=\mathrm{t}(\mathbf{w}) \in \operatorname{sim}(\mathbf{w})$. Then there exists some word $\overline{\mathbf{w}}$ in canonical form with $t=\mathrm{t}(\overline{\mathbf{w}}) \in \operatorname{sim}(\overline{\mathbf{w}})$ such that the identities (13.1) imply the identity $\mathbf{w} \approx \overline{\mathbf{w}}$. 
Proof. The letter $t$ is simple in $\mathbf{w}$, so that ini( $\mathbf{w})=x_{1} \ldots x_{m} t$ for some $x_{1}, \ldots, x_{m} \in \mathcal{X}$. It is clear that $\mathbf{w}$ can be written in the form (13.2) with (I) satisfied and $\mathbf{w}_{i} \in\left\{x_{1}, \ldots, x_{i}\right\}^{*}$ for all $i$. Since the letters in each $\mathbf{w}_{i}$ are non-first occurrences in $\mathbf{w}$, the identities (13.1c) can be used to order them so that $\mathbf{w}_{i} \in\left\{x_{1}^{e_{1}} \ldots x_{i}^{e_{i}} \mid e_{1}, \ldots, e_{i} \geqslant 0\right\}$. The identities $\{(13.1 \mathrm{a}),(13.1 \mathrm{~b})\}$ can then be used to reduce each exponent $e_{i}$ to a number in $\{0,1\}$. Hence (II) is satisfied.

\subsection{Proof of Proposition 13.1}

Let $\mathbf{X}_{(13.1)}$ denote the variety defined by the identities (13.1). It is routinely checked that $J, O \in \mathbf{X}_{(13.1)}$, so that $\mathbf{J} \vee \mathbf{O}=\mathbf{X}_{(13.1)} \Sigma$ for some set $\Sigma$ of identities. Let $\mathbf{w} \approx \mathbf{w}^{\prime}$ be any identity in $\Sigma$. The subsemigroup $\{a, b a, 1\}$ of $O$ is isomorphic to $L_{2}^{1}$, hence by Lemma 2.1(iii),

(a) $\operatorname{ini}(\mathbf{w})=\operatorname{ini}\left(\mathbf{w}^{\prime}\right)$.

Since $J$ satisfies the identity $\mathbf{w} \approx \mathbf{w}^{\prime}$, it follows from Lemma 2.2 that one of the following holds:

(b) $\mathrm{t}(\mathbf{w}) \in \operatorname{sim}(\mathbf{w}), \mathrm{t}\left(\mathbf{w}^{\prime}\right) \in \operatorname{sim}\left(\mathbf{w}^{\prime}\right)$, and $\mathrm{t}(\mathbf{w})=\mathrm{t}\left(\mathbf{w}^{\prime}\right)$;

(c) $\mathrm{t}(\mathbf{w}) \notin \operatorname{sim}(\mathbf{w})$ and $\mathrm{t}\left(\mathbf{w}^{\prime}\right) \notin \operatorname{sim}\left(\mathbf{w}^{\prime}\right)$.

If (c) holds, then Lemma 13.3 implies that the identity $\mathbf{w} \approx \mathbf{w}^{\prime}$ in $\Sigma$ can be replaced by one that satisfies (b) without changing the definition of $\mathbf{X}_{(13.1)} \Sigma$. Therefore the identity $\mathbf{w} \approx \mathbf{w}^{\prime}$ can be assumed to satisfy both (a) and (b). By Lemma 13.4, the words $\mathbf{w}$ and $\mathbf{w}^{\prime}$ can be chosen to be in canonical form. It then follows from (a) and (b) that

$$
\mathbf{w}=\left(\prod_{i=1}^{m}\left(x_{i} \mathbf{w}_{i}\right)\right) t \quad \text { and } \quad \mathbf{w}^{\prime}=\left(\prod_{i=1}^{m}\left(x_{i} \mathbf{w}_{i}^{\prime}\right)\right) t .
$$

Let $\ell$ be the least integer such that $\operatorname{con}\left(\mathbf{w}_{\ell}\right) \neq \operatorname{con}\left(\mathbf{w}_{\ell}^{\prime}\right)$, say $x_{k} \in \operatorname{con}\left(\mathbf{w}_{\ell}\right) \backslash \operatorname{con}\left(\mathbf{w}_{\ell}^{\prime}\right)$ for some $k \leqslant \ell$. Then it follows from (II) that $\mathbf{w}_{i}=\mathbf{w}_{i}^{\prime}$ for all $i<\ell$. But now Lemma 13.2 is violated since $\operatorname{occ}\left(x_{k}, t, \mathbf{w}\right) \not \equiv \operatorname{occ}\left(x_{k}, t, \mathbf{w}^{\prime}\right)(\bmod 2)$ if $\ell=m$, and $\operatorname{occ}\left(x_{k}, x_{\ell+1}, \mathbf{w}\right) \not \equiv \operatorname{occ}\left(x_{k}, x_{\ell+1}, \mathbf{w}^{\prime}\right)$ $(\bmod 2)$ if $\ell<m$. Therefore the integer $\ell$ does not exist, whence $\mathbf{w}_{i}=\mathbf{w}_{i}^{\prime}$ for all $i$. The identity $\mathbf{w} \approx \mathbf{w}^{\prime}$ is thus trivial and is satisfied by $\mathbf{X}_{(13.1)}$. Since $\mathbf{w} \approx \mathbf{w}^{\prime}$ is arbitrary in $\Sigma$, it follows that $\mathbf{X}_{(13.1)}=\mathbf{X}_{(13.1)} \Sigma=\mathbf{J} \vee \mathbf{O}$.

$$
\text { 14. } \mathscr{A}_{4}, \mathscr{A}_{7}, \mathscr{B}_{2}, \mathscr{G}_{3}
$$

This section establishes the finite basis property of the following semigroups.

\begin{tabular}{c|ccccccc|cccccccc|ccccccc|cccccc}
$\mathscr{A}_{4}$ & 1 & 2 & 3 & 4 & 5 & 6 & $\mathscr{A}_{7}$ & 1 & 2 & 3 & 4 & 5 & 6 & & $\mathscr{B}_{2}$ & 1 & 2 & 3 & 4 & 5 & 6 & $\mathscr{G}_{3}$ & 1 & 2 & 3 & 4 & 5 & 6 \\
\hline 1 & 1 & 1 & 1 & 1 & 1 & 1 & 1 & 1 & 1 & 1 & 1 & 1 & 1 & 1 & 1 & 1 & 1 & 1 & 1 & 1 & 1 & 1 & 1 & 1 & 1 & 1 & 1 \\
2 & 1 & 1 & 1 & 1 & 1 & 1 & 2 & 1 & 1 & 1 & 1 & 1 & 1 & 2 & 1 & 1 & 1 & 1 & 1 & 1 & 2 & 1 & 2 & 1 & 2 & 5 & 6 \\
3 & 1 & 1 & 3 & 1 & 5 & 6 & 3 & 1 & 2 & 3 & 1 & 5 & 6 & 3 & 3 & 3 & 3 & 3 & 3 & 3 & 3 & 3 & 3 & 3 & 3 & 3 & 3 \\
4 & 4 & 4 & 4 & 4 & 4 & 4 & 4 & 4 & 4 & 4 & 4 & 4 & 4 & 4 & 3 & 3 & 3 & 4 & 5 & 6 & 4 & 1 & 2 & 1 & 2 & 5 & 6 \\
5 & 1 & 1 & 3 & 6 & 5 & 6 & 5 & 1 & 2 & 3 & 6 & 5 & 6 & 5 & 5 & 5 & 5 & 5 & 5 & 5 & 5 & 1 & 2 & 6 & 2 & 5 & 6 \\
6 & 6 & 6 & 6 & 6 & 6 & 6 & 6 & 6 & 6 & 6 & 6 & 6 & 6 & 6 & 5 & 5 & 3 & 4 & 5 & 6 & 6 & 6 & 6 & 6 & 6 & 6 & 6
\end{tabular}

\subsection{Finite basis property of $\mathscr{A}_{7}$}

Proposition 14.1. The variety generated by $\mathscr{A}_{7}$ is defined by the identities

$$
\begin{aligned}
x^{2} y & \approx x y, \\
x y x^{2} & \approx x y x, \\
x \mathrm{H} y \mathrm{~K} x y & \approx x \mathrm{H} y \mathrm{~K} y, \\
x \mathrm{H} y \mathrm{~K} y x & \approx x \mathrm{H} y \mathrm{~K} x .
\end{aligned}
$$


Let $\mathbf{w}$ be any word such that ini $(\mathbf{w})=x_{0} \ldots x_{m}$ with $m \geqslant 1$. In this section, such a word $\mathbf{w}$ is said to be in $\alpha$-canonical form if

$$
\mathbf{w}=x_{0} \prod_{i=1}^{m}\left(x_{i} \mathbf{w}_{i}\right)
$$

where the following are satisfied:

(I) $\mathbf{w}_{m} \in\left\{\emptyset, x_{0}, \ldots, x_{m}\right\}$;

(II) $\mathbf{w}_{i} \in\left\{\emptyset, x_{0}, \ldots, x_{i-1}\right\}$ for each $i$ such that $1 \leqslant i<m$.

Lemma 14.2. Let $\mathbf{w}$ be any word that contains at least two distinct letters. Then there exists some word $\overline{\mathbf{w}}$ in $\alpha$-canonical form such that the identities (14.1) imply the identity $\mathbf{w} \approx \overline{\mathbf{w}}$.

Proof. It suffices to convert $\mathbf{w}$, using the identities (14.1), into a word in $\alpha$-canonical form. Since $\mathbf{w}$ contains at least two distinct letters, generality is not lost by assuming $\operatorname{ini}(\mathbf{w})=x_{0} \ldots x_{m}$ with $m \geqslant 1$. Then $\mathbf{w}$ can be written in the form $\mathbf{w}=\prod_{i=0}^{m}\left(x_{i} \mathbf{w}_{i}\right)$ with $\mathbf{w}_{i} \in\left\{x_{0}, \ldots, x_{i}\right\}^{*}$. If $\mathbf{w}_{0}$ is nonempty, then $\mathbf{w}_{0}=x_{0}$ and the identity (14.1a) can be used to eliminate $\mathbf{w}_{0}=x_{0}$ from $\mathbf{w}$. Hence $\mathbf{w}$ is of the form (14.2).

Suppose $\mathbf{w}_{m} \neq \emptyset$. Then $\mathbf{w}_{m}=z_{1} \ldots z_{s}$ for some $z_{1}, \ldots, z_{s} \in\left\{x_{0}, \ldots, x_{m}\right\}$ with $s \geqslant 1$. Hence $\mathbf{w}=\left(x_{0} \prod_{i=1}^{m-1}\left(x_{i} \mathbf{w}_{i}\right)\right) x_{m} z_{1} \ldots z_{s}$. The letters $z_{1}, \ldots, z_{s}$ are non-first occurrences in $\mathbf{w}$. Therefore if $s \geqslant 2$, then the identities (14.1b)-(14.1d) can be used to eliminate $z_{1}, \ldots, z_{s-1}$ from $\mathbf{w}_{m}$. Hence (I) is satisfied.

Suppose $\mathbf{w}_{i} \neq \emptyset$ for some $i<m$. Then $\mathbf{w}_{i}=y_{1} \ldots y_{r}$ for some $y_{1}, \ldots, y_{r} \in\left\{x_{0}, \ldots, x_{i}\right\}$ with $r \geqslant 1$. Hence

$$
\mathbf{w}=\mathbf{p} x_{i} \underbrace{y_{1} \ldots y_{r}}_{\mathbf{w}_{i}} \mathbf{q}
$$

where $\mathbf{p}=x_{0} \prod_{j=1}^{i-1}\left(x_{j} \mathbf{w}_{j}\right)$ and $\mathbf{q}=\prod_{j=i+1}^{m}\left(x_{j} \mathbf{w}_{j}\right)$. The letters $y_{1}, \ldots, y_{r}$ are non-first occurrences in $\mathbf{w}$. Therefore if $r \geqslant 2$, then the identities (14.1b)-(14.1d) can be used to eliminate the letters $y_{1}, \ldots, y_{r-1}$ from $\mathbf{w}_{i}$. Hence $\mathbf{w} \stackrel{(14.1)}{\approx} \mathbf{p} x_{i} y_{r} \mathbf{q}$. If $y_{r}=x_{i}$, then the identity (14.1a) can be used to further eliminate $y_{r}$ from $\mathbf{w}_{i}$. Therefore $\mathbf{w}_{i}$ is converted into a word in $\left\{\emptyset, x_{1}, \ldots, x_{i-1}\right\}$, whence (II) is satisfied.

Lemma 14.3. Suppose that $\mathbf{w} \approx \mathbf{w}^{\prime}$ is any identity satisfied by $\mathscr{A}_{7}$. Then:

(i) $\operatorname{ini}(\mathbf{w})=\operatorname{ini}\left(\mathbf{w}^{\prime}\right)$ and $\mathrm{t}(\mathbf{w})=\mathrm{t}\left(\mathbf{w}^{\prime}\right)$;

(ii) $\mathrm{t}(\mathbf{w}) \in \operatorname{sim}(\mathbf{w})$ if and only if $\mathrm{t}\left(\mathbf{w}^{\prime}\right) \in \operatorname{sim}\left(\mathbf{w}^{\prime}\right)$.

Proof. This follows from Lemmas 2.1 and 2.2 because the subsemigroups $\{1,3,6\},\{3,5\}$, and $\{1,2,5\}$ of $\mathscr{A}_{7}$ are isomorphic to $L_{2}^{1}, R_{2}$, and $J$, respectively.

Proof of Proposition 14.1. It is routinely checked that $\mathscr{A}_{7}$ satisfies the identities (14.1). Hence it suffices to show that any identity $\mathbf{w} \approx \mathbf{w}^{\prime}$ satisfied by $\mathscr{A}_{7}$ is implied by the identities (14.1). If either $\mathbf{w}$ or $\mathbf{w}^{\prime}$ involves only one letter, say $\operatorname{con}(\mathbf{w})=\{x\}$, then it is easily shown by Lemma 14.3 that the identity $\mathbf{w} \approx \mathbf{w}^{\prime}$ is implied by the identity (14.1a). Therefore assume that $\mathbf{w}$ and $\mathbf{w}^{\prime}$ each contains at least two distinct letters and so by Lemma 14.2, can be chosen to be in $\alpha$-canonical form. Since ini $(\mathbf{w})=$ ini $\left(\mathbf{w}^{\prime}\right)$ by Lemma 14.3(i), generality is not lost by assuming

$$
\mathbf{w}=x_{0} \prod_{i=1}^{m}\left(x_{i} \mathbf{w}_{i}\right) \quad \text { and } \quad \mathbf{w}^{\prime}=x_{0} \prod_{i=1}^{m}\left(x_{i} \mathbf{w}_{i}^{\prime}\right) \quad \text { for some } m \geqslant 1 .
$$


Suppose $\mathbf{w}_{m} \neq \mathbf{w}_{m}^{\prime}$. Then since $\mathrm{t}(\mathbf{w})=\mathrm{t}\left(\mathbf{w}^{\prime}\right)$ by Lemma 14.3(i), it follows from (I) that $\left(\mathbf{w}_{m}, \mathbf{w}_{m}^{\prime}\right) \in\left\{\left(x_{m}, \emptyset\right),\left(\emptyset, x_{m}\right)\right\}$, say $\left(\mathbf{w}_{m}, \mathbf{w}_{m}^{\prime}\right)=\left(x_{m}, \emptyset\right)$. Hence $\mathbf{w}=\left(x_{0} \prod_{i=1}^{m-1}\left(x_{i} \mathbf{w}_{i}\right)\right) x_{m}^{2}$ and $\mathbf{w}^{\prime}=\left(x_{0} \prod_{i=1}^{m-1}\left(x_{i} \mathbf{w}_{i}^{\prime}\right)\right) x_{m}$. But now Lemma 14.3(ii) is violated because $\mathrm{t}(\mathbf{w}) \notin \operatorname{sim}(\mathbf{w})$ and $\mathrm{t}\left(\mathbf{w}^{\prime}\right) \in \operatorname{sim}\left(\mathbf{w}^{\prime}\right)$. Therefore $\mathbf{w}_{m}=\mathbf{w}_{m}^{\prime}$.

Suppose $\mathbf{w}_{\ell} \neq \mathbf{w}_{\ell}^{\prime}$ for some $\ell<m$, so that $\mathbf{w}_{\ell}, \mathbf{w}_{\ell}^{\prime} \in\left\{\emptyset, x_{0}, \ldots, x_{\ell-1}\right\}$ by (II). Then there are two cases.

Case 1: $\mathbf{w}_{\ell} \neq \emptyset \neq \mathbf{w}_{\ell}^{\prime}$. Then $\mathbf{w}_{\ell}=x_{j}$ and $\mathbf{w}_{\ell}^{\prime}=x_{k}$ for some $j, k \in\{0, \ldots, \ell-1\}$ with $j \neq k$. By symmetry, it suffices to assume $0 \leqslant j<k<\ell$. Then

$$
\mathbf{w}=\mathbf{p} x_{\ell} x_{j} \mathbf{q} \quad \text { and } \quad \mathbf{w}^{\prime}=\mathbf{p}^{\prime} x_{\ell} x_{k} \mathbf{q}^{\prime}
$$

where $\mathbf{p}=x_{0} \prod_{i=1}^{\ell-1}\left(x_{i} \mathbf{w}_{i}\right), \mathbf{q}=\prod_{i=\ell+1}^{m}\left(x_{i} \mathbf{w}_{i}\right), \mathbf{p}^{\prime}=x_{0} \prod_{i=1}^{\ell-1}\left(x_{i} \mathbf{w}_{i}^{\prime}\right)$, and $\mathbf{q}^{\prime}=\prod_{i=\ell+1}^{m}\left(x_{i} \mathbf{w}_{i}^{\prime}\right)$. Let $\varphi: \mathcal{X} \rightarrow \mathscr{A}_{7}$ denote the substitution

$$
z \mapsto \begin{cases}5 & \text { if } z=x_{j} \\ 4 & \text { if } z=x_{\ell+1} \\ 3 & \text { otherwise. }\end{cases}
$$

Then $\mathbf{w} \varphi \neq \mathbf{w}^{\prime} \varphi$ because

$$
\mathbf{w} \varphi=\mathbf{p} \varphi \cdot x_{\ell} \varphi \cdot x_{j} \varphi \cdot \mathbf{q} \varphi \in\{3,5\}^{*} \cdot 3 \cdot 5 \cdot 4 \ldots=\{6\}
$$

and

$$
\mathbf{w}^{\prime} \varphi=\mathbf{p}^{\prime} \varphi \cdot x_{\ell} \varphi \cdot x_{k} \varphi \cdot \mathbf{q}^{\prime} \varphi \in\{3,5\}^{*} \cdot 3 \cdot 3 \cdot 4 \ldots=\{1\} .
$$

Case 2 : $\mathbf{w}_{\ell}=\emptyset \neq \mathbf{w}_{\ell}^{\prime}$ or $\mathbf{w}_{\ell} \neq \emptyset=\mathbf{w}_{\ell}^{\prime}$. Then by symmetry, it suffices to assume $\mathbf{w}_{\ell}=\emptyset$ and $\mathbf{w}_{\ell}^{\prime}=x_{j}$ for some $j \in\{0, \ldots, \ell-1\}$. Hence

$$
\mathbf{w}=\mathbf{p} x_{\ell} \mathbf{q} \quad \text { and } \quad \mathbf{w}^{\prime}=\mathbf{p}^{\prime} x_{\ell} x_{j} \mathbf{q}^{\prime}
$$

where $\mathbf{p}, \mathbf{q}, \mathbf{p}^{\prime}$, and $\mathbf{q}^{\prime}$ are as defined in Case 1 . Therefore $\mathbf{w} \varphi \neq \mathbf{w}^{\prime} \varphi$ because

$$
\mathbf{w} \varphi=\mathbf{p} \varphi \cdot x_{\ell} \varphi \cdot \mathbf{q} \varphi \in\{3,5\}^{*} \cdot 3 \cdot 4 \ldots=\{1\}
$$

and

$$
\mathbf{w}^{\prime} \varphi=\mathbf{p}^{\prime} \varphi \cdot x_{\ell} \varphi \cdot x_{j} \varphi \cdot \mathbf{q}^{\prime} \varphi \in\{3,5\}^{*} \cdot 3 \cdot 5 \cdot 4 \ldots=\{6\} .
$$

Since both cases are impossible, the integer $\ell$ does not exist. Therefore the identity $\mathbf{w} \approx \mathbf{w}^{\prime}$ is trivial and so is implied by the identities (14.1).

14.2. Finite basis property of $\mathscr{A}_{4}, \mathscr{B}_{2}$, and $\mathscr{G}_{3}$

Proposition 14.4. The variety generated by any $S \in\left\{\mathscr{A}_{4}, \mathscr{B}_{2}, \mathscr{G}_{3}\right\}$ is defined by the identities

$$
\begin{aligned}
x^{2} y & \approx x y, \\
x y^{2} & \approx x y, \\
x \mathrm{H} y \mathrm{~K} x y & \approx x \mathrm{H} y \mathrm{~K} y, \\
x \mathrm{H} y \mathrm{~K} y x & \approx x \mathrm{H} y \mathrm{~K} x .
\end{aligned}
$$


Let $\mathbf{w}$ be any word such that ini $(\mathbf{w})=x_{0} \ldots x_{m}$ with $m \geqslant 1$. In this section, such a word $\mathbf{w}$ is said to be in $\beta$-canonical form if

$$
\mathbf{w}=x_{0} \prod_{i=1}^{m}\left(x_{i} \mathbf{w}_{i}\right)
$$

where $\mathbf{w}_{i} \in\left\{\emptyset, x_{1}, \ldots, x_{i-1}\right\}$ for all $i$.

Lemma 14.5. Let $\mathbf{w}$ be any word that contains at least two distinct letters. Then there exists some word $\overline{\mathbf{w}}$ in $\beta$-canonical form such that the identities (14.3) imply the identity $\mathbf{w} \approx \overline{\mathbf{w}}$.

Proof. It suffices to convert $\mathbf{w}$, using the identities (14.3), into a word in $\beta$-canonical form. It is easily seen that the identities (14.3) imply the identities (14.1). Therefore by Lemma 14.2, the identities (14.3) can be used to convert $\mathbf{w}$ into a word in $\alpha$-canonical form, that is, a word of the form (14.4) such that $\mathbf{w}_{m} \in\left\{\emptyset, x_{0}, \ldots, x_{m}\right\}$ and $\mathbf{w}_{i} \in\left\{\emptyset, x_{0}, \ldots, x_{i-1}\right\}$ for all $i$ with $1 \leqslant i<m$. If $\mathbf{w}_{m}=x_{m}$, then the identity (14.3b) can be used to eliminate $\mathbf{w}_{m}=x_{m}$ from $\mathbf{w}$. The resulting word is in $\beta$-canonical form.

Lemma 14.6. Let $S \in\left\{\mathscr{A}_{4}, \mathscr{B}_{2}, \mathscr{G}_{3}\right\}$. Suppose that $\mathbf{w} \approx \mathbf{w}^{\prime}$ is any identity satisfied by $S$. Then $\operatorname{ini}(\mathbf{w})=\operatorname{ini}\left(\mathbf{w}^{\prime}\right)$ and $\mathrm{t}(\mathbf{w})=\mathrm{t}\left(\mathbf{w}^{\prime}\right)$.

Proof. The subsemigroups $\{3,5\} \subset \mathscr{A}_{4},\{4,6\} \subset \mathscr{B}_{2}$, and $\{2,5\} \subset \mathscr{G}_{3}$ are isomorphic to $R_{2}$, while the subsemigroups $\{1,3,6\} \subset \mathscr{A}_{4},\{3,4,5\} \subset \mathscr{B}_{2}$, and $\{1,2,6\} \subset \mathscr{G}_{3}$ are isomorphic to $L_{2}^{1}$. Hence the result follows from Lemma 2.1 parts (ii) and (iii).

Proof of Proposition 14.4. Let $S \in\left\{\mathscr{A}_{4}, \mathscr{B}_{2}, \mathscr{G}_{3}\right\}$. It is routinely checked that $S$ satisfies the identities (14.3). Hence it suffices to show that any identity $\mathbf{w} \approx \mathbf{w}^{\prime}$ satisfied by $S$ is implied by the identities (14.3). If either $\mathbf{w}$ or $\mathbf{w}^{\prime}$ involves only one letter, say $\operatorname{con}(\mathbf{w})=\{x\}$, then it is easily shown by Lemma 14.6 that the identity $\mathbf{w} \approx \mathbf{w}^{\prime}$ is implied by the identity (14.3a). Therefore assume that $\mathbf{w}$ and $\mathbf{w}^{\prime}$ each contains at least two distinct letters and so, by Lemma 14.5, can be chosen to be in $\beta$-canonical form. Since ini( $\mathbf{w})=\operatorname{ini}\left(\mathbf{w}^{\prime}\right)$ by Lemma 14.6, generality is not lost by assuming

$$
\mathbf{w}=x_{0} \prod_{i=1}^{m}\left(x_{i} \mathbf{w}_{i}\right) \quad \text { and } \quad \mathbf{w}^{\prime}=x_{0} \prod_{i=1}^{m}\left(x_{i} \mathbf{w}_{i}^{\prime}\right) \quad \text { for some } m \geqslant 1 .
$$

Since $\mathrm{t}(\mathbf{w})=\mathrm{t}\left(\mathbf{w}^{\prime}\right)$ by Lemma 14.6 and $\mathbf{w}_{m}, \mathbf{w}_{m}^{\prime} \in\left\{\emptyset, x_{0}, \ldots, x_{m-1}\right\}$, it follows that $\mathbf{w}_{m}=\mathbf{w}_{m}^{\prime}$. Suppose $\mathbf{w}_{\ell} \neq \mathbf{w}_{\ell}^{\prime}$ for some $\ell<m$, so that $\mathbf{w}_{\ell}, \mathbf{w}_{\ell}^{\prime} \in\left\{\emptyset, x_{1}, \ldots, x_{\ell-1}\right\}$. Then there are two cases.

Case $1: \mathbf{w}_{\ell} \neq \emptyset \neq \mathbf{w}_{\ell}^{\prime}$. Then $\mathbf{w}_{\ell}=x_{j}$ and $\mathbf{w}_{\ell}^{\prime}=x_{k}$ for some $j, k \in\{0, \ldots, \ell-1\}$ with $j \neq k$. By symmetry, it suffices to assume $0 \leqslant j<k<\ell$. Then

$$
\mathbf{w}=\mathbf{p} x_{\ell} x_{j} \mathbf{q} \quad \text { and } \quad \mathbf{w}^{\prime}=\mathbf{p}^{\prime} x_{\ell} x_{k} \mathbf{q}^{\prime}
$$

where $\mathbf{p}=x_{0} \prod_{i=1}^{\ell-1}\left(x_{i} \mathbf{w}_{i}\right), \mathbf{q}=\prod_{i=\ell+1}^{m}\left(x_{i} \mathbf{w}_{i}\right), \mathbf{p}^{\prime}=x_{0} \prod_{i=1}^{\ell-1}\left(x_{i} \mathbf{w}_{i}^{\prime}\right)$, and $\mathbf{q}^{\prime}=\prod_{i=\ell+1}^{m}\left(x_{i} \mathbf{w}_{i}^{\prime}\right)$. Let $\varphi_{1}, \varphi_{2}$, and $\varphi_{3}$ denote the following substitutions into $\mathscr{A}_{4}, \mathscr{B}_{2}$, and $\mathscr{G}_{3}$, respectively: 


$$
z \mapsto\left\{\begin{array} { l l } 
{ 5 } & { \text { if } z = x _ { j } , } \\
{ 4 } & { \text { if } z = x _ { \ell + 1 } , } \\
{ 3 } & { \text { otherwise; } }
\end{array} \quad z \mapsto \left\{\begin{array} { l l } 
{ 6 } & { \text { if } z = x _ { j } , } \\
{ 1 } & { \text { if } z = x _ { \ell + 1 } , } \\
{ 4 } & { \text { otherwise; } }
\end{array} \quad z \mapsto \left\{\begin{array}{ll}
5 & \text { if } z=x_{j}, \\
3 & \text { if } z=x_{\ell+1} \\
2 & \text { otherwise. }
\end{array}\right.\right.\right.
$$

Then $\mathbf{w} \varphi_{1} \neq \mathbf{w}^{\prime} \varphi_{1}, \mathbf{w} \varphi_{2} \neq \mathbf{w}^{\prime} \varphi_{2}$, and $\mathbf{w} \varphi_{3} \neq \mathbf{w}^{\prime} \varphi_{3}$ because

$$
\mathbf{w} \varphi_{1}=\mathbf{p} \varphi_{1} \cdot x_{\ell} \varphi_{1} \cdot x_{j} \varphi_{1} \cdot \mathbf{q} \varphi_{1} \in\{3,5\}^{*} \cdot 3 \cdot 5 \cdot 4 \ldots=\{6\}
$$

and

$$
\begin{aligned}
\mathbf{w}^{\prime} \varphi_{1} & =\mathbf{p}^{\prime} \varphi_{1} \cdot x_{\ell} \varphi_{1} \cdot x_{k} \varphi_{1} \cdot \mathbf{q}^{\prime} \varphi_{1} \in\{3,5\}^{*} \cdot 3 \cdot 3 \cdot 4 \ldots=\{1\} \\
\mathbf{w} \varphi_{2} & =\mathbf{p} \varphi_{2} \cdot x_{\ell} \varphi_{2} \cdot x_{j} \varphi_{2} \cdot \mathbf{q} \varphi_{2} \in\{4,6\}^{*} \cdot 4 \cdot 6 \cdot 1 \ldots=\{5\}
\end{aligned}
$$

and

$$
\begin{aligned}
\mathbf{w}^{\prime} \varphi_{2} & =\mathbf{p}^{\prime} \varphi_{2} \cdot x_{\ell} \varphi_{2} \cdot x_{k} \varphi_{2} \cdot \mathbf{q}^{\prime} \varphi_{2} \in\{4,6\}^{*} \cdot 4 \cdot 4 \cdot 1 \ldots=\{3\} \\
\mathbf{w} \varphi_{3} & =\mathbf{p} \varphi_{3} \cdot x_{\ell} \varphi_{3} \cdot x_{j} \varphi_{3} \cdot \mathbf{q} \varphi_{3} \in\{2,5\}^{*} \cdot 2 \cdot 5 \cdot 3 \ldots=\{6\}
\end{aligned}
$$

and

$$
\mathbf{w}^{\prime} \varphi_{3}=\mathbf{p}^{\prime} \varphi_{3} \cdot x_{\ell} \varphi_{3} \cdot x_{k} \varphi_{3} \cdot \mathbf{q}^{\prime} \varphi_{3} \in\{2,5\}^{*} \cdot 2 \cdot 2 \cdot 3 \ldots=\{1\}
$$

Case 2: $\mathbf{w}_{\ell}=\emptyset \neq \mathbf{w}_{\ell}^{\prime}$ or $\mathbf{w}_{\ell} \neq \emptyset=\mathbf{w}_{\ell}^{\prime}$. Then by symmetry, it suffices to assume $\mathbf{w}_{\ell}=\emptyset$ and $\mathbf{w}_{\ell}^{\prime}=x_{j}$ for some $j \in\{0, \ldots, \ell-1\}$. Hence

$$
\mathbf{w}=\mathbf{p} x_{\ell} \mathbf{q} \quad \text { and } \quad \mathbf{w}^{\prime}=\mathbf{p}^{\prime} x_{\ell} x_{j} \mathbf{q}^{\prime}
$$

where $\mathbf{p}, \mathbf{q}, \mathbf{p}^{\prime}$, and $\mathbf{q}^{\prime}$ are as defined in Case 1 . It follows that

$$
\mathbf{w} \varphi_{1}=\mathbf{p} \varphi_{1} \cdot x_{\ell} \varphi_{1} \cdot \mathbf{q} \varphi_{1} \in\{3,5\}^{*} \cdot 3 \cdot 4 \ldots=\{1\}
$$

and

$$
\begin{gathered}
\mathbf{w}^{\prime} \varphi_{1}=\mathbf{p}^{\prime} \varphi_{1} \cdot x_{\ell} \varphi_{1} \cdot x_{j} \varphi_{1} \cdot \mathbf{q}^{\prime} \varphi_{1} \in\{3,5\}^{*} \cdot 3 \cdot 5 \cdot 4 \ldots=\{6\} \\
\mathbf{w} \varphi_{2}=\mathbf{p} \varphi_{2} \cdot x_{\ell} \varphi_{2} \cdot \mathbf{q} \varphi_{2} \in\{4,6\}^{*} \cdot 4 \cdot 1 \ldots=\{3\}
\end{gathered}
$$

and

$$
\begin{gathered}
\mathbf{w}^{\prime} \varphi_{2}=\mathbf{p}^{\prime} \varphi_{2} \cdot x_{\ell} \varphi_{2} \cdot x_{j} \varphi_{2} \cdot \mathbf{q}^{\prime} \varphi_{2} \in\{4,6\}^{*} \cdot 4 \cdot 6 \cdot 1 \ldots=\{5\} \\
\mathbf{w} \varphi_{3}=\mathbf{p} \varphi_{3} \cdot x_{\ell} \varphi_{3} \cdot \mathbf{q} \varphi_{3} \in\{2,5\}^{*} \cdot 2 \cdot 3 \ldots=\{1\}
\end{gathered}
$$

and

$$
\mathbf{w}^{\prime} \varphi_{3}=\mathbf{p}^{\prime} \varphi_{3} \cdot x_{\ell} \varphi_{3} \cdot x_{j} \varphi_{3} \cdot \mathbf{q}^{\prime} \varphi_{3} \in\{2,5\}^{*} \cdot 2 \cdot 5 \cdot 3 \ldots=\{6\} .
$$

Therefore $\mathbf{w} \varphi_{1} \neq \mathbf{w}^{\prime} \varphi_{1}, \mathbf{w} \varphi_{2} \neq \mathbf{w}^{\prime} \varphi_{2}$, and $\mathbf{w} \varphi_{3} \neq \mathbf{w}^{\prime} \varphi_{3}$.

Since both cases are impossible, the integer $\ell$ does not exist. Therefore the identity $\mathbf{w} \approx \mathbf{w}^{\prime}$ is trivial and so is implied by the identities (14.3). 
15. $\mathscr{B}_{4}, \mathscr{B}_{5}, \mathscr{B}_{6}, \mathscr{B}_{9}, \mathscr{B}_{10}, \mathscr{E}_{1}$

This section establishes the finite basis property of the following semigroups.

\begin{tabular}{|c|c|c|c|c|c|c|c|c|c|c|c|c|c|c|c|c|c|c|c|c|}
\hline $\mathscr{B}_{4}$ & 1 & 2 & 3 & 4 & 5 & 0 & $\mathscr{B}_{5}$ & & & & & & 6 & $\mathscr{B}_{6}$ & & 2 & & & & \\
\hline 1 & 1 & 1 & 1 & 1 & 1 & 1 & 1 & 1 & 1 & 1 & 1 & 1 & 1 & 1 & 1 & 1 & 1 & 1 & 1 & 1 \\
\hline 2 & 1 & 1 & 1 & 1 & 1 & 2 & 2 & 1 & 1 & 1 & 1 & 1 & 2 & 2 & 1 & 1 & 1 & 1 & 1 & 2 \\
\hline 3 & 1 & 1 & 1 & 1 & 1 & 3 & 3 & 1 & 1 & 1 & 1 & 1 & 3 & 3 & 1 & 1 & 1 & 1 & 1 & 3 \\
\hline 4 & 1 & 1 & 1 & 2 & 1 & 4 & 4 & 1 & 1 & 1 & 2 & 1 & 4 & 4 & 1 & 1 & 1 & 2 & 1 & 4 \\
\hline 5 & 1 & 1 & 2 & 1 & 1 & 1 & 5 & 1 & 1 & 2 & 1 & 1 & 1 & 5 & 1 & 1 & 2 & 1 & 1 & 2 \\
\hline 6 & 1 & 2 & 1 & 4 & 5 & 6 & 6 & 1 & 2 & 2 & 4 & 5 & 6 & 6 & 1 & 2 & 2 & 4 & 5 & 6 \\
\hline $\mathscr{B}_{9}$ & 1 & 2 & 3 & 4 & 5 & 6 & $\mathscr{B}_{10}$ & 1 & 2 & 3 & 4 & 5 & 6 & $\mathscr{E}_{1}$ & 1 & 2 & 3 & 4 & 5 & 6 \\
\hline 1 & 1 & 1 & 1 & 1 & 1 & 1 & 1 & & 1 & 1 & 1 & 1 & 1 & 1 & 1 & 1 & 1 & 1 & 1 & 1 \\
\hline 2 & 1 & 1 & 1 & 1 & 1 & 2 & 2 & 1 & 1 & 1 & 1 & 1 & 2 & 2 & 1 & 1 & 1 & 1 & 1 & 2 \\
\hline 3 & 1 & 1 & 1 & 1 & 1 & 3 & 3 & 1 & 1 & 1 & 1 & 1 & 3 & 3 & 1 & 1 & 2 & 1 & 2 & 5 \\
\hline 4 & 1 & 1 & 1 & 2 & 2 & 4 & 4 & 1 & 1 & 1 & 2 & 2 & 4 & 4 & 1 & 1 & 2 & 2 & 2 & 4 \\
\hline 5 & 1 & 1 & 2 & 2 & 2 & 4 & 5 & 1 & 1 & 2 & 2 & 2 & 4 & 5 & 1 & 1 & 2 & 2 & 2 & 5 \\
\hline 6 & 1 & 2 & 1 & 4 & 5 & 6 & 6 & 1 & 2 & 2 & 4 & 5 & 6 & 6 & 1 & 2 & 3 & 5 & 5 & 6 \\
\hline
\end{tabular}

Proposition 15.1. The variety generated by any $S \in\left\{\mathscr{B}_{4}, \mathscr{B}_{5}, \mathscr{B}_{6}, \mathscr{B}_{9}, \mathscr{B}_{10}, \mathscr{E}_{1}\right\}$ is defined by the identities

$$
\begin{aligned}
& x^{2} \mathrm{H} x \mathrm{~K} x \approx x \mathrm{H} x \mathrm{~K} x, \quad x \mathrm{H} x^{2} \mathrm{~K} x \approx x \mathrm{H} x \mathrm{~K} x, \quad x \mathrm{H} x \mathrm{~K} x^{2} \approx x \mathrm{H} x \mathrm{~K} x, \\
& x \mathrm{H} y \mathrm{~K} x \mathrm{\top} y \approx y \mathrm{H} x \mathrm{~K} x \mathrm{\top} y, \quad x \mathrm{H} y \mathrm{~K} x \mathrm{\top} y \approx x \mathrm{H} x \mathrm{~K} y \mathrm{\top} y, \quad x \mathrm{H} y \mathrm{~K} x \mathrm{\top} y \approx x \mathrm{H} y \mathrm{~K} y \mathrm{\top} x, \\
& x^{2} h y \mathrm{~K} y \approx x h x y \mathrm{~K} y, \quad x^{2} \mathrm{H} y \mathrm{~K} y \approx x \mathrm{H} y \mathrm{~K} x y, \quad y \mathrm{H} x^{2} k y \approx y \mathrm{H} x k x y, \\
& x \mathrm{H} x k y^{2} \approx x \mathrm{H} x y k y, \quad x \mathrm{H} x \mathrm{~K} y^{2} \approx x y \mathrm{H} x \mathrm{~K} y, \quad x h y^{2} \mathrm{~K} x \approx x y h y \mathrm{~K} x, \\
& h x h y k \mathrm{~T} k \approx h y h x k \mathrm{~T} k, \quad k \mathrm{~T} k x h y h \approx k \mathrm{~T} k y h x h, \\
& h x h y h \approx h y h x h .
\end{aligned}
$$

The proof of Proposition 15.1 is given in $\S 15.4$.

15.1. Identities satisfied by $\mathscr{B}_{4}, \mathscr{B}_{5}, \mathscr{B}_{6}, \mathscr{B}_{9}, \mathscr{B}_{10}$, and $\mathscr{E}_{1}$

For any word $\mathbf{w}$, let $\operatorname{con}_{k}(\mathbf{w})$ denote the set of letters that occur exactly $k$ times in $\mathbf{w}$ :

$$
\operatorname{con}_{k}(\mathbf{w})=\{x \in \mathcal{X} \mid \operatorname{occ}(x, \mathbf{w})=k\}
$$

Let $\operatorname{con}_{3}^{\infty}(\mathbf{w})=\bigcup_{k \geqslant 3} \operatorname{con}_{k}(\mathbf{w})$. Note that $\operatorname{con}(\mathbf{w})=\operatorname{sim}(\mathbf{w}) \cup \operatorname{con}_{2}(\mathbf{w}) \cup \operatorname{con}_{3}^{\infty}(\mathbf{w})$ is a disjoint union.

Lemma 15.2. Let $S \in\left\{\mathscr{B}_{4}, \mathscr{B}_{5}, \mathscr{B}_{6}, \mathscr{B}_{9}, \mathscr{B}_{10}, \mathscr{E}_{1}\right\}$. Suppose that $\mathbf{w} \approx \mathbf{w}^{\prime}$ is any identity satisfied by $S$. Then:

(i) $\operatorname{sim}(\mathbf{w})=\operatorname{sim}\left(\mathbf{w}^{\prime}\right), \operatorname{con}_{2}(\mathbf{w})=\operatorname{con}_{2}\left(\mathbf{w}^{\prime}\right)$, and $\operatorname{con}_{3}^{\infty}(\mathbf{w})=\operatorname{con}_{3}^{\infty}\left(\mathbf{w}^{\prime}\right)$;

(ii) either (a) $\mathrm{t}(\mathbf{w}) \in \operatorname{sim}(\mathbf{w}), \mathrm{t}\left(\mathbf{w}^{\prime}\right) \in \operatorname{sim}\left(\mathbf{w}^{\prime}\right)$, and $\mathrm{t}(\mathbf{w})=\mathrm{t}\left(\mathbf{w}^{\prime}\right)$, or (b) $\mathrm{t}(\mathbf{w}) \notin \operatorname{sim}(\mathbf{w})$ and $\mathrm{t}\left(\mathbf{w}^{\prime}\right) \notin \operatorname{sim}\left(\mathbf{w}^{\prime}\right)$; 
(iii) either (a) $\mathrm{h}(\mathbf{w}) \in \operatorname{sim}(\mathbf{w}), \mathrm{h}\left(\mathbf{w}^{\prime}\right) \in \operatorname{sim}\left(\mathbf{w}^{\prime}\right)$, and $\mathrm{h}(\mathbf{w})=\mathrm{h}\left(\mathbf{w}^{\prime}\right)$, or (b) $\mathrm{h}(\mathbf{w}) \notin \operatorname{sim}(\mathbf{w})$ and $\mathrm{h}\left(\mathbf{w}^{\prime}\right) \notin \operatorname{sim}\left(\mathbf{w}^{\prime}\right)$;

(iv) $\mathrm{F}_{\mathrm{SS}}(\mathbf{w})=\mathrm{F}_{\mathrm{SS}}\left(\mathbf{w}^{\prime}\right)$.

Proof. (i) The subsemigroup $\{1,2,4,6\}$ of $S^{\prime} \in\left\{\mathscr{B}_{4}, \mathscr{B}_{5}, \mathscr{B}_{6}, \mathscr{B}_{9}, \mathscr{B}_{10}\right\}$ and the divisor $\{1,2,3,5,6\} /\{3,5\}$ of $\mathscr{E}_{1}$ are isomorphic to $N_{3}^{1}$. Hence the result follows from Lemma 2.1(vii).

(ii) The subsemigroups $\{1,5,6\} \subset \mathscr{B}_{4}$ and $\{1,5,6\} \subset \mathscr{B}_{5}$ and the quotients $\mathscr{B}_{6} /\{1,2,3,4\}$, $\mathscr{B}_{9} /\{1,2,3,4\}, \mathscr{B}_{10} /\{1,2,3,4\}$, and $\mathscr{E}_{1} /\{1,2,4,5\}$ are isomorphic to $J$. Therefore the result follows from Lemma 2.2 .

(iii) The subsemigroup $\{1,3,6\} \subset \mathscr{B}_{4}$ and the quotients $\mathscr{B}_{5} /\{1,2,4,5\}, \mathscr{B}_{6} /\{1,2,4,5\}$, $\mathscr{B}_{9} /\{1,2,4,5\}, \mathscr{B}_{10} /\{1,2,4,5\}$, and $\mathscr{E}_{1} /\{1,2,3,5\}$ are isomorphic to the dual semigroup of $J$. Therefore the result follows from the dual result of Lemma 2.2 .

(iv) The semigroup $S$ does not satisfy the identity (2.3) with $n=3$ because

$$
6^{3} \cdot 5 \cdot 6^{3} \cdot 3 \cdot 6^{3} \neq 6^{3} \cdot 5 \cdot 3 \cdot 6^{3} \quad \text { in } S^{\prime} \in\left\{\mathscr{B}_{4}, \mathscr{B}_{5}, \mathscr{B}_{6}, \mathscr{B}_{9}, \mathscr{B}_{10}\right\}
$$

and

$$
6^{3} \cdot 3 \cdot 6^{3} \cdot 4 \cdot 6^{3} \neq 6^{3} \cdot 3 \cdot 4 \cdot 6^{3} \quad \text { in } \mathscr{E}_{1} .
$$

Therefore $\mathrm{F}_{\mathrm{SS}}(\mathbf{w})=\mathrm{F}_{\mathrm{SS}}\left(\mathbf{w}^{\prime}\right)$ by Lemma 2.10 and part (i).

\section{2. $\alpha$-canonical form}

Let $\mathbf{w}$ be any word with $\operatorname{con}_{2}(\mathbf{w})=\left\{x_{1}, \ldots, x_{m}\right\}$ for some $m \geqslant 1$ and $\operatorname{con}_{3}^{\infty}(\mathbf{w})=\emptyset$. Suppose that the letters $x_{1}, \ldots, x_{m}$ are in alphabetical order. In this section, such a word $\mathbf{w}$ is said to be in $\alpha$-canonical form if

$$
\begin{aligned}
\mathbf{w} & =\mathbf{p}\left(\prod_{i=1}^{r}\left(x_{i} \mathbf{s}_{i} x_{i} \mathbf{t}_{i}\right)\right)\left(\prod_{i=r+1}^{m} x_{i}^{2}\right) \mathbf{q} \\
& =\mathbf{p} \cdot x_{1} \mathbf{s}_{1} x_{1} \mathbf{t}_{1} \ldots x_{r} \mathbf{s}_{r} x_{r} \mathbf{t}_{r} \cdot x_{r+1}^{2} \ldots x_{m}^{2} \cdot \mathbf{q}
\end{aligned}
$$

where all of the following are satisfied:

(I) $r \in\{0, \ldots, m\}$;

(II) the letters of $\mathbf{p}, \mathbf{q}, \mathbf{t}_{r} \in \mathcal{X}^{*}$ and $\mathbf{s}_{1}, \ldots, \mathbf{s}_{r}, \mathbf{t}_{1}, \ldots, \mathbf{t}_{r-1} \in \mathcal{X}^{+}$are simple in $\mathbf{w}$;

(III) if $r=m$, then $\mathbf{q}=\emptyset$.

Note the extreme cases:

$$
\mathbf{w}= \begin{cases}\mathbf{p} \cdot x_{1}^{2} \ldots x_{m}^{2} \cdot \mathbf{q} & \text { if } r=0 \\ \mathbf{p} \cdot x_{1} \mathbf{s}_{1} x_{1} \mathbf{t}_{1} \ldots x_{m} \mathbf{s}_{m} x_{m} \mathbf{t}_{m} & \text { if } r=m\end{cases}
$$

Lemma 15.3. Let $\mathbf{w}$ be any non-simple word such that $\operatorname{con}_{3}^{\infty}(\mathbf{w})=\emptyset$. Then there exists some word $\overline{\mathbf{w}}$ in $\alpha$-canonical form such that the identities (15.1) imply the identity $\mathbf{w} \approx \overline{\mathbf{w}}$.

Proof. It suffices to convert $\mathbf{w}$, using the identities (15.1), into a word in $\alpha$-canonical form. Since $\mathbf{w}$ is non-simple, generality is not lost by assuming $\operatorname{con}_{2}(\mathbf{w})=\left\{x_{1}, \ldots, x_{m}\right\}$ with $x_{1}, \ldots, x_{m}$ in alphabetical order. The identities (15.1b) can be used to interchange any two occurrences of non-simple letters in $\mathbf{w}$. In particular, the identities (15.1b) can be used to arrange the non-simple letters of $\mathbf{w}$ so that they occur in alphabetical order, that is,

$$
\mathbf{w} \stackrel{(15.1 \mathrm{~b})}{\approx} \underbrace{\left(\ldots x_{1} \ldots x_{1} \ldots\right)\left(\ldots x_{2} \ldots x_{2} \ldots\right) \ldots\left(\ldots x_{m} \ldots x_{m} \ldots\right)}_{\mathbf{w}^{\prime}} .
$$


Consider the factorization of $\mathbf{w}^{\prime}$ that displays all occurrences of $x_{i}$ and $x_{i+1}$ individually:

$$
\mathbf{w}^{\prime}=\mathbf{a} x_{i} \mathbf{b} x_{i} \mathbf{c} x_{i+1} \mathbf{d} x_{i+1} \mathbf{e}
$$

where $\mathbf{a}, \mathbf{b}, \mathbf{c}, \mathbf{d}, \mathbf{e} \in \mathcal{X}^{*}$. There are two cases.

Case 1: $\mathbf{b}=\emptyset \neq \mathbf{c}$, that is, the two occurrences of $x_{i}$ are adjacent but the second $x_{i}$ is not adjacent with the first $x_{i+1}$. Then the two occurrences of $x_{i}$ can be separated by the identities (15.1c):

$$
\mathbf{w}^{\prime}=\mathbf{a} x_{i}^{2} \mathbf{c} x_{i+1} \mathbf{d} x_{i+1} \mathbf{e} \stackrel{(15.1 \mathrm{c})}{\approx} \mathbf{a} x_{i} \mathbf{c} x_{i} x_{i+1} \mathbf{d} x_{i+1} \mathbf{e} .
$$

Case 2: $\mathbf{c}=\emptyset \neq \mathbf{d}$, that is, the second $x_{i}$ is adjacent with the first $x_{i+1}$ but the two occurrences of $x_{i+1}$ are not adjacent. Then the second $x_{i}$ and the first $x_{i+1}$ can be separated by the identities (15.1d):

$$
\mathbf{w}^{\prime}=\mathbf{a} x_{i} \mathbf{b} x_{i} x_{i+1} \mathbf{d} x_{i+1} \mathbf{e} \stackrel{(15.1 \mathrm{~d})}{\approx} \mathbf{a} x_{i} \mathbf{b} x_{i} \mathbf{d} x_{i+1}^{2} \mathbf{e} .
$$

It is easily seen how the procedures in Cases 1 and 2 can be repeated until $\mathbf{w}^{\prime}$ is converted by the identities $\{(15.1 \mathrm{c}),(15.1 \mathrm{~d})\}$ into a word in $\alpha$-canonical form.

\section{3. $\beta$-canonical form}

Let $\mathbf{w}$ be any word with $\operatorname{con}_{2}(\mathbf{w})=\left\{x_{1}, \ldots, x_{m}\right\}$ for some $m \geqslant 0$ and $\operatorname{con}_{3}^{\infty}(\mathbf{w})=\left\{y_{1}, \ldots, y_{n}\right\}$ for some $n \geqslant 1$. Suppose that the letters $x_{1}, \ldots, x_{m}$ are in alphabetical order and the letters $y_{1}, \ldots, y_{n}$ are in alphabetical order. In this section, such a word $\mathbf{w}$ is said to be in $\beta$-canonical form if

where all of the following are satisfied:

$$
\mathbf{w}=\mathbf{p x} \prod_{i=1}^{r}\left(\mathbf{y} \mathbf{s}_{i}\right)=\mathbf{p x} \cdot \mathbf{y s}_{1} \ldots \mathbf{y} \mathbf{s}_{r},
$$

(I) $r \geqslant 1$;

(II) the letters of $\mathbf{p}, \mathbf{s}_{r} \in \mathcal{X}^{*}$ and $\mathbf{s}_{1}, \ldots, \mathbf{s}_{r-1} \in \mathcal{X}^{+}$are simple in $\mathbf{w}$;

(III) $\mathbf{x}=x_{1}^{2} \ldots x_{m}^{2}$;

(IV) $\mathbf{y}=y_{1}^{3} \ldots y_{n}^{3}$.

Note that $\mathbf{x}$ is empty if $m=0$, but $\mathbf{y}$ is nonempty because $\operatorname{con}_{3}^{\infty}(\mathbf{w}) \neq \emptyset$.

Lemma 15.4. Let $\mathbf{w}$ be any non-simple word such that $\operatorname{con}_{3}^{\infty}(\mathbf{w}) \neq \emptyset$. Then there exists some word $\mathbf{w}$ in $\beta$-canonical form such that the identities (15.1) imply the identity $\mathbf{w} \approx \overline{\mathbf{w}}$.

Proof. It suffices to convert $\mathbf{w}$, using the identities (15.1), into a word in $\beta$-canonical form. Since $\mathbf{w}$ is non-simple, generality is not lost by assuming $\operatorname{con}_{2}(\mathbf{w})=\left\{x_{1}, \ldots, x_{m}\right\}$ with $x_{1}, \ldots, x_{m}$ in alphabetical order, and $\operatorname{con}_{3}^{\infty}(\mathbf{w})=\left\{y_{1}, \ldots, y_{n}\right\}$ with $y_{1}, \ldots, y_{n}$ in alphabetical order. Then $\mathbf{w}$ can be written in the form

$$
\mathbf{w}=\mathbf{p w}_{1} \mathbf{s}_{1} \ldots \mathbf{w}_{r} \mathbf{s}_{r},
$$

where (I) and (II) are satisfied and the letters of $\mathbf{w}_{1}, \ldots, \mathbf{w}_{r} \in \mathcal{X}^{+}$are all non-simple in $\mathbf{w}$. Let $k \in\{1, \ldots, n\}$. By assumption, $y_{k} \in \operatorname{con}\left(\mathbf{w}_{i}\right)$ for some $i$. Suppose $y_{k} \notin \operatorname{con}\left(\mathbf{w}_{i+1}\right)$. Then

$$
\begin{array}{rll}
\mathbf{w} & \ldots \overbrace{\mathbf{a} y_{k} \mathbf{b}} \mathbf{s}_{i} \cdot \mathbf{w}_{i+1} \mathbf{s}_{i+1} \ldots & \text { for some } \mathbf{a}, \mathbf{b} \in\left\{x_{1}, \ldots, x_{m}, y_{1}, \ldots, y_{n}\right\}^{*} \\
\stackrel{\mathbf{w}_{i}}{\stackrel{(15.1 \mathrm{a})}{\approx}} \ldots \mathbf{a} y_{k}^{2} \mathbf{b} \mathbf{s}_{i} \cdot \mathbf{w}_{i+1} \mathbf{s}_{i+1} \ldots & \text { since occ }\left(y_{k}, \mathbf{w}\right) \geqslant 3 \\
\stackrel{(15.1 \mathrm{c})}{\approx} \ldots \mathbf{a} y_{k} \mathbf{b} \mathbf{b s}_{i} \cdot y_{k} \mathbf{w}_{i+1} \mathbf{s}_{i+1} \ldots & \text { since } \mathrm{h}\left(\mathbf{w}_{i+1}\right) \text { is non-simple in } \mathbf{w} \\
=\ldots \mathbf{w}_{i} \mathbf{s}_{i} \cdot\left(y_{k} \mathbf{w}_{i+1}\right) \mathbf{s}_{i+1} \ldots, &
\end{array}
$$


that is, $\mathbf{w}_{i+1}$ is converted by the identities $\{(15.1 \mathrm{a}),(15.1 \mathrm{c})\}$ into $y_{k} \mathbf{w}_{i+1}$. If $y_{k} \notin \operatorname{con}\left(\mathbf{w}_{i-1}\right)$, then by a symmetrical argument, $\mathbf{w}_{i-1}$ can be converted by the identities $\{(15.1 \mathrm{a}),(15.1 \mathrm{~d})\}$ into $\mathbf{w}_{i-1} y_{k}$. Since $k$ is arbitrary in $\{1, \ldots, n\}$, it is easily seen how this procedure can be repeated until each $\mathbf{w}_{i}$ is converted into a word that contains all the letters $y_{1}, \ldots, y_{n}$. Now for each $i$, the letters of $\mathbf{w}_{i}$ are non-simple in $\mathbf{w}$ and so can be arranged by the identities (15.1b) in any manner. Hence each $\mathbf{w}_{i}$ can be converted by the identities (15.1b) into a word of the form $\mathbf{x}_{i} y_{1}^{e_{1}} \ldots y_{n}^{e_{n}}$ with $e_{1}, \ldots, e_{n} \geqslant 1$ and $\mathbf{x}_{i} \in\left\{x_{1}, \ldots, x_{m}\right\}^{*}$. Since $\operatorname{occ}\left(y_{1}, \mathbf{w}\right), \ldots, \operatorname{occ}\left(y_{n}, \mathbf{w}\right) \geqslant 3$, the identities (15.1a) can be used to replace each exponent $e_{i}$ by 3 . Hence the identities (15.1) can be used to convert $\mathbf{w}$ into the word

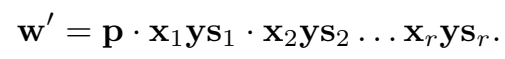

If the factor $\mathbf{x}_{2}$ of $\mathbf{w}^{\prime}$ is nonempty, then it can be moved to the left by the identities (15.1) until it immediately follows the factor $\mathbf{x}_{1}$ :

$$
\begin{aligned}
& \mathbf{w}^{\prime}=\mathbf{p} \cdot \mathbf{x}_{1} \mathbf{y s}_{1} \cdot \overbrace{z_{1} \ldots z_{p}}^{\mathbf{x}_{2}} \mathbf{y s}_{2} \ldots \quad \text { for some } z_{1}, \ldots, z_{p} \in\left\{x_{1}, \ldots, x_{m}\right\} \\
& \stackrel{(15.1 \mathrm{a})}{\approx} \mathbf{p} \cdot \mathbf{x}_{1} y_{1}^{p} \mathbf{y s}_{1} \cdot z_{1} \ldots z_{p} \mathbf{y} \mathbf{s}_{2} \ldots \quad \text { since } y_{1}=\mathrm{h}(\mathbf{y}) \text { and } \operatorname{occ}\left(y_{1}, \mathbf{w}^{\prime}\right) \geqslant 3 \\
& \stackrel{(15.1 \mathrm{~b})}{\approx} \mathbf{p} \cdot \mathbf{x}_{1} z_{1} \ldots z_{p} \mathbf{\mathbf { y s } _ { 1 }} \cdot y_{1}^{p} \mathbf{y s}_{2} \ldots \quad \text { since } y_{1}, z_{1}, \ldots, z_{p} \text { are non-simple in } \mathbf{w}^{\prime} \\
& \stackrel{(15.1 \mathrm{a})}{\approx} \mathbf{p} \cdot \mathbf{x}_{1} \mathbf{x}_{2} \mathbf{y} \mathbf{s}_{1} \cdot \mathbf{y} \mathbf{s}_{2} \ldots
\end{aligned}
$$

By the same argument, if the factor $\mathbf{x}_{3}$ is nonempty, then it can be moved to the left by the identities (15.1) until it immediately follows the factor $\mathbf{x}_{1} \mathbf{x}_{2}$. Continuing in this manner, $\mathbf{w}^{\prime}$ can be converted by the identities (15.1) into the word

$$
\mathbf{w}^{\prime \prime}=\mathbf{p}\left(\mathbf{x}_{1} \mathbf{x}_{2} \ldots \mathbf{x}_{r}\right) \cdot \mathbf{y s}_{1} \cdot \mathbf{y s}_{2} \ldots \mathbf{y} \mathbf{s}_{r} .
$$

It is easily seen that when converting $\mathbf{w}$ into $\mathbf{w}^{\prime \prime}$, the number of occurrences of each $x_{i}$ remained unchanged throughout, that is, $\operatorname{occ}\left(x_{i}, \mathbf{x}_{1} \mathbf{x}_{2} \ldots \mathbf{x}_{r}\right)=\operatorname{occ}\left(x_{i}, \mathbf{w}\right)=2$. Therefore if the letters of the factor $\mathbf{x}_{1} \mathbf{x}_{2} \ldots \mathbf{x}_{r}$ are alphabetically ordered by the identities (15.1b), then it becomes $\mathbf{x}=x_{1}^{2} \ldots x_{m}^{2}$. The resulting word is in $\beta$-canonical form.

\subsection{Proof of Proposition 15.1}

Let $S \in\left\{\mathscr{B}_{4}, \mathscr{B}_{5}, \mathscr{B}_{6}, \mathscr{B}_{9}, \mathscr{B}_{10}, \mathscr{E}_{1}\right\}$. It is routinely checked that $S$ satisfies the identities (15.1). Hence it suffices to show that any identity $\mathbf{w} \approx \mathbf{w}^{\prime}$ satisfied by $S$ is implied by the identities (15.1). If either $\mathbf{w}$ or $\mathbf{w}^{\prime}$ is a simple word, then it follows from Lemma 15.2 parts (i) and (iv) that the identity $\mathbf{w} \approx \mathbf{w}^{\prime}$ is trivial and so is vacuously implied by the identities (15.1). Therefore assume that $\mathbf{w}$ and $\mathbf{w}^{\prime}$ are both non-simple words. By Lemma 15.2(i), it can further be assumed that:

(a) $\operatorname{con}_{2}(\mathbf{w})=\operatorname{con}_{2}\left(\mathbf{w}^{\prime}\right)=\left\{x_{1}, \ldots, x_{m}\right\}$ where $x_{1}, \ldots, x_{m}$ are in alphabetical order;

(b) $\operatorname{con}_{3}^{\infty}(\mathbf{w})=\operatorname{con}_{3}^{\infty}\left(\mathbf{w}^{\prime}\right)=\left\{y_{1}, \ldots, y_{n}\right\}$ where $y_{1}, \ldots, y_{n}$ are in alphabetical order.

There are two cases.

Case 1: $\operatorname{con}_{3}^{\infty}(\mathbf{w})=\operatorname{con}_{3}^{\infty}\left(\mathbf{w}^{\prime}\right)=\emptyset$. Then by Lemma 15.3, the words $\mathbf{w}$ and $\mathbf{w}^{\prime}$ can be chosen to be in $\alpha$-canonical form, whence by (a),

$$
\mathbf{w}=\mathbf{p} \cdot x_{1} \mathbf{s}_{1} x_{1} \mathbf{t}_{1} \ldots x_{r} \mathbf{s}_{r} x_{r} \mathbf{t}_{r} \cdot x_{r+1}^{2} \ldots x_{m}^{2} \cdot \mathbf{q}
$$

and

$$
\mathbf{w}^{\prime}=\mathbf{p}^{\prime} \cdot x_{1} \mathbf{s}_{1}^{\prime} x_{1} \mathbf{t}_{1}^{\prime} \ldots x_{r^{\prime}} \mathbf{s}_{r^{\prime}}^{\prime} x_{r^{\prime}} \mathbf{t}_{r^{\prime}}^{\prime} \cdot x_{r^{\prime}+1}^{2} \ldots x_{m}^{2} \cdot \mathbf{q}^{\prime}
$$


Therefore $\mathbf{p}=\mathbf{p}^{\prime}$ and $\mathbf{q}=\mathbf{q}^{\prime}$ by parts (ii)-(iv) of Lemma 15.2, and

(c) $\left\{\mathbf{s}_{1}, \mathbf{t}_{1}, \ldots, \mathbf{s}_{r}, \mathbf{t}_{r}\right\}=\left\{\mathbf{s}_{1}^{\prime}, \mathbf{t}_{1}^{\prime}, \ldots, \mathbf{s}_{r^{\prime}}^{\prime}, \mathbf{t}_{r^{\prime}}^{\prime}\right\}$

by part (iv) of the same lemma, whence $r=r^{\prime}$. Consequently,

$$
\mathbf{w}^{\prime}=\mathbf{p} \cdot x_{1} \mathbf{s}_{1}^{\prime} x_{1} \mathbf{t}_{1}^{\prime} \ldots x_{r} \mathbf{s}_{r}^{\prime} x_{r} \mathbf{t}_{r}^{\prime} \cdot x_{r+1}^{2} \ldots x_{m}^{2} \cdot \mathbf{q} .
$$

It now follows from (c) that $\mathbf{w}^{\prime}$ can be converted by the identities (15.1e) into $\mathbf{w}$.

Case 2: $\operatorname{con}_{3}^{\infty}(\mathbf{w})=\operatorname{con}_{3}^{\infty}\left(\mathbf{w}^{\prime}\right) \neq \emptyset$. Then by Lemma 15.4 , the words $\mathbf{w}$ and $\mathbf{w}^{\prime}$ can be chosen to be in $\beta$-canonical form, whence by (a) and (b),

$$
\mathbf{w}=\mathbf{p x} \cdot \mathbf{y} \mathbf{s}_{1} \ldots \mathbf{y} \mathbf{s}_{r-1} \cdot \mathbf{y} \mathbf{s}_{r} \quad \text { and } \quad \mathbf{w}^{\prime}=\mathbf{p}^{\prime} \mathbf{x} \cdot \mathbf{y s}_{1}^{\prime} \ldots \mathbf{y} \mathbf{s}_{r^{\prime}-1}^{\prime} \cdot \mathbf{y} \mathbf{s}_{r^{\prime}}^{\prime}
$$

where $\mathbf{x}=x_{1}^{2} \ldots x_{m}^{2}$ and $\mathbf{y}=y_{1}^{3} \ldots y_{n}^{3}$. Therefore $\mathbf{p}=\mathbf{p}^{\prime}$ and $\mathbf{s}_{r}=\mathbf{s}_{r^{\prime}}^{\prime}$ by parts (ii)-(iv) of Lemma 15.2, and

(d) $\left\{\mathbf{s}_{1}, \ldots, \mathbf{s}_{r-1}\right\}=\left\{\mathbf{s}_{1}^{\prime}, \ldots, \mathbf{s}_{r^{\prime}-1}^{\prime}\right\}$

by part (iv) of the same lemma, whence $r=r^{\prime}$. Consequently,

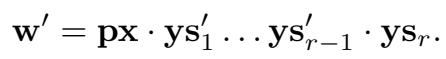

It now follows from (d) that $\mathbf{w}^{\prime}$ can be converted by the identity (15.1f) into $\mathbf{w}$.

$$
\text { 16. } \mathscr{C}_{1}, \mathscr{C}_{2}, \mathscr{C}_{3}, \mathscr{C}_{4}, \mathscr{D}_{3}, \mathscr{D}_{5}
$$

This section establishes the finite basis property of the following semigroups.

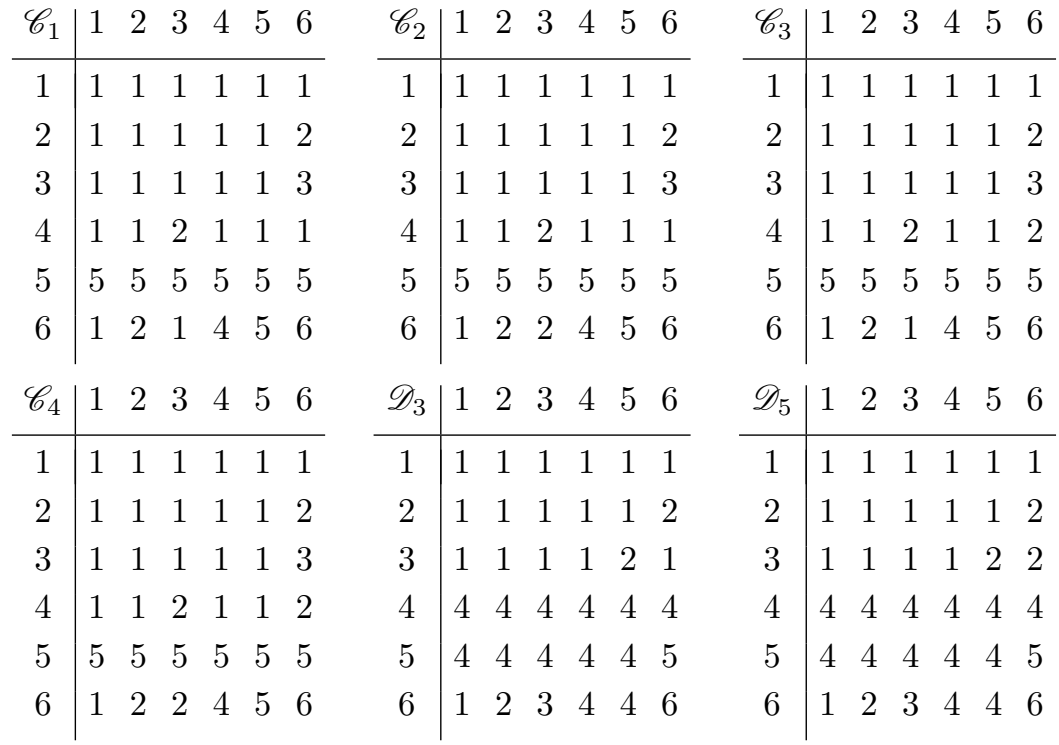

Proposition 16.1. The variety generated by any $S \in\left\{\mathscr{C}_{1}, \mathscr{C}_{2}, \mathscr{C}_{3}, \mathscr{C}_{4}, \mathscr{D}_{3}, \mathscr{D}_{5}\right\}$ is defined by the identities

$$
\begin{aligned}
x^{3} \approx x^{2}, \quad x^{2} y x & \approx x y x, \quad x y x^{2} \approx x y x, \\
x y x z^{2} & \approx x^{2} y z^{2}, \\
x^{2} \mathrm{H} y^{2} & \approx x \mathrm{H} y^{2} x, \\
x \mathrm{H} y \mathrm{~K} x y & \approx x \mathrm{H} y \mathrm{~K} y x .
\end{aligned}
$$

The proof of Proposition 16.1 is given in $\S 16.3$. 
16.1. Identities satisfied by $\mathscr{C}_{1}, \mathscr{C}_{2}, \mathscr{C}_{3}, \mathscr{C}_{4}, \mathscr{D}_{3}$, and $\mathscr{D}_{5}$

Lemma 16.2. Let $S \in\left\{\mathscr{C}_{1}, \mathscr{C}_{2}, \mathscr{C}_{3}, \mathscr{C}_{4}, \mathscr{D}_{3}, \mathscr{D}_{5}\right\}$. Suppose that $\mathbf{w} \approx \mathbf{w}^{\prime}$ is any identity satisfied by $S$. Then:

(i) $\operatorname{ini}(\mathbf{w})=\operatorname{ini}\left(\mathbf{w}^{\prime}\right)$;

(ii) $\operatorname{con}(\mathbf{w})=\operatorname{con}\left(\mathbf{w}^{\prime}\right)$ and $\operatorname{sim}(\mathbf{w})=\operatorname{sim}\left(\mathbf{w}^{\prime}\right)$;

(iii) $\mathrm{t}(\mathbf{w}) \in \operatorname{sim}(\mathbf{w})$ if and only if $\mathrm{t}\left(\mathbf{w}^{\prime}\right) \in \operatorname{sim}\left(\mathbf{w}^{\prime}\right)$;

(iv) $\mathrm{F}_{\mathrm{SS}}(\mathbf{w})=\mathrm{F}_{\mathrm{SS}}\left(\mathbf{w}^{\prime}\right)$.

Proof. (i) The subsemigroup $\{1,5,6\}$ of $S^{\prime} \in\left\{\mathscr{C}_{1}, \mathscr{C}_{2}, \mathscr{C}_{3}, \mathscr{C}_{4}\right\}$ and the subsemigroup $\{1,4,6\}$ of $S^{\prime \prime} \in\left\{\mathscr{D}_{3}, \mathscr{D}_{5}\right\}$ are isomorphic to $L_{2}^{1}$. Therefore the result follows from Lemma 2.1(iii).

(ii) This follows from Lemma 2.1(v) since the subsemigroup $\{1,2,6\}$ of $S$ is isomorphic to $N_{2}^{1}$.

(iii) This follows from Lemma 2.2 since the subsemigroup $\{1,4,6\}$ of $S^{\prime} \in\left\{\mathscr{C}_{1}, \mathscr{C}_{2}\right\}$, the divisor $\{1,2,4,6\} /\{1,2\}$ of $S^{\prime \prime} \in\left\{\mathscr{C}_{3}, \mathscr{C}_{4}\right\}$, the subsemigroup $\{1,3,6\}$ of $\mathscr{D}_{3}$, and the divisor $\{1,2,3,6\} /\{1,2\}$ of $\mathscr{D}_{5}$ are isomorphic to $J$.

(iv) The semigroup $S$ does not satisfy the identity (2.3) with $n=2$ because

$$
6^{2} \cdot 4 \cdot 6^{2} \cdot 3 \cdot 6^{2} \neq 6^{2} \cdot 4 \cdot 3 \cdot 6^{2} \quad \text { in } S^{\prime} \in\left\{\mathscr{C}_{1}, \mathscr{C}_{2}, \mathscr{C}_{3}, \mathscr{C}_{4}\right\}
$$

and

$$
6^{2} \cdot 3 \cdot 6^{2} \cdot 5 \cdot 6^{2} \neq 6^{2} \cdot 3 \cdot 5 \cdot 6^{2} \quad \text { in } S^{\prime \prime} \in\left\{\mathscr{D}_{3}, \mathscr{D}_{4}\right\} .
$$

Therefore $F_{\mathrm{SS}}(\mathbf{w})=\mathrm{F}_{\mathrm{SS}}\left(\mathbf{w}^{\prime}\right)$ by part (ii) and Lemma 2.10 .

\subsection{A canonical form}

Let $\mathbf{w}$ be any non-simple word and let $x_{0}$ be the first non-simple letter of $\mathbf{w}$. Then there exist $\mathbf{p} \in \mathcal{X}^{*}$ and $\mathbf{q} \in \mathcal{X}^{+}$such that

$$
\mathbf{w}=\mathbf{p} x_{0} \mathbf{q}
$$

where the prefix $\mathbf{p}$, if nonempty, consists of simple letters of $\mathbf{w}$. Note that the suffix $\mathbf{q}$ is nonempty since it contains all non-first occurrences of $x_{0}$. In this section, such a non-simple word $\mathbf{w}$ is said to be in canonical form if

$$
\mathbf{w}=\mathbf{p} x_{0}^{2} \prod_{i=1}^{m}\left(x_{i}^{e_{i}} x_{0}^{f_{i}}\right)=\mathbf{p} x_{0}^{2} \cdot x_{1}^{e_{1}} x_{0}^{f_{1}} \ldots x_{m}^{e_{m}} x_{0}^{f_{m}},
$$

where all of the following are satisfied:

(I) the letters $x_{0}, x_{1}, \ldots, x_{m}$ are all distinct;

(II) $e_{1}, \ldots, e_{m} \in\{1,2\}$;

(III) $f_{1}, \ldots, f_{m} \in\{0,2\}$;

(IV) if $f_{i}=2$, then $e_{i}=e_{i+1}=1$.

Note that $\operatorname{occ}\left(x_{i}, \mathbf{w}\right)=e_{i}$ for each $i \in\{1, \ldots, m\}$. Therefore

(V) for each $i \in\{1, \ldots, m\}$, the letter $x_{i}$ is simple in $\mathbf{w}$ if and only if $e_{i}=1$.

Lemma 16.3. Let $\mathbf{w}$ be any non-simple word. Then there exists some word $\overline{\mathbf{w}}$ in canonical form such that the identities (16.1) imply the identity $\mathbf{w} \approx \overline{\mathbf{w}}$.

Proof. It suffices to convert $\mathbf{w}$ in (16.2), using the identities (16.1), into a word in canonical form. Suppose ini $\left(x_{0} \mathbf{q}\right)=x_{0} x_{1} \ldots x_{m}$ for some $m \geqslant 0$. Then $\mathbf{w}$ can be written in the form

$$
\mathbf{w}=\mathbf{p} x_{0}^{r} \prod_{i=1}^{m}\left(x_{i} \mathbf{w}_{i}\right)=\mathbf{p} x_{0}^{r} \cdot x_{1} \mathbf{w}_{1} \ldots x_{m} \mathbf{w}_{m},
$$


where $r \geqslant 1$ and $\mathbf{w}_{i} \in\left\{x_{0}, \ldots, x_{i}\right\}^{*}$. Since the letter $x_{0}$ is non-simple in $\mathbf{w}$, the identities (16.1a) can be used to replace the exponent $r$ by 2 . For each $i$, since the letters of $\mathbf{w}_{i}$ are non-first occurrences, the identities (16.1d) can be used to arrange them, within $\mathbf{w}_{i}$, in any order. Specifically, any occurrence of $x_{i}$ in $\mathbf{w}_{i}$ can be moved to the left and grouped with the $x_{i}$ in $\mathbf{w}$ that immediately precedes $\mathbf{w}_{i}$. Hence

$$
\mathbf{w} \stackrel{(16.1 \mathrm{~d})}{\approx} \underbrace{\mathbf{p} x_{0}^{2} \cdot x_{1}^{e_{1}} \mathbf{w}_{1}^{\prime} \ldots x_{m}^{e_{m}} \mathbf{w}_{m}^{\prime}}_{\mathbf{w}^{\prime}}
$$

for some $e_{i} \geqslant 1$ and $\mathbf{w}_{i}^{\prime} \in\left\{x_{0}, \ldots, x_{i-1}\right\}^{*}$.

Consider an arbitrary $k \in\{1, \ldots, m\}$ such that $\mathbf{w}_{k}^{\prime} \neq \emptyset$. Then $\mathbf{w}_{k}^{\prime}=y_{1} \ldots y_{n}$ for some $y_{1}, \ldots, y_{n} \in\left\{x_{0}, \ldots, x_{k-1}\right\}$. Write

$$
\mathbf{w}^{\prime}=\mathbf{p} x_{0}^{2} \cdot \mathbf{a} \cdot x_{k}^{e_{k}} \mathbf{w}_{k}^{\prime} \cdot \mathbf{b}
$$

where $\mathbf{a}=\prod_{i=1}^{k-1}\left(x_{i}^{e_{i}} \mathbf{w}_{i}^{\prime}\right)$ and $\mathbf{b}=\prod_{i=k+1}^{m}\left(x_{i}^{e_{i}} \mathbf{w}_{i}^{\prime}\right)$. Since $y_{n}$ is non-simple in $\mathbf{w}^{\prime}$,

$$
\begin{aligned}
& \mathbf{w}^{\prime} \stackrel{(16.1 \mathrm{a})}{\approx} \mathbf{p} x_{0}^{2} \cdot \mathbf{a} \cdot x_{k}^{e_{k}} y_{1} \ldots y_{n-1} y_{n}^{2} \cdot \mathbf{b} \\
& \stackrel{(16.1 \mathrm{c})}{\approx} \mathbf{p} x_{0} \cdot \mathbf{a} \cdot x_{k}^{e_{k}} y_{1} \ldots y_{n-1} y_{n}^{2} x_{0} \cdot \mathbf{b} \\
& \stackrel{(16.1 \mathrm{a}))}{\mathbf{p} x_{0}^{2} \cdot \mathbf{a} \cdot x_{k}^{e_{k}} y_{1} \ldots y_{n-1} y_{n} x_{0}^{2} \cdot \mathbf{b}} .
\end{aligned} \underbrace{}_{\mathbf{w}^{\prime \prime}} .
$$

If the letter $y_{n}$ in $\mathbf{w}^{\prime \prime}$ is $x_{0}$, then it can be eliminated by the first identity from (16.1a). If the letter $y_{n}$ in $\mathbf{w}^{\prime \prime}$ is not $x_{0}$, then it is one of $x_{1}, \ldots, x_{k-1}$ and hence can be gathered, using the identity (16.1b), with one of the factors $x_{1}^{e_{1}}, \ldots, x_{k-1}^{e_{k-1}}$ in $\mathbf{a}$. In any case, the letter $y_{n}$ in $\mathbf{w}^{\prime \prime}$ is eliminated from $\mathbf{w}_{k}^{\prime}$. Repeat the same argument to eliminate the letters $y_{n-1}, y_{n-2}, \ldots, y_{1}$ from $\mathbf{w}_{k}^{\prime}$. Hence

$$
\mathbf{w}^{\prime \prime} \stackrel{(16.1)}{\approx} \mathbf{p} x_{0}^{2} \cdot \mathbf{a} \cdot x_{k}^{e_{k}} x_{0}^{2} \cdot \mathbf{b}
$$

that is, the factor $\mathbf{w}_{k}^{\prime}$ of $\mathbf{w}$ is converted into $x_{0}^{2}$. Since $k \in\{1, \ldots, m\}$ is arbitrary, the same argument can be repeated on any $\mathbf{w}_{i}^{\prime}$ that is nonempty. Therefore $\mathbf{w}$ can be converted by the identities (16.1) into the word (16.3) with (I) and (III) satisfied and $e_{1}, \ldots, e_{m} \geqslant 1$. If $e_{i} \geqslant 3$, then the identities (16.1a) can be used to reduce $e_{i}$ to 2 . Hence (II) is satisfied.

If $e_{i}=2$ and $f_{i}=2$, then

$$
\begin{gathered}
\mathbf{w}=\mathbf{p} x_{0}^{2} \ldots x_{i}^{e_{i-1}} x_{0}^{f_{i-1}} \cdot x_{i}^{2} x_{0}^{2} \cdot x_{i+1}^{e_{i+1}} x_{0}^{f_{i+1}} \ldots \\
\stackrel{(16.1 \mathrm{c})}{\approx} \mathbf{p} x_{0}^{4} \ldots x_{i}^{e_{i-1}} x_{0}^{f_{i-1}} \cdot x_{i}^{2} \cdot x_{i+1}^{e_{i+1}} x_{0}^{f_{i+1}} \ldots \\
\stackrel{(16.1 \mathrm{a})}{\approx} \mathbf{p} x_{0}^{2} \ldots x_{i}^{e_{i-1}} x_{0}^{f_{i-1}} \cdot x_{i}^{2} \cdot x_{i+1}^{e_{i+1}} x_{0}^{f_{i+1}} \ldots,
\end{gathered}
$$

so that $f_{i}$ is reduced to 0 . Similarly, if $e_{i+1}=2$ and $f_{i}=2$, then

$$
\begin{gathered}
\mathbf{w}=\mathbf{p} x_{0}^{2} \ldots x_{i}^{e_{i-1}} x_{0}^{f_{i-1}} \cdot x_{i}^{e_{i}} x_{0}^{2} \cdot x_{i+1}^{2} x_{0}^{f_{i+1}} \ldots \\
\stackrel{(16.1 \mathrm{~b})}{\approx} \mathbf{p} x_{0}^{4} \ldots x_{i}^{e_{i-1}} x_{0}^{f_{i-1}} \cdot x_{i}^{e_{i}} \cdot x_{i+1}^{2} x_{0}^{f_{i+1}} \ldots \\
\stackrel{(16.1 \mathrm{a})}{\approx} \mathbf{p} x_{0}^{2} \ldots x_{i}^{e_{i-1}} x_{0}^{f_{i-1}} \cdot x_{i}^{e_{i}} \cdot x_{i+1}^{2} x_{0}^{f_{i+1}} \ldots,
\end{gathered}
$$

so that $f_{i}$ is reduced to 0 . Consequently, (IV) is satisfied. 


\subsection{Proof of Proposition 16.1}

Let $S \in\left\{\mathscr{C}_{1}, \mathscr{C}_{2}, \mathscr{C}_{3}, \mathscr{C}_{4}, \mathscr{D}_{3}, \mathscr{D}_{5}\right\}$. It is routinely checked that $S$ satisfies the identities (16.1). Hence it suffices to show that any identity $\mathbf{w} \approx \mathbf{w}^{\prime}$ satisfied by $S$ is implied by the identities (16.1). Note that $\operatorname{ini}(\mathbf{w})=\operatorname{ini}\left(\mathbf{w}^{\prime}\right)$ and $\operatorname{sim}(\mathbf{w})=\operatorname{sim}\left(\mathbf{w}^{\prime}\right)$ by Lemma 16.2 parts (i) and (ii). Therefore if either $\mathbf{w}$ or $\mathbf{w}^{\prime}$ is a simple word, then the identity $\mathbf{w} \approx \mathbf{w}^{\prime}$ is trivial and so is vacuously implied by the identities (16.1). Hence assume that $\mathbf{w}$ and $\mathbf{w}^{\prime}$ are both non-simple words and so, by Lemma 16.3, can be chosen to be in canonical form. Since ini $(\mathbf{w})=\operatorname{ini}\left(\mathbf{w}^{\prime}\right)$ and $\operatorname{sim}(\mathbf{w})=\operatorname{sim}\left(\mathbf{w}^{\prime}\right)$, it follows from $(\mathrm{V})$ that

$$
\mathbf{w}=\mathbf{p} x_{0}^{2} \cdot x_{1}^{e_{1}} x_{0}^{f_{1}} \ldots x_{m}^{e_{m}} x_{0}^{f_{m}} \quad \text { and } \quad \mathbf{w}^{\prime}=\mathbf{p} x_{0}^{2} \cdot x_{1}^{e_{1}} x_{0}^{f_{1}^{\prime}} \ldots x_{m}^{e_{m}} x_{0}^{f_{m}^{\prime}} .
$$

Case 1: $f_{m} \neq f_{m}^{\prime}$, say $\left(f_{m}, f_{m}^{\prime}\right)=(2,0)$. Then $e_{m}=1$ by $(\mathrm{IV})$, so that

$$
\mathbf{w}=\mathbf{p} x_{0}^{2} \cdot x_{1}^{e_{1}} x_{0}^{f_{1}} \ldots x_{m-1}^{e_{m-1}} x_{0}^{f_{m-1}} \cdot x_{m} x_{0}^{2} \quad \text { and } \quad \mathbf{w}^{\prime}=\mathbf{p} x_{0}^{2} \cdot x_{1}^{e_{1}} x_{0}^{f_{1}^{\prime}} \ldots x_{m-1}^{e_{m-1}} x_{0}^{f_{m-1}^{\prime}} \cdot x_{m} .
$$

But $\mathrm{t}(\mathbf{w})=x_{0} \notin \operatorname{sim}(\mathbf{w})$ and $\mathrm{t}\left(\mathbf{w}^{\prime}\right)=x_{m} \in \operatorname{sim}\left(\mathbf{w}^{\prime}\right)$ violate Lemma 16.2(iii).

Case 2: $f_{i} \neq f_{i}^{\prime}$ for some $i<m$, say $\left(f_{i}, f_{i}^{\prime}\right)=(2,0)$. Then $e_{i}=e_{i+1}=1$ by (IV), so that

$$
\mathbf{w}=\mathbf{p} x_{0}^{2} \cdot x_{1}^{e_{1}} x_{0}^{f_{1}} \ldots x_{i} x_{0}^{2} \cdot x_{i+1} x_{0}^{f_{i+1}} \ldots \quad \text { and } \quad \mathbf{w}^{\prime}=\mathbf{p} x_{0}^{2} \cdot x_{1}^{e_{1}} x_{0}^{f_{1}^{\prime}} \ldots x_{i} \cdot x_{i+1} x_{0}^{f_{i+1}^{\prime}} \ldots
$$

But $x_{i} x_{i+1} \notin \mathrm{FSS}(\mathbf{w})$ and $x_{i} x_{i+1} \in \mathrm{F}_{\mathrm{SS}}\left(\mathbf{w}^{\prime}\right)$ violate Lemma 16.2(iv).

Since both cases are impossible, $f_{i}=f_{i}^{\prime}$ for all $i$. Therefore the identity $\mathbf{w} \approx \mathbf{w}^{\prime}$ is trivial and so is implied by the identities (16.1).

$$
\text { 17. } \mathscr{C}_{5}, \mathscr{C}_{6}, \mathscr{C}_{10}, \mathscr{D}_{1}
$$

This section establishes the finite basis property of the following semigroups.

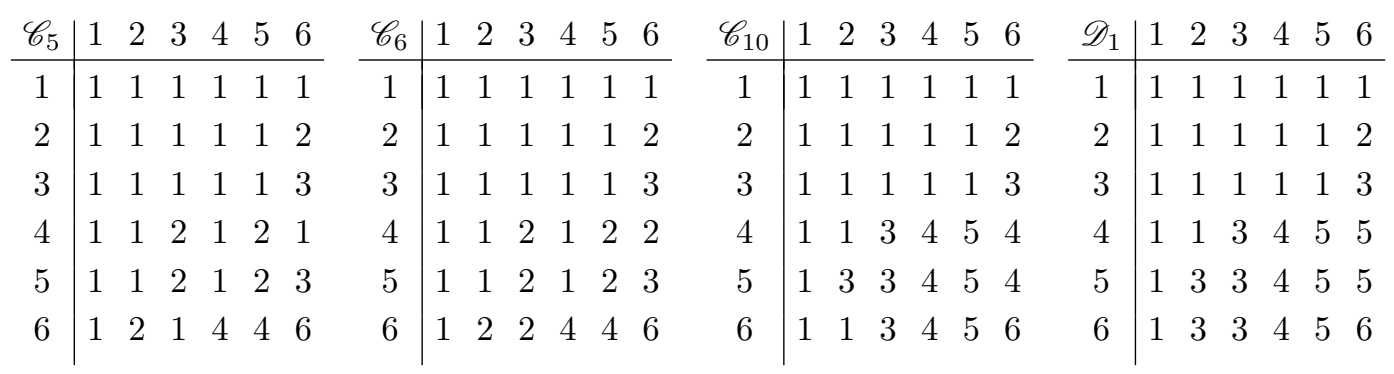

17.1. Finite basis property of $\mathscr{C}_{5}$ and $\mathscr{C}_{6}$

Proposition 17.1. The variety generated by $S \in\left\{\mathscr{C}_{5}, \mathscr{C}_{6}\right\}$ is defined by the identities

$$
\begin{aligned}
x^{2} y x \approx x y x, & x y x^{2} \approx x y x, \\
x^{2} \mathrm{H} y^{2} & \approx y^{2} \mathrm{H} x^{2}, \\
x^{3} \mathrm{H} y^{2} & \approx y^{2} \mathrm{H} x^{3}, \\
x^{3} \mathrm{H} y^{3} & \approx y^{3} \mathrm{H} x^{3}, \\
x^{3} y h^{2} & \approx x^{3} y x^{3} h^{2}, \\
h^{2} y x^{3} & \approx h^{2} x^{3} y x^{3},
\end{aligned}
$$




$$
\begin{aligned}
h^{2} x^{2} y k^{2} & \approx h^{2} y x^{2} k^{2}, \\
h x h y h & \approx h y h x h, \\
h^{2} x k^{2} y t^{2} & \approx h^{2} y k^{2} x t^{2} .
\end{aligned}
$$

A non-simple letter $x$ of a word $\mathbf{w}$ is said to be restricted if $\mathbf{w}=\mathbf{a} x^{2} \mathbf{b}$ for some $\mathbf{a}, \mathbf{b} \in \mathcal{X}^{*}$ such that $x \notin \operatorname{con}(\mathbf{a b})$; in this case, $x^{2}$ is called a restricted square of $\mathbf{w}$. A non-simple letter that is not restricted is said to be unrestricted. For any word $\mathbf{w}$, let res $(\mathbf{w})$ denote the set of restricted letters of $\mathbf{w}$ and let unres( $\mathbf{w})$ denote the set of unrestricted letters of $\mathbf{w}$. Note that the disjoint union res $(\mathbf{w}) \cup$ unres $(\mathbf{w})$ coincides with the set of non-simple letters of $\mathbf{w}$.

Lemma 17.2. Let $S \in\left\{\mathscr{C}_{5}, \mathscr{C}_{6}\right\}$. Suppose that $\mathbf{w} \approx \mathbf{w}^{\prime}$ is any identity satisfied by $S$. Then:

(i) $\operatorname{con}(\mathbf{w})=\operatorname{con}\left(\mathbf{w}^{\prime}\right)$ and $\operatorname{sim}(\mathbf{w})=\operatorname{sim}\left(\mathbf{w}^{\prime}\right)$;

(ii) either (a) $\mathrm{t}(\mathbf{w}) \in \operatorname{sim}(\mathbf{w}), \mathrm{t}\left(\mathbf{w}^{\prime}\right) \in \operatorname{sim}\left(\mathbf{w}^{\prime}\right)$, and $\mathrm{t}(\mathbf{w})=\mathrm{t}\left(\mathbf{w}^{\prime}\right)$, or (b) $\mathrm{t}(\mathbf{w}) \notin \operatorname{sim}(\mathbf{w})$ and $\mathrm{t}\left(\mathbf{w}^{\prime}\right) \notin \operatorname{sim}\left(\mathbf{w}^{\prime}\right)$;

(iii) either (a) $\mathrm{h}(\mathbf{w}) \in \operatorname{sim}(\mathbf{w}), \mathrm{h}\left(\mathbf{w}^{\prime}\right) \in \operatorname{sim}\left(\mathbf{w}^{\prime}\right)$, and $\mathrm{h}(\mathbf{w})=\mathrm{h}\left(\mathbf{w}^{\prime}\right)$, or (b) $\mathrm{h}(\mathbf{w}) \notin \operatorname{sim}(\mathbf{w})$ and $\mathrm{h}\left(\mathbf{w}^{\prime}\right) \notin \operatorname{sim}\left(\mathbf{w}^{\prime}\right)$;

(iv) $\operatorname{res}(\mathbf{w})=\operatorname{res}\left(\mathbf{w}^{\prime}\right)$ and unres $(\mathbf{w})=\operatorname{unres}\left(\mathbf{w}^{\prime}\right)$;

(v) $\mathrm{F}_{\mathrm{SS}}(\mathrm{w})=\mathrm{F}_{\mathrm{SS}}\left(\mathrm{w}^{\prime}\right)$.

Proof. (i) This follows from Lemma 2.1(v) since the subsemigroup $\{1,2,6\}$ of $S$ is isomorphic to $N_{2}^{1}$.

(ii) This follows from Lemma 2.2 since the subsemigroup $\{1,4,6\}$ of $\mathscr{C}_{5}$ and the divisor $\{1,2,4,6\} /\{1,2\}$ of $\mathscr{C}_{6}$ are isomorphic to $J$.

(iii) This follows from the dual result of Lemma 2.2 since the subsemigroup $\{1,3,6\}$ of $\mathscr{C}_{5}$ and the divisor $\{1,2,3,6\} /\{1,2\}$ of $\mathscr{C}_{6}$ are isomorphic to the dual semigroup of $J$.

(iv) By part (i), the set of non-simple letters of $\mathbf{w}$ coincides with the set of non-simple letters of $\mathbf{w}^{\prime}$. Therefore it suffices to verify that $\operatorname{res}(\mathbf{w})=\operatorname{res}\left(\mathbf{w}^{\prime}\right)$. Suppose $x \in \operatorname{res}(\mathbf{w})$. Then $\mathbf{w}=\mathbf{a} x^{2} \mathbf{b}$ for some $\mathbf{a}, \mathbf{b} \in \mathcal{X}^{*}$ such that $x \notin \operatorname{con}(\mathbf{a b})$. Let $\varphi: \mathcal{X} \rightarrow S$ denote the substitution

$$
z \mapsto \begin{cases}5 & \text { if } z=x, \\ 6 & \text { otherwise. }\end{cases}
$$

Then $\mathbf{w} \varphi=\ldots 5^{2} \ldots=2$. By part (i), the letter $x$ is non-simple in $\mathbf{w}^{\prime}, \operatorname{so} \operatorname{occ}\left(x, \mathbf{w}^{\prime}\right)=k \geqslant 2$. If two occurrences of $x$ in $\mathbf{w}^{\prime}$ sandwich some other letter, say $\mathbf{w}^{\prime}=\mathbf{c} x \mathbf{d} x \mathbf{e}$ for some $\mathbf{c}, \mathbf{e} \in \mathcal{X}^{*}$ and $\mathbf{d} \in \mathcal{X}^{+}$such that $x \notin \operatorname{con}(\mathbf{d})$, then $\mathbf{w}^{\prime} \varphi=\ldots 5 \cdot 6 \cdot 5 \ldots=1$ is a contradiction. Therefore all $k$ occurrences of $x$ form the factor $x^{k}$ in $\mathbf{w}^{\prime}$, whence $\mathbf{w}^{\prime}=\mathbf{f} x^{k} \mathbf{g}$ for some $\mathbf{f}, \mathbf{g} \in \mathcal{X}^{*}$ such that $x \notin \operatorname{con}(\mathbf{f g})$. If $k \geqslant 3$, then $\mathbf{w}^{\prime} \varphi=\ldots 5^{k} \ldots=1$ is a contradiction. It follows that $k=2$ and $x \in \operatorname{res}\left(\mathbf{w}^{\prime}\right)$. Consequently, the inclusion $\operatorname{res}(\mathbf{w}) \subseteq \operatorname{res}\left(\mathbf{w}^{\prime}\right)$ holds. The inclusion $\operatorname{res}\left(\mathbf{w}^{\prime}\right) \subseteq \operatorname{res}(\mathbf{w})$ holds by symmetry.

(v) The identity $(2.3)$ with $n=3$ is not satisfied by $S$ since $6^{3} \cdot 4 \cdot 6^{3} \cdot 3 \cdot 6^{3} \neq 6^{3} \cdot 4 \cdot 3 \cdot 6^{3}$. Therefore $\mathrm{F}_{\mathrm{SS}}(\mathbf{w})=\mathrm{F}_{\mathrm{SS}}\left(\mathbf{w}^{\prime}\right)$ by Lemma 2.10 and part (i).

Let $\mathbf{w}$ be any word with $\operatorname{res}(\mathbf{w})=\left\{x_{1}, \ldots, x_{m}\right\}$ for some $m \geqslant 1$ and unres $(\mathbf{w})=\emptyset$. Suppose that the letters $x_{1}, \ldots, x_{m}$ are in alphabetical order. In this subsection, such a word $\mathbf{w}$ is said to be in $\alpha$-canonical form if

$$
\begin{aligned}
\mathbf{w} & =\mathbf{p}\left(\prod_{i=1}^{r}\left(x_{i}^{2} \mathbf{s}_{i}\right)\right)\left(\prod_{i=r+1}^{m} x_{i}^{2}\right) \mathbf{q} \\
& =\mathbf{p} \cdot x_{1}^{2} \mathbf{s}_{1} \ldots x_{r}^{2} \mathbf{s}_{r} \cdot x_{r+1}^{2} \ldots x_{m}^{2} \cdot \mathbf{q},
\end{aligned}
$$

where all of the following are satisfied: 
(I) $r \in\{0, \ldots, m\}$;

(II) the letters of $\mathbf{p}, \mathbf{q} \in \mathcal{X}^{*}$ and $\mathbf{s}_{1}, \ldots, \mathbf{s}_{r} \in \mathcal{X}^{+}$are simple in $\mathbf{w}$;

(III) if $r=m$, then $\mathbf{q}=\emptyset$.

Note the extreme cases:

$$
\mathbf{w}= \begin{cases}\mathbf{p} \cdot x_{1}^{2} \ldots x_{m}^{2} \cdot \mathbf{q} & \text { if } r=0 \\ \mathbf{p} \cdot x_{1}^{2} \mathbf{s}_{1} \ldots x_{m}^{2} \mathbf{s}_{m} & \text { if } r=m\end{cases}
$$

Lemma 17.3. Let $\mathbf{w}$ be any non-simple word such that unres $(\mathbf{w})=\emptyset$. Then there exists some word $\overline{\mathbf{w}}$ in $\alpha$-canonical form such that the identities (17.1) imply the identity $\mathbf{w} \approx \overline{\mathbf{w}}$.

Proof. It suffices to convert $\mathbf{w}$, using the identities (17.1), into a word in $\alpha$-canonical form. Generality is not lost by assuming res $(\mathbf{w})=\left\{x_{1}, \ldots, x_{m}\right\}$ with $x_{1}, \ldots, x_{m}$ in alphabetical order. Hence $\mathbf{w}$ is a product, in some order, of the restricted squares $x_{1}^{2}, \ldots, x_{m}^{2}$ and some simple letters. The identities (17.1b) can be used to arrange these restricted squares so that they appear in alphabetical order, that is,

$$
\mathbf{w} \stackrel{(17.1 \mathrm{~b})}{\approx} \underbrace{\ldots x_{1}^{2} \ldots x_{2}^{2} \ldots x_{m}^{2} \ldots}_{\mathbf{w}^{\prime}} \in \mathcal{X}^{*} x_{1}^{2} \mathcal{X}^{*} x_{2}^{2} \mathcal{X}^{*} \ldots \mathcal{X}^{*} x_{m}^{2} \mathcal{X}^{*} .
$$

If the squares $x_{i}^{2}$ and $x_{i+1}^{2}$ are adjacent but the squares $x_{i+1}^{2}$ and $x_{i+2}^{2}$ are not adjacent, so that $\mathbf{w}^{\prime}=\mathbf{a} x_{i}^{2} x_{i+1}^{2} \mathbf{b} x_{i+2}^{2} \mathbf{c}$ for some $\mathbf{a}, \mathbf{c} \in \mathcal{X}^{*}$ and $\mathbf{b} \in \mathcal{X}^{+}$, then the squares $x_{i}^{2}$ and $x_{i+1}^{2}$ can be separated by the identity $(17.1 \mathrm{~g})$ :

$$
\mathbf{w}^{\prime}=\mathbf{a} x_{i}^{2} x_{i+1}^{2} \mathbf{b} x_{i+2}^{2} \mathbf{c} \stackrel{(17.1 \mathrm{~g})}{\approx} \mathbf{a} x_{i}^{2} \mathbf{b} x_{i+1}^{2} x_{i+2}^{2} \mathbf{c} .
$$

It is easily seen how this procedure can be repeated until $\mathbf{w}^{\prime}$ is converted by the identity $(17.1 \mathrm{~g})$ into a word in $\alpha$-canonical form.

Let $\mathbf{w}$ be any word with $\operatorname{res}(\mathbf{w})=\left\{x_{1}, \ldots, x_{m}\right\}$ for some $m \geqslant 0$ and unres $(\mathbf{w})=\left\{y_{1}, \ldots, y_{n}\right\}$ for some $n \geqslant 1$. Suppose that the letters $x_{1}, \ldots, x_{m}$ are in alphabetical order and the letters $y_{1}, \ldots, y_{n}$ are in alphabetical order. In this subsection, such a word $\mathbf{w}$ is said to be in $\beta$ canonical form if

$$
\mathbf{w}=\mathbf{p x} \prod_{i=1}^{r}\left(\mathbf{y s} \mathbf{s}_{i}\right)=\mathbf{p} \mathbf{x} \cdot \mathbf{y} \mathbf{s}_{1} \ldots \mathbf{y} \mathbf{s}_{r}
$$

where all of the following are satisfied:

(I) $r \geqslant 1$;

(II) the letters of $\mathbf{p}, \mathbf{s}_{r} \in \mathcal{X}^{*}$ and $\mathbf{s}_{1}, \ldots, \mathbf{s}_{r-1} \in \mathcal{X}^{+}$are simple in $\mathbf{w}$;

(III) $\mathbf{x}=x_{1}^{2} \ldots x_{m}^{2}$

(IV) $\mathbf{y}=y_{1}^{3} \ldots y_{n}^{3}$.

Note that $\mathbf{x}$ is empty if $m=0$, but $\mathbf{y}$ is nonempty because unres $(\mathbf{w}) \neq \emptyset$.

Lemma 17.4. Let $\mathbf{w}$ be any non-simple word such that unres $(\mathbf{w}) \neq \emptyset$. Then there exists some word $\overline{\mathbf{w}}$ in $\beta$-canonical form such that the identities (17.1) imply the identity $\mathbf{w} \approx \overline{\mathbf{w}}$.

Proof. It suffices to convert $\mathbf{w}$, using the identities (17.1), into a word in $\beta$-canonical form. Since $\mathbf{w}$ is non-simple, generality is not lost by assuming $\operatorname{res}(\mathbf{w})=\left\{x_{1}, \ldots, x_{m}\right\}$ with $x_{1}, \ldots, x_{m}$ in alphabetical order, and unres $(\mathbf{w})=\left\{y_{1}, \ldots, y_{n}\right\}$ with $y_{1}, \ldots, y_{n}$ in alphabetical order. Then $\mathbf{w}$ can be written in the form

$$
\mathbf{w}=\mathbf{p w}_{1} \mathbf{s}_{1} \ldots \mathbf{w}_{r} \mathbf{s}_{r}
$$


where (I) and (II) are satisfied and the letters of $\mathbf{w}_{1}, \ldots, \mathbf{w}_{r} \in \mathcal{X}^{+}$are all non-simple in $\mathbf{w}$. The identities (17.1a) can be used to replace each unrestricted letter $y_{i}$ by $y_{i}^{3}$. Hence it can further be assumed that $\mathbf{w}_{i} \in\left\{x_{1}^{2}, \ldots, x_{m}^{2}, y_{1}^{3}, \ldots, y_{n}^{3}\right\}^{+}$for all $i$.

Let $k \in\{1, \ldots, n\}$. By assumption, $y_{k}^{3}$ is a factor of some $\mathbf{w}_{i}$, so that $\mathbf{w}_{i}=\mathbf{a} y_{k}^{3} \mathbf{b}$ for some $\mathbf{a}, \mathbf{b} \in\left\{x_{1}^{2}, \ldots, x_{m}^{2}, y_{1}^{3}, \ldots, y_{n}^{3}\right\}^{*}$. Suppose that $y_{k}^{3}$ is not a factor of $\mathbf{w}_{i+1}$. Let $h=\mathbf{h}\left(\mathbf{w}_{i+1}\right)$. Then $\mathbf{w}_{i+1}$ begins with $h^{2}$ or $h^{3}$ depending on whether or not $h$ is restricted. In any case, $\mathbf{w}_{i+1}=h^{2} \mathbf{w}_{i+1}^{\prime}$ for some $\mathbf{w}_{i+1}^{\prime} \in \mathcal{X}^{*}$. Hence

$$
\begin{aligned}
\mathbf{w} & =\ldots \overbrace{\mathbf{a} y_{k}^{3} \mathbf{b}}^{\mathbf{w}_{i}} \mathbf{s}_{i} \cdot \overbrace{h^{2} \mathbf{w}_{i+1}^{\prime}}^{\mathbf{w}_{i+1}} \mathbf{s}_{i+1} \ldots \stackrel{\sim}{\approx}^{(17.1 \mathrm{e})} \ldots \mathbf{a} y_{k}^{3} \mathbf{b} \mathbf{s}_{i} \cdot y_{k}^{3} h^{2} \mathbf{w}_{i+1}^{\prime} \mathbf{s}_{i+1} \ldots \\
& =\ldots \mathbf{w}_{i} \mathbf{s}_{i} \cdot\left(y_{k}^{3} \mathbf{w}_{i+1}\right) \mathbf{s}_{i+1} \ldots,
\end{aligned}
$$

that is, $\mathbf{w}_{i+1}$ is converted by the identity (17.1e) into $y_{k}^{3} \mathbf{w}_{i+1}$. If $y_{k}^{3}$ is not a factor of $\mathbf{w}_{i-1}$, then by a symmetrical argument, $\mathbf{w}_{i-1}$ can be converted by the identity (17.1f) into $\mathbf{w}_{i-1} y_{k}^{3}$. Since $k$ is arbitrary in $\{1, \ldots, n\}$, it is easily seen how this procedure can be repeated until

(a) every $\mathbf{w}_{i}$ is converted into a word that contains all the factors $y_{1}^{3}, \ldots, y_{n}^{3}$.

Now the letters $x_{1}, \ldots, x_{m}$ are restricted, so that each of the restricted squares $x_{1}^{2}, \ldots, x_{m}^{2}$ occurs precisely once in $\mathbf{w}$ as a factor of some $\mathbf{w}_{i}$. The identities $\{(17.1 \mathrm{~b}),(17.1 \mathrm{c}),(17.1 \mathrm{~g})\}$ can thus be used to move the squares $x_{1}^{2}, \ldots, x_{m}^{2}$ to the left until they are grouped with $\mathbf{w}_{1}$. Therefore in view of (a):

(b) $\mathbf{w}_{1}$ is converted into a product involving $x_{1}^{2}, \ldots, x_{m}^{2}, y_{1}^{3}, \ldots, y_{n}^{3}$;

(c) each of $\mathbf{w}_{2}, \ldots, \mathbf{w}_{r}$ is converted into a product involving only $y_{1}^{3}, \ldots, y_{n}^{3}$.

It then easily follows from (b) and (c) that the identities (17.1a)-(17.1d) can be used to convert $\mathbf{w}_{1}$ into $\mathbf{x y}$, and every one of $\mathbf{w}_{2}, \ldots, \mathbf{w}_{r}$ to $\mathbf{y}$. Hence (III) and (IV) are satisfied, and the resulting word is in $\beta$-canonical form.

Proof of Proposition 17.1. Let $S \in\left\{\mathscr{C}_{5}, \mathscr{C}_{6}\right\}$. It is routinely checked that $S$ satisfies the identities (17.1). Hence it suffices to show that any identity $\mathbf{w} \approx \mathbf{w}^{\prime}$ satisfied by $S$ is implied by the identities (17.1). If either $\mathbf{w}$ or $\mathbf{w}^{\prime}$ is a simple word, then it follows from Lemma 17.2 parts (i) and (v) that the identity $\mathbf{w} \approx \mathbf{w}^{\prime}$ is trivial and so is vacuously implied by (17.1). Therefore assume that $\mathbf{w}$ and $\mathbf{w}^{\prime}$ are both non-simple words. By Lemma 17.2 parts (i) and (iv), it can further be assumed that:

(a) $\operatorname{res}(\mathbf{w})=\operatorname{res}\left(\mathbf{w}^{\prime}\right)=\left\{x_{1}, \ldots, x_{m}\right\}$ where $x_{1}, \ldots, x_{m}$ are in alphabetical order;

(b) unres $(\mathbf{w})=$ unres $\left(\mathbf{w}^{\prime}\right)=\left\{y_{1}, \ldots, y_{n}\right\}$ where $y_{1}, \ldots, y_{n}$ are in alphabetical order.

There are two cases.

Case 1: unres $(\mathbf{w})=\operatorname{unres}\left(\mathbf{w}^{\prime}\right)=\emptyset$. Then by Lemma 17.3, the words $\mathbf{w}$ and $\mathbf{w}^{\prime}$ can be chosen to be in $\alpha$-canonical form, whence by (a),

$$
\mathbf{w}=\mathbf{p} \cdot x_{1}^{2} \mathbf{s}_{1} \ldots x_{r}^{2} \mathbf{s}_{r} \cdot x_{r+1}^{2} \ldots x_{m}^{2} \cdot \mathbf{q} \quad \text { and } \quad \mathbf{w}^{\prime}=\mathbf{p}^{\prime} \cdot x_{1}^{2} \mathbf{s}_{1}^{\prime} \ldots x_{r^{\prime}}^{2} \mathbf{s}_{r^{\prime}}^{\prime} \cdot x_{r^{\prime}+1}^{2} \ldots x_{m}^{2} \cdot \mathbf{q}^{\prime} .
$$

Therefore $\mathbf{p}=\mathbf{p}^{\prime}$ and $\mathbf{q}=\mathbf{q}^{\prime}$ by parts (ii), (iii), and (v) of Lemma 17.2, and

(c) $\left\{\mathbf{s}_{1}, \ldots, \mathbf{s}_{r}\right\}=\left\{\mathbf{s}_{1}^{\prime}, \ldots, \mathbf{s}_{r^{\prime}}^{\prime}\right\}$

by part (v) of the same lemma, whence $r=r^{\prime}$. Thus $\mathbf{w}^{\prime}=\mathbf{p} \cdot x_{1}^{2} \mathbf{s}_{1}^{\prime} \ldots x_{r}^{2} \mathbf{s}_{r}^{\prime} \cdot x_{r+1}^{2} \ldots x_{m}^{2} \cdot \mathbf{q}$. It now follows from (c) that $\mathbf{w}^{\prime}$ can be converted by the identity (17.1i) into $\mathbf{w}$.

Case 2: unres $(\mathbf{w})=$ unres $\left(\mathbf{w}^{\prime}\right) \neq \emptyset$. Then by Lemma 17.4 , the words $\mathbf{w}$ and $\mathbf{w}^{\prime}$ can be chosen to be in $\beta$-canonical form, whence by (a) and (b),

$$
\mathbf{w}=\mathbf{p x} \cdot \mathbf{y} \mathbf{s}_{1} \ldots \mathbf{y} \mathbf{s}_{r-1} \cdot \mathbf{y} \mathbf{s}_{r} \quad \text { and } \quad \mathbf{w}^{\prime}=\mathbf{p}^{\prime} \mathbf{x} \cdot \mathbf{y s}_{1}^{\prime} \ldots \mathbf{y} \mathbf{s}_{r^{\prime}-1}^{\prime} \cdot \mathbf{y} \mathbf{s}_{r^{\prime}}^{\prime}
$$


where $\mathbf{x}=x_{1}^{2} \ldots x_{m}^{2}$ and $\mathbf{y}=y_{1}^{3} \ldots y_{n}^{3}$. Therefore $\mathbf{p}=\mathbf{p}^{\prime}$ and $\mathbf{s}_{r}=\mathbf{s}_{r^{\prime}}^{\prime}$ by parts (ii), (iii), and $(\mathrm{v})$ of Lemma 17.2, and

(d) $\left\{\mathbf{s}_{1}, \ldots, \mathbf{s}_{r-1}\right\}=\left\{\mathbf{s}_{1}^{\prime}, \ldots, \mathbf{s}_{r^{\prime}-1}^{\prime}\right\}$

by part (v) of the same lemma, whence $r=r^{\prime}$. Thus $\mathbf{w}^{\prime}=\mathbf{p x} \cdot \mathbf{y s}_{1}^{\prime} \ldots \mathbf{y} \mathbf{s}_{r-1}^{\prime} \cdot \mathbf{y} \mathbf{s}_{r}$. It now follows from $(\mathrm{d})$ that $\mathbf{w}^{\prime}$ can be converted by the identity (17.1h) into $\mathbf{w}$.

17.2. Finite basis property of $\mathscr{C}_{10}$ and $\mathscr{D}_{1}$

Proposition 17.5. The variety generated by $S \in\left\{\mathscr{C}_{10}, \mathscr{D}_{1}\right\}$ is defined by the identities

$$
\begin{aligned}
x^{3} \approx x^{2}, \quad x^{2} y x & \approx x y x, \quad x y x^{2} \approx x y x, \\
x^{2} y^{2} z^{2} & \approx y^{2} x^{2} z^{2}, \\
x^{2} \mathrm{H} y^{2} & \approx y x^{2} \mathrm{H} y, \\
x y \mathrm{H} x \mathrm{~K} y & \approx y x \mathrm{H} x \mathrm{~K} y .
\end{aligned}
$$

For any word $\mathbf{w}$, let $F_{L S}(\mathbf{w})$ denote the set of factors of $\mathbf{w}$ of length two that begin with the last occurrence of some non-simple letter and end with a simple letter:

$$
\mathrm{F}_{\mathrm{LS}}(\mathbf{w})=\left\{x y \in \mathcal{X}^{2} \mid \mathbf{w} \in \mathcal{X}^{*} x y(\mathcal{X} \backslash\{x\})^{*}, x \notin \operatorname{sim}(\mathbf{w}), y \in \operatorname{sim}(\mathbf{w})\right\} .
$$

Lemma 17.6. Let $S \in\left\{\mathscr{C}_{10}, \mathscr{D}_{1}\right\}$. Suppose that $\mathbf{w} \approx \mathbf{w}^{\prime}$ is any identity satisfied by $S$. Then:

(i) $\mathrm{t}(\mathbf{w})=\mathrm{t}\left(\mathbf{w}^{\prime}\right)$;

(ii) $\operatorname{con}(\mathbf{w})=\operatorname{con}\left(\mathbf{w}^{\prime}\right)$ and $\operatorname{sim}(\mathbf{w})=\operatorname{sim}\left(\mathbf{w}^{\prime}\right)$;

(iii) for any $x \in \operatorname{con}(\mathbf{w})=\operatorname{con}\left(\mathbf{w}^{\prime}\right)$ and $y \in \operatorname{sim}(\mathbf{w})=\operatorname{sim}\left(\mathbf{w}^{\prime}\right)$, the conditions $x \prec_{\mathbf{w}} y$ and $x \prec_{\mathbf{w}^{\prime}} y$ are equivalent;

(iv) $\mathbf{w}_{\text {sim }}=\mathbf{w}_{\text {sim }}^{\prime}$;

(v) $\mathrm{F}_{\mathrm{LS}}(\mathbf{w})=\mathrm{F}_{\mathrm{LS}}\left(\mathbf{w}^{\prime}\right)$;

(vi) $\mathrm{F}_{\mathrm{SS}}(\mathbf{w})=\mathrm{F}_{\mathrm{SS}}\left(\mathbf{w}^{\prime}\right)$.

Proof. The subsemigroup $\{4,5\}$ of $S$ is isomorphic to $R_{2}$, so part (i) holds by Lemma 2.1(ii). Parts (ii)-(iv) hold by Lemma 2.3 because the subsemigroup $\{1,3,4,6\}$ of $\mathscr{C}_{10}$ and the divisor $\{1,3,4,5,6\} /\{4,5\}$ of $\mathscr{D}_{1}$ are isomorphic to $J^{1}$.

(v) Suppose $\mathrm{F}_{\mathrm{LS}}(\mathbf{w}) \neq \mathrm{F}_{\mathrm{LS}}\left(\mathbf{w}^{\prime}\right)$, say $x y \in \mathrm{F}_{\mathrm{LS}}(\mathbf{w}) \backslash \mathrm{F}_{\mathrm{LS}}\left(\mathbf{w}^{\prime}\right)$. Then

(a) $\mathbf{w}=\mathbf{a} x y \mathbf{b}$ for some $\mathbf{a}, \mathbf{b} \in \mathcal{X}^{*}$ such that $x \in \operatorname{con}(\mathbf{a}) \backslash \operatorname{con}(\mathbf{b})$ and $y \notin \operatorname{con}(\mathbf{a b})$.

By part (ii), the letter $x$ is non-simple in both $\mathbf{w}$ and $\mathbf{w}^{\prime}$, while the letter $y$ is simple in both $\mathbf{w}$ and $\mathbf{w}^{\prime}$. Further, since $x \prec_{\mathbf{w}} y$, it follows from part (iii) that $x \prec_{\mathbf{w}^{\prime}} y$. Hence

(b) $\mathbf{w}^{\prime}=\mathbf{a}^{\prime} t y \mathbf{b}^{\prime}$ for some $t \in \mathcal{X}$ and some $\mathbf{a}^{\prime}, \mathbf{b}^{\prime} \in \mathcal{X}^{*}$ such that $x \in \operatorname{con}\left(\mathbf{a}^{\prime}\right) \backslash \operatorname{con}\left(t \mathbf{b}^{\prime}\right)$ and $y \notin \operatorname{con}\left(\mathbf{a}^{\prime} t \mathbf{b}^{\prime}\right)$.

Let $\varphi_{1}$ and $\varphi_{2}$ denote the following substitutions into $\mathscr{C}_{10}$ and $\mathscr{D}_{1}$, respectively:

$$
z \mapsto\left\{\begin{array} { l l } 
{ 5 } & { \text { if } z = x , } \\
{ 2 } & { \text { if } z = y , } \\
{ 6 } & { \text { otherwise; } }
\end{array} \quad z \mapsto \left\{\begin{array}{ll}
4 & \text { if } z=x, \\
2 & \text { if } z=y, \\
6 & \text { otherwise. }
\end{array}\right.\right.
$$

Then $\mathbf{a} \varphi_{1} \in\{5,6\}^{+} \subseteq\{4,5,6\}$ and $\mathbf{b} \varphi_{1} \in\{6\}^{*}$ by (a), so that

$$
\mathbf{w} \varphi_{1}=\mathbf{a} \varphi_{1} \cdot 5 \cdot 2 \cdot \mathbf{b} \varphi_{1}=3 \quad \text { and } \quad \mathbf{w}^{\prime} \varphi_{1}=\ldots 6 \cdot 2 \ldots=1,
$$

and $\mathbf{a}^{\prime} \varphi_{2} \in\{4,6\}^{+} \subseteq\{4,5,6\}$ and $\mathbf{b}^{\prime} \varphi_{2} \in\{6\}^{*}$ by (b), so that

$$
\mathbf{w}^{\prime} \varphi_{2}=\mathbf{a}^{\prime} \varphi_{2} \cdot 6 \cdot 2 \cdot \mathbf{b}^{\prime} \varphi_{2}=3 \quad \text { and } \quad \mathbf{w} \varphi_{2}=\ldots 4 \cdot 2 \ldots=1 .
$$

Both of these are impossible. Hence $F_{\mathrm{LS}}(\mathbf{w})=\mathrm{F}_{\mathrm{LS}}\left(\mathbf{w}^{\prime}\right)$. 
(vi) Suppose $\mathrm{F}_{\mathrm{SS}}(\mathbf{w}) \neq \mathrm{F}_{\mathrm{SS}}\left(\mathbf{w}^{\prime}\right)$, say $x y \in \mathrm{F}_{\mathrm{SS}}(\mathbf{w}) \backslash \mathrm{F}_{\mathrm{SS}}\left(\mathbf{w}^{\prime}\right)$. Then it is easily seen from part (ii) that $x y \in \mathrm{F}_{\mathrm{LS}}(x \mathbf{w}) \backslash \mathrm{F}_{\mathrm{LS}}\left(x \mathbf{w}^{\prime}\right)$. It then follows from part (v) that $S$ does not satisfy the identity $x \mathbf{w} \approx x \mathbf{w}^{\prime}$, which is impossible.

Let $\mathbf{w}$ be any non-simple word with distinct non-simple letters $x_{1}, \ldots, x_{r}$. In this subsection, such a word is said to be in canonical form if

$$
\mathbf{w}=\prod_{i=1}^{m}\left(\mathbf{s}_{i} \mathbf{w}_{i}\right),
$$

where all of the following are satisfied:

(I) the letters of $\mathbf{s}_{1} \in \mathcal{X}^{*}$ and $\mathbf{s}_{2}, \ldots, \mathbf{s}_{m} \in \mathcal{X}^{+}$are simple in $\mathbf{w}$;

(II) $\mathbf{w}_{1}, \ldots, \mathbf{w}_{m-1} \in \mathcal{X}^{+}$and $\mathbf{w}_{m} \in \mathcal{X}^{*}$ are such that

$$
\mathbf{w}_{1}, \ldots, \mathbf{w}_{m} \in\left\{y_{1}^{2} \ldots y_{\ell}^{2} \mid y_{1}, \ldots, y_{\ell} \text { are distinct in }\left\{x_{1}, \ldots, x_{r}\right\} \text { with } \ell \geqslant 0\right\} ;
$$

(III) $\operatorname{con}\left(\mathbf{w}_{1}\right) \supseteq \ldots \supseteq \operatorname{con}\left(\mathbf{w}_{m}\right)$.

Lemma 17.7. Let $\mathbf{w}$ be any non-simple word. Then there exists some word $\overline{\mathbf{w}}$ in canonical form such that the identities (17.2) imply the identity $\mathbf{w} \approx \overline{\mathbf{w}}$.

Proof. It suffices to convert $\mathbf{w}$, using the identities (17.2), into a word in canonical form. Clearly $\mathbf{w}$ can be written in the form (17.3) with (I) satisfied, $\mathbf{w}_{1}, \ldots, \mathbf{w}_{m-1} \in\left\{x_{1}, \ldots, x_{r}\right\}^{+}$, and $\mathbf{w}_{m} \in\left\{x_{1}, \ldots, x_{r}\right\}^{*}$. If $y \in \operatorname{con}\left(\mathbf{w}_{i+1}\right) \backslash \operatorname{con}\left(\mathbf{w}_{i}\right)$, say $\mathbf{w}_{i+1}=\mathbf{a} y \mathbf{b}$ for some $\mathbf{a}, \mathbf{b} \in \mathcal{X}^{*}$, then the identities (17.2) can be used to convert $\mathbf{w}_{i}$ into $y \mathbf{w}_{i}$ :

$$
\begin{aligned}
& \mathbf{w}=\ldots \mathbf{s}_{i} \mathbf{w}_{i} \cdot \mathbf{s}_{i+1} \mathbf{w}_{i+1} \ldots \stackrel{(17.2 \mathrm{a})}{\approx} \ldots \mathbf{s}_{i}\left(\mathrm{~h}\left(\mathbf{w}_{i}\right)\right)^{2} \mathbf{w}_{i} \cdot \mathbf{s}_{i+1} \mathbf{a} y^{2} \mathbf{b} \ldots \\
& \stackrel{(17.2 \mathrm{c})}{\approx} \ldots \mathbf{s}_{i} y\left(\mathrm{~h}\left(\mathbf{w}_{i}\right)\right)^{2} \mathbf{w}_{i} \cdot \mathbf{s}_{i+1} \mathbf{a} y \mathbf{b} \ldots \stackrel{(17.2 \mathrm{a})}{\approx} \ldots \mathbf{s}_{i}\left(y \mathbf{w}_{i}\right) \cdot \mathbf{s}_{i+1} \mathbf{w}_{i+1} \ldots
\end{aligned}
$$

Hence (III) is satisfied by applying the identities (17.2).

Choose any $k \in\{1, \ldots, m\}$. It is convenient to let $\mathbf{p}=\prod_{i=1}^{k-1}\left(\mathbf{s}_{i} \mathbf{w}_{i}\right)$ and $\mathbf{q}=\prod_{i=k+1}^{m}\left(\mathbf{s}_{i} \mathbf{w}_{i}\right)$, so that $\mathbf{w}=\mathbf{p s}_{k} \mathbf{w}_{k} \mathbf{q}$. Suppose $t=\mathrm{t}\left(\mathbf{w}_{k}\right)$ and $\operatorname{con}\left(\mathbf{w}_{k}\right)=\left\{y_{1}, \ldots, y_{\ell}, t\right\} \subseteq\left\{x_{1}, \ldots, x_{r}\right\}$. Let $\varphi$ denote the substitution $x \mapsto x^{2}$ for all $x \in \mathcal{X}$. Since the letters of $\mathbf{w}_{k}$ are non-simple in $\mathbf{w}$,

$$
\begin{aligned}
& \mathbf{w} \stackrel{(17.2 \mathrm{a})}{\sim} \mathbf{p s}_{k}\left(\mathbf{w}_{k} \varphi\right) \mathbf{q} \stackrel{(17.2 \mathrm{~b})}{\approx} \mathbf{p s}_{k} y_{1}^{2 e_{1}} \ldots y_{\ell}^{2 e_{\ell}} t^{2 e} \mathbf{q} \text { for some } e_{1}, \ldots, e_{\ell}, e \geqslant 1 \\
& \stackrel{(17.2 \mathrm{a})}{\approx} \mathbf{p s}_{k} y_{1}^{2} \ldots y_{\ell}^{2} t^{2} \mathbf{q} .
\end{aligned}
$$

Hence $\mathbf{w}_{k}$ satisfies (II).

Lemma 17.8. Let $S \in\left\{\mathscr{C}_{10}, \mathscr{D}_{1}\right\}$. Suppose that $S$ satisfies an identity $\mathbf{w} \approx \mathbf{w}^{\prime}$, where

$$
\mathbf{w}=\prod_{i=1}^{m}\left(\mathbf{s}_{i} \mathbf{w}_{i}\right) \quad \text { and } \quad \mathbf{w}^{\prime}=\prod_{i=1}^{m}\left(\mathbf{s}_{i} \mathbf{w}_{i}^{\prime}\right)
$$

are in canonical form. Then $\operatorname{con}\left(\mathbf{w}_{i}\right)=\operatorname{con}\left(\mathbf{w}_{i}^{\prime}\right)$ for all $i$.

Proof. First note that $\bigcup_{i=1}^{m} \operatorname{con}\left(\mathbf{w}_{i}\right)=\bigcup_{i=1}^{m} \operatorname{con}\left(\mathbf{w}_{i}^{\prime}\right)$ holds by Lemma 17.6(ii). Suppose $\operatorname{con}\left(\mathbf{w}_{1}\right) \neq \operatorname{con}\left(\mathbf{w}_{1}^{\prime}\right)$, say $x \in \operatorname{con}\left(\mathbf{w}_{1}\right) \backslash \operatorname{con}\left(\mathbf{w}_{1}^{\prime}\right)$. Since the letter $x$ is non-simple in $\mathbf{w}$ by (II), it is also non-simple in $\mathbf{w}^{\prime}$ by Lemma 17.6(ii). Hence by (II), there exists some $i>1$ such that $x \in \operatorname{con}\left(\mathbf{w}_{i}^{\prime}\right)$. But then (III) implies $x \in \operatorname{con}\left(\mathbf{w}_{1}^{\prime}\right)$, contradicting the assumption. Therefore $\operatorname{con}\left(\mathbf{w}_{1}\right)=\operatorname{con}\left(\mathbf{w}_{1}^{\prime}\right)$. 
Now suppose $\operatorname{con}\left(\mathbf{w}_{k}\right) \neq \operatorname{con}\left(\mathbf{w}_{k}^{\prime}\right)$ for some $k>1$, say $x \in \operatorname{con}\left(\mathbf{w}_{k}\right) \backslash \operatorname{con}\left(\mathbf{w}_{k}^{\prime}\right)$. Then (III) implies $x \in \operatorname{con}\left(\mathbf{w}_{1} \ldots \mathbf{w}_{k}\right)$ and $x \notin \operatorname{con}\left(\mathbf{w}_{k}^{\prime} \ldots \mathbf{w}_{m}^{\prime}\right)$, whence $x \nprec_{\mathbf{w}} \mathrm{h}\left(\mathbf{s}_{k}\right)$ and $x \prec_{\mathbf{w}^{\prime}} \mathrm{h}\left(\mathbf{s}_{k}\right)$. But this violates Lemma 17.6(iii).

Lemma 17.9. Let $S \in\left\{\mathscr{C}_{10}, \mathscr{D}_{1}\right\}$. Suppose that $S$ satisfies an identity $\mathbf{w} \approx \mathbf{w}^{\prime}$, where

$$
\mathbf{w}=\prod_{i=1}^{m}\left(\mathbf{s}_{i} \mathbf{w}_{i}\right) \quad \text { and } \quad \mathbf{w}^{\prime}=\prod_{i=1}^{m}\left(\mathbf{s}_{i} \mathbf{w}_{i}^{\prime}\right)
$$

are in canonical form. Then the identities (17.2) imply the identity $\mathbf{w} \approx \mathbf{w}^{\prime}$.

Proof. It suffices to show that for any $k \in\{1, \ldots, m\}$, the identities (17.2) can be used to convert the factor $\mathbf{w}_{k}$ of $\mathbf{w}$ into $\mathbf{w}_{k}^{\prime}$. For convenience, write

$$
\mathbf{w}=\mathbf{p s}_{k} \mathbf{w}_{k} \mathbf{q} \quad \text { and } \quad \mathbf{w}^{\prime}=\mathbf{p}^{\prime} \mathbf{s}_{k} \mathbf{w}_{k}^{\prime} \mathbf{q}^{\prime}
$$

where $\mathbf{p}=\prod_{i=1}^{k-1}\left(\mathbf{s}_{i} \mathbf{w}_{i}\right), \mathbf{q}=\prod_{i=k+1}^{m}\left(\mathbf{s}_{i} \mathbf{w}_{i}\right), \mathbf{p}^{\prime}=\prod_{i=1}^{k-1}\left(\mathbf{s}_{i} \mathbf{w}_{i}^{\prime}\right)$, and $\mathbf{q}^{\prime}=\prod_{i=k+1}^{m}\left(\mathbf{s}_{i} \mathbf{w}_{i}^{\prime}\right)$. Then Lemma 17.8 and the assumption of this lemma imply

(a) $\operatorname{con}\left(\mathbf{w}_{k}\right)=\operatorname{con}\left(\mathbf{w}_{k}^{\prime}\right) \subseteq\left\{x_{1}, \ldots, x_{r}\right\}$,

(b) $\operatorname{con}(\mathbf{q})=\operatorname{con}\left(\mathbf{q}^{\prime}\right)$,

and $\operatorname{con}(\mathbf{p})=\operatorname{con}\left(\mathbf{p}^{\prime}\right)$. There are two cases.

Case 1: $\mathrm{t}\left(\mathbf{w}_{k}\right)=\mathrm{t}\left(\mathbf{w}_{k}^{\prime}\right)=t . \quad$ By (a) and (II), there exist distinct $y_{1}, \ldots, y_{\ell} \in\left\{x_{1}, \ldots, x_{r}\right\}$ with $\ell \geqslant 0$ such that $\mathbf{w}_{k}=y_{1}^{2} \ldots y_{\ell}^{2} t^{2}$ and $\mathbf{w}_{k}^{\prime}=y_{1 \pi}^{2} \ldots y_{\ell \pi}^{2} t^{2}$, where $\pi$ is some permutation on $\{1, \ldots, \ell\}$. Then

$$
\mathbf{w}=\mathbf{p s}_{k} y_{1}^{2} \ldots y_{\ell}^{2} t^{2} \mathbf{q} \stackrel{(17.2 \mathrm{~b})}{\approx} \mathbf{p s}_{k} y_{1 \pi}^{2} \ldots y_{\ell \pi}^{2} t^{2} \mathbf{q}=\mathbf{p s}_{k} \mathbf{w}_{k}^{\prime} \mathbf{q}
$$

Case 2: $\mathrm{t}\left(\mathbf{w}_{k}\right)=t \neq t^{\prime}=\mathrm{t}\left(\mathbf{w}_{k}^{\prime}\right)$. Since $\mathrm{t}\left(\mathbf{w}_{m}\right)=\mathrm{t}(\mathbf{w})=\mathrm{t}\left(\mathbf{w}^{\prime}\right)=\mathrm{t}\left(\mathbf{w}_{m}^{\prime}\right)$ by Lemma 17.6(i), it follows that $k<m$ and $\mathbf{q} \neq \emptyset$. Recall that $\mathrm{F}_{\mathrm{LS}}(\mathbf{w})=\mathrm{F}_{\mathrm{LS}}\left(\mathbf{w}^{\prime}\right)$ by Lemma 17.6(v). Hence if

(c) the last occurrence of $t$ in $\mathbf{w}_{k}$ is the last occurrence of $t$ in $\mathbf{w}$, so that $t \mathrm{~h}\left(\mathbf{s}_{k+1}\right) \in \mathrm{F}_{\mathrm{LS}}(\mathbf{w})$, then $t \mathrm{~h}\left(\mathbf{s}_{k+1}\right) \in \mathrm{F}_{\mathrm{LS}}\left(\mathbf{w}^{\prime}\right)$ is a contradiction because the letter in $\mathbf{w}^{\prime}$ that immediately precedes the simple letter $\mathrm{h}\left(\mathbf{s}_{k+1}\right)$ is $t^{\prime}=\mathrm{t}\left(\mathbf{w}_{k}^{\prime}\right)$. By symmetry, the same contradiction is obtained if

(d) the last occurrence of $t^{\prime}$ in $\mathbf{w}_{k}^{\prime}$ is the last occurrence of $t^{\prime}$ in $\mathbf{w}^{\prime}$.

Therefore neither (c) nor (d) holds, whence $t, t^{\prime} \in \operatorname{con}(\mathbf{q})$ by (b). Now by (a) and (II), there exist distinct letters $y_{1}, \ldots, y_{\ell}, z_{1}, \ldots, z_{s} \in\left\{x_{1}, \ldots, x_{r}\right\}$, where $\ell, s \geqslant 0$, such that

$$
\mathbf{w}_{k}=y_{1}^{2} \ldots y_{\ell}^{2}\left(t^{\prime}\right)^{2} z_{1}^{2} \ldots z_{s}^{2} t^{2}
$$

and $\mathbf{w}_{k}^{\prime}$ is a product of $y_{1}^{2}, \ldots, y_{\ell}^{2}, z_{1}^{2}, \ldots, z_{s}^{2}, t^{2},\left(t^{\prime}\right)^{2}$ in some order ending with $\left(t^{\prime}\right)^{2}$. Then

$$
\begin{aligned}
& \mathbf{w}=\mathbf{p s}_{k} y_{1}^{2} \ldots y_{\ell}^{2}\left(t^{\prime}\right)^{2} z_{1}^{2} \ldots z_{s}^{2} t^{2} \mathbf{q} \stackrel{(17.2 \mathrm{~b})}{\approx} \mathbf{p s}_{k} y_{1}^{2} \ldots y_{\ell}^{2} z_{1}^{2} \ldots z_{s}^{2}\left(t^{\prime}\right)^{2} t^{2} \mathbf{q} \\
& \stackrel{(17.2 \mathrm{~d})}{\approx} \mathbf{p s}_{k} \underbrace{y_{1}^{2} \ldots y_{\ell}^{2} z_{1}^{2} \ldots z_{s}^{2} t^{2}\left(t^{\prime}\right)^{2}}_{\mathbf{u}} \mathbf{q} \quad \text { because } t, t^{\prime} \in \operatorname{con}(\mathbf{q}) .
\end{aligned}
$$

Since $\operatorname{con}(\mathbf{u})=\operatorname{con}\left(\mathbf{w}_{k}^{\prime}\right)$ and $\mathrm{t}(\mathbf{u})=\mathrm{t}\left(\mathbf{w}_{k}^{\prime}\right)$, the procedure in Case 1 can be repeated to give $\mathbf{p s}_{k} \mathbf{u q} \stackrel{(17.2)}{\approx} \mathbf{p s}_{k} \mathbf{w}_{k}^{\prime} \mathbf{q}$.

Proof of Proposition 17.5. Let $S \in\left\{\mathscr{C}_{10}, \mathscr{D}_{1}\right\}$. It is routinely checked that $S$ satisfies the identities (17.2). Hence it suffices to show that any identity $\mathbf{w} \approx \mathbf{w}^{\prime}$ satisfied by $S$ is implied by the identities (17.2). If either $\mathbf{w}$ or $\mathbf{w}^{\prime}$ is a simple word, then it follows from Lemma 17.6 parts (ii) and (iv) that the identity $\mathbf{w} \approx \mathbf{w}^{\prime}$ is trivial and so is vacuously implied by (17.2). 
Therefore assume that $\mathbf{w}$ and $\mathbf{w}^{\prime}$ are both non-simple and so by Lemma 17.7, can be chosen to be in canonical form. Hence

$$
\mathbf{w}=\prod_{i=1}^{m}\left(\mathbf{s}_{i} \mathbf{w}_{i}\right) \quad \text { and } \quad \mathbf{w}^{\prime}=\prod_{i=1}^{m^{\prime}}\left(\mathbf{s}_{i}^{\prime} \mathbf{w}_{i}^{\prime}\right) .
$$

Since $\mathbf{s}_{1} \ldots \mathbf{s}_{m}=\mathbf{w}_{\text {sim }}=\mathbf{w}_{\text {sim }}^{\prime}=\mathbf{s}_{1}^{\prime} \ldots \mathbf{s}_{m^{\prime}}^{\prime}$ by Lemma 17.6(iv), it follows from Lemma 17.6(vi) that $m=m^{\prime}$ and $\mathbf{s}_{i}=\mathbf{s}_{i}^{\prime}$ for all $i$, that is, $\mathbf{w}^{\prime}=\prod_{i=1}^{m}\left(\mathbf{s}_{i} \mathbf{w}_{i}^{\prime}\right)$. By Lemma 17.9, the identities (17.2) imply the identity $\mathbf{w} \approx \mathbf{w}^{\prime}$.

\section{8. $\mathscr{C}_{7}$}

This section establishes the finite basis property of the following semigroup.

\begin{tabular}{c|cccccc}
$\mathscr{C}_{7}$ & 1 & 2 & 3 & 4 & 5 & 6 \\
\hline 1 & 1 & 1 & 1 & 1 & 1 & 1 \\
2 & 1 & 1 & 1 & 1 & 1 & 2 \\
3 & 1 & 1 & 1 & 1 & 1 & 3 \\
4 & 1 & 1 & 2 & 1 & 4 & 1 \\
5 & 1 & 1 & 3 & 1 & 5 & 1 \\
6 & 1 & 2 & 1 & 4 & 1 & 6
\end{tabular}

Proposition 18.1. The variety generated by $\mathscr{C}_{7}$ is defined by the identities

$$
\begin{aligned}
x^{3} \approx x^{2}, \quad x^{2} y x & \approx x y x, \quad x y x^{2} \approx x y x, \\
x^{2} y^{2} & \approx y^{2} x^{2}, \\
h x^{2} y h & \approx h y x^{2} h, \\
h x h y h & \approx h y h x h, \\
x \mathrm{H} y \mathrm{~K} x \mathrm{\top} y & \approx x \mathrm{H} y \mathrm{~K} x \mathrm{\top} y x, \\
x \mathrm{H} y \mathrm{~K} x \mathrm{\top} y & \approx y x \mathrm{H} y \mathrm{~K} x \mathrm{\top} y .
\end{aligned}
$$

The proof of Proposition 18.1 is given in $\S 18.3$.

18.1. A canonical form

Lemma 18.2. Let $\mathbf{w}=\mathbf{s}_{1} \mathbf{w}_{1} \mathbf{s}_{2}$ with $\mathbf{s}_{1}, \mathbf{s}_{2} \in \mathcal{X}^{*}$ and $\mathbf{w}_{1} \in \mathcal{X}^{+}$. Suppose that the letters of $\mathbf{w}_{1}$ are non-simple in $\mathbf{w}$ with $\operatorname{con}\left(\mathbf{w}_{1}\right)=\left\{x_{1}, \ldots, x_{r}\right\}$. Then for any permutation $\pi$ on $\{1, \ldots, r\}$, the identities (18.1) imply the identity

$$
\mathbf{s}_{1} \mathbf{w}_{1} \mathbf{s}_{2} \approx \mathbf{s}_{1} x_{1 \pi}^{2} \ldots x_{r \pi}^{2} \mathbf{s}_{2} .
$$

Proof. Suppose $\mathbf{w}_{1}=y_{1} \ldots y_{m}$ where $y_{1}, \ldots, y_{m} \in\left\{x_{1}, \ldots, x_{r}\right\}$. Then since $y_{1}, \ldots, y_{m}$ are non-simple letters in $\mathbf{w}$,

$$
\begin{gathered}
\mathbf{s}_{1} \mathbf{w}_{1} \mathbf{s}_{2} \stackrel{(18.1 \mathrm{a})}{\stackrel{\sim}{\approx}} \mathbf{s}_{1} y_{1}^{2} \ldots y_{m}^{2} \mathbf{s}_{2} \stackrel{(18.1 \mathrm{~b})}{\approx} \mathbf{s}_{1} x_{1 \pi}^{2 e_{1}} \ldots x_{r \pi}^{2 e_{r}} \mathbf{s}_{2} \quad \text { for some } e_{1}, \ldots, e_{r} \geqslant 1 \\
\stackrel{(18.1 \mathrm{a})}{\approx} \mathbf{s}_{1} x_{1 \pi}^{2} \ldots x_{r \pi}^{2} \mathbf{s}_{2} .
\end{gathered}
$$

Therefore the identities (18.1) imply the identity (18.2).

Corollary 18.3. Suppose that $\mathbf{w}$ and $\mathbf{w}^{\prime}$ are any words such that $\operatorname{con}(\mathbf{w})=\operatorname{con}\left(\mathbf{w}^{\prime}\right)$ and $\operatorname{sim}(\mathbf{w})=\operatorname{sim}\left(\mathbf{w}^{\prime}\right)=\emptyset$. Then the identities (18.1) imply the identity $\mathbf{w} \approx \mathbf{w}^{\prime}$. 
Lemma 18.4. Let $\mathbf{w}=\mathbf{s}_{1} \mathbf{w}_{1} \mathbf{s}_{2} \mathbf{w}_{2} \mathbf{s}_{3}$ where the letters of $\mathbf{s}_{1}, \mathbf{s}_{3} \in \mathcal{X}^{*}$ and $\mathbf{s}_{2} \in \mathcal{X}^{+}$are simple in $\mathbf{w}$ and the letters of $\mathbf{w}_{1}, \mathbf{w}_{2} \in \mathcal{X}^{+}$are non-simple in $\mathbf{w}$. Suppose $\operatorname{con}\left(\mathbf{w}_{1}\right) \cap \operatorname{con}\left(\mathbf{w}_{2}\right) \neq \emptyset$. Then the identities (18.1) imply the identity

$$
\mathbf{s}_{1} \mathbf{w}_{1} \mathbf{s}_{2} \mathbf{w}_{2} \mathbf{s}_{3} \approx \mathbf{s}_{1} \mathbf{w}_{1} \mathbf{w}_{2} \mathbf{s}_{2} \mathbf{w}_{1} \mathbf{w}_{2} \mathbf{s}_{3}
$$

Proof. Generality is not lost by assuming

$$
\operatorname{con}\left(\mathbf{w}_{1}\right)=\left\{x_{1}, \ldots, x_{k}, y_{1}, \ldots, y_{m}\right\} \quad \text { and } \operatorname{con}\left(\mathbf{w}_{2}\right)=\left\{x_{1}, \ldots, x_{k}, z_{1}, \ldots, z_{n}\right\},
$$

where $k \geqslant 1$ and $m, n \geqslant 0$ with $y_{i} \neq z_{j}$ for all $i$ and $j$. For brevity, write $\mathbf{y}=y_{1}^{2} \ldots y_{m}^{2}$ and $\mathbf{z}=z_{1}^{2} \ldots z_{n}^{2}$. Then

$$
\begin{aligned}
& \mathbf{s}_{1} \mathbf{w}_{1} \mathbf{s}_{2} \mathbf{w}_{2} \mathbf{s}_{3} \stackrel{(18.2)}{\approx} \mathbf{s}_{1} \cdot x_{1}^{2} \ldots x_{k}^{2} \mathbf{y} \cdot \mathbf{s}_{2} \cdot x_{1}^{2} \ldots x_{k-1}^{2} x_{k}^{2} \mathbf{z} \cdot \mathbf{s}_{3} \\
& \stackrel{(18.2)}{\approx} \mathbf{s}_{1} \cdot \mathbf{y} x_{1}^{2} \ldots x_{k}^{2} \mathbf{y}^{2} \cdot \mathbf{s}_{2} \cdot \mathbf{z} x_{1}^{2} \ldots x_{k-1}^{2} x_{k}^{2} \mathbf{z}^{2} \cdot \mathbf{s}_{3} \\
& \stackrel{(18.1 \mathrm{c})}{\approx} \mathbf{s}_{1} \cdot \mathbf{y} x_{1}^{2} \ldots x_{k}^{2} \cdot \mathbf{s}_{2} \cdot \mathbf{z} x_{1}^{2} \ldots x_{k-1}^{2} \mathbf{y}^{2} x_{k}^{2} \mathbf{z}^{2} \cdot \mathbf{s}_{3} \\
& \stackrel{(18.1 \mathrm{~b})}{\stackrel{(16}{\approx}} \mathbf{s}_{1} \cdot \mathbf{y} x_{1}^{2} \ldots x_{k}^{2} \cdot \mathbf{s}_{2} \cdot \mathbf{z} x_{1}^{2} \ldots x_{k-1}^{2} \mathbf{y}^{2} \mathbf{z}^{2} x_{k}^{2} \cdot \mathbf{s}_{3} \\
& \stackrel{(18.1 \mathrm{c})}{\approx} \mathbf{s}_{1} \cdot \mathbf{y} x_{1}^{2} \ldots x_{k}^{2} \mathbf{z}^{2} \cdot \mathbf{s}_{2} \cdot \mathbf{z} x_{1}^{2} \ldots x_{k-1}^{2} \mathbf{y}^{2} x_{k}^{2} \cdot \mathbf{s}_{3} \\
& \stackrel{(18.2)}{\approx} \mathbf{s}_{1} \cdot x_{1}^{2} \ldots x_{k}^{2} \mathbf{y z} \cdot \mathbf{s}_{2} \cdot x_{1}^{2} \ldots x_{k}^{2} \mathbf{y z} \cdot \mathbf{s}_{3} \\
& \stackrel{(18.2)}{\approx} \mathbf{s}_{1} \mathbf{w}_{1} \mathbf{w}_{2} \mathbf{s}_{2} \mathbf{w}_{1} \mathbf{w}_{2} \mathbf{s}_{3} .
\end{aligned}
$$

Therefore the identities (18.1) imply the identity (18.3).

Let $\mathbf{w}$ be any non-simple word such that $\operatorname{sim}(\mathbf{w}) \neq \emptyset$ and $\mathrm{h}(\mathbf{w}), \mathrm{t}(\mathbf{w}) \notin \operatorname{sim}(\mathbf{w})$, and let $x_{1}, \ldots, x_{r}$ be the distinct non-simple letters of $\mathbf{w}$ listed in alphabetical order. In this section, such a word $\mathbf{w}$ is said to be in canonical form if

$$
\mathbf{w}=\mathbf{w}_{0} \prod_{i=1}^{m}\left(\mathbf{s}_{i} \mathbf{w}_{i}\right)
$$

for some $m \geqslant 1$, where the following are satisfied:

(I) the letters of $\mathbf{s}_{1}, \ldots, \mathbf{s}_{m} \in \mathcal{X}^{+}$are simple in $\mathbf{w}$;

(II) $\mathbf{w}_{0}, \ldots, \mathbf{w}_{m} \in\left\{x_{n_{1}}^{2} \ldots x_{n_{k}}^{2} \mid 1 \leqslant n_{1}<\ldots<n_{k} \leqslant r\right.$ and $\left.k \geqslant 1\right\}$;

(III) if $i \neq j$, then either $\mathbf{w}_{i}=\mathbf{w}_{j}$ or $\operatorname{con}\left(\mathbf{w}_{i}\right) \cap \operatorname{con}\left(\mathbf{w}_{j}\right)=\emptyset$;

(IV) if $\mathbf{w}_{i}=\mathbf{w}_{k}$ and $\mathbf{w}_{j}=\mathbf{w}_{\ell}$ where $i<j<k<\ell$, then $\mathbf{w}_{i}=\mathbf{w}_{j}=\mathbf{w}_{k}=\mathbf{w}_{\ell}$.

Lemma 18.5. Let $\mathbf{w}$ be any non-simple word such that $\operatorname{sim}(\mathbf{w}) \neq \emptyset$. Suppose that $\mathrm{h}(\mathbf{w})$ and $\mathrm{t}(\mathbf{w})$ are non-simple letters of $\mathbf{w}$. Then there exists some word $\overline{\mathbf{w}}$ in canonical form such that the identities (18.1) imply the identity $\mathbf{w} \approx \overline{\mathbf{w}}$.

Proof. Suppose that $x_{1}, \ldots, x_{r}$ are the distinct non-simple letters of $\mathbf{w}$ listed in alphabetical order. By Lemmas 18.2 and 18.4, it suffices to convert w, using the identities (18.1)-(18.3), into a word in canonical form. It is easily seen that $\mathbf{w}$ can be written as a word of the form $\mathbf{w}=\mathbf{w}_{0} \prod_{i=1}^{m}\left(\mathbf{s}_{i} \mathbf{w}_{i}\right)$ with (I) satisfied and $\mathbf{w}_{i} \in\left\{x_{1}, \ldots, x_{r}\right\}^{+}$. The letters of $\mathbf{w}_{i}$ are non-simple in $\mathbf{w}$, so that (II) is satisfied by applying the identity (18.2).

Suppose that the factors $\mathbf{w}_{i}$ and $\mathbf{w}_{j}$, where $i<j$, do not satisfy (III). Then $\mathbf{w}_{i} \neq \mathbf{w}_{j}$ and $\operatorname{con}\left(\mathbf{w}_{i}\right) \cap \operatorname{con}\left(\mathbf{w}_{j}\right) \neq \emptyset$. Generality is not lost by assuming $\operatorname{con}\left(\mathbf{w}_{i} \mathbf{w}_{j}\right)=\left\{x_{n_{1}}, \ldots, x_{n_{k}}\right\}$ where 
$1 \leqslant n_{1}<\ldots<n_{k} \leqslant r$. Since

$$
\begin{aligned}
\mathbf{w} & =\ldots \mathbf{s}_{i} \mathbf{w}_{i} \ldots \mathbf{s}_{j} \mathbf{w}_{j} \ldots \quad\left(\text { the prefix } \ldots \mathbf{s}_{i} \text { is empty if } i=0\right) \\
\stackrel{(18.3)}{\approx} & \ldots \mathbf{s}_{i} \mathbf{w}_{i} \mathbf{w}_{j} \ldots \mathbf{s}_{j} \mathbf{w}_{i} \mathbf{w}_{j} \ldots \\
\stackrel{(18.2)}{\approx} & \ldots \mathbf{s}_{i}\left(x_{n_{1}}^{2} \ldots x_{n_{k}}^{2}\right) \ldots \mathbf{s}_{j}\left(x_{n_{1}}^{2} \ldots x_{n_{k}}^{2}\right) \ldots,
\end{aligned}
$$

the factors $\mathbf{w}_{i}$ and $\mathbf{w}_{j}$ are converted by the identities $\{(18.2),(18.3)\}$ into the same word $x_{n_{1}}^{2} \ldots x_{n_{k}}^{2}$. Hence (III) is satisfied.

Suppose that $\mathbf{w}_{i}, \mathbf{w}_{j}, \mathbf{w}_{k}$, and $\mathbf{w}_{\ell}$, where $i<j<k<\ell$, violate (IV). Then $\mathbf{w}_{i}=\mathbf{w}_{k}=\mathbf{a}$ and $\mathbf{w}_{j}=\mathbf{w}_{\ell}=\mathbf{b}$ with $\mathbf{a} \neq \mathbf{b}$. Generality is not lost by assuming con(ab) $=\left\{x_{n_{1}}, \ldots, x_{n_{k}}\right\}$ where $1 \leqslant n_{1}<\ldots<n_{k} \leqslant r$. Then

$$
\begin{aligned}
\mathbf{w} & \left.=\ldots \mathbf{s}_{i} \mathbf{w}_{i} \ldots \mathbf{s}_{j} \mathbf{w}_{j} \ldots \mathbf{s}_{k} \mathbf{w}_{k} \ldots \mathbf{s}_{\ell} \mathbf{w}_{\ell} \ldots \quad \text { (the prefix } \ldots \mathbf{s}_{i} \text { is empty if } i=0\right) \\
& =\ldots \mathbf{s}_{i} \mathbf{a} \ldots \mathbf{s}_{j} \mathbf{b} \ldots \mathbf{s}_{k} \mathbf{a} \ldots \mathbf{s}_{\ell} \mathbf{b} \ldots \\
\stackrel{(18.1 \mathrm{e})}{\approx} & \ldots \mathbf{s}_{i} \mathbf{a} \ldots \mathbf{s}_{j} \mathbf{b} \ldots \mathbf{s}_{k} \mathbf{a} \ldots \mathbf{s}_{\ell} \mathbf{b} \mathbf{b} \ldots \\
\stackrel{(18.1 \mathrm{f})}{\approx} & \ldots \mathbf{s}_{i} \mathbf{b} \mathbf{b} \ldots \mathbf{s}_{j} \mathbf{b} \ldots \mathbf{s}_{k} \mathbf{a} \ldots \mathbf{s}_{\ell} \mathbf{b a} \ldots \\
\stackrel{(18.3)}{\approx} & \ldots \mathbf{s}_{i} \mathbf{b} \mathbf{b} \mathbf{b} \ldots \mathbf{s}_{j} \mathbf{b a b} \ldots \mathbf{s}_{k} \mathbf{a} \ldots \mathbf{s}_{\ell} \mathbf{b} \mathbf{b} \ldots \quad \text { since } \operatorname{con}(\mathbf{b a}) \cap \operatorname{con}(\mathbf{b}) \neq \emptyset \\
\stackrel{(18.3)}{\approx} & \ldots \mathbf{s}_{i} \mathbf{b a b} \ldots \mathbf{s}_{j} \mathbf{b a b} \ldots \mathbf{s}_{k} \mathbf{a b a} \ldots \mathbf{s}_{\ell} \mathbf{a b a} \ldots \quad \text { since con }(\mathbf{a}) \cap \operatorname{con}(\mathbf{b a}) \neq \emptyset \\
\stackrel{(18.2)}{\approx} & \ldots \mathbf{s}_{i} \mathbf{c} \ldots \mathbf{s}_{j} \mathbf{c} \ldots \mathbf{s}_{k} \mathbf{c} \ldots \mathbf{s}_{\ell} \mathbf{c} \ldots \quad \text { where } \mathbf{c}=x_{n_{1}}^{2} \ldots x_{n_{k}}^{2} .
\end{aligned}
$$

Hence the factors $\mathbf{w}_{i}, \mathbf{w}_{j}, \mathbf{w}_{k}$ and $\mathbf{w}_{\ell}$ are converted by the identities (18.1)-(18.3) into the same word $\mathbf{c}$, whence (IV) is no longer violated.

LEMMA 18.6. Let $\mathbf{w}$ be any product of pairwise disjoint connected words with $\operatorname{sim}(\mathbf{w}) \neq \emptyset$. Then there exists some connected word $\overline{\mathbf{w}}=\mathbf{w}_{0} \prod_{i=1}^{m}\left(\mathbf{s}_{i} \mathbf{w}_{i}\right)$ in canonical form, with $\mathbf{w}_{0}=\mathbf{w}_{m}$, such that the identities (18.1) imply the identity $\mathbf{w} \approx \overline{\mathbf{w}}$.

Proof. By Lemmas 18.2 and 18.4, it suffices to convert w, using the identities (18.1)-(18.3), into a connected word in canonical form. First suppose that $\mathbf{w}$ is a single connected word with $\operatorname{sim}(\mathbf{w}) \neq \emptyset$. Then there exists a sequence $\mathrm{h}(\mathbf{w})=x_{1}, x_{2}, \ldots, x_{m}=\mathrm{t}(\mathbf{w})$ of non-simple letters of $\mathbf{w}$ occurring in an overlapping pattern such that the first $x_{i+1}$ occurs between two occurrences of $x_{i}$ and the last $x_{i+1}$ occurs to the right of all occurrences of $x_{i}$. This sequence can be chosen to have minimal length among all such sequences, so that

$$
\mathbf{w}=x_{1} \mathbf{p}_{1} x_{2} \mathbf{p}_{2} x_{1} \mathbf{q}_{1} x_{3} \mathbf{p}_{3} x_{2} \mathbf{q}_{2} x_{4} \mathbf{p}_{4} x_{3} \mathbf{q}_{3} \ldots \mathbf{q}_{m-1} x_{m}
$$

for some $\mathbf{p}_{1}, \ldots, \mathbf{p}_{m}, \mathbf{q}_{1}, \ldots, \mathbf{q}_{m-1} \in \mathcal{X}^{*}$. (Note that the factor $\mathbf{p}_{i}$ follows the first $x_{i}$, the factor $\mathbf{q}_{i}$ follows the last $x_{i}$, and at least one simple letter of $\mathbf{w}$ belongs to some $\mathbf{p}_{i}$ or $\mathbf{q}_{i}$.) Then

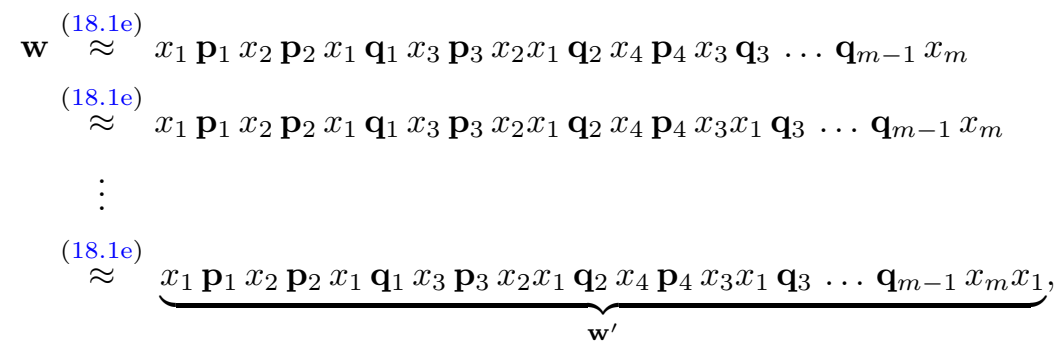


where $\mathbf{w}^{\prime}$ is a word that satisfies $\operatorname{sim}\left(\mathbf{w}^{\prime}\right) \neq \emptyset$ and $\mathrm{h}\left(\mathbf{w}^{\prime}\right)=\mathrm{t}\left(\mathbf{w}^{\prime}\right)$. Repeat the proof of Lemma 18.5 to convert $\mathbf{w}^{\prime}$, using the identities (18.1)-(18.3), into a word $\mathbf{w}=\mathbf{w}_{0} \prod_{i=1}^{m}\left(\mathbf{s}_{i} \mathbf{w}_{i}\right)$ in canonical form. The letter $x_{1}$ is easily seen to belong to both $\mathbf{w}_{0}$ and $\mathbf{w}_{m}$, so that $\mathbf{w}_{0}=\mathbf{w}_{m}$ by (III). It follows that $\overline{\mathbf{w}}$ is connected.

Now suppose that $\mathbf{w}$ is a product of pairwise disjoint connected words with $\operatorname{sim}(\mathbf{w}) \neq \emptyset$. Then $\mathbf{w}=\mathbf{c}_{1} \ldots \mathbf{c}_{n}$ where $\mathbf{c}_{1}, \ldots, \mathbf{c}_{n}$ are pairwise disjoint connected words. For each $i$, since $\mathbf{c}_{i}$ is connected, the letters $h_{i}=\mathrm{h}\left(\mathbf{c}_{i}\right)$ and $t_{i}=\mathrm{t}\left(\mathbf{c}_{i}\right)$ are non-simple in $\mathbf{c}_{i}$. Therefore

$$
\begin{gathered}
\mathbf{w} \stackrel{(18.1 \mathrm{a})}{\approx} h_{1}^{2} \mathbf{c}_{1} t_{1}^{2} \cdot h_{2}^{2} \mathbf{c}_{2} t_{2}^{2} \cdot h_{3}^{2} \mathbf{c}_{3} t_{3}^{2} \ldots h_{n-1}^{2} \mathbf{c}_{n-1} t_{n-1}^{2} \cdot h_{n}^{2} \mathbf{c}_{n} t_{n}^{2} \\
\stackrel{(18.1 \mathrm{~b})}{\approx} \underbrace{h_{1}^{2} \mathbf{c}_{1} h_{2}^{2} \cdot t_{1}^{2} \mathbf{c}_{2} h_{3}^{2} \cdot t_{2}^{2} \mathbf{c}_{3} h_{4}^{2} \ldots t_{n-2}^{2} \mathbf{c}_{n-1} h_{n}^{2} \cdot t_{n-1}^{2} \mathbf{c}_{n} t_{n}^{2}}_{\mathbf{w}^{\prime \prime}},
\end{gathered}
$$

that is, the word $\mathbf{w}$ is converted by the identities (18.1) into the connected word $\mathbf{w}^{\prime \prime}$ with $\operatorname{sim}\left(\mathbf{w}^{\prime \prime}\right) \neq \emptyset$. The procedure in the previous paragraph can then be repeated to convert $\mathbf{w}^{\prime \prime}$ into a word with the required properties.

\subsection{Identities satisfied by $\mathscr{C}_{7}$}

Lemma 18.7. Suppose that $\mathbf{w} \approx \mathbf{w}^{\prime}$ is any identity satisfied by $\mathscr{C}_{7}$. Then:

(i) $\operatorname{con}(\mathbf{w})=\operatorname{con}\left(\mathbf{w}^{\prime}\right)$ and $\operatorname{sim}(\mathbf{w})=\operatorname{sim}\left(\mathbf{w}^{\prime}\right)$;

(ii) $\mathrm{F}_{\mathrm{SS}}(\mathbf{w})=\mathrm{F}_{\mathrm{SS}}\left(\mathbf{w}^{\prime}\right)$.

Proof. (i) This follows from Lemma $2.1(\mathrm{v})$ because the subsemigroup $\{1,2,6\}$ of $\mathscr{C}_{7}$ is isomorphic to $N_{2}^{1}$.

(ii) Since $6^{2} \cdot 4 \cdot 6^{2} \cdot 3 \cdot 6^{2} \neq 6^{2} \cdot 4 \cdot 3 \cdot 6^{2}$ in $\mathscr{C}_{7}$, the identity (2.3) with $n=2$ is not satisfied by $\mathscr{C}_{7}$. Therefore $\mathrm{F}_{\mathrm{SS}}(\mathbf{w})=\mathrm{F}_{\mathrm{SS}}\left(\mathbf{w}^{\prime}\right)$ by Lemma 2.10 and part (i).

For any word

$$
\mathbf{w}=\mathbf{w}_{0} \prod_{i=1}^{m}\left(\mathbf{s}_{i} \mathbf{w}_{i}\right)
$$

in canonical form, it is convenient to call $\mathbf{s}_{1}, \ldots, \mathbf{s}_{m}$ the maximal simple factors of $\mathbf{w}$, and call $\mathbf{w}_{0}, \ldots, \mathbf{w}_{m}$ the maximal non-simple factors of $\mathbf{w}$. Recall from (III) that any two maximal non-simple factors of $\mathbf{w}$ are either identical or disjoint.

For any distinct maximal non-simple factors $\mathbf{a}$ and $\mathbf{b}$ of the word $\mathbf{w}$ in (18.4), write $\mathbf{a} \Subset_{\mathbf{w}} \mathbf{b}$ to indicate that within $\mathbf{w}$, all occurrences of $\mathbf{a}$ are sandwiched between two occurrences of $\mathbf{b}$. Note that if $\mathbf{a} \Subset_{\mathbf{w}} \mathbf{b}$, then (IV) implies that no occurrence of $\mathbf{b}$ is sandwiched between any two occurrences of $\mathbf{a}$.

Lemma 18.8. Suppose that $\mathbf{w} \approx \mathbf{w}^{\prime}$ is any identity satisfied by $\mathscr{C}_{7}$, where

$$
\mathbf{w}=\mathbf{w}_{0} \prod_{i=1}^{m}\left(\mathbf{s}_{i} \mathbf{w}_{i}\right) \quad \text { and } \quad \mathbf{w}^{\prime}=\mathbf{w}_{0}^{\prime} \prod_{i=1}^{m^{\prime}}\left(\mathbf{s}_{i}^{\prime} \mathbf{w}_{i}^{\prime}\right)
$$

are in canonical form. Then:

(i) $m=m^{\prime}$;

(ii) $\left(\mathbf{s}_{1}, \ldots, \mathbf{s}_{m}\right)=\left(\mathbf{s}_{1 \pi}^{\prime}, \ldots, \mathbf{s}_{m \pi}^{\prime}\right)$ for some permutation $\pi$ on $\{1, \ldots, m\}$;

(iii) $\left(\mathbf{w}_{0}, \ldots, \mathbf{w}_{m}\right)=\left(\mathbf{w}_{0 \tau}^{\prime}, \ldots, \mathbf{w}_{m \tau}^{\prime}\right)$ for some permutation $\tau$ on $\{0, \ldots, m\}$;

(iv) $\mathbf{s}_{i} \mathbf{w}_{j}$ is a factor of $\mathbf{w}$ if and only if $\mathbf{s}_{i} \mathbf{w}_{j}$ is a factor of $\mathbf{w}^{\prime}$;

(v) $\mathbf{w}_{i} \mathbf{s}_{j}$ is a factor of $\mathbf{w}$ if and only if $\mathbf{w}_{i} \mathbf{s}_{j}$ is a factor of $\mathbf{w}^{\prime}$. 
Further, if the words $\mathbf{w}$ and $\mathbf{w}^{\prime}$ are connected, then

(vi) $\mathbf{w}_{0}=\mathbf{w}_{m}=\mathbf{w}_{0}^{\prime}=\mathbf{w}_{m}^{\prime}$.

Proof. Parts (i) and (ii) follow from Lemma 18.7.

Suppose that $x^{2}$ and $y^{2}$ are factors of different maximal non-simple factors of $\mathbf{w}$. Generality is not lost by assuming that $x^{2}$ is a factor of $\mathbf{a}$ and $y^{2}$ is a factor of $\mathbf{b}$ with $\mathbf{b} \notin_{\mathbf{w}}$ a. Let $p$ be the least integer such that $\mathbf{w}_{p}=\mathbf{a}$ and let $q$ be the greatest integer such that $\mathbf{w}_{q}=\mathbf{a}$. If $\mathbf{w}_{i}=\mathbf{b}$ for some $i$ with $p<i<q$, then it follows from (IV) that all occurrences of $\mathbf{b}$ are sandwiched between $\mathbf{w}_{p}=\mathbf{a}$ and $\mathbf{w}_{q}=\mathbf{a}$, whence the contradiction $\mathbf{b} \Subset_{\mathbf{w}} \mathbf{a}$ is deduced. Therefore $\mathbf{b} \notin\left\{\mathbf{w}_{p}, \ldots, \mathbf{w}_{q}\right\}$, so that $\mathbf{b} \in\left\{\mathbf{w}_{0}, \ldots, \mathbf{w}_{p-1}\right\} \cup\left\{\mathbf{w}_{q+1}, \ldots, \mathbf{w}_{m}\right\}$, whence $(p, q) \neq$ $(0, m)$. Let $\varphi_{1}: \mathcal{X} \rightarrow \mathscr{C}_{7}$ denote the substitution

$$
z \mapsto \begin{cases}4 & \text { if } z=\mathrm{h}\left(\mathbf{s}_{p}\right) \\ 3 & \text { if } z=\mathrm{t}\left(\mathbf{s}_{q+1}\right) \\ 5 & \text { if } z \in \operatorname{con}\left(\mathbf{s}_{p} \mathbf{w}_{p} \mathbf{s}_{p+1} \mathbf{w}_{p+1} \ldots \mathbf{s}_{q} \mathbf{w}_{q} \mathbf{s}_{q+1}\right) \text { and } z \neq \mathrm{h}\left(\mathbf{s}_{p}\right), \mathrm{t}\left(\mathbf{s}_{q+1}\right), \\ 6 & \text { otherwise. }\end{cases}
$$

(Note that $\mathbf{s}_{p}=\emptyset$ if $p=0$, and $\mathbf{s}_{q+1}=\emptyset$ if $q=m$.) If $p=0$ and $q<m$, then

$$
\begin{aligned}
\mathbf{w} \varphi_{1} & =\left(\mathbf{w}_{0} \prod_{i=1}^{q}\left(\mathbf{s}_{i} \mathbf{w}_{i}\right)\right) \varphi_{1} \cdot \mathbf{s}_{q+1} \varphi_{1} \cdot\left(\mathbf{w}_{q+1} \prod_{i=q+2}^{m}\left(\mathbf{s}_{i} \mathbf{w}_{i}\right)\right) \varphi_{1} \\
& =(5 \ldots 5) \cdot(5 \ldots 5 \cdot 3) \cdot(6 \ldots 6)=3 .
\end{aligned}
$$

If $0<p$ and $q=m$, then

$$
\begin{aligned}
\mathbf{w} \varphi_{1} & =\left(\mathbf{w}_{0} \prod_{i=1}^{p-1}\left(\mathbf{s}_{i} \mathbf{w}_{i}\right)\right) \varphi_{1} \cdot \mathbf{s}_{p} \varphi_{1} \cdot\left(\mathbf{w}_{p} \prod_{i=p+1}^{m}\left(\mathbf{s}_{i} \mathbf{w}_{i}\right)\right) \varphi_{1} \\
& =(6 \ldots 6) \cdot(4 \cdot 5 \ldots 5) \cdot(5 \ldots 5)=4 .
\end{aligned}
$$

If $0<p$ and $q<m$, then

$$
\begin{aligned}
\mathbf{w} \varphi_{1} & =\left(\mathbf{w}_{0} \prod_{i=1}^{p-1}\left(\mathbf{s}_{i} \mathbf{w}_{i}\right)\right) \varphi_{1} \cdot \mathbf{s}_{p} \varphi_{1} \cdot\left(\mathbf{w}_{p} \prod_{i=p+1}^{q}\left(\mathbf{s}_{i} \mathbf{w}_{i}\right)\right) \varphi_{1} \cdot \mathbf{s}_{q+1} \varphi_{1} \cdot\left(\mathbf{w}_{q+1} \prod_{i=q+2}^{m}\left(\mathbf{s}_{i} \mathbf{w}_{i}\right)\right) \varphi_{1} \\
& =(6 \ldots 6) \cdot(4 \cdot 5 \ldots 5) \cdot(5 \ldots 5) \cdot(5 \ldots 5 \cdot 3) \cdot(6 \ldots 6)=2 .
\end{aligned}
$$

Therefore $\mathbf{w} \varphi_{1} \neq 1$. Now it follows from Lemma 18.7(i), (I), and (II) that $x^{2}$ and $y^{2}$ are factors of $\mathbf{w}^{\prime}$. If $x^{2}$ and $y^{2}$ are factors of the same maximal non-simple factor of $\mathbf{w}^{\prime}$, say $\mathbf{w}_{k}^{\prime}=\ldots x^{2} \ldots y^{2} \ldots$, then

$$
\mathbf{w}^{\prime} \varphi_{1} \in \ldots \underbrace{\{5,6\}^{*} \cdot 5^{2} \cdot\{5,6\}^{*} \cdot 6^{2} \cdot\{5,6\}^{*}}_{\mathbf{w}_{k}^{\prime} \varphi_{1}} \ldots=\{1\}
$$

is a contradiction. Therefore $x^{2}$ and $y^{2}$ are factors of different maximal non-simple factors of $\mathbf{w}^{\prime}$. By symmetry, if $x^{2}$ and $y^{2}$ are factors of different maximal non-simple factors of $\mathbf{w}^{\prime}$, then they are factors of different maximal non-simple factors of $\mathbf{w}$. Consequently,

(a) $x^{2}$ and $y^{2}$ are factors of the same $\mathbf{w}_{i}$ if and only if $x^{2}$ and $y^{2}$ are factors of the same $\mathbf{w}_{j}^{\prime}$.

Let $x^{2} y^{2}$ be a factor of some $\mathbf{w}_{i}$. Then $\mathbf{w}_{i}=\ldots x^{2} y^{2} \ldots$ and $x$ alphabetically precedes $y$ by (II). By (a), the words $x^{2}$ and $y^{2}$ are factors of some $\mathbf{w}_{j}^{\prime}$. Suppose that $x^{2} y^{2}$ is not a factor of $\mathbf{w}_{j}^{\prime}$. Then $\mathbf{w}_{j}^{\prime}=\ldots x^{2} \cdot z_{1}^{2} z_{2}^{2} \ldots z_{k}^{2} \cdot y^{2} \ldots$ for some letters $z_{1}, z_{2}, \ldots, z_{k}$ such that 
$x, z_{1}, z_{2}, \ldots, z_{k}, y$ are in alphabetical order. By (II), the letter $z_{1}$ cannot belong to $\mathbf{w}_{i}$, so that $x^{2}$ and $z_{1}^{2}$ are factors of different maximal non-simple factors of $\mathbf{w}$. But this contradicts (a) since $x^{2}$ and $z_{1}^{2}$ are factors of $\mathbf{w}_{j}^{\prime}$. Therefore $x^{2} y^{2}$ is a factor of $\mathbf{w}_{j}^{\prime}$. By symmetry, if $x^{2} y^{2}$ is a factor of some $\mathbf{w}_{j}^{\prime}$, then it is a factor of some $\mathbf{w}_{i}$. Consequently, $x^{2} y^{2}$ is a factor of some $\mathbf{w}_{i}$ if and only if $x^{2} y^{2}$ is a factor of some $\mathbf{w}_{j}^{\prime}$. It then follows from Lemma 18.7(i), (I), and (II) that $\mathbf{w}$ and $\mathbf{w}^{\prime}$ share the same set of maximal non-simple factors, that is,

(b) $\bigcup_{i=0}^{m}\left\{\mathbf{w}_{i}\right\}=\bigcup_{i=0}^{m}\left\{\mathbf{w}_{i}^{\prime}\right\}$.

Let $\mathbf{s}_{i} \mathbf{a}$ be a factor of $\mathbf{w}$ and $\mathbf{s}_{i} \mathbf{b}$ be a factor of $\mathbf{w}^{\prime}$, where $\mathbf{a}, \mathbf{b} \in \bigcup_{i=0}^{m}\left\{\mathbf{w}_{i}\right\}$. Suppose $\mathbf{a} \neq \mathbf{b}$. Choose any letter $z \notin \operatorname{con}(\mathbf{w})=\operatorname{con}\left(\mathbf{w}^{\prime}\right)$ that alphabetically precedes all of $x_{1}, \ldots, x_{r}$. Let $\varphi_{2}$ denote the substitution $\mathrm{t}\left(\mathbf{s}_{i}\right) \mapsto \mathrm{t}\left(\mathbf{s}_{i}\right) z^{2}$. Then $\mathbf{w} \varphi_{2}=\ldots z^{2} \mathbf{a} \ldots$ and $\mathbf{w}^{\prime} \varphi_{2}=\ldots z^{2} \mathbf{b} \ldots$ Following the proof of Lemma 18.6, the words $\mathbf{w} \varphi_{2}$ and $\mathbf{w}^{\prime} \varphi_{2}$ can be converted by the identities (18.1) into words $\overline{\mathbf{w} \varphi_{1}}$ and $\overline{\mathbf{w}^{\prime} \varphi_{2}}$ in canonical form. Since the identities (18.1) are satisfied by $\mathscr{C}_{7}$, the identity $\overline{\mathbf{w} \varphi_{2}} \approx \overline{\mathbf{w}^{\prime} \varphi_{2}}$ is also satisfied by $\mathscr{C}_{7}$. It is easily seen that $\overline{\mathbf{w} \varphi_{2}}$ is obtained from $\mathbf{w}$ by replacing all occurrences of $\mathbf{a}$ with $z^{2} \mathbf{a}$, while $\overline{\mathbf{w}^{\prime} \varphi_{2}}$ is obtained from $\mathbf{w}^{\prime}$ by replacing all occurrences of $\mathbf{b}$ with $z^{2} \mathbf{b}$. It follows that $\overline{\mathbf{w} \varphi_{2}}$ and $\overline{\mathbf{w}^{\prime} \varphi_{2}}$ do not share the same maximal non-simple factors, and this contradicts the result obtained in the previous paragraph. Therefore $\mathbf{a}=\mathbf{b}$. Consequently, for any $\mathbf{a} \in \bigcup_{i=0}^{m}\left\{\mathbf{w}_{i}\right\}=\bigcup_{i=0}^{m}\left\{\mathbf{w}_{i}^{\prime}\right\}$,

(c) $\mathbf{s}_{i} \mathbf{a}$ is a factor of $\mathbf{w}$ if and only if $\mathbf{s}_{i} \mathbf{a}$ is a factor of $\mathbf{w}^{\prime}$.

By a symmetrical argument,

(d) $\mathbf{a s}_{i}$ is a factor of $\mathbf{w}$ if and only if $\mathbf{a s}_{i}$ is a factor of $\mathbf{w}^{\prime}$.

Parts (iii)-(v) now follow from (b)-(d).

Finally, suppose that $\mathbf{w}$ and $\mathbf{w}^{\prime}$ are connected. Then $\mathbf{w}_{0}=\mathbf{w}_{m}$ and $\mathbf{w}_{0}^{\prime}=\mathbf{w}_{m}^{\prime}$ by Lemma 18.6. Suppose $\mathbf{a}=\mathbf{w}_{0}=\mathbf{w}_{m}$ and $\mathbf{b}=\mathbf{w}_{0}^{\prime}=\mathbf{w}_{m}^{\prime}$ with $\mathbf{a} \neq \mathbf{b}$. Then by picking any distinct letters $x, y, z \notin \operatorname{con}(\mathbf{w})=\operatorname{con}\left(\mathbf{w}^{\prime}\right)$, the words $\widetilde{\mathbf{w}}=x y \mathbf{w} z x$ and $\widetilde{\mathbf{w}}^{\prime}=x y \mathbf{w}^{\prime} z x$ are in canonical form such that the identity $\widetilde{\mathbf{w}} \approx \widetilde{\mathbf{w}}^{\prime}$ is satisfied by $\mathscr{C}_{7}$. Now $y \in \operatorname{sim}(\widetilde{\mathbf{w}})=\operatorname{sim}\left(\widetilde{\mathbf{w}}^{\prime}\right)$ and $y$ a is a factor of $\widetilde{\mathbf{w}}$ that is not a factor of $\widetilde{\mathbf{w}}^{\prime}$. But this is impossible by part (iv). Therefore $\mathbf{a}=\mathbf{b}$, so that part (vi) holds.

Lemma 18.9. The semigroup $\mathscr{C}_{7}$ has a basis of the form $\{(18.1)\} \cup \bar{\Sigma}$, where $\bar{\Sigma}$ is some set of identities formed by connected words in canonical form.

Proof. It is easily verified that $\mathscr{C}_{7}$ satisfies the identities (18.1) and is idempotent-separable. Let $\mathbf{V}$ denote the variety generated by $\mathscr{C}_{7}$. Then $B_{0} \in \mathbf{V}$ because the subsemigroup $\{1,3,5,6\}$ of $\mathscr{C}_{7}$ is isomorphic to $B_{0}$. The semigroup $A_{0}$ does not satisfy the identity (18.1b) because $a^{2} b^{2} \neq b^{2} a^{2}$ in $A_{0}$. Therefore $A_{0} \notin \mathbf{V}$, and it follows from Lemma 2.6(ii) that $\mathscr{C}_{7}$ has a basis $\Sigma$ that consists of identities formed by words that are products of pairwise disjoint connected words. Hence $\{(18.1)\} \cup \Sigma$ is also a basis for $\mathscr{C}_{7}$.

Let $\mathbf{w} \approx \mathbf{w}^{\prime}$ be any identity in $\Sigma$. Then $\operatorname{con}(\mathbf{w})=\operatorname{con}\left(\mathbf{w}^{\prime}\right)$ and $\operatorname{sim}(\mathbf{w})=\operatorname{sim}\left(\mathbf{w}^{\prime}\right)$ by Lemma 18.7(i). If $\operatorname{sim}(\mathbf{w})=\operatorname{sim}\left(\mathbf{w}^{\prime}\right)=\emptyset$, then by Corollary 18.3 , the identity $\mathbf{w} \approx \mathbf{w}^{\prime}$ is implied by the identities (18.1) and hence is redundant in the basis of $\mathscr{C}_{7}$. Therefore it can further be assumed that $\operatorname{sim}(\mathbf{w})=\operatorname{sim}\left(\mathbf{w}^{\prime}\right) \neq \emptyset$. By Lemma 18.6, there exist connected words $\overline{\mathbf{w}}$ and $\overline{\mathbf{w}}^{\prime}$ in canonical form such that the identities (18.1) imply the identities $\mathbf{w} \approx \overline{\mathbf{w}}$ and $\mathbf{w}^{\prime} \approx \overline{\mathbf{w}}^{\prime}$. Hence the sets $\left\{(18.1), \mathbf{w} \approx \mathbf{w}^{\prime}\right\}$ and $\left\{(18.1), \overline{\mathbf{w}} \approx \overline{\mathbf{w}}^{\prime}\right\}$ define the same variety.

Now since the identity $\mathbf{w} \approx \mathbf{w}^{\prime}$ is arbitrary in $\Sigma$, the construction of $\overline{\mathbf{w}} \approx \overline{\mathbf{w}}^{\prime}$ from $\mathbf{w} \approx \mathbf{w}^{\prime}$ in the preceding paragraph can be repeated on every other identity in $\Sigma$ to obtain the set $\bar{\Sigma}=\left\{\overline{\mathbf{w}} \approx \overline{\mathbf{w}}^{\prime} \mid \mathbf{w} \approx \mathbf{w}^{\prime} \in \Sigma\right\}$ with the property that $\{(18.1)\} \cup \bar{\Sigma}$ is a basis for $\mathscr{C}_{7}$.

\subsection{Proof of Proposition 18.1}

By Lemma 18.9, the semigroup $\mathscr{C}_{7}$ has a basis of the form $\{(18.1)\} \cup \bar{\Sigma}$ where $\bar{\Sigma}$ is some set of identities formed by connected words in canonical form. Let $\mathbf{w} \approx \mathbf{w}^{\prime}$ be any identity from $\bar{\Sigma}$. 
Then by Lemma 18.8 parts (i)-(iii),

$$
\mathbf{w}=\mathbf{w}_{0} \prod_{i=1}^{m}\left(\mathbf{s}_{i} \mathbf{w}_{i}\right) \quad \text { and } \quad \mathbf{w}^{\prime}=\mathbf{w}_{0}^{\prime} \prod_{i=1}^{m}\left(\mathbf{s}_{i}^{\prime} \mathbf{w}_{i}^{\prime}\right)
$$

where:

- $\left(\mathbf{s}_{1}, \ldots, \mathbf{s}_{m}\right)=\left(\mathbf{s}_{1 \pi}^{\prime}, \ldots, \mathbf{s}_{m \pi}^{\prime}\right)$ for some permutation $\pi$ on $\{1, \ldots, m\}$;

- $\left(\mathbf{w}_{0}, \ldots, \mathbf{w}_{m}\right)=\left(\mathbf{w}_{0 \tau}^{\prime}, \ldots, \mathbf{w}_{m \tau}^{\prime}\right)$ for some permutation $\tau$ on $\{0, \ldots, m\}$.

In the remainder of this proof, it is shown that the identities (18.1) can be used to convert $\mathbf{w}^{\prime}$ into $\mathbf{w}$. It follows that the identity $\mathbf{w} \approx \mathbf{w}^{\prime}$ is implied by the identities (18.1). Consequently, the identities (18.1) imply every identity in $\bar{\Sigma}$ and so constitute a basis for $\mathscr{C}_{7}$.

Since the words $\mathbf{w}$ and $\mathbf{w}^{\prime}$ are connected, $\mathbf{w}_{0}=\mathbf{w}_{0}^{\prime}$ by Lemma 18.8(vi). Suppose that $\ell \geqslant 1$ is such that $\left(\mathbf{s}_{1} \mathbf{w}_{1}, \ldots, \mathbf{s}_{\ell-1} \mathbf{w}_{\ell-1}\right)=\left(\mathbf{s}_{1}^{\prime} \mathbf{w}_{1}^{\prime}, \ldots, \mathbf{s}_{\ell-1}^{\prime} \mathbf{w}_{\ell-1}^{\prime}\right)$ and $\mathbf{s}_{\ell} \mathbf{w}_{\ell} \neq \mathbf{s}_{\ell}^{\prime} \mathbf{w}_{\ell}^{\prime}$. Then

$$
\mathbf{w}=\operatorname{has}_{\ell} \mathbf{w}_{\ell} \ldots \mathbf{s}_{m} \mathbf{w}_{m} \quad \text { and } \quad \mathbf{w}^{\prime}=\mathbf{h a s}_{\ell}^{\prime} \mathbf{w}_{\ell}^{\prime} \ldots \mathbf{s}_{m}^{\prime} \mathbf{w}_{m}^{\prime}
$$

where $\mathbf{h}=\mathbf{w}_{0}\left(\prod_{i=1}^{\ell-2}\left(\mathbf{s}_{i} \mathbf{w}_{i}\right)\right) \mathbf{s}_{\ell-1}=\mathbf{w}_{0}^{\prime}\left(\prod_{i=1}^{\ell-2}\left(\mathbf{s}_{i}^{\prime} \mathbf{w}_{i}^{\prime}\right)\right) \mathbf{s}_{\ell-1}^{\prime}$ and $\mathbf{a}=\mathbf{w}_{\ell-1}=\mathbf{w}_{\ell-1}^{\prime}$. (Note that if $\ell=1$, then $\mathbf{h}=\emptyset$ and $\mathbf{a}=\mathbf{w}_{0}=\mathbf{w}_{0}^{\prime}$.) If $\mathbf{s}_{\ell} \neq \mathbf{s}_{\ell}^{\prime}$, then it is shown in Lemma 18.10 below that the identities (18.1) can be used to convert $\mathbf{w}^{\prime}$ into a word $\mathbf{w}^{\prime}=\mathbf{h a s} \ell \ldots$ in canonical form. Hence it can be assumed that $\mathbf{s}_{\ell}=\mathbf{s}_{\ell}^{\prime}$. It then follows from Lemma 18.8(iv) that $\mathbf{w}_{\ell}=\mathbf{w}_{\ell}^{\prime}$, whence $\mathbf{s}_{\ell} \mathbf{w}_{\ell}=\mathbf{s}_{\ell}^{\prime} \mathbf{w}_{\ell}^{\prime}$. The argument in this paragraph can be repeated until $\mathbf{w}^{\prime}$ is converted by the identities (18.1) into $\mathbf{w}$.

Lemma 18.10. Suppose $\mathbf{s}_{\ell} \neq \mathbf{s}_{\ell}^{\prime}$ in (18.5). Then the identities (18.1) can be used to convert $\mathbf{w}^{\prime}$

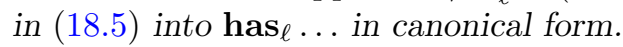

Proof. By assumption, $\mathbf{a s}_{\ell} \mathbf{w}_{\ell} \neq \mathbf{a s}_{\ell}^{\prime} \mathbf{w}_{\ell}^{\prime}$ where $\mathbf{a s}_{\ell} \mathbf{w}_{\ell}$ is a factor of $\mathbf{w}$ and $\mathbf{a s}_{\ell}^{\prime} \mathbf{w}_{\ell}^{\prime}$ is a factor of $\mathbf{w}^{\prime}$. Then it follows from Lemma 18.8 parts (iv) and (v) that:

- $\mathbf{a s}_{\ell}^{\prime} \mathbf{w}_{\ell}^{\prime}$ is a factor of $\mathbf{w}$, say $\mathbf{a s}_{\ell}^{\prime} \mathbf{w}_{\ell}^{\prime}=\mathbf{w}_{p} \mathbf{s}_{p+1} \mathbf{w}_{p+1}$ for some $p \geqslant \ell$;

- $\mathbf{a s}_{\ell} \mathbf{w}_{\ell}$ is a factor of $\mathbf{w}^{\prime}$, say $\mathbf{a s}_{\ell} \mathbf{w}_{\ell}=\mathbf{w}_{q}^{\prime} \mathbf{s}_{q+1}^{\prime} \mathbf{w}_{q+1}^{\prime}$ for some $q \geqslant \ell$. Thus

$$
\mathbf{w}=\mathbf{h a} \cdot \mathbf{s}_{\ell} \mathbf{w}_{\ell} \cdot \mathbf{s}_{\ell+1} \mathbf{w}_{\ell+1} \ldots \mathbf{s}_{p} \overbrace{\mathbf{a} \cdot \mathbf{s}_{\ell}^{\prime} \mathbf{w}_{\ell}^{\prime}}^{\mathbf{w}_{p} \cdot \mathbf{s}_{p+1} \mathbf{w}_{p+1}} \cdot \mathbf{s}_{p+2} \mathbf{w}_{p+2} \ldots \mathbf{s}_{m} \mathbf{w}_{m}
$$

and

$$
\mathbf{w}^{\prime}=\mathbf{h a} \cdot \mathbf{s}_{\ell}^{\prime} \mathbf{w}_{\ell}^{\prime} \cdot \mathbf{s}_{\ell+1}^{\prime} \mathbf{w}_{\ell+1}^{\prime} \ldots \mathbf{s}_{q}^{\prime} \underbrace{\mathbf{a} \cdot \mathbf{s}_{\ell} \mathbf{w}_{\ell}}_{\mathbf{w}_{q}^{\prime} \cdot \mathbf{s}_{q+1}^{\prime} \mathbf{w}_{q+1}^{\prime}} \cdot \mathbf{s}_{q+2}^{\prime} \mathbf{w}_{q+2}^{\prime} \ldots \mathbf{s}_{m}^{\prime} \mathbf{w}_{m}^{\prime} .
$$

Note that by $(\mathrm{I})$,

(a) $\operatorname{con}(\mathbf{h}) \cap \operatorname{con}\left(\mathbf{s}_{\ell} \mathbf{s}_{\ell+1} \ldots \mathbf{s}_{p}\right)=\emptyset$.

Further, the maximal non-simple factors $\mathbf{w}_{\ell}^{\prime}, \mathbf{w}_{\ell+1}^{\prime}, \ldots, \mathbf{w}_{q-1}^{\prime}$ of $\mathbf{w}^{\prime}$ are sandwiched between $\mathbf{w}_{\ell-1}^{\prime}=\mathbf{a}$ and $\mathbf{w}_{q}^{\prime}=\mathbf{a}$. Therefore by (IV),

(b) $\left\{\mathbf{w}_{\ell}^{\prime}, \mathbf{w}_{\ell+1}^{\prime}, \ldots, \mathbf{w}_{q-1}^{\prime}\right\} \cap\left\{\mathbf{w}_{q+1}^{\prime}, \mathbf{w}_{q+2}^{\prime}, \ldots, \mathbf{w}_{m}^{\prime}\right\}$ is either $\{\mathbf{a}\}$ or empty.

Suppose that

$(\dagger) \mathbf{a}$ is not equal to any of the factors $\mathbf{w}_{q+1}^{\prime}, \mathbf{w}_{q+2}^{\prime}, \ldots, \mathbf{w}_{m}^{\prime}$ of $\mathbf{w}^{\prime}$.

By Lemma 18.8(iv), the factor $\mathbf{s}_{p} \mathbf{a}$ of $\mathbf{w}$ is a factor of $\mathbf{w}^{\prime}$. Hence by (a) and $(\dagger)$,

(c) $\mathbf{s}_{p}$ is a factor of $\mathbf{s}_{\ell}^{\prime} \mathbf{w}_{\ell}^{\prime} \cdot \mathbf{s}_{\ell+1}^{\prime} \mathbf{w}_{\ell+1}^{\prime} \ldots \mathbf{s}_{q}^{\prime} \mathbf{w}_{q}^{\prime}$.

By Lemma 18.8(v), the factor $\mathbf{w}_{p-1} \mathbf{s}_{p}$ of $\mathbf{w}$ is a factor of $\mathbf{w}^{\prime}$. Hence (c) implies that

(d) $\mathbf{w}_{p-1}$ is a factor of $\mathbf{a} \cdot \mathbf{s}_{\ell}^{\prime} \mathbf{w}_{\ell}^{\prime} \cdot \mathbf{s}_{\ell+1}^{\prime} \mathbf{w}_{\ell+1}^{\prime} \ldots \mathbf{s}_{q}^{\prime} \mathbf{w}_{q}^{\prime}$.

If $\mathbf{w}_{p-1}=\mathbf{a}$, then $(\dagger)$ implies that

(e) $\mathbf{w}_{p-1}$ is not equal to any of $\mathbf{w}_{q+1}^{\prime}, \mathbf{w}_{q+2}^{\prime}, \ldots, \mathbf{w}_{m}^{\prime}$. 
If $\mathbf{w}_{p-1} \neq \mathbf{a}$, then $\mathbf{a}=\mathbf{w}_{q}^{\prime}$ and (d) imply $\mathbf{w}_{p-1} \in\left\{\mathbf{w}_{\ell}^{\prime}, \mathbf{w}_{\ell+1}^{\prime}, \ldots, \mathbf{w}_{q-1}^{\prime}\right\}$, which together with (b), imply (e). Therefore (e) holds in any case. By Lemma 18.8(iv), the factor $\mathbf{s}_{p-1} \mathbf{w}_{p-1}$ of $\mathbf{w}$ is a factor of $\mathbf{w}^{\prime}$. Hence (d) and (e) imply that the factor $\mathbf{s}_{p-1}$ of $\mathbf{w}$ is a factor of $\mathbf{s}_{\ell}^{\prime} \mathbf{w}_{\ell}^{\prime} \cdot \mathbf{s}_{\ell+1}^{\prime} \mathbf{w}_{\ell+1}^{\prime} \ldots \mathbf{s}_{q}^{\prime} \mathbf{w}_{q}^{\prime}$.

The argument in the previous paragraph can be repeated to deduce that the factors $\mathbf{s}_{p-2}, \mathbf{s}_{p-3}, \ldots, \mathbf{s}_{\ell+1}$ of $\mathbf{w}$ are factors of $\mathbf{s}_{\ell}^{\prime} \mathbf{w}_{\ell}^{\prime} \cdot \mathbf{s}_{\ell+1}^{\prime} \mathbf{w}_{\ell+1}^{\prime} \ldots \mathbf{s}_{q}^{\prime} \mathbf{w}_{q}^{\prime}$. It follows that $\mathbf{s}_{\ell} \mathbf{w}_{\ell} \mathbf{s}_{\ell+1}$ is not a factor of $\mathbf{w}^{\prime}$. However, $\mathbf{s}_{\ell} \mathbf{w}_{\ell} \mathbf{s}_{\ell+1}$ is a factor of $\mathbf{w}$, whence Lemma 18.8 parts (iv) and (v) are violated. Therefore the assumption $(\dagger)$ cannot hold, whence $\mathbf{a}=\mathbf{w}_{j}^{\prime}$ for some $j \in\{q+1, q+2, \ldots, m\}$. Consequently,

$$
\begin{array}{r}
\mathbf{w}^{\prime}=\mathbf{h}\left(\mathbf{a} \cdot \mathbf{s}_{\ell}^{\prime} \mathbf{w}_{\ell}^{\prime} \ldots \mathbf{s}_{q}^{\prime} \cdot \mathbf{a} \cdot \mathbf{s}_{\ell} \mathbf{w}_{\ell} \ldots \mathbf{s}_{j}^{\prime} \cdot \mathbf{a}\right) \mathbf{s}_{j+1}^{\prime} \mathbf{w}_{j+1}^{\prime} \ldots \mathbf{s}_{m}^{\prime} \mathbf{w}_{m}^{\prime} \\
\stackrel{(18.1 \mathrm{~d})}{\approx} \mathbf{h}\left(\mathbf{a} \cdot \mathbf{s}_{\ell} \mathbf{w}_{\ell} \ldots \mathbf{s}_{j}^{\prime} \cdot \mathbf{a} \cdot \mathbf{s}_{\ell}^{\prime} \mathbf{w}_{\ell}^{\prime} \ldots \mathbf{s}_{q}^{\prime} \cdot \mathbf{a}\right) \mathbf{s}_{j+1}^{\prime} \mathbf{w}_{j+1}^{\prime} \ldots \mathbf{s}_{m}^{\prime} \mathbf{w}_{m}^{\prime},
\end{array}
$$

where the latter word begins with has $\boldsymbol{s}_{\ell}$ and is in canonical form.

19. $\mathscr{C}_{8}$

This section establishes the finite basis property of the following semigroup.

\begin{tabular}{c|cccccc}
$\mathscr{C}_{8}$ & 1 & 2 & 3 & 4 & 5 & 6 \\
\hline 1 & 1 & 1 & 1 & 1 & 1 & 1 \\
2 & 1 & 1 & 1 & 1 & 1 & 2 \\
3 & 1 & 1 & 1 & 1 & 1 & 3 \\
4 & 1 & 1 & 2 & 1 & 4 & 1 \\
5 & 1 & 1 & 3 & 1 & 5 & 1 \\
6 & 1 & 2 & 2 & 4 & 4 & 6
\end{tabular}

Proposition 19.1. The variety generated by $\mathscr{C}_{8}$ is defined by the identities

$$
\begin{aligned}
x^{3} \approx x^{2}, \quad x^{2} y x & \approx x y x, \quad x y x^{2} \approx x y x, \\
h x h y h & \approx h y h x h, \\
x \mathrm{H} y \mathrm{~K} x \mathrm{~T} y & \approx x \mathrm{H} x \mathrm{~K} x \mathrm{~T} y^{2} x, \\
x \mathrm{H} y^{2} x \approx y^{2} x \mathrm{H} y^{2} x, \quad x \mathrm{H} y^{2} x \mathrm{~K} x \approx x \mathrm{H} y^{2} x \mathrm{~K} y^{2} x, & x \mathrm{H} y \mathrm{~K} x y \approx x \mathrm{H} y \mathrm{~K} y x, \quad x \mathrm{H} y \mathrm{~K} x y \approx y \mathrm{H} x \mathrm{~K} x y, \\
x \mathrm{H} y^{2} x \mathrm{~K} y \approx x \mathrm{H} y^{2} x \mathrm{~K} x, \quad y \mathrm{H} x \mathrm{~K} y^{2} x & \approx x \mathrm{H} x \mathrm{~K} y^{2} x, \quad x \mathrm{H} y \mathrm{~K} y^{2} x \approx x \mathrm{H} x \mathrm{~K} y^{2} x .
\end{aligned}
$$

The proof of Proposition 19.1 is given in $\S 19.4$.

\section{1. $\quad \mathcal{Z}$-perfect words}

For any nonempty set $\mathcal{Z}=\left\{z_{1}, \ldots, z_{r}\right\}$ of letters where $z_{1}, \ldots, z_{r}$ are in alphabetical order, define the perfect $\mathcal{Z}$-square to be the word

$$
\mathcal{Z}^{\bigotimes}=\left(z_{1} \ldots z_{r}\right)^{2} .
$$

More generally, a perfect square is a perfect $\mathcal{Z}$-square for some nonempty set $\mathcal{Z}$ of letters.

Let $\mathbf{w}$ be any non-simple word and let $\mathcal{Z}$ be some nonempty set of non-simple letters of $\mathbf{w}$. Then $\mathbf{w}$ can be written in the form

$$
\mathbf{w}=\mathbf{a}_{0} \prod_{i=1}^{n}\left(\mathbf{z}_{i} \mathbf{a}_{i}\right)=\mathbf{a}_{0} \mathbf{z}_{1} \mathbf{a}_{1} \mathbf{z}_{2} \mathbf{a}_{2} \ldots \mathbf{z}_{n} \mathbf{a}_{n}
$$


for some $n \geqslant 1$, where $\mathbf{a}_{0}, \mathbf{a}_{n} \in \mathcal{X}^{*}, \mathbf{a}_{1}, \ldots, \mathbf{a}_{n-1} \in \mathcal{X}^{+}$, and $\mathbf{z}_{1}, \ldots, \mathbf{z}_{n} \in \mathcal{Z}^{+}$are such that $\operatorname{con}\left(\mathbf{a}_{0} \ldots \mathbf{a}_{n}\right) \cap \mathcal{Z}=\emptyset$. In this case, the words $\mathbf{z}_{1}, \ldots, \mathbf{z}_{n}$ are called maximal $\mathcal{Z}$-factors of $\mathbf{w}$. If $\mathbf{z}_{1}=\ldots=\mathbf{z}_{n}=\mathcal{Z}^{\square}$, then $\mathbf{w}$ is said to be $\mathcal{Z}$-perfect.

For any distinct non-simple letters $x$ and $y$ of a word $\mathbf{w}$, write $x \sim_{\mathbf{w}} y$ to indicate that any one of the following conditions holds:

(C1) $\mathbf{w} \in \mathcal{X}^{*} x \mathcal{X}^{*} y x \mathcal{X}^{*}$ or $\mathbf{w} \in \mathcal{X}^{*} y \mathcal{X}^{*} x y \mathcal{X}^{*}$;

$(\mathrm{C} 2) \mathbf{w} \in \mathcal{X}^{*} x \mathcal{X}^{*} y \mathcal{X}^{*} x \mathcal{X}^{*} y \mathcal{X}^{*}$ or $\mathbf{w} \in \mathcal{X}^{*} y \mathcal{X}^{*} x \mathcal{X}^{*} y \mathcal{X}^{*} x \mathcal{X}^{*}$.

Note that $x \sim_{\mathbf{w}} y$ if and only if $y \sim_{\mathbf{w}} x$.

Lemma 19.2. Let $\mathbf{w}$ be any non-simple word. Suppose that $z_{1}$ and $z_{2}$ are any distinct nonsimple letters of $\mathbf{w}$ such that $z_{1} \sim_{\mathbf{w}} z_{2}$. Then there exists some $\left\{z_{1}, z_{2}\right\}$-perfect word $\mathbf{w}^{\prime}$ such that the identities (19.1) imply the identity $\mathbf{w} \approx \mathbf{w}^{\prime}$.

Proof. By assumption,

$$
\mathbf{w}=\mathbf{a}_{0} \prod_{i=1}^{n}\left(\mathbf{z}_{i} \mathbf{a}_{i}\right)=\mathbf{a}_{0} \mathbf{z}_{1} \mathbf{a}_{1} \mathbf{z}_{2} \mathbf{a}_{2} \ldots \mathbf{z}_{n} \mathbf{a}_{n}
$$

for some $n \geqslant 1$, where $\mathbf{a}_{0}, \mathbf{a}_{n} \in \mathcal{X}^{*}, \mathbf{a}_{1}, \ldots, \mathbf{a}_{n-1} \in \mathcal{X}^{+}$, and $\mathbf{z}_{1}, \ldots, \mathbf{z}_{n} \in\left\{z_{1}, z_{2}\right\}^{+}$are such that $z_{1}, z_{2} \notin \operatorname{con}\left(\mathbf{a}_{0} \ldots \mathbf{a}_{n}\right)$. Generality is not lost by assuming that $z_{1}$ alphabetically precedes $z_{2}$, so that $\left\{z_{1}, z_{2}\right\}^{\otimes}=\left(z_{1} z_{2}\right)^{2}$. Then it suffices to convert $\mathbf{w}$, using the identities (19.1), into the $\left\{z_{1}, z_{2}\right\}$-perfect word

$$
\mathbf{w}^{\prime}=\mathbf{a}_{0} \prod_{i=1}^{n}\left(\left(z_{1} z_{2}\right)^{2} \mathbf{a}_{i}\right) .
$$

There are two cases depending on whether $(\mathrm{C} 1)$ or $(\mathrm{C} 2)$ holds.

Case 1: (C1) holds. Then $\mathbf{w} \in \mathcal{X}^{*} z_{j} \mathcal{X}^{*} z_{k} z_{j} \mathcal{X}^{*}$ where $\left\{z_{j}, z_{k}\right\}=\left\{z_{1}, z_{2}\right\}$.

1.1. $n=1$. Then

$$
\mathbf{w}=\mathbf{a}_{0} \underbrace{\mathbf{b} z_{j} \mathbf{c} z_{k} z_{j} \mathbf{d}}_{\mathbf{z}_{1}} \mathbf{a}_{1}
$$

for some $\mathbf{b}, \mathbf{c}, \mathbf{d} \in\left\{z_{1}, z_{2}\right\}^{*}$. Hence

$$
\begin{aligned}
& \mathbf{w} \stackrel{(19.1 \mathrm{a})}{\underset{\sim}{\approx}} \mathbf{a}_{0} \mathbf{b} z_{j} \mathbf{c} z_{k}^{2} z_{j} \mathbf{d} \mathbf{a}_{1} \stackrel{(19.1 \mathrm{f})}{\approx} \mathbf{a}_{0} z_{j}^{|\mathbf{b}|} z_{j} z_{j}^{|\mathbf{c}|} z_{k}^{2} z_{j} z_{j}^{|\mathbf{d}|} \mathbf{a}_{1} \stackrel{(19.1 \mathrm{a})}{\approx} \mathbf{a}_{0} z_{j} z_{k}^{2} z_{j} \mathbf{a}_{1} \\
& \stackrel{(19.1 \mathrm{e})}{\approx} \mathbf{a}_{0}\left(z_{1} z_{2}\right)^{2} \mathbf{a}_{1}=\mathbf{w}^{\prime} .
\end{aligned}
$$

1.2. $n \geqslant 2$. Then

$$
\mathbf{w}=\mathbf{a}_{0}\left(\prod_{i=1}^{\ell-1}\left(\mathbf{z}_{i} \mathbf{a}_{i}\right)\right) \underbrace{\mathbf{b} z_{k} z_{j}}_{\mathbf{z}_{\ell}} \mathbf{c} \mathbf{a}_{\ell}\left(\prod_{i=\ell+1}^{n}\left(\mathbf{z}_{i} \mathbf{a}_{i}\right)\right)
$$

for some $\mathbf{b}, \mathbf{c} \in\left\{z_{1}, z_{2}\right\}^{*}$ with $\mathbf{a}_{0}\left(\prod_{i=1}^{\ell-1}\left(\mathbf{z}_{i} \mathbf{a}_{i}\right)\right) \mathbf{b} \in \mathcal{X}^{*} z_{j} \mathcal{X}^{*}$. Hence

$$
\begin{aligned}
& \mathbf{w} \stackrel{(19.1 \mathrm{a})}{\approx} \mathbf{a}_{0}\left(\prod_{i=1}^{\ell-1}\left(\mathbf{z}_{i} \mathbf{a}_{i}\right)\right) \mathbf{b} z_{k}^{2} z_{j} \mathbf{c} \mathbf{a}_{\ell}\left(\prod_{i=\ell+1}^{n}\left(\mathbf{z}_{i} \mathbf{a}_{i}\right)\right) \\
& \stackrel{(19.1 \mathrm{f})}{\approx} \mathbf{a}_{0}\left(\prod_{i=1}^{\ell-1}\left(z_{j}^{\left|\mathbf{z}_{i}\right|} \mathbf{a}_{i}\right)\right) z_{j}^{|\mathbf{b}|} z_{k}^{2} z_{j} z_{j}^{|\mathbf{c}|} \mathbf{a}_{\ell}\left(\prod_{i=\ell+1}^{n}\left(z_{j}^{\left|\mathbf{z}_{i}\right|} \mathbf{a}_{i}\right)\right)
\end{aligned}
$$




$$
\begin{aligned}
& \stackrel{(19.1 \mathrm{~d})}{\approx} \mathbf{a}_{0}\left(\prod_{i=1}^{\ell-1}\left(z_{k}^{2} z_{j}^{\left|\mathbf{z}_{i}\right|} \mathbf{a}_{i}\right)\right) z_{j}^{|\mathbf{b}|} z_{k}^{2} z_{j} z_{j}^{|\mathbf{c}|} \mathbf{a}_{\ell}\left(\prod_{i=\ell+1}^{n}\left(z_{k}^{2} z_{j}^{\left|\mathbf{z}_{i}\right|} \mathbf{a}_{i}\right)\right) \\
& \stackrel{(19.1 \mathrm{e})}{\approx} \mathbf{a}_{0}\left(\prod_{i=1}^{\ell-1}\left(z_{k}^{2} z_{j}^{\left|\mathbf{z}_{i}\right|} \mathbf{a}_{i}\right)\right) z_{k}^{2} z_{j}^{|\mathbf{b}|+1+|\mathbf{c}|} \mathbf{a}_{\ell}\left(\prod_{i=\ell+1}^{n}\left(z_{k}^{2} z_{j}^{\left|\mathbf{z}_{i}\right|} \mathbf{a}_{i}\right)\right) \\
& \stackrel{(19.1 \mathrm{a})}{\approx} \mathbf{a}_{0}\left(\prod_{i=1}^{\ell-1}\left(z_{k}^{2} z_{j}^{2} \mathbf{a}_{i}\right)\right) z_{k}^{2} z_{j}^{2} \mathbf{a}_{\ell}\left(\prod_{i=\ell+1}^{n}\left(z_{k}^{2} z_{j}^{2} \mathbf{a}_{i}\right)\right) \\
& \stackrel{(19.1 \mathrm{e})}{\approx} \mathbf{a}_{0}\left(\prod_{i=1}^{\ell-1}\left(\left(z_{1} z_{2}\right)^{2} \mathbf{a}_{i}\right)\right)\left(z_{1} z_{2}\right)^{2} \mathbf{a}_{\ell}\left(\prod_{i=\ell+1}^{n}\left(\left(z_{1} z_{2}\right)^{2} \mathbf{a}_{i}\right)\right)=\mathbf{w}^{\prime} .
\end{aligned}
$$

Case 2: (C2) holds. Then $\mathbf{w} \in \mathcal{X}^{*} z_{j} \mathcal{X}^{*} z_{k} \mathcal{X}^{*} z_{j} \mathcal{X}^{*} z_{k} \mathcal{X}^{*}$ where $\left\{z_{j}, z_{k}\right\}=\left\{z_{1}, z_{2}\right\}$. Hence

$$
\mathbf{w}=\ldots z_{j} \ldots z_{k} \ldots z_{j} \ldots z_{k} \ldots \stackrel{(19.1 \mathrm{c})}{\approx} \underbrace{\ldots z_{j} \ldots z_{j} \ldots z_{j} \ldots z_{k}^{2} z_{j} \ldots}_{\mathbf{u}},
$$

where $\mathbf{u}$ belongs to $\mathcal{X}^{*} z_{j} \mathcal{X}^{*} z_{k} z_{j} \mathcal{X}^{*}$. By Case 1 , the word $\mathbf{u}$ can be converted by the identities (19.1) into $\mathbf{w}^{\prime}$.

Let $\mathbf{w}$ be any non-simple word and let $\mathcal{Z}$ and $\mathcal{Z}^{\prime}$ be some disjoint nonempty sets of nonsimple letters of $\mathbf{w}$. Then $\sim_{\mathbf{w}}$ can be generalized: for any perfect $\mathcal{Z}$-square $\mathbf{x}$ and any perfect $\mathcal{Z}^{\prime}$-square $\mathbf{y}$, write $\mathbf{x} \sim_{\mathbf{w}} \mathbf{y}$ whenever one of the following generalized conditions holds:

(G1) $\mathbf{w} \in \mathcal{X}^{*} \mathbf{x} \mathcal{X}^{*} \mathbf{y} \mathbf{x} \mathcal{X}^{*}$ or $\mathbf{w} \in \mathcal{X}^{*} \mathbf{y} \mathcal{X}^{*} \mathbf{x y} \mathcal{X}^{*}$

(G2) $\mathbf{w} \in \mathcal{X}^{*} \mathbf{x} \mathcal{X}^{*} \mathbf{y} \mathcal{X}^{*} \mathbf{x} \mathcal{X}^{*} \mathbf{y} \mathcal{X}^{*}$ or $\mathbf{w} \in \mathcal{X}^{*} \mathbf{y} \mathcal{X}^{*} \mathbf{x} \mathcal{X}^{*} \mathbf{y} \mathcal{X}^{*} \mathbf{x} \mathcal{X}^{*}$

\subsection{A canonical form}

Let $\mathbf{w}$ be any connected word and let $x_{1}, \ldots, x_{t}$ be the distinct non-simple letters of $\mathbf{w}$ listed in alphabetical order. In this section, such a word $\mathbf{w}$ is said to be in canonical form if some partition $\mathcal{Z}_{1}, \ldots, \mathcal{Z}_{r}$ of $\left\{x_{1}, \ldots, x_{t}\right\}$ exists and

$$
\mathbf{w}=\mathbf{z}_{0} \prod_{i=1}^{n}\left(\mathbf{s}_{i} \mathbf{z}_{i}\right)
$$

for some $n \geqslant 0$ such that the following are satisfied:

(I) the letters of $\mathbf{s}_{1}, \ldots, \mathbf{s}_{n} \in \mathcal{X}^{*}$ are simple in $\mathbf{w}$;

(II) the word $\mathbf{w}$ is $\mathcal{Z}_{i}$-perfect for each $i \in\{1, \ldots, r\}$ and $\mathbf{z}_{0}, \ldots, \mathbf{z}_{n} \in\left\{\mathcal{Z}_{1}^{\bigotimes}, \ldots, \mathcal{Z}_{r}^{\bigotimes}\right\}$ (so that $\left.\operatorname{con}\left(\mathbf{z}_{0} \ldots \mathbf{z}_{n}\right)=\left\{x_{1}, \ldots, x_{t}\right\}\right) ;$

(III) if $\mathbf{z}_{i} \neq \mathbf{z}_{j}$, then $\mathbf{z}_{i} \chi_{\mathbf{w}} \mathbf{z}_{j}$;

(IV) if $\mathbf{z}_{i}=\mathbf{z}_{j}$ with $i<j$, then $\mathbf{s}_{j} \neq \emptyset$;

(V) $\mathbf{z}_{0}=\mathbf{z}_{n}$.

Note that since $\mathcal{Z}_{1}, \ldots, \mathcal{Z}_{r}$ form a partition of $\left\{x_{1}, \ldots, x_{t}\right\}$, it follows from (II) that

(VI) for any $i$ and $j$, either $\mathbf{z}_{i}=\mathbf{z}_{j}$ or $\operatorname{con}\left(\mathbf{z}_{i}\right) \cap \operatorname{con}\left(\mathbf{z}_{j}\right)=\emptyset$.

In view of (I) and (II), it is convenient to call the words $\mathbf{s}_{1}, \ldots, \mathbf{s}_{n}$ maximal simple factors of $\mathbf{w}$.

Lemma 19.3. Let $\mathbf{w}$ be any connected word. Then there exists some word $\overline{\mathbf{w}}$ in canonical form such that the identities (19.1) imply the identity $\mathbf{w} \approx \overline{\mathbf{w}}$. 
Proof. It suffices to convert $\mathbf{w}$, using the identities (19.1), into a word in canonical form. Let $x_{1}, \ldots, x_{t}$ be the distinct non-simple letters of $\mathbf{w}$ listed in alphabetical order. The proof is trivial if $t=1$. Therefore assume $t \geqslant 2$.

Since $\mathbf{w}$ is connected, there exist distinct non-simple letters $\mathrm{h}(\mathbf{w})=x_{r_{1}}, x_{r_{2}}, \ldots, x_{r_{\ell}}=\mathrm{t}(\mathbf{w})$ from $\left\{x_{1}, \ldots, x_{t}\right\}$ occurring in $\mathbf{w}$ in an overlapping pattern such that the first $x_{r_{i+1}}$ occurs between two occurrences of $x_{r_{i}}$ and the last $x_{r_{i+1}}$ occurs to the right of all occurrences of $x_{r_{i}}$. This sequence can be chosen to have minimal length among all such sequences, so that

$$
\mathbf{w}=x_{r_{1}} \mathbf{p}_{1} x_{r_{2}} \mathbf{p}_{2} x_{r_{1}} \mathbf{q}_{1} x_{r_{3}} \mathbf{p}_{3} x_{r_{2}} \mathbf{q}_{2} x_{r_{4}} \mathbf{p}_{4} x_{r_{3}} \mathbf{q}_{3} \ldots \mathbf{q}_{\ell-1} x_{r_{\ell}}
$$

for some $\mathbf{p}_{1}, \ldots, \mathbf{p}_{\ell}, \mathbf{q}_{1}, \ldots, \mathbf{q}_{\ell-1} \in \mathcal{X}^{*}$. (Note that the factor $\mathbf{p}_{i}$ follows the first $x_{r_{i}}$ and the factor $\mathbf{q}_{i}$ follows the last $x_{r_{i}}$.) Then $x_{r_{1}} \sim_{\mathbf{w}} x_{r_{2}} \sim_{\mathbf{w}} \ldots \sim_{\mathbf{w}} x_{r_{\ell}}$ by (G2). By Lemma 19.2, the identities (19.1) can be used to convert $\mathbf{w}$ into some $\left\{x_{r_{1}}, x_{r_{2}}\right\}$-perfect word $\mathbf{w}^{\prime}$. Note that $\mathbf{w}^{\prime}$ is obtained by replacing all maximal $\left\{x_{r_{1}}, x_{r_{2}}\right\}$-factors of $\mathbf{w}$ by the perfect square $\left\{x_{r_{1}}, x_{r_{2}}\right\}^{\bigotimes}=$ $\left(x_{r_{1}} x_{r_{2}}\right)^{2}$. It is clear that $\left\{x_{r_{1}}, x_{r_{2}}\right\}^{\bigotimes} \sim_{\mathbf{w}^{\prime}} x_{r_{3}}$. Therefore the proof of Lemma 19.2 can be repeated to convert $\mathbf{w}^{\prime}$, using the identities (19.1), into some $\left\{x_{r_{1}}, x_{r_{2}}, x_{r_{3}}\right\}$-perfect word. Continuing in this manner, $\mathbf{w}$ is converted by the identities (19.1) into an $\left\{x_{r_{1}}, \ldots, x_{r_{\ell}}\right\}$ perfect word. This can be further repeated until $\mathbf{w}$ is converted by the identities (19.1) into a $\mathcal{Z}_{1}$-perfect word, where $\mathcal{Z}_{1}$ is some subset of $\left\{x_{1}, \ldots, x_{t}\right\}$ that contains $x_{r_{1}}, \ldots, x_{r_{\ell}}$ and

(a) $\mathcal{Z}_{1}^{\bigotimes} \sim_{\mathbf{w}} x_{i}$ if and only if $x_{i} \in \mathcal{Z}_{1}$.

Hence it can be assumed that

$$
\mathbf{w}=\mathcal{Z}_{1}^{\otimes} \mathbf{w}_{1} \mathcal{Z}_{1}^{\otimes} \mathbf{w}_{2} \ldots \mathcal{Z}_{1}^{\otimes} \mathbf{w}_{m} \mathcal{Z}_{1}^{\otimes}
$$

where

(b) $\mathbf{w}_{1}, \ldots, \mathbf{w}_{m} \in \mathcal{X}^{+}$are such that $\operatorname{con}\left(\mathbf{w}_{1} \ldots \mathbf{w}_{m}\right) \cap \mathcal{Z}_{1}=\emptyset$.

If $x \in \operatorname{con}\left(\mathbf{w}_{i}\right) \cap \operatorname{con}\left(\mathbf{w}_{j}\right)$ for some distinct $i$ and $j$, then $\mathcal{Z}_{1}^{\otimes} \sim_{\mathbf{w}} x$ by (G2), and this contradicts (a) and (b). If $\mathrm{t}\left(\mathbf{w}_{i}\right) \notin \operatorname{sim}(\mathbf{w})$ for some $i$, then $\mathcal{Z}_{1}^{\bigotimes} \sim_{\mathbf{w}} \mathrm{t}\left(\mathbf{w}_{i}\right)$ by (G1), and this again contradicts (a) and (b). Therefore

(c) $\mathbf{w}_{1}, \ldots, \mathbf{w}_{m}$ are pairwise disjoint and $\mathrm{t}\left(\mathbf{w}_{1}\right), \ldots, \mathrm{t}\left(\mathbf{w}_{m}\right) \in \operatorname{sim}(\mathbf{w})$.

If $x_{i} \sim_{\mathbf{w}} x_{j}$ for some $x_{i}, x_{j} \in \operatorname{con}\left(\mathbf{w}_{k}\right)$ (so that $x_{i}, x_{j} \notin \mathcal{Z}_{1}$ by (c)), then by Lemma 19.2, the identities (19.1) can be used to convert $\mathbf{w}$ into some $\left\{x_{i}, x_{j}\right\}$-perfect word. This can be repeated until the word $\mathbf{w}$ in (19.3) is converted into a $\mathcal{Z}_{2}$-perfect word, where $\mathcal{Z}_{2}$ is some subset of $\left\{x_{1}, \ldots, x_{t}\right\} \backslash \mathcal{Z}_{1}$ such that $\mathcal{Z}_{2}^{\bigotimes} \sim_{\mathbf{w}} x_{i}$ if and only if $x_{i} \in \mathcal{Z}_{2}$. (Note that by (c), all perfect $\mathcal{Z}_{2}$-squares $\mathcal{Z}_{2}^{\bigotimes}$ are factors of some $\mathbf{w}_{k}$.) It is easily seen how this argument can be repeated until a partition $\mathcal{Z}_{1}, \ldots, \mathcal{Z}_{r}$ of $\left\{x_{1}, \ldots, x_{t}\right\}$ is obtained, and the word $\mathbf{w}$ in (19.3) is converted by the identities (19.1) into a word $\overline{\mathbf{w}}$ that is simultaneously $\mathcal{Z}_{i}$-perfect for all $i$ with $\mathcal{Z}_{i}^{\bigotimes} \chi_{\overline{\mathbf{w}}} \mathcal{Z}_{j}^{\bigotimes}$ whenever $i \neq j$.

Consequently, $\overline{\mathbf{w}}$ is of the form (19.2) with (I), (II), (III), and (V) satisfied. Suppose $\mathbf{z}_{i}=\mathbf{z}_{j}$ with $i<j$ and $\mathbf{s}_{j}=\emptyset$, so that $\overline{\mathbf{w}}=\ldots \mathbf{s}_{i} \mathbf{z}_{i} \ldots \mathbf{s}_{j-1} \mathbf{z}_{j-1} \cdot \mathbf{z}_{i} \ldots$ If $i<j-1$, then $\mathbf{z}_{i} \sim \overline{\mathbf{w}} \mathbf{z}_{j-1}$ by (G1), which contradicts (III). Therefore $i=j-1$, whence

$$
\overline{\mathbf{w}}=\ldots \mathbf{s}_{i} \mathbf{z}_{i} \cdot \mathbf{z}_{i} \ldots \stackrel{(19.1 \mathrm{a})}{\approx} \ldots \mathbf{s}_{i} \mathbf{z}_{i} \ldots
$$

that is, the factor $\mathbf{z}_{j}$ is eliminated from $\overline{\mathbf{w}}$. Hence (IV) is satisfied.

Lemma 19.4. The semigroup $\mathscr{C}_{8}$ has a basis of the form $\{(19.1)\} \cup \Sigma$, where $\Sigma$ is some set of identities formed by connected words in canonical form.

Proof. It is routinely verified that $\mathscr{C}_{8}$ satisfies the identities (19.1) and is idempotentseparable. Since the subsemigroup $\{1,4,5,6\}$ of $\mathscr{C}_{8}$ is isomorphic to $A_{0}$, the variety generated by $\mathscr{C}_{8}$ contains $A_{0}$. It follows from Lemma 2.6(i) that $\mathscr{C}_{8}$ has a basis $\Sigma$ that consists of 
identities formed by connected words. Therefore the set $\{(19.1)\} \cup \Sigma$ is also a basis for $\mathscr{C}_{8}$. By Lemma 19.3, the words that form the identities in $\Sigma$ can be chosen to be connected and in canonical form.

\subsection{Identities satisfied by $\mathscr{C}_{8}$}

Let $\mathbf{x}$ and $\mathbf{y}$ be any distinct perfect squares of a word $\mathbf{w}$ in canonical form. Then write $\mathbf{x} \Subset_{\mathbf{w}} \mathbf{y}$ to indicate that within $\mathbf{w}$, all occurrences of $\mathbf{x}$ are sandwiched between two occurrences of $\mathbf{y}$. Note that if $\mathbf{x} \Subset_{\mathbf{w}} \mathbf{y}$, then (III) implies that no occurrence of $\mathbf{y}$ is sandwiched between any two occurrences of $\mathbf{x}$.

A generalized version of the order $\prec_{\mathbf{w}}$ introduced in $\S 2$ is also required: write $\mathbf{x} \prec_{\mathbf{w}} \mathbf{y}$ to indicate that within $\mathbf{w}$, each occurrence of $\mathbf{x}$ precedes the first occurrence of $\mathbf{y}$.

REMARK 19.5. Suppose that $\mathbf{x}$ and $\mathbf{y}$ are distinct perfect squares of a word $\mathbf{w}$ in canonical form. Then $\mathbf{x} \varkappa_{\mathbf{w}} \mathbf{y}$ by (III), whence by (G1) and (G2), precisely one of the following holds:

$\left(1_{\chi}\right) \mathbf{x} \prec_{\mathbf{w}} \mathbf{y}$;

$(2 \nsim) \mathbf{y} \prec_{\mathbf{w}} \mathbf{x}$;

$(3 \nsim) \mathbf{x} \Subset_{\mathbf{w}} \mathbf{y}$ and $\mathbf{x y}$ is not a factor of $\mathbf{w}$;

$(4 \nsim) \mathbf{y} \Subset_{\mathbf{w}} \mathbf{x}$ and $\mathbf{y x}$ is not a factor of $\mathbf{w}$.

For the remainder of this subsection, suppose that $\mathbf{w} \approx \mathbf{w}^{\prime}$ is any identity satisfied by the semigroup $\mathscr{C}_{8}$, where the words

$$
\mathbf{w}=\mathbf{z}_{0} \prod_{i=1}^{n}\left(\mathbf{s}_{i} \mathbf{z}_{i}\right) \quad \text { and } \quad \mathbf{w}^{\prime}=\mathbf{z}_{0}^{\prime} \prod_{i=1}^{n^{\prime}}\left(\mathbf{s}_{i}^{\prime} \mathbf{z}_{i}^{\prime}\right)
$$

are in canonical form. Let

$$
\mathbf{Z}=\bigcup_{i=0}^{n}\left\{\mathbf{z}_{i}\right\}, \quad \mathbf{S}=\bigcup_{i=1}^{n}\left\{\mathbf{s}_{i}\right\}, \quad \mathbf{Z}^{\prime}=\bigcup_{i=0}^{n^{\prime}}\left\{\mathbf{z}_{i}^{\prime}\right\} \quad \text { and } \quad \mathbf{S}^{\prime}=\bigcup_{i=1}^{n^{\prime}}\left\{\mathbf{s}_{i}^{\prime}\right\} .
$$

Note that there are repetitions in $\mathbf{z}_{0}, \ldots, \mathbf{z}_{n}$ and in $\mathbf{z}_{0}^{\prime}, \ldots, \mathbf{z}_{n^{\prime}}^{\prime}$, since $\mathbf{z}_{0}=\mathbf{z}_{n}$ and $\mathbf{z}_{0}^{\prime}=\mathbf{z}_{n^{\prime}}^{\prime}$ by $(\mathrm{V})$. But by (I), the only repetition that can possibly occur in $S$ and in $S^{\prime}$ is when two or more words equal the empty word.

Lemma 19.6. The equality $\mathrm{S}=\mathrm{S}^{\prime}$ holds.

Proof. The subsemigroup $\{1,2,6\}$ of $\mathscr{C}_{8}$ is isomorphic to $N_{2}^{1}$. Therefore $\operatorname{con}(\mathbf{w})=\operatorname{con}\left(\mathbf{w}^{\prime}\right)$ and $\operatorname{sim}(\mathbf{w})=\operatorname{sim}\left(\mathbf{w}^{\prime}\right)$ by Lemma $2.1(\mathrm{v})$. Further, $\mathscr{C}_{8}$ does not satisfy the identity (2.3) with $n=2$ because $6^{2} \cdot 4 \cdot 6^{2} \cdot 3 \cdot 6^{2} \neq 6^{2} \cdot 4 \cdot 3 \cdot 6^{2}$, so that $\mathrm{F}_{\mathrm{SS}}(\mathbf{w})=\mathrm{F}_{\mathrm{SS}}\left(\mathbf{w}^{\prime}\right)$ by Lemma 2.10 . The present lemma thus follows.

Lemma 19.7. The equality $\mathrm{Z}=\mathrm{Z}^{\prime}$ holds.

Proof. Since $\operatorname{con}(\mathbf{w})=\operatorname{con}\left(\mathbf{w}^{\prime}\right)$ and $\operatorname{sim}(\mathbf{w})=\operatorname{sim}\left(\mathbf{w}^{\prime}\right)$ by the proof of Lemma 19.6,

(a) $\operatorname{con}\left(\mathbf{z}_{0} \ldots \mathbf{z}_{n}\right)=\operatorname{con}\left(\mathbf{z}_{0}^{\prime} \ldots \mathbf{z}_{n^{\prime}}^{\prime}\right)$ and $\operatorname{con}\left(\mathbf{s}_{1} \ldots \mathbf{s}_{n}\right)=\operatorname{con}\left(\mathbf{s}_{1}^{\prime} \ldots \mathbf{s}_{n^{\prime}}^{\prime}\right)$.

Let $x$ and $y$ be any distinct letters of some perfect square $\mathbf{z}^{\prime}$ from $Z^{\prime}$, say

$$
\mathbf{z}^{\prime}=(\ldots x \ldots y \ldots)^{2} .
$$

It is shown in the remainder of this proof that the letters $x$ and $y$ belong to the same perfect square in Z. Hence

(b) if $x, y \in \operatorname{con}\left(\mathbf{z}^{\prime}\right)$ for some $\mathbf{z}^{\prime} \in \mathbf{Z}^{\prime}$, then $x, y \in \operatorname{con}(\mathbf{z})$ for some $\mathbf{z} \in \mathbf{Z}$.

The converse of (b) holds by symmetry. It then follows from (a), (II), and (VI) that $Z=Z^{\prime}$. 
Suppose that $x$ and $y$ are letters from distinct perfect squares $\mathbf{x}, \mathbf{y} \in \mathbf{Z}$, that is, $\mathbf{x} \neq \mathbf{y}$ with $\mathbf{x}=(\ldots x \ldots)^{2}$ and $\mathbf{y}=(\ldots y \ldots)^{2}$. Then $\mathbf{x} \chi_{\mathbf{w}} \mathbf{y}$ by $(\mathrm{III})$ and $\operatorname{con}(\mathbf{x}) \cap \operatorname{con}(\mathbf{y})=\emptyset$ by $(\mathrm{VI})$. As observed in Remark 19.5, both $(3 \nsim)$ and $\left(4_{\chi}\right)$ cannot simultaneously hold. By symmetry, assume that $\left(4_{\chi}\right)$ does not hold. Hence it follows from either $\left(1_{\chi}\right),\left(2_{\chi}\right)$, or $\left(3_{\chi}\right)$ that

(c) no occurrence of $\mathbf{y}$ in $\mathbf{w}$ is sandwiched between any two occurrences of $\mathbf{x}$.

Let $p$ be the least integer such that $\mathbf{z}_{p}=\mathbf{x}$ and let $q$ be the greatest integer such that $\mathbf{z}_{q}=\mathbf{x}$. Then

$$
\mathbf{w}=\underbrace{\mathbf{z}_{0}\left(\prod_{i=1}^{p-1}\left(\mathbf{s}_{i} \mathbf{z}_{i}\right)\right) \mathbf{s}_{p}}_{\mathbf{a}} \mathbf{x} \underbrace{\left.\mathbf{x} \prod_{i=p+1}^{q-1}\left(\mathbf{s}_{i} \mathbf{z}_{i}\right)\right) \mathbf{s}_{q} \mathbf{x}}_{\mathbf{b}} \underbrace{\left(\prod_{i=q+1}^{n}\left(\mathbf{s}_{i} \mathbf{z}_{i}\right)\right)}_{\mathbf{c}} .
$$

By (V), either $p=0$ or $q=n$ implies $\mathbf{z}_{0}=\mathbf{z}_{n}=\mathbf{x}$, whence (c) is violated. It thus follows that $0<p \leqslant q<n$ and $\mathbf{a} \neq \emptyset \neq \mathbf{c}$. Now $\operatorname{con}(\mathbf{x}) \cap \operatorname{con}(\mathbf{a c})=\emptyset$ by the choice of $p$ and $q$. Further, since (III) implies $\mathbf{x} \not_{\mathbf{w}} \mathbf{h}$ for all $\mathbf{h} \in \mathbf{Z} \backslash\{\mathbf{x}\}$, it follows from (I) and (G2) that

(d) $\operatorname{con}(\mathbf{b}) \cap \operatorname{con}(\mathbf{a c})=\emptyset$.

Since $\mathbf{z}_{0}=\mathbf{z}_{n}$ by $(\mathrm{V})$, the sets $\left\{\mathbf{z}_{0}, \ldots, \mathbf{z}_{p-1}\right\}$ and $\left\{\mathbf{z}_{q+1}, \ldots, \mathbf{z}_{n}\right\}$ share some common element. Let $m \in\{0, \ldots, p-1\}$ be the greatest integer such that

$$
\mathbf{z}_{m} \in\left\{\mathbf{z}_{0}, \ldots, \mathbf{z}_{p-1}\right\} \cap\left\{\mathbf{z}_{q+1}, \ldots, \mathbf{z}_{n}\right\},
$$

and let $r \in\{q+1, \ldots, n\}$ be the least integer such that $\mathbf{z}_{m}=\mathbf{z}_{r}$. Then

$$
\mathbf{w}=\underbrace{\mathbf{z}_{0}\left(\prod_{i=1}^{m-1}\left(\mathbf{s}_{i} \mathbf{z}_{i}\right)\right) \mathbf{s}_{m} \mathbf{u}\left(\prod_{i=m+1}^{p-1}\left(\mathbf{s}_{i} \mathbf{z}_{i}\right)\right) \mathbf{s}_{p}}_{\mathbf{a}} \mathbf{b} \underbrace{\left(\prod_{i=q+1}^{r-1}\left(\mathbf{s}_{i} \mathbf{z}_{i}\right)\right)}_{\mathbf{c}^{\prime}} \mathbf{s}_{r} \underbrace{\mathbf{u}\left(\prod_{i=r+1}^{n}\left(\mathbf{s}_{i} \mathbf{z}_{i}\right)\right)}_{\mathbf{c}^{\prime \prime}}
$$

where $\mathbf{u}=\mathbf{z}_{m}=\mathbf{z}_{r}$ and $\mathbf{c}=\mathbf{c}^{\prime} \mathbf{s}_{r} \mathbf{c}^{\prime \prime}$. Note that by (IV),

(e) $\mathbf{s}_{r} \neq \emptyset$.

Now the choice of $m$ and $r$ implies that:

(f) $\mathbf{u} \notin\left\{\mathbf{z}_{m+1}, \ldots, \mathbf{z}_{p-1}\right\} \cup\left\{\mathbf{z}_{q+1}, \ldots, \mathbf{z}_{r-1}\right\}$;

(g) $\left\{\mathbf{z}_{m+1}, \ldots, \mathbf{z}_{p-1}\right\} \cap\left\{\mathbf{z}_{q+1}, \ldots, \mathbf{z}_{r-1}\right\}=\emptyset$.

If there exists some $\mathbf{k} \in\left\{\mathbf{z}_{0}, \ldots, \mathbf{z}_{m-1}\right\} \cap\left\{\mathbf{z}_{q+1}, \ldots, \mathbf{z}_{r-1}\right\}$, then $\mathbf{k} \neq \mathbf{u}$ by (f) so that $\mathbf{k} \sim_{\mathbf{w}} \mathbf{u}$ by (G2). If there exists some $\mathbf{k}^{\prime} \in\left\{\mathbf{z}_{q+1}, \ldots, \mathbf{z}_{r-1}\right\} \cap\left\{\mathbf{z}_{r+1}, \ldots, \mathbf{z}_{n}\right\}$, then $\mathbf{k}^{\prime} \neq \mathbf{u}$ by (f) so that $\mathbf{k}^{\prime} \sim_{\mathbf{w}} \mathbf{u}$ by (G2). But either of these contradicts (III). Thus $\mathbf{k}$ and $\mathbf{k}^{\prime}$ do not exist, whence

$$
\left\{\mathbf{z}_{0}, \ldots, \mathbf{z}_{m-1}\right\} \cap\left\{\mathbf{z}_{q+1}, \ldots, \mathbf{z}_{r-1}\right\}=\left\{\mathbf{z}_{q+1}, \ldots, \mathbf{z}_{r-1}\right\} \cap\left\{\mathbf{z}_{r+1}, \ldots, \mathbf{z}_{n}\right\}=\emptyset .
$$

It then follows from (I), (d), (f), and (g) that

(h) $\operatorname{con}\left(\mathbf{c}^{\prime}\right) \cap \operatorname{con}\left(\mathbf{a b s}_{r} \mathbf{c}^{\prime \prime}\right)=\emptyset$.

Let $\varphi: \mathcal{X} \rightarrow \mathscr{C}_{8}$ denote the substitution

$$
z \mapsto \begin{cases}5 & \text { if } z \in \operatorname{con}\left(\mathbf{c}^{\prime}\right) \\ 3 & \text { if } z=\mathrm{h}\left(\mathbf{s}_{r}\right) \\ 6 & \text { otherwise }\end{cases}
$$

Then by (d), (e), and (h),

$$
\begin{aligned}
\mathbf{w} \varphi & = \begin{cases}(\mathbf{a b}) \varphi \cdot \mathbf{c}^{\prime} \varphi \cdot \mathbf{s}_{r} \varphi \cdot \mathbf{c}^{\prime \prime} \varphi & \text { if } \mathbf{c}^{\prime} \neq \emptyset \\
(\mathbf{a b}) \varphi \cdot \mathbf{s}_{r} \varphi \cdot \mathbf{c}^{\prime \prime} \varphi & \text { if } \mathbf{c}^{\prime}=\emptyset\end{cases} \\
& = \begin{cases}(6 \ldots 6) \cdot(5 \ldots 5) \cdot(3 \cdot 6 \ldots 6) \cdot(6 \ldots 6) & \text { if } \mathbf{c}^{\prime} \neq \emptyset \\
(6 \ldots 6) \cdot(3 \cdot 6 \ldots 6) \cdot(6 \ldots 6) & \text { if } \mathbf{c}^{\prime}=\emptyset\end{cases} \\
& =2 .
\end{aligned}
$$


But $\mathbf{z}^{\prime} \varphi \in\left(\{5,6\}^{*} \cdot 6 \cdot\{5,6\}^{*} \cdot 5 \cdot\{5,6\}^{*}\right)^{2}=\{1\}$ implies the contradiction $\mathbf{w}^{\prime} \varphi=1$. Therefore the assumption that the letters $x$ and $y$ belong to the distinct perfect squares $\mathbf{x}, \mathbf{y} \in \mathbf{Z}$ does not hold. Consequently, the letters $x$ and $y$ belong to the same perfect square in $\mathrm{Z}$.

Corollary 19.8. Let $\varphi$ be any endomorphism of $\mathcal{X}^{+}$. Suppose that the words $\mathbf{w} \varphi$ and $\mathbf{w}^{\prime} \varphi$ are converted by the identities (19.1) into the words $\overline{\mathbf{w} \varphi}$ and $\overline{\mathbf{w}^{\prime} \varphi}$ in canonical form. Then the set of perfect squares of $\overline{\mathbf{w} \varphi}$ is equal to the set of perfect squares of $\overline{\mathbf{w}^{\prime} \varphi}$.

Proof. As observed in the proof of Lemma 19.4, the semigroup $\mathscr{C}_{8}$ satisfies the identities (19.1). Then by assumption, $\mathscr{C}_{8}$ satisfies the identity $\mathbf{w} \varphi \approx \mathbf{w}^{\prime} \varphi$. Hence the result follows from Lemma 19.7 .

Lemma 19.9. Suppose that $\mathbf{z} \in \mathbf{Z}=\mathbf{Z}^{\prime}$ and $\mathbf{s} \in \mathbf{S}=\mathrm{S}^{\prime}$ with $\mathbf{s} \neq \emptyset$. Then $\mathbf{s z}$ is a factor of $\mathbf{w}$ if and only if $\mathbf{s z}$ is a factor of $\mathbf{w}^{\prime}$.

Proof. Let $\mathbf{s z}$ be a factor of $\mathbf{w}$. Suppose that $\mathbf{s z}$ is not a factor of $\mathbf{w}^{\prime}$. Then there exists some $\mathbf{z}^{\prime} \in \mathbf{Z}=\mathbf{Z}^{\prime}$ with $\mathbf{z}^{\prime} \neq \mathbf{z}$ such that $\mathbf{s z}^{\prime}$ is a factor of $\mathbf{w}^{\prime}$. Hence $\mathbf{z} \chi_{\mathbf{w}} \mathbf{z}^{\prime}$ and $\mathbf{z} \mathcal{\psi}_{\mathbf{w}^{\prime}} \mathbf{z}^{\prime}$ by (III), and $\operatorname{con}(\mathbf{z}) \cap \operatorname{con}\left(\mathbf{z}^{\prime}\right)=\emptyset$ by (VI). Since $\mathbf{s z}=\mathbf{s}_{k} \mathbf{z}_{k}$ for some $k \in\{1, \ldots, n\}$ and $\mathbf{s z}^{\prime}=\mathbf{s}_{k^{\prime}}^{\prime} \mathbf{z}_{k^{\prime}}^{\prime}$ for some $k^{\prime} \in\left\{1, \ldots, n^{\prime}\right\}$,

$$
\mathbf{w}=\mathbf{z}_{0}\left(\prod_{i=1}^{k-1}\left(\mathbf{s}_{i} \mathbf{z}_{i}\right)\right) \mathbf{s z}\left(\prod_{i=k+1}^{n}\left(\mathbf{s}_{i} \mathbf{z}_{i}\right)\right) \quad \text { and } \quad \mathbf{w}^{\prime}=\mathbf{z}_{0}^{\prime}\left(\prod_{i=1}^{k^{\prime}-1}\left(\mathbf{s}_{i}^{\prime} \mathbf{z}_{i}^{\prime}\right)\right) \mathbf{s z}^{\prime}\left(\prod_{i=k^{\prime}+1}^{n^{\prime}}\left(\mathbf{s}_{i}^{\prime} \mathbf{z}_{i}^{\prime}\right)\right) .
$$

Choose any letter $x \notin \operatorname{con}\left(\mathbf{w} \mathbf{w}^{\prime}\right)$ and let $\varphi_{1}$ denote the substitution $\mathrm{h}(\mathbf{s}) \mapsto x \mathbf{z} \mathrm{h}(\mathbf{s})$. Then $\mathscr{C}_{8}$ satisfies the identity $\mathbf{w} \varphi_{1} \approx \mathbf{w}^{\prime} \varphi_{1}$. It is easily seen that the set of perfect squares of

$$
\mathbf{w} \varphi_{1}=\mathbf{z}_{0}\left(\prod_{i=1}^{k-1}\left(\mathbf{s}_{i} \mathbf{z}_{i}\right)\right) x \mathbf{z s z}\left(\prod_{i=k+1}^{n}\left(\mathbf{s}_{i} \mathbf{z}_{i}\right)\right)
$$

is equal to the set $\mathbf{Z}$ of perfect squares of $\mathbf{w}$. Since (III) implies $\mathbf{z} \chi_{\mathbf{w}} \mathbf{x}$ for any $\mathbf{x} \in \mathbf{Z} \backslash\{\mathbf{z}\}$, it is easily shown that $\mathbf{z} \chi_{\mathbf{w} \varphi_{1}} \mathbf{x}$ for any $\mathbf{x} \in \mathbf{Z} \backslash\{\mathbf{z}\}$. Therefore the word $\mathbf{w} \varphi_{1}=\overline{\mathbf{w} \varphi_{1}}$ is already in canonical form and so its set of perfect squares is equal to Z. Similarly, the set of perfect squares of

$$
\mathbf{w}^{\prime} \varphi_{1}=\mathbf{z}_{0}^{\prime}\left(\prod_{i=1}^{k^{\prime}-1}\left(\mathbf{s}_{i}^{\prime} \mathbf{z}_{i}^{\prime}\right)\right) x \mathbf{z s} \mathbf{z}^{\prime}\left(\prod_{i=k^{\prime}+1}^{n^{\prime}}\left(\mathbf{s}_{i}^{\prime} \mathbf{z}_{i}^{\prime}\right)\right)
$$

is equal to the set $\mathbf{Z}$ of perfect squares of $\mathbf{w}^{\prime}$. Suppose $\mathbf{z} \sim_{\mathbf{w}^{\prime} \varphi_{1}} \mathbf{y}$ for some $\mathbf{y} \in \mathbf{Z} \backslash\{\mathbf{z}\}$, so that (III) is violated by $\mathbf{w}^{\prime} \varphi_{1}$. Then the word $\mathbf{w}^{\prime} \varphi_{1}$ is not in canonical form and so by Lemma 19.3, can be converted by the identities (19.1) into some word $\overline{\mathbf{w}^{\prime} \varphi_{1}}$ in canonical form. Since $\mathbf{z} \sim_{\mathbf{w}^{\prime} \varphi_{1}} \mathbf{y}$, the conversion of $\mathbf{w}^{\prime} \varphi_{1}$ into $\overline{\mathbf{w}^{\prime} \varphi_{1}}$ will result in the letters of $\mathbf{z}$ and $\mathbf{y}$ being grouped into the same perfect square. It follows that the set $Z$ of perfect squares of $\overline{\mathbf{w} \varphi_{1}}$ is not equal to the set of perfect squares of $\overline{\mathbf{w}^{\prime} \varphi_{1}}$, whence Corollary 19.8 is contradicted. Therefore $\mathbf{y}$ does not exist, so that $\mathbf{z} \chi_{\mathbf{w}^{\prime} \varphi_{1}} \mathbf{x}$ for all $\mathbf{x} \in \mathbf{Z} \backslash\{\mathbf{z}\}$. Consequently, $\mathbf{w}^{\prime} \varphi_{1}$ is in canonical form.

Now let $\varphi_{2}$ denote the substitution $\mathrm{t}(\mathbf{s}) \mapsto \mathrm{t}(\mathbf{s}) \mathbf{z}^{\prime}$. Then $\mathscr{C}_{8}$ satisfies $\mathbf{w} \varphi_{1} \varphi_{2} \approx \mathbf{w}^{\prime} \varphi_{1} \varphi_{2}$. Since

$$
\mathbf{w}^{\prime} \varphi_{1} \varphi_{2}=\mathbf{z}_{0}^{\prime}\left(\prod_{i=1}^{k^{\prime}-1}\left(\mathbf{s}_{i}^{\prime} \mathbf{z}_{i}^{\prime}\right)\right) x \mathbf{z} \mathbf{s z} \mathbf{z}^{\prime} \mathbf{z}^{\prime}\left(\prod_{i=k^{\prime}+1}^{n^{\prime}}\left(\mathbf{s}_{i}^{\prime} \mathbf{z}_{i}^{\prime}\right)\right) \stackrel{(19.1 \mathrm{a})}{\approx} \mathbf{w}^{\prime} \varphi_{1}
$$

where the word $\mathbf{w}^{\prime} \varphi_{1}$ is in canonical form, the set of perfect squares of $\overline{\mathbf{w}^{\prime} \varphi_{1} \varphi_{2}}=\mathbf{w}^{\prime} \varphi_{1}$ is 
equal to Z. On the other hand, the word

$$
\mathbf{w} \varphi_{1} \varphi_{2}=\mathbf{z}_{0}\left(\prod_{i=1}^{k-1}\left(\mathbf{s}_{i} \mathbf{z}_{i}\right)\right) x \mathbf{z s z} \mathbf{z}^{\prime} \mathbf{z}\left(\prod_{i=k+1}^{n}\left(\mathbf{s}_{i} \mathbf{z}_{i}\right)\right)
$$

is not in canonical form because $\mathbf{z} \sim_{\mathbf{w} \varphi_{1} \varphi_{2}} \mathbf{z}^{\prime}$ by (G1). Therefore when the word $\mathbf{w} \varphi_{1} \varphi_{2}$ is converted by the identities (19.1) into the word $\overline{\mathbf{w} \varphi_{1} \varphi_{2}}$ in canonical form, the letters of $\mathbf{z}$ and $\mathbf{z}^{\prime}$ are grouped into the same perfect square. It follows that the set of perfect squares of $\overline{\mathbf{w} \varphi_{1} \varphi_{2}}$ is not equal to the set $\mathbf{Z}$ of perfect squares of $\overline{\mathbf{w}^{\prime} \varphi_{1} \varphi_{2}}$, whence Corollary 19.8 is contradicted. Consequently, the assumption that $\mathbf{s z}$ is not a factor of $\mathbf{w}^{\prime}$ does not hold.

Lemma 19.10. Suppose that $\mathbf{z} \in \mathbf{Z}=\mathbf{Z}^{\prime}$ and $\mathbf{s} \in \mathbf{S}=\mathrm{S}^{\prime}$ with $\mathbf{s} \neq \emptyset$. Then $\mathbf{z s}$ is a factor of $\mathbf{w}$ if and only if $\mathbf{z s}$ is a factor of $\mathbf{w}^{\prime}$.

Proof. Let $\mathbf{z s}$ be a factor of $\mathbf{w}$. Suppose that $\mathbf{z s}$ is not a factor of $\mathbf{w}^{\prime}$. Then there exists some $\mathbf{z}^{\prime} \in \mathbf{Z}=\mathbf{Z}^{\prime}$ with $\mathbf{z}^{\prime} \neq \mathbf{z}$ such that $\mathbf{z}^{\prime} \mathbf{s}$ is a factor of $\mathbf{w}^{\prime}$. Hence $\mathbf{z} \chi_{\mathbf{w}} \mathbf{z}^{\prime}$ and $\mathbf{z} \chi_{\mathbf{w}^{\prime}} \mathbf{z}^{\prime}$ by (III), and $\operatorname{con}(\mathbf{z}) \cap \operatorname{con}\left(\mathbf{z}^{\prime}\right)=\emptyset$ by (VI). Now $\mathbf{z s}=\mathbf{z}_{k} \mathbf{s}_{k+1}$ for some $k \in\{0, \ldots, n-1\}$ and $\mathbf{z}^{\prime} \mathbf{s}=\mathbf{z}_{k^{\prime}}^{\prime} \mathbf{s}_{k^{\prime}+1}^{\prime}$ for some $k^{\prime} \in\left\{0, \ldots, n^{\prime}-1\right\}$. Further, $\mathbf{z}_{k+1}=\mathbf{z}_{k^{\prime}+1}^{\prime}$ by Lemma 19.9. Thus

$$
\mathbf{w}=\mathbf{z}_{0}\left(\prod_{i=1}^{k-1}\left(\mathbf{s}_{i} \mathbf{z}_{i}\right)\right) \mathbf{s}_{k} \cdot \mathbf{z s} \cdot \mathbf{x}\left(\prod_{i=k+2}^{n}\left(\mathbf{s}_{i} \mathbf{z}_{i}\right)\right)
$$

and

$$
\mathbf{w}^{\prime}=\mathbf{z}_{0}^{\prime}\left(\prod_{i=1}^{k^{\prime}-1}\left(\mathbf{s}_{i}^{\prime} \mathbf{z}_{i}^{\prime}\right)\right) \mathbf{s}_{k^{\prime}}^{\prime} \cdot \mathbf{z}^{\prime} \mathbf{s} \cdot \mathbf{x}\left(\prod_{i=k^{\prime}+2}^{n^{\prime}}\left(\mathbf{s}_{i}^{\prime} \mathbf{z}_{i}^{\prime}\right)\right)
$$

where $\mathbf{x}=\mathbf{z}_{k+1}=\mathbf{z}_{k^{\prime}+1}^{\prime}$.

Suppose $\mathbf{z} \in\left\{\mathbf{z}_{0}^{\prime}, \ldots, \mathbf{z}_{k^{\prime}-1}^{\prime}\right\}$. Let $\varphi_{1}$ denote the substitution $\mathrm{h}(\mathbf{s}) \mapsto \mathbf{z h}(\mathbf{s})$. Then $\mathscr{C}_{8}$ satisfies the identity $\mathbf{w} \varphi_{1} \approx \mathbf{w}^{\prime} \varphi_{1}$. Since

$$
\mathbf{w} \varphi_{1}=\mathbf{z}_{0}\left(\prod_{i=1}^{k-1}\left(\mathbf{s}_{i} \mathbf{z}_{i}\right)\right) \mathbf{s}_{k} \cdot \mathbf{z z s} \cdot \mathbf{x}\left(\prod_{i=k+2}^{n}\left(\mathbf{s}_{i} \mathbf{z}_{i}\right)\right) \stackrel{(19.1 \mathrm{a})}{\approx} \mathbf{w},
$$

where the word $\mathbf{w}$ is in canonical form, the set of perfect squares of $\overline{\mathbf{w} \varphi_{1}}=\mathbf{w}$ is equal to $\mathbf{Z}$. On the other hand,

$$
\mathbf{w}^{\prime} \varphi_{1}=\mathbf{z}_{0}^{\prime}\left(\prod_{i=1}^{k^{\prime}-1}\left(\mathbf{s}_{i}^{\prime} \mathbf{z}_{i}^{\prime}\right)\right) \mathbf{s}_{k^{\prime}}^{\prime} \cdot \mathbf{z}^{\prime} \mathbf{z s} \cdot \mathbf{x}\left(\prod_{i=k^{\prime}+2}^{n^{\prime}}\left(\mathbf{s}_{i}^{\prime} \mathbf{z}_{i}^{\prime}\right)\right)
$$

and $\mathbf{z} \in\left\{\mathbf{z}_{0}^{\prime}, \ldots, \mathbf{z}_{k^{\prime}-1}^{\prime}\right\}$ imply $\mathbf{z} \sim_{\mathbf{w}^{\prime} \varphi_{1}} \mathbf{z}^{\prime}$ by (G1). Hence the word $\mathbf{w}^{\prime} \varphi_{1}$ is not in canonical form, and when it is converted by the identities (19.1) into the word $\overline{\mathbf{w}^{\prime} \varphi_{1}}$ in canonical form, the letters of $\mathbf{z}$ and $\mathbf{z}^{\prime}$ are grouped into the same perfect square. It follows that the set of perfect squares of $\overline{\mathbf{w}^{\prime} \varphi_{1}}$ is not equal to the set $\mathbf{Z}$ of perfect squares of $\overline{\mathbf{w} \varphi_{1}}$, whence Corollary 19.8 is contradicted. Thus $\mathbf{z} \notin\left\{\mathbf{z}_{0}^{\prime}, \ldots, \mathbf{z}_{k^{\prime}-1}^{\prime}\right\}$. A symmetrical argument shows $\mathbf{z}^{\prime} \notin\left\{\mathbf{z}_{0}, \ldots, \mathbf{z}_{k-1}\right\}$.

Suppose $\mathbf{x}=\mathbf{z}^{\prime}$. Let $\varphi_{2}$ denote the substitution $\mathrm{t}(\mathbf{s}) \mapsto \mathrm{t}(\mathbf{s}) \mathbf{z}$. Then

$$
\mathbf{w} \varphi_{2}=\mathbf{z}_{0}\left(\prod_{i=1}^{k-1}\left(\mathbf{s}_{i} \mathbf{z}_{i}\right)\right) \mathbf{s}_{k} \cdot \mathbf{z s z} \cdot \mathbf{z}^{\prime}\left(\prod_{i=k+2}^{n}\left(\mathbf{s}_{i} \mathbf{z}_{i}\right)\right) .
$$


It is clear that the set of perfect squares of $\mathbf{w} \varphi_{2}$ is equal to $\mathbf{Z}$. Further, $\mathbf{z} \chi_{\mathbf{w} \varphi_{2}} \mathbf{z}^{\prime}$ because $\mathbf{z}^{\prime} \notin\left\{\mathbf{z}_{0}, \ldots, \mathbf{z}_{k-1}\right\}$ and $\mathbf{z} \chi_{\mathbf{w}} \mathbf{z}^{\prime}$. It follows that the word $\mathbf{w} \varphi_{2}=\overline{\mathbf{w} \varphi_{2}}$ is in canonical form and its set of perfect squares is equal to $Z$. On the other hand, the word

$$
\mathbf{w}^{\prime} \varphi_{2}=\mathbf{z}_{0}^{\prime}\left(\prod_{i=1}^{k^{\prime}-1}\left(\mathbf{s}_{i}^{\prime} \mathbf{z}_{i}^{\prime}\right)\right) \mathbf{s}_{k^{\prime}}^{\prime} \cdot \mathbf{z}^{\prime} \mathbf{s z} \cdot \mathbf{z}^{\prime}\left(\prod_{i=k^{\prime}+2}^{n^{\prime}}\left(\mathbf{s}_{i}^{\prime} \mathbf{z}_{i}^{\prime}\right)\right)
$$

is not in canonical form because $\mathbf{z} \sim_{\mathbf{w}^{\prime} \varphi_{2}} \mathbf{z}^{\prime}$ by (G1). Therefore when the word $\mathbf{w}^{\prime} \varphi_{2}$ is converted by the identities (19.1) into the word $\overline{\mathbf{w}^{\prime} \varphi_{2}}$ in canonical form, the letters of $\mathbf{z}$ and $\mathbf{z}^{\prime}$ are grouped into the same perfect square. It follows that the set of perfect squares of $\overline{\mathbf{w}^{\prime} \varphi_{2}}$ is not equal to the set $\mathbf{Z}$ of perfect squares of $\overline{\mathbf{w} \varphi_{2}}$, whence Corollary 19.8 is contradicted. Hence $\mathbf{x} \neq \mathbf{z}^{\prime}$. A symmetrical argument shows $\mathbf{x} \neq \mathbf{z}$.

Now the word $\mathbf{z}^{\prime}$ is a perfect square of $\mathbf{w}$ because $\mathbf{z}^{\prime} \in Z^{\prime}=Z$. But since it was shown that $\mathbf{z}^{\prime} \notin\left\{\mathbf{z}_{0}, \ldots, \mathbf{z}_{k-1}\right\}$ and $\mathbf{z}^{\prime} \neq \mathbf{x}$, each factor $\mathbf{z}^{\prime}$ of $\mathbf{w}$ is one of $\mathbf{z}_{k+2}, \ldots, \mathbf{z}_{n}$. Let $p \in\{k+2, \ldots, n\}$ be the least such that $\mathbf{z}_{p}=\mathbf{z}^{\prime}$ and let $q \in\{k+2, \ldots, n\}$ be the greatest such that $\mathbf{z}_{q}=\mathbf{z}^{\prime}$. Then

$$
\mathbf{w}=\underbrace{\mathbf{z}_{0}\left(\prod_{i=1}^{k-1}\left(\mathbf{s}_{i} \mathbf{z}_{i}\right)\right) \mathbf{s}_{k} \mathbf{z s}}_{\mathbf{a}} \mathbf{x} \underbrace{\mathbf{( \prod _ { i = k + 2 } ^ { p - 1 } ( \mathbf { s } _ { i } \mathbf { z } _ { i } ) ) \mathbf { s } _ { p }}}_{\mathbf{b}} \underbrace{\mathbf{z}^{\prime}\left(\prod_{i=p+1}^{q-1}\left(\mathbf{s}_{i} \mathbf{z}_{i}\right)\right) \mathbf{s}_{q} \mathbf{z}^{\prime}}_{\mathbf{c}} \underbrace{\left(\prod_{i=q+1}^{n}\left(\mathbf{s}_{i} \mathbf{z}_{i}\right)\right)}_{\mathbf{d}}
$$

where

(a) any perfect square $\mathbf{z}^{\prime}$ of $\mathbf{w}$ is a factor of $\mathbf{c}$.

Further, it follows from (G2), (I), and (III) that

(b) $\operatorname{con}(\mathbf{c}) \cap \operatorname{con}(\mathbf{a b d})=\emptyset$.

Suppose that the factors $\mathbf{b}$ and $\mathbf{d}$ share some common perfect square. Then there exists the least $r \in\{q+1, \ldots, n\}$ such that $\mathbf{z}_{r} \in\left\{\mathbf{x}, \mathbf{z}_{k+2}, \ldots, \mathbf{z}_{p-1}\right\}$, whence $\mathbf{s}_{r} \neq \emptyset$ by (IV). Since $\left\{\mathbf{z}_{q+1}, \ldots, \mathbf{z}_{r-1}\right\} \cap\left\{\mathbf{x}, \mathbf{z}_{k+2}, \ldots, \mathbf{z}_{p-1}\right\}=\emptyset$ by the minimality of $r$, it follows from (I) that

(c) $\operatorname{con}(\mathbf{b}) \cap \operatorname{con}\left(\prod_{i=q+1}^{r-1}\left(\mathbf{s}_{i} \mathbf{z}_{i}\right)\right)=\emptyset$.

Further, since $\mathbf{z}_{r} \in\left\{\mathbf{x}, \mathbf{z}_{k+2}, \ldots, \mathbf{z}_{p-1}\right\}$, it follows from (G2), (I), and (III) that

(d) $\operatorname{con}(\mathbf{a}) \cap \operatorname{con}\left(\prod_{i=q+1}^{r-1}\left(\mathbf{s}_{i} \mathbf{z}_{i}\right)\right)=\operatorname{con}\left(\prod_{i=q+1}^{r-1}\left(\mathbf{s}_{i} \mathbf{z}_{i}\right)\right) \cap \operatorname{con}\left(\prod_{i=r+1}^{n}\left(\mathbf{s}_{i} \mathbf{z}_{i}\right)\right)=\emptyset$.

Let $\varphi_{3}: \mathcal{X} \rightarrow \mathscr{C}_{8}$ denote the substitution

$$
z \mapsto \begin{cases}5 & \text { if } z \in \operatorname{con}\left(\mathbf{c} \prod_{i=q+1}^{r-1}\left(\mathbf{s}_{i} \mathbf{z}_{i}\right)\right) \\ 3 & \text { if } z=\mathrm{h}\left(\mathbf{s}_{r}\right) \\ 6 & \text { otherwise }\end{cases}
$$

Then by (a)-(d),

$$
\begin{aligned}
\mathbf{w} \varphi_{3} & =(\mathbf{a b}) \varphi_{3} \cdot\left(\mathbf{c} \prod_{i=q+1}^{r-1}\left(\mathbf{s}_{i} \mathbf{z}_{i}\right)\right) \varphi_{3} \cdot \mathbf{s}_{r} \varphi_{3} \cdot\left(\mathbf{z}_{r} \prod_{i=r+1}^{n}\left(\mathbf{s}_{i} \mathbf{z}_{i}\right)\right) \varphi_{3} \\
& =(6 \ldots 6) \cdot(5 \ldots 5) \cdot(3 \cdot 6 \ldots 6) \cdot(6 \ldots 6)=2 .
\end{aligned}
$$

But $\left(\mathbf{z}^{\prime} \mathbf{s}\right) \varphi_{3}=(5 \ldots 5) \cdot(6 \ldots 6)=1$ so that $\mathbf{w}^{\prime} \varphi_{3}=1$, which is impossible. Hence $\mathbf{b}$ and $\mathbf{d}$ do not share any common perfect squares. It then follows from (I) that

(e) $\operatorname{con}(\mathbf{b}) \cap \operatorname{con}(\mathbf{d})=\emptyset$.

Now since $\mathbf{z}_{0}=\mathbf{z}_{n}$ by $(\mathrm{V})$, the factors $\mathbf{a}$ and $\mathbf{d}$ share at least one common perfect square. Let $t \in\{q+1, \ldots, n\}$ be the least such that $\mathbf{z}_{t} \in\left\{\mathbf{z}_{0}, \ldots, \mathbf{z}_{k-1}, \mathbf{z}\right\}$. Then $\mathbf{s}_{t} \neq \emptyset$ by (IV). Since 
$\left\{\mathbf{z}_{q+1}, \ldots, \mathbf{z}_{t-1}\right\} \cap\left\{\mathbf{z}_{0}, \ldots, \mathbf{z}_{k-1}, \mathbf{z}\right\}=\emptyset$ by the minimality of $t$, it follows from (I) that

(f) $\operatorname{con}(\mathbf{a}) \cap \operatorname{con}\left(\prod_{i=q+1}^{t-1}\left(\mathbf{s}_{i} \mathbf{z}_{i}\right)\right)=\emptyset$.

Let $\varphi_{4}: \mathcal{X} \rightarrow \mathscr{C}_{8}$ denote the substitution

$$
z \mapsto \begin{cases}5 & \text { if } z \in \operatorname{con}\left(\mathbf{c} \prod_{i=q+1}^{t-1}\left(\mathbf{s}_{i} \mathbf{z}_{i}\right)\right) \\ 3 & \text { if } z=\mathrm{h}\left(\mathbf{s}_{t}\right) \\ 6 & \text { otherwise. }\end{cases}
$$

Then by (a), (b), (e), and (f),

$$
\begin{aligned}
\mathbf{w} \varphi_{4} & =(\mathbf{a b}) \varphi_{4} \cdot\left(\mathbf{c} \prod_{i=q+1}^{t-1}\left(\mathbf{s}_{i} \mathbf{z}_{i}\right)\right) \varphi_{4} \cdot \mathbf{s}_{t} \varphi_{4} \cdot\left(\mathbf{z}_{t} \prod_{i=t+1}^{n}\left(\mathbf{s}_{i} \mathbf{z}_{i}\right)\right) \varphi_{4} \\
& =(6 \ldots 6) \cdot(5 \ldots 5) \cdot(3 \cdot 6 \ldots 6) \cdot(6 \ldots 6)=2 .
\end{aligned}
$$

$\operatorname{But}\left(\mathbf{z}^{\prime} \mathbf{s}\right) \varphi_{4}=(5 \ldots 5) \cdot(6 \ldots 6)=1$ so that $\mathbf{w}^{\prime} \varphi_{4}=1$, which is impossible. Consequently, the assumption that $\mathbf{z s}$ is not a factor of $\mathbf{w}^{\prime}$ does not hold.

\section{Lemma 19.11. Let $\mathbf{x}, \mathbf{y} \in \mathbf{Z}=\mathbf{Z}^{\prime}$ with $\mathbf{x} \neq \mathbf{y}$. Then $\mathbf{x} \Subset_{\mathbf{w}} \mathbf{y}$ if and only if $\mathbf{x} \Subset_{\mathbf{w}^{\prime}} \mathbf{y}$.}

Proof. By symmetry, it suffices to show $\mathbf{x} \Subset_{\mathbf{w}} \mathbf{y}$ implies $\mathbf{x} \Subset_{\mathbf{w}^{\prime}} \mathbf{y}$. Suppose $\mathbf{x} \Subset_{\mathbf{w}} \mathbf{y}$. Then all occurrences of $\mathbf{x}$ in $\mathbf{w}$ are sandwiched between two occurrences of $\mathbf{y}$. Hence there exist $p$ and $q$ with $p \leqslant q$ such that $\mathbf{z}_{p}=\mathbf{z}_{q}=\mathbf{x}$, and any occurrence of $\mathbf{x}$ in $\mathbf{w}$ is one of $\mathbf{z}_{p}, \ldots, \mathbf{z}_{q}$. Let $k \in\{0, \ldots, p-1\}$ be the greatest such that $\mathbf{z}_{k}=\mathbf{y}$ and let $r \in\{q+1, \ldots, m\}$ be the least such that $\mathbf{z}_{r}=\mathbf{y}$. Then

$$
\begin{aligned}
& \mathbf{w}=\overbrace{\left(\mathbf{z}_{0} \prod_{i=1}^{k-1}\left(\mathbf{s}_{i} \mathbf{z}_{i}\right)\right) \mathbf{s}_{k}}^{\mathbf{a}} \mathbf{y} \overbrace{\left(\prod_{i=k+1}^{p-1}\left(\mathbf{s}_{i} \mathbf{z}_{i}\right)\right) \mathbf{s}_{p}}^{\mathbf{b}} \mathbf{x} \overbrace{\left(\prod_{i=p+1}^{q-1}\left(\mathbf{s}_{i} \mathbf{z}_{i}\right)\right) \mathbf{s}_{q}}^{\mathbf{c}} \mathbf{x} \\
& \underbrace{\left(\prod_{i=q+1}^{r-1}\left(\mathbf{s}_{i} \mathbf{z}_{i}\right)\right) \mathbf{s}_{r}}_{\mathbf{d}} \mathbf{y} \underbrace{\prod_{i=r+1}^{n}\left(\mathbf{s}_{i} \mathbf{z}_{i}\right)}_{\mathbf{e}}
\end{aligned}
$$

with

(a) $\operatorname{con}(\mathbf{x}) \cap \operatorname{con}($ abde $)=\operatorname{con}(\mathbf{y}) \cap \operatorname{con}(\mathbf{b c d})=\emptyset$.

Further, $\mathbf{s}_{r} \neq \emptyset$ by (IV), and

(b) $\operatorname{con}(\mathbf{b}) \cap \operatorname{con}(\mathbf{a c e})=\operatorname{con}(\mathbf{c}) \cap \operatorname{con}(\mathbf{a b d e})=\operatorname{con}(\mathbf{d}) \cap \operatorname{con}(\mathbf{a c e})=\emptyset$

by (G2), (I), and (III). Suppose $\mathbf{x} \notin_{\mathbf{w}^{\prime}} \mathbf{y}$. Then by Remark 19.5 , either $\left(1_{\chi}\right),(2 \nsim)$, or $(4 \nsim)$ holds. But it is shown in the following that none of these is possible. Hence $\mathbf{x} \Subset_{\mathbf{w}^{\prime}} \mathbf{y}$.

Case 1: $\mathbf{y} \Subset_{\mathbf{w}^{\prime}} \mathbf{x}$. By Lemma 19.9, the factor $\mathbf{s}_{r} \mathbf{y}$ of $\mathbf{w}$ is also a factor of $\mathbf{w}^{\prime}$. By the assumption of this case, all occurrences of $\mathbf{y}$ in $\mathbf{w}^{\prime}$ are sandwiched between two occurrences of $\mathbf{x}$. Therefore the factor $\mathbf{s}_{r} \mathbf{y}$ is also sandwiched between those two occurrences of $\mathbf{x}$, whence $\mathbf{w}^{\prime}=\ldots \mathbf{x} \ldots \mathbf{s}_{r} \mathbf{y} \ldots \mathbf{x} \ldots$ Let $\varphi_{1}: \mathcal{X} \rightarrow \mathscr{C}_{8}$ denote the substitution

$$
z \mapsto \begin{cases}5 & \text { if } z \in \operatorname{con}\left(\mathbf{c x} \prod_{i=q+1}^{r-1}\left(\mathbf{s}_{i} \mathbf{z}_{i}\right)\right) \\ 3 & \text { if } z=\mathrm{h}\left(\mathbf{s}_{r}\right) \\ 6 & \text { otherwise. }\end{cases}
$$


Then by (a) and (b),

$$
\begin{aligned}
\mathbf{w} \varphi_{1} & =(\mathbf{a y b}) \varphi_{1} \cdot\left(\mathbf{x c x} \prod_{i=q+1}^{r-1}\left(\mathbf{s}_{i} \mathbf{z}_{i}\right)\right) \varphi_{1} \cdot \mathbf{s}_{r} \varphi_{1} \cdot(\mathbf{y e}) \varphi_{1} \\
& =(6 \ldots 6) \cdot(5 \ldots 5) \cdot(3 \cdot 6 \ldots 6) \cdot(6 \ldots 6)=2 .
\end{aligned}
$$

It is routinely shown that $\{5,6\}^{*} \cdot 5 \cdot\{5,6\}^{*}=\{1,4,5\}$ in $\mathscr{C}_{8}$. Therefore

$$
\begin{aligned}
\mathbf{w}^{\prime} \varphi_{1} & =\left(\ldots \mathbf{x} \ldots \mathbf{s}_{r} \mathbf{y} \ldots \mathbf{x} \ldots\right) \varphi_{1} \\
& \in\{5,6\}^{*} \cdot(5 \ldots 5) \cdot\{5,6\}^{*} \cdot(3 \cdot 6 \ldots 6) \cdot(6 \ldots 6) \cdot\{5,6\}^{*} \cdot(5 \ldots 5) \cdot\{5,6\}^{*} \\
& =\{1,4,5\} \cdot 3 \cdot\{1,4,5\}=\{1\},
\end{aligned}
$$

whence the contradiction $\mathbf{w} \varphi_{1} \neq \mathbf{w}^{\prime} \varphi_{1}$ is obtained.

Case 2: $\mathbf{y} \prec_{\mathbf{w}^{\prime}} \mathbf{x}$. Then each occurrence of $\mathbf{y}$ in $\mathbf{w}^{\prime}$ precedes every occurrence of $\mathbf{x}$. As observed in Case 1, the factor $\mathbf{s}_{r} \mathbf{y}$ of $\mathbf{w}$ is also a factor of $\mathbf{w}^{\prime}$. Hence

$$
\begin{aligned}
\mathbf{w}^{\prime} \varphi_{1} & =\left(\ldots \mathbf{s}_{r} \mathbf{y} \ldots \mathbf{x} \ldots\right) \varphi_{1} \in\{5,6\}^{*} \cdot(3 \cdot 6 \ldots 6) \cdot(6 \ldots 6) \cdot\{5,6\}^{*} \cdot(5 \ldots 5) \cdot\{5,6\}^{*} \\
& =\{1,4,5,6\} \cdot 3 \cdot\{1,4,5\}=\{1\},
\end{aligned}
$$

which implies the same contradiction as in Case 1.

Case 3: $\mathbf{x} \prec_{\mathbf{w}^{\prime}} \mathbf{y}$. Then each occurrence of $\mathbf{x}$ in $\mathbf{w}^{\prime}$ precedes every occurrence of $\mathbf{y}$. Hence there exist $\ell^{\prime}, m^{\prime}, p^{\prime}$, and $q^{\prime}$ with $0 \leqslant \ell^{\prime} \leqslant m^{\prime}<p^{\prime} \leqslant q^{\prime} \leqslant n^{\prime}$ such that:

- $\mathbf{z}_{\ell^{\prime}}^{\prime}=\mathbf{z}_{m^{\prime}}^{\prime}=\mathbf{x}$ and any occurrence of $\mathbf{x}$ in $\mathbf{w}^{\prime}$ is one of $\mathbf{z}_{\ell^{\prime}}^{\prime}, \ldots, \mathbf{z}_{m^{\prime}}^{\prime}$;

- $\mathbf{z}_{p^{\prime}}^{\prime}=\mathbf{z}_{q^{\prime}}^{\prime}=\mathbf{y}$ and any occurrence of $\mathbf{y}$ in $\mathbf{w}^{\prime}$ is one of $\mathbf{z}_{p^{\prime}}^{\prime}, \ldots, \mathbf{z}_{q^{\prime}}^{\prime}$.

Note that $0<\ell^{\prime}$ and $q^{\prime}<n^{\prime}$ because $\mathbf{z}_{0}^{\prime}=\mathbf{z}_{n}^{\prime}$ by $(\mathrm{V})$. It follows that

$$
\begin{aligned}
& \mathbf{w}^{\prime}=\overbrace{\left(\mathbf{z}_{0}^{\prime} \prod_{i=1}^{\ell^{\prime}-1}\left(\mathbf{s}_{i}^{\prime} \mathbf{z}_{i}^{\prime}\right)\right) \mathbf{s}_{\ell^{\prime}}^{\prime}}^{\mathbf{a}^{\prime}} \mathbf{x} \overbrace{\left.\prod_{i=\ell^{\prime}+1}^{m^{\prime}-1}\left(\mathbf{s}_{i}^{\prime} \mathbf{z}_{i}^{\prime}\right)\right) \mathbf{s}_{m^{\prime}}^{\prime}}^{\mathbf{b}^{\prime}} \mathbf{x} \\
& \underbrace{\left(\prod_{i=m^{\prime}+1}^{p^{\prime}-1}\left(\mathbf{s}_{i}^{\prime} \mathbf{z}_{i}^{\prime}\right)\right) \mathbf{s}_{p^{\prime}}^{\prime}}_{\mathbf{c}^{\prime}} \mathbf{y} \underbrace{\left(\prod_{i=p^{\prime}+1}^{q^{\prime}-1}\left(\mathbf{s}_{i}^{\prime} \mathbf{z}_{i}^{\prime}\right)\right) \mathbf{s}_{q^{\prime}}^{\prime}}_{\mathbf{d}^{\prime}} \mathbf{y} \underbrace{\prod_{i=q^{\prime}+1}^{n^{\prime}}\left(\mathbf{s}_{i}^{\prime} \mathbf{z}_{i}^{\prime}\right)}_{\mathbf{e}^{\prime}}
\end{aligned}
$$

with

(c) $\operatorname{con}(\mathbf{x}) \cap \operatorname{con}\left(\mathbf{a}^{\prime} \mathbf{c}^{\prime} \mathbf{d}^{\prime} \mathbf{e}^{\prime}\right)=\operatorname{con}(\mathbf{y}) \cap \operatorname{con}\left(\mathbf{a}^{\prime} \mathbf{b}^{\prime} \mathbf{c}^{\prime} \mathbf{e}^{\prime}\right)=\emptyset$.

It follows from (G2), (I), and (III) that

(d) $\operatorname{con}\left(\mathbf{b}^{\prime}\right) \cap \operatorname{con}\left(\mathbf{a}^{\prime} \mathbf{c}^{\prime} \mathbf{d}^{\prime} \mathbf{e}^{\prime}\right)=\operatorname{con}\left(\mathbf{d}^{\prime}\right) \cap \operatorname{con}\left(\mathbf{a}^{\prime} \mathbf{b}^{\prime} \mathbf{c}^{\prime} \mathbf{e}^{\prime}\right)=\emptyset$.

There are two subcases.

3.1. $\operatorname{con}\left(\mathbf{c}^{\prime}\right) \cap \operatorname{con}\left(\mathbf{e}^{\prime}\right)=\emptyset$. Since $\mathbf{z}_{0}^{\prime}=\mathbf{z}_{n^{\prime}}^{\prime}$ by $(V)$, it follows that the sets $\left\{\mathbf{z}_{0}^{\prime}, \ldots, \mathbf{z}_{\ell^{\prime}-1}^{\prime}\right\}$ and $\left\{\mathbf{z}_{q^{\prime}+1}^{\prime}, \ldots, \mathbf{z}_{n}^{\prime}\right\}$ are not disjoint. Let $t^{\prime} \in\left\{q^{\prime}+1, \ldots, n^{\prime}\right\}$ be the least such that

$$
\mathbf{z}_{t^{\prime}}^{\prime} \in\left\{\mathbf{z}_{0}^{\prime}, \ldots, \mathbf{z}_{\ell^{\prime}-1}^{\prime}\right\} \cap\left\{\mathbf{z}_{q^{\prime}+1}^{\prime}, \ldots, \mathbf{z}_{n}^{\prime}\right\},
$$

and let $k^{\prime} \in\left\{0, \ldots, \ell^{\prime}-1\right\}$ be the greatest such that $\mathbf{z}_{k^{\prime}}^{\prime}=\mathbf{z}_{t^{\prime}}^{\prime}$. Then

$$
\mathbf{w}^{\prime}=\mathbf{a}^{\prime} \mathbf{x} \mathbf{b}^{\prime} \mathbf{x} \mathbf{c}^{\prime} \mathbf{y} \mathbf{d}^{\prime} \mathbf{y} \underbrace{\left(\prod_{i=q^{\prime}+1}^{t^{\prime}-1}\left(\mathbf{s}_{i}^{\prime} \mathbf{z}_{i}^{\prime}\right)\right)}_{\mathbf{e}_{1}^{\prime}} \mathbf{s}_{t^{\prime}}^{\prime} \mathbf{z}_{t^{\prime}}^{\prime} \underbrace{\left(\prod_{i=t^{\prime}+1}^{n^{\prime}}\left(\mathbf{s}_{i}^{\prime} \mathbf{z}_{i}^{\prime}\right)\right)}_{\mathbf{e}_{2}^{\prime}}
$$


where $\mathbf{z}_{k^{\prime}}^{\prime}$ is a factor of $\mathbf{a}^{\prime}$. Note that

(e) $\mathbf{s}_{t^{\prime}}^{\prime} \neq \emptyset$

by (IV), and that $\left\{\mathbf{z}_{q^{\prime}+1}^{\prime}, \ldots, \mathbf{z}_{t^{\prime}-1}^{\prime}\right\} \cap\left\{\mathbf{z}_{0}^{\prime}, \ldots, \mathbf{z}_{\ell^{\prime}-1}^{\prime}\right\}=\emptyset$ by (I) and the minimality of $t^{\prime}$. Hence

(f) $\operatorname{con}\left(\mathbf{e}_{1}^{\prime}\right) \cap \operatorname{con}\left(\mathbf{a}^{\prime} \mathbf{s}_{t^{\prime}}^{\prime} \mathbf{z}_{t^{\prime}}^{\prime}\right)=\emptyset$.

If $\operatorname{con}\left(\mathbf{e}_{1}^{\prime}\right) \cap \operatorname{con}\left(\mathbf{e}_{2}^{\prime}\right) \neq \emptyset$ so that $\mathbf{z}_{j}^{\prime} \in\left\{\mathbf{z}_{q^{\prime}+1}^{\prime}, \ldots, \mathbf{z}_{t^{\prime}-1}^{\prime}\right\} \cap\left\{\mathbf{z}_{t^{\prime}+1}^{\prime}, \ldots, \mathbf{z}_{n^{\prime}}^{\prime}\right\}$ for some $j$, then $\mathbf{z}_{j}^{\prime} \sim_{\mathbf{w}^{\prime}} \mathbf{z}_{t^{\prime}}^{\prime}$ by $(\mathrm{G} 2)$ due to $\mathbf{z}_{t^{\prime}}^{\prime} \in\left\{\mathbf{z}_{0}^{\prime}, \ldots, \mathbf{z}_{\ell^{\prime}-1}^{\prime}\right\}$, whence (III) is violated. Hence

(g) $\operatorname{con}\left(\mathbf{e}_{1}^{\prime}\right) \cap \operatorname{con}\left(\mathbf{e}_{2}^{\prime}\right)=\emptyset$.

Let $\varphi_{2}: \mathcal{X} \rightarrow \mathscr{C}_{8}$ denote the substitution

$$
z \mapsto \begin{cases}5 & \text { if } z \in \operatorname{con}\left(\mathbf{d}^{\prime} \mathbf{y e} \mathbf{e}_{1}^{\prime}\right) \\ 3 & \text { if } z=\mathrm{h}\left(\mathbf{s}_{t^{\prime}}^{\prime}\right) \\ 6 & \text { otherwise. }\end{cases}
$$

Then $\mathbf{w} \varphi_{2}=\mathbf{w}^{\prime} \varphi_{2}$ in $\mathscr{C}_{8}$. By (c)-(g) and the assumption of this subcase,

$$
\begin{aligned}
\mathbf{w}^{\prime} \varphi_{2} & =\left(\mathbf{a}^{\prime} \mathbf{x} \mathbf{b}^{\prime} \mathbf{x} \mathbf{c}^{\prime}\right) \varphi_{2} \cdot\left(\mathbf{y} \mathbf{d}^{\prime} \mathbf{y} \mathbf{e}_{1}\right) \varphi_{2} \cdot \mathbf{s}_{t^{\prime}}^{\prime} \varphi_{2} \cdot\left(\mathbf{z}_{t^{\prime}}^{\prime} \mathbf{e}_{2}^{\prime}\right) \varphi_{2} \\
& =(6 \ldots 6) \cdot(5 \ldots 5) \cdot(3 \cdot 6 \ldots 6) \cdot(6 \ldots 6)=2 .
\end{aligned}
$$

Therefore $\mathbf{w} \varphi_{2}=2$. More specifically,

$$
\mathbf{w} \varphi_{2}=\mathbf{a} \varphi_{2} \cdot 5 \cdot \mathbf{b} \varphi_{2} \cdot 6 \cdot \mathbf{c} \varphi_{2} \cdot 6 \cdot \mathbf{d} \varphi_{2} \cdot 5 \cdot \mathbf{e} \varphi_{2}=2 .
$$

By Lemma 19.6, the simple letter $\mathrm{h}\left(\mathbf{s}_{t^{\prime}}^{\prime}\right)$ of $\mathbf{w}^{\prime}$ is also a simple letter in $\mathbf{w}$. Since $\mathbf{z}_{0}=\mathbf{z}_{n}$ by $(\mathrm{V})$, the letter $\mathrm{h}\left(\mathbf{s}_{t^{\prime}}^{\prime}\right)$ cannot be the first or last letter of $\mathbf{w}$. Further, $\mathbf{x}$ and $\mathbf{y}$ are perfect squares of $\mathbf{w}$. Therefore $\mathbf{w} \varphi_{2}$ does not begin or end with 3 , and it is a product, in some order, of more than one 5 , more than one 6 , and precisely one 3 . Since $3 \cdot 5=3 \cdot 6 \cdot 5=5 \cdot 6=1$ in $\mathscr{C}_{8}$, it is routinely shown that the only way for $\mathbf{w} \varphi_{2}$ to equal 2 is

$$
\mathbf{w} \varphi_{2}=(6 \ldots 6) \cdot(5 \ldots 5) \cdot 3 \cdot(6 \ldots 6) .
$$

But this is impossible in view of (19.4).

3.2. $\operatorname{con}\left(\mathbf{c}^{\prime}\right) \cap \operatorname{con}\left(\mathbf{e}^{\prime}\right) \neq \emptyset$. Then there exists the least $r^{\prime} \in\left\{q^{\prime}+1, \ldots, n^{\prime}\right\}$ such that

$$
\mathbf{z}_{r^{\prime}}^{\prime} \in\left\{\mathbf{z}_{m^{\prime}+1}^{\prime}, \ldots, \mathbf{z}_{p^{\prime}-1}^{\prime}\right\} \cap\left\{\mathbf{z}_{q^{\prime}+1}^{\prime}, \ldots, \mathbf{z}_{n}^{\prime}\right\},
$$

and there exists the greatest $h^{\prime} \in\left\{\mathbf{z}_{m^{\prime}+1}^{\prime}, \ldots, \mathbf{z}_{p^{\prime}-1}^{\prime}\right\}$ such that $\mathbf{z}_{h^{\prime}}^{\prime}=\mathbf{z}_{r^{\prime}}^{\prime}$. Hence

$$
\begin{aligned}
& \mathbf{w}^{\prime}= \mathbf{a}^{\prime} \mathbf{x} \mathbf{b}^{\prime} \mathbf{x} \overbrace{\left.\prod_{i=m^{\prime}+1}^{h^{\prime}-1}\left(\mathbf{s}_{i}^{\prime} \mathbf{z}_{i}^{\prime}\right)\right) \mathbf{s}_{h^{\prime}}^{\prime}}^{\mathbf{c}_{1}^{\prime}} \mathbf{v} \overbrace{\left(\prod_{i=h^{\prime}+1}^{p^{\prime}-1}\left(\mathbf{s}_{i}^{\prime} \mathbf{z}_{i}^{\prime}\right)\right) \mathbf{s}_{p^{\prime}+1}^{\prime}}^{\mathbf{c}_{2}^{\prime}} \mathbf{y d}^{\prime} \mathbf{y} \\
& \underbrace{\left(\prod_{i=q^{\prime}+1}^{r^{\prime}-1}\left(\mathbf{s}_{i}^{\prime} \mathbf{z}_{i}^{\prime}\right)\right)}_{\mathbf{e}_{3}^{\prime}} \mathbf{s}_{r^{\prime}}^{\prime} \mathbf{v} \\
& \underbrace{\left.\prod_{i=r^{\prime}+1}^{n^{\prime}}\left(\mathbf{s}_{i}^{\prime} \mathbf{z}_{i}^{\prime}\right)\right)}_{\mathbf{e}_{4}^{\prime}}
\end{aligned}
$$

where $\mathbf{v}=\mathbf{z}_{h^{\prime}}^{\prime}=\mathbf{z}_{r^{\prime}}^{\prime}$. Note that (IV) implies

(h) $\mathbf{s}_{r^{\prime}}^{\prime} \neq \emptyset$,

and (I) and the choice of $h^{\prime}$ and $r^{\prime}$ imply that

(i) $\mathbf{c}_{2}^{\prime}, \mathbf{e}_{3}^{\prime}$, and $\mathbf{v}$ are pairwise disjoint. 
If $\mathbf{f}$ is a perfect square that is shared by $\mathbf{c}_{2}^{\prime}$ and either $\mathbf{a}^{\prime}, \mathbf{b}^{\prime}, \mathbf{c}_{1}^{\prime}$, or $\mathbf{e}_{4}^{\prime}$, then $\mathbf{f} \neq \mathbf{v}$ by (i), so that $\mathbf{f} \sim_{\mathbf{w}^{\prime}} \mathbf{v}$ by (G2). If $\mathbf{g}$ is a perfect square that is shared by $\mathbf{e}_{3}^{\prime}$ and either $\mathbf{a}^{\prime}, \mathbf{b}^{\prime}, \mathbf{c}_{1}^{\prime}$, or $\mathbf{e}_{4}^{\prime}$, then $\mathbf{g} \neq \mathbf{v}$ by (i), so that $\mathbf{g} \sim_{\mathbf{w}^{\prime}} \mathbf{v}$ by (G2). But either of these contradicts (III). Therefore both $\mathbf{f}$ and $\mathbf{g}$ do not exist, whence

(j) $\operatorname{con}\left(\mathbf{c}_{2}^{\prime}\right) \cap \operatorname{con}\left(\mathbf{a}^{\prime} \mathbf{b}^{\prime} \mathbf{c}_{1}^{\prime} \mathbf{e}_{4}^{\prime}\right)=\operatorname{con}\left(\mathbf{e}_{3}^{\prime}\right) \cap \operatorname{con}\left(\mathbf{a}^{\prime} \mathbf{b}^{\prime} \mathbf{c}_{1}^{\prime} \mathbf{e}_{4}^{\prime}\right)=\emptyset$.

Let $\varphi_{3}: \mathcal{X} \rightarrow \mathscr{C}_{8}$ denote the substitution

$$
z \mapsto \begin{cases}5 & \text { if } z \in \operatorname{con}\left(\mathbf{d}^{\prime} \mathbf{y} \mathbf{e}_{3}^{\prime}\right), \\ 3 & \text { if } z=\mathrm{h}\left(\mathbf{s}_{r^{\prime}}^{\prime}\right), \\ 6 & \text { otherwise. }\end{cases}
$$

Then by (c), (d), (h), and (j),

$$
\begin{aligned}
\mathbf{w}^{\prime} \varphi_{3} & =\left(\mathbf{a}^{\prime} \mathbf{x} \mathbf{b}^{\prime} \mathbf{x} \mathbf{c}^{\prime}\right) \varphi_{3} \cdot\left(\mathbf{y d}^{\prime} \mathbf{y} \mathbf{e}_{3}^{\prime}\right) \varphi_{3} \cdot \mathbf{s}_{r^{\prime}}^{\prime} \varphi_{3} \cdot\left(\mathbf{v} \mathbf{e}_{4}^{\prime}\right) \varphi_{3} \\
& =(6 \ldots 6) \cdot(5 \ldots 5) \cdot(3 \cdot 6 \ldots 6) \cdot(6 \ldots 6)=2 .
\end{aligned}
$$

But following the same argument as in Subcase 3.1, it is impossible for $\mathbf{w} \varphi_{3}$ to equal 2.

\subsection{Proof of Proposition 19.1}

REMARK 19.12. Let $\mathbf{w}$ be any word in canonical form that contains two or more distinct perfect squares. By (V), the word $\mathbf{w}$ begins and ends with some perfect square $\mathbf{z}$. Hence by (IV),

$$
\mathbf{w}=\mathbf{z} \prod_{i=1}^{n}\left(\mathbf{p}_{i} \mathbf{s}_{i} \mathbf{z}\right)=\mathbf{z p}_{1} \mathbf{s}_{1} \mathbf{z} \ldots \mathbf{p}_{n} \mathbf{s}_{n} \mathbf{z}
$$

for some $n \geqslant 1$, where the following holds for each $i$ :

(Re1) $\mathbf{s}_{i}$ is a nonempty maximal simple factor of $\mathbf{w}$;

$(\operatorname{Re} 2) \mathbf{p}_{i}$ is a product of some of the following: maximal simple factors of $\mathbf{w}$ and perfect squares of $\mathbf{w}$ different from $\mathbf{z}$;

$(\operatorname{Re} 3)$ if $\mathbf{p}_{i}$ is nonempty, then it ends with some perfect square of $\mathbf{w}$.

Note that (I), (III), and (G2) imply that:

$(\operatorname{Re} 4) \mathbf{z}, \mathbf{p}_{1}, \mathbf{s}_{1}, \ldots, \mathbf{p}_{n}, \mathbf{s}_{n}$ are pairwise disjoint;

$(\operatorname{Re} 5)$ if $\mathbf{x}$ is any perfect square of $\mathbf{w}$ different from $\mathbf{z}$, then $\mathbf{x} \Subset_{\mathbf{w}} \mathbf{z}$.

By Lemma 19.4, the semigroup $\mathscr{C}_{8}$ has a basis of the form $\{(19.1)\} \cup \Sigma$, where $\Sigma$ is some set of identities formed by connected words in canonical form. Let $\mathbf{w} \approx \mathbf{w}^{\prime}$ be any identity from $\Sigma$. By Lemmas 19.6 and 19.7, the set $\mathbf{Z}$ of perfect squares of $\mathbf{w}$ is equal to the set of perfect squares of $\mathbf{w}^{\prime}$, and the set $\mathbf{S}$ of maximal simple factors of $\mathbf{w}$ is equal to the set of maximal simple factors of $\mathbf{w}^{\prime}$. The remainder of this subsection is devoted to the verification of the following statement for each $k \geqslant 1$ :

$\left(\dagger_{k}\right)$ if $\mathbf{w} \approx \mathbf{w}^{\prime}$ is any identity in $\Sigma$ that involves at most $k$ distinct perfect squares, then the identities (19.1) imply the identity $\mathbf{w} \approx \mathbf{w}^{\prime}$.

Consequently, the identities (19.1) imply every identity in $\Sigma$ and so form a basis for $\mathscr{C}_{8}$.

First consider the case when the identity $\mathbf{w} \approx \mathbf{w}^{\prime}$ from $\Sigma$ involves $k=1$ perfect square $\mathbf{z}$. Then $\mathbf{w}=\mathbf{z} \prod_{i=1}^{n}\left(\mathbf{s}_{i} \mathbf{z}\right)=\mathbf{z s}_{1} \mathbf{z} \ldots \mathbf{s}_{n} \mathbf{z}$ where $\mathbf{Z}=\{\mathbf{z}\}$ and $\mathbf{S}=\left\{\mathbf{s}_{1}, \ldots, \mathbf{s}_{n}\right\}$. By Lemmas 19.9 and 19.10, there exists a permutation $\pi$ on $\{1, \ldots, n\}$ such that $\mathbf{w}^{\prime}=\mathbf{z s}_{1 \pi} \mathbf{z} \ldots \mathbf{s}_{n \pi} \mathbf{z}$. It is then easily seen that the identity $(19.1 \mathrm{~b})$ implies the identity $\mathbf{w} \approx \mathbf{w}^{\prime}$. Hence statement $\left(\dagger_{1}\right)$ holds.

Now suppose that statement $\left(\dagger_{k-1}\right)$ holds for some $k>1$ and that the identity $\mathbf{w} \approx \mathbf{w}^{\prime}$ from $\Sigma$ contains $k$ distinct perfect squares. By Remark 19.12, the word $\mathbf{w}$ is of the form

$$
\mathbf{w}=\mathbf{z} \mathbf{p}_{1} \mathbf{s}_{1} \mathbf{z} \mathbf{p}_{2} \mathbf{s}_{2} \mathbf{z} \ldots \mathbf{p}_{n} \mathbf{s}_{n} \mathbf{z}
$$


where (Re1)-(Re5) are satisfied. By (V), the word $\mathbf{w}^{\prime}$ begins and ends with the same perfect square; by Lemma 19.11 this perfect square must be $\mathbf{z}$. Hence it follows from Lemma 19.9 and Remark 19.12 that $\mathbf{w}^{\prime}=\mathbf{z} \mathbf{p}_{1}^{\prime} \mathbf{s}_{1 \pi} \mathbf{z} \mathbf{p}_{2}^{\prime} \mathbf{s}_{2 \pi} \mathbf{z} \ldots \mathbf{p}_{n}^{\prime} \mathbf{s}_{n \pi} \mathbf{z}$ for some permutation $\pi$ on $\{1, \ldots, n\}$. Since the identity (19.1b) can be used to interchange any two factors $\mathbf{p}_{i}^{\prime} \mathbf{s}_{i \pi}$ and $\mathbf{p}_{j}^{\prime} \mathbf{s}_{j \pi}$, generality is not lost by assuming that

$$
\mathbf{w}^{\prime}=\mathbf{z} \mathbf{p}_{1}^{\prime} \mathbf{s}_{1} \mathbf{z p}_{2}^{\prime} \mathbf{s}_{2} \mathbf{z} \ldots \mathbf{p}_{n}^{\prime} \mathbf{s}_{n} \mathbf{z}
$$

to begin with. By Lemmas 19.13 and 19.14 below, the identities (19.1) imply $\mathbf{w} \approx \mathbf{w}^{\prime}$.

Lemma 19.13. If $n=1$, then the identities (19.1) imply the identity $\mathbf{w} \approx \mathbf{w}^{\prime}$.

Proof. Generality is not lost by writing

$$
\mathbf{w}=\mathbf{z p s z} \quad \text { and } \quad \mathbf{w}^{\prime}=\mathbf{z p}^{\prime} \mathbf{s z}
$$

where $\mathbf{z}, \mathbf{p}, \mathbf{p}^{\prime}$, and $\mathbf{s}$ satisfy (Re1)-(Re4). It then follows that $\operatorname{con}(\mathbf{p})=\operatorname{con}\left(\mathbf{p}^{\prime}\right)$ by Lemmas 19.6 and 19.7. If $\mathbf{p}=\mathbf{p}^{\prime}=\emptyset$, then the identity $\mathbf{w} \approx \mathbf{w}^{\prime}$ is trivial and so is clearly implied by the identities (19.1). Therefore suppose that the factors $\mathbf{p}$ and $\mathbf{p}^{\prime}$ are nonempty. Note that $\mathbf{p}$ can be written as a product $\mathbf{p}=\mathbf{a}_{r} \mathbf{a}_{r-1} \ldots \mathbf{a}_{2} \mathbf{a}_{1}$ of pairwise disjoint words $\mathbf{a}_{r}, \mathbf{a}_{r-1}, \ldots, \mathbf{a}_{2}, \mathbf{a}_{1} \in \mathcal{X}^{+}$ where each $\mathbf{a}_{i}$ is either connected or a maximal simple factor of $\mathbf{w}$. If $\mathbf{a}_{i}$ is connected, then since $\mathbf{x} \varkappa_{\mathbf{w}} \mathbf{y}$ for any distinct perfect squares $\mathbf{x}$ and $\mathbf{y}$ of $\mathbf{w}$, it follows from $(\operatorname{Re} 2)$ that $\mathbf{a}_{i}$ begins and ends with the same perfect square of $\mathbf{w}$. The same applies to connected factors of the word $\mathbf{p}^{\prime}$. Therefore in summary, the words $\mathbf{p}$ and $\mathbf{p}^{\prime}$ can be written as

$$
\mathbf{p}=\mathbf{a}_{r} \mathbf{a}_{r-1} \ldots \mathbf{a}_{2} \mathbf{a}_{1} \quad \text { and } \quad \mathbf{p}^{\prime}=\mathbf{a}_{r^{\prime}}^{\prime} \mathbf{a}_{r^{\prime}-1}^{\prime} \ldots \mathbf{a}_{2}^{\prime} \mathbf{a}_{1}^{\prime}
$$

for some $r, r^{\prime} \geqslant 1$, where:

(a) $\mathbf{a}_{i}$ is either a maximal simple factor of $\mathbf{w}$ or a word that begins and ends with the same perfect square of $\mathbf{w}$

(b) $\mathbf{a}_{i}^{\prime}$ is either a maximal simple factor of $\mathbf{w}^{\prime}$ or a word that begins and ends with the same perfect square of $\mathbf{w}^{\prime}$

(c) $\mathbf{a}_{r}, \ldots, \mathbf{a}_{1}$ are pairwise disjoint and $\mathbf{a}_{r^{\prime}}^{\prime}, \ldots, \mathbf{a}_{1}^{\prime}$ are pairwise disjoint.

Note that (c) and (Re2) imply that

(d) $\mathbf{a}_{r}, \ldots, \mathbf{a}_{1}, \mathbf{s}, \mathbf{z}$ are pairwise disjoint and $\mathbf{a}_{r^{\prime}}^{\prime}, \ldots, \mathbf{a}_{1}^{\prime}, \mathbf{s}, \mathbf{z}$ are pairwise disjoint.

Further, since the word $\mathbf{w}$ is in canonical form,

(e) any two maximal simple factors of $\mathbf{w}$ must sandwich at least one perfect square of $\mathbf{w}$.

First, it is shown that the identities (19.1) imply the identity $\mathbf{a}_{1} \approx \mathbf{a}_{1}^{\prime}$. To achieve this goal, observe that by (e), the word $\mathbf{a}_{1}$ is not a maximal simple factor of $\mathbf{w}$ since it is followed by the maximal simple factor $\mathbf{s}$. Therefore by (a), the word $\mathbf{a}_{1}$ begins and ends with the same perfect square of $\mathbf{w}$. Hence $\mathbf{w}$ is of the form

$$
\mathbf{w}=\mathbf{z} \mathbf{a}_{r} \ldots \mathbf{a}_{2} \underbrace{\mathbf{z}_{1} \mathbf{b}_{1} \mathbf{z}_{1}}_{\mathbf{a}_{1}} \mathbf{s z}
$$

where $\mathbf{z}_{1}$ is some perfect square of $\mathbf{w}$. By the same argument, the word $\mathbf{a}_{1}^{\prime}$ also begins and ends with the same perfect square of $\mathbf{w}^{\prime}$; by Lemma 19.10, this perfect square must equal $\mathbf{z}_{1}$. Hence $\mathbf{w}^{\prime}$ is of the form

$$
\mathbf{w}^{\prime}=\mathbf{z} \mathbf{a}_{r^{\prime}}^{\prime} \ldots \mathbf{a}_{2}^{\prime} \underbrace{\mathbf{z}_{1} \mathbf{b}_{1}^{\prime} \mathbf{z}_{1}}_{\mathbf{a}_{1}^{\prime}} \mathbf{s z} .
$$

Suppose that $\mathbf{y}$ is a perfect square of $\mathbf{w}$ that is a factor of $\mathbf{b}_{1}$. If $\mathbf{y}=\mathbf{z}_{1}$, then $\mathbf{y}$ is clearly a factor of $\mathbf{a}_{1}^{\prime}$. If $\mathbf{y} \neq \mathbf{z}_{1}$, then $\mathbf{y} \Subset_{\mathbf{w}} \mathbf{z}_{1}$ by $(\mathrm{d})$, so that $\mathbf{y} \Subset_{\mathbf{w}^{\prime}} \mathbf{z}_{1}$ by Lemma 19.11. Therefore in 
any case, $\mathbf{y}$ is a factor of $\mathbf{a}_{1}^{\prime}$. By symmetry, any perfect square of $\mathbf{w}^{\prime}$ that is a factor of $\mathbf{a}_{1}^{\prime}$ is also a factor of $\mathbf{a}_{1}$. Hence

(f) $\mathbf{a}_{1}$ and $\mathbf{a}_{1}^{\prime}$ share the same set of perfect squares.

Suppose that $\mathbf{t}$ is a maximal simple factor of $\mathbf{w}$ that is a factor of $\mathbf{b}_{1}$. Then $\mathbf{t}$ is immediately preceded and followed by perfect squares of $\mathbf{w}$ that occur in $\mathbf{a}_{1}=\mathbf{z}_{1} \mathbf{b}_{1} \mathbf{z}_{1}$. More specifically, $\mathbf{y}_{1} \mathbf{t} \mathbf{y}_{2}$ is a factor of $\mathbf{a}_{1}=\mathbf{z}_{1} \mathbf{b}_{1} \mathbf{z}_{1}$ for some perfect squares $\mathbf{y}_{1}$ and $\mathbf{y}_{2}$. It follows from (f) and Lemmas 19.9 and 19.10 that $\mathbf{y}_{1} \mathbf{t y}_{2}$ is a factor of $\mathbf{a}_{1}^{\prime}=\mathbf{z}_{1} \mathbf{b}_{1}^{\prime} \mathbf{z}_{1}$, whence $\mathbf{t}$ is a factor of $\mathbf{b}_{1}^{\prime}$. By symmetry, any maximal simple factor of $\mathbf{w}^{\prime}$ that is a factor of $\mathbf{b}_{1}^{\prime}$ is also a factor of $\mathbf{b}_{1}$. Hence

(g) $\mathbf{b}_{1}$ and $\mathbf{b}_{1}^{\prime}$ share the same maximal simple factors.

It then follows from (f) and (g) that $\operatorname{con}\left(\mathbf{a}_{1}\right)=\operatorname{con}\left(\mathbf{a}_{1}^{\prime}\right)$. Let $\chi_{1}$ denote the substitution $x \mapsto \mathbf{z}_{1}$ for all $x \notin \operatorname{con}\left(\mathbf{a}_{1}\right)=\operatorname{con}\left(\mathbf{a}_{1}^{\prime}\right)$. Then the deductions $\mathbf{w} \chi_{1} \stackrel{(19.1 \mathrm{a})}{\approx} \mathbf{a}_{1}$ and $\mathbf{w}^{\prime} \chi_{1} \stackrel{(19.1 \mathrm{a})}{\approx} \mathbf{a}_{1}^{\prime}$ imply that $\mathscr{C}_{8}$ satisfies the identity $\mathbf{a}_{1} \approx \mathbf{a}_{1}^{\prime}$. The identity $\mathbf{a}_{1} \approx \mathbf{a}_{1}^{\prime}$ involves less than $k$ distinct perfect squares. Therefore by $\left(\dagger_{k-1}\right)$, the identities (19.1) imply the identity $\mathbf{a}_{1} \approx \mathbf{a}_{1}^{\prime}$.

Next, it is shown that the identities (19.1) imply the identity $\mathbf{a}_{2} \approx \mathbf{a}_{2}^{\prime}$. Based on (a), there are two cases.

Case 1: $\mathbf{a}_{2}$ is a maximal simple factor of $\mathbf{w}$. Then by Lemma 19.9, the factor $\mathbf{a}_{2} \mathbf{z}_{1}$ of $\mathbf{w}$ is also a factor of $\mathbf{w}^{\prime}$. But since $\operatorname{con}\left(\mathbf{a}_{1}\right)=\operatorname{con}\left(\mathbf{a}_{1}^{\prime}\right)$, it follows from (d) that $\mathbf{a}_{2}$ is not a factor of $\mathbf{a}_{1}^{\prime}$. Therefore the only way for $\mathbf{a}_{2} \mathbf{z}_{1}$ to be a factor of $\mathbf{w}^{\prime}$ is when $\mathbf{a}_{2}=\mathbf{a}_{2}^{\prime}$. The identity $\mathbf{a}_{2} \approx \mathbf{a}_{2}^{\prime}$ is trivial and so is implied by the identities (19.1).

Case 2: $\mathbf{a}_{2}$ begins and ends with the same perfect square of $\mathbf{w}$. Then $\mathbf{w}$ is of the form

$$
\mathbf{w}=\mathbf{z a}_{r} \ldots \mathbf{a}_{3} \underbrace{\mathbf{z}_{2} \mathbf{b}_{2} \mathbf{z}_{2}}_{\mathbf{a}_{2}} \mathbf{a}_{1} \mathbf{s z},
$$

where $\mathbf{z}_{2}$ is some perfect square of $\mathbf{w}$. If $\mathbf{a}_{2}^{\prime}$ is a maximal simple factor of $\mathbf{w}^{\prime}$, then the argument in Case 1 shows $\mathbf{a}_{2}=\mathbf{a}_{2}^{\prime}$, which is impossible. Therefore by (b), the word $\mathbf{a}_{2}^{\prime}$ begins and ends with the same perfect square of $\mathbf{w}^{\prime}$, whence

$$
\mathbf{w}^{\prime}=\mathbf{z} \mathbf{a}_{r^{\prime}}^{\prime} \ldots \mathbf{a}_{3}^{\prime} \underbrace{\mathbf{z}_{2}^{\prime} \mathbf{b}_{2}^{\prime} \mathbf{z}_{2}^{\prime}}_{\mathbf{a}_{2}^{\prime}} \mathbf{a}_{1}^{\prime} \mathbf{s z}
$$

for some perfect square $\mathbf{z}_{2}^{\prime}$ of $\mathbf{w}^{\prime}$. Suppose $\mathbf{z}_{2} \neq \mathbf{z}_{2}^{\prime}$. Since the set of perfect squares of $\mathbf{w}$ is equal to the set of perfect squares of $\mathbf{w}^{\prime}$, the perfect square $\mathbf{z}_{2}$ of $\mathbf{w}$ is also a perfect square of $\mathbf{w}^{\prime}$. If $\mathbf{z}_{2}$ is a factor of $\mathbf{b}_{2}^{\prime}$, then $\mathbf{z}_{2} \Subset_{\mathbf{w}^{\prime}} \mathbf{z}_{2}^{\prime}$, so that $\mathbf{z}_{2} \Subset_{\mathbf{w}} \mathbf{z}_{2}^{\prime}$ by Lemma 19.11 ; but $\mathbf{z}_{2} \Subset_{\mathbf{w}} \mathbf{z}_{2}^{\prime}$ implies that $\mathbf{z}_{2}^{\prime}$ is a factor shared by both the prefix $\mathbf{z a}_{r} \ldots \mathbf{a}_{3}$ and suffix $\mathbf{a}_{1} \mathbf{s z}$ of $\mathbf{w}$, and this is impossible due to (d). Therefore

(h) $\mathbf{z}_{2}$ is not a factor of $\mathbf{a}_{2}^{\prime}$, but is a factor of one of $\mathbf{a}_{r^{\prime}}^{\prime}, \ldots, \mathbf{a}_{3}^{\prime}$.

By symmetry,

(i) $\mathbf{z}_{2}^{\prime}$ is not a factor of $\mathbf{a}_{2}$, but is a factor of one of $\mathbf{a}_{r}, \ldots, \mathbf{a}_{3}$.

Let $\varphi: \mathcal{X} \rightarrow \mathscr{C}_{8}$ denote the substitution

$$
z \mapsto \begin{cases}5 & \text { if } z \in \operatorname{con}\left(\mathbf{a}_{2} \mathbf{a}_{1}\right) \\ 3 & \text { if } z=\mathrm{h}(\mathbf{s}) \\ 6 & \text { otherwise. }\end{cases}
$$

Then

$$
\mathbf{w} \varphi=\left(\mathbf{z a}_{r} \ldots \mathbf{a}_{3}\right) \varphi \cdot\left(\mathbf{a}_{2} \mathbf{a}_{1}\right) \varphi \cdot \mathbf{s} \varphi \cdot \mathbf{z} \varphi=(6 \ldots 6) \cdot(5 \ldots 5) \cdot(3 \cdot 6 \ldots 6) \cdot(6 \ldots 6)=2 .
$$


But (d) and (i) imply $\mathbf{z}_{2}^{\prime} \varphi=6$, so that (h) implies

$$
\begin{aligned}
\left(\mathbf{z} \mathbf{a}_{r^{\prime}}^{\prime} \ldots \mathbf{a}_{3}^{\prime} \mathbf{z}_{2}^{\prime}\right) \varphi & =\mathbf{z} \varphi \cdot\left(\mathbf{a}_{r^{\prime}}^{\prime} \ldots \mathbf{a}_{3}^{\prime}\right) \varphi \cdot \mathbf{z}_{2}^{\prime} \varphi=\mathbf{z} \varphi \ldots \mathbf{z}_{2} \varphi \ldots \mathbf{z}_{2}^{\prime} \varphi \\
& \in 6 \cdot\{5,6\}^{*} \cdot 5 \cdot\{5,6\}^{*} \cdot 6=\{1\}
\end{aligned}
$$

Hence the contradiction $\mathbf{w}^{\prime} \varphi=1$ is obtained. Therefore the assumption $\mathbf{z}_{2} \neq \mathbf{z}_{2}^{\prime}$ does not hold, whence

$$
\mathbf{w}^{\prime}=\mathbf{z a}_{r^{\prime}}^{\prime} \ldots \mathbf{a}_{3}^{\prime} \underbrace{\mathbf{z}_{2} \mathbf{b}_{2}^{\prime} \mathbf{z}_{2}}_{\mathbf{a}_{2}^{\prime}} \mathbf{a}_{1}^{\prime} \mathbf{s z} .
$$

It can then be shown, by the arguments used in establishing (f) and $(\mathrm{g})$, that $\operatorname{con}\left(\mathbf{a}_{2}\right)=\operatorname{con}\left(\mathbf{a}_{2}^{\prime}\right)$. Let $\chi_{2}$ denote the substitution $x \mapsto \mathbf{z}_{2}$ for all $x \notin \operatorname{con}\left(\mathbf{a}_{2}\right)=\operatorname{con}\left(\mathbf{a}_{2}^{\prime}\right)$. Then the deductions $\mathbf{w} \chi_{2} \stackrel{(19.1 \mathrm{a})}{\approx} \mathbf{a}_{2}$ and $\mathbf{w}^{\prime} \chi_{2} \stackrel{(19.1 \mathrm{a})}{\approx} \mathbf{a}_{2}^{\prime}$ imply that $\mathscr{C}_{8}$ satisfies the identity $\mathbf{a}_{2} \approx \mathbf{a}_{2}^{\prime}$. The identity $\mathbf{a}_{2} \approx \mathbf{a}_{2}^{\prime}$ involves less than $k$ distinct perfect squares. Therefore by $\left(\dagger_{k-1}\right)$, the identities (19.1) imply the identity $\mathbf{a}_{2} \approx \mathbf{a}_{2}^{\prime}$.

In the two cases just considered, the identities (19.1) imply the identity $\mathbf{a}_{2} \approx \mathbf{a}_{2}^{\prime}$. The arguments in Cases 1 and 2 can be repeated to show that the identities (19.1) imply the identity $\mathbf{a}_{3} \approx \mathbf{a}_{3}^{\prime}$. This can be continued so that the identities (19.1) imply the identity $\mathbf{a}_{i} \approx \mathbf{a}_{i}^{\prime}$ for all $i$ with $r=r^{\prime}$. It is then easily seen that the identities (19.1) imply $\mathbf{w} \approx \mathbf{w}^{\prime}$.

Lemma 19.14. If $n>1$, then the identities (19.1) imply the identity $\mathbf{w} \approx \mathbf{w}^{\prime}$.

Proof. Let $\ell$ be the least integer such that $\operatorname{con}\left(\mathbf{p}_{\ell}\right) \neq \operatorname{con}\left(\mathbf{p}_{\ell}^{\prime}\right)$. Then generality is not lost by assuming that $x \in \operatorname{con}\left(\mathbf{p}_{\ell}^{\prime}\right) \backslash \operatorname{con}\left(\mathbf{p}_{\ell}\right)$, so that $x \in \operatorname{con}\left(\mathbf{p}_{r}\right)$ for some $r>\ell$. Let $\varphi: \mathcal{X} \rightarrow \mathscr{C}_{8}$ denote the substitution

$$
z \mapsto \begin{cases}5 & \text { if } z \in \operatorname{con}\left(\mathbf{p}_{r}\right) \\ 3 & \text { if } z=\mathrm{h}\left(\mathbf{s}_{r}\right) \\ 6 & \text { otherwise }\end{cases}
$$

Then

$$
\begin{aligned}
\mathbf{w} \varphi & =\left(\mathbf{z} \prod_{i=1}^{r-1}\left(\mathbf{p}_{i} \mathbf{s}_{i} \mathbf{z}\right)\right) \varphi \cdot \mathbf{p}_{r} \varphi \cdot \mathbf{s}_{r} \varphi \cdot\left(\mathbf{z} \prod_{i=1}^{r+1}\left(\mathbf{p}_{i} \mathbf{s}_{i} \mathbf{z}\right)\right) \varphi \\
& =(6 \ldots 6) \cdot(5 \ldots 5) \cdot(3 \cdot 6 \ldots 6) \cdot(6 \ldots 6)=2 .
\end{aligned}
$$

But $x \in \operatorname{con}\left(\mathbf{p}_{\ell}^{\prime}\right)$ implies

$$
\left(\mathbf{z p}_{\ell}^{\prime} \mathbf{s}_{\ell} \mathbf{z}\right) \varphi=\mathbf{z} \varphi \ldots x \varphi \ldots \mathbf{z} \varphi \in 6 \cdot\{5,6\}^{*} \cdot 5 \cdot\{5,6\}^{*} \cdot 6=\{1\}
$$

Hence the contradiction $\mathbf{w}^{\prime} \varphi=1$ is obtained. Therefore the integer $\ell$ does not exist, whence $\operatorname{con}\left(\mathbf{p}_{i}\right)=\operatorname{con}\left(\mathbf{p}_{i}^{\prime}\right)$ for all $i \in\{1, \ldots, n\}$.

For each $i \in\{1, \ldots, n\}$, let $\chi_{i}$ denote the substitution $z \mapsto \mathbf{z}$ for all $z \notin \operatorname{con}\left(\mathbf{p}_{i} \mathbf{s}_{i}\right)=\operatorname{con}\left(\mathbf{p}_{i}^{\prime} \mathbf{s}_{i}\right)$.

Then the deductions $\mathbf{w} \chi_{i} \stackrel{(19.1 \mathrm{a})}{\approx} \mathbf{z p}_{i} \mathbf{s}_{i} \mathbf{z}$ and $\mathbf{w}^{\prime} \chi_{i} \stackrel{(19.1 \mathrm{a})}{\approx} \mathbf{z p}_{i}^{\prime} \mathbf{s}_{i} \mathbf{z}$ imply that $\mathscr{C}_{8}$ satisfies the identity $\mathbf{z p}_{i} \mathbf{s}_{i} \mathbf{z} \approx \mathbf{z p}_{i}^{\prime} \mathbf{s}_{i} \mathbf{z}$. By Lemma 19.13, the identities (19.1) imply $\mathbf{z p}_{i} \mathbf{s}_{i} \mathbf{z} \approx \mathbf{z p}_{i}^{\prime} \mathbf{s}_{i} \mathbf{z}$. It is then easily seen that the identities (19.1) imply $\mathbf{w} \approx \mathbf{w}^{\prime}$. 
20. $\mathscr{D}_{2}, \mathscr{D}_{4}, \mathscr{E}_{4}$

This section establishes the finite basis property of the following semigroups.

\begin{tabular}{c|cccccc}
$\mathscr{D}_{2}$ & 1 & 2 & 3 & 4 & 5 & 6 \\
\hline 1 & 1 & 1 & 1 & 1 & 1 & 1 \\
2 & 1 & 1 & 1 & 1 & 1 & 2 \\
3 & 1 & 1 & 1 & 1 & 2 & 1 \\
4 & 1 & 2 & 3 & 4 & 4 & 4 \\
5 & 1 & 2 & 3 & 4 & 4 & 5 \\
6 & 1 & 2 & 3 & 4 & 4 & 6
\end{tabular}

\begin{tabular}{c|cccccc}
$\mathscr{D}_{4}$ & 1 & 2 & 3 & 4 & 5 & 6 \\
\hline 1 & 1 & 1 & 1 & 1 & 1 & 1 \\
2 & 1 & 1 & 1 & 1 & 1 & 2 \\
3 & 1 & 1 & 1 & 1 & 2 & 2 \\
4 & 1 & 2 & 3 & 4 & 4 & 4 \\
5 & 1 & 2 & 3 & 4 & 4 & 5 \\
6 & 1 & 2 & 3 & 4 & 4 & 6
\end{tabular}

\begin{tabular}{c|cccccc}
$\mathscr{E}_{4}$ & 1 & 2 & 3 & 4 & 5 & 6 \\
\hline 1 & 1 & 1 & 1 & 1 & 1 & 1 \\
2 & 1 & 1 & 1 & 1 & 1 & 3 \\
3 & 1 & 1 & 1 & 1 & 3 & 3 \\
4 & 1 & 1 & 1 & 3 & 4 & 4 \\
5 & 1 & 2 & 3 & 4 & 5 & 5 \\
6 & 1 & 2 & 3 & 4 & 6 & 6
\end{tabular}

20.1. Finite basis property of $\mathscr{D}_{2}$ and $\mathscr{D}_{4}$

Proposition 20.1. The variety generated by $S \in\left\{\mathscr{D}_{2}, \mathscr{D}_{4}\right\}$ is defined by the identities

$$
\begin{aligned}
x^{2} \mathrm{H} x & \approx x \mathrm{H} x, \\
x^{2} y^{2} & \approx y^{2} x^{2}, \\
x \mathrm{H} x y \mathrm{~K} y^{2} & \approx x \mathrm{H} x \mathrm{~K} y^{2}, \\
x \mathrm{H} y \mathrm{~K} y \mathrm{\top} x & \approx y \mathrm{H} y \mathrm{~K} y x \mathrm{\top} x, \\
y \mathrm{H} x \mathrm{~K} y \mathrm{\top} x & \approx y \mathrm{H} y \mathrm{~K} y x \mathrm{\top} x, \\
x \mathrm{H} y \mathrm{~K} y \mathrm{\top} x & \approx y \mathrm{H} x \mathrm{~K} y \mathrm{\top} x .
\end{aligned}
$$

For any word $\mathbf{w}$, let $\mathrm{F}_{\mathrm{SL}}(\mathbf{w})$ denote the set of factors of $\mathbf{w}$ of length two that begin with a simple letter and end with the last occurrence of some non-simple letter:

$$
\mathrm{F}_{\mathrm{SL}}(\mathbf{w})=\left\{x y \in \mathcal{X}^{2} \mid \mathbf{w} \in \mathcal{X}^{*} x y(\mathcal{X} \backslash\{y\})^{*}, x \in \operatorname{sim}(\mathbf{w}), y \notin \operatorname{sim}(\mathbf{w})\right\} .
$$

Lemma 20.2. Let $S \in\left\{\mathscr{D}_{2}, \mathscr{D}_{4}\right\}$. Suppose that $\mathbf{w} \approx \mathbf{w}^{\prime}$ is any identity satisfied by $S$. Then:

(i) $\operatorname{con}(\mathbf{w})=\operatorname{con}\left(\mathbf{w}^{\prime}\right)$ and $\operatorname{sim}(\mathbf{w})=\operatorname{sim}\left(\mathbf{w}^{\prime}\right)$;

(ii) for any letters $x \in \operatorname{con}(\mathbf{w})=\operatorname{con}\left(\mathbf{w}^{\prime}\right)$ and $y \in \operatorname{sim}(\mathbf{w})=\operatorname{sim}\left(\mathbf{w}^{\prime}\right)$, the conditions $x \prec_{\mathbf{w}} y$ and $x \prec_{\mathbf{w}^{\prime}} y$ are equivalent;

(iii) $\mathbf{w}_{\text {sim }}=\mathbf{w}_{\text {sim }}^{\prime}$;

(iv) $\mathrm{F}_{\mathrm{SL}}(\mathbf{w})=\mathrm{F}_{\mathrm{SL}}\left(\mathbf{w}^{\prime}\right)$;

(v) $\mathrm{F}_{\mathrm{SS}}(\mathbf{w})=\mathrm{F}_{\mathrm{SS}}\left(\mathbf{w}^{\prime}\right)$.

Proof. The subsemigroup $\{1,2,4,6\}$ of $S$ is isomorphic to $J^{1}$, so that parts (i)-(iii) hold by Lemma 2.3.

(iv) Seeking a contradiction, suppose $\mathrm{F}_{\mathrm{SL}}(\mathbf{w}) \neq \mathrm{F}_{\mathrm{SL}}\left(\mathbf{w}^{\prime}\right)$. By symmetry, it suffices to assume that $x y \in \mathrm{F}_{\mathrm{SL}}(\mathbf{w}) \backslash \mathrm{F}_{\mathrm{SL}}\left(\mathbf{w}^{\prime}\right)$. Then

(a) $\mathbf{w}=\mathbf{a} x y \mathbf{b}$ for some $\mathbf{a}, \mathbf{b} \in \mathcal{X}^{*}$ such that $x \notin \operatorname{con}(\mathbf{a b})$ and $y \in \operatorname{con}(\mathbf{a}) \backslash \operatorname{con}(\mathbf{b})$.

By part (i),

(b) $x$ is simple in both $\mathbf{w}$ and $\mathbf{w}^{\prime}$, while $y$ is non-simple in both $\mathbf{w}$ and $\mathbf{w}^{\prime}$.

Since $y \nprec_{\mathrm{w}} x$, it follows from part (ii) that

(c) $y \nprec_{\mathbf{w}^{\prime}} x$.

Let $\varphi: \mathcal{X} \rightarrow S$ denote the substitution

$$
z \mapsto \begin{cases}3 & \text { if } z=x, \\ 5 & \text { if } z=y, \\ 6 & \text { otherwise. }\end{cases}
$$


Then it follows from (a) that $\mathbf{w} \varphi \in\{5,6\}^{+} \cdot 3 \cdot 5 \cdot\{6\}^{*}=\{2\}$. This leads to the required contradiction, as shown in the following collectively exhaustive cases.

Case 1: $x y$ is a factor of $\mathbf{w}^{\prime}$. Since $x y \notin \mathrm{F}_{\mathrm{SL}}\left(\mathbf{w}^{\prime}\right)$, the $y$ that immediately follows $x$ is not the last occurrence; this, together with (b), imply $\mathbf{w}^{\prime}=\mathbf{a}^{\prime} x y \mathbf{b}^{\prime} y \mathbf{c}^{\prime}$ for some $\mathbf{a}^{\prime}, \mathbf{b}^{\prime}, \mathbf{c}^{\prime} \in \mathcal{X}^{*}$ with $x \notin \operatorname{con}\left(\mathbf{a}^{\prime} \mathbf{b}^{\prime} \mathbf{c}^{\prime}\right)$. Therefore

$$
\mathbf{w}^{\prime} \varphi \in\{5,6\}^{*} \cdot 3 \cdot 5 \cdot\{5,6\}^{*} \cdot 5 \cdot\{5,6\}^{*}=\{1\} .
$$

Case 2: $x y$ is not a factor of $\mathbf{w}^{\prime}$. Then it follows from (c) that $\mathbf{w}^{\prime}=\mathbf{a}^{\prime} x h \mathbf{b}^{\prime} y \mathbf{c}^{\prime}$ for some $\mathbf{a}^{\prime}, \mathbf{b}^{\prime}, \mathbf{c}^{\prime} \in \mathcal{X}^{*}$ and $h \in \mathcal{X}$ such that $x \notin \operatorname{con}\left(\mathbf{a}^{\prime} \mathbf{b}^{\prime} \mathbf{c}^{\prime}\right)$ and $x \neq h \neq y$. Hence

$$
\mathbf{w}^{\prime} \varphi \in\{5,6\}^{*} \cdot 3 \cdot 6 \cdot\{5,6\}^{*} \cdot 5 \cdot\{5,6\}^{*}=\{1\} .
$$

(v) Suppose $\mathrm{F}_{\mathrm{SS}}(\mathbf{w}) \neq \mathrm{F}_{\mathrm{SS}}\left(\mathbf{w}^{\prime}\right)$, say $x y \in \mathrm{F}_{\mathrm{SS}}(\mathbf{w}) \backslash \mathrm{F}_{\mathrm{SS}}\left(\mathbf{w}^{\prime}\right)$. Then it is easily seen from part (i) that $x y \in \mathrm{F}_{\mathrm{SL}}(y \mathbf{w}) \backslash \mathrm{F}_{\mathrm{SL}}\left(y \mathbf{w}^{\prime}\right)$. It then follows from part (iv) that $S$ does not satisfy the identity $y \mathbf{w} \approx y \mathbf{w}^{\prime}$, which is impossible.

For any letter $x \in \mathcal{X}$, define an $x$-block to be a non-simple word of the form

$$
\mathbf{x}=\left(\prod_{i=1}^{r}\left(x \mathbf{s}_{i}\right)\right) x^{e}=x \mathbf{s}_{1} x \mathbf{s}_{2} \ldots x \mathbf{s}_{r} x^{e}
$$

where $\mathbf{s}_{1}, \ldots, \mathbf{s}_{r} \in \mathcal{X}^{+}, e \in\{1,2\}$, and $r \geqslant 0$ satisfy the following:

(B1) the letters of $\mathbf{s}_{1}, \ldots, \mathbf{s}_{r}$ are all simple in $\mathbf{x}$;

(B2) if $r=0$, then $e=2$.

REMARK 20.3. (i) In any $x$-block, the letter $x$ is the only non-simple letter.

(ii) If $r=0$ in (20.2), then the prefix $\prod_{i=1}^{r}\left(x \mathbf{s}_{i}\right)$ of $\mathbf{x}$ is empty, so that $\mathbf{x}=x^{2}$ by (B2).

Let $\mathbf{w}$ be any non-simple word and let $x$ be any non-simple letter of $\mathbf{w}$ with $\operatorname{occ}(x, \mathbf{w})=r \geqslant 2$, so that

$$
\mathbf{w}=\mathbf{w}_{0} x \mathbf{w}_{1} x \mathbf{w}_{2} \ldots x \mathbf{w}_{r}
$$

for some $\mathbf{w}_{0}, \ldots, \mathbf{w}_{r} \in \mathcal{X}^{*}$ with $x \notin \operatorname{con}\left(\mathbf{w}_{0} \ldots \mathbf{w}_{r}\right)$. Then the letter $x$ is said to be linked in $\mathbf{w}$ if $\operatorname{con}\left(\mathbf{w}_{1} \ldots \mathbf{w}_{r-1}\right) \subseteq \operatorname{sim}(\mathbf{w})$. In other words, a non-simple letter $x$ of $\mathbf{w}$ is linked if any other non-simple letter of $\mathbf{w}$ is not sandwiched between any two occurrences of $x$. A non-simple word is linked if each of its non-simple letters is linked.

Lemma 20.4. Let $\mathbf{w}$ be any non-simple word. Then there exists some linked word $\overline{\mathbf{w}}$ such that the identities (20.1) imply the identity $\mathbf{w} \approx \overline{\mathbf{w}}$.

Proof. It suffices to convert $\mathbf{w}$, using the identities (20.1), into a linked word. Let $x$ be the last non-simple letter of $\mathbf{w}$ that is not linked. Then

$$
\mathbf{w}=\mathbf{p} x \mathbf{q}
$$

for some $\mathbf{p}, \mathbf{q} \in \mathcal{X}^{*}$ such that $x \in \operatorname{con}(\mathbf{p}) \backslash \operatorname{con}(\mathbf{q})$ and any non-simple letter of $\mathbf{w}$ that occurs in $\mathbf{q}$ is linked. It follows that $\mathbf{p} x$ and $\mathbf{q}$ are disjoint words. Since the letter $x$ is not linked and $x \in \operatorname{con}(\mathbf{p}) \backslash \operatorname{con}(\mathbf{q})$, some other non-simple letter $y$ of $\mathbf{w}$ occurring in $\mathbf{p}$ is sandwiched between two occurrences of $x$, that is,

$$
\mathbf{w}=\underbrace{\mathbf{a} x \mathbf{b} y \mathbf{c}}_{\mathbf{p}} x \mathbf{q}
$$


for some $\mathbf{a}, \mathbf{b}, \mathbf{c} \in \mathcal{X}^{*}$ such that $y \in \operatorname{con}(\mathbf{a b}) \backslash \operatorname{con}(\mathbf{c q})$. If $y \in \operatorname{con}(\mathbf{a})$, then $\mathbf{a}=\mathbf{a}^{\prime} y \mathbf{a}^{\prime \prime}$ for some $\mathbf{a}^{\prime}, \mathbf{a}^{\prime \prime} \in \mathcal{X}^{*}$, so that

$$
\mathbf{w}=\mathbf{a}^{\prime} y \mathbf{a}^{\prime \prime} x \mathbf{b} y \mathbf{c} x \mathbf{q} \stackrel{(20.1 \mathrm{e})}{\approx} \mathbf{a}^{\prime} y \mathbf{a}^{\prime \prime} y \mathbf{b} y x \mathbf{c} x \mathbf{q}=\mathbf{a} y \mathbf{b} y x \mathbf{c} x \mathbf{q} .
$$

If $y \in \operatorname{con}(\mathbf{b})$, then $\mathbf{b}=\mathbf{b}^{\prime} y \mathbf{b}^{\prime \prime}$ for some $\mathbf{b}^{\prime}, \mathbf{b}^{\prime \prime} \in \mathcal{X}^{*}$, so that

$$
\mathbf{w}=\mathbf{a} x \mathbf{b}^{\prime} y \mathbf{b}^{\prime \prime} y \mathbf{c} x \mathbf{q} \stackrel{(20.1 \mathrm{~d})}{\approx} \mathbf{a} y \mathbf{b}^{\prime} y \mathbf{b}^{\prime \prime} y x \mathbf{c} x \mathbf{q}=\mathbf{a} y \mathbf{b} y x \mathbf{c} x \mathbf{q} .
$$

Therefore in any case, the two occurrences of $x$ appearing in (20.3) no longer sandwich $y$. This argument can be repeated until a word of the form

$$
\mathbf{w}^{\prime}=\mathbf{p}^{\prime} x \mathbf{s}_{1} x \mathbf{s}_{2} \ldots x \mathbf{s}_{m} x \mathbf{q}
$$

is obtained, where $\mathbf{p}^{\prime}, \mathbf{s}_{1}, \ldots, \mathbf{s}_{m} \in \mathcal{X}^{*}$ are such that $\operatorname{con}\left(\mathbf{s}_{1} \ldots \mathbf{s}_{m}\right) \subseteq \operatorname{sim}\left(\mathbf{w}^{\prime}\right)$ and $x \notin \operatorname{con}\left(\mathbf{p}^{\prime}\right)$. In particular, the letter $x$ is linked in $\mathbf{w}^{\prime}$.

The procedure in the previous paragraph can be repeated on $\mathbf{p}^{\prime}$ to convert $\mathbf{w}^{\prime}$ into a word where all non-simple letters are linked.

In this subsection, a non-simple word $\mathbf{w}$ is said to be in canonical form if

$$
\mathbf{w}=\mathbf{p x}_{1} \ldots \mathbf{x}_{m} \mathbf{q}
$$

where $\mathbf{p}, \mathbf{q} \in \mathcal{X}^{*}, \mathbf{x}_{1}, \ldots, \mathbf{x}_{m} \in \mathcal{X}^{+}$, and $m \geqslant 1$ satisfy the following:

(I) $\mathbf{x}_{i}$ is an $x_{i}$-block;

(II) the letters of $\mathbf{p}$ and $\mathbf{q}$ are simple in $\mathbf{w}$;

(III) $\mathbf{p}, \mathbf{x}_{1}, \ldots, \mathbf{x}_{m}, \mathbf{q}$ are pairwise disjoint;

(IV) if $x_{i}^{2}$ is a suffix of $\mathbf{x}_{i}$ and $\mathbf{x}_{i+1}=x_{i+1}^{2}$, then $x_{i}$ alphabetically precedes $x_{i+1}$.

Note that $x_{1}, \ldots, x_{m}$ are precisely all non-simple letters of the word $\mathbf{w}$ in (20.4).

Lemma 20.5. Let $\mathbf{w}$ be any non-simple word. Then there exists some word $\overline{\mathbf{w}}$ in canonical form such that the identities (20.1) imply the identity $\mathbf{w} \approx \overline{\mathbf{w}}$.

Proof. It suffices to convert $\mathbf{w}$, using the identities (20.1), into a word in canonical form. By Lemma 20.4, the word $\mathbf{w}$ can be assumed linked, say

$$
\mathbf{w}=\mathbf{p} \mathbf{x}_{1} \mathbf{t}_{1} \mathbf{x}_{2} \mathbf{t}_{2} \ldots \mathbf{x}_{m-1} \mathbf{t}_{m-1} \mathbf{x}_{m} \mathbf{q}
$$

where the letters of $\mathbf{p}, \mathbf{t}_{1}, \ldots, \mathbf{t}_{m-1}, \mathbf{q} \in \mathcal{X}^{*}$ are all simple in $\mathbf{w}$, each $\mathbf{x}_{i}$ is a linked word that begins and ends with $x_{i}$, and $x_{1}, \ldots, x_{m}$ are precisely the non-simple letters of $\mathbf{w}$. It is clearly seen that the identities (20.1a) can be used to convert each $\mathbf{x}_{i}$ into an $x_{i}$-block. If $\mathbf{t}_{i} \neq \emptyset$, then

$$
\begin{gathered}
\mathbf{w} \stackrel{(20.1 \mathrm{a})}{\stackrel{\sim}{\approx}} \mathbf{p} \mathbf{x}_{1} \mathbf{t}_{1} \ldots \mathbf{x}_{i} \mathbf{t}_{i} x_{i+1}^{2} \mathbf{x}_{i+1} \ldots \mathbf{t}_{m-1} \mathbf{x}_{m} \mathbf{q} \\
\stackrel{(20.1 \mathrm{c})}{\approx} \mathbf{p x}_{1} \mathbf{t}_{1} \ldots \mathbf{x}_{i} x_{i+1} \mathbf{t}_{i} x_{i+1}^{2} \mathbf{x}_{i+1} \ldots \mathbf{t}_{m-1} \mathbf{x}_{m} \mathbf{q} \\
\stackrel{(20.1 \mathrm{a})}{\approx} \mathbf{p x}_{1} \mathbf{t}_{1} \ldots \mathbf{x}_{i} \underbrace{x_{i+1} \mathbf{t}_{i} \mathbf{x}_{i+1}}_{\mathbf{x}_{i+1}^{\prime}} \ldots \mathbf{t}_{m-1} \mathbf{x}_{m} \mathbf{q}
\end{gathered}
$$

where $\mathbf{x}_{i+1}^{\prime}$ is an $x_{i+1}$-block. Therefore $\mathbf{w}$ can be converted into a word of the form (20.4) with (I)-(III) satisfied.

It remains to convert $\mathbf{w}$ into a word that satisfies (IV). Suppose that $x_{i}^{2}$ is a suffix of $\mathbf{x}_{i}$ and $\mathbf{x}_{i+1}=x_{i+1}^{2}$, where $x_{i+1}$ alphabetically precedes $x_{i}$. Then $\mathbf{x}_{i}=x_{i} \mathbf{s}_{1} x_{i} \mathbf{s}_{2} \ldots x_{i} \mathbf{s}_{r} x_{i}^{2}$. In what 
follows, it is shown that the relabeling $x_{i} \leftrightarrow x_{i+1}$ can be performed on $\mathbf{w}$, so that (IV) is satisfied. If $r=0$, then $\mathbf{x}_{i}=x_{i}^{2}$ so that

$$
\mathbf{w}=\ldots \underbrace{x_{i}^{2}}_{\mathbf{x}_{i}} \cdot \underbrace{x_{i+1}^{2}}_{\mathbf{x}_{i+1}} \ldots \stackrel{(20.1 \mathrm{~b})}{\approx} \ldots x_{i+1}^{2} \cdot x_{i}^{2} \ldots
$$

If $r \geqslant 1$, then

$$
\begin{aligned}
& \mathbf{w}=\ldots \overbrace{x_{i} \mathbf{s}_{1} x_{i} \mathbf{s}_{2} \ldots x_{i} \mathbf{s}_{r} x_{i}^{2}} \\
& \begin{array}{l}
(20.1 \mathrm{~b}) \\
\stackrel{\mathbf{x}_{i}}{\approx}
\end{array} \overbrace{x_{i+1}^{2} \ldots x_{i} \mathbf{s}_{1} x_{i} \mathbf{s}_{2} \ldots x_{i} \mathbf{s}_{r} \cdot x_{i+1}^{2} x_{i}^{2} \ldots}^{\mathbf{x}_{i+1}} \ldots \\
& \stackrel{(20.1 \mathrm{a})}{\approx} \ldots x_{i} \mathbf{s}_{1} x_{i} \mathbf{s}_{2} \ldots x_{i} \mathbf{s}_{r} x_{i+1}^{r} \cdot x_{i+1}^{2} x_{i}^{2} \ldots \\
& \stackrel{(20.1 \mathrm{f})}{\approx} \ldots x_{i+1} \mathbf{s}_{1} x_{i+1} \mathbf{s}_{2} \ldots x_{i+1} \mathbf{s}_{r} x_{i}^{r} \cdot x_{i+1}^{2} x_{i}^{2} \ldots \\
& \stackrel{(20.1 \mathrm{a})}{\approx} \ldots x_{i+1} \mathbf{s}_{1} x_{i+1} \mathbf{s}_{2} \ldots x_{i+1} \mathbf{s}_{r} x_{i}^{2} \cdot x_{i+1}^{2} x_{i}^{2} \ldots \\
& \stackrel{(20.1 \mathrm{~b})}{\approx} \ldots x_{i+1} \mathbf{s}_{1} x_{i+1} \mathbf{s}_{2} \ldots x_{i+1} \mathbf{s}_{r} x_{i+1}^{2} \cdot x_{i}^{2} x_{i}^{2} \ldots \\
&(20.1 \mathrm{a}) \\
& \stackrel{\approx}{\approx} \ldots x_{i+1} \mathbf{s}_{1} x_{i+1} \mathbf{s}_{2} \ldots x_{i+1} \mathbf{s}_{r} x_{i+1}^{2} \cdot x_{i}^{2} \ldots
\end{aligned}
$$

as required.

Lemma 20.6. Let $S \in\left\{\mathscr{D}_{2}, \mathscr{D}_{4}\right\}$ and let

$$
\mathbf{w}=\mathbf{p x}_{1} \ldots \mathbf{x}_{m} \mathbf{q} \quad \text { and } \quad \mathbf{w}^{\prime}=\mathbf{p y}_{1} \ldots \mathbf{y}_{m} \mathbf{q}
$$

be any words in canonical form such that each $\mathbf{x}_{i}$ is an $x_{i}$-block and each $\mathbf{y}_{i}$ is a $y_{i}$-block with $\left\{x_{1}, \ldots, x_{m}\right\}=\left\{y_{1}, \ldots, y_{m}\right\}$. Suppose that $S$ satisfies the identity $\mathbf{w} \approx \mathbf{w}^{\prime}$. Then $\left(x_{1}, \ldots, x_{m}\right)=\left(y_{1}, \ldots, y_{m}\right)$.

Proof. By assumption, $x_{1} \prec_{\mathbf{w}} \ldots \prec_{\mathbf{w}} x_{m}$. Let $i \in\{1, \ldots, m-1\}$. Then it suffices to show $x_{i} \prec_{\mathbf{w}^{\prime}} x_{i+1}$.

Case 1: $\mathbf{x}_{i+1}$ contains some simple letter, say $h$, of $\mathbf{w}$. Then

(a) $x_{i+1} \nprec_{\mathbf{w}} h$

and $x_{i} \prec_{\mathbf{w}} h$, so that $x_{i} \prec_{\mathbf{w}^{\prime}} h$ by Lemma 20.2(ii). If $x_{i} \nprec_{\mathbf{w}^{\prime}} x_{i+1}$, then $x_{i+1} \prec_{\mathbf{w}^{\prime}} x_{i} \prec_{\mathbf{w}^{\prime}} h$; this contradicts Lemma 20.2(ii) in view of (a). Hence $x_{i} \prec_{\mathbf{w}^{\prime}} x_{i+1}$.

Case 2: $\mathbf{x}_{i+1}$ does not contain any simple letter of $\mathbf{w}$. Then $\mathbf{x}_{i+1}=x_{i+1}^{2}$ by (B2). Since $\mathbf{x}_{i}$ is an $x_{i}$-block, it is of the form

$$
\mathbf{x}_{i}=x_{i} \mathbf{s}_{1} x_{i} \mathbf{s}_{2} \ldots x_{i} \mathbf{s}_{r} x_{i}^{e}
$$

where $e \in\{1,2\}$ and the letters of $\mathbf{s}_{1}, \ldots, \mathbf{s}_{r} \in \mathcal{X}^{+}$are all simple in $\mathbf{w}$. There are two subcases.

2.1. $e=1$. Then $r \geqslant 1$ by (B2). Let $s=\mathrm{t}\left(\mathbf{s}_{r}\right)$. Since $s \prec_{\mathbf{w}} x_{i+1}$ and $s x_{i} \in \mathrm{F}_{\mathrm{SL}}(\mathbf{w})$,

(b) $x_{i+1} \nprec_{\mathbf{w}^{\prime}} s$

and $s x_{i} \in \mathrm{F}_{\mathrm{SL}}\left(\mathbf{w}^{\prime}\right)$ by Lemma 20.2 parts (ii) and (iv). The letter $s$ thus belongs to the $x_{i}$-block in $\mathbf{w}^{\prime}$. Suppose $x_{i} \nprec_{\mathbf{w}^{\prime}} x_{i+1}$, so that $x_{i+1} \prec \mathbf{w}^{\prime} x_{i}$. Then within $\mathbf{w}^{\prime}$, the $x_{i+1}$-block occurs before the $x_{i}$-block, so that $x_{i+1} \prec_{\mathbf{w}^{\prime}} s$. In view of (b), this is impossible. Hence $x_{i} \prec_{\mathbf{w}^{\prime}} x_{i+1}$.

2.2. $e=2$. Then (IV) implies that

(c) $x_{i}$ alphabetically precedes $x_{i+1}$. 
Seeking a contradiction with (c), suppose $x_{i} \nprec_{\mathbf{w}^{\prime}} x_{i+1}$. Then $x_{i+1} \prec_{\mathbf{w}^{\prime}} x_{i}$, whence there exist $k$ and $\ell$ with $1 \leqslant k<\ell \leqslant m$ such that $\mathbf{y}_{k}$ is an $x_{i+1}$-block (so that $y_{k}=x_{i+1}$ ) and $\mathbf{y}_{\ell}$ is an $x_{i}$-block (so that $y_{\ell}=x_{i}$ ), that is,

$$
\mathbf{w}^{\prime}=\mathbf{p y}_{1} \ldots \mathbf{y}_{k} \mathbf{y}_{k+1} \ldots \mathbf{y}_{\ell} \ldots \mathbf{y}_{m} \mathbf{q}
$$

Suppose that the factor $\mathbf{y}_{k+1} \ldots \mathbf{y}_{\ell}$ of $\mathbf{w}^{\prime}$ contains some simple letter $h$. Then $x_{i+1}=y_{k} \prec_{\mathbf{w}^{\prime}} h$ and

(d) $x_{i}=y_{\ell} \nprec_{\mathbf{w}^{\prime}} h$,

whence $x_{i+1} \prec_{\mathbf{w}} h$ by Lemma 20.2(ii). The assumption $x_{i} \prec_{\mathbf{w}} x_{i+1}$ implies $x_{i} \prec_{\mathbf{w}} h$. But then Lemma 20.2(ii) implies $x_{i} \prec_{\mathbf{w}^{\prime}} h$, which contradicts (d). Therefore the factor $\mathbf{y}_{k+1} \ldots \mathbf{y}_{\ell}$ of $\mathbf{w}^{\prime}$ cannot contain any simple letter. Since each $\mathbf{y}_{i}$ is a $y_{i}$-block, it follows from (B2) that

$$
\left(\mathbf{y}_{k+1}, \ldots, \mathbf{y}_{\ell}\right)=\left(y_{k+1}^{2}, \ldots, y_{\ell}^{2}\right) \text {. }
$$

By (IV), the letters $y_{k+1}, \ldots, y_{\ell}$ are in alphabetical order. Specifically,

(e) $y_{k+1}$ alphabetically precedes $y_{\ell}=x_{i}$.

Now since $\mathbf{y}_{k}$ is an $x_{i+1}$-block, it is of the form

$$
\mathbf{y}_{k}=x_{i+1} \mathbf{t}_{1} x_{i+1} \mathbf{t}_{2} \ldots x_{i+1} \mathbf{t}_{r} x_{i+1}^{f},
$$

where $f \in\{1,2\}$ and the letters of $\mathbf{t}_{1}, \ldots, \mathbf{t}_{r} \in \mathcal{X}^{+}$are all simple in $\mathbf{w}^{\prime}$. Suppose $f=1$. Then $r \geqslant 1$ by (B2). Let $t=\mathrm{t}\left(\mathbf{t}_{r}\right)$. Since $t \prec_{\mathbf{w}^{\prime}} y_{\ell}=x_{i}$ and $t x_{i+1} \in \mathrm{F}_{\mathrm{SL}}\left(\mathbf{w}^{\prime}\right)$,

(f) $x_{i} \nprec_{\mathbf{w}} t$

and $t x_{i+1} \in \mathrm{F}_{\mathrm{SL}}(\mathbf{w})$ by Lemma 20.2 parts (ii) and (iv). Therefore the simple letter $t$ of $\mathbf{w}$ occurs in the $x_{i+1}$-block $\mathbf{x}_{i+1}$, whence $x_{i} \prec_{\mathbf{w}} t$. In view of (f), this is impossible. Hence $f=2$. Now by (IV), the letter $x_{i+1}=y_{k}$ alphabetically precedes $y_{k+1}$. Therefore by (e), the letter $x_{i+1}$ alphabetically precedes $x_{i}$. This is the required contradiction with (c).

Proof of Proposition 20.1. Let $S \in\left\{\mathscr{D}_{2}, \mathscr{D}_{4}\right\}$. It is routinely checked that $S$ satisfies the identities (20.1). Hence it suffices to show that any identity $\mathbf{w} \approx \mathbf{w}^{\prime}$ satisfied by $S$ is implied by the identities (20.1). If either $\mathbf{w}$ or $\mathbf{w}^{\prime}$ is a simple word, then it follows from Lemma 20.2 parts (i) and (iii) that the identity $\mathbf{w} \approx \mathbf{w}^{\prime}$ is trivial and so is vacuously implied by the identities (20.1). Therefore assume that $\mathbf{w}$ and $\mathbf{w}^{\prime}$ are both non-simple words and so by Lemma 20.5, can be chosen to be in canonical form. Since $\operatorname{con}(\mathbf{w})=\operatorname{con}\left(\mathbf{w}^{\prime}\right)$ and $\operatorname{sim}(\mathbf{w})=\operatorname{sim}\left(\mathbf{w}^{\prime}\right)$ by part (i) of Lemma 20.2, it follows from parts (iii) and (v) of the same lemma that

$$
\mathbf{w}=\mathbf{p x}_{1} \ldots \mathbf{x}_{m} \mathbf{q} \text { and } \quad \mathbf{w}^{\prime}=\mathbf{p y}_{1} \ldots \mathbf{y}_{m} \mathbf{q},
$$

where each $\mathbf{x}_{i}$ is an $x_{i}$-block and each $\mathbf{y}_{i}$ is a $y_{i}$-block with $\left\{x_{1}, \ldots, x_{m}\right\}=\left\{y_{1}, \ldots, y_{m}\right\}$. It then follows from Lemma 20.6 that each $\mathbf{y}_{i}$ is an $x_{i}$-block. Let

$$
\mathbf{p}, \mathbf{s}_{1}, \ldots, \mathbf{s}_{n}, \mathbf{q}
$$

be all the maximal factors of $\mathbf{w}$, in order of appearance, that consist of simple letters, so that $\mathbf{w}_{\text {sim }}=\mathbf{p s}_{1} \ldots \mathbf{s}_{n} \mathbf{q}$. By part (v) of Lemma 20.2, the maximal factors of $\mathbf{w}^{\prime}$, in order of appearance, that consist of simple letters coincide with the words from (20.5); by part (iii) of the same lemma, $\mathbf{w}_{\text {sim }}^{\prime}=\mathbf{p s}_{1} \ldots \mathbf{s}_{n} \mathbf{q}$. Hence

$$
\mathbf{w}=\mathbf{p} \underbrace{x_{1} \mathbf{s}_{1} x_{1} \mathbf{s}_{2} \ldots x_{1} \mathbf{s}_{j} x_{1}^{e}}_{\mathbf{x}_{1}} \mathbf{x}_{2} \ldots \mathbf{x}_{m} \mathbf{q} \quad \text { and } \quad \mathbf{w}^{\prime}=\mathbf{p} \underbrace{x_{1} \mathbf{s}_{1} x_{1} \mathbf{s}_{2} \ldots x_{1} \mathbf{s}_{k} x_{1}^{f}}_{\mathbf{y}_{1}} \mathbf{y}_{2} \ldots \mathbf{y}_{m} \mathbf{q} .
$$

Suppose $j \neq k$, say $j<k$. Then $x_{1} \nprec_{\mathbf{w}^{\prime}} h$ where $h=\mathrm{h}\left(\mathbf{s}_{k}\right)$. But $\mathbf{s}_{k}$ is a factor of $\mathbf{x}_{2} \ldots \mathbf{x}_{m}$ and so $x_{1} \prec_{\mathbf{w}} h$, contradicting Lemma 20.2(ii). Hence $j=k$. Let $t=\mathrm{t}\left(\mathbf{s}_{j}\right)=\mathrm{t}\left(\mathbf{s}_{k}\right)$. If $e \neq f$, say $(e, f)=(1,2)$, then $t x_{1} \in \mathrm{F}_{\mathrm{SL}}(\mathbf{w})$ and $t x_{1} \notin \mathrm{F}_{\mathrm{SL}}\left(\mathbf{w}^{\prime}\right)$, contradicting Lemma 20.2(iv). Therefore $e=f$, whence $\mathbf{x}_{1}=\mathbf{y}_{1}$. Similarly, it can be shown that $\mathbf{x}_{i}=\mathbf{y}_{i}$ for each $i>1$, so that $\mathbf{w}=\mathbf{w}^{\prime}$. Consequently, the identity $\mathbf{w} \approx \mathbf{w}^{\prime}$ is implied by the identities (20.1). 


\subsection{Finite basis property of $\mathscr{E}_{4}$}

Proposition 20.7. The variety generated by $\mathscr{E}_{4}$ is defined by the identities

$$
\begin{aligned}
x \mathrm{H} x \mathrm{~K} x^{2} & \approx x \mathrm{H} x \mathrm{~K} x, \\
x \mathrm{H} x \mathrm{~K} x & \approx x \mathrm{~K} x \mathrm{H} x, \\
x \mathrm{H} x \cdot \mathrm{K} y \cdot \mathrm{T} y & \approx x \mathrm{H} x \cdot \mathrm{T} y \cdot \mathrm{K} y, \\
x \cdot \mathrm{H} y \cdot \mathrm{K} x \cdot \mathrm{T} y & \approx x \cdot \mathrm{K} x \cdot \mathrm{H} y \cdot \mathrm{T} y, \\
x \cdot \mathrm{H} y \mathrm{~K} y \cdot \mathrm{T} x & \approx x \cdot \mathrm{T} x \cdot \mathrm{H} y \mathrm{~K} y, \\
x \mathrm{H} x \cdot \mathrm{K}_{1} y \mathrm{~K}_{2} y \cdot \mathrm{T}_{1} z \mathrm{~T}_{2} z & \approx x \mathrm{H} x \cdot \mathrm{T}_{1} z \mathrm{~T}_{2} z \cdot \mathrm{K}_{1} y \mathrm{~K}_{2} y .
\end{aligned}
$$

For any word $\mathbf{w}$, let $\mathrm{F}_{\mathrm{SN}}(\mathbf{w})$ denote the set of factors of $\mathbf{w}$ of length two that begin with a simple letter and end with a non-simple letter:

$$
\mathrm{F}_{\mathrm{SN}}(\mathbf{w})=\left\{x y \in \mathcal{X}^{2} \mid \mathbf{w} \in \mathcal{X}^{*} x y \mathcal{X}^{*}, x \in \operatorname{sim}(\mathbf{w}), y \notin \operatorname{sim}(\mathbf{w})\right\} .
$$

Lemma 20.8. Suppose that $\mathbf{w} \approx \mathbf{w}^{\prime}$ is any identity satisfied by $\mathscr{E}_{4}$. Then:

(i) for each $x \in \mathcal{X}$, either $\operatorname{occ}(x, \mathbf{w})=\operatorname{occ}\left(x, \mathbf{w}^{\prime}\right) \leqslant 2$ or $\operatorname{occ}(x, \mathbf{w}), \operatorname{occ}\left(x, \mathbf{w}^{\prime}\right) \geqslant 3$;

(ii) $\mathrm{h}(\mathbf{w})=\mathrm{h}\left(\mathbf{w}^{\prime}\right)$;

(iii) $\mathrm{F}_{\mathrm{SN}}(\mathbf{w})=\mathrm{F}_{\mathrm{SN}}\left(\mathbf{w}^{\prime}\right)$;

(iv) $\mathrm{F}_{\mathrm{SS}}(\mathbf{w})=\mathrm{F}_{\mathrm{SS}}\left(\mathbf{w}^{\prime}\right)$.

Proof. (i) This follows from Lemma 2.1 (vii) since the subsemigroup $\{1,3,4,5\}$ of $\mathscr{E}_{4}$ is isomorphic to $N_{3}^{1}$.

(ii) This follows from Lemma 2.1(i) since the subsemigroup $\{5,6\}$ of $\mathscr{E}_{4}$ is isomorphic to $L_{2}$.

(iii) Suppose $\mathrm{F}_{\mathrm{SN}}(\mathbf{w}) \neq \mathrm{F}_{\mathrm{SN}}\left(\mathbf{w}^{\prime}\right)$, say $x y \in \mathrm{F}_{\mathrm{SN}}(\mathbf{w}) \backslash \mathrm{F}_{\mathrm{SN}}\left(\mathbf{w}^{\prime}\right)$. Then it follows from part (i) that $x$ is simple in $\mathbf{w}^{\prime}$ and $y$ is non-simple in $\mathbf{w}^{\prime}$, whence $x y$ is not a factor of $\mathbf{w}^{\prime}$. Let $\varphi: \mathcal{X} \rightarrow \mathscr{E}_{4}$ denote the substitution

$$
z \mapsto \begin{cases}2 & \text { if } z=x \\ 5 & \text { if } z=y \\ 6 & \text { otherwise }\end{cases}
$$

Then $\mathbf{w} \varphi=\ldots 2 \cdot 5 \ldots=1$ and $\mathbf{w}^{\prime} \varphi \in\{5,6\}^{*} \cdot 2 \cdot 6 \cdot\{5,6\}^{*}=\{3\}$, which is impossible. Hence $\mathrm{F}_{\mathrm{SN}}(\mathbf{w})=\mathrm{F}_{\mathrm{SN}}\left(\mathbf{w}^{\prime}\right)$

(iv) It follows from part (i) that $\operatorname{con}(\mathbf{w})=\operatorname{con}\left(\mathbf{w}^{\prime}\right)$ and $\operatorname{sim}(\mathbf{w})=\operatorname{sim}\left(\mathbf{w}^{\prime}\right)$. The identity $(2.3)$ with $n=3$ is not satisfied by the semigroup $\mathscr{E}_{4}$ because $5^{3} \cdot 2 \cdot 5^{3} \cdot 6 \cdot 5^{3} \neq 5^{3} \cdot 2 \cdot 6 \cdot 5^{3}$. Therefore $\mathrm{F}_{\mathrm{SS}}(\mathbf{w})=\mathrm{F}_{\mathrm{SS}}\left(\mathbf{w}^{\prime}\right)$ by Lemma 2.10 .

For any letter $x \in \mathcal{X}$, define an $x$-rigid block to be a non-simple word of the form

$$
\mathbf{x}=\mathbf{s}_{1} x \mathbf{s}_{2} x \ldots \mathbf{s}_{r-1} x \mathbf{s}_{r} x^{e},
$$

where $\mathbf{s}_{1} \in \mathcal{X}^{*}, \mathbf{s}_{2}, \ldots, \mathbf{s}_{r} \in \mathcal{X}^{+}, e \in\{1,2,3\}$, and $r \geqslant 1$ satisfy the following:

(Ri1) the letters of $\mathbf{s}_{1}, \ldots, \mathbf{s}_{r}$ are all simple in $\mathbf{x}$;

(Ri2) the letters $\mathrm{h}\left(\mathbf{s}_{2}\right), \ldots, \mathrm{h}\left(\mathbf{s}_{r}\right)$ are in strict alphabetical order;

(Ri3) if $r=1$, then $e \in\{2,3\}$;

(Ri4) if $r=2$, then $e \in\{1,2\}$;

$(\operatorname{Ri} 5)$ if $r \geqslant 3$, then $e=1$.

The $x$-rigid block in (20.7) is fully $x$-rigid if

(Ri6) $\mathbf{s}_{1} \neq \emptyset$ implies that the letters $\mathrm{h}\left(\mathbf{s}_{1}\right), \ldots, \mathrm{h}\left(\mathbf{s}_{r}\right)$ are in strict alphabetical order. 
REMARK 20.9. (i) In any $x$-rigid block, the letter $x$ is the only non-simple letter.

(ii) The $x$-rigid block in (20.7) is vacuously fully $x$-rigid if $\mathbf{s}_{1}=\emptyset$.

Lemma 20.10. Let $\mathbf{h}$ and $\mathbf{x}$ be any non-simple words that end with non-simple letters. Suppose that $x$ is the only non-simple letter of $\mathbf{x}$ and that $\mathrm{t}(\mathbf{x})=x$. Then:

(i) there exists an $x$-rigid block $\widetilde{\mathbf{x}}$ such that the identities (20.6) imply the identity $\mathbf{x} \approx \widetilde{\mathbf{x}}$;

(ii) there exists a fully $x$-rigid block $\widetilde{\mathbf{x}}$ such that the identities (20.6) imply the identity $\mathbf{h x} \approx \mathbf{h} \widetilde{\mathbf{x}}$.

Proof. (i) It suffices to convert $\mathbf{x}$, using the identities (20.6), into an $x$-rigid block. By assumption, $\mathbf{x}=\mathbf{s}_{1} x \mathbf{s}_{2} x \ldots \mathbf{s}_{k-1} x \mathbf{s}_{k} x$ for some $\mathbf{s}_{1}, \ldots, \mathbf{s}_{k} \in \mathcal{X}^{*}$ with $\operatorname{con}\left(\mathbf{s}_{1} \ldots \mathbf{s}_{k}\right)=\operatorname{sim}(\mathbf{x})$. The identities (20.6b) can be used to arrange the factors $\mathbf{s}_{2} x, \ldots, \mathbf{s}_{k} x$ in any order. In particular, the factors from $\mathbf{s}_{2} x, \ldots, \mathbf{s}_{k} x$ with empty $\mathbf{s}_{i}$ can be gathered to the right, resulting in a word of the form (20.7) with (Ri1) satisfied and $e \geqslant 1$. Then the identities (20.6b) can be applied to arrange the factors $\mathbf{s}_{2} x, \ldots, \mathbf{s}_{r} x$ in (20.7) until (Ri2) is satisfied. Finally, the identities (20.6a) can be applied until (Ri3)-(Ri5) are satisfied.

(ii) It suffices to convert the word $\mathbf{h x}$, using the identities (20.6), into a word $\mathbf{h} \widetilde{\mathbf{x}}$ where $\widetilde{\mathbf{x}}$ is a fully $x$-rigid block. By assumption, $\mathbf{h}=\mathbf{a} h \mathbf{b} h$ for some $\mathbf{a}, \mathbf{b} \in \mathcal{X}^{*}$ and $h \in \mathcal{X}$. By part (i), the identities (20.6) can be used to convert $\mathbf{x}$ into an $x$-rigid block, say the one in (20.7). Hence

$$
\mathbf{h x} \stackrel{(20.6)}{\approx} \mathbf{a} h \mathbf{b} h \underbrace{\mathbf{s}_{1} x \mathbf{s}_{2} x \ldots \mathbf{s}_{r-1} x \mathbf{s}_{r} x^{e}}_{\widetilde{\mathbf{x}}}
$$

where $\widetilde{\mathbf{x}}$ satisfies (Ri1)-(Ri5). If $\mathbf{s}_{1} \neq \emptyset$, then the identities $\{(20.6 \mathrm{~b}),(20.6 \mathrm{c})\}$ can be applied until (Ri6) is additionally satisfied.

Lemma 20.11. Let $\mathbf{x}_{i}$ be any $x_{i}$-rigid block, where $i \in\{1,2,3\}$. Then the identities (20.6f) imply the identity $\mathbf{x}_{1} \mathbf{x}_{2} \mathbf{x}_{3} \approx \mathbf{x}_{1} \mathbf{x}_{3} \mathbf{x}_{2}$.

Proof. By assumption, $\mathbf{x}_{i}=\mathbf{a}_{i} x_{i} \mathbf{b}_{i} x_{i}$ for some $\mathbf{a}_{i}, \mathbf{b}_{i} \in \mathcal{X}^{*}$. Since

$$
\begin{aligned}
\mathbf{x}_{1} \mathbf{x}_{2} \mathbf{x}_{3} & =\mathbf{a}_{1} x_{1} \mathbf{b}_{1} x_{1} \cdot \mathbf{a}_{2} x_{2} \mathbf{b}_{2} x_{2} \cdot \mathbf{a}_{3} x_{3} \mathbf{b}_{3} x_{3} \\
\stackrel{(20.6 \mathrm{f})}{\approx} & \mathbf{a}_{1} x_{1} \mathbf{b}_{1} x_{1} \cdot \mathbf{a}_{3} x_{3} \mathbf{b}_{3} x_{3} \cdot \mathbf{a}_{2} x_{2} \mathbf{b}_{2} x_{2}=\mathbf{x}_{1} \mathbf{x}_{3} \mathbf{x}_{2},
\end{aligned}
$$

the identities (20.6f) imply the identity $\mathbf{x}_{1} \mathbf{x}_{2} \mathbf{x}_{3} \approx \mathbf{x}_{1} \mathbf{x}_{3} \mathbf{x}_{2}$.

In this subsection, a non-simple word $\mathbf{w}$ is said to be in canonical form if

$$
\mathbf{w}=\mathbf{x}_{1} \ldots \mathbf{x}_{m} \mathbf{z}
$$

where $\mathbf{x}_{1}, \ldots, \mathbf{x}_{m} \in \mathcal{X}^{+}$and $\mathbf{z} \in \mathcal{X}^{*}$ are such that:

(I) $\mathbf{x}_{1}$ is an $x_{1}$-rigid block;

(II) $\mathbf{x}_{i}$ is a fully $x_{i}$-rigid block for each $i \in\{2, \ldots, m\}$;

(III) the letters $x_{2}, \ldots, x_{m}$ are in strict alphabetical order;

(IV) the letters of $\mathbf{z}$ are simple in $\mathbf{w}$;

(V) $\mathbf{x}_{1}, \ldots, \mathbf{x}_{m}, \mathbf{z}$ are pairwise disjoint.

Lemma 20.12. Let $\mathbf{w}$ be any non-simple word. Then there exists some word $\mathbf{w}$ in canonical form such that the identities (20.6) imply the identity $\mathbf{w} \approx \overline{\mathbf{w}}$.

Proof. It suffices to convert $\mathbf{w}$, using the identities (20.6), into a word in canonical form. Consider a factorization of $\mathbf{w}$ that displays all of its non-simple letters individually, that is, 
$\mathbf{w}=\mathbf{s}_{1} x_{1} \mathbf{s}_{2} x_{2} \ldots \mathbf{s}_{k} x_{k} \mathbf{z}$ where the letters $x_{1}, \ldots, x_{k}$ are non-simple in $\mathbf{w}$ and the letters in the factors $\mathbf{s}_{1}, \ldots, \mathbf{s}_{k}, \mathbf{z} \in \mathcal{X}^{*}$ are simple in $\mathbf{w}$. Each letter in the list $x_{1}, \ldots, x_{k}$ is non-simple in $\mathbf{w}$ and thus appears at least twice in the list, whence $k \geqslant 2$.

Let $r \geqslant 2$ be such that $x_{1}=x_{2}=\ldots=x_{r-1}$ and $x_{1} \neq x_{r}$. Suppose that $\ell>r$ is the least integer such that $x_{1}=x_{\ell}$. Then

$$
\mathbf{w}=\left(\prod_{i=1}^{r-1}\left(\mathbf{s}_{i} x_{1}\right)\right) \underbrace{\left(\prod_{i=r}^{\ell-1}\left(\mathbf{s}_{i} x_{i}\right)\right)}_{\mathbf{a}} \mathbf{s}_{\ell} x_{1} \underbrace{\left(\prod_{i=\ell+1}^{k}\left(\mathbf{s}_{i} x_{i}\right)\right)}_{\mathbf{b}} \mathbf{z} .
$$

Note that the factor $\mathbf{a}$ is nonempty because $x_{r} \in \operatorname{con}(\mathbf{a})$, and that $\mathrm{t}(\mathbf{a})=x_{\ell-1} \neq x_{1}$ by the minimality of $\ell$. Since the letter $\mathrm{t}(\mathbf{a})=x_{\ell-1}$ is non-simple in $\mathbf{w}$, either $\operatorname{occ}\left(x_{\ell-1}, \mathbf{a}\right) \geqslant 2$ or $x_{\ell-1} \in \operatorname{con}(\mathbf{b})$. Hence

$$
\begin{aligned}
\mathbf{w} & =\left(\prod_{i=1}^{r-1}\left(\mathbf{s}_{i} x_{1}\right)\right) \cdot \mathbf{a} \cdot\left(\mathbf{s}_{\ell} x_{1}\right) \cdot \mathbf{b} \cdot \mathbf{z} \stackrel{(20.6 \mathrm{~d}),(20.6 \mathrm{e})}{\approx}\left(\prod_{i=1}^{r-1}\left(\mathbf{s}_{i} x_{1}\right)\right) \cdot \mathbf{s}_{\ell} x_{1} \cdot \mathbf{a} \cdot \mathbf{b} \cdot \mathbf{z} \\
& =\left(\prod_{i=1}^{r-1}\left(\mathbf{s}_{i} x_{1}\right)\right) \cdot \mathbf{s}_{\ell} x_{1} \cdot\left(\prod_{i=r}^{\ell-1}\left(\mathbf{s}_{i} x_{i}\right)\right)\left(\prod_{i=\ell+1}^{k}\left(\mathbf{s}_{i} x_{i}\right)\right) \cdot \mathbf{z},
\end{aligned}
$$

that is, the identities $\{(20.6 \mathrm{~d}),(20.6 \mathrm{e})\}$ can be used to move the factor $\mathbf{s}_{\ell} x_{1}$ to the left until it immediately follows the prefix $\prod_{i=1}^{r-1}\left(\mathbf{s}_{i} x_{1}\right)$.

Now $x_{1} \notin\left\{x_{r}, \ldots, x_{\ell-1}\right\}$ by the minimality of $\ell$. But if $x_{1} \in\left\{x_{\ell+1}, \ldots, x_{k}\right\}$, say $x_{1}=x_{j}$ for some least $j \in\{\ell+1, \ldots, k\}$, then the procedure in the previous paragraph can be repeated to move the factor $\mathbf{s}_{j} x_{1}$ in $\prod_{i=\ell+1}^{k}\left(\mathbf{s}_{i} x_{i}\right)$ to the left until it immediately follows the prefix $\left(\prod_{i=1}^{r-1}\left(\mathbf{s}_{i} x_{1}\right)\right) \mathbf{s}_{\ell} x_{1}$. It is easily seen how this can be repeated until $\mathbf{w}$ is converted into a word of the form $\mathbf{x}_{1} \ldots \mathbf{x}_{m} \mathbf{z}$ with (IV) and (V) satisfied, and each $\mathbf{x}_{i}$ is a word that contains precisely one non-simple letter, say $x_{i}$, with $\mathrm{t}\left(\mathbf{x}_{i}\right)=x_{i}$. Then it follows from Lemma 20.10 that (I) and (II) are satisfied by applying the identities (20.6). By Lemma 20.11, the factors $\mathbf{x}_{2}, \ldots, \mathbf{x}_{m}$ can be arranged by the identities (20.6f) in any manner. Hence (III) is satisfied.

Proof of Proposition 20.7. It is routinely checked that $\mathscr{E}_{4}$ satisfies the identities (20.6). Hence it suffices to show that any identity $\mathbf{w} \approx \mathbf{w}^{\prime}$ satisfied by $\mathscr{E}_{4}$ is implied by the identities (20.6). If either $\mathbf{w}$ or $\mathbf{w}^{\prime}$ is a simple word, then it follows from Lemma 20.8 parts (i) and (iv) that the identity $\mathbf{w} \approx \mathbf{w}^{\prime}$ is trivial and so is vacuously implied by (20.6). Therefore assume that $\mathbf{w}$ and $\mathbf{w}^{\prime}$ are both non-simple words and so, by Lemma 20.12, can be chosen to be in canonical form. Lemma 20.8(i) implies $\operatorname{con}(\mathbf{w})=\operatorname{con}\left(\mathbf{w}^{\prime}\right)$ and $\operatorname{sim}(\mathbf{w})=\operatorname{sim}\left(\mathbf{w}^{\prime}\right)$. Hence

$$
\mathbf{w}=\mathbf{x}_{1} \ldots \mathbf{x}_{m} \mathbf{z} \quad \text { and } \quad \mathbf{w}^{\prime}=\mathbf{x}_{1}^{\prime} \ldots \mathbf{x}_{m}^{\prime} \mathbf{z}^{\prime}
$$

where $\mathbf{x}_{1}$ is an $x_{1}$-rigid block, $\mathbf{x}_{i}$ is a fully $x_{i}$-rigid block for each $i \in\{2, \ldots, m\}, \mathbf{x}_{1}^{\prime}$ is an $x_{1}^{\prime}$-rigid block, and $\mathbf{x}_{i}^{\prime}$ is a fully $x_{i}^{\prime}$-rigid block for each $i \in\{2, \ldots, m\}$.

By definition, $\mathbf{x}_{1}$ and $\mathbf{x}_{1}^{\prime}$ are of the form

$$
\mathbf{x}_{1}=\mathbf{s}_{1} x_{1} \mathbf{s}_{2} x_{1} \ldots \mathbf{s}_{r-1} x_{1} \mathbf{s}_{r} x_{1}^{e} \quad \text { and } \quad \mathbf{x}_{1}^{\prime}=\mathbf{s}_{1}^{\prime} x_{1}^{\prime} \mathbf{s}_{2}^{\prime} x_{1}^{\prime} \ldots \mathbf{s}_{r^{\prime}-1}^{\prime} x_{1}^{\prime} \mathbf{s}_{r^{\prime}}^{\prime}\left(x_{1}^{\prime}\right)^{e^{\prime}}
$$

Then Lemma 20.8 parts (ii)-(iv) imply $\mathbf{s}_{1} x_{1}=\mathbf{s}_{1}^{\prime} x_{1}^{\prime}$, and parts (iii) and (iv) of the same lemma imply $\left\{\mathbf{s}_{2}, \ldots, \mathbf{s}_{r}\right\}=\left\{\mathbf{s}_{2}^{\prime}, \ldots, \mathbf{s}_{r^{\prime}}^{\prime}\right\}$. Since $\mathbf{x}_{1}$ and $\mathbf{x}_{1}^{\prime}$ are $x_{1}$-rigid blocks, it follows from (Ri2) that $\left(\mathbf{s}_{2}, \ldots, \mathbf{s}_{r}\right)=\left(\mathbf{s}_{2}^{\prime}, \ldots, \mathbf{s}_{r^{\prime}}^{\prime}\right)$. It is then easily shown by $(\mathrm{Ri} 3)-(\operatorname{Ri} 5)$ and Lemma $20.8(\mathrm{i})$ that $e=e^{\prime}$. Hence $\mathbf{x}_{1}=\mathbf{x}_{1}^{\prime}$. Now since $\mathbf{w}$ and $\mathbf{w}^{\prime}$ share the same set of non-simple letters, it follows from (III) that $x_{i}=x_{i}^{\prime}$ for all $i \in\{2, \ldots, m\}$. 
Now consider any $i \in\{2, \ldots, m\}$. Then $\mathbf{x}_{i}$ and $\mathbf{x}_{i}^{\prime}$ are of the form

$$
\mathbf{x}_{i}=\mathbf{s}_{1} x_{i} \mathbf{s}_{2} x_{i} \ldots \mathbf{s}_{r-1} x_{i} \mathbf{s}_{r} x_{i}^{e} \quad \text { and } \quad \mathbf{x}_{i}^{\prime}=\mathbf{s}_{1}^{\prime} x_{i} \mathbf{s}_{2}^{\prime} x_{i} \ldots \mathbf{s}_{r^{\prime}-1}^{\prime} x_{i} \mathbf{s}_{r^{\prime}}^{\prime} x_{i}^{e^{\prime}} .
$$

Lemma 20.8 parts (iii) and (iv) imply $\left\{\mathbf{s}_{1}, \ldots, \mathbf{s}_{r}\right\}=\left\{\mathbf{s}_{1}^{\prime}, \ldots, \mathbf{s}_{r^{\prime}}^{\prime}\right\}$. Since $\mathbf{x}_{i}$ and $\mathbf{x}_{i}^{\prime}$ are fully $x_{i}$-rigid, it follows from (Ri2) and (Ri6) that $\left(\mathbf{s}_{1}, \ldots, \mathbf{s}_{r}\right)=\left(\mathbf{s}_{1}^{\prime}, \ldots, \mathbf{s}_{r^{\prime}}^{\prime}\right)$. It is then easily shown by $(\operatorname{Ri} 3)-(\operatorname{Ri} 5)$ and Lemma 20.8(i) that $e=e^{\prime}$. Therefore $\mathbf{x}_{i}=\mathbf{x}_{i}^{\prime}$. Since $i \in\{2, \ldots, m\}$ is arbitrary, $\mathbf{x}_{1} \ldots \mathbf{x}_{m}=\mathbf{x}_{1}^{\prime} \ldots \mathbf{x}_{m}^{\prime}$. Finally, $\mathbf{z}=\mathbf{z}^{\prime}$ by Lemma 20.8(iv). Consequently, the identity $\mathbf{w} \approx \mathbf{w}^{\prime}$ is trivial and is implied by the identities (20.6).

$$
\text { 21. } \mathscr{D}_{6}, \mathscr{D}_{7}, \mathscr{D}_{8}, \mathscr{D}_{9}, \mathscr{E}_{2}, \mathscr{E}_{3}
$$

\begin{tabular}{|c|c|c|c|c|c|c|c|c|c|c|c|c|c|c|c|c|c|c|c|}
\hline$y_{6}$ & 1 & 2 & 3 & 4 & & & $\mathscr{D}_{7}$ & & & & & & & $\mathscr{D}_{8}$ & & 2 & & & \\
\hline 1 & 1 & 1 & 1 & 1 & 1 & 1 & 1 & 1 & 1 & 1 & 1 & 1 & & 1 & 1 & 1 & 1 & 1 & 1 \\
\hline 2 & 1 & 1 & 1 & 1 & 1 & 2 & 2 & 1 & 1 & 1 & 1 & 1 & 2 & 2 & 1 & 1 & 1 & 1 & 1 \\
\hline 3 & 1 & 1 & 1 & 1 & 4 & 1 & 3 & 1 & 1 & 1 & 1 & 4 & 1 & 3 & 1 & 1 & 1 & 1 & 42 \\
\hline 4 & 4 & 4 & 4 & 4 & 4 & 4 & 4 & 4 & 4 & 4 & 4 & 4 & 4 & 4 & & 4 & 4 & 4 & 4 \\
\hline 5 & 5 & 5 & 5 & 5 & 5 & 5 & 5 & 5 & 5 & 5 & 5 & 5 & 5 & 5 & 5 & 5 & 5 & & 5 \\
\hline 6 & 1 & 2 & 3 & 4 & 1 & 6 & 6 & 1 & 2 & 3 & 4 & 4 & 6 & 6 & 1 & 2 & 3 & 4 & 16 \\
\hline $\mathscr{D}_{9}$ & 1 & 2 & 3 & 4 & 5 & 6 & $\mathscr{E}_{2}$ & 1 & 2 & 3 & 4 & 5 & 6 & $\mathscr{E}_{3}$ & 1 & 2 & 3 & 4 & 56 \\
\hline 1 & 1 & 1 & 1 & 1 & 1 & 1 & 1 & 1 & 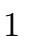 & 1 & 1 & 1 & & 1 & 1 & 1 & 1 & 1 & 1 \\
\hline 2 & 1 & 1 & 1 & 1 & 1 & 2 & 2 & 1 & 1 & 1 & 1 & 1 & 2 & 2 & 1 & 1 & 1 & 1 & 12 \\
\hline 3 & 1 & 1 & 1 & 1 & 4 & 2 & 3 & 3 & 3 & 3 & 3 & 3 & 3 & 3 & 3 & 3 & 3 & 3 & 3 \\
\hline 4 & 4 & 4 & 4 & 4 & 4 & 4 & 4 & 3 & 3 & 3 & 3 & 1 & 3 & 4 & 3 & 3 & 3 & 3 & 1 \\
\hline 5 & 5 & 5 & 5 & 5 & 5 & 5 & 5 & 5 & 5 & 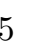 & 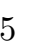 & 5 & 5 & 5 & 5 & 5 & 5 & 5 & 5 \\
\hline 6 & 1 & 2 & 3 & 4 & 4 & 6 & 6 & 1 & 2 & 3 & 4 & 1 & 6 & 6 & 1 & 2 & 3 & 4 & 3 \\
\hline
\end{tabular}

This section establishes the finite basis property of the following semigroups.

Proposition 21.1. The variety generated by any $S \in\left\{\mathscr{D}_{6}, \mathscr{D}_{7}, \mathscr{D}_{8}, \mathscr{D}_{9}, \mathscr{E}_{2}, \mathscr{E}_{3}\right\}$ is defined by the identities

$$
\begin{aligned}
x \mathrm{H} x^{2} & \approx x \mathrm{H} x, \\
x \mathrm{H} x \mathrm{~K} y^{2} x & \approx x \mathrm{H} x \mathrm{~K} y^{2}, \\
x \mathrm{H} y \mathrm{~K} x \mathrm{\top} y & \approx x \mathrm{H} y \mathrm{~K} y \mathrm{\top} x .
\end{aligned}
$$

The proof of Proposition 21.1 is given in $\S 21.3$.

21.1. Identities satisfied by $\mathscr{D}_{6}, \mathscr{D}_{7}, \mathscr{D}_{8}, \mathscr{D}_{9}, \mathscr{E}_{2}$, and $\mathscr{E}_{3}$

Lemma 21.2. The identities (21.1) imply the identity

$$
x \mathbf{a b h} x \approx x \mathbf{a} x h x \mathbf{b}
$$

for any $\mathbf{a}, \mathbf{b} \in \mathcal{X}^{+}$such that $\operatorname{con}(\mathbf{b}) \subseteq \operatorname{con}(x \mathbf{a})$.

Proof. This is proved by induction on $|\mathbf{b}|$. If $|\mathbf{b}|=1$, say $\mathbf{b}=y \in \operatorname{con}(x \mathbf{a})$, then

$$
x \mathbf{a b h} x=x \mathbf{a} y h x \stackrel{(21.1 \mathrm{a})}{\approx} x \mathbf{a} y h x x \stackrel{(21.1 \mathrm{c})}{\approx} x \mathbf{a} x h x y=x \mathbf{a} x h x \mathbf{b} .
$$


Assume that the identities (21.1) imply the identity (21.2) whenever $1 \leqslant|\mathbf{b}|<n$. If $|\mathbf{b}|=n$, say $\mathbf{b}=\mathbf{b}^{\prime} \mathbf{b}^{\prime \prime}$ for some $\mathbf{b}^{\prime}, \mathbf{b}^{\prime \prime} \in \mathcal{X}^{+}$with $\left|\mathbf{b}^{\prime}\right|+\left|\mathbf{b}^{\prime \prime}\right|=n$, then

$$
\begin{gathered}
x \mathbf{a b h x}=x \mathbf{a b}^{\prime} \mathbf{b}^{\prime \prime} h x \stackrel{(21.1)}{\approx} x \mathbf{a} \mathbf{b}^{\prime} x h x \mathbf{b}^{\prime \prime} \stackrel{(21.1)}{\approx} x \mathbf{a} x x h x \mathbf{b}^{\prime} \mathbf{b}^{\prime \prime} \\
\stackrel{(21.1 \mathrm{a})}{\approx} x \mathbf{a} x h x \mathbf{b}^{\prime} \mathbf{b}^{\prime \prime}=x \mathbf{a} x h x \mathbf{b},
\end{gathered}
$$

where the two deductions in (21.3) hold by induction hypothesis.

For any word $\mathbf{w}$, let $\mathrm{F}_{11}(\mathbf{w})$ denote the set of factors of $\mathbf{w}$ of length two that consist of two first occurrences in $\mathbf{w}$ :

$$
\mathrm{F}_{11}(\mathbf{w})=\left\{x y \in \mathcal{X}^{2} \mid \mathbf{w} \in(\mathcal{X} \backslash\{x, y\})^{*} x y \mathcal{X}^{*} \text { and } x \neq y\right\} .
$$

Lemma 21.3. Let $S \in\left\{\mathscr{D}_{6}, \mathscr{D}_{7}, \mathscr{D}_{8}, \mathscr{D}_{9}, \mathscr{E}_{2}, \mathscr{E}_{3}\right\}$. Suppose that $\mathbf{w} \approx \mathbf{w}^{\prime}$ is any identity satisfied by $S$. Then:

(i) $\operatorname{ini}(\mathbf{w})=\operatorname{ini}\left(\mathbf{w}^{\prime}\right)$;

(ii) $\operatorname{sim}(\mathbf{w})=\operatorname{sim}\left(\mathbf{w}^{\prime}\right)$;

(iii) $\mathrm{t}(\mathbf{w}) \in \operatorname{sim}(\mathbf{w})$ if and only if $\mathrm{t}\left(\mathbf{w}^{\prime}\right) \in \operatorname{sim}\left(\mathbf{w}^{\prime}\right)$;

(iv) $\mathrm{F}_{11}(\mathbf{w})=\mathrm{F}_{11}\left(\mathbf{w}^{\prime}\right)$.

Proof. (i) The subsemigroup $\{1,4,6\}$ of $S^{\prime} \in\left\{\mathscr{D}_{6}, \mathscr{D}_{7}, \mathscr{D}_{8}, \mathscr{D}_{9}\right\}$ and the subsemigroup $\{1,3,6\}$ of $S^{\prime \prime} \in\left\{\mathscr{E}_{2}, \mathscr{E}_{3}\right\}$ are isomorphic to $L_{2}^{1}$. Therefore the result holds by Lemma 2.1(iii).

(ii) This holds by Lemma 2.1(v) since the subsemigroup $\{1,2,6\}$ of $S$ is isomorphic to $N_{2}^{1}$.

(iii) This follows from Lemma 2.2 since the subsemigroup $\{1,3,6\}$ of $S^{\prime} \in\left\{\mathscr{D}_{6}, \mathscr{D}_{7}\right\}$, the subsemigroup $\{3,4,6\}$ of $S^{\prime \prime} \in\left\{\mathscr{E}_{2}, \mathscr{E}_{3}\right\}$, and the divisor $\{1,2,3,6\} /\{1,2\}$ of $S^{\prime \prime \prime} \in\left\{\mathscr{D}_{8}, \mathscr{D}_{9}\right\}$ are isomorphic to $J$.

(iv) Suppose $\mathrm{F}_{11}(\mathbf{w}) \neq \mathrm{F}_{11}\left(\mathbf{w}^{\prime}\right)$, say with $x y \in \mathrm{F}_{11}(\mathbf{w}) \backslash \mathrm{F}_{11}\left(\mathbf{w}^{\prime}\right)$. Then $\mathbf{w}=\mathbf{a} x y \mathbf{b}$ for some $\mathbf{a}, \mathbf{b} \in \mathcal{X}^{*}$ such that $x, y \notin \operatorname{con}(\mathbf{a})$. Since $\operatorname{ini}\left(\mathbf{w}^{\prime}\right)=\operatorname{ini}(\mathbf{w})$ by part (i) and $\operatorname{ini}(\mathbf{w})=\ldots x y \ldots$, it follows that $\mathbf{w}^{\prime}=\mathbf{c} x \mathbf{d} y \mathbf{e}$ for some $\mathbf{c}, \mathbf{e} \in \mathcal{X}^{*}$ and $\mathbf{d} \in \mathcal{X}^{+}$such that $x \notin \operatorname{con}(\mathbf{c})$ and $y \notin \operatorname{con}(\mathbf{c d})$. Let $\varphi_{1}$ and $\varphi_{2}$ denote the following substitutions into $S^{\prime} \in\left\{\mathscr{D}_{6}, \mathscr{D}_{7}, \mathscr{D}_{8}, \mathscr{D}_{9}\right\}$ and $S^{\prime \prime} \in\left\{\mathscr{E}_{2}, \mathscr{E}_{3}\right\}$, respectively:

$$
z \mapsto\left\{\begin{array} { l l } 
{ 3 } & { \text { if } z = x , } \\
{ 5 } & { \text { if } z = y , } \\
{ 6 } & { \text { otherwise; } }
\end{array} \quad z \mapsto \left\{\begin{array}{ll}
4 & \text { if } z=x, \\
5 & \text { if } z=y, \\
6 & \text { otherwise. }
\end{array}\right.\right.
$$

Then the following contradictions are obtained:

$$
\begin{aligned}
& \mathbf{w} \varphi_{1} \in\{6\}^{*} \cdot 3 \cdot 5 \cdot \mathbf{b} \varphi_{1}=\{4\} \quad \text { and } \quad \mathbf{w}^{\prime} \varphi_{1} \in\{6\}^{*} \cdot 3 \cdot\{3,6\}^{+} \cdot 5 \cdot \mathbf{e} \varphi_{1}=\{1\} \\
& \mathbf{w} \varphi_{2} \in\{6\}^{*} \cdot 4 \cdot 5 \cdot \mathbf{b} \varphi_{2}=\{1\} \quad \text { and } \quad \mathbf{w}^{\prime} \varphi_{2} \in\{6\}^{*} \cdot 4 \cdot\{4,6\}^{+} \cdot 5 \cdot \mathbf{e} \varphi_{2}=\{3\}
\end{aligned}
$$

Hence $\mathrm{F}_{11}(\mathbf{w})=\mathrm{F}_{11}\left(\mathbf{w}^{\prime}\right)$.

\subsection{A canonical form}

Any non-simple word $\mathbf{w}$ can be written in the form $\mathbf{w}=\mathbf{p u q}$, where $\mathbf{p}, \mathbf{q} \in \mathcal{X}^{*}$ and $\mathbf{u} \in \mathcal{X}^{+}$ are such that $\operatorname{con}(\mathbf{p q}) \subseteq \operatorname{sim}(\mathbf{w})$ and $\mathrm{h}(\mathbf{u}), \mathrm{t}(\mathbf{u}) \notin \operatorname{sim}(\mathbf{w})$. Generality is not lost by assuming $\operatorname{ini}(\mathbf{u})=x_{1} \ldots x_{m}$ for some $m \geqslant 1$, so that ini $(\mathbf{w})=\mathbf{p} x_{1} \ldots x_{m} \mathbf{q}$. In this section, such a word $\mathbf{w}$ is said to be in canonical form if

$$
\mathbf{w}=\mathbf{p}\left(\prod_{i=1}^{m}\left(x_{i} \mathbf{x}_{i}\right)\right) \mathbf{q}
$$


where all of the following are satisfied:

(I) $\mathbf{x}_{1}, \ldots, \mathbf{x}_{m-1} \in\left\{\emptyset, x_{1}\right\}$;

(II) $\mathbf{x}_{m}=x_{1}^{e_{1}} \ldots x_{m}^{e_{m}}$ for some $e_{1}, \ldots, e_{m} \in\{0,1\}$ with $\left(e_{1}, \ldots, e_{m}\right) \neq(0, \ldots, 0)$;

(III) if $\left(\mathbf{x}_{1}, \ldots, \mathbf{x}_{m-1}\right) \neq(\emptyset, \ldots, \emptyset)$ and $\left(e_{2}, \ldots, e_{m}\right) \neq(0, \ldots, 0)$, then $e_{1}=0$.

REMARK 21.4. Consider the word $\mathbf{w}$ in (21.4).

(i) If $m=1$, then $\mathbf{w}=\mathbf{p} x_{1} \mathbf{x}_{1} \mathbf{q}=\mathbf{p} x_{1}^{2} \mathbf{q}$ by (II).

(ii) The assumption $x_{1}=\mathrm{h}(\mathbf{u}) \notin \operatorname{sim}(\mathbf{w})$ implies

$$
2 \leqslant \operatorname{occ}\left(x_{1}, \mathbf{w}\right)=1+\left|\mathbf{x}_{1} \ldots \mathbf{x}_{m-1}\right|+e_{1} .
$$

Therefore if $\left(\mathbf{x}_{1}, \ldots, \mathbf{x}_{m-1}\right)=(\emptyset, \ldots, \emptyset)$, then $e_{1}=1$.

Lemma 21.5. Let $\mathbf{w}$ be any non-simple word. Then there exists some word $\overline{\mathbf{w}}$ in canonical form such that the identities (21.1) imply the identity $\mathbf{w} \approx \overline{\mathbf{w}}$.

Proof. By Lemma 21.2, it suffices to convert w, using the identities $\{(21.1),(21.2)\}$, into a word in canonical form. As observed in the beginning of this subsection, the word $\mathbf{w}$ can be written as $\mathbf{w}=$ puq where $\mathbf{p}, \mathbf{q} \in \mathcal{X}^{*}$ and $\mathbf{u} \in \mathcal{X}^{+}$are such that $\operatorname{con}(\mathbf{p q}) \subseteq \operatorname{sim}(\mathbf{w})$ and $\mathrm{h}(\mathbf{u}), \mathrm{t}(\mathbf{u}) \notin \operatorname{sim}(\mathbf{w})$. Let $\operatorname{ini}(\mathbf{u})=x_{1} \ldots x_{m}$. Then $\mathbf{u}$ can be written as $\mathbf{u}=\prod_{i=1}^{m}\left(x_{i} \mathbf{w}_{i}\right)$, where $\mathbf{w}_{i} \in\left\{x_{1}, \ldots, x_{i}\right\}^{*}$ and $\mathbf{w}_{m} \neq \emptyset$. The letter $t=\mathrm{t}\left(\mathbf{w}_{m}\right)$ and any other letter in $\mathbf{w}_{m}$ are clearly non-simple in $\mathbf{w}$. By assumption, the letter $x_{1}=\mathrm{h}(\mathbf{u})$ is also non-simple in $\mathbf{w}$. Therefore

$$
\begin{aligned}
\mathbf{w} & \stackrel{(21.1 \mathrm{a})}{\approx} \mathbf{p}\left(\prod_{i=1}^{m-1}\left(x_{i} \mathbf{w}_{i}\right)\right) x_{m} \mathbf{w}_{m} t^{2} \mathbf{q} \stackrel{(21.1 \mathrm{~b})}{\approx} \mathbf{p}\left(\prod_{i=1}^{m-1}\left(x_{i} \mathbf{w}_{i}\right)\right) x_{m} \mathbf{w}_{m} t^{2} x_{1} \mathbf{q} \\
\stackrel{(21.1 \mathrm{a})}{\approx} & \mathbf{p}\left(\prod_{i=1}^{m-1}\left(x_{i} \mathbf{w}_{i}\right)\right) x_{m} \mathbf{w}_{m} x_{1} \mathbf{q} \stackrel{(21.1 \mathrm{c})}{\approx} \mathbf{p}\left(\prod_{i=1}^{m-1}\left(x_{i} \mathbf{w}_{i}\right)\right) x_{m}\left(x_{1} \mathbf{w}_{m}\right) \mathbf{q} .
\end{aligned}
$$

Hence generality is not lost by assuming $\mathrm{h}\left(\mathbf{w}_{m}\right)=x_{1}$ to begin with, so that $\mathbf{w}_{m}=x_{1} \mathbf{w}_{m}^{\prime}$ for some $\mathbf{w}_{m}^{\prime} \in\left\{x_{1}, \ldots, x_{m}\right\}^{*}$.

If $\mathbf{w}_{1} \neq \emptyset$, then since $\mathbf{w}_{1} \in\left\{x_{1}\right\}^{*}$, the identity $x^{3} \approx x^{2}$ from (21.1a) can be used to reduce the factor $x_{1} \mathbf{w}_{1}$ of $\mathbf{w}$ to $x_{1} x_{1}$. In other words, the word $\mathbf{w}_{1}$ can be converted into $x_{1}=\mathbf{x}_{1}$. Now suppose $\mathbf{w}_{k} \neq \emptyset$ for some $k \in\{2, \ldots, m-1\}$. Since $\operatorname{con}\left(\mathbf{w}_{k}\right) \subseteq \operatorname{con}\left(\left(\prod_{i=1}^{k-1}\left(x_{i} \mathbf{w}_{i}\right)\right) x_{k}\right)$ and $\mathrm{h}\left(\mathbf{w}_{m}\right)=x_{1}$, it follows from Lemma 21.2 that

$$
\begin{array}{r}
\mathbf{w}=\mathbf{p} x_{1} \mathbf{w}_{1}\left(\prod_{i=2}^{k-1}\left(x_{i} \mathbf{w}_{i}\right)\right) x_{k} \mathbf{w}_{k}\left(\prod_{i=k+1}^{m-1}\left(x_{i} \mathbf{w}_{i}\right)\right) x_{m} \overbrace{x_{1} \mathbf{w}_{m}^{\prime}}^{\mathbf{w}_{m}} \mathbf{q} \\
\stackrel{(21.2)}{\approx} \mathbf{p} x_{1} \mathbf{w}_{1}\left(\prod_{i=2}^{k-1}\left(x_{i} \mathbf{w}_{i}\right)\right) x_{k} x_{1}\left(\prod_{i=k+1}^{m-1}\left(x_{i} \mathbf{w}_{i}\right)\right) x_{m} x_{1} \mathbf{w}_{k} \mathbf{w}_{m}^{\prime} \mathbf{q},
\end{array}
$$

that is, the identities (21.2) can be used to simultaneously replace the factor $\mathbf{w}_{k}$ by $x_{1}=\mathbf{x}_{i}$ and insert a copy of $\mathbf{w}_{k}$ between the $x_{1}$ and $\mathbf{w}_{m}^{\prime}$ in $\mathbf{w}_{m}$. It is easily seen how this can be repeated until $\mathbf{w}$ is converted into the word

$$
\mathbf{w}=\mathbf{p}\left(\prod_{i=1}^{m}\left(x_{i} \mathbf{x}_{i}\right)\right) x_{m} \underbrace{x_{1} \mathbf{w}_{2} \ldots \mathbf{w}_{m-1} \mathbf{w}_{m}^{\prime}}_{\mathbf{w}_{m}^{\prime \prime}} \mathbf{q}
$$

where $\mathbf{x}_{i} \in\left\{\emptyset, x_{1}\right\}$. Since the letters of $\mathbf{w}_{m}^{\prime \prime}$ are non-first occurrences in $\mathbf{w}$, the identities (21.1c) can be used to rearrange them into $\mathbf{x}_{m}=x_{1}^{e_{1}} \ldots x_{m}^{e_{m}}$, where $e_{1}, \ldots, e_{m} \geqslant 0$ are such that 
$\left(e_{1}, \ldots, e_{m}\right) \neq(0, \ldots, 0)$. The identities $(21.1 \mathrm{a})$ can then be applied to reduce any nonzero exponent $e_{i}$ to 1 . Hence (I) and (II) are satisfied. Since $x_{1}$ is a letter of $\mathbf{w}_{m}^{\prime \prime}$, it is also a letter of $\mathbf{x}_{m}$. Therefore $e_{1}=1$.

Suppose $\mathbf{x}_{k} \neq \emptyset$ for some $k<m$ and $e_{\ell} \neq 0$ for some $\ell>1$. Then $\mathbf{x}_{k}=x_{1}$ and $\mathbf{x}_{m}=x_{1} \mathbf{a} x_{\ell} \mathbf{b}$, where $\mathbf{a}=\prod_{i=2}^{\ell-1} x_{i}^{e_{i}}$ and $\mathbf{b}=\prod_{i=\ell+1}^{m} x_{i}^{e_{i}}$. Since the letters of $\mathbf{x}_{m}$ are non-first occurrences in $\mathbf{w}$,

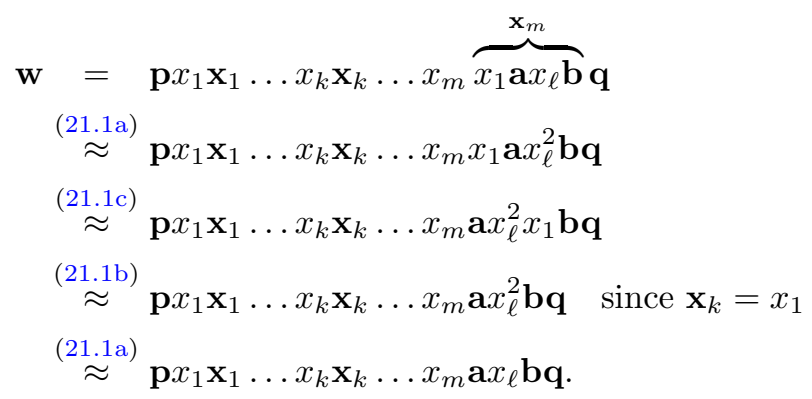

The first letter $x_{1}$ of $\mathbf{w}_{m}$ is thus eliminated, resulting in $e_{1}=0$. Hence (III) is satisfied.

\subsection{Proof of Proposition 21.1}

Let $S \in\left\{\mathscr{D}_{6}, \mathscr{D}_{7}, \mathscr{D}_{8}, \mathscr{D}_{9}, \mathscr{E}_{2}, \mathscr{E}_{3}\right\}$. It is routinely checked that $S$ satisfies the identities (21.1). Hence it suffices to show that any identity $\mathbf{w} \approx \mathbf{w}^{\prime}$ satisfied by $S$ is implied by the identities (21.1). If either $\mathbf{w}$ or $\mathbf{w}^{\prime}$ is a simple word, then it follows from Lemma 21.3 parts (i) and (ii) that the identity $\mathbf{w} \approx \mathbf{w}^{\prime}$ is trivial and so is vacuously implied by the identities (21.1). Therefore assume that $\mathbf{w}$ and $\mathbf{w}^{\prime}$ are both non-simple words and so by Lemma 21.5, can be chosen to be in canonical form. Hence

$$
\mathbf{w}=\mathbf{p}\left(\prod_{i=1}^{m}\left(x_{i} \mathbf{x}_{i}\right)\right) \mathbf{q} \quad \text { and } \quad \mathbf{w}^{\prime}=\mathbf{p}^{\prime}\left(\prod_{i=1}^{m^{\prime}}\left(x_{i}^{\prime} \mathbf{x}_{i}^{\prime}\right)\right) \mathbf{q}^{\prime} .
$$

Then by Lemma 21.3(i),

(a) $\mathbf{p} x_{1} \ldots x_{m} \mathbf{q}=\operatorname{ini}(\mathbf{w})=\operatorname{ini}\left(\mathbf{w}^{\prime}\right)=\mathbf{p}^{\prime} x_{1}^{\prime} \ldots x_{m^{\prime}}^{\prime} \mathbf{q}^{\prime}$.

Since $x_{1} \notin \operatorname{sim}(\mathbf{w})$ by assumption and $\operatorname{sim}(\mathbf{w})=\operatorname{sim}\left(\mathbf{w}^{\prime}\right)$ by Lemma 21.3(ii), the letter $x_{1}$ is non-simple in $\mathbf{w}^{\prime}$, so that $x_{1} \notin \operatorname{con}\left(\mathbf{p}^{\prime}\right)$. By a symmetrical argument, $x_{1}^{\prime} \notin \operatorname{sim}(\mathbf{p})$. It then follows from (a) that

(b) $\mathbf{p}=\mathbf{p}^{\prime}$.

If either $\mathbf{q}$ or $\mathbf{q}^{\prime}$ is empty, then $\mathbf{q}=\mathbf{q}^{\prime}=\emptyset$ by Lemma 21.3(iii). Therefore suppose that $\mathbf{q}$ and $\mathbf{q}^{\prime}$ are nonempty. Seeking a contradiction, suppose $\mathbf{q} \neq \mathbf{q}^{\prime}$. Then by (a), either $\mathbf{q}$ is a proper suffix of $\mathbf{q}^{\prime}$ or $\mathbf{q}^{\prime}$ is a proper suffix of $\mathbf{q}$. By symmetry, it suffices to assume that $\mathbf{q}$ is a proper suffix of $\mathbf{q}^{\prime}$. It then follows from (a) that $x_{m} \mathbf{q}$ is a suffix of $\mathbf{q}^{\prime}$, whence $x_{m} \in \operatorname{sim}\left(\mathbf{w}^{\prime}\right)$ and $x_{m} \mathbf{h}(\mathbf{q}) \in \mathrm{F}_{11}\left(\mathbf{q}^{\prime}\right)$. However, $\mathbf{x}_{m} \neq \emptyset$ by (II) so that $x_{m} \mathbf{h}(\mathbf{q}) \notin \mathrm{F}_{11}(\mathbf{q})$, and this contradicts Lemma 21.3(iv). Therefore

(c) $\mathbf{q}=\mathbf{q}^{\prime}$.

It now follows from (a)-(c) that $m=m^{\prime}$ and $x_{i}=x_{i}^{\prime}$ for all $i$. If $\mathbf{x}_{i} \neq \mathbf{x}_{i}^{\prime}$ for some $i<m$, say $\mathbf{x}_{i}=\emptyset$ and $\mathbf{x}_{i}^{\prime}=x_{1}$, then $x_{i} x_{i+1} \in \mathrm{F}_{11}(\mathbf{w}) \backslash \mathrm{F}_{11}\left(\mathbf{w}^{\prime}\right)$, contradicting Lemma 21.3(iv). Therefore $\mathbf{x}_{i}=\mathbf{x}_{i}^{\prime}$ for all $i<m$, whence

$$
\mathbf{w}=\mathbf{p} \mathbf{z} x_{m} \underbrace{x_{1}^{e_{1}} x_{2}^{e_{2}} \ldots x_{m}^{e_{m}}}_{\mathbf{x}_{m}} \mathbf{q} \text { and } \mathbf{w}^{\prime}=\mathbf{p z} x_{m} \underbrace{x_{1}^{e_{1}^{\prime}} x_{2}^{e_{2}^{\prime}} \ldots x_{m}^{e_{m}^{\prime}}}_{\mathbf{x}_{m}^{\prime}} \mathbf{q}
$$

with $\mathbf{z}=\prod_{i=1}^{m-1}\left(x_{i} \mathbf{x}_{i}\right)$. Now (I) and (II) $\operatorname{imply} \operatorname{occ}\left(x_{i}, \mathbf{w}\right)=1+e_{i}$ and $\operatorname{occ}\left(x_{i}, \mathbf{w}^{\prime}\right)=1+e_{i}^{\prime}$ for each $i>1$. Hence $\left(e_{2}, \ldots, e_{m}\right)=\left(e_{2}^{\prime}, \ldots, e_{m}^{\prime}\right)$ by Lemma 21.3(ii). In each of the following two 
cases, it is shown that $e_{1}=e_{1}^{\prime}$. Consequently, the identity $\mathbf{w} \approx \mathbf{w}^{\prime}$ is trivial and is implied by the identities (21.1).

Case 1: $\left(e_{2}, \ldots, e_{m}\right)=(0, \ldots, 0)$. Then $e_{1}=1=e_{1}^{\prime}$ by $(\mathrm{II})$.

Case $2:\left(e_{2}, \ldots, e_{m}\right) \neq(0, \ldots, 0)$. If $\left(\mathbf{x}_{1}, \ldots, \mathbf{x}_{m-1}\right) \neq(\emptyset, \ldots, \emptyset)$, then $e_{1}=0=e_{1}^{\prime}$ by (III). If $\left(\mathbf{x}_{1}, \ldots, \mathbf{x}_{m-1}\right)=(\emptyset, \ldots, \emptyset)$, then $e_{1}=1=e_{1}^{\prime}$ by Remark 21.4(ii).

\section{2. $\mathscr{E}_{5}, \mathscr{E}_{6}, \mathscr{E}_{7}$}

This section establishes the finite basis property of the following semigroups.

\begin{tabular}{c|cccccc}
$\mathscr{E}_{5}$ & 1 & 2 & 3 & 4 & 5 & 6 \\
\hline 1 & 1 & 1 & 1 & 1 & 1 & 1 \\
2 & 1 & 1 & 1 & 1 & 1 & 3 \\
3 & 1 & 1 & 1 & 1 & 3 & 3 \\
4 & 1 & 2 & 3 & 4 & 4 & 4 \\
5 & 1 & 2 & 3 & 4 & 5 & 5 \\
6 & 1 & 2 & 3 & 4 & 6 & 6
\end{tabular}

\begin{tabular}{c|cccccc}
$\mathscr{E}_{6}$ & 1 & 2 & 3 & 4 & 5 & 6 \\
\hline 1 & 1 & 1 & 1 & 1 & 1 & 1 \\
2 & 1 & 1 & 1 & 1 & 1 & 3 \\
3 & 1 & 1 & 1 & 1 & 3 & 3 \\
4 & 4 & 4 & 4 & 4 & 4 & 4 \\
5 & 1 & 2 & 3 & 4 & 5 & 5 \\
6 & 1 & 2 & 3 & 4 & 6 & 6
\end{tabular}

\begin{tabular}{c|cccccc}
$\mathscr{E}_{7}$ & 1 & 2 & 3 & 4 & 5 & 6 \\
\hline 1 & 1 & 1 & 1 & 1 & 1 & 1 \\
2 & 1 & 1 & 1 & 1 & 1 & 3 \\
3 & 3 & 3 & 3 & 3 & 3 & 3 \\
4 & 1 & 2 & 3 & 4 & 5 & 1 \\
5 & 1 & 2 & 3 & 4 & 5 & 3 \\
6 & 6 & 6 & 6 & 6 & 6 & 6
\end{tabular}

22.1. Finite basis property of $\mathscr{E}_{5}$

Proposition 22.1. The variety generated by $\mathscr{E}_{5}$ is defined by the identities

$$
\begin{aligned}
x^{3} \approx x^{2}, \quad x^{2} y x & \approx x y x, \quad x y x^{2} \approx x y x, \\
h^{2} x y x & \approx h^{2} y x^{2}, \\
h^{2} x^{2} y^{2} & \approx h^{2} y^{2} x^{2} .
\end{aligned}
$$

For any word $\mathbf{w}$, let $\mathrm{F}_{\mathrm{SN}}(\mathbf{w})$ denote the set of factors of $\mathbf{w}$ of length two that begin with a simple letter and end with a non-simple letter:

$$
\mathrm{F}_{\mathrm{SN}}(\mathbf{w})=\left\{x y \in \mathcal{X}^{2} \mid \mathbf{w} \in \mathcal{X}^{*} x y \mathcal{X}^{*}, x \in \operatorname{sim}(\mathbf{w}), y \notin \operatorname{sim}(\mathbf{w})\right\} .
$$

Lemma 22.2. Suppose that $\mathbf{w} \approx \mathbf{w}^{\prime}$ is any identity satisfied by $\mathscr{E}_{5}$. Then:

(i) $\mathrm{h}(\mathbf{w})=\mathrm{h}\left(\mathbf{w}^{\prime}\right)$;

(ii) $\operatorname{con}(\mathbf{w})=\operatorname{con}\left(\mathbf{w}^{\prime}\right)$ and $\operatorname{sim}(\mathbf{w})=\operatorname{sim}\left(\mathbf{w}^{\prime}\right)$;

(iii) for any letters $x \in \operatorname{con}(\mathbf{w})=\operatorname{con}\left(\mathbf{w}^{\prime}\right)$ and $y \in \operatorname{sim}(\mathbf{w})=\operatorname{sim}\left(\mathbf{w}^{\prime}\right)$, the conditions $x \prec_{\mathbf{w}} y$ and $x \prec_{\mathbf{w}^{\prime}} y$ are equivalent;

(iv) $\mathbf{w}_{\text {sim }}=\mathbf{w}_{\text {sim }}^{\prime}$;

(v) $\mathrm{F}_{\mathrm{SN}}(\mathbf{w})=\mathrm{F}_{\mathrm{SN}}\left(\mathbf{w}^{\prime}\right)$.

Proof. Parts (i)-(iv) follow from Lemmas 2.1(i) and 2.3 since the subsemigroups $\{5,6\}$ and $\{1,3,4,5\}$ of $\mathscr{E}_{5}$ are isomorphic to $L_{2}$ and $J^{1}$, respectively.

(v) Suppose $\mathrm{F}_{\mathrm{SN}}(\mathbf{w}) \neq \mathrm{F}_{\mathrm{SN}}\left(\mathbf{w}^{\prime}\right)$, say $x y \in \mathrm{F}_{\mathrm{SN}}(\mathbf{w}) \backslash \mathrm{F}_{\mathrm{SN}}\left(\mathbf{w}^{\prime}\right)$. Then $x \in \operatorname{sim}(\mathbf{w})=\operatorname{sim}\left(\mathbf{w}^{\prime}\right)$ by part (ii). Therefore $\mathbf{w}=\mathbf{a} x y \mathbf{b}$ and $\mathbf{w}^{\prime}=\mathbf{a}^{\prime} x \mathbf{b}^{\prime}$ for some $\mathbf{a}, \mathbf{b}, \mathbf{a}^{\prime}, \mathbf{b}^{\prime} \in \mathcal{X}^{*}$ such that $\mathbf{h}\left(\mathbf{b}^{\prime}\right) \neq y$ and $x \notin \operatorname{con}\left(\mathbf{a b a}^{\prime} \mathbf{b}^{\prime}\right)$. Let $\varphi: \mathcal{X} \rightarrow \mathscr{E}_{5}$ denote the substitution

$$
z \mapsto \begin{cases}2 & \text { if } z=x, \\ 6 & \text { if } z=y, \\ 5 & \text { otherwise. }\end{cases}
$$


Then $5 \cdot \mathbf{a} \varphi=5 \cdot \mathbf{a}^{\prime} \varphi=\mathbf{b}^{\prime} \varphi \cdot 5=5$ and $6 \cdot \mathbf{b} \varphi \cdot 5=6$, so that

$$
5 \cdot \mathbf{w} \varphi \cdot 5=(5 \cdot \mathbf{a} \varphi) \cdot 2 \cdot(6 \cdot \mathbf{b} \varphi \cdot 5)=5 \cdot 2 \cdot 6=3
$$

and

$$
5 \cdot \mathbf{w}^{\prime} \varphi \cdot 5=\left(5 \cdot \mathbf{a}^{\prime} \varphi\right) \cdot 2 \cdot\left(\mathbf{b}^{\prime} \varphi \cdot 5\right)=5 \cdot 2 \cdot 5=1,
$$

which is impossible.

In this subsection, a non-simple word $\mathbf{w}$ is said to be in canonical form if

$$
\mathbf{w}=\prod_{i=1}^{m}\left(\mathbf{s}_{i} \mathbf{w}_{i}\right)
$$

where all of the following are satisfied:

(I) the letters of $\mathbf{s}_{1} \in \mathcal{X}^{*}$ and $\mathbf{s}_{2}, \ldots, \mathbf{s}_{m} \in \mathcal{X}^{+}$are simple in $\mathbf{w}$;

(II) the letters of $\mathbf{w}_{1}, \ldots, \mathbf{w}_{m-1} \in \mathcal{X}^{+}$and $\mathbf{w}_{m} \in \mathcal{X}^{*}$ are non-simple in $\mathbf{w}$;

(III) $\operatorname{con}\left(\mathbf{w}_{1}\right) \supseteq \ldots \supseteq \operatorname{con}\left(\mathbf{w}_{m}\right)$.

Lemma 22.3. Let $\mathbf{w}$ be any non-simple word. Then there exists some word $\overline{\mathbf{w}}$ in canonical form such that the identities (22.1) imply the identity $\mathbf{w} \approx \overline{\mathbf{w}}$.

Proof. It suffices to convert $\mathbf{w}$, using the identities (22.1), into a word in canonical form. It is easily seen that $\mathbf{w}$ can be written as a word of the form (22.2) with (I) and (II) satisfied. If $x \in \operatorname{con}\left(\mathbf{w}_{i+1}\right) \backslash \operatorname{con}\left(\mathbf{w}_{i}\right)$, say $\mathbf{w}_{i+1}=\mathbf{a} x \mathbf{b}$ for some $\mathbf{a}, \mathbf{b} \in \mathcal{X}^{*}$, then the identities (22.1) can be used to convert $\mathbf{w}_{i}$ into $\mathbf{w}_{i} x$ :

$$
\begin{aligned}
& \mathbf{w}=\ldots \mathbf{s}_{i} \mathbf{w}_{i} \cdot \mathbf{s}_{i+1} \mathbf{w}_{i+1} \ldots \stackrel{(22.1 \mathrm{a})}{\approx} \ldots \mathbf{s}_{i} \mathbf{w}_{i}\left(\mathrm{t}\left(\mathbf{w}_{i}\right)\right)^{2} \cdot \mathbf{s}_{i+1} \mathbf{a} x^{2} \mathbf{b} \ldots \\
& \stackrel{(22.1 \mathrm{~b})}{\approx} \ldots \mathbf{s}_{i} \mathbf{w}_{i}\left(\mathrm{t}\left(\mathbf{w}_{i}\right)\right)^{2} x \cdot \mathbf{s}_{i+1} \mathbf{a} x \mathbf{b} \ldots \stackrel{(22.1 \mathrm{a})}{\approx} \ldots \mathbf{s}_{i}\left(\mathbf{w}_{i} x\right) \cdot \mathbf{s}_{i+1} \mathbf{w}_{i+1} \ldots
\end{aligned}
$$

Hence (III) is satisfied by applying the identities (22.1).

Lemma 22.4. Suppose that $\mathscr{E}_{5}$ satisfies an identity $\mathbf{w} \approx \mathbf{w}^{\prime}$, where

$$
\mathbf{w}=\prod_{i=1}^{m}\left(\mathbf{s}_{i} \mathbf{w}_{i}\right) \quad \text { and } \quad \mathbf{w}^{\prime}=\prod_{i=1}^{m}\left(\mathbf{s}_{i} \mathbf{w}_{i}^{\prime}\right)
$$

are in canonical form. Then $\operatorname{con}\left(\mathbf{w}_{i}\right)=\operatorname{con}\left(\mathbf{w}_{i}^{\prime}\right)$ for all $i$.

Proof. First note that $\bigcup_{i=1}^{m} \operatorname{con}\left(\mathbf{w}_{i}\right)=\bigcup_{i=1}^{m} \operatorname{con}\left(\mathbf{w}_{i}^{\prime}\right)$ holds by Lemma 22.2(ii). Suppose $\operatorname{con}\left(\mathbf{w}_{1}\right) \neq \operatorname{con}\left(\mathbf{w}_{1}^{\prime}\right)$, say $x \in \operatorname{con}\left(\mathbf{w}_{1}\right) \backslash \operatorname{con}\left(\mathbf{w}_{1}^{\prime}\right)$. Then by (II), the letter $x$ is non-simple in $\mathbf{w}$ and so by Lemma 22.2(ii), it is also non-simple in $\mathbf{w}^{\prime}$. By (II), there exists some $i>1$ such that $x \in \operatorname{con}\left(\mathbf{w}_{i}^{\prime}\right)$. But then (III) implies $x \in \operatorname{con}\left(\mathbf{w}_{1}^{\prime}\right)$, contradicting the assumption. Therefore $\operatorname{con}\left(\mathbf{w}_{1}\right)=\operatorname{con}\left(\mathbf{w}_{1}^{\prime}\right)$.

Now suppose $\operatorname{con}\left(\mathbf{w}_{k}\right) \neq \operatorname{con}\left(\mathbf{w}_{k}^{\prime}\right)$ for some $k>1$, say $x \in \operatorname{con}\left(\mathbf{w}_{k}\right) \backslash \operatorname{con}\left(\mathbf{w}_{k}^{\prime}\right)$. Then (III) implies $x \in \operatorname{con}\left(\mathbf{w}_{1} \ldots \mathbf{w}_{k}\right)$ and $x \notin \operatorname{con}\left(\mathbf{w}_{k}^{\prime} \ldots \mathbf{w}_{m}^{\prime}\right)$, whence $x \nprec_{\mathbf{w}} \mathrm{h}\left(\mathbf{s}_{k}\right)$ and $x \prec_{\mathbf{w}^{\prime}} \mathrm{h}\left(\mathbf{s}_{k}\right)$. But this violates Lemma 22.2(iii).

Proof of Proposition 22.1. It is routinely checked that $\mathscr{E}_{5}$ satisfies the identities (22.1). Hence it suffices to show that any identity $\mathbf{w} \approx \mathbf{w}^{\prime}$ satisfied by $\mathscr{E}_{5}$ is implied by the identities $(22.1)$. If either $\mathbf{w}$ or $\mathbf{w}^{\prime}$ is a simple word, then it follows from Lemma 22.2 that the identity $\mathbf{w} \approx \mathbf{w}^{\prime}$ 
is trivial and so is vacuously implied by (22.1). Therefore assume that $\mathbf{w}$ and $\mathbf{w}^{\prime}$ are both non-simple words and so by Lemma 22.3, can be chosen to be in canonical form. Hence

$$
\mathbf{w}=\prod_{i=1}^{m}\left(\mathbf{s}_{i} \mathbf{w}_{i}\right) \quad \text { and } \quad \mathbf{w}^{\prime}=\prod_{i=1}^{m^{\prime}}\left(\mathbf{s}_{i}^{\prime} \mathbf{w}_{i}^{\prime}\right) .
$$

Since $\mathbf{s}_{1} \ldots \mathbf{s}_{m}=\mathbf{w}_{\text {sim }}=\mathbf{w}_{\text {sim }}^{\prime}=\mathbf{s}_{1}^{\prime} \ldots \mathbf{s}_{m^{\prime}}^{\prime}$ by Lemma 22.2 part (iv), it follows from parts (i) and (v) of the same lemma that $m=m^{\prime}, \mathbf{s}_{i}=\mathbf{s}_{i}^{\prime}$, and $\mathrm{h}\left(\mathbf{w}_{i}\right)=\mathrm{h}\left(\mathbf{w}_{i}^{\prime}\right)$ for all $i$. Therefore $\mathbf{w}^{\prime}=\prod_{i=1}^{m}\left(\mathbf{s}_{i} \mathbf{w}_{i}^{\prime}\right)$. Now Lemma 22.4 implies $\operatorname{con}\left(\mathbf{w}_{i}\right)=\operatorname{con}\left(\mathbf{w}_{i}^{\prime}\right)$ for all $i$. Hence it can be assumed that $h_{i}=\mathrm{h}\left(\mathbf{w}_{i}\right)=\mathrm{h}\left(\mathbf{w}_{i}^{\prime}\right)$ and $\operatorname{con}\left(\mathbf{w}_{i}\right)=\operatorname{con}\left(\mathbf{w}_{i}^{\prime}\right)=\left\{h_{i}, x_{i, 1}, \ldots, x_{i, r_{i}}\right\}$. Let $\varphi$ denote the substitution $x \mapsto x^{2}$ for all $x \in \mathcal{X}$. Then it follows from (II) that

$$
\begin{aligned}
& \mathbf{w} \stackrel{(22.1 \mathrm{a})}{\approx} \prod_{i=1}^{m}\left(\mathbf{s}_{i}\left(\mathbf{w}_{i} \varphi\right)\right) \stackrel{(22.1 \mathrm{c})}{\approx} \prod_{i=1}^{m}\left(\mathbf{s}_{i} h_{i}^{e_{i, 0}} x_{i, 1}^{e_{i, 1}} \ldots x_{i, r_{i}}^{e_{i, r_{i}}}\right) \quad \text { for some } e_{i, j} \in\{2,4,6, \ldots\} \\
& \stackrel{(22.1 \mathrm{a})}{\approx} \prod_{i=1}^{m}\left(\mathbf{s}_{i} h_{i}^{2} x_{i, 1}^{2} \ldots x_{i, r_{i}}^{2}\right) .
\end{aligned}
$$

Similarly, the deduction $\mathbf{w}^{\prime} \stackrel{(22.1)}{\approx} \prod_{i=1}^{m}\left(\mathbf{s}_{i} h_{i}^{2} x_{i, 1}^{2} \ldots x_{i, r_{i}}^{2}\right)$ also holds. Consequently, the identity $\mathbf{w} \approx \mathbf{w}^{\prime}$ is implied by the identities (22.1).

\subsection{Finite basis property of $\mathscr{E}_{6}$}

Proposition 22.5. The variety generated by $\mathscr{E}_{6}$ is defined by the identities

$$
\begin{aligned}
x^{3} \approx x^{2}, \quad x^{2} y x & \approx x y x, \quad x y x^{2} \approx x y x, \\
x \mathrm{H} y^{2} x & \approx x^{2} \mathrm{H} y^{2} .
\end{aligned}
$$

Lemma 22.6. Suppose that $\mathbf{w} \approx \mathbf{w}^{\prime}$ is any identity satisfied by $\mathscr{E}_{6}$. Then $\operatorname{ini}(\mathbf{w})=\operatorname{ini}\left(\mathbf{w}^{\prime}\right)$ and $\operatorname{sim}(\mathbf{w})=\operatorname{sim}\left(\mathbf{w}^{\prime}\right)$.

Proof. This follows from Lemma 2.1 since the subsemigroups $\{1,4,5\}$ and $\{1,3,5\}$ of $\mathscr{E}_{6}$ are isomorphic to $L_{2}^{1}$ and $N_{2}^{1}$, respectively.

In this subsection, a word $\mathbf{w}$ with $\operatorname{ini}(\mathbf{w})=x_{1} \ldots x_{m}$ is said to be in canonical form if

$$
\mathbf{w}=\prod_{i=1}^{m}\left(x_{i}^{e_{i}} \mathbf{w}_{i}\right)
$$

where all of the following are satisfied:

(I) $\mathbf{w}_{i} \in\left\{\emptyset, x_{1}, \ldots, x_{i-1}\right\}$;

(II) $e_{1}, \ldots, e_{m} \in\{1,2\}$;

(III) if $x_{i} \notin \operatorname{sim}(\mathbf{w})$, then $\mathbf{w}_{i}=\emptyset$;

(IV) if $x_{i} \notin \operatorname{sim}(\mathbf{w})$ and $x_{i}=\mathbf{w}_{j}$ for some $j>i$, then $e_{i}=1$.

Note that (IV) implies that the word $\mathbf{w}$ in (22.4) does not contain a factor of the form $x^{2} \mathbf{a} x$ where $\mathbf{a} \in \mathcal{X}^{+}$.

Lemma 22.7. Let $\mathbf{w}$ be any word. Then there exists some word $\overline{\mathbf{w}}$ in canonical form such that the identities (22.3) imply the identity $\mathbf{w} \approx \overline{\mathbf{w}}$. 
Proof. It suffices to convert $\mathbf{w}$, using the identities (22.3), into a word in canonical form. Suppose that $x$ and $y$ are distinct non-simple letters of $\mathbf{w}$ such that $\mathbf{w}=\mathbf{a} x \mathbf{b} y x \mathbf{c}$ for some $\mathbf{a}, \mathbf{b}, \mathbf{c} \in \mathcal{X}^{*}$ with $y \in \operatorname{con}(\mathbf{a b c})$. Then

$$
\mathbf{w} \stackrel{(22.3 \mathrm{a})}{\approx} \mathbf{a} x \mathbf{b} y^{2} x \mathbf{c} \stackrel{(22.3 \mathrm{~b})}{\approx} \mathbf{a} x^{2} \mathbf{b} y^{2} \mathbf{c} \stackrel{(22.3 \mathrm{a})}{\approx} \mathbf{a} x^{2} \mathbf{b} y \mathbf{c}
$$

that is, using the identities (22.3), any non-first occurrence of $x$ in $\mathbf{w}$ that immediately follows another non-simple letter can be gathered with the first $x$ in $\mathbf{w}$. It follows that $\mathbf{w}$ can be converted into a word of the form (22.4) with (I) and (III) satisfied. Then (II) and (IV) are satisfied by applying the identities (22.3a).

Lemma 22.8. Suppose that $\mathscr{E}_{6}$ satisfies an identity $\mathbf{w} \approx \mathbf{w}^{\prime}$, where

$$
\mathbf{w}=\prod_{i=1}^{m}\left(x_{i}^{e_{i}} \mathbf{w}_{i}\right) \quad \text { and } \quad \mathbf{w}^{\prime}=\prod_{i=1}^{m}\left(x_{i}^{e_{i}^{\prime}} \mathbf{w}_{i}^{\prime}\right)
$$

are in canonical form. Then $e_{i}=e_{i}^{\prime}$ for all $i$.

Proof. Since $\operatorname{occ}\left(x_{m}, \mathbf{w}\right)=e_{m}$ and $\operatorname{occ}\left(x_{m}, \mathbf{w}^{\prime}\right)=e_{m}^{\prime}$ by (I), it follows from (II) and Lemma 22.6 that $e_{m}=e_{m}^{\prime}$. Suppose $e_{j} \neq e_{j}^{\prime}$ for some $j<m$, say $\left(e_{j}, e_{j}^{\prime}\right)=(1,2)$. Then $x_{j} \notin \operatorname{sim}\left(\mathbf{w}^{\prime}\right)$, so that $x_{j} \notin \operatorname{sim}(\mathbf{w})$ by Lemma 22.6. The second $x_{j}$ in $\mathbf{w}$ coincides with $\mathbf{w}_{k}$ for some $k>j$, so that $\mathbf{w}_{k} \neq \emptyset$. Hence $x_{k} \in \operatorname{sim}(\mathbf{w})$ by (III), and

$$
\mathbf{w}=\mathbf{a} x_{j}^{e_{j}} \mathbf{b} x_{k} \mathbf{w}_{k} \mathbf{c}=\mathbf{a} x_{j} \mathbf{b} x_{k} x_{j} \mathbf{c}
$$

for some $\mathbf{a}, \mathbf{b}, \mathbf{c} \in \mathcal{X}^{*}$ with $x_{j} \notin \operatorname{con}(\mathbf{a b})$ and $x_{k} \notin \operatorname{con}(\mathbf{a b c})$. By Lemma 22.6, the letter $x_{k}$ is also simple in $\mathbf{w}^{\prime}$. Since $e_{j}^{\prime}=2$, it follows from (I) and (IV) that $\operatorname{occ}\left(x_{j}, \mathbf{w}^{\prime}\right)=2$, whence

$$
\mathbf{w}^{\prime}=\mathbf{a}^{\prime} x_{j}^{e_{j}^{\prime}} \mathbf{b}^{\prime} x_{k} \mathbf{c}^{\prime}=\mathbf{a}^{\prime} x_{j}^{2} \mathbf{b}^{\prime} x_{k} \mathbf{c}^{\prime}
$$

for some $\mathbf{a}^{\prime}, \mathbf{b}^{\prime}, \mathbf{c}^{\prime} \in \mathcal{X}^{*}$ with $x_{j}, x_{k} \notin \operatorname{con}\left(\mathbf{a}^{\prime} \mathbf{b}^{\prime} \mathbf{c}^{\prime}\right)$. Let $\varphi: \mathcal{X} \rightarrow \mathscr{E}_{6}$ denote the substitution

$$
z \mapsto \begin{cases}5 & \text { if } z=x_{j} \\ 2 & \text { if } z=x_{k} \\ 6 & \text { otherwise }\end{cases}
$$

Note that $x_{j}, x_{k} \notin \operatorname{con}\left(\mathbf{a b a}^{\prime} \mathbf{b}^{\prime} \mathbf{c}^{\prime}\right)$, so $6 \cdot \mathbf{a} \varphi=6 \cdot \mathbf{a}^{\prime} \varphi=\mathbf{c}^{\prime} \varphi \cdot 6=6$ and $5 \cdot \mathbf{b} \varphi=5^{2} \cdot \mathbf{b}^{\prime} \varphi=5$. Hence

$$
6 \cdot \mathbf{w} \varphi \cdot 6=(6 \cdot \mathbf{a} \varphi) \cdot(5 \cdot \mathbf{b} \varphi) \cdot(2 \cdot 5) \cdot \mathbf{c} \varphi \cdot 6=6 \cdot 5 \cdot 1 \cdot \mathbf{c} \varphi \cdot 6=1
$$

and

$$
6 \cdot \mathbf{w}^{\prime} \varphi \cdot 6=\left(6 \cdot \mathbf{a}^{\prime} \varphi\right) \cdot\left(5^{2} \cdot \mathbf{b}^{\prime} \varphi\right) \cdot 2 \cdot\left(\mathbf{c}^{\prime} \varphi \cdot 6\right)=6 \cdot 5 \cdot 2 \cdot 6=3,
$$

which is a contradiction. Consequently, $j$ does not exist, so that $e_{i}=e_{i}^{\prime}$ for all $i$.

Proof of Proposition 22.5. It is routinely checked that $\mathscr{E}_{6}$ satisfies the identities (22.3). Hence it suffices to show that any identity $\mathbf{w} \approx \mathbf{w}^{\prime}$ satisfied by $\mathscr{E}_{6}$ is implied by the identities (22.3). By Lemma 22.7, the words $\mathbf{w}$ and $\mathbf{w}^{\prime}$ can be assumed to be in canonical form. Since ini $(\mathbf{w})=$ $\operatorname{ini}\left(\mathbf{w}^{\prime}\right)$ by Lemma 22.6 , it follows from Lemma 22.8 that

$$
\mathbf{w}=\prod_{i=1}^{m}\left(x_{i}^{e_{i}} \mathbf{w}_{i}\right) \quad \text { and } \quad \mathbf{w}^{\prime}=\prod_{i=1}^{m}\left(x_{i}^{e_{i}} \mathbf{w}_{i}^{\prime}\right) .
$$


Suppose that $\ell$ is the least integer such that $\mathbf{w}_{\ell} \neq \mathbf{w}_{\ell}^{\prime}$. Then $\left(\mathbf{w}_{\ell}, \mathbf{w}_{\ell}^{\prime}\right) \neq(\emptyset, \emptyset)$, say $\mathbf{w}_{\ell} \neq \emptyset$. It follows from (I) that $\mathbf{w}_{\ell}=x_{k}$ for some $k<\ell$, whence $x_{\ell} \in \operatorname{sim}(\mathbf{w})$ and $e_{\ell}=1$ by (III). Thus

$$
\mathbf{w}=\mathbf{p} x_{\ell}^{e_{\ell}} \mathbf{w}_{\ell} \mathbf{q}=\mathbf{p} x_{\ell} x_{k} \mathbf{q}
$$

where $\mathbf{p}=\prod_{i=1}^{\ell-1}\left(x_{i}^{e_{i}} \mathbf{w}_{i}\right)$ and $\mathbf{q}=\prod_{i=\ell+1}^{m}\left(x_{i}^{e_{i}} \mathbf{w}_{i}\right)$, and

$$
\mathbf{w}^{\prime}=\mathbf{p} x_{\ell}^{e_{\ell}} \mathbf{w}_{\ell}^{\prime} \mathbf{q}^{\prime}=\mathbf{p} x_{\ell} \mathbf{w}_{\ell}^{\prime} \mathbf{q}^{\prime}
$$

where $\mathbf{q}^{\prime}=\prod_{i=\ell+1}^{m}\left(x_{i}^{e_{i}} \mathbf{w}_{i}^{\prime}\right)$ and $\mathbf{w}_{\ell}^{\prime} \in\left\{\emptyset, x_{1}, \ldots, x_{\ell-1}\right\} \backslash\left\{x_{k}\right\}$. Further, $\operatorname{sim}(\mathbf{w})=\operatorname{sim}\left(\mathbf{w}^{\prime}\right)$ by Lemma 22.6, so that $x_{\ell} \notin \operatorname{con}\left(\mathbf{p q} \mathbf{w}_{\ell}^{\prime} \mathbf{q}^{\prime}\right)$. Let $\varphi: \mathcal{X} \rightarrow \mathscr{E}_{6}$ denote the substitution

$$
z \mapsto \begin{cases}5 & \text { if } z=x_{k} \\ 2 & \text { if } z=x_{\ell} \\ 6 & \text { otherwise. }\end{cases}
$$

Then $6 \cdot \mathbf{w} \varphi \cdot 6=(6 \cdot \mathbf{p} \varphi) \cdot 2 \cdot(5 \cdot \mathbf{q} \varphi \cdot 6)=6 \cdot 2 \cdot 5=1$. Note that if $\mathbf{q}^{\prime} \neq \emptyset$, then $\mathrm{h}\left(\mathbf{q}^{\prime}\right)=x_{\ell+1}$ and $x_{\ell} \notin \operatorname{con}\left(\mathbf{q}^{\prime}\right)$, so that $\mathbf{q}^{\prime} \varphi \cdot 6=6$. Therefore

$$
\begin{aligned}
6 \cdot \mathbf{w}^{\prime} \varphi \cdot 6 & = \begin{cases}(6 \cdot \mathbf{p} \varphi) \cdot 2 \cdot 6 \cdot\left(\mathbf{q}^{\prime} \varphi \cdot 6\right) & \text { if } \mathbf{w}_{\ell}^{\prime} \neq \emptyset \\
(6 \cdot \mathbf{p} \varphi) \cdot 2 \cdot\left(\mathbf{q}^{\prime} \varphi \cdot 6\right) & \text { if } \mathbf{w}_{\ell}^{\prime}=\emptyset\end{cases} \\
& =6 \cdot 2 \cdot 6=3,
\end{aligned}
$$

and the contradiction $6 \cdot \mathbf{w} \varphi \cdot 6 \neq 6 \cdot \mathbf{w}^{\prime} \varphi \cdot 6$ is established. Hence the integer $\ell$ does not exist. Consequently, the identity $\mathbf{w} \approx \mathbf{w}^{\prime}$ is trivial and is implied by the identities (22.3).

\subsection{Finite basis property of $\mathscr{E}_{7}$}

Proposition 22.9. The variety generated by $\mathscr{E}_{7}$ is defined by the identities

$$
x \mathrm{H} x^{2} \approx x \mathrm{H} x, \quad x \mathrm{H} y \mathrm{~K} x y \approx x \mathrm{H} y \mathrm{~K} y, \quad x \mathrm{H} y \mathrm{~K} y x \approx x \mathrm{H} y \mathrm{~K} x .
$$

In this subsection, a word $\mathbf{w}$ with $\operatorname{ini}(\mathbf{w})=x_{1} \ldots x_{m}$ is said to be in canonical form if

$$
\mathbf{w}=\prod_{i=1}^{m}\left(x_{i} \mathbf{w}_{i}\right)
$$

where $\mathbf{w}_{i} \in\left\{\emptyset, x_{1}, \ldots, x_{i}\right\}$.

Lemma 22.10. Let $\mathbf{w}$ be any word. Then there exists some word $\overline{\mathbf{w}}$ in canonical form such that the identities (22.5) imply the identity $\mathbf{w} \approx \overline{\mathbf{w}}$.

Proof. It suffices to convert $\mathbf{w}$, using the identities (22.5), into a word in canonical form. Suppose ini $(\mathbf{w})=x_{1} \ldots x_{m}$. Then $\mathbf{w}$ can be written in the form (22.6) with $\mathbf{w}_{i} \in\left\{x_{1}, \ldots, x_{i}\right\}^{*}$. Since any letter in $\mathbf{w}_{i}$ is a non-first occurrence in $\mathbf{w}$, the identities (22.5) can be used to eliminate all except the last letter of $\mathbf{w}_{i}$. Therefore $\mathbf{w}_{i} \in\left\{\emptyset, x_{1}, \ldots, x_{i}\right\}$.

Proof of Proposition 22.9. It is routinely checked that $\mathscr{E}_{7}$ satisfies the identities (22.5). Hence it suffices to show that any identity $\mathbf{w} \approx \mathbf{w}^{\prime}$ satisfied by $\mathscr{E}_{7}$ is implied by the identities (22.5). By Lemma 22.10, the words $\mathbf{w}$ and $\mathbf{w}^{\prime}$ can be assumed to be in canonical form. Now the subsemigroup $\{1,3,5\}$ of $\mathscr{E}_{7}$ is isomorphic to $L_{2}^{1}$, so that ini $(\mathbf{w})=\operatorname{ini}\left(\mathbf{w}^{\prime}\right)$ by Lemmas 2.1(iii). Hence

$$
\mathbf{w}=\prod_{i=1}^{m}\left(x_{i} \mathbf{w}_{i}\right) \quad \text { and } \quad \mathbf{w}^{\prime}=\prod_{i=1}^{m}\left(x_{i} \mathbf{w}_{i}^{\prime}\right) .
$$


Suppose that $\ell$ is the least integer such that $\mathbf{w}_{\ell} \neq \mathbf{w}_{\ell}^{\prime}$. Then

$$
\mathbf{w}=\mathbf{p} x_{\ell} \mathbf{w}_{\ell} \mathbf{q} \quad \text { and } \quad \mathbf{w}^{\prime}=\mathbf{p} x_{\ell} \mathbf{w}_{\ell}^{\prime} \mathbf{q}^{\prime}
$$

where $\mathbf{p}=\prod_{i=1}^{\ell-1}\left(x_{i} \mathbf{w}_{i}\right)=\prod_{i=1}^{\ell-1}\left(x_{i} \mathbf{w}_{i}^{\prime}\right), \mathbf{q}=\prod_{i=\ell+1}^{m}\left(x_{i} \mathbf{w}_{i}\right)$, and $\mathbf{q}^{\prime}=\prod_{i=\ell+1}^{m}\left(x_{i} \mathbf{w}_{i}^{\prime}\right)$. By symmetry, there are three cases.

Case 1: $\left(\mathbf{w}_{\ell}, \mathbf{w}_{\ell}^{\prime}\right)=\left(\emptyset, x_{\ell}\right)$. Let $\varphi_{1}: \mathcal{X} \rightarrow \mathscr{E}_{7}$ denote the substitution

$$
z \mapsto \begin{cases}2 & \text { if } z=x_{\ell}, \\ 6 & \text { if } z=x_{\ell+1}, \\ 4 & \text { otherwise. }\end{cases}
$$

Then $x_{\ell}, x_{\ell+1} \notin \operatorname{con}(\mathbf{p})$, so that $4 \cdot \mathbf{p} \varphi_{1}=4$. Hence

$$
4 \cdot \mathbf{w} \varphi_{1} \cdot 6=\left(4 \cdot \mathbf{p} \varphi_{1}\right) \cdot 2 \cdot\left(\mathbf{q} \varphi_{1} \cdot 6\right)=4 \cdot 2 \cdot 6=3
$$

and

$$
4 \cdot \mathbf{w}^{\prime} \varphi_{1} \cdot 6=\left(4 \cdot \mathbf{p} \varphi_{1}\right) \cdot 2 \cdot 2 \cdot\left(\mathbf{q}^{\prime} \varphi_{1} \cdot 6\right)=4 \cdot 2 \cdot 2 \cdot 6=1
$$

Case 2: $\left(\mathbf{w}_{\ell}, \mathbf{w}_{\ell}^{\prime}\right)=\left(\emptyset, x_{k}\right)$ with $k<\ell$. Let $\varphi_{2}: \mathcal{X} \rightarrow \mathscr{E}_{7}$ denote the substitution

$$
z \mapsto \begin{cases}5 & \text { if } z=x_{k}, \\ 6 & \text { if } z=x_{\ell+1}, \\ 4 & \text { otherwise. }\end{cases}
$$

Then $x_{\ell+1} \notin \operatorname{con}(\mathbf{p})$, so that $4 \cdot \mathbf{p} \varphi_{2} \in\{4,5\}$. Hence

$$
4 \cdot \mathbf{w} \varphi_{2} \cdot 6=\left(4 \cdot \mathbf{p} \varphi_{2}\right) \cdot 4 \cdot\left(\mathbf{q} \varphi_{2} \cdot 6\right) \in\{4,5\} \cdot 4 \cdot 6=\{1\}
$$

and

$$
4 \cdot \mathbf{w}^{\prime} \varphi_{2} \cdot 6=\left(4 \cdot \mathbf{p} \varphi_{2}\right) \cdot 4 \cdot 5 \cdot\left(\mathbf{q}^{\prime} \varphi_{2} \cdot 6\right) \in\{4,5\} \cdot 4 \cdot 5 \cdot 6=\{3\}
$$

Case 3: $\left(\mathbf{w}_{\ell}, \mathbf{w}_{\ell}^{\prime}\right)=\left(x_{j}, x_{k}\right)$ with $j<k \leqslant \ell$. Then since $x_{\ell+1} \notin \operatorname{con}(\mathbf{p})$, it follows that $\left(\mathbf{p} x_{\ell}\right) \varphi_{2} \in\{4,5\} \cdot 4=\{4\}$. Hence

$$
\mathbf{w} \varphi_{2} \cdot 6=\left(\mathbf{p} x_{\ell}\right) \varphi_{2} \cdot 4 \cdot\left(\mathbf{q} \varphi_{2} \cdot 6\right)=4 \cdot 4 \cdot 6=1
$$

and

$$
\mathbf{w}^{\prime} \varphi_{2} \cdot 6=\left(\mathbf{p} x_{\ell}\right) \varphi_{2} \cdot 5 \cdot\left(\mathbf{q}^{\prime} \varphi_{2} \cdot 6\right)=4 \cdot 5 \cdot 6=3 .
$$

Since all of the above cases are impossible, the integer $\ell$ does not exist. Consequently, the identity $\mathbf{w} \approx \mathbf{w}^{\prime}$ is trivial and so is implied by the identities (22.5).

$$
\text { 23. } \mathscr{E}_{8}, \mathscr{E}_{9}, \mathscr{E}_{10}, \mathscr{F}_{5}
$$

This section establishes the finite basis property of the following semigroups.

\begin{tabular}{c|ccccccc|cccccccc|ccccccc|cccccc}
$\mathscr{E}_{8}$ & 1 & 2 & 3 & 4 & 5 & 6 & $\mathscr{E}_{9}$ & 1 & 2 & 3 & 4 & 5 & 6 & & $\mathscr{E}_{10}$ & 1 & 2 & 3 & 4 & 5 & 6 & $\mathscr{F}_{5}$ & 1 & 2 & 3 & 4 & 5 & 6 \\
\hline 1 & 1 & 1 & 1 & 1 & 1 & 1 & 1 & 1 & 1 & 1 & 1 & 1 & 1 & & 1 & 1 & 1 & 1 & 1 & 1 & 1 & 1 & 1 & 1 & 1 & 1 & 1 & 1 \\
2 & 1 & 1 & 1 & 1 & 1 & 3 & 2 & 1 & 1 & 1 & 1 & 1 & 3 & 2 & 1 & 1 & 1 & 1 & 2 & 2 & 2 & 1 & 1 & 1 & 1 & 2 & 2 \\
3 & 3 & 3 & 3 & 3 & 3 & 3 & 3 & 3 & 3 & 3 & 3 & 3 & 3 & 3 & 1 & 1 & 1 & 1 & 3 & 3 & 3 & 3 & 3 & 3 & 3 & 3 & 3 \\
4 & 1 & 2 & 3 & 4 & 5 & 1 & 4 & 1 & 2 & 3 & 4 & 5 & 3 & 4 & 4 & 4 & 4 & 4 & 4 & 4 & 4 & 3 & 3 & 3 & 3 & 4 & 4 \\
5 & 1 & 2 & 3 & 5 & 4 & 1 & 5 & 1 & 2 & 3 & 5 & 4 & 3 & 5 & 1 & 1 & 3 & 1 & 5 & 6 & 5 & 1 & 2 & 1 & 1 & 5 & 6 \\
6 & 6 & 6 & 6 & 6 & 6 & 6 & 6 & 6 & 6 & 6 & 6 & 6 & 6 & 6 & 1 & 3 & 3 & 1 & 5 & 6 & 6 & 1 & 2 & 1 & 2 & 5 & 6
\end{tabular}




\subsection{Finite basis property of $\mathscr{E}_{8}$ and $\mathscr{E}_{9}$}

Proposition 23.1. The variety generated by $S \in\left\{\mathscr{E}_{8}, \mathscr{E}_{9}\right\}$ is defined by the identities

$$
\begin{aligned}
x^{2} y x & \approx x^{3} y^{3}, \\
x \mathrm{H} x^{3} & \approx x \mathrm{H} x, \\
x \mathrm{H} y x^{2} & \approx x \mathrm{H} y^{3}, \\
x \mathrm{H} y^{3} x & \approx x \mathrm{H} y x, \\
x \mathrm{H} y \mathrm{~K} x y & \approx x \mathrm{H} y \mathrm{~K} y x, \\
y \mathrm{H} x y \mathrm{~K} z^{2} & \approx y \mathrm{H} x^{2} \mathrm{~K} z^{2} x y .
\end{aligned}
$$

LEMma 23.2. Let $S \in\left\{\mathscr{E}_{8}, \mathscr{E}_{9}\right\}$. Suppose that $\mathbf{w} \approx \mathbf{w}^{\prime}$ is any identity satisfied by $S$. Then $\operatorname{ini}(\mathbf{w})=\operatorname{ini}\left(\mathbf{w}^{\prime}\right)$ and $\operatorname{occ}(x, \mathbf{w}) \equiv \operatorname{occ}\left(x, \mathbf{w}^{\prime}\right)(\bmod 2)$ for all $x \in \mathcal{X}$.

Proof. This follows from Lemma 2.1 since the subsemigroups $\{1,3,4\}$ and $\{4,5\}$ of $S$ are isomorphic to $L_{2}^{1}$ and $\mathbb{Z}_{2}$, respectively.

Lemma 23.3. Let $S \in\left\{\mathscr{E}_{8}, \mathscr{E}_{9}\right\}$. Then $S$ does not satisfy the identity

$$
x^{2} y^{3} z^{2} \approx x^{2} y z^{2} .
$$

Proof. This holds because $4^{2} \cdot 2^{3} \cdot 6^{2}=1 \neq 3=4^{2} \cdot 2 \cdot 6^{2}$ in $S$.

In this subsection, a non-simple word $\mathbf{w}$ with $\operatorname{ini}(\mathbf{w})=x_{0} \ldots x_{m}$ is said to be in canonical form if

$$
\mathbf{w}=x_{0}^{e_{0}} \ldots x_{r}^{e_{r}} \cdot x_{0}^{f_{0}} \ldots x_{r-1}^{f_{r-1}} \cdot x_{r+1} \ldots x_{m}
$$

for some $r \in\{0, \ldots, m\}$ such that all of the following are satisfied:

(I) $e_{0}, \ldots, e_{r} \in\{1,2,3\}$;

(II) $f_{0}, \ldots, f_{r-1} \in\{0,1\}$;

(III) if $e_{i} \geqslant 2$ and $i \in\{0, \ldots, r-1\}$, then $f_{i}=0$;

(IV) if $e_{r}=1$, then $\left(f_{0}, \ldots, f_{r-1}\right) \neq(0, \ldots, 0)$;

(V) if $r \geqslant 1$ and $\left(f_{0}, \ldots, f_{r-1}\right) \neq(0, \ldots, 0)$, then $e_{r} \leqslant 2$.

REMARK 23.4. (i) The word $\mathbf{w}$ in (23.3) satisfies $\operatorname{occ}(x, \mathbf{w}) \leqslant 3$ for all $x \in \mathcal{X}$.

(ii) If $r=0$, then the word $\mathbf{w}$ in $(23.3)$ is $x_{0}^{e_{0}} x_{1} \ldots x_{m}$.

Lemma 23.5. Let $\mathbf{w}$ be any non-simple word. Then there exists some word $\overline{\mathbf{w}}$ in canonical form such that the identities (23.1) imply the identity $\mathbf{w} \approx \overline{\mathbf{w}}$.

Proof. It suffices to convert $\mathbf{w}$, using the identities (23.1), into a word in canonical form. Suppose $\operatorname{ini}(\mathbf{w})=x_{0} \ldots x_{m}$. Then $\mathbf{w}$ can be written as a word of the form $\mathbf{w}=\prod_{i=0}^{m}\left(x_{i} \mathbf{w}_{i}\right)$ with $\mathbf{w}_{i} \in\left\{x_{0}, \ldots, x_{i}\right\}^{*}$. Let $r$ be the largest integer for which $\mathbf{w}_{r} \neq \emptyset$, so that $\mathbf{w}_{j}=\emptyset$ for all $j>r$. Then $\mathbf{w}=\left(\prod_{i=0}^{r}\left(x_{i} \mathbf{w}_{i}\right)\right)\left(\prod_{i=r+1}^{m} x_{i}\right)$. Since the letters of $\mathbf{w}_{i}$ are non-first occurrences, the identities (23.1e) can be used to arrange them, within $\mathbf{w}_{i}$, in any order. Hence it can be assumed that

$$
\mathbf{w}=x_{0}^{e_{0}} \cdot x_{1}^{e_{1}} \mathbf{w}_{1}^{\prime} \ldots x_{r}^{e_{r}} \mathbf{w}_{r}^{\prime} \cdot x_{r+1} \ldots x_{m}
$$

where $e_{0}, \ldots, e_{r} \geqslant 1$ and $\mathbf{w}_{i}^{\prime} \in\left\{x_{0}, \ldots, x_{i-1}\right\}^{*}$. The assumption $\mathbf{w}_{r} \neq \emptyset$ implies that either $e_{r} \geqslant 2$ or $\mathbf{w}_{r}^{\prime} \neq \emptyset$. Therefore

$$
\mathbf{w} \stackrel{(23.1 \mathrm{~b})}{\approx} \begin{cases}x_{0}^{e_{0}} \cdot x_{1}^{e_{1}} \mathbf{w}_{1}^{\prime} \ldots x_{r-1}^{e_{r}-1} \mathbf{w}_{r-1}^{\prime} \cdot x_{r}^{e_{r}} \mathbf{w}_{r}^{\prime} x_{r}^{2} \cdot x_{r+1} \ldots x_{m} & \text { if } \mathbf{w}_{r}^{\prime}=\emptyset, \\ x_{0}^{e_{0}} \cdot x_{1}^{e_{1}} \mathbf{w}_{1}^{\prime} \ldots x_{r-1}^{e_{r-1}} \mathbf{w}_{r-1}^{\prime} \cdot x_{r}^{e_{r}} \mathbf{w}_{r}^{\prime}\left(\mathbf{t}\left(\mathbf{w}_{r}^{\prime}\right)\right)^{2} \cdot x_{r+1} \ldots x_{m} & \text { if } \mathbf{w}_{r}^{\prime} \neq \emptyset .\end{cases}
$$


In either case, there exists some $z \in\left\{x_{0}, \ldots, x_{r}\right\}$ such that

$$
\mathbf{w} \stackrel{(23.1 \mathrm{~b})}{\approx} x_{0}^{e_{0}} \cdot x_{1}^{e_{1}} \mathbf{w}_{1}^{\prime} \ldots x_{r-1}^{e_{r-1}} \mathbf{w}_{r-1}^{\prime} \cdot x_{r}^{e_{r}} \mathbf{w}_{r}^{\prime} z^{2} \cdot x_{r+1} \ldots x_{m}
$$

Suppose $\mathbf{w}_{k}^{\prime} \neq \emptyset$ for some $k<r$. Then $\mathbf{w}_{k}^{\prime}=y_{1} y_{2} \ldots y_{p}$ for some $y_{i} \in\left\{x_{0}, \ldots, x_{k-1}\right\}$, whence

$$
\mathbf{w}=\mathbf{p} \cdot x_{k}^{e_{k}} y_{1} y_{2} \ldots y_{p} \cdot \mathbf{q} \cdot x_{r}^{e_{r}} \mathbf{w}_{r}^{\prime} \cdot x_{r+1} \ldots x_{m}
$$

where $\mathbf{p}=x_{0}^{e_{0}} \prod_{i=1}^{k-1}\left(x_{i}^{e_{i}} \mathbf{w}_{i}^{\prime}\right)$ and $\mathbf{q}=\prod_{i=k+1}^{r-1}\left(x_{i}^{e_{i}} \mathbf{w}_{i}^{\prime}\right)$. Since the prefix $\mathbf{p}$ contains the letters $y_{1}, \ldots, y_{p}$,

$$
\begin{aligned}
& \mathbf{w} \stackrel{(23.1 \mathrm{~b})}{\approx} \mathbf{p} \cdot x_{k}^{e_{k}} y_{1} y_{2} \ldots y_{p} \cdot \mathbf{q} \cdot x_{r}^{e_{r}} \mathbf{w}_{r}^{\prime} z^{2} \cdot x_{r+1} \ldots x_{m} \\
& \stackrel{(23.1 \mathrm{f})}{\approx} \mathbf{p} \cdot x_{k}^{e_{k}+1} y_{2} y_{3} \ldots y_{p} \cdot \mathbf{q} \cdot x_{r}^{e_{r}} \mathbf{w}_{r}^{\prime} z^{2} \cdot x_{k} y_{1} \cdot x_{r+1} \ldots x_{m} \\
& \stackrel{(23.1 \mathrm{f})}{\approx} \mathbf{p} \cdot x_{k}^{e_{k}+2} y_{3} y_{4} \ldots y_{p} \cdot \mathbf{q} \cdot x_{r}^{e_{r}} \mathbf{w}_{r}^{\prime} z^{2} \cdot x_{k} y_{2} \cdot x_{k} y_{1} \cdot x_{r+1} \ldots x_{m} \\
& \quad \vdots \\
& \quad \\
& \quad{ }_{(23.1 \mathrm{f})}^{\approx} \mathbf{p} \cdot x_{k}^{e_{k}+p} \cdot \mathbf{q} \cdot x_{r}^{e_{r}} \mathbf{w}_{r}^{\prime} z^{2} \cdot x_{k} y_{p} \cdot x_{k} y_{p-1} \ldots x_{k} y_{1} \cdot x_{r+1} \ldots x_{m} \\
& \stackrel{(23.1 \mathrm{e})}{\approx} \mathbf{p} \cdot x_{k}^{e_{k}+p} \cdot \mathbf{q} \cdot x_{r}^{e_{r}} \mathbf{w}_{r}^{\prime} z^{2} \cdot x_{k}^{p} y_{1} y_{2} \ldots y_{p} \cdot x_{r+1} \ldots x_{m} \\
& \quad \mathbf{p} \cdot x_{k}^{e_{k}+p} \cdot \mathbf{q} \cdot x_{r}^{e_{r}} \mathbf{w}_{r}^{\prime} \cdot x_{k}^{p} y_{1} y_{2} \ldots y_{p} \cdot x_{r+1} \ldots x_{m} \\
& \quad=\mathbf{p} \cdot x_{k}^{e_{k}+\left|\mathbf{w}_{k}^{\prime}\right|} \cdot \mathbf{q} \cdot x_{r}^{e_{r}} \mathbf{w}_{r}^{\prime} x_{k}^{\left|\mathbf{w}_{k}^{\prime}\right|} \mathbf{w}_{k}^{\prime} \cdot x_{r+1} \ldots x_{m} .
\end{aligned}
$$

Hence the identities (23.1) can be used to move the factor $\mathbf{w}_{k}^{\prime}$ to the right until it immediately precedes the letter $x_{r+1}$ (while simultaneously introducing two copies of $x_{k}^{\left|\mathbf{w}_{k}^{\prime}\right|}$ to the word), that is,

$$
\begin{aligned}
& \mathbf{w}=\mathbf{p} \cdot x_{k}^{e_{k}} \mathbf{w}_{k}^{\prime} \cdot \mathbf{q} \cdot x_{r}^{e_{r}} \mathbf{w}_{r}^{\prime} \cdot x_{r+1} \ldots x_{m} \\
& \stackrel{(23.1)}{\approx} \mathbf{p} \cdot x_{k}^{e_{k}+\left|\mathbf{w}_{k}^{\prime}\right|} \cdot \mathbf{q} \cdot x_{r}^{e_{r}} \mathbf{w}_{r}^{\prime} x_{k}^{\left|\mathbf{w}_{k}^{\prime}\right|} \mathbf{w}_{k}^{\prime} \cdot x_{r+1} \ldots x_{m} \text {. }
\end{aligned}
$$

The above deduction holds vacuously if $\mathbf{w}_{k}^{\prime}=\emptyset$. Since $k<r$ is arbitrary, this deduction can be repeated on every $\mathbf{w}_{1}^{\prime}, \ldots, \mathbf{w}_{r-1}^{\prime}$, resulting in

$$
\mathbf{w} \stackrel{(23.1)}{\approx} x_{0}^{e_{0}}\left(\prod_{i=1}^{r-1} x_{i}^{e_{i}+\left|\mathbf{w}_{i}^{\prime}\right|}\right) x_{r}^{e_{r}} \mathbf{w}_{r}^{\prime}\left(\prod_{i=1}^{r-1}\left(x_{i}^{\left|\mathbf{w}_{i}^{\prime}\right|} \mathbf{w}_{i}^{\prime}\right)\right) x_{r+1} \ldots x_{m} .
$$

Since the letters in $\mathbf{w}_{r}^{\prime}\left(\prod_{i=1}^{r-1}\left(x_{i}^{\left|\mathbf{w}_{i}^{\prime}\right|} \mathbf{w}_{i}^{\prime}\right)\right)$ are non-first occurrences in $\mathbf{w}$, the identities $(23.1 \mathrm{e})$ can be used to order them. Hence $\mathbf{w}$ can be converted by the identities (23.1) into the word in (23.3). As observed earlier, either $e_{r} \geqslant 2$ or $\mathbf{w}_{r}^{\prime} \neq \emptyset$. Hence (IV) is satisfied. The identities $\{(23.1 \mathrm{~b}),(23.1 \mathrm{c})\}$ can be used to reduce the exponents $f_{r-1}, \ldots, f_{0}$ to numbers in $\{0,1\}$, so that (II) is satisfied.

Suppose $e_{i} \geqslant 2$ and $f_{i}=1$ for some $i<r$. Then

$$
\mathbf{w}=x_{0}^{e_{0}} \cdot \mathbf{a} \cdot x_{i}^{e_{i}} x_{i+1}^{e_{i+1}} \ldots x_{r}^{e_{r}} \cdot x_{0}^{f_{0}} \ldots x_{i-1}^{f_{i-1}} x_{i} \cdot \mathbf{b}
$$


where $\mathbf{a}=x_{1}^{e_{1}} \ldots x_{i-1}^{e_{i-1}}$ and $\mathbf{b}=x_{i+1}^{f_{i+1}} \ldots x_{r-1}^{f_{r-1}} \cdot x_{r+1} \ldots x_{m}$, whence

$$
\begin{aligned}
& \mathbf{w} \stackrel{(23.1 \mathrm{e})}{\stackrel{2}{\approx}} x_{0}^{e_{0}} \cdot \mathbf{a} \cdot\left(x_{i}^{e_{i}} x_{i+1}^{e_{i+1}} \ldots x_{r}^{e_{r}} x_{i}\right) \cdot x_{0}^{f_{0}} \ldots x_{i-1}^{f_{i-1}} \cdot \mathbf{b} \\
& \quad \stackrel{(23.1 \mathrm{a})}{\approx} x_{0}^{e_{0}} \cdot \mathbf{a} \cdot x_{i}^{e_{i}+1}\left(x_{i+1}^{e_{i+1}} \ldots x_{r}^{e_{r}}\right)^{3} \cdot x_{0}^{f_{0}} \ldots x_{i-1}^{f_{i-1}} \cdot \mathbf{b} \\
& \quad=x_{0}^{e_{0}} \cdot \mathbf{a} \cdot x_{i}^{e_{i}+1} x_{i+1}^{e_{i+1}} \ldots x_{r-1}^{e_{r}-1} x_{r}^{e_{r}}\left(x_{i+1}^{e_{i+1}} \ldots x_{r-1}^{e_{r}-1} x_{r}^{e_{r}}\right)^{2} \cdot x_{0}^{f_{0}} \ldots x_{i-1}^{f_{i-1}} \cdot \mathbf{b} \\
& \quad \stackrel{(23.1 \mathrm{e})}{\approx} x_{0}^{e_{0}} \cdot \mathbf{a} \cdot x_{i}^{e_{i}+1} x_{i+1}^{e_{i+1}} \ldots x_{r-1}^{e_{r-1}} x_{r}^{e_{r}} x_{i+1}^{2 e_{i+1}} \ldots x_{r-1}^{2 e_{r-1}} x_{r}^{2 e_{r}} \cdot x_{0}^{f_{0}} \ldots x_{i-1}^{f_{i-1}} \cdot \mathbf{b} \\
& \quad \stackrel{(23.1 \mathrm{~b})}{\approx} x_{0}^{e_{0}} \cdot \mathbf{a} \cdot x_{i}^{e_{i}+1} x_{i+1}^{e_{i+1}} \ldots x_{r-1}^{e_{r-1}} x_{r}^{e_{r}} x_{i+1}^{2} \ldots x_{r-1}^{2} x_{r}^{2} \cdot x_{0}^{f_{0}} \ldots x_{i-1}^{f_{i-1}} \cdot \mathbf{b} \\
& \quad \stackrel{(23.1 \mathrm{c})}{\approx} x_{0}^{e_{0}} \cdot \mathbf{a} \cdot x_{i}^{e_{i}+1} x_{i+1}^{e_{i+1}} \ldots x_{r-1}^{e_{r-1}} x_{r}^{e_{r}+2(r-i)} x_{0}^{f_{0}} \ldots x_{i-1}^{f_{i-1}} \cdot \mathbf{b} \\
& x_{0}^{e_{0}} \cdot \mathbf{a} \cdot x_{i}^{e_{i}+1} x_{i+1}^{e_{i+1}} \ldots x_{r-1}^{e_{r-1}} x_{r}^{e_{r}+2} x_{0}^{f_{0}} \ldots x_{i-1}^{f_{i-1}} \cdot \mathbf{b} .
\end{aligned}
$$

Therefore the identities (23.1) can be used to reduce the exponent $f_{i}$ from 1 to 0 . Since $i$ is arbitrary, this argument can be repeated until (III) is satisfied. It is then easily shown that (I) is satisfied by applying the identities (23.1b). Finally, (V) is satisfied by applying the identities (23.1d).

Lemma 23.6. Let $S \in\left\{\mathscr{E}_{8}, \mathscr{E}_{9}\right\}$. Suppose that $\mathbf{w} \approx \mathbf{w}^{\prime}$ is any nontrivial identity satisfied by $S$. Then the words $\mathbf{w}$ and $\mathbf{w}^{\prime}$ are both non-simple.

Proof. By Lemma 23.2, it can be assumed that ini $(\mathbf{w})=\operatorname{ini}\left(\mathbf{w}^{\prime}\right)=x_{0} \ldots x_{m}$. Therefore if the words $\mathbf{w}$ and $\mathbf{w}^{\prime}$ are both simple, then the identity $\mathbf{w} \approx \mathbf{w}^{\prime}$ is contradictorily trivial. Hence it suffices to assume that $\mathbf{w}$ is non-simple. By Lemma 23.5, there exists a word $\overline{\mathbf{w}}$ in canonical form such that the identities (23.1) imply the identity $\mathbf{w} \approx \overline{\mathbf{w}}$. It is routinely checked that $S$ satisfies the identities (23.1), whence $S$ satisfies $\overline{\mathbf{w}} \approx \mathbf{w}^{\prime}$.

Suppose that the word $\mathbf{w}^{\prime}$ is simple. Then $\mathbf{w}^{\prime}=\operatorname{ini}\left(\mathbf{w}^{\prime}\right)=x_{0} \ldots x_{m}$. Let $x_{i}$ be any non-simple letter in $\overline{\mathbf{w}}$. Since $\operatorname{occ}\left(x_{i}, \overline{\mathbf{w}}\right) \equiv \operatorname{occ}\left(x_{i}, \mathbf{w}^{\prime}\right)(\bmod 2)$ by Lemma 23.2 , it follows from (I)-(III) that $\operatorname{occ}\left(x_{i}, \overline{\mathbf{w}}\right)=3$ and $x_{i}^{3}$ is a factor of $\overline{\mathbf{w}}$. Let $\varphi$ denote the substitution $x_{i} \mapsto x_{i}^{3}$. The deduction $\overline{\mathbf{w}} \varphi \stackrel{(23.1 \mathrm{~b})}{\approx} \overline{\mathbf{w}}$ is easily verified. Since

$$
x_{0} \ldots x_{m}=\mathbf{w}^{\prime} \stackrel{S}{\approx} \overline{\mathbf{w}}^{(23.1 \mathrm{~b})} \overline{\mathbf{w}} \varphi \stackrel{S}{\approx} \mathbf{w}^{\prime} \varphi=x_{0} \ldots x_{i-1} x_{i}^{3} x_{i+1} \ldots x_{m}
$$

the semigroup $S$ satisfies the identity $x_{0} \ldots x_{m} \approx x_{0} \ldots x_{i-1} x_{i}^{3} x_{i+1} \ldots x_{m}$. It is then easily shown that $S$ satisfies the identity (23.2), contradicting Lemma 23.3. Consequently, $\mathbf{w}^{\prime}$ cannot be simple.

Lemma 23.7. Let $S \in\left\{\mathscr{E}_{8}, \mathscr{E}_{9}\right\}$. Suppose that $S$ satisfies an identity $\mathbf{w} \approx \mathbf{w}^{\prime}$, where

$$
\mathbf{w}=x_{0}^{e_{0}} \ldots x_{r}^{e_{r}} \cdot x_{0}^{f_{0}} \ldots x_{r-1}^{f_{r-1}} \cdot x_{r+1} \ldots x_{m}
$$

and

$$
\mathbf{w}^{\prime}=x_{0}^{e_{0}^{\prime}} \ldots x_{r^{\prime}}^{e_{r^{\prime}}^{\prime}} \cdot x_{0}^{f_{0}^{\prime}} \ldots x_{r^{\prime}-1}^{f_{r^{\prime}-1}^{\prime}} \cdot x_{r^{\prime}+1} \ldots x_{m}
$$

are in canonical form. Then $r=r^{\prime}$.

Proof. Seeking a contradiction, suppose $r>r^{\prime}$. Then the letter $x_{r}$ occurs in the suffix $x_{r^{\prime}+1} \ldots x_{m}$ of $\mathbf{w}^{\prime}$, so that $\operatorname{occ}\left(x_{r}, \mathbf{w}^{\prime}\right)=1$. Hence $e_{r}=\operatorname{occ}\left(x_{r}, \mathbf{w}\right) \in\{1,3\}$ by Lemma 23.2 
and Remark 23.4(i). Let $\varphi$ denote the following substitution into $S$ :

$$
z \mapsto \begin{cases}4 & \text { if } z \in\left\{x_{0}, \ldots, x_{r-1}\right\}, \\ 2 & \text { if } z=x_{r} \\ 6 & \text { if } z \in\left\{x_{r+1}, \ldots, x_{m}\right\} .\end{cases}
$$

Then $4 \cdot \mathbf{w}^{\prime} \varphi \cdot 6=4 \cdot 2 \cdot 6=3$ and

$$
4 \cdot \mathbf{w} \varphi \cdot 6=4 \cdot x_{r}^{e_{r}} \varphi \cdot\left(x_{0}^{f_{0}} \ldots x_{r-1}^{f_{r-1}}\right) \varphi \cdot 6 .
$$

If $e_{r}=3$, then $4 \cdot \mathbf{w} \varphi \cdot 6=4 \cdot 2^{3} \ldots 6=1$. If $e_{r}=1$, then $\left(f_{0}, \ldots, f_{r-1}\right) \neq(0, \ldots, 0)$ by (IV), so that $4 \cdot \mathbf{w} \varphi \cdot 6=4 \cdot 2 \cdot 4 \cdot 6=1$. In either case, the contradiction $4 \cdot \mathbf{w} \varphi \cdot 6 \neq 4 \cdot \mathbf{w}^{\prime} \varphi \cdot 6$ is obtained.

Lemma 23.8. Let $S \in\left\{\mathscr{E}_{8}, \mathscr{E}_{9}\right\}$. Suppose that $S$ satisfies an identity $\mathbf{w} \approx \mathbf{w}^{\prime}$, where

$$
\mathbf{w}=x_{0}^{e_{0}} \ldots x_{r}^{e_{r}} \cdot x_{0}^{f_{0}} \ldots x_{r-1}^{f_{r-1}} \cdot x_{r+1} \ldots x_{m}
$$

and

$$
\mathbf{w}^{\prime}=x_{0}^{e_{0}^{\prime}} \ldots x_{r}^{e_{r}^{\prime}} \cdot x_{0}^{f_{0}^{\prime}} \ldots x_{r-1}^{f_{r-1}^{\prime}} \cdot x_{r+1} \ldots x_{m}
$$

are in canonical form. Then:

(i) $\left(f_{0}, \ldots, f_{r-1}\right)=\left(f_{0}^{\prime}, \ldots, f_{r-1}^{\prime}\right)$;

(ii) $\left(e_{0}, \ldots, e_{r}\right)=\left(e_{0}^{\prime}, \ldots, e_{r}^{\prime}\right)$.

Consequently, $\mathbf{w}=\mathbf{w}^{\prime}$.

Proof. (i) Seeking a contradiction, suppose $f_{i}>f_{i}^{\prime}$ for some $i \in\{0, \ldots, r-1\}$. Then $\left(f_{i}, f_{i}^{\prime}\right)=$ $(1,0)$ by (II), so that $e_{i}=1$ by (I) and (III). Since occ $\left(x_{i}, \mathbf{w}\right)=e_{i}+f_{i}=2$, it follows from Remark 23.4(i) and Lemma 23.2 that $2=\operatorname{occ}\left(x_{i}, \mathbf{w}^{\prime}\right)=e_{i}^{\prime}+f_{i}^{\prime}=e_{i}^{\prime}$. Let $\varphi: \mathcal{X} \rightarrow S$ denote the substitution

$$
z \mapsto \begin{cases}4 & \text { if } z \in\left\{x_{0}, \ldots, x_{i-1}\right\} \\ 2 & \text { if } z=x_{i} \\ 6 & \text { otherwise }\end{cases}
$$

Then $e_{i}=1$ and $e_{i}^{\prime}=2$ imply that

$$
4 \cdot \mathbf{w} \varphi \cdot 6=4 \cdot\left(x_{0}^{e_{0}} \ldots x_{i-1}^{e_{i-1}}\right) \varphi \cdot 2 \cdot\left(x_{i+1}^{e_{i+1}} \ldots x_{r}^{e_{r}}\right) \varphi \ldots=4 \cdot 2 \cdot 6 \ldots=3
$$

and

$$
4 \cdot \mathbf{w}^{\prime} \varphi \cdot 6=4 \cdot\left(x_{0}^{e_{0}^{\prime}} \ldots x_{i-1}^{e_{i-1}^{\prime}}\right) \varphi \cdot 2^{2} \cdot\left(x_{i+1}^{e_{i+1}^{\prime}} \ldots x_{r}^{e_{r}^{\prime}}\right) \varphi \ldots=4 \cdot 1 \cdot 6 \ldots=1,
$$

whence the contradiction $\mathbf{w} \varphi \neq \mathbf{w}^{\prime} \varphi$ is deduced.

(ii) Seeking a contradiction, suppose $e_{i}>e_{i}^{\prime}$ for some $i \in\{0, \ldots, r\}$.

Case 1: $e_{r}>e_{r}^{\prime}$. Since $\operatorname{occ}\left(x_{r}, \mathbf{w}\right)=e_{r}$ and $\operatorname{occ}\left(x_{r}, \mathbf{w}^{\prime}\right)=e_{r}^{\prime}$, it follows from (I) and Lemma 23.2 that $\left(e_{r}, e_{r}^{\prime}\right)=(3,1)$. If both $r \geqslant 1$ and $\left(f_{0}, \ldots, f_{r-1}\right) \neq(0, \ldots, 0)$ hold, then the contradiction $e_{r} \leqslant 2$ follows from $(\mathrm{V})$. Therefore either $r=0$ or $\left(f_{0}, \ldots, f_{r-1}\right)=(0, \ldots, 0)$, whence the identity $\mathbf{w} \approx \mathbf{w}^{\prime}$ is either

$$
x_{0}^{3} x_{1} \ldots x_{m} \approx x_{0} x_{1} \ldots x_{m} \quad \text { or } \quad x_{0} \ldots x_{r-1} x_{r}^{3} x_{r+1} \ldots x_{m} \approx x_{0} \ldots x_{m} .
$$

In both cases, $S$ satisfies the identity (23.2), which is impossible by Lemma 23.3. 
Case 2: $e_{i}>e_{i}^{\prime}$ for some $i<r$. Since $f_{i}=f_{i}^{\prime}$ by part (i) with $\operatorname{occ}\left(x_{i}, \mathbf{w}\right)=e_{i}+f_{i}$ and $\operatorname{occ}\left(x_{i}, \mathbf{w}^{\prime}\right)=e_{i}^{\prime}+f_{i}^{\prime}$, it follows from (I) and Lemma 23.2 that $\left(e_{i}, e_{i}^{\prime}\right)=(3,1)$. Let $\varphi: \mathcal{X} \rightarrow S$ denote the substitution given in part (i). Then

$$
4 \cdot \mathbf{w} \varphi \cdot 6=4 \cdot\left(x_{0}^{e_{0}} \ldots x_{i-1}^{e_{i-1}}\right) \varphi \cdot 2^{3} \cdot\left(x_{i+1}^{e_{i+1}} \ldots x_{r}^{e_{r}}\right) \varphi \ldots=4 \cdot 1 \cdot 6 \ldots=1
$$

and

$$
4 \cdot \mathbf{w}^{\prime} \varphi \cdot 6=4 \cdot\left(x_{0}^{e_{0}^{\prime}} \ldots x_{i-1}^{e_{i-1}^{\prime}}\right) \varphi \cdot 2 \cdot\left(x_{i+1}^{e_{i+1}^{\prime}} \ldots x_{r}^{e_{r}^{\prime}}\right) \varphi \ldots=4 \cdot 2 \cdot 6 \ldots=3,
$$

which is impossible.

Proof of Proposition 23.1. Let $S \in\left\{\mathscr{E}_{8}, \mathscr{E}_{9}\right\}$. It is routinely checked that $S$ satisfies the identities (23.1). Hence it suffices to show that any nontrivial identity $\mathbf{w} \approx \mathbf{w}^{\prime}$ satisfied by $S$ is implied by the identities (23.1). By Lemma 23.6, the words $\mathbf{w}$ and $\mathbf{w}^{\prime}$ are non-simple. Therefore by Lemma 23.5 , there exist words $\overline{\mathbf{w}}$ and $\overline{\mathbf{w}}^{\prime}$ in canonical form such that the identities (23.1) imply the identities $\mathbf{w} \approx \overline{\mathbf{w}}$ and $\mathbf{w}^{\prime} \approx \overline{\mathbf{w}}^{\prime}$. Since ini $(\overline{\mathbf{w}})=\operatorname{ini}\left(\overline{\mathbf{w}}^{\prime}\right)$ by Lemma 23.2 ,

$$
\overline{\mathbf{w}}=x_{0}^{e_{0}} \ldots x_{r}^{e_{r}} \cdot x_{0}^{f_{0}} \ldots x_{r-1}^{f_{r-1}} \cdot x_{r+1} \ldots x_{m}
$$

and

$$
\overline{\mathbf{w}}^{\prime}=x_{0}^{e_{0}^{\prime}} \ldots x_{r^{\prime}}^{e_{r^{\prime}}^{\prime}} \cdot x_{0}^{f_{0}^{\prime}} \ldots x_{r^{\prime}-1}^{f_{r^{\prime}-1}^{\prime}} \cdot x_{r^{\prime}+1} \ldots x_{m} .
$$

Then $r=r^{\prime}$ by Lemma 23.7, and $\overline{\mathbf{w}}=\overline{\mathbf{w}}^{\prime}$ by Lemma 23.8. Consequently, the identities (23.1) imply the identity $\mathbf{w} \approx \mathbf{w}^{\prime}$.

23.2. Finite basis property of $\mathscr{E}_{10}$ and $\mathscr{F}_{5}$

Proposition 23.9. The variety generated by $S \in\left\{\mathscr{E}_{10}, \mathscr{F}_{5}\right\}$ is defined by the identities

$$
\begin{aligned}
x^{3} \approx x^{2}, \quad x^{2} y x & \approx x y x, \quad x y x^{2} \approx x y x, \\
h x^{2} k y^{2} t z^{2} & \approx h y^{2} t x^{2} k z^{2} .
\end{aligned}
$$

Lemma 23.10. For all $\mathbf{h}, \mathbf{x}, \mathbf{y}, \mathbf{z} \in \mathcal{X}^{+}$such that $\mathrm{h}(\mathbf{x}), \mathrm{h}(\mathbf{y}), \mathrm{h}(\mathbf{z}) \notin \operatorname{sim}(\mathbf{h x y z})$, the identities (23.4) imply the identity

$$
\text { hxyz } \approx \text { hyxz. }
$$

Proof. If $x=\mathrm{h}(\mathbf{x}), y=\mathrm{h}(\mathbf{y})$, and $z=\mathrm{h}(\mathbf{z})$ are non-simple letters of $\mathbf{h x y z}$, then

$$
\mathbf{h x y z} \stackrel{(23.4 \mathrm{a})}{\approx} \mathbf{h} x^{2} \mathbf{x} y^{2} \mathbf{y} z^{2} \mathbf{z} \stackrel{(23.4 \mathrm{~b})}{\approx} \mathbf{h} y^{2} \mathbf{y} x^{2} \mathbf{x} z^{2} \mathbf{z} \stackrel{(23.4 \mathrm{a})}{\approx} \mathbf{h y x z}
$$

Hence the identities (23.4) imply the identity (23.5).

For any word $\mathbf{w}$, let $\mathrm{F}_{\mathrm{NS}}(\mathbf{w})$ denote the set of factors of $\mathbf{w}$ of length two that begin with a non-simple letter and end with a simple letter:

$$
\mathrm{F}_{\mathrm{NS}}(\mathbf{w})=\left\{x y \in \mathcal{X}^{2} \mid \mathbf{w} \in \mathcal{X}^{*} x y \mathcal{X}^{*}, x \notin \operatorname{sim}(\mathbf{w}), y \in \operatorname{sim}(\mathbf{w})\right\}
$$

Lemma 23.11. Let $S \in\left\{\mathscr{E}_{10}, \mathscr{F}_{5}\right\}$. Suppose that $\mathbf{w} \approx \mathbf{w}^{\prime}$ is any identity satisfied by $S$. Then:

(i) $\operatorname{con}(\mathbf{w})=\operatorname{con}\left(\mathbf{w}^{\prime}\right), \operatorname{sim}(\mathbf{w})=\operatorname{sim}\left(\mathbf{w}^{\prime}\right), \mathrm{h}(\mathbf{w})=\mathrm{h}\left(\mathbf{w}^{\prime}\right)$, and $\mathrm{t}(\mathbf{w})=\mathrm{t}\left(\mathbf{w}^{\prime}\right)$;

(ii) $\mathrm{F}_{\mathrm{NS}}(\mathbf{w})=\mathrm{F}_{\mathrm{NS}}\left(\mathbf{w}^{\prime}\right)$;

(iii) $\mathrm{F}_{\mathrm{SS}}(\mathbf{w})=\mathrm{F}_{\mathrm{SS}}\left(\mathbf{w}^{\prime}\right)$. 
Proof. (i) This follows from Lemma 2.1 since the subsemigroups $\{1,4\},\{5,6\}$, and $\{1,3,5\}$ of $\mathscr{E}_{10}$ are isomorphic to $L_{2}, R_{2}$, and $N_{2}^{1}$, respectively, while the subsemigroups $\{1,3\},\{5,6\}$, and $\{1,2,5\}$ of $\mathscr{F}_{5}$ are isomorphic to $L_{2}, R_{2}$, and $N_{2}^{1}$, respectively.

(ii) Suppose $\mathrm{F}_{\mathrm{NS}}(\mathbf{w}) \neq \mathrm{F}_{\mathrm{NS}}\left(\mathbf{w}^{\prime}\right)$, say $x y \in \mathrm{F}_{\mathrm{NS}}(\mathbf{w}) \backslash \mathrm{F}_{\mathrm{NS}}\left(\mathbf{w}^{\prime}\right)$. Then it follows from part (i) that $x$ is non-simple in $\mathbf{w}^{\prime}$ and $y$ is simple in $\mathbf{w}^{\prime}$, whence $x y$ is not a factor of $\mathbf{w}^{\prime}$. Let $\varphi_{1}$ and $\varphi_{2}$ denote the following substitutions into $\mathscr{E}_{10}$ and $\mathscr{F}_{5}$, respectively:

$$
z \mapsto\left\{\begin{array} { l l } 
{ 5 } & { \text { if } z = x , } \\
{ 2 } & { \text { if } z = y , } \\
{ 6 } & { \text { otherwise; } }
\end{array} \quad z \mapsto \left\{\begin{array}{ll}
5 & \text { if } z=x, \\
4 & \text { if } z=y, \\
6 & \text { otherwise. }
\end{array}\right.\right.
$$

Then the following contradictions are obtained:

$$
\begin{aligned}
& \mathbf{w} \varphi_{1} \in\{5,6\}^{*} \cdot 5 \cdot 2 \cdot\{5,6\}^{*}=\{1\} \quad \text { and } \quad \mathbf{w}^{\prime} \varphi_{1} \in\{5,6\}^{*} \cdot 6 \cdot 2 \cdot\{5,6\}^{*}=\{3\} ; \\
& \mathbf{w} \varphi_{2} \in\{5,6\}^{*} \cdot 5 \cdot 4 \cdot\{5,6\}^{*}=\{1\} \quad \text { and } \quad \mathbf{w}^{\prime} \varphi_{2} \in\{5,6\}^{*} \cdot 6 \cdot 4 \cdot\{5,6\}^{*}=\{2\} .
\end{aligned}
$$

Hence $\mathrm{F}_{\mathrm{NS}}(\mathbf{w})=\mathrm{F}_{\mathrm{NS}}\left(\mathbf{w}^{\prime}\right)$.

(iii) Suppose $\mathrm{F}_{\mathrm{SS}}(\mathbf{w}) \neq \mathrm{F}_{\mathrm{SS}}\left(\mathbf{w}^{\prime}\right)$, say $x y \in \mathrm{F}_{\mathrm{SS}}(\mathbf{w}) \backslash \mathrm{F}_{\mathrm{SS}}\left(\mathbf{w}^{\prime}\right)$. Then $x, y \in \operatorname{sim}(\mathbf{w})=\operatorname{sim}\left(\mathbf{w}^{\prime}\right)$ by part (i) so that $x y \in \mathrm{F}_{\mathrm{NS}}(x \mathbf{w}) \backslash \mathrm{F}_{\mathrm{NS}}\left(x \mathbf{w}^{\prime}\right)$. Therefore by part (ii), the semigroup $S$ does not satisfy the identity $x \mathbf{w} \approx x \mathbf{w}^{\prime}$, but this is impossible.

For any non-simple word $\mathbf{w}$, define $\overrightarrow{\mathbf{w}}=y_{1}^{2} \ldots y_{k}^{2}$ where $y_{1}, \ldots, y_{k}$ are precisely all the distinct non-simple letters of $\mathbf{w}$ listed in alphabetical order. In this subsection, a non-simple word $\mathbf{w}$ is said to be in canonical form if it can be written as

$$
\mathbf{w}=\mathbf{w}_{0} \cdot \overrightarrow{\mathbf{w}} \cdot x_{1} \mathbf{w}_{1} \ldots x_{m} \mathbf{w}_{m},
$$

where $\mathbf{w}_{0}, \ldots, \mathbf{w}_{m} \in \mathcal{X}^{+}$and $x_{1}, \ldots, x_{m} \in \mathcal{X}$ satisfy the following:

(I) the letters of $\mathbf{w}_{0}, \ldots, \mathbf{w}_{m}$ are precisely all simple letters of $\mathbf{w}$;

(II) the letters $x_{1}, \ldots, x_{m}$ are non-simple in $\mathbf{w}$;

(III) the letters $x_{1}, \ldots, x_{m-1}$ are in alphabetical order.

Note that the letters $x_{1}, \ldots, x_{m}$ need not be distinct, but it follows from (I) that the words $x_{1} \mathbf{w}_{1}, \ldots, x_{m} \mathbf{w}_{m}$ are distinct.

Lemma 23.12. Let $\mathbf{w}$ be any non-simple word such that $\mathrm{h}(\mathbf{w}), \mathrm{t}(\mathbf{w}) \in \operatorname{sim}(\mathbf{w})$. Then there exists some word $\overline{\mathbf{w}}$ in canonical form such that the identities (23.4) imply the identity $\mathbf{w} \approx \overline{\mathbf{w}}$.

Proof. By Lemma 23.10, it suffices to convert w, using the identities $\{(23.4),(23.5)\}$, into a word in canonical form. Consider a factorization of $\mathbf{w}$ that displays all of its non-simple letters individually, that is, $\mathbf{w}=\mathbf{w}_{0} x_{1} \mathbf{w}_{1} \ldots x_{r} \mathbf{w}_{r}$ where the letters $x_{1}, \ldots, x_{r}$ are non-simple in $\mathbf{w}$ and the letters in the factors $\mathbf{w}_{0}, \ldots, \mathbf{w}_{r} \in \mathcal{X}^{*}$ are simple in $\mathbf{w}$. The assumption $\mathrm{h}(\mathbf{w}), \mathrm{t}(\mathbf{w}) \in$ $\operatorname{sim}(\mathbf{w})$ implies $\mathbf{w}_{0}, \mathbf{w}_{r} \neq \emptyset$. Further, each letter in the list $x_{1}, \ldots, x_{r}$ is non-simple in $\mathbf{w}$ and thus appears at least twice in the list, whence $r \geqslant 2$.

Now the words $x_{1} \mathbf{w}_{1}, \ldots, x_{r} \mathbf{w}_{r}$ begin with non-simple letters of $\mathbf{w}$. Therefore by applying the identity (23.5) sufficiently many times, the factors

$$
x_{1} \mathbf{w}_{1}, \ldots, x_{r-1} \mathbf{w}_{r-1}
$$

of $\mathbf{w}$ can be rearranged in any manner. In particular, the factors from (23.7) with nonempty $\mathbf{w}_{i}$ can be gathered to the right, resulting in a word of the form

$$
\mathbf{w}_{0}\left(\prod_{i \in I_{1}} x_{i}\right)\left(\prod_{i \in I_{2}}\left(x_{i} \mathbf{w}_{i}\right)\right) x_{r} \mathbf{w}_{r}
$$


for some $I_{1}$ and $I_{2}$ such that $I_{1} \cup I_{2}=\{1, \ldots, r-1\}$ and $I_{1} \cap I_{2}=\emptyset$. Hence

$$
\begin{aligned}
& \mathbf{w} \stackrel{(23.5)}{\approx} \mathbf{w}_{0}\left(\prod_{i \in I_{1}} x_{i}\right)\left(\prod_{i \in I_{2}}\left(x_{i} \mathbf{w}_{i}\right)\right) x_{r} \mathbf{w}_{r} \stackrel{(23.4 \mathrm{a})}{\approx} \mathbf{w}_{0}\left(\prod_{i \in I_{1}} x_{i}^{2}\right)\left(\prod_{i \in I_{2}}\left(x_{i}^{2} x_{i} \mathbf{w}_{i}\right)\right) x_{r}^{2} x_{r} \mathbf{w}_{r} \\
& \stackrel{(23.5)}{\approx} \mathbf{w}_{0}\left(\prod_{i=1}^{r} x_{i}^{2}\right)\left(\prod_{i \in I_{2}}\left(x_{i} \mathbf{w}_{i}\right)\right) x_{r} \mathbf{w}_{r} .
\end{aligned}
$$

The identities $\{(23.4),(23.5)\}$ can be used to convert the factor $\prod_{i=1}^{r} x_{i}^{2}$ of the latter word into $\overrightarrow{\mathbf{w}}$, resulting in a word of the form (23.6) with (I) and (II) satisfied. The identities (23.5) can then be used to rearrange the factors $x_{i} \mathbf{w}_{i}$ in $\prod_{i \in I_{2}}\left(x_{i} \mathbf{w}_{i}\right)$ until (III) is satisfied.

Let $\mathbf{X}_{(23.4)}$ denote the variety defined by the identities (23.4).

Lemma 23.13. Suppose that $\mathbf{w}, \mathbf{w}^{\prime} \in \mathcal{X}^{+}$and $x, y \in \mathcal{X}$ are such that $\operatorname{con}(\mathbf{w})=\operatorname{con}\left(\mathbf{w}^{\prime}\right)$, $\operatorname{sim}(\mathbf{w})=\operatorname{sim}\left(\mathbf{w}^{\prime}\right), h=\mathrm{h}(\mathbf{w})=\mathrm{h}\left(\mathbf{w}^{\prime}\right), t=\mathrm{t}(\mathbf{w})=\mathrm{t}\left(\mathbf{w}^{\prime}\right)$, and $x, y \notin \operatorname{con}(\mathbf{w})=\operatorname{con}\left(\mathbf{w}^{\prime}\right)$. Then $\mathbf{X}_{(23.4)}\left\{\mathbf{w} \approx \mathbf{w}^{\prime}\right\}=\mathbf{X}_{(23.4)}\{\sigma\}$ where the identity

$$
\sigma \text { is } \begin{cases}x \mathbf{w} \approx x \mathbf{w}^{\prime} & \text { if } h \notin \operatorname{sim}(\mathbf{w})=\operatorname{sim}\left(\mathbf{w}^{\prime}\right) \text { and } t \in \operatorname{sim}(\mathbf{w})=\operatorname{sim}\left(\mathbf{w}^{\prime}\right), \\ \mathbf{w} y \approx \mathbf{w}^{\prime} y & \text { if } h \in \operatorname{sim}(\mathbf{w})=\operatorname{sim}\left(\mathbf{w}^{\prime}\right) \text { and } t \notin \operatorname{sim}(\mathbf{w})=\operatorname{sim}\left(\mathbf{w}^{\prime}\right), \\ x \mathbf{w} y \approx x \mathbf{w}^{\prime} y & \text { if } h, t \notin \operatorname{sim}(\mathbf{w})=\operatorname{sim}\left(\mathbf{w}^{\prime}\right) .\end{cases}
$$

Proof. It suffices to consider the case when $h \notin \operatorname{sim}(\mathbf{w})$ and $t \in \operatorname{sim}(\mathbf{w})$ since the other two cases can be established similarly. Let $\sigma$ denote the identity $x \mathbf{w} \approx x \mathbf{w}^{\prime}$. Then the inclusion $\mathbf{X}_{(23.4)}\left\{\mathbf{w} \approx \mathbf{w}^{\prime}\right\} \subseteq \mathbf{X}_{(23.4)}\{\sigma\}$ holds trivially. Conversely, since the words $\mathbf{w}$ and $\mathbf{w}^{\prime}$ begin with the non-simple letter $h$,

$$
\mathbf{w} \stackrel{(23.4 \mathrm{a})}{\approx} h \mathbf{w} \stackrel{\sigma}{\approx} h \mathbf{w}^{\prime} \stackrel{(23.4 a)}{\approx} \mathbf{w}^{\prime}
$$

Hence the inclusion $\mathbf{X}_{(23.4)}\{\sigma\} \subseteq \mathbf{X}_{(23.4)}\left\{\mathbf{w} \approx \mathbf{w}^{\prime}\right\}$ holds.

Proof of Proposition 23.9. Let $S \in\left\{\mathscr{E}_{10}, \mathscr{F}_{5}\right\}$. It is routinely verified that $S$ satisfies the identities (23.4). Therefore there exists some set $\Sigma$ of identities satisfied by $S$ such that $\{(23.4)\} \cup \Sigma$ is a basis for $S$. If $\mathbf{w} \approx \mathbf{w}^{\prime}$ is an identity in $\Sigma$ such that either $\mathbf{w}$ or $\mathbf{w}^{\prime}$ is a simple word, then it follows from Lemma 23.11(i) that the identity $\mathbf{w} \approx \mathbf{w}^{\prime}$ is trivial. Hence generality is not lost by assuming that all identities in $\Sigma$ are formed by non-simple words.

Let $\mathbf{w} \approx \mathbf{w}^{\prime}$ be any identity from $\Sigma$. By Lemma 23.11(i),

(a) $\operatorname{con}(\mathbf{w})=\operatorname{con}\left(\mathbf{w}^{\prime}\right), \operatorname{sim}(\mathbf{w})=\operatorname{sim}\left(\mathbf{w}^{\prime}\right), \mathrm{h}(\mathbf{w})=\mathrm{h}\left(\mathbf{w}^{\prime}\right)$, and $\mathrm{t}(\mathbf{w})=\mathrm{t}\left(\mathbf{w}^{\prime}\right)$.

By Lemma 23.13, the words forming the identities in $\Sigma$ can be chosen to begin and end with simple letters. Therefore by Lemma 23.12,

(b) the words forming the identities in $\Sigma$ can be chosen to be in canonical form.

It then follows from (a) and (b) that $\mathbf{w}$ and $\mathbf{w}^{\prime}$ are of the form

$$
\mathbf{w}=\mathbf{w}_{0} \cdot \overrightarrow{\mathbf{w}} \cdot x_{1} \mathbf{w}_{1} \ldots x_{m} \mathbf{w}_{m} \quad \text { and } \quad \mathbf{w}^{\prime}=\mathbf{w}_{0}^{\prime} \cdot \overrightarrow{\mathbf{w}} \cdot y_{1} \mathbf{w}_{1}^{\prime} \ldots y_{n} \mathbf{w}_{n}^{\prime},
$$

where $\operatorname{con}\left(\mathbf{w}_{0} \ldots \mathbf{w}_{m}\right)=\operatorname{sim}(\mathbf{w})=\operatorname{sim}\left(\mathbf{w}^{\prime}\right)=\operatorname{con}\left(\mathbf{w}_{0}^{\prime} \ldots \mathbf{w}_{n}^{\prime}\right), \mathrm{h}\left(\mathbf{w}_{0}\right)=\mathrm{h}\left(\mathbf{w}_{0}^{\prime}\right), \mathrm{t}\left(\mathbf{w}_{m}\right)=\mathrm{t}\left(\mathbf{w}_{n}^{\prime}\right)$, and $x_{1}, \ldots, x_{m}, y_{1}, \ldots, y_{n} \in \operatorname{con}(\overrightarrow{\mathbf{w}})$. Then by Lemma 23.11:

(c) $\mathbf{w}_{0}=\mathbf{w}_{0}^{\prime}$;

(d) $x_{m} \mathbf{w}_{m}=y_{n} \mathbf{w}_{n}^{\prime}$;

(e) $\left\{x_{1} \mathbf{w}_{1}, \ldots, x_{m-1} \mathbf{w}_{m-1}\right\}=\left\{y_{1} \mathbf{w}_{1}^{\prime}, \ldots, y_{n-1} \mathbf{w}_{n-1}^{\prime}\right\}$.

Therefore $\left(x_{1} \mathbf{w}_{1}, \ldots, x_{m-1} \mathbf{w}_{m-1}\right)=\left(y_{1} \mathbf{w}_{1}^{\prime}, \ldots, y_{n-1} \mathbf{w}_{n-1}^{\prime}\right)$ by (c)-(e) and (III), whence the identity $\mathbf{w} \approx \mathbf{w}^{\prime}$ is trivial. Since the identity $\mathbf{w} \approx \mathbf{w}^{\prime}$ is arbitrary in $\Sigma$, the identities (23.4) constitute a basis for $S$. 


$$
\text { 24. } \mathscr{F}_{1}, \mathscr{F}_{2}
$$

This section establishes the finite basis property of the following semigroups.

\begin{tabular}{c|cccccc}
$\mathscr{F}_{1}$ & 1 & 2 & 3 & 4 & 5 & 6 \\
\hline 1 & 1 & 1 & 1 & 1 & 1 & 1 \\
2 & 1 & 1 & 1 & 1 & 2 & 2 \\
3 & 1 & 1 & 1 & 1 & 3 & 3 \\
4 & 4 & 4 & 4 & 4 & 4 & 4 \\
5 & 1 & 1 & 3 & 4 & 5 & 6 \\
6 & 1 & 3 & 3 & 4 & 5 & 6
\end{tabular}

\begin{tabular}{c|cccccc}
$\mathscr{F}_{2}$ & 1 & 2 & 3 & 4 & 5 & 6 \\
\hline 1 & 1 & 1 & 1 & 1 & 1 & 1 \\
2 & 1 & 1 & 1 & 1 & 2 & 2 \\
3 & 1 & 1 & 2 & 1 & 3 & 3 \\
4 & 4 & 4 & 4 & 4 & 4 & 4 \\
5 & 1 & 2 & 3 & 4 & 5 & 6 \\
6 & 1 & 2 & 3 & 4 & 5 & 6
\end{tabular}

\subsection{Finite basis property of $\mathscr{F}_{1}$}

Proposition 24.1. The variety generated by $\mathscr{F}_{1}$ is defined by the identities

$$
\begin{aligned}
x^{3} \approx x^{2}, \quad x^{2} y x & \approx x y x, \quad x y x^{2} \approx x y x, \\
x y x z^{2} & \approx x^{2} y z^{2} .
\end{aligned}
$$

Lemma 24.2. Suppose that $\mathbf{w} \approx \mathbf{w}^{\prime}$ is any identity satisfied by $\mathscr{F}_{1}$. Then $\operatorname{ini}(\mathbf{w})=\operatorname{ini}\left(\mathbf{w}^{\prime}\right)$, $\operatorname{sim}(\mathbf{w})=\operatorname{sim}\left(\mathbf{w}^{\prime}\right)$, and $\mathrm{t}(\mathbf{w})=\mathrm{t}\left(\mathbf{w}^{\prime}\right)$.

Proof. This follows from Lemma 2.1 since the subsemigroups $\{1,4,6\},\{1,3,5\}$, and $\{5,6\}$ of $\mathscr{F}_{1}$ are isomorphic to $L_{2}^{1}, N_{2}^{1}$, and $R_{2}$, respectively.

In this subsection, a word $\mathbf{w}$ with $\operatorname{ini}(\mathbf{w})=x_{1} \ldots x_{m}$ is said to be in canonical form if

$$
\mathbf{w}=\prod_{i=1}^{m}\left(x_{i}^{e_{i}} \mathbf{w}_{i}\right),
$$

where all of the following are satisfied:

(I) $\mathbf{w}_{i} \in\left\{\emptyset, x_{1}, \ldots, x_{i-1}\right\}$;

(II) $e_{1}, \ldots, e_{m} \in\{1,2\}$;

(III) if $x_{i} \notin \operatorname{sim}(\mathbf{w})$, then $\mathbf{w}_{i-1}=\emptyset$;

(IV) if $x_{i} \notin \operatorname{sim}(\mathbf{w})$ and $x_{i}=\mathbf{w}_{j}$ for some $j>i$, then $e_{i}=1$.

Note that (I) and (IV) imply that the word $\mathbf{w}$ in (24.2) does not contain a factor of the form $x^{2} \mathbf{a} x$ where $\mathbf{a} \in \mathcal{X}^{*}$.

Lemma 24.3. Let $\mathbf{w}$ be any word. Then there exists some word $\overline{\mathbf{w}}$ in canonical form such that the identities (24.1) imply the identity $\mathbf{w} \approx \overline{\mathbf{w}}$.

Proof. It suffices to convert $\mathbf{w}$, using the identities (24.1), into a word in canonical form. Suppose that $x$ and $y$ are non-simple letters of $\mathbf{w}$ such that $\mathbf{w}=\mathbf{a} x \mathbf{b} x y \mathbf{c}$ for some $\mathbf{a}, \mathbf{b}, \mathbf{c} \in \mathcal{X}^{*}$ with $y \in \operatorname{con}(\mathbf{a b c})$. Then

$$
\mathbf{w} \stackrel{(24.1 \mathrm{a})}{\approx} \mathbf{a} x \mathbf{b} x y^{2} \mathbf{c} \stackrel{(24.1 \mathrm{~b})}{\approx} \mathbf{a} x^{2} \mathbf{b} y^{2} \mathbf{c} \stackrel{(24.1 \mathrm{a})}{\approx} \mathbf{a} x^{2} \mathbf{b} y \mathbf{c}
$$

that is, using the identities (24.1), any non-first occurrence of $x$ in $\mathbf{w}$ that immediately precedes another non-simple letter can be gathered with the first $x$ in $\mathbf{w}$. It follows that $\mathbf{w}$ can be converted into a word of the form (24.2) with (I) and (III) satisfied. Then (II) and (IV) are satisfied by applying the identities (24.1a). 
Lemma 24.4. Suppose that $\mathscr{F}_{1}$ satisfies an identity $\mathbf{w} \approx \mathbf{w}^{\prime}$, where

$$
\mathbf{w}=\prod_{i=1}^{m}\left(x_{i}^{e_{i}} \mathbf{w}_{i}\right) \quad \text { and } \quad \mathbf{w}^{\prime}=\prod_{i=1}^{m}\left(x_{i}^{e_{i}^{\prime}} \mathbf{w}_{i}^{\prime}\right)
$$

are words in canonical form. Then $e_{i}=e_{i}^{\prime}$ for all $i$.

Proof. Observe that $\operatorname{occ}\left(x_{m}, \mathbf{w}\right)=e_{m}$ and $\operatorname{occ}\left(x_{m}, \mathbf{w}^{\prime}\right)=e_{m}^{\prime}$ by (I) and that

(a) $\operatorname{sim}(\mathbf{w})=\operatorname{sim}\left(\mathbf{w}^{\prime}\right)$

by Lemma 24.2. Therefore $e_{m}=e_{m}^{\prime}$ by (II). Seeking a contradiction, suppose $e_{r} \neq e_{r}^{\prime}$ for some $r<m$, say $\left(e_{r}, e_{r}^{\prime}\right)=(1,2)$. Then $x_{r} \notin \operatorname{sim}\left(\mathbf{w}^{\prime}\right)$, whence $x_{r} \notin \operatorname{sim}(\mathbf{w})$ by (a). Since $\mathbf{w}$ is in canonical form, the second $x_{r}$ in $\mathbf{w}$ coincides with one of $\mathbf{w}_{r+1}, \ldots, \mathbf{w}_{m}$, say $x_{r}=\mathbf{w}_{\ell}$. Then $x_{r} \neq x_{\ell}$ by $(\mathrm{I})$, and the word $\mathbf{w}$ can be written as

$$
\mathbf{w}=\mathbf{p} x_{r} \mathbf{w}_{r} \mathbf{q} x_{\ell}^{e_{\ell}} \underbrace{x_{r}}_{\mathbf{w}_{\ell}} \mathbf{u},
$$

where $\mathbf{p}=\prod_{i=1}^{r-1}\left(x_{i}^{e_{i}} \mathbf{w}_{i}\right), \mathbf{q}=\prod_{i=r+1}^{\ell-1}\left(x_{i}^{e_{i}} \mathbf{w}_{i}\right)$, and $\mathbf{u}=\prod_{i=\ell+1}^{m}\left(x_{i}^{e_{i}} \mathbf{w}_{i}\right)$ with

(b) $x_{r} \notin \operatorname{con}\left(\mathbf{p w}_{r} \mathbf{q} x_{\ell}\right)$.

As for the word $\mathbf{w}^{\prime}$, since $e_{r}^{\prime}=2$, it can be written as

$$
\mathbf{w}^{\prime}=\mathbf{p}^{\prime} x_{r}^{2} \mathbf{w}_{r}^{\prime} \mathbf{q}^{\prime} x_{\ell}^{e_{\ell}^{\prime}} \mathbf{w}_{\ell}^{\prime} \mathbf{u}^{\prime},
$$

where $\mathbf{p}^{\prime}=\prod_{i=1}^{r-1}\left(x_{i}^{e_{i}^{\prime}} \mathbf{w}_{i}^{\prime}\right), \mathbf{q}^{\prime}=\prod_{i=r+1}^{\ell-1}\left(x_{i}^{e_{i}^{\prime}} \mathbf{w}_{i}^{\prime}\right)$, and $\mathbf{u}^{\prime}=\prod_{i=\ell+1}^{m}\left(x_{i}^{e_{i}^{\prime}} \mathbf{w}_{i}^{\prime}\right)$ with $x_{r} \notin \operatorname{con}\left(\mathbf{p}^{\prime}\right)$ and $x_{\ell} \notin \operatorname{con}\left(\mathbf{p}^{\prime} x_{r} \mathbf{w}_{r}^{\prime} \mathbf{q}^{\prime}\right)$. In fact, it follows from (I) and (IV) that

(c) $x_{r} \notin \operatorname{con}\left(\mathbf{p}^{\prime} \mathbf{w}_{r}^{\prime} \mathbf{q}^{\prime} x_{\ell} \mathbf{w}_{\ell}^{\prime} \mathbf{u}^{\prime}\right)$.

In particular, $\mathrm{t}\left(\mathbf{w}^{\prime}\right) \neq x_{r}$. Since $\mathrm{t}(\mathbf{w})=\mathrm{t}\left(\mathbf{w}^{\prime}\right)$ by Lemma 24.2 , it follows that $\mathbf{u} \neq \emptyset$, whence

(d) $\mathrm{h}(\mathbf{u})=x_{\ell+1} \in \operatorname{sim}(\mathbf{w})$

by (III), and

(e) $\mathrm{h}\left(\mathbf{u}^{\prime}\right)=x_{\ell+1} \in \operatorname{sim}\left(\mathbf{w}^{\prime}\right)$

by (a). Let $\varphi: \mathcal{X} \rightarrow \mathscr{F}_{1}$ denote the substitution

$$
z \mapsto \begin{cases}6 & \text { if } z=x_{r}, \\ 2 & \text { if } z=x_{\ell+1}, \\ 5 & \text { otherwise. }\end{cases}
$$

Then $\mathbf{u} \varphi=2$ and $\mathbf{u}^{\prime} \varphi=2$ by (d) and (e). Since $x_{r}, x_{\ell+1} \notin \operatorname{con}\left(\mathbf{p} \mathbf{w}_{r} \mathbf{q} x_{\ell}\right)$ by (b) and (d), it follows that $5 \cdot \mathbf{p} \varphi=\left(\mathbf{w}_{r} \mathbf{q} x_{\ell}^{e_{\ell}}\right) \varphi=5$. Thus

$$
5 \cdot \mathbf{w} \varphi=(5 \cdot \mathbf{p} \varphi) \cdot x_{r} \varphi \cdot\left(\mathbf{w}_{r} \mathbf{q} x_{\ell}^{e_{\ell}}\right) \varphi \cdot x_{r} \varphi \cdot \mathbf{u} \varphi=5 \cdot 6 \cdot 5 \cdot 6 \cdot 2=3 .
$$

Similarly, $x_{r}, x_{\ell+1} \notin \operatorname{con}\left(\mathbf{p}^{\prime} \mathbf{w}_{r}^{\prime} \mathbf{q}^{\prime} x_{\ell} \mathbf{w}_{\ell}^{\prime}\right)$ by (c) and (e), so $5 \cdot \mathbf{p}^{\prime} \varphi=\left(\mathbf{w}_{r}^{\prime} \mathbf{q}^{\prime} x_{\ell}^{e_{\ell}^{\prime}} \mathbf{w}_{\ell}^{\prime}\right) \varphi=5$. Hence

$$
5 \cdot \mathbf{w}^{\prime} \varphi=\left(5 \cdot \mathbf{p}^{\prime} \varphi\right) \cdot x_{r}^{2} \varphi \cdot\left(\mathbf{w}_{r}^{\prime} \mathbf{q}^{\prime} x_{\ell}^{e_{\ell}^{\prime}} \mathbf{w}_{\ell}^{\prime}\right) \varphi \cdot \mathbf{u}^{\prime} \varphi=5 \cdot 6 \cdot 5 \cdot 2=1 .
$$

Consequently, the contradiction $5 \cdot \mathbf{w} \varphi \neq 5 \cdot \mathbf{w}^{\prime} \varphi$ is established.

Proof of Proposition 24.1. It is routinely checked that $\mathscr{F}_{1}$ satisfies the identities (24.1). Hence it suffices to show that any identity $\mathbf{w} \approx \mathbf{w}^{\prime}$ satisfied by $\mathscr{F}_{1}$ is implied by the identities (24.1). By Lemma 24.3, the words $\mathbf{w}$ and $\mathbf{w}^{\prime}$ can be assumed to be in canonical form. By Lemmas 24.2 and 24.4,

$$
\mathbf{w}=\prod_{i=1}^{m}\left(x_{i}^{e_{i}} \mathbf{w}_{i}\right) \quad \text { and } \quad \mathbf{w}^{\prime}=\prod_{i=1}^{m}\left(x_{i}^{e_{i}} \mathbf{w}_{i}^{\prime}\right)
$$


with $\mathbf{w}_{m}=\mathbf{w}_{m}^{\prime}$. Let $\ell<m$ be the least integer such that $\mathbf{w}_{\ell} \neq \mathbf{w}_{\ell}^{\prime}$. Then the words $\mathbf{w}$ and $\mathbf{w}^{\prime}$ share the prefix $\mathbf{p}=\prod_{i=1}^{\ell-1}\left(x_{i}^{e_{i}} \mathbf{w}_{i}\right)=\prod_{i=1}^{\ell-1}\left(x_{i}^{e_{i}} \mathbf{w}_{i}^{\prime}\right)$. Since $\left(\mathbf{w}_{\ell}, \mathbf{w}_{\ell}^{\prime}\right) \neq(\emptyset, \emptyset)$, generality is not lost by assuming $\mathbf{w}_{\ell} \neq \emptyset$. Then (I) implies $\mathbf{w}_{\ell}=x_{k}$ for some $k \in\{0, \ldots, \ell-1\}$ and

(a) $\mathbf{w}_{\ell}^{\prime} \in\left\{\emptyset, x_{0}, \ldots, x_{\ell-1}\right\} \backslash\left\{x_{k}\right\}$, whence

$$
\mathbf{w}=\mathbf{p} x_{\ell}^{e_{\ell}} \underbrace{x_{k}}_{\mathbf{w}_{\ell}} x_{\ell+1}^{e_{\ell+1}} \mathbf{q} \quad \text { and } \quad \mathbf{w}^{\prime}=\mathbf{p} x_{\ell}^{e_{\ell}} \mathbf{w}_{\ell}^{\prime} x_{\ell+1}^{e_{\ell+1}} \mathbf{q}^{\prime},
$$

where $\mathbf{q}=\mathbf{w}_{\ell+1} \prod_{i=\ell+2}^{m}\left(x_{i}^{e_{i}} \mathbf{w}_{i}\right)$ and $\mathbf{q}^{\prime}=\mathbf{w}_{\ell+1}^{\prime} \prod_{i=\ell+2}^{m}\left(x_{i}^{e_{i}} \mathbf{w}_{i}^{\prime}\right)$. The conditions $\mathbf{w}_{\ell} \neq \emptyset$ and (III) imply $x_{\ell+1} \in \operatorname{sim}(\mathbf{w})$; since $\operatorname{sim}(\mathbf{w})=\operatorname{sim}\left(\mathbf{w}^{\prime}\right)$ by Lemma 24.2,

(b) $x_{\ell+1} \in \operatorname{sim}(\mathbf{w})=\operatorname{sim}\left(\mathbf{w}^{\prime}\right)$ and $e_{\ell+1}=1$.

Let $\varphi: \mathcal{X} \rightarrow \mathscr{F}_{1}$ denote the substitution

$$
z \mapsto \begin{cases}6 & \text { if } z=x_{k}, \\ 2 & \text { if } z=x_{\ell+1} \\ 5 & \text { otherwise. }\end{cases}
$$

Since $x_{\ell+1} \notin \operatorname{con}\left(\mathbf{p} x_{\ell} \mathbf{q}\right)$ by (b), it follows that $\left(\mathbf{p} x_{\ell}^{e_{\ell}}\right) \varphi=5$ and $\mathbf{q} \varphi \in\{\emptyset, 5,6\}$. Thus

$$
\mathbf{w} \varphi=\left(\mathbf{p} x_{\ell}^{e_{\ell}}\right) \varphi \cdot x_{k} \varphi \cdot x_{\ell+1} \varphi \cdot \mathbf{q} \varphi=5 \cdot 6 \cdot 2 \cdot \mathbf{q} \varphi=3 .
$$

On the other hand, $\mathbf{w}_{\ell}^{\prime} \varphi \in\{\emptyset, 5\}$ by (a), so that

$$
\mathbf{w}^{\prime} \varphi=\left(\mathbf{p} x_{\ell}^{e_{\ell}}\right) \varphi \cdot \mathbf{w}_{\ell}^{\prime} \varphi \cdot x_{\ell+1} \varphi \cdot \mathbf{q}^{\prime} \varphi=5 \cdot \mathbf{w}_{\ell}^{\prime} \varphi \cdot 2 \cdot \mathbf{q}^{\prime} \varphi=1 .
$$

The contradiction $\mathbf{w} \varphi \neq \mathbf{w}^{\prime} \varphi$ implies that the integer $\ell$ does not exist. Consequently, $\mathbf{w}_{i}=\mathbf{w}_{i}^{\prime}$ for all $i$, whence the identity $\mathbf{w} \approx \mathbf{w}^{\prime}$ is trivial and is implied by the identities (24.1).

\subsection{Finite basis property of $\mathscr{F}_{2}$}

Proposition 24.5. The variety generated by $\mathscr{F}_{2}$ is defined by the identities

$$
\begin{aligned}
x^{4} & \approx x^{3}, \\
x^{3} y x & \approx x^{2} y x, \\
x y x z & \approx x^{2} y z .
\end{aligned}
$$

In this subsection, a word $\mathbf{w}$ is said to be in canonical form if

$$
\mathbf{w}=x_{1}^{e_{1}} \ldots x_{m}^{e_{m}} y
$$

where all of the following are satisfied:

(I) $e_{1}, \ldots, e_{m} \in\{1,2,3\}$;

(II) $x_{1}, \ldots, x_{m} \in \mathcal{X}$ are distinct;

(III) either (a) $y=\emptyset$ or (b) $y=x_{\ell}$ for some $\ell<m$ with $e_{\ell} \in\{1,2\}$.

Lemma 24.6. Let $\mathbf{w}$ be any word. Then there exists some word $\overline{\mathbf{w}}$ in canonical form such that the identities (24.3) imply the identity $\mathbf{w} \approx \overline{\mathbf{w}}$.

Proof. It suffices to convert $\mathbf{w}$, using the identities (24.3), into a word in canonical form. This is easily achieved if $|\operatorname{con}(\mathbf{w})|=1$. Hence assume $|\operatorname{con}(\mathbf{w})| \geqslant 2$. Then $\mathbf{w}=\mathbf{p} y$ for some 
$\mathbf{p} \in \mathcal{X}^{+}$with $y=\mathrm{t}(\mathbf{w})$. If a letter $x$ occurs more than once in $\mathbf{p}$, then the identity (24.3c) can be used to gather any non-first $x$ in $\mathbf{p}$ with the first $x$ in $\mathbf{p}$ :

$$
\mathbf{w}=\underbrace{\mathbf{a} x \mathbf{b} x \mathbf{c}}_{\mathbf{p}} y \stackrel{(24.3 \mathbf{c})}{\approx} \mathbf{a} x^{2} \mathbf{b c} y .
$$

Hence the identity (24.3c) can be used to convert $\mathbf{w}$ into a word of the form $\mathbf{w}^{\prime}=x_{1}^{e_{1}} \ldots x_{m}^{e_{m}} y$ that satisfies (II). If $y=x_{m}$, then $\mathbf{w}^{\prime}=x_{1}^{e_{1}} \ldots x_{m-1}^{e_{m-1}} x_{m}^{e_{m}+1}$ and the identity (24.3a) can be used to reduce the exponents $e_{1}, \ldots, e_{m-1}, e_{m}+1$ in $\mathbf{w}^{\prime}$ to numbers in $\{1,2,3\}$, whence (I) and (IIIa) are satisfied. If $y=x_{\ell}$ for some $\ell<m$, then (I) and (IIIb) are satisfied by applying the identities $\{(24.3 \mathrm{a}),(24.3 \mathrm{~b})\}$.

Proof of Proposition 24.5. It is routinely checked that $\mathscr{F}_{2}$ satisfies the identities (24.3). Therefore it suffices to show that any identity $\mathbf{w} \approx \mathbf{w}^{\prime}$ satisfied by $\mathscr{F}_{2}$ is implied by the identities (24.3). By Lemma 24.6, the words $\mathbf{w}$ and $\mathbf{w}^{\prime}$ can be assumed to be in canonical form. Since the subsemigroups $\{1,4,6\}$ and $\{5,6\}$ of $\mathscr{F}_{2}$ are isomorphic to $L_{2}^{1}$ and $R_{2}$, respectively, it follows from Lemma 2.1 that $\mathbf{w}=x_{1}^{e_{1}} \ldots x_{m}^{e_{m}} y$ and $\mathbf{w}^{\prime}=x_{1}^{e_{1}^{\prime}} \ldots x_{m}^{e_{m}^{\prime}} y$. Since the subsemigroup $\{1,2,3,5\}$ of $\mathscr{F}_{2}$ is isomorphic to $N_{3}^{1}$, it follows from (I), (III), and Lemma 2.1(vii) that $e_{i}=e_{i}^{\prime}$ for all $i$. Hence the identity $\mathbf{w} \approx \mathbf{w}^{\prime}$ is trivial and so is implied by the identities (24.3).

$$
\text { 25. } \mathscr{F}_{3}, \mathscr{F}_{4}
$$

This section establishes the finite basis property of the following semigroups.

\begin{tabular}{c|cccccc}
$\mathscr{F}_{3}$ & 1 & 2 & 3 & 4 & 5 & 6 \\
\hline 1 & 1 & 1 & 1 & 1 & 1 & 1 \\
2 & 1 & 1 & 1 & 1 & 2 & 2 \\
3 & 1 & 2 & 3 & 1 & 1 & 1 \\
4 & 4 & 4 & 4 & 4 & 4 & 4 \\
5 & 1 & 1 & 1 & 4 & 5 & 6 \\
6 & 4 & 4 & 4 & 1 & 6 & 5
\end{tabular}

\begin{tabular}{c|cccccc}
$\mathscr{F}_{4}$ & 1 & 2 & 3 & 4 & 5 & 6 \\
\hline 1 & 1 & 1 & 1 & 1 & 1 & 1 \\
2 & 1 & 1 & 1 & 1 & 2 & 2 \\
3 & 1 & 2 & 3 & 1 & 2 & 2 \\
4 & 4 & 4 & 4 & 4 & 4 & 4 \\
5 & 1 & 1 & 1 & 4 & 5 & 6 \\
6 & 4 & 4 & 4 & 1 & 6 & 5
\end{tabular}

Let $\mathbf{W}$ denote the variety generated by $\overleftarrow{J}$ and $O$, where $\overleftarrow{J}$ is the dual semigroup of $J$ and $O$ is the semigroup from Remark 6.6.

Lemma 25.1. The variety $\mathbf{W}$ is defined by the identities

$$
\begin{aligned}
x y^{3} & \approx x y, \\
x^{3} y & \approx x y x^{2}, \\
x y x y & \approx x y^{2} x .
\end{aligned}
$$

Proof. Let $\mathbf{X}_{(25.1)}$ denote the variety defined by the identities (25.1). It is routinely checked that $\overleftarrow{J}, O \in \mathbf{X}_{(25.1)}$. Results from Edmunds et al. $[\mathbf{9}, \S 6]$ (specifically, the proofs of Lemmas 6.3, 6.4, and 6.9-6.11) imply that any proper subvariety of $\mathbf{X}_{(25.1)}$ must satisfy either $x^{3} y \approx x y$ or $x y x \approx x^{2} y$; it is routinely verified that these two identities are not satisfied by $\overleftarrow{J}$ and $O$, respectively. Consequently, $\mathbf{W}=\mathbf{X}_{(25.1)}$.

A finite basis for the variety $\mathbf{A}_{\mathbf{0}} \vee \mathbf{W}$ is established in $\S 25.1$. It is then routinely checked that $\mathscr{F}_{4} \in \mathbf{A}_{\mathbf{0}} \vee \mathbf{W}$. But since the subsemigroups $\{1,2,3,5\},\{1,2,5\}$, and $\{1,4,5,6\}$ of $\mathscr{F}_{4}$ 
are isomorphic to $A_{0}, \overleftarrow{J}$, and $O$, respectively, the semigroup $\mathscr{F}_{4}$ generates the variety $\mathbf{A}_{\mathbf{0}} \vee \mathbf{W}$ and so is finitely based.

Similarly, a finite basis for the variety $\mathbf{B}_{\mathbf{0}} \vee \mathbf{W}$ is established in $\S 25.2$, from which the condition $\mathscr{F}_{3} \in \mathbf{B}_{\mathbf{0}} \vee \mathbf{W}$ can be routinely checked. The subsemigroups $\{1,2,3,5\},\{1,2,5\}$, and $\{1,4,5,6\}$ of $\mathscr{F}_{3}$ are isomorphic to $B_{0}, \overleftarrow{J}$, and $O$, respectively, whence $\mathscr{F}_{3}$ generates the variety $\mathbf{B}_{\mathbf{0}} \vee \mathbf{W}$ and is finitely based.

\subsection{A basis for $\mathbf{A}_{\mathbf{0}} \vee \mathbf{W}$}

Proposition 25.2. The variety $\mathbf{A}_{\mathbf{0}} \vee \mathbf{W}$ is defined by the identities

$$
\begin{aligned}
x \mathrm{H} x^{2} \mathrm{~K} x & \approx x \mathrm{HK} x, \\
x \mathrm{H} y \mathrm{~K} y^{2} \mathrm{~T} x & \approx x \mathrm{H} y \mathrm{KT} x, \\
x \mathrm{H} y \mathrm{~K} x^{2} \mathrm{~T} y & \approx x \mathrm{H} y \mathrm{KT} y x^{2}, \\
x \mathrm{H} y \mathrm{~K} x y & \approx x \mathrm{H} y \mathrm{~K} y x .
\end{aligned}
$$

Lemma 25.3. Suppose that $\mathbf{w}$ is any connected word. Then there exists some word $\widetilde{\mathbf{w}}$ with $\operatorname{con}(\mathbf{w})=\operatorname{con}(\widetilde{\mathbf{w}})$ and $\mathrm{h}(\mathbf{w})=\mathrm{h}(\widetilde{\mathbf{w}})=\mathrm{t}(\widetilde{\mathbf{w}})$ such that the identities (25.2) imply $\mathbf{w} \approx \widetilde{\mathbf{w}}$.

Proof. If $\mathrm{h}(\mathbf{w})=\mathrm{t}(\mathbf{w})$, then the lemma holds vacuously with $\widetilde{\mathbf{w}}=\mathbf{w}$. Therefore assume $\mathrm{h}(\mathbf{w}) \neq \mathrm{t}(\mathbf{w})$. Since $\mathbf{w}$ is connected, there exists a sequence $\mathrm{h}(\mathbf{w})=x_{1}, x_{2}, \ldots, x_{m}=\mathrm{t}(\mathbf{w})$ of non-simple letters of $\mathbf{w}$ occurring in an overlapping pattern such that the first $x_{i+1}$ occurs between two occurrences of $x_{i}$ and the last $x_{i+1}$ occurs to the right of all occurrences of $x_{i}$. This sequence can be chosen to have minimal length among all such sequences, so that

$$
\mathbf{w}=x_{1} \mathbf{p}_{1} x_{2} \mathbf{p}_{2} x_{1} \mathbf{q}_{1} x_{3} \mathbf{p}_{3} x_{2} \mathbf{q}_{2} x_{4} \mathbf{p}_{4} x_{3} \mathbf{q}_{3} \ldots \mathbf{q}_{m-1} x_{m}
$$

for some $\mathbf{p}_{1}, \ldots, \mathbf{p}_{m}, \mathbf{q}_{1}, \ldots, \mathbf{q}_{m-1} \in \mathcal{X}^{*}$. (Note that $\mathbf{p}_{i}$ follows the first $x_{i}$ while $\mathbf{q}_{i}$ follows the last $x_{i}$.) Then

$$
\begin{aligned}
& \mathbf{w}=\left(x_{1} \mathbf{p}_{1} x_{2} \mathbf{p}_{2} x_{1}\right) \mathbf{q}_{1} x_{3} \mathbf{p}_{3} x_{2} \mathbf{q}_{2} x_{4} \mathbf{p}_{4} x_{3} \mathbf{q}_{3} \ldots \mathbf{q}_{m-1} x_{m} \\
& \stackrel{(25.2 \mathrm{a})}{\approx}\left(x_{1} \mathbf{p}_{1} x_{2} \mathbf{p}_{2} x_{1}^{3} \mathbf{q}_{1} x_{3} \mathbf{p}_{3} x_{2}\right) \mathbf{q}_{2} x_{4} \mathbf{p}_{4} x_{3} \mathbf{q}_{3} \ldots \mathbf{q}_{m-1} x_{m} \\
& \stackrel{(25.2 \mathrm{c})}{\approx}\left(x_{1} \mathbf{p}_{1} x_{2} \mathbf{p}_{2} x_{1} \mathbf{q}_{1} x_{3} \mathbf{p}_{3} x_{2} x_{1}^{2} \mathbf{q}_{2} x_{4} \mathbf{p}_{4} x_{3}\right) \mathbf{q}_{3} \ldots \mathbf{q}_{m-1} x_{m} \\
& \stackrel{(25.2 \mathrm{c})}{\approx} x_{1} \mathbf{p}_{1} x_{2} \mathbf{p}_{2} x_{1} \mathbf{q}_{1} x_{3} \mathbf{p}_{3} x_{2} \mathbf{q}_{2} x_{4} \mathbf{p}_{4} x_{3} x_{1}^{2} \mathbf{q}_{3} \ldots \mathbf{q}_{m-1} x_{m} \\
& \vdots \\
& \stackrel{(25.2 \mathrm{c})}{\approx} \underbrace{x_{1} \mathbf{p}_{1} x_{2} \mathbf{p}_{2} x_{1} \mathbf{q}_{1} x_{3} \mathbf{p}_{3} x_{2} \mathbf{q}_{2} x_{4} \mathbf{p}_{4} x_{3} \mathbf{q}_{3} \ldots \mathbf{q}_{m-1} x_{m} x_{1}^{2}}_{\widetilde{\mathbf{w}}},
\end{aligned}
$$

where the word $\widetilde{\mathbf{w}}$ satisfies the required properties.

Let $\mathbf{X}_{(25.2)}$ denote the variety defined by the identities (25.2).

Lemma 25.4. Let $\mathbf{w} \approx \mathbf{w}^{\prime}$ be any identity satisfied by $A_{0}, \overleftarrow{J}$, and $O$. Then

$$
\mathbf{X}_{(25.2)}\left\{\mathbf{w} \approx \mathbf{w}^{\prime}\right\}=\mathbf{X}_{(25.2)}\left\{\mathbf{w}_{i} \approx \mathbf{w}_{i}^{\prime} \mid 1 \leqslant i \leqslant m\right\}
$$

for some identities $\mathbf{w}_{i} \approx \mathbf{w}_{i}^{\prime}$ such that $\mathrm{h}\left(\mathbf{w}_{i}\right)=\mathrm{t}\left(\mathbf{w}_{i}\right)=\mathrm{h}\left(\mathbf{w}_{i}^{\prime}\right)=\mathrm{t}\left(\mathbf{w}_{i}^{\prime}\right)$. 
Proof. Since $A_{0}$ satisfies $\mathbf{w} \approx \mathbf{w}^{\prime}$, it follows from Lee and Volkov [21, Proposition 3.2(i)] that

$$
\mathbf{w}=\mathbf{s}_{0} \prod_{i=1}^{m}\left(\mathbf{w}_{i} \mathbf{s}_{i}\right) \quad \text { and } \quad \mathbf{w}^{\prime}=\mathbf{s}_{0} \prod_{i=1}^{m}\left(\mathbf{w}_{i}^{\prime} \mathbf{s}_{i}\right),
$$

where:

(a) the letters of $\mathbf{s}_{0}, \mathbf{s}_{m} \in \mathcal{X}^{*}$ and $\mathbf{s}_{1}, \ldots, \mathbf{s}_{m-1} \in \mathcal{X}^{+}$are simple in $\mathbf{w}$ and $\mathbf{w}^{\prime}$;

(b) $\mathbf{w}_{1}, \ldots, \mathbf{w}_{m}, \mathbf{w}_{1}^{\prime}, \ldots, \mathbf{w}_{m}^{\prime} \in \mathcal{X}^{+}$are connected;

(c) $\mathbf{s}_{0}, \mathbf{w}_{1}, \mathbf{s}_{1}, \ldots, \mathbf{w}_{m}, \mathbf{s}_{m}$ are pairwise disjoint;

(d) $\mathbf{s}_{0}, \mathbf{w}_{1}^{\prime}, \mathbf{s}_{1}, \ldots, \mathbf{w}_{m}^{\prime}, \mathbf{s}_{m}$ are pairwise disjoint;

(e) $\operatorname{con}\left(\mathbf{w}_{i}\right)=\operatorname{con}\left(\mathbf{w}_{i}^{\prime}\right)$ and $A_{0}$ satisfies $\mathbf{w}_{i} \approx \mathbf{w}_{i}^{\prime}$ for all $i$.

It is easily seen that the inclusion $\mathbf{X}_{(25.2)}\left\{\mathbf{w}_{i} \approx \mathbf{w}_{i}^{\prime} \mid 1 \leqslant i \leqslant m\right\} \subseteq \mathbf{X}_{(25.2)}\left\{\mathbf{w} \approx \mathbf{w}^{\prime}\right\}$ holds.

Consider any $i \in\{1, \ldots, m\}$. The subsemigroup $\{a, b a, 1\}$ of $O$ is isomorphic to $L_{2}^{1}$. Therefore $\operatorname{ini}\left(\mathbf{w}_{i}\right)=\operatorname{ini}\left(\mathbf{w}_{i}^{\prime}\right)$ by Lemma 2.1 (iii); in particular, $\mathrm{h}\left(\mathbf{w}_{i}\right)=\mathrm{h}\left(\mathbf{w}_{i}^{\prime}\right)$. Further, by Lemma 25.3 , the words $\mathbf{w}_{i}$ and $\mathbf{w}_{i}^{\prime}$ can be chosen to satisfy $\mathrm{h}\left(\mathbf{w}_{i}\right)=\mathrm{t}\left(\mathbf{w}_{i}\right)$ and $\mathrm{h}\left(\mathbf{w}_{i}^{\prime}\right)=\mathrm{t}\left(\mathbf{w}_{i}^{\prime}\right)$. For convenience, let $h_{i}=\mathrm{h}\left(\mathbf{w}_{i}\right)=\mathrm{t}\left(\mathbf{w}_{i}\right)=\mathrm{h}\left(\mathbf{w}_{i}^{\prime}\right)=\mathrm{t}\left(\mathbf{w}_{i}^{\prime}\right)$. Let $\varphi$ denote the substitution $x \mapsto h_{i}^{2}$ for all $x \notin \operatorname{con}\left(\mathbf{w}_{i}\right)=\operatorname{con}\left(\mathbf{w}_{i}^{\prime}\right)$. Then the deductions $\mathbf{w} \varphi \stackrel{(25.2 \mathrm{a})}{\approx} \mathbf{w}_{i}$ and $\mathbf{w}^{\prime} \varphi \stackrel{(25.2 \mathrm{a})}{\approx} \mathbf{w}_{i}^{\prime}$ hold, so that the identities $\left\{(25.2), \mathbf{w} \approx \mathbf{w}^{\prime}\right\}$ imply $\mathbf{w}_{i} \approx \mathbf{w}_{i}^{\prime}$. Since $i \in\{1, \ldots, m\}$ is arbitrary, the inclusion $\mathbf{X}_{(25.2)}\left\{\mathbf{w} \approx \mathbf{w}^{\prime}\right\} \subseteq \mathbf{X}_{(25.2)}\left\{\mathbf{w}_{i} \approx \mathbf{w}_{i}^{\prime} \mid 1 \leqslant i \leqslant m\right\}$ holds.

Proof of Proposition 25.2. It is routinely checked that $A_{0}, \overleftarrow{J}$, and $O$ satisfy the identities (25.2). Hence $\mathbf{A}_{\mathbf{0}} \vee \mathbf{W}=\mathbf{X}_{(25.2)} \Sigma$ for some set $\Sigma$ of identities. By Lemma 25.4, each identity $\mathbf{w} \approx \mathbf{w}^{\prime}$ in $\Sigma$ can be chosen to satisfy $\mathrm{h}(\mathbf{w})=\mathrm{t}(\mathbf{w})=\mathrm{h}\left(\mathbf{w}^{\prime}\right)=\mathrm{t}\left(\mathbf{w}^{\prime}\right)$. Let $\mathbf{w} \approx \mathbf{w}^{\prime}$ be any such identity with $h=\mathrm{h}(\mathbf{w})=\mathrm{t}(\mathbf{w})=\mathrm{h}\left(\mathbf{w}^{\prime}\right)=\mathrm{t}\left(\mathbf{w}^{\prime}\right)$. Since $\overleftarrow{J}$ and $O$ satisfy the identity $\mathbf{w} \approx \mathbf{w}^{\prime}$, it follows from Lemma 25.1 and Birkhoff's completeness theorem of equational logic $[\mathbf{3}]$ that there exists a deduction sequence

$$
\mathbf{w}=\mathbf{z}_{0} \Rightarrow \mathbf{z}_{1} \Rightarrow \cdots \Rightarrow \mathbf{z}_{r}=\mathbf{w}^{\prime}
$$

where each deduction $\mathbf{z}_{i} \Rightarrow \mathbf{z}_{i+1}$ involves an identity from (25.1), that is, there exist $\mathbf{e}_{i}, \mathbf{f}_{i} \in \mathcal{X}^{*}$, an identity $\mathbf{u}_{i} \approx \mathbf{v}_{i}$ from (25.1), and an endomorphism $\varphi_{i}$ of $\mathcal{X}^{+}$such that $\mathbf{z}_{i}=\mathbf{e}_{i}\left(\mathbf{u}_{i} \varphi_{i}\right) \mathbf{f}_{i}$ and $\mathbf{z}_{i+1}=\mathbf{e}_{i}\left(\mathbf{v}_{i} \varphi_{i}\right) \mathbf{f}_{i}$. In what follows, it is shown that each deduction $\mathbf{z}_{i} \Rightarrow \mathbf{z}_{i+1}$ in (25.3) can be replaced by a deduction sequence that involves only identities from (25.2), whence the variety $\mathbf{X}_{(25.2)}$ satisfies the identity $\mathbf{w} \approx \mathbf{w}^{\prime}$. Since the identity $\mathbf{w} \approx \mathbf{w}^{\prime}$ is arbitrary in $\Sigma$, it follows that $\mathbf{X}_{(25.2)}=\mathbf{X}_{(25.2)} \Sigma=\mathbf{A}_{\mathbf{0}} \vee \mathbf{W}$.

Multiplying each word in (25.3) by $h^{2}$ on both the left and the right results in

$$
h^{2} \mathbf{w} h^{2}=h^{2} \mathbf{z}_{0} h^{2} \Rightarrow h^{2} \mathbf{z}_{1} h^{2} \Rightarrow \cdots \Rightarrow h^{2} \mathbf{z}_{r} h^{2}=h^{2} \mathbf{w}^{\prime} h^{2} .
$$

Since the identities $h^{2} \mathbf{w} h^{2} \approx \mathbf{w}$ and $h^{2} \mathbf{w}^{\prime} h^{2} \approx \mathbf{w}^{\prime}$ are implied by the identities (25.2a) of $\mathbf{X}_{(25.2)}$, generality is not lost if every word in (25.3) is assumed to begin and end with the letter $h$. Let $i$ be any fixed number from $\{0, \ldots, r-1\}$.

Case 1: $\mathbf{z}_{i} \Rightarrow \mathbf{z}_{i+1}$ involves (25.1a). Then $\left\{\mathbf{z}_{i}, \mathbf{z}_{i+1}\right\}=\left\{\mathbf{e a b}^{3} \mathbf{f}, \mathbf{e a b f}\right\}$ for some $\mathbf{a}, \mathbf{b} \in \mathcal{X}^{+}$ and $\mathbf{e}, \mathbf{f} \in \mathcal{X}^{*}$. Since

$$
\mathbf{e a b}^{3} \mathbf{f} \stackrel{(25.2 \mathrm{a})}{\approx} h^{2} \mathbf{e a b}^{3} \mathbf{f} h^{2} \stackrel{(25.2 \mathrm{~b})}{\approx} h^{2} \mathbf{e a b f} h^{2} \stackrel{(25.2 \mathrm{a})}{\approx} \mathbf{e a b f}
$$

the deduction $\mathbf{z}_{i} \Rightarrow \mathbf{z}_{i+1}$ can be replaced by a deduction sequence that involves identities from (25.2). 
Case 2: $\mathbf{z}_{i} \Rightarrow \mathbf{z}_{i+1}$ involves (25.1b). Then $\left\{\mathbf{z}_{i}, \mathbf{z}_{i+1}\right\}=\left\{\mathbf{e a}^{3} \mathbf{b f}, \mathbf{e a b a}^{2} \mathbf{f}\right\}$ for some $\mathbf{a}, \mathbf{b} \in \mathcal{X}^{+}$ and $\mathbf{e}, \mathbf{f} \in \mathcal{X}^{*}$. Since

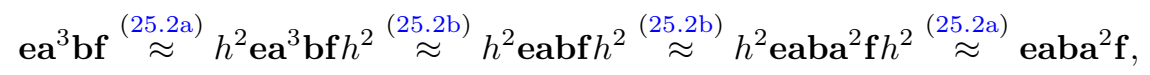

the deduction $\mathbf{z}_{i} \Rightarrow \mathbf{z}_{i+1}$ can be replaced by a deduction sequence that involves identities from (25.2).

Case 3: $\mathbf{z}_{i} \Rightarrow \mathbf{z}_{i+1}$ involves (25.1c). Since the identity (25.1c) belongs to (25.2d), the deduction $\mathbf{z}_{i} \Rightarrow \mathbf{z}_{i+1}$ can be replaced by a deduction that involves an identity from (25.2).

\subsection{A basis for $\mathbf{B}_{\mathbf{0}} \vee \mathbf{W}$}

Proposition 25.5. The variety $\mathbf{B}_{\mathbf{0}} \vee \mathbf{W}$ is defined by the identities

$$
\begin{aligned}
x \mathrm{H} x^{2} \mathrm{~K} x & \approx x \mathrm{HK} x, \\
x \mathrm{H} y \mathrm{~K} y^{2} \mathrm{~T} x & \approx x \mathrm{H} y \mathrm{KT} x, \\
x \mathrm{H} y \mathrm{~K} x^{2} \mathrm{~T} y & \approx x \mathrm{H} y \mathrm{KT} y x^{2}, \\
x \mathrm{H} y \mathrm{~K} x y & \approx x \mathrm{H} y \mathrm{~K} y x, \\
x^{2} y^{2} x^{2} y^{2} & \approx x^{2} y^{2} .
\end{aligned}
$$

Lemma 25.6. Let $\mathbf{w}=\mathbf{w}_{1} \ldots \mathbf{w}_{m}$ where $\mathbf{w}_{1}, \ldots, \mathbf{w}_{m}$ are pairwise disjoint connected words. Then there exists some word $\widetilde{\mathbf{w}}$ with $\operatorname{con}(\mathbf{w})=\operatorname{con}(\widetilde{\mathbf{w}})$ and $\mathrm{h}(\mathbf{w})=\mathrm{h}(\widetilde{\mathbf{w}})=\mathrm{t}(\widetilde{\mathbf{w}})$ such that the identities (25.4) imply the identity $\mathbf{w} \approx \widetilde{\mathbf{w}}$.

Proof. It suffices to verify the lemma for $m=2$ since the general case can be obtained by induction. Since the word $\mathbf{w}_{1}$ is connected, the letter $t=\mathrm{t}\left(\mathbf{w}_{1}\right)$ occurs at least twice in $\mathbf{w}_{1}$. Hence $\mathbf{w}_{1}=\mathbf{a} t \mathbf{b} t$ for some $\mathbf{a}, \mathbf{b} \in \mathcal{X}^{*}$. Similarly, the letter $h=\mathrm{h}\left(\mathbf{w}_{2}\right)$ occurs at least twice in $\mathbf{w}_{2}$, so that $\mathbf{w}_{2}=h \mathbf{c} h \mathbf{d}$ for some $\mathbf{c}, \mathbf{d} \in \mathcal{X}^{*}$. Then $\mathbf{w}_{1} h^{2} t^{2} \mathbf{w}_{2}$ is a connected word such that

$$
\mathbf{w}_{1} h^{2} t^{2} \mathbf{w}_{2} \stackrel{(25.4 \mathrm{a})}{\approx} \mathbf{w}_{1} t^{2} h^{2} t^{2} h^{2} \mathbf{w}_{2} \stackrel{(25.4 \mathrm{e})}{\approx} \mathbf{w}_{1} t^{2} h^{2} \mathbf{w}_{2} \stackrel{(25.4 \mathrm{a})}{\approx} \mathbf{w}_{1} \mathbf{w}_{2}
$$

Since the identities (25.2) belong to (25.4), the proof of Lemma 25.3 can be repeated to convert the connected word $\mathbf{w}_{1} h^{2} t^{2} \mathbf{w}_{2}$, using the identities (25.4), into a word $\widetilde{\mathbf{w}}$ with $\operatorname{con}(\mathbf{w})=\operatorname{con}(\widetilde{\mathbf{w}})$ and $\mathrm{h}(\mathbf{w})=\mathrm{h}(\widetilde{\mathbf{w}})=\mathrm{t}(\widetilde{\mathbf{w}})$.

Let $\mathbf{X}_{(25.4)}$ denote the variety defined by the identities (25.4).

LEMma 25.7. Let $\mathbf{w} \approx \mathbf{w}^{\prime}$ be any identity satisfied by $B_{0}, \overleftarrow{J}$, and $O$. Then

$$
\mathbf{X}_{(25.4)}\left\{\mathbf{w} \approx \mathbf{w}^{\prime}\right\}=\mathbf{X}_{(25.4)}\left\{\mathbf{w}_{i} \approx \mathbf{w}_{i}^{\prime} \mid 1 \leqslant i \leqslant m\right\}
$$

for some identities $\mathbf{w}_{i} \approx \mathbf{w}_{i}^{\prime}$ such that $\mathrm{h}\left(\mathbf{w}_{i}\right)=\mathrm{t}\left(\mathbf{w}_{i}\right)=\mathrm{h}\left(\mathbf{w}_{i}^{\prime}\right)=\mathrm{t}\left(\mathbf{w}_{i}^{\prime}\right)$.

Proof. Since $B_{0}$ satisfies $\mathbf{w} \approx \mathbf{w}^{\prime}$, it follows from Lee and Volkov [21, Proposition 3.2(ii)] that

$$
\mathbf{w}=\mathbf{s}_{0} \prod_{i=1}^{m}\left(\mathbf{w}_{i} \mathbf{s}_{i}\right) \quad \text { and } \quad \mathbf{w}^{\prime}=\mathbf{s}_{0} \prod_{i=1}^{m}\left(\mathbf{w}_{i}^{\prime} \mathbf{s}_{i}\right),
$$


where the following holds:

(a) the letters of $\mathbf{s}_{0}, \mathbf{s}_{m} \in \mathcal{X}^{*}$ and $\mathbf{s}_{1}, \ldots, \mathbf{s}_{m-1} \in \mathcal{X}^{+}$are simple in $\mathbf{w}$ and $\mathbf{w}^{\prime}$;

(b) each $\mathbf{w}_{1}, \ldots, \mathbf{w}_{m}, \mathbf{w}_{1}^{\prime}, \ldots, \mathbf{w}_{m}^{\prime} \in \mathcal{X}^{+}$is a product of pairwise disjoint connected words;

(c) $\mathbf{s}_{0}, \mathbf{w}_{1}, \mathbf{s}_{1}, \ldots, \mathbf{w}_{m}, \mathbf{s}_{m}$ are pairwise disjoint;

(d) $\mathbf{s}_{0}, \mathbf{w}_{1}^{\prime}, \mathbf{s}_{1}, \ldots, \mathbf{w}_{m}^{\prime}, \mathbf{s}_{m}$ are pairwise disjoint;

(e) $\operatorname{con}\left(\mathbf{w}_{i}\right)=\operatorname{con}\left(\mathbf{w}_{i}^{\prime}\right)$ and $B_{0}$ satisfies $\mathbf{w}_{i} \approx \mathbf{w}_{i}^{\prime}$ for all $i$.

By Lemma 25.6, each word in (b) can be replaced with a connected word. Since the identities (25.2) belong to (25.4), the proof of Lemma 25.4 can be repeated to establish the present lemma.

Proof of Proposition 25.5. It is routinely shown that $B_{0}, \overleftarrow{J}$, and $O$ satisfy the identities $\{(25.2),(25.4)\}$. Therefore $\mathbf{B}_{\mathbf{0}} \vee \mathbf{W}=\mathbf{X}_{(25.4)} \Sigma$ for some set $\Sigma$ of identities. By Lemma 25.7, each identity $\mathbf{w} \approx \mathbf{w}^{\prime}$ in $\Sigma$ can be chosen to satisfy $\mathrm{h}(\mathbf{w})=\mathrm{t}(\mathbf{w})=\mathrm{h}\left(\mathbf{w}^{\prime}\right)=\mathrm{t}\left(\mathbf{w}^{\prime}\right)$. Let $\mathbf{w} \approx \mathbf{w}^{\prime}$ be any such identity with $h=\mathrm{h}(\mathbf{w})=\mathrm{t}(\mathbf{w})=\mathrm{h}\left(\mathbf{w}^{\prime}\right)=\mathrm{t}\left(\mathbf{w}^{\prime}\right)$. Then the proof of Proposition 25.2 can be repeated to show that the identity $\mathbf{w} \approx \mathbf{w}^{\prime}$ is deducible from the identities (25.2). Since the identities (25.2) belong to (25.4), the identity $\mathbf{w} \approx \mathbf{w}^{\prime}$ is also deducible from the identities (25.4). Consequently, the variety $\mathbf{X}_{(25.4)}$ satisfies the identity $\mathbf{w} \approx \mathbf{w}^{\prime}$. Since the identity $\mathbf{w} \approx \mathbf{w}^{\prime}$ is arbitrary in $\Sigma$, it follows that $\mathbf{X}_{(25.4)}=\mathbf{X}_{(25.4)} \Sigma=$ $\mathbf{B}_{0} \vee \mathbf{W}$.

$$
\text { 26. } \mathscr{F}_{6}, \mathscr{F}_{7}, \mathscr{F}_{8}
$$

This section establishes the finite basis property of the following semigroups.

\begin{tabular}{c|cccccc}
$\mathscr{F}_{6}$ & 1 & 2 & 3 & 4 & 5 & 6 \\
\hline 1 & 1 & 1 & 1 & 1 & 1 & 1 \\
2 & 1 & 1 & 1 & 1 & 2 & 2 \\
3 & 3 & 3 & 3 & 3 & 3 & 3 \\
4 & 4 & 4 & 4 & 4 & 4 & 4 \\
5 & 1 & 1 & 1 & 4 & 5 & 6 \\
6 & 1 & 1 & 4 & 4 & 5 & 6
\end{tabular}

\begin{tabular}{c|cccccc}
$\mathscr{F}_{7}$ & 1 & 2 & 3 & 4 & 5 & 6 \\
\hline 1 & 1 & 1 & 1 & 1 & 1 & 1 \\
2 & 1 & 1 & 1 & 1 & 2 & 2 \\
3 & 3 & 3 & 3 & 3 & 3 & 3 \\
4 & 4 & 4 & 4 & 4 & 4 & 4 \\
5 & 1 & 2 & 1 & 4 & 5 & 6 \\
6 & 1 & 2 & 4 & 4 & 5 & 6
\end{tabular}

\begin{tabular}{c|cccccc}
$\mathscr{F}_{8}$ & 1 & 2 & 3 & 4 & 5 & 6 \\
\hline 1 & 1 & 1 & 1 & 1 & 1 & 1 \\
2 & 1 & 1 & 1 & 1 & 2 & 2 \\
3 & 3 & 3 & 3 & 3 & 3 & 3 \\
4 & 4 & 4 & 4 & 4 & 4 & 4 \\
5 & 3 & 3 & 3 & 4 & 5 & 6 \\
6 & 4 & 4 & 3 & 4 & 5 & 6
\end{tabular}

26.1. Finite basis property of $\mathscr{F}_{7}$

Proposition 26.1. The variety generated by $\mathscr{F}_{7}$ is defined by the identities

$$
\begin{aligned}
x^{2} \mathrm{H} x & \approx x \mathrm{H} x, \\
x \mathrm{H} x^{2} & \approx x \mathrm{H} x, \\
x \mathrm{H} y \mathrm{~K} x y & \approx x^{2} \mathrm{H} y \mathrm{~K} y, \\
x \mathrm{H} y z y x & \approx x \mathrm{H} y^{2} z x .
\end{aligned}
$$

In this section, a word $\mathbf{w}$ with ini $(\mathbf{w})=x_{0} \ldots x_{m}$ is said to be in $\alpha$-canonical form if

$$
\mathbf{w}=x_{0}^{e_{0}} \prod_{i=1}^{m}\left(x_{i}^{e_{i}} \mathbf{w}_{i}\right),
$$

where all of the following are satisfied:

(I) $\mathbf{w}_{i} \in\left\{\emptyset, x_{0}, \ldots, x_{i-1}\right\}$;

(II) $e_{0}, \ldots, e_{m} \in\{1,2\}$;

(III) $\mathbf{w}$ does not contain a factor of the form $x^{2} \mathbf{a} x$ where $\mathbf{a} \in \mathcal{X}^{+}$. 
Note that (I) and (II) imply that the word $\mathbf{w}$ in (26.2) does not contain a factor of the form $x \mathbf{a} x^{2}$ where $\mathbf{a} \in \mathcal{X}^{*}$.

Lemma 26.2. Let $\mathbf{w}$ be any word. Then there exists some word $\overline{\mathbf{w}}$ in $\alpha$-canonical form such that the identities (26.1) imply the identity $\mathbf{w} \approx \overline{\mathbf{w}}$.

Proof. It suffices to convert $\mathbf{w}$, using the identities (26.1), into a word in $\alpha$-canonical form. Suppose $\operatorname{ini}(\mathbf{w})=x_{0} \ldots x_{m}$. Then $\mathbf{w}$ can be written as a word of the form $\mathbf{w}=\prod_{i=0}^{m}\left(x_{i} \mathbf{w}_{i}\right)$ with $\mathbf{w}_{i} \in\left\{x_{0}, \ldots, x_{i}\right\}^{*}$. Suppose $\left|\mathbf{w}_{r}\right| \geqslant 2$ for some $r \in\{0, \ldots, m\}$. Then $\mathbf{w}_{r}=\mathbf{w}_{r}^{\prime} x_{k} x_{\ell}$ for some $\mathbf{w}_{r}^{\prime} \in \mathcal{X}^{*}$ with $\operatorname{con}\left(\mathbf{w}_{r}^{\prime} x_{k} x_{\ell}\right) \subseteq\left\{x_{0}, \ldots, x_{r}\right\}$. Hence

$$
\mathbf{w}=\mathbf{p} x_{r} \mathbf{w}_{r} \mathbf{q}
$$

where $\mathbf{p}=\prod_{i=0}^{r-1}\left(x_{i} \mathbf{w}_{i}\right)$ and $\mathbf{q}=\prod_{i=r+1}^{m}\left(x_{i} \mathbf{w}_{i}\right)$. Since the letters $x_{k}$ and $x_{\ell}$ in $\mathbf{w}_{r}$ are non-first occurrences, the first $x_{k}$ and $x_{\ell}$ of $\mathbf{w}$ occur somewhere in the prefix $\mathbf{p} x_{r}$. If $k=\ell$, then

$$
\mathbf{w}=\underbrace{\ldots x_{\ell} \ldots}_{\mathbf{p} x_{r}} \underbrace{\mathbf{w}_{r}^{\prime} x_{\ell}^{2}}_{\mathbf{w}_{r}} \mathbf{q} \stackrel{(26.1 \mathrm{~b})}{\approx} \ldots x_{\ell} \ldots \mathbf{w}_{r}^{\prime} x_{\ell} \mathbf{q} .
$$

If $k<\ell$, then

$$
\mathbf{w}=\underbrace{\ldots x_{k} \ldots x_{\ell} \ldots}_{\mathbf{p} x_{r}} \underbrace{\mathbf{w}_{r}^{\prime} x_{k} x_{\ell}}_{\mathbf{w}_{r}} \mathbf{q} \stackrel{(26.1 \mathrm{c})}{\approx} \ldots x_{k}^{2} \ldots x_{\ell} \ldots \mathbf{w}_{r}^{\prime} x_{\ell} \mathbf{q} .
$$

If $\ell<k$, then

$$
\mathbf{w}=\underbrace{\ldots x_{\ell} \ldots x_{k} \ldots}_{\mathbf{p} x_{r}} \underbrace{\mathbf{w}_{r}^{\prime} x_{k} x_{\ell}}_{\mathbf{w}_{r}} \mathbf{q} \stackrel{(26.1 \mathrm{~d})}{\approx} \ldots x_{\ell} \ldots x_{k}^{2} \ldots \mathbf{w}_{r}^{\prime} x_{\ell} \mathbf{q} .
$$

In each of the three cases just considered, an identity from (26.1) has been used to convert the factor $\mathbf{w}_{r}=\mathbf{w}_{r}^{\prime} x_{k} x_{\ell}$ of $\mathbf{w}$ into $\mathbf{w}_{r}^{\prime} x_{\ell}$, that is, the length of $\mathbf{w}_{r}$ is reduced by one. This argument can be repeated until the length of $\mathbf{w}_{r}$ is reduced to one. Hence it is easily shown that $\mathbf{w}$ can be converted by the identities (26.1) into the form (26.2) with (I) satisfied. The identities (26.1a) can then be used to convert this word into one that satisfies (II) and (III).

Lemma 26.3. Suppose that $\mathbf{w} \approx \mathbf{w}^{\prime}$ is any identity satisfied by $\mathscr{F}_{7}$. Then $\operatorname{ini}(\mathbf{w})=\operatorname{ini}\left(\mathbf{w}^{\prime}\right)$, $\operatorname{sim}(\mathbf{w})=\operatorname{sim}\left(\mathbf{w}^{\prime}\right)$, and $\mathrm{t}(\mathbf{w})=\mathrm{t}\left(\mathbf{w}^{\prime}\right)$.

Proof. This follows from Lemma 2.1 since the subsemigroups $\{1,4,5\},\{1,2,5\}$, and $\{5,6\}$ of $\mathscr{F}_{7}$ are isomorphic to $L_{2}^{1}, N_{2}^{1}$, and $R_{2}$, respectively.

Lemma 26.4. Suppose that $\mathscr{F}_{7}$ satisfies an identity $\mathbf{w} \approx \mathbf{w}^{\prime}$, where

$$
\mathbf{w}=x_{0}^{e_{0}} \prod_{i=1}^{m}\left(x_{i}^{e_{i}} \mathbf{w}_{i}\right) \quad \text { and } \quad \mathbf{w}^{\prime}=x_{0}^{e_{0}^{\prime}} \prod_{i=1}^{m}\left(x_{i}^{e_{i}^{\prime}} \mathbf{w}_{i}^{\prime}\right)
$$

are words in $\alpha$-canonical form. Then $e_{i}=e_{i}^{\prime}$ for all $i$.

Proof. Seeking a contradiction, suppose $e_{r} \neq e_{r}^{\prime}$ for some $r$, say $\left(e_{r}, e_{r}^{\prime}\right)=(1,2)$. Then the letter $x_{r}$ is non-simple in $\mathbf{w}^{\prime}$. By Lemma 26.3, the letter $x_{r}$ is also non-simple in $\mathbf{w}$. Since $\mathbf{w}$ 
is in $\alpha$-canonical form, the second $x_{r}$ in $\mathbf{w}$ coincides with one of $\mathbf{w}_{r+1}, \ldots, \mathbf{w}_{m}$, say $x_{r}=\mathbf{w}_{\ell}$. Then $x_{r} \neq x_{\ell}$ by (I), and the word $\mathbf{w}$ can be written as

$$
\mathbf{w}=\mathbf{p} x_{r} \mathbf{w}_{r} \mathbf{q} x_{\ell}^{e_{\ell}} \underbrace{x_{r}}_{\mathbf{w}_{\ell}} \mathbf{u}
$$

where $\mathbf{p}=x_{0}^{e_{0}} \prod_{i=1}^{r-1}\left(x_{i}^{e_{i}} \mathbf{w}_{i}\right), \mathbf{q}=\prod_{i=r+1}^{\ell-1}\left(x_{i}^{e_{i}} \mathbf{w}_{i}\right)$, and $\mathbf{u}=\prod_{i=\ell+1}^{m}\left(x_{i}^{e_{i}} \mathbf{w}_{i}\right)$ with

(a) $x_{r} \notin \operatorname{con}\left(\mathbf{p w}_{r} \mathbf{q} x_{\ell}\right)$.

As for the word $\mathbf{w}^{\prime}$, since $e_{r}^{\prime}=2$, it can be written as

$$
\mathbf{w}^{\prime}=\mathbf{p}^{\prime} x_{r}^{2} \mathbf{w}_{r}^{\prime} \mathbf{q}^{\prime} x_{\ell}^{e_{\ell}^{\prime}} \mathbf{w}_{\ell}^{\prime} \mathbf{u}^{\prime}
$$

where $\mathbf{p}^{\prime}=x_{0}^{e_{0}^{\prime}} \prod_{i=1}^{r-1}\left(x_{i}^{e_{i}^{\prime}} \mathbf{w}_{i}^{\prime}\right), \mathbf{q}^{\prime}=\prod_{i=r+1}^{\ell-1}\left(x_{i}^{e_{i}^{\prime}} \mathbf{w}_{i}^{\prime}\right)$, and $\mathbf{u}^{\prime}=\prod_{i=\ell+1}^{m}\left(x_{i}^{e_{i}^{\prime}} \mathbf{w}_{i}^{\prime}\right)$ with $x_{r} \notin \operatorname{con}\left(\mathbf{p}^{\prime}\right)$ and $x_{\ell} \notin \operatorname{con}\left(\mathbf{p}^{\prime} x_{r} \mathbf{w}_{r}^{\prime} \mathbf{q}^{\prime}\right)$. In fact, it follows from (I) and (III) that

(b) $x_{r} \notin \operatorname{con}\left(\mathbf{p}^{\prime} \mathbf{w}_{r}^{\prime} \mathbf{q}^{\prime} x_{\ell} \mathbf{w}_{\ell}^{\prime} \mathbf{u}^{\prime}\right)$.

In particular, $\mathrm{t}\left(\mathbf{w}^{\prime}\right) \neq x_{r}$. Since $\mathrm{t}(\mathbf{w})=\mathrm{t}\left(\mathbf{w}^{\prime}\right)$ by Lemma 26.3 , it follows that $\mathbf{u} \neq \emptyset$, whence

(c) $\mathrm{h}(\mathbf{u})=x_{\ell+1} \notin \operatorname{con}\left(\mathbf{p} x_{r} \mathbf{w}_{r} \mathbf{q} x_{\ell}\right)$

and $\operatorname{ini}(\mathbf{w})=\ldots x_{\ell} x_{\ell+1} \ldots$ Further, ini $(\mathbf{w})=\operatorname{ini}\left(\mathbf{w}^{\prime}\right)$ by Lemma 26.3 , so that $\mathbf{u}^{\prime} \neq \emptyset$ and

(d) $\mathrm{h}\left(\mathbf{u}^{\prime}\right)=x_{\ell+1} \notin \operatorname{con}\left(\mathbf{p}^{\prime} x_{r} \mathbf{w}_{r}^{\prime} \mathbf{q}^{\prime} x_{\ell} \mathbf{w}_{\ell}^{\prime}\right)$.

Let $\varphi: \mathcal{X} \rightarrow \mathscr{F}_{7}$ denote the substitution

$$
z \mapsto \begin{cases}6 & \text { if } z=x_{r}, \\ 3 & \text { if } z=x_{\ell+1}, \\ 5 & \text { otherwise. }\end{cases}
$$

Since $x_{r}, x_{\ell+1} \notin \operatorname{con}\left(\mathbf{p w}_{r} \mathbf{q} x_{\ell}\right)$ by (a) and (c), it follows that $\mathbf{p} \varphi \in\{\emptyset, 5\}$ and $\left(\mathbf{w}_{r} \mathbf{q} x_{\ell}^{\ell_{\ell}}\right) \varphi=5$. Thus

$$
\mathbf{w} \varphi=\mathbf{p} \varphi \cdot x_{r} \varphi \cdot\left(\mathbf{w}_{r} \mathbf{q} x_{\ell}^{e_{\ell}}\right) \varphi \cdot x_{r} \varphi \cdot \mathbf{u} \varphi=\mathbf{p} \varphi \cdot 6 \cdot 5 \cdot 6 \cdot 3 \ldots=4 .
$$

Similarly, since $x_{r}, x_{\ell+1} \notin \operatorname{con}\left(\mathbf{p}^{\prime} \mathbf{w}_{r}^{\prime} \mathbf{q}^{\prime} x_{\ell} \mathbf{w}_{\ell}^{\prime}\right)$ by (b) and (d), it follows that $\mathbf{p}^{\prime} \varphi \in\{\emptyset, 5\}$ and $\left(\mathbf{w}_{r}^{\prime} \mathbf{q}^{\prime} x_{\ell}^{e_{\ell}^{\prime}} \mathbf{w}_{\ell}^{\prime}\right) \varphi=5$. Hence

$$
\mathbf{w}^{\prime} \varphi=\mathbf{p}^{\prime} \varphi \cdot x_{r}^{2} \varphi \cdot\left(\mathbf{w}_{r}^{\prime} \mathbf{q}^{\prime} x_{\ell}^{e_{\ell}^{\prime}} \mathbf{w}_{\ell}^{\prime}\right) \varphi \cdot \mathbf{u}^{\prime} \varphi=\mathbf{p}^{\prime} \varphi \cdot 6 \cdot 5 \cdot 3 \ldots=1 .
$$

Consequently, the contradiction $\mathbf{w} \varphi \neq \mathbf{w}^{\prime} \varphi$ is established.

Proof of Proposition 26.1. It is routinely checked that $\mathscr{F}_{7}$ satisfies the identities (26.1). Hence it suffices to show that any identity $\mathbf{w} \approx \mathbf{w}^{\prime}$ satisfied by $\mathscr{F}_{7}$ is implied by the identities (26.1). By Lemma 26.2, the words $\mathbf{w}$ and $\mathbf{w}^{\prime}$ can be chosen to be in $\alpha$-canonical form. By Lemmas 26.3 and 26.4,

$$
\mathbf{w}=x_{0}^{e_{0}} \prod_{i=1}^{m}\left(x_{i}^{e_{i}} \mathbf{w}_{i}\right) \quad \text { and } \quad \mathbf{w}^{\prime}=x_{0}^{e_{0}} \prod_{i=1}^{m}\left(x_{i}^{e_{i}} \mathbf{w}_{i}^{\prime}\right)
$$

with $\mathbf{w}_{m}=\mathbf{w}_{m}^{\prime}$. Let $\ell<m$ be the least integer such that $\mathbf{w}_{\ell} \neq \mathbf{w}_{\ell}^{\prime}$. Then it is convenient to write

(a) $\mathbf{p}=x_{0}^{e_{0}} \prod_{i=1}^{\ell-1}\left(x_{i}^{e_{i}} \mathbf{w}_{i}\right)=x_{0}^{e_{0}} \prod_{i=1}^{\ell-1}\left(x_{i}^{e_{i}} \mathbf{w}_{i}^{\prime}\right)$.

Since $\left(\mathbf{w}_{\ell}, \mathbf{w}_{\ell}^{\prime}\right) \neq(\emptyset, \emptyset)$, generality is not lost by assuming $\mathbf{w}_{\ell} \neq \emptyset$. Then (I) implies $\mathbf{w}_{\ell}=x_{k}$ for some $k \in\{0, \ldots, \ell-1\}$ and

(b) $\mathbf{w}_{\ell}^{\prime} \in\left\{\emptyset, x_{0}, \ldots, x_{\ell-1}\right\} \backslash\left\{x_{k}\right\}$, 
whence

$$
\mathbf{w}=\mathbf{p} x_{\ell}^{e_{\ell}} \underbrace{x_{k}}_{\mathbf{w}_{\ell}} x_{\ell+1}^{e_{\ell+1}} \mathbf{q} \text { and } \mathbf{w}^{\prime}=\mathbf{p} x_{\ell}^{e_{\ell}} \mathbf{w}_{\ell}^{\prime} x_{\ell+1}^{e_{\ell+1}} \mathbf{q}^{\prime},
$$

where $\mathbf{q}=\mathbf{w}_{\ell+1} \prod_{i=\ell+2}^{m}\left(x_{i}^{e_{i}} \mathbf{w}_{i}\right)$ and $\mathbf{q}^{\prime}=\mathbf{w}_{\ell+1}^{\prime} \prod_{i=\ell+2}^{m}\left(x_{i}^{e_{i}} \mathbf{w}_{i}^{\prime}\right)$. Let $\varphi: \mathcal{X} \rightarrow \mathscr{F}_{7}$ denote the substitution

$$
z \mapsto \begin{cases}6 & \text { if } z=x_{k}, \\ 3 & \text { if } z=x_{\ell+1}, \\ 5 & \text { otherwise. }\end{cases}
$$

Since the prefix $\mathbf{p}$ contains the first $x_{k}$ of $\mathbf{w}$ and $x_{\ell+1} \notin \operatorname{con}\left(\mathbf{p} x_{\ell}\right)$ by (I) and (a), it follows that $\left(\mathbf{p} x_{\ell}^{e_{\ell}}\right) \varphi=5$. Hence

$$
\mathbf{w} \varphi=\left(\mathbf{p} x_{\ell}^{e_{\ell}}\right) \varphi \cdot x_{k} \varphi \cdot x_{\ell+1}^{e_{\ell+1}} \varphi \cdot \mathbf{q} \varphi=5 \cdot 6 \cdot 3 \cdot \mathbf{q} \varphi=4 .
$$

On the other hand, $\mathbf{w}_{\ell}^{\prime} \varphi \in\{\emptyset, 5\}$ by (b), so that

$$
\mathbf{w}^{\prime} \varphi=\left(\mathbf{p} x_{\ell}^{e_{\ell}}\right) \varphi \cdot \mathbf{w}_{\ell}^{\prime} \varphi \cdot x_{\ell+1}^{e_{\ell+1}} \varphi \cdot \mathbf{q}^{\prime} \varphi=5 \cdot \mathbf{w}_{\ell}^{\prime} \varphi \cdot 3 \cdot \mathbf{q}^{\prime} \varphi=1 .
$$

The contradiction $\mathbf{w} \varphi \neq \mathbf{w}^{\prime} \varphi$ implies that the integer $\ell$ does not exist. Consequently, $\mathbf{w}_{i}=\mathbf{w}_{i}^{\prime}$ for all $i$, whence the identity $\mathbf{w} \approx \mathbf{w}^{\prime}$ is trivial and so is implied by the identities (26.1).

\subsection{Finite basis property of $\mathscr{F}_{6}$ and $\mathscr{F}_{8}$}

The semigroup $\mathscr{F}_{8}^{\text {iso }}$ given by the following multiplication table is isomorphic to the semigroup $\mathscr{F}_{8}$ via the relabeling $1 \leftrightarrow 3$.

\begin{tabular}{c|cccccc}
$\mathscr{F}_{8}^{\text {iso }}$ & 1 & 2 & 3 & 4 & 5 & 6 \\
\hline 1 & 1 & 1 & 1 & 1 & 1 & 1 \\
2 & 3 & 3 & 3 & 3 & 2 & 2 \\
3 & 3 & 3 & 3 & 3 & 3 & 3 \\
4 & 4 & 4 & 4 & 4 & 4 & 4 \\
5 & 1 & 1 & 1 & 4 & 5 & 6 \\
6 & 1 & 4 & 4 & 4 & 5 & 6
\end{tabular}

It turns out that arguments in the present subsection will become simpler if $\mathscr{F}_{8}^{\text {iso }}$ is considered instead of $\mathscr{F}_{8}$.

Proposition 26.5. The variety generated by $S \in\left\{\mathscr{F}_{6}, \mathscr{F}_{8}^{\text {iso }}\right\}$ is defined by the identities

$$
\begin{aligned}
x^{2} \mathrm{H} x & \approx x \mathrm{H} x, \\
x \mathrm{H} x^{2} & \approx x \mathrm{H} x, \\
x \mathrm{H} y \mathrm{~K} x y & \approx x^{2} \mathrm{H} y \mathrm{~K} y, \\
x \mathrm{H} y z y x & \approx x \mathrm{H} y z x, \\
x y^{2} & \approx x y .
\end{aligned}
$$

In this section, a word $\mathbf{w}$ with ini $(\mathbf{w})=x_{0} \ldots x_{m}$ is said to be in $\beta$-canonical form if

$$
\mathbf{w}=x_{0}^{e} \prod_{i=1}^{m}\left(x_{i} \mathbf{w}_{i}\right),
$$


where all of the following are satisfied:

(I) $\mathbf{w}_{i} \in\left\{\emptyset, x_{0}, \ldots, x_{i-1}\right\}$;

(II) $e \in\{1,2\}$;

(III) if $\mathbf{w}_{i}=x_{0}$ for some $i \geqslant 1$, then $e=1$.

It is easily shown that a word in $\beta$-canonical form is also in $\alpha$-canonical form.

Lemma 26.6. Let $\mathbf{w}$ be any word. Then there exists some word $\overline{\mathbf{w}}$ in $\beta$-canonical form such that the identities (26.3) imply the identity $\mathbf{w} \approx \overline{\mathbf{w}}$.

Proof. It suffices to convert $\mathbf{w}$, using the identities (26.3), into a word in $\beta$-canonical form. Let ini $(\mathbf{w})=x_{0} \ldots x_{m}$. Note that the identities (26.3) imply the identities (26.1). Hence by Lemma 26.2, the identities (26.3) can be used to convert $\mathbf{w}$ into a word in $\alpha$-canonical form, that is, a word of the form $x_{0}^{e_{0}} \prod_{i=1}^{m}\left(x_{i}^{e_{i}} \mathbf{w}_{i}\right)$ that satisfies (I). The identity (26.3e) can be used to reduce the exponents $e_{1}, \ldots, e_{m}$ to 1 . It is easily seen that (II) and (III) are satisfied by applying the identities (26.3a).

Lemma 26.7. Let $S \in\left\{\mathscr{F}_{6}, \mathscr{F}_{8}^{\text {iso }}\right\}$. Suppose that $\mathbf{w} \approx \mathbf{w}^{\prime}$ is any identity satisfied by $S$. Then $\operatorname{ini}(\mathbf{w})=\operatorname{ini}\left(\mathbf{w}^{\prime}\right)$ and $\mathrm{t}(\mathbf{w})=\mathrm{t}\left(\mathbf{w}^{\prime}\right)$.

Proof. This follows from Lemma 2.1 since the subsemigroups $\{1,4,5\}$ and $\{5,6\}$ of $S$ are isomorphic to $L_{2}^{1}$ and $R_{2}$, respectively.

Lemma 26.8. Let $S \in\left\{\mathscr{F}_{6}, \mathscr{F}_{8}^{\text {iso }}\right\}$. Suppose that $S$ satisfies an identity $\mathbf{w} \approx \mathbf{w}^{\prime}$, where

$$
\mathbf{w}=x_{0}^{e} \prod_{i=1}^{m}\left(x_{i} \mathbf{w}_{i}\right) \quad \text { and } \quad \mathbf{w}^{\prime}=x_{0}^{e^{\prime}} \prod_{i=1}^{m}\left(x_{i} \mathbf{w}_{i}^{\prime}\right)
$$

are words in $\beta$-canonical form. Then $e=e^{\prime}$.

Proof. Let $\mathbf{u}=\prod_{i=1}^{m}\left(x_{i} \mathbf{w}_{i}\right)$ and $\mathbf{u}^{\prime}=\prod_{i=1}^{m}\left(x_{i} \mathbf{w}_{i}^{\prime}\right)$. Seeking a contradiction, suppose $e \neq e^{\prime}$, say $\left(e, e^{\prime}\right)=(1,2)$. Then $x_{0} \notin \operatorname{con}\left(\mathbf{u}^{\prime}\right)$ by (I) and (III). There are two cases to consider.

Case 1: $x_{0} \notin \operatorname{con}(\mathbf{u}) . \quad$ Let $\varphi_{1}: \mathcal{X} \rightarrow S$ denote the substitution

$$
z \mapsto \begin{cases}2 & \text { if } z=x_{0} \\ 5 & \text { otherwise }\end{cases}
$$

Then $\mathbf{w} \varphi_{1}=2 \cdot \mathbf{u} \varphi_{1}=2$ and $\mathbf{w}^{\prime} \varphi_{1}=x_{0}^{2} \varphi_{1} \cdot \mathbf{u}^{\prime} \varphi_{1} \in\{1,3\}$, whence $\mathbf{w} \varphi_{1} \neq \mathbf{w}^{\prime} \varphi_{1}$ is impossible.

Case 2: $x_{0} \in \operatorname{con}(\mathbf{u})$. Then $x_{0}$ is non-simple in $\mathbf{w}$. Since the word $\mathbf{w}$ is in $\beta$-canonical form, the second $x_{0}$ in $\mathbf{w}$ coincides with one of $\mathbf{w}_{1}, \ldots, \mathbf{w}_{m}$, say $x_{0}=\mathbf{w}_{\ell}$ for some least possible $\ell \in\{1, \ldots, m\}$. Then $x_{0} \neq x_{\ell}$ by (I), and $\mathbf{w}$ can be written as

$$
\mathbf{w}=x_{0} \mathbf{p} x_{\ell} \underbrace{x_{0}}_{\mathbf{w}_{\ell}} \mathbf{q}
$$

where $\mathbf{p}=\prod_{i=1}^{\ell-1}\left(x_{i} \mathbf{w}_{i}\right)$ and $\mathbf{q}=\prod_{i=\ell+1}^{m}\left(x_{i} \mathbf{w}_{i}\right)$ with $x_{\ell} \notin \operatorname{con}\left(x_{0} \mathbf{p}\right)$ and

(a) $x_{0} \notin \operatorname{con}\left(\mathbf{p} x_{\ell}\right)$.

As for the word $\mathbf{w}^{\prime}$, since $e^{\prime}=2$, it can be written as

$$
\mathbf{w}^{\prime}=x_{0}^{2} \mathbf{p}^{\prime} x_{\ell} \mathbf{w}_{\ell}^{\prime} \mathbf{q}^{\prime}
$$


where $\mathbf{p}^{\prime}=\prod_{i=1}^{\ell-1}\left(x_{i} \mathbf{w}_{i}^{\prime}\right)$ and $\mathbf{q}^{\prime}=\prod_{i=\ell+1}^{m}\left(x_{i} \mathbf{w}_{i}^{\prime}\right)$ with $x_{\ell} \notin \operatorname{con}\left(x_{0} \mathbf{p}^{\prime}\right)$. By (I) and (III),

(b) $x_{0} \notin \operatorname{con}\left(\mathbf{p}^{\prime} x_{\ell} \mathbf{w}_{\ell}^{\prime} \mathbf{q}^{\prime}\right)$.

In particular, $\mathrm{t}\left(\mathbf{w}^{\prime}\right) \neq x_{0}$. Since $\mathrm{t}(\mathbf{w})=\mathrm{t}\left(\mathbf{w}^{\prime}\right)$ by Lemma 26.7 , it follows that $\mathrm{t}(\mathbf{w}) \neq x_{0}$. Therefore $\mathbf{q} \neq \emptyset$, whence

(c) $\mathrm{h}(\mathbf{q})=x_{\ell+1} \notin \operatorname{con}\left(x_{0} \mathbf{p} x_{\ell}\right)$

and $\operatorname{ini}(\mathbf{w})=\ldots x_{\ell} x_{\ell+1} \ldots$ Further, ini $(\mathbf{w})=\operatorname{ini}\left(\mathbf{w}^{\prime}\right)$ by Lemma 26.7 , and this implies $\mathbf{q}^{\prime} \neq \emptyset$ and

(d) $\mathrm{h}\left(\mathbf{q}^{\prime}\right)=x_{\ell+1} \notin \operatorname{con}\left(x_{0} \mathbf{p}^{\prime} x_{\ell} \mathbf{w}_{\ell}^{\prime}\right)$.

Let $\varphi_{2}: \mathcal{X} \rightarrow S$ denote the substitution

$$
z \mapsto \begin{cases}6 & \text { if } z=x_{0}, \\ 3 & \text { if } z=x_{\ell+1}, \\ 5 & \text { otherwise. }\end{cases}
$$

Since $x_{0}, x_{\ell+1} \notin \operatorname{con}\left(\mathbf{p} x_{\ell}\right)$ by (a) and (c), it follows that $\left(\mathbf{p} x_{\ell}\right) \varphi_{2}=5$. Hence

$$
\mathbf{w} \varphi_{2}=x_{0} \varphi_{2} \cdot\left(\mathbf{p} x_{\ell}\right) \varphi_{2} \cdot x_{0} \varphi_{2} \cdot \mathbf{q} \varphi_{2}=6 \cdot 5 \cdot 6 \cdot 3 \ldots=4 .
$$

On the other hand, $x_{0}, x_{\ell+1} \notin \operatorname{con}\left(\mathbf{p}^{\prime} x_{\ell} \mathbf{w}_{\ell}^{\prime}\right)$ by (b) and (d), so that $\left(\mathbf{p}^{\prime} x_{\ell} \mathbf{w}_{\ell}^{\prime}\right) \varphi_{2}=5$. Therefore

$$
\mathbf{w}^{\prime} \varphi_{2}=x_{0}^{2} \varphi_{2} \cdot\left(\mathbf{p}^{\prime} x_{\ell} \mathbf{w}_{\ell}^{\prime}\right) \varphi_{2} \cdot \mathbf{q}^{\prime} \varphi_{2}=6 \cdot 5 \cdot 3 \ldots=1 .
$$

Consequently, the contradiction $\mathbf{w} \varphi_{2} \neq \mathbf{w}^{\prime} \varphi_{2}$ is deduced.

Proof of Proposition 26.5. Let $S \in\left\{\mathscr{F}_{6}, \mathscr{F}_{8}^{\text {iso }}\right\}$. It is routinely checked that $S$ satisfies the identities (26.3). Hence it suffices to show that any identity $\mathbf{w} \approx \mathbf{w}^{\prime}$ satisfied by $S$ is implied by the identities (26.3). By Lemma 26.6, the words $\mathbf{w}$ and $\mathbf{w}^{\prime}$ can be assumed to be in $\beta$-canonical form. By Lemmas 26.7 and 26.8,

$$
\mathbf{w}=x_{0}^{e} \prod_{i=1}^{m}\left(x_{i} \mathbf{w}_{i}\right) \quad \text { and } \quad \mathbf{w}^{\prime}=x_{0}^{e} \prod_{i=1}^{m}\left(x_{i} \mathbf{w}_{i}^{\prime}\right)
$$

with $\mathbf{w}_{m}=\mathbf{w}_{m}^{\prime}$. Let $\ell<m$ be the least integer such that $\mathbf{w}_{\ell} \neq \mathbf{w}_{\ell}^{\prime}$. For convenience, write

(a) $\mathbf{p}=x_{0}^{e} \prod_{i=1}^{\ell-1}\left(x_{i} \mathbf{w}_{i}\right)=x_{0}^{e} \prod_{i=1}^{\ell-1}\left(x_{i} \mathbf{w}_{i}^{\prime}\right)$.

Since $\left(\mathbf{w}_{\ell}, \mathbf{w}_{\ell}^{\prime}\right) \neq(\emptyset, \emptyset)$, generality is not lost by assuming $\mathbf{w}_{\ell} \neq \emptyset$. Then (I) implies $\mathbf{w}_{\ell}=x_{k}$ for some $k \in\{0, \ldots, \ell-1\}$ and

(b) $\mathbf{w}_{\ell}^{\prime} \in\left\{\emptyset, x_{0}, \ldots, x_{\ell-1}\right\} \backslash\left\{x_{k}\right\}$, whence

$$
\mathbf{w}=\mathbf{p} x_{\ell} \underbrace{x_{k}}_{\mathbf{w}_{\ell}} x_{\ell+1} \mathbf{q} \text { and } \mathbf{w}^{\prime}=\mathbf{p} x_{\ell} \mathbf{w}_{\ell}^{\prime} x_{\ell+1} \mathbf{q}^{\prime},
$$

where $\mathbf{q}=\mathbf{w}_{\ell+1} \prod_{i=\ell+2}^{m}\left(x_{i} \mathbf{w}_{i}\right)$ and $\mathbf{q}^{\prime}=\mathbf{w}_{\ell+1}^{\prime} \prod_{i=\ell+2}^{m}\left(x_{i} \mathbf{w}_{i}^{\prime}\right)$. Let $\varphi: \mathcal{X} \rightarrow S$ denote the substitution

$$
z \mapsto \begin{cases}6 & \text { if } z=x_{k}, \\ 3 & \text { if } z=x_{\ell+1}, \\ 5 & \text { otherwise. }\end{cases}
$$

Since $\mathbf{p}$ contains the first $x_{k}$ of $\mathbf{w}$ and $x_{\ell+1} \notin \operatorname{con}\left(\mathbf{p} x_{\ell}\right)$ by (I) and (a), it follows that $\left(\mathbf{p} x_{\ell}\right) \varphi=5$. Hence

$$
\mathbf{w} \varphi=\left(\mathbf{p} x_{\ell}\right) \varphi \cdot x_{k} \varphi \cdot x_{\ell+1} \varphi \cdot \mathbf{q} \varphi=5 \cdot 6 \cdot 3 \cdot \mathbf{q} \varphi=4 .
$$

On the other hand, $\mathbf{w}_{\ell}^{\prime} \varphi \in\{\emptyset, 5\}$ by (b), so that

$$
\mathbf{w}^{\prime} \varphi=\left(\mathbf{p} x_{\ell}\right) \varphi \cdot \mathbf{w}_{\ell}^{\prime} \varphi \cdot x_{\ell+1} \varphi \cdot \mathbf{q}^{\prime} \varphi=5 \cdot \mathbf{w}_{\ell}^{\prime} \varphi \cdot 3 \cdot \mathbf{q}^{\prime} \varphi=1 .
$$

The contradiction $\mathbf{w} \varphi \neq \mathbf{w}^{\prime} \varphi$ implies that the integer $\ell$ does not exist. Consequently, $\mathbf{w}_{i}=\mathbf{w}_{i}^{\prime}$ for all $i$, whence the identity $\mathbf{w} \approx \mathbf{w}^{\prime}$ is trivial and so is implied by the identities (26.3). 
27. $\mathscr{F}_{9}, \mathscr{F}_{10}, \mathscr{G}_{1}, \mathscr{G}_{2}$

This section establishes the finite basis property of the following semigroups.

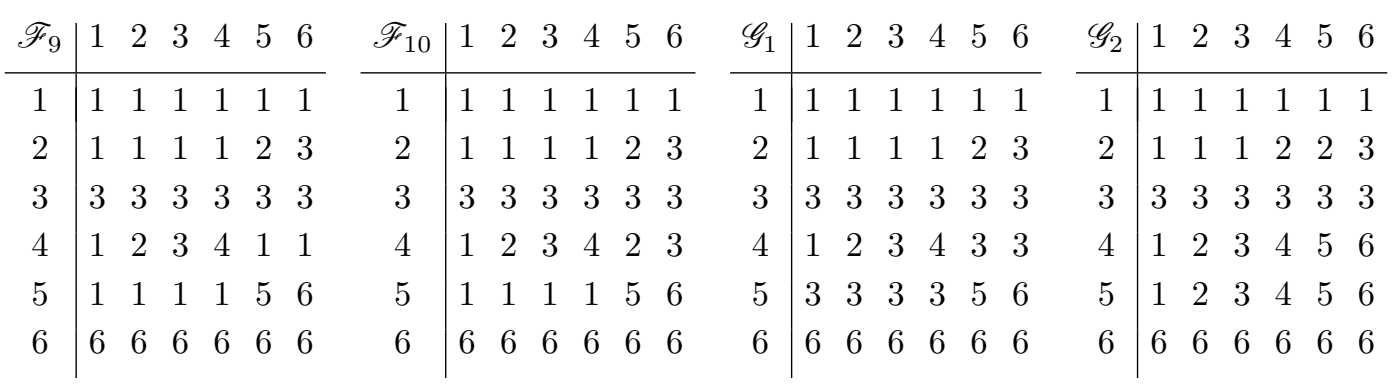

27.1. Finite basis property of $\mathscr{F}_{10}$

Proposition 27.1. The variety generated by $\mathscr{F}_{10}$ is defined by the identities

$$
\begin{aligned}
x \mathrm{H} x^{2} & \approx x \mathrm{H} x, \\
x \mathrm{H} y \mathrm{~K} x y & \approx x \mathrm{H} y \mathrm{~K} y x, \\
x \mathrm{H} y \mathrm{~K} y x & \approx x \mathrm{H} y \mathrm{~K} x, \\
x \mathrm{H} y \mathrm{~K} x \mathrm{\top} y & \approx x \mathrm{H} y \mathrm{~K} x \mathrm{~T} x .
\end{aligned}
$$

In this section, a word $\mathbf{w}$ with $\operatorname{ini}(\mathbf{w})=x_{1} \ldots x_{m}$ is said to be in $\alpha$-canonical form if

$$
\mathbf{w}=\prod_{i=1}^{m}\left(x_{i} \mathbf{w}_{i}\right),
$$

where:

(I) $\mathbf{w}_{i} \in\left\{\emptyset, x_{1}, \ldots, x_{i}\right\}$;

(II) $\mathbf{w}$ does not contain any factor of the form $x_{j} \mathbf{a} x_{k} \mathbf{b} x_{j} \mathbf{c} x_{k}$ where $\mathbf{a}, \mathbf{b}, \mathbf{c} \in \mathcal{X}^{*}$ and $j<k$.

Lemma 27.2. Let $\mathbf{w}$ be any word. Then there exists some word $\overline{\mathbf{w}}$ in $\alpha$-canonical form such that the identities (27.1) imply the identity $\mathbf{w} \approx \overline{\mathbf{w}}$.

Proof. It suffices to convert $\mathbf{w}$, using the identities (27.1), into a word in $\alpha$-canonical form. Let ini $(\mathbf{w})=x_{1} \ldots x_{m}$, so that $\mathbf{w}=\prod_{i=1}^{m}\left(x_{i} \mathbf{w}_{i}\right)$ for some $\mathbf{w}_{i} \in\left\{x_{1}, \ldots, x_{i}\right\}^{*}$. Suppose $\mathbf{w}_{i} \neq \emptyset$. Then there exists a least integer $\ell$ such that $x_{\ell} \in \operatorname{con}\left(\mathbf{w}_{i}\right)$, whence $\operatorname{con}\left(\mathbf{w}_{i}\right) \subseteq\left\{x_{\ell}, \ldots, x_{i}\right\}$. Since the letters of $\mathbf{w}_{i}$ are non-first occurrences in $\mathbf{w}$, the identities $(27.1 \mathrm{~b})$ can be used to arrange them, within $\mathbf{w}_{i}$, in any order. In particular, the identities $(27.1 \mathrm{~b})$ can be used to move all occurrences of $x_{\ell}$ in $\mathbf{w}_{i}$ to the right, so that

$$
\mathbf{w}=\ldots x_{\ell} \mathbf{w}_{\ell} \ldots x_{i} \mathbf{w}_{i} \ldots \stackrel{(27.1 \mathrm{~b})}{\approx} \ldots x_{\ell} \mathbf{w}_{\ell} \ldots x_{i} \mathbf{w}_{i}^{\prime} x_{\ell}^{e} \ldots
$$

where $e=\operatorname{occ}\left(x_{\ell}, \mathbf{w}_{i}\right)$ and $\mathbf{w}_{i}^{\prime}$ is obtained from $\mathbf{w}_{i}$ by removing all occurrences of $x_{\ell}$. Note that $\operatorname{con}\left(\mathbf{w}_{i}^{\prime}\right) \subseteq\left\{x_{\ell+1}, \ldots, x_{i}\right\}$ and any letter in $\mathbf{w}_{i}^{\prime}$ is a non-first occurrence in $\mathbf{w}$ that is sandwiched between two occurrences of $x_{\ell}$. The identities (27.1c) can be used to remove the letters of $\mathbf{w}_{i}^{\prime}$ one by one starting from the right, and the identities $(27.1 \mathrm{a})$ can be used to reduce the exponent $e$ to 1 :

$$
\ldots x_{\ell} \mathbf{w}_{\ell} \ldots x_{i} \mathbf{w}_{i}^{\prime} x_{\ell}^{e} \ldots \stackrel{(27.1 \mathrm{c})}{\approx} \ldots x_{\ell} \mathbf{w}_{\ell} \ldots x_{i} x_{\ell}^{e} \ldots \stackrel{(27.1 \mathrm{a})}{\approx} \ldots x_{\ell} \mathbf{w}_{\ell} \ldots x_{i} x_{\ell} \ldots
$$


Therefore the factor $\mathbf{w}_{i}$ is converted into $x_{\ell}$, whence (I) is satisfied. Since

$$
\mathbf{w}=\ldots x_{j} \mathbf{a} x_{k} \mathbf{b} x_{j} \mathbf{c} x_{k} \ldots \stackrel{(27.1 \mathrm{~d})}{\approx} \ldots x_{j} \mathbf{a} x_{k} \mathbf{b} x_{j} \mathbf{c} x_{j} \ldots
$$

the identities (27.1d) can be used to convert $\mathbf{w}$ into a word that satisfies (II).

Proof of Proposition 27.1. It is routinely checked that $\mathscr{F}_{10}$ satisfies the identities (27.1). Hence it suffices to show that any identity $\mathbf{w} \approx \mathbf{w}^{\prime}$ satisfied by $\mathscr{F}_{10}$ is implied by the identities (27.1). By Lemma 27.2, the words $\mathbf{w}$ and $\mathbf{w}^{\prime}$ can be assumed to be in $\alpha$-canonical form. Since the subsemigroup $\{1,3,4\}$ of $\mathscr{F}_{10}$ is isomorphic to $L_{2}^{1}$, it follows from Lemma 2.1(iii) that

$$
\mathbf{w}=\prod_{i=1}^{m}\left(x_{i} \mathbf{w}_{i}\right) \quad \text { and } \quad \mathbf{w}^{\prime}=\prod_{i=1}^{m}\left(x_{i} \mathbf{w}_{i}^{\prime}\right) .
$$

Let $\ell$ be the least integer such that $\mathbf{w}_{\ell} \neq \mathbf{w}_{\ell}^{\prime}$. Multiplying both sides of the identity $\mathbf{w} \approx \mathbf{w}^{\prime}$ on the right by $x_{m+1}^{2}$ if necessary, generality is not lost by further assuming $\ell<m$. Hence

$$
\mathbf{w}=\mathbf{p} x_{\ell} \mathbf{w}_{\ell} x_{\ell+1} \mathbf{q} \text { and } \quad \mathbf{w}^{\prime}=\mathbf{p} x_{\ell} \mathbf{w}_{\ell}^{\prime} x_{\ell+1} \mathbf{q}^{\prime},
$$

where $\mathbf{p}=\prod_{i=1}^{\ell-1}\left(x_{i} \mathbf{w}_{i}\right), \mathbf{q}=\mathbf{w}_{\ell+1} \prod_{i=\ell+2}^{m}\left(x_{i} \mathbf{w}_{i}\right)$, and $\mathbf{q}^{\prime}=\mathbf{w}_{\ell+1}^{\prime} \prod_{i=\ell+2}^{m}\left(x_{i} \mathbf{w}_{i}^{\prime}\right)$. By symmetry, there are four cases to consider.

Case 1: $\left(\mathbf{w}_{\ell}, \mathbf{w}_{\ell}^{\prime}\right)=\left(x_{\ell}, \emptyset\right)$. Then

$$
\mathbf{w}=\mathbf{p} x_{\ell}^{2} x_{\ell+1} \mathbf{q} \quad \text { and } \quad \mathbf{w}^{\prime}=\mathbf{p} x_{\ell} x_{\ell+1} \mathbf{q}^{\prime}
$$

with $x_{\ell}, x_{\ell+1} \notin \operatorname{con}(\mathbf{p})$. Let $\varphi_{1}: \mathcal{X} \rightarrow \mathscr{F}_{10}$ denote the substitution

$$
z \mapsto \begin{cases}4 & \text { if } z \in \operatorname{con}(\mathbf{p}) \\ 2 & \text { if } z=x_{\ell} \\ 6 & \text { otherwise }\end{cases}
$$

Then $\mathbf{w} \varphi_{1}=\mathbf{p} \varphi_{1} \cdot 2^{2} \cdot 6 \cdot \mathbf{q} \varphi_{1}=1$ and $\mathbf{w}^{\prime} \varphi_{1}=\mathbf{p} \varphi_{1} \cdot 2 \cdot 6 \cdot \mathbf{q}^{\prime} \varphi_{1}=3$, whence the contradiction $\mathbf{w} \varphi_{1} \neq \mathbf{w}^{\prime} \varphi_{1}$ is deduced.

Case 2: $\left(\mathbf{w}_{\ell}, \mathbf{w}_{\ell}^{\prime}\right)=\left(x_{i}, \emptyset\right)$ for some $i<\ell$. Then

$$
\mathbf{w}=\underbrace{\mathbf{a} x_{i} \mathbf{b}}_{\mathbf{p}} x_{\ell} x_{i} x_{\ell+1} \mathbf{q} \quad \text { and } \quad \mathbf{w}^{\prime}=\underbrace{\mathbf{a} x_{i} \mathbf{b}}_{\mathbf{p}} x_{\ell} x_{\ell+1} \mathbf{q}^{\prime} \quad \text { for some } \mathbf{a}, \mathbf{b} \in \mathcal{X}^{*}
$$

such that $x_{i} \notin \operatorname{con}(\mathbf{a}), x_{\ell} \notin \operatorname{con}\left(\mathbf{a} x_{i} \mathbf{b}\right)$, and $x_{\ell+1} \notin \operatorname{con}\left(\mathbf{a} x_{i} \mathbf{b} x_{\ell}\right)$. Let $\varphi_{2}: \mathcal{X} \rightarrow \mathscr{F}_{10}$ denote the substitution

$$
z \mapsto \begin{cases}4 & \text { if } z \in \operatorname{con}\left(\mathbf{a} x_{i} \mathbf{b}\right) \\ 2 & \text { if } z=x_{\ell} \\ 6 & \text { otherwise }\end{cases}
$$

Then $\mathbf{w} \varphi_{2}=4 \cdot 2 \cdot 4 \cdot 6 \cdot \mathbf{q} \varphi_{2}=1$ and $\mathbf{w}^{\prime} \varphi_{2}=4 \cdot 2 \cdot 6 \cdot \mathbf{q}^{\prime} \varphi_{2}=3$, whence the contradiction $\mathbf{w} \varphi_{2} \neq \mathbf{w}^{\prime} \varphi_{2}$ is deduced. 
Case 3: $\left(\mathbf{w}_{\ell}, \mathbf{w}_{\ell}^{\prime}\right)=\left(x_{i}, x_{\ell}\right)$ for some $i<\ell$. Then

$$
\mathbf{w}=\underbrace{\mathbf{c} x_{i} \mathbf{d}}_{\mathbf{p}} x_{\ell} x_{i} x_{\ell+1} \mathbf{q} \quad \text { and } \quad \mathbf{w}^{\prime}=\underbrace{\mathbf{c} x_{i} \mathbf{d}}_{\mathbf{p}} x_{\ell}^{2} x_{\ell+1} \mathbf{q}^{\prime} \quad \text { for some } \mathbf{c}, \mathbf{d} \in \mathcal{X}^{*}
$$

such that $x_{i} \notin \operatorname{con}(\mathbf{c}), x_{\ell} \notin \operatorname{con}\left(\mathbf{c} x_{i} \mathbf{d}\right)$, and $x_{\ell+1} \notin \operatorname{con}\left(\mathbf{c} x_{i} \mathbf{d} x_{\ell}\right)$. Let $\varphi_{3}: \mathcal{X} \rightarrow \mathscr{F}_{10}$ denote the substitution

$$
z \mapsto \begin{cases}4 & \text { if } z \in \operatorname{con}\left(\mathbf{c} x_{i} \mathbf{d}\right), \\ 5 & \text { if } z=x_{\ell} \\ 6 & \text { otherwise. }\end{cases}
$$

Then $\mathbf{w} \varphi_{3}=4 \cdot 5 \cdot 4 \cdot 6 \cdot \mathbf{q} \varphi_{3}=1$ and $\mathbf{w}^{\prime} \varphi_{3}=4 \cdot 5^{2} \cdot 6 \cdot \mathbf{q}^{\prime} \varphi_{3}=3$, whence the contradiction $\mathbf{w} \varphi_{3} \neq \mathbf{w}^{\prime} \varphi_{3}$ is deduced.

Case 4: $\left(\mathbf{w}_{\ell}, \mathbf{w}_{\ell}^{\prime}\right)=\left(x_{i}, x_{j}\right)$ for some $i$ and $j$ with $i<j<\ell$. Then

$$
\mathbf{w}=\underbrace{\mathbf{e} x_{i} \mathbf{f} x_{j} \mathbf{g}}_{\mathbf{p}} x_{\ell} x_{i} x_{\ell+1} \mathbf{q} \text { and } \mathbf{w}^{\prime}=\underbrace{\mathbf{e} x_{i} \mathbf{f} x_{j} \mathbf{g}}_{\mathbf{p}} x_{\ell} x_{j} x_{\ell+1} \mathbf{q}^{\prime} \quad \text { for some } \mathbf{e}, \mathbf{f}, \mathbf{g} \in \mathcal{X}^{*}
$$

such that $x_{i} \notin \operatorname{con}(\mathbf{e}), x_{j} \notin \operatorname{con}\left(\mathbf{e} x_{i} \mathbf{f}\right), x_{\ell} \notin \operatorname{con}\left(\mathbf{e} x_{i} \mathbf{f} x_{j} \mathbf{g}\right)$, and $x_{\ell+1} \notin \operatorname{con}\left(\mathbf{e} x_{i} \mathbf{f} x_{j} \mathbf{g} x_{\ell}\right)$. If the words $\mathbf{e} x_{i} \mathbf{f}$ and $\mathbf{g} x_{\ell}$ share a common letter, then (II) is violated by the word $\mathbf{w}^{\prime}$. It follows that $\operatorname{con}\left(\mathbf{e} x_{i} \mathbf{f}\right) \cap \operatorname{con}\left(x_{j} \mathbf{g} x_{\ell}\right)=\emptyset$. Let $\varphi_{4}: \mathcal{X} \rightarrow \mathscr{F}_{10}$ denote the substitution

$$
z \mapsto \begin{cases}4 & \text { if } z \in \operatorname{con}\left(\mathbf{e} x_{i} \mathbf{f}\right), \\ 5 & \text { if } z \in \operatorname{con}\left(x_{j} \mathbf{g} x_{\ell}\right), \\ 6 & \text { otherwise. }\end{cases}
$$

Then the contradiction $\mathbf{w} \varphi_{4} \neq \mathbf{w}^{\prime} \varphi_{4}$ is deduced because

$$
\mathbf{w} \varphi_{4}=\left(\mathbf{e} x_{i} \mathbf{f}\right) \varphi_{4} \cdot\left(x_{j} \mathbf{g} x_{\ell}\right) \varphi_{4} \cdot x_{i} \varphi_{4} \cdot x_{\ell+1} \varphi_{4} \cdot \mathbf{q} \varphi_{4}=4 \cdot 5 \cdot 4 \cdot 6 \cdot \mathbf{q} \varphi_{4}=1
$$

and

$$
\mathbf{w}^{\prime} \varphi_{4}=\left(\mathbf{e} x_{i} \mathbf{f}\right) \varphi_{4} \cdot\left(x_{j} \mathbf{g} x_{\ell} x_{j}\right) \varphi_{4} \cdot x_{\ell+1} \varphi_{4} \cdot \mathbf{q}^{\prime} \varphi_{4}=4 \cdot 5 \cdot 6 \cdot \mathbf{q}^{\prime} \varphi_{4}=3 .
$$

Since none of the four cases just considered is possible, the integer $\ell$ does not exist. Consequently, $\mathbf{w}_{i}=\mathbf{w}_{i}^{\prime}$ for all $i$, whence the identity $\mathbf{w} \approx \mathbf{w}^{\prime}$ is trivial and so is implied by the identities (27.1).

27.2. Finite basis property of $\mathscr{F}_{9}$ and $\mathscr{G}_{1}$

Proposition 27.3. The variety generated by $S \in\left\{\mathscr{F}_{9}, \mathscr{G}_{1}\right\}$ is defined by the identities

$$
\begin{aligned}
x \mathrm{H} x^{2} & \approx x \mathrm{H} x, \\
x \mathrm{H} y \mathrm{~K} x y & \approx x \mathrm{H} y \mathrm{~K} y x, \\
x \mathrm{H} y \mathrm{~K} y x & \approx x \mathrm{H} y \mathrm{~K} x, \\
x \mathrm{H} y \mathrm{~K} x \mathrm{~T} y & \approx x \mathrm{H} y \mathrm{~K} x \mathrm{~T} x, \\
x \mathrm{H} x y \mathrm{~K} y & \approx x \mathrm{H} x y \mathrm{~K} x .
\end{aligned}
$$

In this section, a word $\mathbf{w}$ with ini $(\mathbf{w})=x_{1} \ldots x_{m}$ is said to be in $\beta$-canonical form if

$$
\mathbf{w}=\prod_{i=1}^{m}\left(x_{i} \mathbf{w}_{i}\right),
$$


where all of the following are satisfied:

(I) $\mathbf{w}_{i} \in\left\{\emptyset, x_{1}, \ldots, x_{i}\right\}$;

(II) $\mathbf{w}$ does not contain any factor of the form $x_{j} \mathbf{a} x_{k} \mathbf{b} x_{j} \mathbf{c} x_{k}$ where $\mathbf{a}, \mathbf{b}, \mathbf{c} \in \mathcal{X}^{*}$ and $j<k$;

(III) $\mathbf{w}$ does not contain any factor of the form $x_{j} \mathbf{d} x_{j} x_{k} \mathbf{e} x_{k}$ where $\mathbf{d}, \mathbf{e} \in \mathcal{X}^{*}$ and $j<k$.

Observe that a word in $\beta$-canonical form is also in $\alpha$-canonical form.

Lemma 27.4. Let $\mathbf{w}$ be any word. Then there exists some word $\overline{\mathbf{w}}$ in $\beta$-canonical form such that the identities (27.2) imply the identity $\mathbf{w} \approx \overline{\mathbf{w}}$.

Proof. It suffices to convert $\mathbf{w}$, using the identities (27.2), into a word in $\beta$-canonical form. Let $\operatorname{ini}(\mathbf{w})=x_{1} \ldots x_{m}$. Then $\mathbf{w}$ can be written as $\mathbf{w}=\prod_{i=1}^{m}\left(x_{i} \mathbf{w}_{i}\right)$ where $\mathbf{w}_{i} \in\left\{x_{1}, \ldots, x_{i}\right\}^{*}$. Note that the identities (27.2) contain the identities (27.1). Hence by Lemma 27.2, the identities $(27.2)$ can be used to convert $\mathbf{w}$ into a word in $\alpha$-canonical form, that is, a word that satisfies (I) and (II). Since

$$
\mathbf{w}=\ldots x_{j} \mathbf{d} x_{j} x_{k} \mathbf{e}_{1} x_{k} \mathbf{e}_{2} x_{k} \ldots \mathbf{e}_{r} x_{k} \ldots \stackrel{(27.2 \mathrm{e})}{\approx} \ldots x_{j} \mathbf{d} x_{j} x_{k} \mathbf{e}_{1} x_{j} \mathbf{e}_{2} x_{j} \ldots \mathbf{e}_{r} x_{j} \ldots
$$

the identities (27.2e) can then be used to convert $\mathbf{w}$ into a word that satisfies (III).

Proof of Proposition 27.3. Let $S \in\left\{\mathscr{F}_{9}, \mathscr{G}_{1}\right\}$. It is routinely checked that $S$ satisfies the identities (27.2). Hence it suffices to show that any identity $\mathbf{w} \approx \mathbf{w}^{\prime}$ satisfied by $S$ is implied by the identities (27.2). By Lemma 27.4, the words $\mathbf{w}$ and $\mathbf{w}^{\prime}$ can be assumed to be in $\beta$ canonical form. Since the subsemigroup $\{1,3,4\}$ of $S$ is isomorphic to $L_{2}^{1}$, it follows from Lemma 2.1(iii) that

$$
\mathbf{w}=\prod_{i=1}^{m}\left(x_{i} \mathbf{w}_{i}\right) \quad \text { and } \quad \mathbf{w}^{\prime}=\prod_{i=1}^{m}\left(x_{i} \mathbf{w}_{i}^{\prime}\right) .
$$

Let $\ell$ be the least integer such that $\mathbf{w}_{\ell} \neq \mathbf{w}_{\ell}^{\prime}$. Multiplying both sides of the identity $\mathbf{w} \approx \mathbf{w}^{\prime}$ on the right by $x_{m+1}^{2}$ if necessary, generality is not lost by further assuming $\ell<m$. Hence

$$
\mathbf{w}=\mathbf{p} x_{\ell} \mathbf{w}_{\ell} x_{\ell+1} \mathbf{q} \text { and } \quad \mathbf{w}^{\prime}=\mathbf{p} x_{\ell} \mathbf{w}_{\ell}^{\prime} x_{\ell+1} \mathbf{q}^{\prime},
$$

where $\mathbf{p}=\prod_{i=1}^{\ell-1}\left(x_{i} \mathbf{w}_{i}\right), \mathbf{q}=\mathbf{w}_{\ell+1} \prod_{i=\ell+2}^{m}\left(x_{i} \mathbf{w}_{i}\right)$, and $\mathbf{q}^{\prime}=\mathbf{w}_{\ell+1}^{\prime} \prod_{i=\ell+2}^{m}\left(x_{i} \mathbf{w}_{i}^{\prime}\right)$. By symmetry, there are three cases to consider.

Case 1: $\left(\mathbf{w}_{\ell}, \mathbf{w}_{\ell}^{\prime}\right)=\left(x_{i}, \emptyset\right)$ for some $i \leqslant \ell$. Then a contradiction is obtained by the same arguments as in Cases 1 and 2 of the proof of Proposition 27.1.

Case 2: $\left(\mathbf{w}_{\ell}, \mathbf{w}_{\ell}^{\prime}\right)=\left(x_{i}, x_{\ell}\right)$ for some $i<\ell$. Then

$$
\mathbf{w}=\underbrace{\mathbf{c} x_{i} \mathbf{d}}_{\mathbf{p}} x_{\ell} x_{i} x_{\ell+1} \mathbf{q} \quad \text { and } \quad \mathbf{w}^{\prime}=\underbrace{\mathbf{c} x_{i} \mathbf{d}}_{\mathbf{p}} x_{\ell}^{2} x_{\ell+1} \mathbf{q}^{\prime} \quad \text { for some } \mathbf{c}, \mathbf{d} \in \mathcal{X}^{*}
$$

such that $x_{i} \notin \operatorname{con}(\mathbf{c}), x_{\ell} \notin \operatorname{con}\left(\mathbf{c} x_{i} \mathbf{d}\right)$, and $x_{\ell+1} \notin \operatorname{con}\left(\mathbf{c} x_{i} \mathbf{d} x_{\ell}\right)$. There are two subcases.

2.1. $\mathbf{d} \neq \emptyset$. Then $\mathbf{d}=\mathbf{u} t$ for some $\mathbf{u} \in \mathcal{X}^{*}$ with $t=\mathrm{t}(\mathbf{d})$, whence

$$
\mathbf{w}=\underbrace{\mathbf{c} x_{i} \mathbf{u} t}_{\mathbf{p}} x_{\ell} x_{i} x_{\ell+1} \mathbf{q} \text { and } \mathbf{w}^{\prime}=\underbrace{\mathbf{c} x_{i} \mathbf{u} t}_{\mathbf{p}} x_{\ell}^{2} x_{\ell+1} \mathbf{q}^{\prime} .
$$

If $t \in \operatorname{con}\left(\mathbf{c} x_{i} \mathbf{u}\right)$, then (III) is violated by the word $\mathbf{w}^{\prime}$. Hence $t \notin \operatorname{con}\left(\mathbf{c} x_{i} \mathbf{u}\right)$. Let $\varphi_{1}: \mathcal{X} \rightarrow S$ denote the substitution

$$
z \mapsto \begin{cases}4 & \text { if } z \in \operatorname{con}\left(\mathbf{c} x_{i} \mathbf{u}\right) \\ 2 & \text { if } z=t \\ 5 & \text { if } z=x_{\ell} \\ 6 & \text { otherwise }\end{cases}
$$


Then $\mathbf{w} \varphi_{1}=4 \cdot 2 \cdot 5 \cdot 4 \cdot 6 \cdot \mathbf{q} \varphi_{1}=1$ and $\mathbf{w}^{\prime} \varphi_{1}=4 \cdot 2 \cdot 5^{2} \cdot 6 \cdot \mathbf{q}^{\prime} \varphi_{1}=3$, whence the contradiction $\mathbf{w} \varphi_{1} \neq \mathbf{w}^{\prime} \varphi_{1}$ is deduced.

2.2. $\mathbf{d}=\emptyset$. Then

$$
\mathbf{w}=\underbrace{\mathbf{c} x_{i}}_{\mathbf{p}} x_{\ell} x_{i} x_{\ell+1} \mathbf{q} \text { and } \mathbf{w}^{\prime}=\underbrace{\mathbf{c} x_{i}}_{\mathbf{p}} x_{\ell}^{2} x_{\ell+1} \mathbf{q}^{\prime} .
$$

Let $\varphi_{2}: \mathcal{X} \rightarrow S$ denote the substitution

$$
z \mapsto \begin{cases}4 & \text { if } z \in \operatorname{con}(\mathbf{c}), \\ 2 & \text { if } z=x_{i} \\ 5 & \text { if } z=x_{\ell} \\ 6 & \text { otherwise. }\end{cases}
$$

Then $\mathbf{w} \varphi_{2}=\mathbf{c} \varphi_{2} \cdot 2 \cdot 5 \cdot 2 \cdot 6 \cdot \mathbf{q} \varphi_{2}=1$ and $\mathbf{w}^{\prime} \varphi_{2}=\mathbf{c} \varphi_{2} \cdot 2 \cdot 5^{2} \cdot 6 \cdot \mathbf{q}^{\prime} \varphi_{2}=3$, whence the contradiction $\mathbf{w} \varphi_{2} \neq \mathbf{w}^{\prime} \varphi_{2}$ is deduced.

Case 3: $\left(\mathbf{w}_{\ell}, \mathbf{w}_{\ell}^{\prime}\right)=\left(x_{i}, x_{j}\right)$ for some $i$ and $j$ with $i<j<\ell$. Then

$$
\mathbf{w}=\underbrace{\mathbf{e} x_{i} \mathbf{f} x_{j} \mathbf{g}}_{\mathbf{p}} x_{\ell} x_{i} x_{\ell+1} \mathbf{q} \quad \text { and } \quad \mathbf{w}^{\prime}=\underbrace{\mathbf{e} x_{i} \mathbf{f} x_{j} \mathbf{g}}_{\mathbf{p}} x_{\ell} x_{j} x_{\ell+1} \mathbf{q}^{\prime} \quad \text { for some } \mathbf{e}, \mathbf{f}, \mathbf{g} \in \mathcal{X}^{*}
$$

such that $x_{i} \notin \operatorname{con}(\mathbf{e}), x_{j} \notin \operatorname{con}\left(\mathbf{e} x_{i} \mathbf{f}\right), x_{\ell} \notin \operatorname{con}\left(\mathbf{e} x_{i} \mathbf{f} x_{j} \mathbf{g}\right)$, and $x_{\ell+1} \notin \operatorname{con}\left(\mathbf{e} x_{i} \mathbf{f} x_{j} \mathbf{g} x_{\ell}\right)$. If the words $\mathbf{e} x_{i} \mathbf{f}$ and $\mathbf{g} x_{\ell}$ share a common letter, then (II) is violated by the word $\mathbf{w}^{\prime}$. It follows that $\operatorname{con}\left(\mathbf{e} x_{i} \mathbf{f}\right) \cap \operatorname{con}\left(x_{j} \mathbf{g} x_{\ell}\right)=\emptyset$. There are two subcases.

3.1. $\mathbf{f} \neq \emptyset$. Then $\mathbf{f}=\mathbf{v} t$ for some $\mathbf{v} \in \mathcal{X}^{*}$ with $t=\mathrm{t}(\mathbf{f})$, whence

$$
\mathbf{w}=\underbrace{\mathbf{e} x_{i} \mathbf{v} t x_{j} \mathbf{g}}_{\mathbf{p}} x_{\ell} x_{i} x_{\ell+1} \mathbf{q} \text { and } \mathbf{w}^{\prime}=\underbrace{\mathbf{e} x_{i} \mathbf{v} t x_{j} \mathbf{g}}_{\mathbf{p}} x_{\ell} x_{j} x_{\ell+1} \mathbf{q}^{\prime} .
$$

If $t \in \operatorname{con}\left(\mathbf{e} x_{i} \mathbf{v}\right)$, then (III) is violated by $\mathbf{w}^{\prime}$. Hence the observation $\operatorname{con}\left(\mathbf{e} x_{i} \mathbf{f}\right) \cap \operatorname{con}\left(x_{j} \mathbf{g} x_{\ell}\right)=\emptyset$ from above implies $t \notin \operatorname{con}\left(\mathbf{e} x_{i} \mathbf{v} x_{j} \mathbf{g} x_{\ell}\right)$. Let $\varphi_{3}: \mathcal{X} \rightarrow S$ denote the substitution

$$
z \mapsto \begin{cases}4 & \text { if } z \in \operatorname{con}\left(\mathbf{e} x_{i} \mathbf{v}\right), \\ 2 & \text { if } z=t \\ 5 & \text { if } z \in \operatorname{con}\left(x_{j} \mathbf{g} x_{\ell}\right), \\ 6 & \text { otherwise. }\end{cases}
$$

Then the contradiction $\mathbf{w} \varphi_{3} \neq \mathbf{w}^{\prime} \varphi_{3}$ is deduced because

$$
\mathbf{w} \varphi_{3}=\left(\mathbf{e} x_{i} \mathbf{v}\right) \varphi_{3} \cdot t \varphi_{3} \cdot\left(x_{j} \mathbf{g} x_{\ell}\right) \varphi_{3} \cdot x_{i} \varphi_{3} \cdot x_{\ell+1} \varphi_{3} \cdot \mathbf{q} \varphi_{3}=4 \cdot 2 \cdot 5 \cdot 4 \cdot 6 \cdot \mathbf{q} \varphi_{3}=1
$$

and

$$
\mathbf{w}^{\prime} \varphi_{3}=\left(\mathbf{e} x_{i} \mathbf{v}\right) \varphi_{3} \cdot t \varphi_{3} \cdot\left(x_{j} \mathbf{g} x_{\ell} x_{j}\right) \varphi_{3} \cdot x_{\ell+1} \varphi_{3} \cdot \mathbf{q}^{\prime} \varphi_{3}=4 \cdot 2 \cdot 5 \cdot 6 \cdot \mathbf{q}^{\prime} \varphi_{3}=3 .
$$

3.2. $\mathbf{f}=\emptyset$. Then

$$
\mathbf{w}=\underbrace{\mathbf{e} x_{i} x_{j} \mathbf{g}}_{\mathbf{p}} x_{\ell} x_{i} x_{\ell+1} \mathbf{q} \text { and } \mathbf{w}^{\prime}=\underbrace{\mathbf{e} x_{i} x_{j} \mathbf{g}}_{\mathbf{p}} x_{\ell} x_{j} x_{\ell+1} \mathbf{q}^{\prime} .
$$

Let $\varphi_{4}: \mathcal{X} \rightarrow S$ denote the substitution

$$
z \mapsto \begin{cases}4 & \text { if } z \in \operatorname{con}(\mathbf{e}) \\ 2 & \text { if } z=x_{i} \\ 5 & \text { if } z \in \operatorname{con}\left(x_{j} \mathbf{g} x_{\ell}\right) \\ 6 & \text { otherwise. }\end{cases}
$$


Then $\mathbf{w} \varphi_{4}=\mathbf{e} \varphi_{4} \cdot 2 \cdot 5 \cdot 2 \cdot 6 \cdot \mathbf{q} \varphi_{4}=1$ and $\mathbf{w}^{\prime} \varphi_{4}=\mathbf{e} \varphi_{4} \cdot 2 \cdot 5 \cdot 6 \cdot \mathbf{q}^{\prime} \varphi_{4}=3$, whence the contradiction $\mathbf{w} \varphi_{4} \neq \mathbf{w}^{\prime} \varphi_{4}$ is deduced.

Since none of the three cases just considered is possible, the integer $\ell$ does not exist. Consequently, $\mathbf{w}_{i}=\mathbf{w}_{i}^{\prime}$ for all $i$, whence the identity $\mathbf{w} \approx \mathbf{w}^{\prime}$ is trivial and so is implied by the identities (27.2).

\subsection{Finite basis property of $\mathscr{G}_{2}$}

Proposition 27.5. The variety generated by $\mathscr{G}_{2}$ is defined by the identities

$$
\begin{aligned}
x \mathrm{H} x^{2} & \approx x \mathrm{H} x, \\
x \mathrm{H} x \mathrm{~K} x t & \approx x \mathrm{H} x \mathrm{~K} t, \\
x \mathrm{H} y \mathrm{~K} x y t & \approx x \mathrm{H} y \mathrm{~K} y x t .
\end{aligned}
$$

Lemma 27.6. Suppose that $\mathbf{w} \approx \mathbf{w}^{\prime}$ is any identity satisfied by $\mathscr{G}_{2}$. Then:

(i) $\operatorname{ini}(\mathbf{w})=\operatorname{ini}\left(\mathbf{w}^{\prime}\right)$;

(ii) $\operatorname{con}(\mathbf{w})=\operatorname{con}\left(\mathbf{w}^{\prime}\right)$ and $\operatorname{sim}(\mathbf{w})=\operatorname{sim}\left(\mathbf{w}^{\prime}\right)$;

(iii) $\mathrm{t}(\mathbf{w})=\mathrm{t}\left(\mathbf{w}^{\prime}\right)$;

(iv) $\mathrm{t}(\mathbf{w}) \in \operatorname{sim}(\mathbf{w})$ if and only if $\mathrm{t}\left(\mathbf{w}^{\prime}\right) \in \operatorname{sim}\left(\mathbf{w}^{\prime}\right)$.

Further, if $\operatorname{occ}(x, \mathbf{w}), \operatorname{occ}\left(x, \mathbf{w}^{\prime}\right) \leqslant 2$ for all $x \in \mathcal{X}$, then

(v) $\operatorname{occ}(y, z, \mathbf{w})=\operatorname{occ}\left(y, z, \mathbf{w}^{\prime}\right)$ for all $y, z \in \mathcal{X}$.

Proof. The subsemigroups $\{1,3,4\},\{1,2,4\}$, and $\{4,5\}$ of $\mathscr{G}_{2}$ are isomorphic to $L_{2}^{1}, N_{2}^{1}$, and $R_{2}$, respectively. Therefore parts (i)-(iii) follow from Lemma 2.1, and part (iv) is a consequence of parts (ii) and (iii).

(v) Suppose $\operatorname{occ}(x, \mathbf{w}), \operatorname{occ}\left(x, \mathbf{w}^{\prime}\right) \leqslant 2$ for all $x \in \mathcal{X}$. Seeking a contradiction, suppose $\operatorname{occ}(y, z, \mathbf{w})<\operatorname{occ}\left(y, z, \mathbf{w}^{\prime}\right)$ for some $y, z \in \mathcal{X}$. Let $p=\operatorname{occ}(y, z, \mathbf{w})$ and $p^{\prime}=\operatorname{occ}\left(y, z, \mathbf{w}^{\prime}\right)$. Then $0 \leqslant p<p^{\prime} \leqslant 2$ so that $\left(p, p^{\prime}\right) \in\{(0,1),(0,2),(1,2)\}$. If $p=0$, then ini(w) $\neq \operatorname{ini}\left(\mathbf{w}^{\prime}\right)$ by (ii), whence (i) is violated. Therefore $\left(p, p^{\prime}\right)=(1,2)$, so that

$$
\mathbf{w}=\mathbf{w}_{1} y \mathbf{w}_{2} z \mathbf{w}_{3} \quad \text { and } \quad \mathbf{w}^{\prime}=\mathbf{w}_{1}^{\prime} y \mathbf{w}_{2}^{\prime} y \mathbf{w}_{3}^{\prime} z \mathbf{w}_{4}^{\prime}
$$

for some $\mathbf{w}_{1}, \mathbf{w}_{2}, \mathbf{w}_{3}, \mathbf{w}_{1}^{\prime}, \mathbf{w}_{2}^{\prime}, \mathbf{w}_{3}^{\prime}, \mathbf{w}_{4}^{\prime} \in \mathcal{X}^{*}$ with $y, z \notin \operatorname{con}\left(\mathbf{w}_{1} \mathbf{w}_{2} \mathbf{w}_{1}^{\prime} \mathbf{w}_{2}^{\prime} \mathbf{w}_{3}^{\prime}\right)$. Let $\varphi: \mathcal{X} \rightarrow \mathscr{G}_{2}$ denote the substitution

$$
x \mapsto \begin{cases}2 & \text { if } x=y, \\ 6 & \text { if } x=z, \\ 4 & \text { otherwise. }\end{cases}
$$

Then $\mathbf{w} \varphi \in\{4\}^{*} \cdot 2 \cdot\{4\}^{*} \cdot 6 \cdot \mathbf{w}_{3} \varphi=\{3\}$ and $\mathbf{w}^{\prime} \varphi \in\{4\}^{*} \cdot 2 \cdot\{4\}^{*} \cdot 2 \cdot\{4\}^{*} \cdot 6 \cdot \mathbf{w}_{4}^{\prime} \varphi=\{1\}$, so the contradiction $\mathbf{w} \varphi \neq \mathbf{w}^{\prime} \varphi$ is deduced.

A word $\mathbf{w}$ with $\mathrm{t}(\mathbf{w}) \in \operatorname{sim}(\mathbf{w})$ is said to be simple-tailed. An identity $\mathbf{w} \approx \mathbf{w}^{\prime}$ is simple-tailed if $\mathbf{w}$ and $\mathbf{w}^{\prime}$ are simple-tailed words.

Lemma 27.7. The variety generated by $\mathscr{G}_{2}$ is defined by the identities (27.3) and some set of simple-tailed identities.

Proof. Let $\mathbf{V}$ denote the variety generated by $\mathscr{G}_{2}$. It is routinely checked that $\mathscr{G}_{2}$ satisfies the identities (27.3), so that $\mathbf{V}$ is defined by (27.3) and some set $\Sigma$ of identities. Suppose that $\sigma: \mathbf{w} \approx \mathbf{w}^{\prime}$ is any identity from $\Sigma$ that is not simple-tailed. Then Lemma 27.6 implies $\mathrm{t}(\mathbf{w})=\mathrm{t}\left(\mathbf{w}^{\prime}\right)=t$ and $t \notin \operatorname{sim}(\mathbf{w})=\operatorname{sim}\left(\mathbf{w}^{\prime}\right)$. Hence $\mathbf{w}=\mathbf{w}_{1} t \mathbf{w}_{2} t$ and $\mathbf{w}^{\prime}=\mathbf{w}_{1}^{\prime} t \mathbf{w}_{2}^{\prime} t$ for some 
$\mathbf{w}_{1}, \mathbf{w}_{2}, \mathbf{w}_{1}^{\prime}, \mathbf{w}_{2}^{\prime} \in \mathcal{X}^{*}$. Choose any letter $z \notin \operatorname{con}(\mathbf{w})=\operatorname{con}\left(\mathbf{w}^{\prime}\right)$. Then the identity $\sigma$ clearly implies the simple-tailed identity $\widetilde{\sigma}: \mathbf{w} z \approx \mathbf{w}^{\prime} z$. Conversely, since

$$
\mathbf{w} \stackrel{(27.3 \mathrm{a})}{\approx} \mathbf{w} t \stackrel{\widetilde{\sigma}}{\approx} \mathbf{w}^{\prime} t \stackrel{(27.3 \mathrm{a})}{\approx} \mathbf{w}^{\prime}
$$

the identities $\{(27.3), \tilde{\sigma}\}$ imply the identity $\sigma$. It follows that if $\sigma$ in $\Sigma$ is replaced by the simple-tailed identity $\widetilde{\sigma}$, then the resulting set, together with (27.3), is still a basis for $\mathbf{V}$.

The same argument can be repeated to replace any non-simple-tailed identity $\sigma$ in $\Sigma$ with a simple-tailed identity $\widetilde{\sigma}$. The resulting set $\widetilde{\Sigma}$ consists of simple-tailed identities; these identities, together with (27.3), constitute a basis for $\mathbf{V}$.

In this subsection, a simple-tailed word $\mathbf{w}$ with $\operatorname{ini}(\mathbf{w})=x_{1} \ldots x_{m} t$ and $t \in \operatorname{sim}(\mathbf{w})$ is said to be in canonical form if

$$
\mathbf{w}=\left(\prod_{i=1}^{m}\left(x_{i} \mathbf{w}_{i}\right)\right) t
$$

where:

(I) $\operatorname{occ}\left(x_{i}, \mathbf{w}\right) \leqslant 2$;

(II) $\mathbf{w}_{i} \in\left\{x_{1}^{e_{1}} \ldots x_{i}^{e_{i}} \mid e_{1}, \ldots, e_{i} \in\{0,1\}\right\}$

(III) $\mathbf{w}_{1}, \ldots, \mathbf{w}_{m}$ are pairwise disjoint.

LEMma 27.8. Let $\mathbf{w}$ be any simple-tailed word. Then there exists some simple-tailed word $\overline{\mathbf{w}}$ in canonical form such that the identities (27.3) imply the identity $\mathbf{w} \approx \overline{\mathbf{w}}$.

Proof. Let $\mathbf{w}$ be any simple-tailed word with ini(w) $=x_{1} \ldots x_{m} t$. Then it suffices to convert $\mathbf{w}$, using the identities (27.3), into a simple-tailed word in canonical form. It is easily shown that $\mathbf{w}$ can be written in the form (27.4) with $\mathbf{w}_{i} \in\left\{x_{1}, \ldots, x_{i}\right\}^{*}$ for all $i$. For each $i$, since the letters of $\mathbf{w}_{i}$ are non-first occurrences, the identities (27.3c) can be used to arrange them within $\mathbf{w}_{i}$ in any order, whence $\mathbf{w}_{i} \in\left\{x_{1}^{e_{1}} \ldots x_{i}^{e_{i}} \mid e_{1}, \ldots, e_{i} \geqslant 0\right\}$. Any $x_{j}$ that is neither a first nor a second occurrence can be eliminated by the identities $(27.3 \mathrm{~b})$, so that (I) is satisfied. It is then easily seen that (II) and (III) are also satisfied.

Proof of Proposition 27.5. By Lemma 27.7, the variety generated by $\mathscr{G}_{2}$ is defined by the identities (27.3) and some set $\Sigma$ of simple-tailed identities. By Lemma 27.8, the simple-tailed words forming the identities in $\Sigma$ can further be chosen to be in canonical form. Let $\mathbf{w} \approx \mathbf{w}^{\prime}$ be any such identity, so that by Lemma 27.6 ,

$$
\mathbf{w}=\left(\prod_{i=1}^{m}\left(x_{i} \mathbf{w}_{i}\right)\right) t \quad \text { and } \quad \mathbf{w}^{\prime}=\left(\prod_{i=1}^{m}\left(x_{i} \mathbf{w}_{i}^{\prime}\right)\right) t .
$$

Suppose that $\ell$ is the least integer such that $\operatorname{con}\left(\mathbf{w}_{\ell}\right) \neq \operatorname{con}\left(\mathbf{w}_{\ell}^{\prime}\right)$. Then by (II), there exists some $k \leqslant \ell$ such that $x_{k}$ belongs to either $\mathbf{w}_{\ell}$ or $\mathbf{w}_{\ell}^{\prime}$ but not both, say $x_{k} \in \operatorname{con}\left(\mathbf{w}_{\ell}\right) \backslash \operatorname{con}\left(\mathbf{w}_{\ell}^{\prime}\right)$. It follows from (II) and the minimality of $\ell$ that $\mathbf{w}_{i}=\mathbf{w}_{i}^{\prime}$ for all $i<\ell$. Hence

$$
\operatorname{occ}\left(x_{k}, \prod_{i=1}^{\ell-1}\left(x_{i} \mathbf{w}_{i}\right)\right)=\operatorname{occ}\left(x_{k}, \prod_{i=1}^{\ell-1}\left(x_{i} \mathbf{w}_{i}^{\prime}\right)\right) .
$$

There are two cases to consider. 
Case 1: $\ell<m$. Then

$$
\operatorname{occ}\left(x_{k}, x_{\ell+1}, \mathbf{w}\right)=\operatorname{occ}\left(x_{k}, \prod_{i=1}^{\ell-1}\left(x_{i} \mathbf{w}_{i}\right)\right)+\operatorname{occ}\left(x_{k}, x_{\ell} \mathbf{w}_{\ell}\right)
$$

and

$$
\operatorname{occ}\left(x_{k}, x_{\ell+1}, \mathbf{w}^{\prime}\right)=\operatorname{occ}\left(x_{k}, \prod_{i=1}^{\ell-1}\left(x_{i} \mathbf{w}_{i}^{\prime}\right)\right)+\operatorname{occ}\left(x_{k}, x_{\ell} \mathbf{w}_{\ell}^{\prime}\right) .
$$

But Lemma 27.6(v) and (27.5) imply $\operatorname{occ}\left(x_{k}, x_{\ell} \mathbf{w}_{\ell}\right)=\operatorname{occ}\left(x_{k}, x_{\ell} \mathbf{w}_{\ell}^{\prime}\right)$, and this is impossible because $x_{k} \in \operatorname{con}\left(\mathbf{w}_{\ell}\right) \backslash \operatorname{con}\left(\mathbf{w}_{\ell}^{\prime}\right)$.

Case 2: $\ell=m$. Then

$$
\operatorname{occ}\left(x_{k}, \mathbf{w}\right)=\operatorname{occ}\left(x_{k}, \prod_{i=1}^{m-1}\left(x_{i} \mathbf{w}_{i}\right)\right)+\operatorname{occ}\left(x_{k}, x_{m} \mathbf{w}_{m}\right)
$$

and

$$
\operatorname{occ}\left(x_{k}, \mathbf{w}^{\prime}\right)=\operatorname{occ}\left(x_{k}, \prod_{i=1}^{m-1}\left(x_{i} \mathbf{w}_{i}^{\prime}\right)\right)+\operatorname{occ}\left(x_{k}, x_{m} \mathbf{w}_{m}^{\prime}\right) .
$$

But Lemma 27.6(ii), (I), and (27.5) imply $\operatorname{occ}\left(x_{k}, x_{m} \mathbf{w}_{m}\right)=\operatorname{occ}\left(x_{k}, x_{m} \mathbf{w}_{m}^{\prime}\right)$, and this is impossible because $x_{k} \in \operatorname{con}\left(\mathbf{w}_{m}\right) \backslash \operatorname{con}\left(\mathbf{w}_{m}^{\prime}\right)$.

Neither of the two cases just considered is possible. Therefore the integer $\ell$ does not exist, whence $\operatorname{con}\left(\mathbf{w}_{i}\right)=\operatorname{con}\left(\mathbf{w}_{i}^{\prime}\right)$ for all $i$. It follows from (II) that $\mathbf{w}_{i}=\mathbf{w}_{i}^{\prime}$ for all $i$. The identity $\mathbf{w} \approx \mathbf{w}^{\prime}$ is thus trivial. Since the identity $\mathbf{w} \approx \mathbf{w}^{\prime}$ is arbitrary in $\Sigma$, every identity in $\Sigma$ is trivial. Consequently, the variety generated by $\mathscr{G}_{2}$ is defined by the identities (27.3).

\section{References}

1. J. Almeida, Finite semigroups and universal algebra (World Scientific, Singapore, 1994).

2. Yu. A. Bahturin and A. Yu. Ol'šAnskil̆, 'Identical relations in finite Lie rings', Math. USSR-Sb. 25 (1975) 507-523 (Engl. transl. of Mat. Sb. 96 (1975) no. 138, 543-559).

3. G. Birkhoff, 'On the structure of abstract algebras', Proc. Cambridge Philos. Soc. 31 (1935) $433-454$.

4. A. D. Bol'вот, 'Finite basing of identities of four-element semigroups', Sib. Math. J. 20 (1979) no. 2, 323.

5. S. Burris and H. P. Sankappanavar, A course in universal algebra (Springer, New York, 1981).

6. A. Distler and T. W. Kelsey, 'The monoids of orders eight, nine \& ten', Ann. Math. Artif. Intell. 56 (2009) 3-25.

7. A. Distler and J. D. Mitchell, 'Smallsemi-a GAP package, version 0.6.2', 2010, http://www.gap-system.org/Packages/smallsemi.html.

8. C. C. Edmunds, 'Varieties generated by semigroups of order four', Semigroup Forum 21 (1980) 67-81.

9. C. C. Edmunds, E. W. H. LeE and K. W. K. LeE, 'Small semigroups generating varieties with continuum many subvarieties', Order 27 (2010) 83-100.

10. È. A. Golubov and M. V. SAPIR, 'Varieties of finitely approximable semigroups', Soviet Math. (Iz. VUZ) 26 (1982) no. 11, 25-36 (Engl. transl. of Izv. Vyssh. Uchebn. Zaved. Mat. (1982) no. 11, 21-29).

11. J. JEŽEK, 'Nonfinitely based three-element idempotent groupoids', Algebra Universalis 20 (1985) $292-301$.

12. J. KARnofsky, 'Finite equational bases for semigroups', Notices Amer. Math. Soc. 17 (1970) 813-814.

13. R. L. KRuse, 'Identities satisfied by a finite ring', J. Algebra 26 (1973) 298-318.

14. E. W. H. LeE, 'Identity bases for some non-exact varieties', Semigroup Forum 68 (2004) 445-457.

15. E. W. H. LEE, 'On identity bases of exclusion varieties for monoids', Comm. Algebra 35 (2007) 2275-2280.

16. E. W. H. LEE, 'Combinatorial Rees-Sushkevich varieties are finitely based', Internat. J. Algebra Comput. 18 (2008) 957-978.

17. E. W. H. LeE, 'Finite basis problem for 2-testable monoids', Cent. Eur. J. Math. 9 (2011) 1-22. 
18. E. W. H. LEE, 'Finite basis problem for semigroups of order five or less: generalization and revisitation', Studia Logica 101 (2013) 95-115.

19. E. W. H. LeE and J. R. Li, 'Minimal non-finitely based monoids', Dissertationes Math. (Rozprawy Mat.) 475 (2011) 3-65.

20. E. W. H. Lee, J. R. Li and W. T. Zhang, 'Minimal non-finitely based semigroups', Semigroup Forum 85 (2012) 577-580

21. E. W. H. LeE and M. V. Volkov, 'On the structure of the lattice of combinatorial Rees-Sushkevich varieties', Semigroups and formal languages, Proceedings of the International Conference, Lisboa, 2005 (eds J. M. André, V. H. Fernandes, M. J. J. Branco, G. M. S. Gomes, J. Fountain and J. C. Meakin; World Scientific, Singapore, 2007) 164-187.

22. E. W. H. LEE and M. V. VOLKOV, 'Limit varieties generated by completely 0-simple semigroups', Internat. J. Algebra. Comput. 21 (2011) 257-294.

23. Y. F. Luo and W. T. ZHANG, 'On the variety generated by all semigroups of order three', J. Algebra 334 (2011) 1-30.

24. I. V. L'vov, 'Varieties of associative rings. I', Algbra. Logic 12 (1973) 150-167 (Engl. transl. of Algebra i Logika 12 (1973) 269-297).

25. R. C. Lyndon, 'Identities in two-valued calculi', Trans. Amer. Math. Soc. 71 (1951) 457-465.

26. R. C. Lyndon, 'Identities in finite algebras', Proc. Amer. Math. Soc. 5 (1954) 8-9.

27. G. I. MashevitskiĬ, 'An example of a finite semigroup without an irreducible basis of identities in the class of completely 0-simple semigroups', Russian Math. Surveys 38 (1983) no. 2, 192-193 (Engl. transl. of Uspekhi Mat. Nauk 38 (1983) no. 2, 211-212).

28. R. McKenzie, 'Equational bases for lattice theories', Math. Scand. 27 (1970) 24-38.

29. V. L. MurskiI, 'The existence in the three-valued logic of a closed class with a finite basis, having no finite complete system of identities', Soviet Math. Dokl. 6 (1965) 1020-1024 (Engl. transl. of Dokl. Akad. Nauk SSSR 163 (1965) 815-818).

30. S. OAtes and M. B. Powell, 'Identical relations in finite groups', J. Algebra 1 (1964) 11-39.

31. P. Perkins, 'Bases for equational theories of semigroups', J. Algebra 11 (1969) 298-314.

32. M. Petrich and N. R. Reilly, Completely regular semigroups (Wiley \& Sons, New York, 1999).

33. R. J. Plemmons, 'There are 15973 semigroups of order 6', Math. Alg. 2 (1967) 2-17.

34. G. Pollák, 'On two classes of hereditarily finitely based semigroup identities', Semigroup Forum 25 (1982) 9-33.

35. G. Pollák, 'Some sufficient conditions for hereditarily finitely based varieties of semigroups', Acta Sci. Math. (Szeged) 50 (1986) no. 3-4, 299-330.

36. G. Pollák and M. V. Volkov, 'On almost simple semigroup identities', Colloq. Math. Soc. János Bolyai 39 (North-Holland, Amsterdam, 1985) 287-323.

37. V. V. RAsin, 'Varieties of orthodox Clifford semigroups', Soviet Math. (Iz. VUZ) 26 (1982) no. 11, 107-110 (Engl. transl. of Izv. Vyssh. Uchebn. Zaved. Mat. (1982) no. 11, 82-85).

38. M. V. SAPIR, 'Problems of Burnside type and the finite basis property in varieties of semigroups', Math. USSR-Izv. 30 (1988) 295-314 (Engl. transl. of Izv. Akad. Nauk SSSR Ser. Mat. 51 (1987) 319-340).

39. L. N. Shevrin and M. V. Volkov, 'Identities of semigroups', Soviet Math. (Iz. VUZ) 29 (1985) no. 11, 1-64 (Engl. transl. of Izv. Vyssh. Uchebn. Zaved. Mat. (1985) no. 11, 3-47).

40. A. TARSKI, 'Equational logic and equational theories of algebras', Contributions to mathematical logic, Proceedings of the Logic Colloquium, Hannover, 1966 (eds H. A. Schmidt, K. Schütte and H. J. Thiele; North-Holland, Amsterdam, 1968) 275-288.

41. A. V. Tishchenko, 'The finiteness of a base of identities for five-element monoids', Semigroup Forum 20 (1980) 171-186.

42. A. N. Trahtman, 'A basis of identities of the five-element Brandt semigroup', Ural. Gos. Univ. Mat. Zap. 12 (1981) no. 3, 147-149 (in Russian).

43. A. N. Trahtman, 'The finite basis question for semigroups of order less than six', Semigroup Forum 27 (1983) 387-389.

44. A. N. Trahtman, 'Some finite infinitely basable semigroups', Ural. Gos. Univ. Mat. Zap. 14 (1987) no. 2, 128-131 (in Russian).

45. A. N. Trahtman, 'Finiteness of identity bases of five-element semigroups', Semigroups and their homomorphisms (ed. E. S. Lyapin; Ross. Gos. Ped. Univ., Leningrad, 1991) 76-97 (in Russian).

46. V. V. VIŠın, 'Identity transformations in a four-valued logic', Soviet Math. Dokl. 4 (1963) 724-726 (Engl transl. of Dokl. Akad. Nauk SSSR 150 (1963) 719-721).

47. M. V. Volkov, 'The finite basis question for varieties of semigroups', Math. Notes 45 (1989) no. 3, 187-194 (Engl. transl. of Mat. Zametki 45 (1989) no. 3, 12-23).

48. M. V. VOLKOV, "Forbidden divisor" characterizations of epigroups with certain properties of group elements', RIMS Kôkyûroku Bessatsu 1166 (2000) 226-234.

49. M. V. Volkov, 'The finite basis problem for finite semigroups', Sci. Math. Jpn. 53 (2001) 171-199.

50. W. T. Zhang and Y. F. Luo, 'A new example of a minimal non-finitely based semigroup', Bull. Aust. Math. Soc. 84 (2011) 484-491. 
Edmond W. H. Lee

Division of Math, Science, and Technology

Nova Southeastern University

Fort Lauderdale, FL 33314

USA

edmond.lee@nova.edu
Wen Ting Zhang

School of Mathematics and Statistics

Lanzhou University

Lanzhou, Gansu 730000

PR China

and

Key Laboratory of Applied Mathematics and Complex Systems

Lanzhou, Gansu 730000

PR China

zhangwt@lzu.edu.cn 